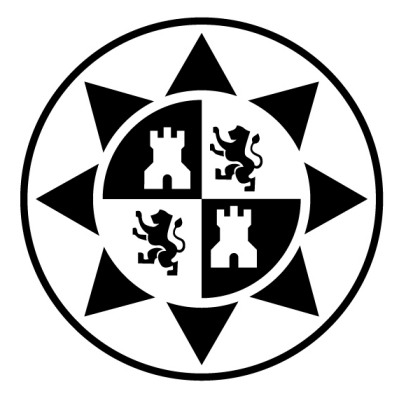

Universidad Politécnica de Cartagena Departamento de Ingeniería Térmica y de Fluidos

\title{
Comportamiento
}

de fluidos no newtonianos en

intercambiadores de calor tubulares

con rascador alternativo

\author{
Damián Crespí Llorens
}

2015 



\title{
TESIS DOCTORAL

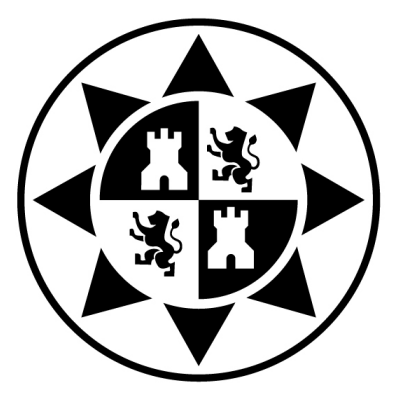

Universidad Politécnica de Cartagena

Departamento de Ingeniería Térmica y de Fluidos

\section{Comportamiento \\ de fluidos no newtonianos en \\ intercambiadores de calor tubulares \\ con rascador alternativo \\ Damián Crespí Llorens}

\author{
Dirigida por \\ Dr. Antonio Viedma Robles \\ Dr. Pedro Ginés Vicente Quiles
}

Presentada para la obtención del grado de

Doctor en el programa de Tecnologías Industriales

Cartagena, enero de 2015 



\section{CONFORMIDAD DE SOLICITUD DEAUTORIZACIÓN DE DEPÓSITO DE TESIS DOCTORAL POR EL/LA DIRECTOR/A DE LA TESIS}

D./D". Antonio Viedma Robles, Director de la Tesis doctoral "Comportamiento de fluidos no newtonianos en intercambiadores de calor tubulares con rascador alternativo".

\section{INFORMA:}

Que la referida Tesis Doctoral, ha sido realizada por D. Damián Crespí Llorens, dentro del programa de doctorado RD 778/1998 de "Tecnologías Industriales" (bienio 2006-2008), dando mi conformidad para que sea presentada ante la Comisión de Doctorado para ser autorizado su depósito.

La rama de conocimiento en la que esta tesis ha sido desarrollada es:

Ciencias

$\square \quad$ Ciencias Sociales y Jurídicas

X Ingeniería y Arquitectura

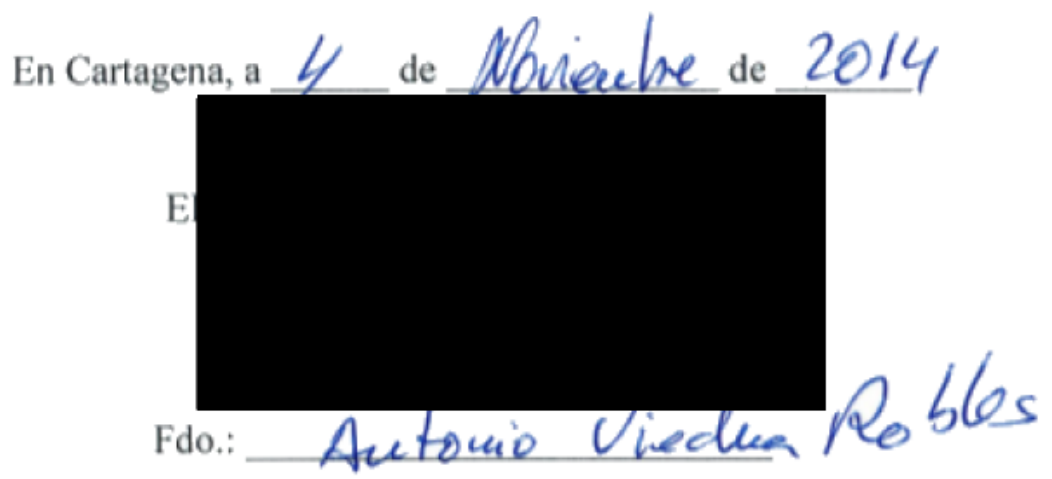




\section{Universidad

\section{CONFORMIDAD DE SOLICITUD DEAUTORIZACIÓN DE DEPÓSITO DE}

TESIS DOCTORAL POR EL/LA DIRECTOR/A DE LA TESIS

D./Dn. Pedro Ginés Vicente Quiles, Profesor Doctor del Area de Máquimas y Motores Térmicos en el Departamento de Ingeniería Mecánica y Energía de la Universidad Miguel Hernández y Director de la Tesis doctoral "Comportamiento de fluidos no newtonianos en intercambiadores de calor tubulares con rascador alternativo".

\section{INFORMA:}

Que la referida Tesis Doctoral, ha sido realizada por D. Damián Crespi Llorens, dentro del programa de doctorado RD 778/1998 de "Tecnologias Industriales" (bienio 2006-2008). dando mi conformidad para que sea presentada ante la Comisión de Doctorado para ser autorizado su depósito.

La rama de conocimiento en la que esta tesis ha sido desarrollada es:

Ciencias

Ciencias Sociales y Jurídicas

$X$ Ingenieria y Arquitectura

En Cartagena, a 4, de Nouremane de $20 / 4$

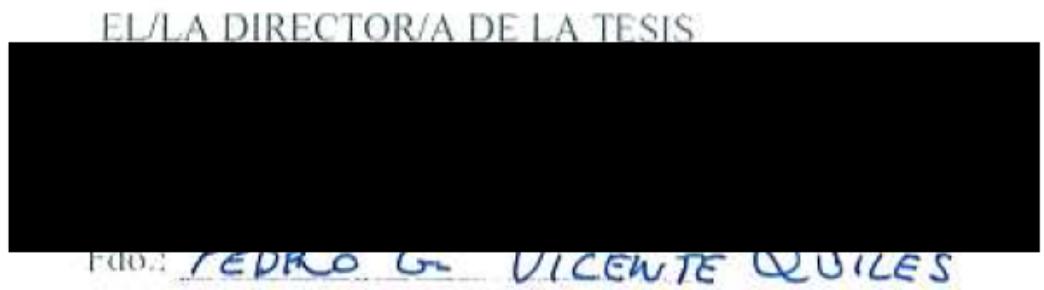




\section{Universidad}

Politécnica

de Cartagena

\section{CONFORMIDAD DE DEPÓSITO DE TESIS DOCTORAL POR LA COMISIÓN ACADÉMICA DEL PROGRAMA}

D. JUAN SUARDIAZ MURO, Presidente/a de la Comisión Académica del Programa de Doctorado de Tecnologias Industriales (RD 778/1998, bienio 2006/08).

\section{INFORMA:}

Que la Tesis Doctoral titulada, "Comportamiento de fluidos no newtonianos en intercambiadores de calor tubulares con rascador alternativo", ha sido realizada, dentro del mencionado programa de doctorado, por D/D". Damián Crespi Llorens,

bajo la dirección y supervisión del Dr. Antonio Viedma Robles y del Dr. Pedro Ginés Vicente Quiles.

En reunión de la Comisión Académica de fecha 31 de octubre de 2014, visto que en la misma se acreditan los indicios de calidad correspondientes y la autorización del Director de la misma, se acordó dar la conformidad, con la finalidad de que sea autorizado su depósito por la Comisión de Doctorado.

La Rama de conocimiento por la que esta tesis ha sido desarrollada es:

$\square$ Ciencias

$\square$ Ciencias Sociales y Jurídicas

凶Ingeniería y Arquitectura

En Cartagena, a 05 de noviembre de 2014

EL PRESIDENTE DE LA COMISIÓN ACADÉMICA DEL PROGRAMA

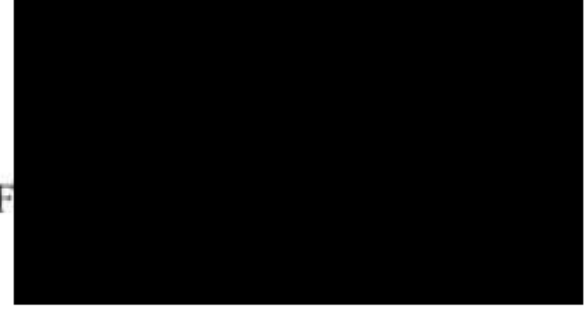

COMISIÓN DE DOCTORADO 



\section{AGRADECIMIENTOS}

A mis directores de Tesis, que tan sabiamente han orientado la investigación, que tanto me han enseñado, y a los que también agradezco su apoyo en los aspectos más cotidianos del trayecto.

A Juan Pedro Solano, por su desinteresada ayuda con las instalaciones experimentales y por sus consejos sobre la investigación y sus divertidas anécdotas sobre la vida.

A Isaac Ballesta Galdeano, por sus instimables aportaciones en cuanto a la visualización del flujo.

A David S. Martínez por las horas de vuelo juntos, por su ayuda con la indomable instalación de ensayos termohidráulicos. Suerte.

A mis padres, que se han esforzado por mi desarrollo profesional y personal. Que me lo han dado todo sin pedir nada a cambio y han sido el mayor refuerzo a lo largo de mi vida. A mi hermana, compañera de viaje durante tantos años. De ella he aprendido a luchar y perseverar cuando peor están las cosas.

A mis abuelas Paquita y Esperanza y a mi tio Pepe, que ya no están aquí y a los que echo de menos y seguro me habrían ayudado mucho estos años.

A mis suegros que me han ofrecido más ayuda de la que podría imaginar y, sobre todo, su afecto. Cuando creía que ya no tenía abuelos, allí aparecieron los yayos, que me acogieron y animaron como si fuese su propio nieto. Gracias José y María.

A mi mujer Mónica, el amor de mi vida, que me ha apoyado en los peores momentos y que tanto ha sufrido mis ausencias. Imposible lograrlo sin ti. Gracias por todos estos felices años y por los que vendrán.

Para mi hijo Damián no tengo palabras. Siempre recordaré los peores días en los que llegaba abatido a casa, hundido, entraba por la puerta y tú sonreías. Mis problemas volaban como el viento, desaparecían como si nunca hubieran existido. Tan pequeño y tan poderoso. También tú eres el amor de mi vida y siempre lo serás, incluso en los peores momentos (recuérdalo). Gracias hijo, también tú me has ayudado a publicar este libro. 



\title{
Non NeWTONian Fluid BehaViour in ENHANCED PIPE HEAT EXCHANGERS WITH A RECIPROCATING SCRAPING DEVICE
}

\begin{abstract}
This work shows a detailed study of the therm-hydraulic behaviour of a smooth pipe with an inserted reciprocating scraper, the working fluid having a non-Newtonian behaviour. A mainly experimental methodology has been followed. For that, it was necessary to obtain the fluid rheological properties. Experimental equations for the friction factor and Nusselt numbers as a function of the involved dimensionless numbers have been proposed. Moreover, visualization experiments in the laminar region of the flow have been carried out. This last information will help to explain fluid behaviour in terms of pressure drop and heat transfer in this region.

In order to explain the non Newtonian fluid behaviour inside the device, the generalization method of the viscosity proposed in the bibliography has been used as a start point. The method is only valid for ducts of constant cross-section, therefore it has been modified for its use in the geometry under study. The generalization method permits us to define the generalized Reynolds number and the generalized Prandtl number. The main advantage of this method is the reduction of one degree of freedom of the hydraulic and thermal problems, which helps simplifying the analysis of the flow.

Firstly the flow has been studied while the insert device is static. Experiments have been performed with the Reynolds number ranging from 0.2 to 600. In pressure drop experiments, three regions of the flow have been observed: laminar region for Reynolds number under 100, transitional region and turbulent one, for Reynolds numbers over 300. In heat transfer experiments two laminar regions have been observed, for Reynolds numbers lower than 4 or for higher values. Transition appears for Reynolds numbers greater than 40 and the turbulent flow shows up for Reynolds numbers greater than 70. This early transition to turbulence results in significant heat enhancement comparing two a situation without the scrapper. The flow pattern in the laminar region has been obtained for a variety of flow conditions by using PIV technique (Particle Image Velocimetry). The exact influence of the pseudoplastic behaviour in the flow pattern could not be quantified due to the several degrees of freedom of the problem. This emphasizes the importance of the generalization method developed which reduces the degrees of freedom.
\end{abstract}


Moreover an the effect of the movement of the scrapper in the problem has been studied. For that, the flow pattern, pressure drop, power demand of the scrapper piston and heat transfer coefficients have been studied as a function of the non-dimensional scrapping velocity. The operation of the scrapper makes the transition region to appear at lower Reynolds numbers and augments the Nusselt number for the same Reynolds number. This effect becomes stronger as the scrapping velocity raises.

The evaluation of the performance of the insert device through the classical criterion of Bergles, shows significant improvements when comparing with a situation of pure forced fully developed convection in a pipe. The operation of the scrapper can improve performance depending on the Reynolds number working range. For high scraping velocities, the consumed power is two high and the system becomes energetically inefficient. Besides, if the thermal entrance length is comparable to the pipe length in the reference geometry, the improvements of the scrapper are not so obvious and the range of operation must be selected with care. However, the study does not take into account the fouling effect.

To conclude, it can be manifested that the convenience of using the insert device in an industrial process should be evaluated in each case. It will depend on the flow characteristics, the admissible fouling level (if any), the required hygiene and the possible alternatives. Thus, the present document provides the necessary tools to evaluate this convenience: friction factors, Nusselt number, scrapper operation power in a wide range of working conditions. Furthermore, the operation of the scrapper is only recommended to eliminate fouling, at low velocity if possible, or to improve heat transfer in certain conditions knowing that power consumption will increase. 


\section{Comportamiento De Fluidos nO NEWTONianos EN INTERCAMBIADORES DE CALOR TUBULARES CON RASCADOR ALTERNATIVO}

\section{Resumen}

La presente tesis doctoral muestra un estudio detallado del comportamiento termohidráulico de un rascador con movimiento alternativo insertado en un tubo liso, teniendo el fluido de trabajo un comportamiento no newtoniano. La metodología de trabajo ha sido principalmente experimental, donde ha sido necesario caracterizar reológicamente el fluido. El factor de fricción y el número de Nusselt han sido correlacionados en función de los números adimensionales de los que depende el problema. Además se han realizado estudios de visualización del flujo en la región laminar, lo cual sirve de base para la explicación de los efectos de pérdida de presión y transmisión de calor en dicha región.

Para la caracterización del comportamiento no newtoniano del fluido se ha partido del método de generalización de la viscosidad existente en la bibliografía y aplicable a geometrías con sección transversal constante. El método ha sido modificado para poder ser aplicado a la geometría estudiada, lo cual permite la definición de los números de Reynolds y Prandtl generalizados. La ventaja de este método consiste en la reducción los grados de libertad en los problemas fluidomecánico y térmico, lo que simplifica el análisis del flujo.

En primer lugar se ha estudiado el flujo en régimen de rascador estático. Los ensayos realizados abarcan el rango de número de Reynolds generalizado entre 0,2 y 600. En los ensayos de pérdida de presión se observan 3 regiones de comportamiento del flujo: la región laminar para Reynolds por debajo de 100, la región de transición y la turbulenta, que aparece para Reynolds mayores de 300. En los ensayos de transmisión de calor se observan dos subregiones laminares, para Reynolds menores que 4 o mayores que dicho valor. En dichos ensayos la transición se adelanta a números de Reynolds mayores de 40 y aparece flujo de características turbulentas para valores por encima de 70 aproximadamente. El adelanto de la transición produce fuertes incrementos del número de Nusselt en comparación con una situación de convección forzada pura y longitud de entrada térmica despreciable en una geometría idéntica donde se retiran los tacos rascadores. Se ha obtenido el patrón de flujo en la región laminar en diferentes condiciones de funcionamiento mediante visualización utilizando 
la técnica de PIV (Velocimetría por Imágenes de Partículas). De dicho estudio no se ha podido extraer una conclusión clara acerca de la influencia del comportamiento seudoplástico en el patrón de flujo, lo cual enfatiza la importancia del método de generalización desarrollado.

Además se ha realizado un estudio del comportamiento del rascador en régimen dinámico. Para ello se han estudiado los mismos parámetros que en el caso estático, añadiendo la velocidad adimensional de rascado y la potencia de accionamiento consumida por el pistón hidráulico que impulsa al rascador. El accionamiento del rascador adelanta la transición y aumenta el número de Nusselt para un mismo número de Reynolds generalizado, siendo el efecto mayor a medida que aumenta la velocidad de rascado.

La comparativa realizada, utilizando los criterios clásicos R3 y R5, muestra mejoras significativas en la transferencia de calor o en la reducción de área de intercambio al comparar con un flujo en tubo liso de longitud infinita donde la convección es puramente forzada. El movimiento del rascador produce mejoras, dependiendo del rango de números de Reynolds y, sobre todo, para velocidades de rascado bajas, las cuales requieren menor potencia de accionamiento. Al aumentar la velocidad de rascado, los requisitos de potencia son demasiado altos y no compensan la mejora en el número de Nusselt obtenida. Además si en la geometría de referencia la longitud de entrada térmica es comparable, se endurecen los requisitos para que el criterio R3 y R5 sean favorables al tubo con rascador. No obstante estos estudios no tienen en cuenta el efecto del ensuciamiento.

De los estudios realizados se concluye que la conveniencia del uso del dispositivo estudiado debe ser estudiada en cada caso concreto, ya que depende de las características del fluido, del ensuciamiento admisible, la higiene necesaria y de las posibles alternativas. Así, en el presente documento se proporcionan las herramientas necesarias para evaluar dicha conveniencia, en forma de correlaciones experimentales para el factor de fricción, el número de Nusselt y la posible potencia de accionamiento, evaluadas en un amplio rango de condiciones de ensayo. Además, se recomienda el accionamiento del rascador únicamente en función de las necesidades de limpieza, a ser posible a baja velocidad, ya que el consumo de potencia es importante y reduce la eficiencia global del sistema. 


\section{Índice general}

\section{Introducción y metodología experimental}

1. Introducción $\quad 23$

1.1. Fluidos no newtonianos. Fundamentos . . . . . . . . . . . . . . . . 24

1.1.1. Comportamientos no newtonianos de los fluidos . . . . . . . . . 25

1.1.1.1. Fluidos independientes del tiempo o inelásticos . . . . . 26

1.1.2. Flujo laminar en tubos de fluidos seudoplásticos . . . . . . . . . . 30

1.1.3. Fluidos no newtonianos en la industria . . . . . . . . . . . . . . 32

1.2. Transferencia de calor mejorada y ensuciamiento . . . . . . . . . . 33

1.2.1. Técnicas de mejora de la transferencia de calor . . . . . . . . . . . 34

1.2.2. Ensuciamiento o fouling . . . . . . . . . . . . . . 36

1.2.3. Transferencia de calor mejorada en fluidos no newtonianos . . . . . . 39

1.3. ICSR como solución tecnológica . . . . . . . . . . . . . . . . . . 40

1.4. Objetivos de la tesis. . . . . . . . . . . . . . . . . . . . . . 42

1.5. Desarrollo de la tesis doctoral . . . . . . . . . . . . . . . . . . . 44

2. Análisis dimensional $\quad 47$

2.1. Problema fluidomecánico . . . . . . . . . . . . . . . . . . . . . . . . 48

2.1.1. Análisis dimensional del problema . . . . . . . . . . . . . . 50

2.2. Problema térmico . . . . . . . . . . . . . . . . . . . 52

2.2.1. Análisis dimensional . . . . . . . . . . . . . . . . . . 53

2.3. Problemas fluidomecánico y térmico con rascador en movimiento . . . . . . . 54

2.4. Viscosidad generalizada y método de generalización . . . . . . . . . . 55

2.4.1. Geometrías con sección transversal constante . . . . . . . . . . 56

2.5. Conclusiones . . . . . . . . . . . . . . . . . . . . . . . . . . 61 
3. Modelo numérico $\quad 63$

3.1. Flujo en tubos concéntricos. Fundamentos . . . . . . . . . . . . . . . . . 64

3.2. Modelo numérico del flujo desarrollado . . . . . . . . . . . . . . . . . 66

3.2.1. Validación del modelo . . . . . . . . . . . . . . . . . . . . 69

3.3. Modelo numérico del desarrollo del flujo . . . . . . . . . . . . . . . . . 71

3.3.1. Ecuaciones del modelo . . . . . . . . . . . . . . . . . . . . . . 72

3.3.2. Método de resolución de las ecuaciones . . . . . . . . . . . . . 75

3.3.3. Validación del modelo . . . . . . . . . . . . . . . . 76

3.3.3.1. Tubo liso . . . . . . . . . . . . . 76

3.3.3.2. Tubos concéntricos . . . . . . . . . . . . . . 83

3.4. Conclusiones . . . . . . . . . . . . . . . . . . 87

4. Instalaciones experimentales $\quad 89$

4.1. Descripción de la geometría . . . . . . . . . . . . . . . . . . . . 89

4.2. Instalación de ensayos termohidráulicos . . . . . . . . . . . . . . . . . . 91

4.3. Instalación de visualización . . . . . . . . . . . . . . . . . . . . 102

4.3.1. Técnica de PIV . . . . . . . . . . . . . . . . . . 107

5. Caracterización del fluido de trabajo 111

5.1. Reología . . . . . . . . . . . . . . . . . . 112

5.1.1. Metodología de medición de las propiedades reológicas . . . . . . . . 114

5.1.2. Variación de las propiedades reológicas del fluido . . . . . . . . . . . 117

5.1.2.1. Degradación del fluido o tixotropía . . . . . . . . . . 118

5.1.2.2. Variación de las propiedades con la temperatura . . . . . . . 120

5.2. Otras propiedades termofísicas del fluido . . . . . . . . . . . . 126

5.2.1. Densidad ......................... 128

5.3. Generalización de la geometría bajo estudio . . . . . . . . . . . . . . 130

5.3.1. Generalización basada en geometría anular . . . . . . . . . . . . . 132

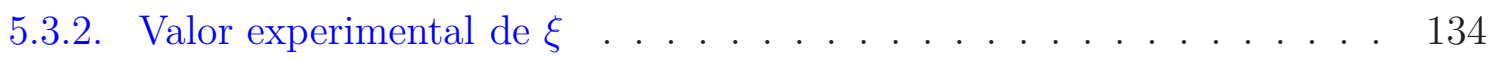

5.3.3. Generalización a partir de correlaciones experimentales . . . . . . . . 136

5.4. Conclusiones . . . . . . . . . . . . . . . . . . . . . . . 139

6. Pto. de ensayo y cálculo de incertidumbres 141

6.1. Ensayos de pérdida de presión . . . . . . . . . . . . . . . . 142

6.1.1. Oscilación del caudal . . . . . . . . . . . . . . . 144 
6.2. Ensayo de calibración de los termopares . . . . . . . . . . . . . . . . 146

6.3. Ensayos de transmisión de calor . . . . . . . . . . . . . . . . . . . . 147

6.3.1. Factor de corrección del número de Nusselt . . . . . . . . . . . . . . 151

6.4. Ensayos de visualización . . . . . . . . . . . . . . . . . . 155

6.4.1. Metodología empleada . . . . . . . . . . . . . . 155

6.4.2. Validación de la metodología . . . . . . . . . . . . . . . . . . . 161

6.5. Cálculo de incertidumbres . . . . . . . . . . . . . . . . . . . . . . 164

6.5.1. Instalación de ensayos termohidráulicos . . . . . . . . . . . . . 164

6.5.1.1. Dimensiones de la geometría . . . . . . . . . . . . . 164

6.5.1.2. Medidas de los sensores . . . . . . . . . . . . . . 165

6.5.1.3. Variables medidas indirectamente . . . . . . . . . . . . 168

6.5.1.4. Propiedades del fluido . . . . . . . . . . . . . . . . . . 169

6.5.1.5. Resultados de pérdida de presión y potencia de accionamiento 170

6.5.1.6. Resultados de transmisión de calor . . . . . . . . . . . . . . 172

6.5.2. Ensayos de visualización . . . . . . . . . . . . . . . . . 175

\section{$\begin{array}{lr}\text { II Resultados y conclusiones } & 179\end{array}$}

7. Visualización del flujo $\quad 181$

7.1. Plano de visualización del flujo . . . . . . . . . . . . . . . . 183

7.2. Régimen de rascador estático . . . . . . . . . . . . . . . . . . . 184

7.2.1. Estructura general del flujo . . . . . . . . . . . . . . . . . 184

7.2.2. Influencia del número de Reynolds y de $n$ en el flujo . . . . . . . . . . 186

7.2.3. Comparación con comportamiento newtoniano en la misma geometría 191

7.3. Régimen de rascador dinámico . . . . . . . . . . . . . . . . . . . 193

7.3.1. Ensayos a caudal cero . . . . . . . . . . . . . . 193

7.3.2. Ensayos dinámicos . . . . . . . . . . . . . . . . . . . . 195

7.3.2.1. Factor de bloqueo nulo . . . . . . . . . . . . . . . . . . . 197

7.3.2.2. Factor de bloqueo positivo . . . . . . . . . . . . . . . 200

7.3.2.3. Factor de bloqueo negativo . . . . . . . . . . . . . 209

7.4. Conclusiones . . . . . . . . . . . . . . . . . . 213

8. Pérdida de presión y potencia de accionamiento 215

8.1. Caída de presión en régimen estático . . . . . . . . . . . . . . . . . . 216 
8.1.1. Resultados de pérdida de presión . . . . . . . . . . . . . . 218

8.1.2. Efecto del comportamiento seudoplástico . . . . . . . . . . . . . . . . 221

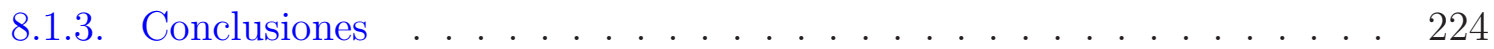

8.2. Caída de presión en régimen dinámico . . . . . . . . . . . . . . . 226

8.2.1. Selección del sistema de bombeo . . . . . . . . . . . . . . . . . . 227

8.2.2. Resultados . . . . . . . . . . . . . . . . . 228

8.2.2.1. Caída de presión en equi-/contracorriente . . . . . . . . . . 229

8.2.2.2. Caída de presión promedio . . . . . . . . . . . . . . . 236

8.2.3. Conclusiones . . . . . . . . . . . . . . . . 238

8.3. Potencia de accionamiento . . . . . . . . . . . . . . . . . . . . . . . 239

8.3.1. Contribución del pistón . . . . . . . . . . . . . . . . . . . . 240

8.3.2. Contribución del rascador . . . . . . . . . . . . . . . 240

8.3.3. Efecto del flujo en la velocidad de rascado . . . . . . . . . . . . . 242

8.4. Conclusiones . . . . . . . . . . . . . . . . . . . . . . . . . . . . . 249

9. Transmisión de calor $\quad 251$

9.1. Geometrías simples. . . . . . . . . . . . . . . . . . 252

9.1.1. Tubo liso ........................ . . . 252

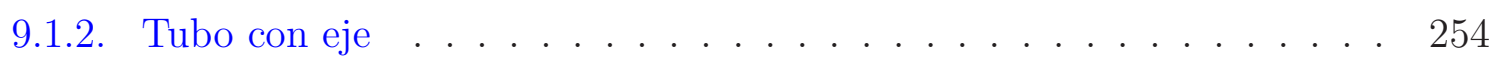

9.2. Régimen estático . . . . . . . . . . . . . . . . . . . 255

9.2.1. Resultados . . . . . . . . . . . . . . . . . 257

9.3. Régimen dinámico . . . . . . . . . . . . . . . . . . . . . 262

9.3.1. Resultados ....................... . . 264

9.4. Evaluación de las prestaciones . . . . . . . . . . . . . . . . . . . 274

9.4.1. Formulación de los criterios de comparación . . . . . . . . . . . . 276

9.4.2. Resultados en régimen estático . . . . . . . . . . . . . . . 278

9.4.2.1. Tubo con eje . . . . . . . . . . . . . . . . 278

9.4.2.2. Rascador frente a tubo liso . . . . . . . . . . . . . . . 284

9.4.3. Resultados en régimen dinámico . . . . . . . . . . . . . . . . . . . . . 284

9.5. Conclusiones . . . . . . . . . . . . . . . . . . . . . . . 292

10.Aplicación industrial y conclusiones finales 295

10.1. Resumen del cumplimiento de los objetivos . . . . . . . . . . . . 295

10.2. Aplicación industrial de los resultados obtenidos . . . . . . . . . . . . 296 
10.2.1. Consulta de los resultados de la investigación. . . . . . . . . . . . . . 297

10.3. Conclusiones finales . . . . . . . . . . . . . . . . . . . . . . . . 302

10.3.1. Descripción experimental del campo de velocidades . . . . . . . . . . 302

10.3.2. Descripción del comportamiento termohidráulico . . . . . . . . . . . . 303

10.3.3. Producción científica . . . . . . . . . . . . . . . . . . 304

\section{$\begin{array}{ll}\text { Bibliografía y Nomenclatura } & 309\end{array}$}

$\begin{array}{ll}\text { Bibliografía } & 309\end{array}$

$\begin{array}{ll}\text { Nomenclatura } & 321\end{array}$

\section{Apéndice $\quad 331$}

A. Cálculos transmisión de calor $\quad 331$

A.1. Procedimiento de cálculo de $\bar{T}_{f}\left(z_{i}\right)$ en las secciones de medida. . . . . . . . 331

A.2. Cálculo de la temperatura de pared interior . . . . . . . . . . . . . . 335

A.3. Algoritmo de Churchill y Chu . . . . . . . . . . . . . . . . . . . . . . 338

A.4. Procedimiento de cálculo para la calibración de los termopares . . . . . . . . 340

A.5. Obtención de $\Delta$ en tubo con eje . . . . . . . . . . . . . . . . . . . . . 341

A.5.1. Obtención de $\Delta \ldots \ldots \ldots$. . . . . . . . . . . . 341

A.5.2. Exponente de $\Delta \ldots \ldots$. . . . . . . . . . . . . . 343

A.6. Definición de criterios R1, R3 y R5 . . . . . . . . . . . . . . . . . . . 343

A.6.1. Tubo con eje . . . . . . . . . . . . . . . . . . . . 347

A.6.1.1. Criterio R1 . . . . . . . . . . . . . . . . 347

A.6.1.2. Criterio R3 . . . . . . . . . . . . . . . . . . 348

A.6.1.3. Criterio R5 . . . . . . . . . . . . . . . . . 348

A.6.2. Tubo liso . . . . . . . . . . . . . . . . . . 349

A.6.2.1. Criterio R1 . . . . . . . . . . . . . . . . . . 350

A.6.2.2. Criterio R3 . . . . . . . . . . . . . . . . . 350

A.6.2.3. Criterio R5 . . . . . . . . . . . . . . . . 351

A.6.3. Rascador en régimen dinámico frente a estático . . . . . . . . . . . . 351

A.6.3.1. Criterio R1 . . . . . . . . . . . . . 352

A.6.3.2. Criterio R3 . . . . . . . . . . . . . . . . . . 352 
A.6.3.3. Criterio R5 . . . . . . . . . . . . . . . . 352

B. Modelo numérico. Discretización. 355

B.1. Discretización de las ecuaciones . . . . . . . . . . . . . . . . . . . . . . 355

B.2. Importancia relativa de los términos de la ecuación de la energía . . . . . . . 357

B.3. Solución analítica del problema térmico en tubo liso . . . . . . . . . . . . . 359

$\begin{array}{ll}\text { C. Visualización. Resultados adicionales. } & 367\end{array}$

D. Transmisión de calor. Resultados adicionales. 373

D.1. Resultados adicionales de transmisión de calor . . . . . . . . . . . . . . . 373

D.2. Evaluación de Prestaciones. . . . . . . . . . . . . . . . . . . . . . . . 381 


\section{Parte I}

\section{Introducción y metodología experimental}





\section{Capítulo 1}

\section{Introducción}

Los procesos de transferencia de calor tienen una gran presencia en la industria. Los intercambiadores de calor más utilizados son los de carcasa y tubo (65\% del mercado), debido a su sencillez, solidez constructiva y a ser menos sensibles al ensuciamiento que los de placas. En la industria de procesos, el tamaño y coste de los intercambiadores de calor, así como los costes de funcionamiento debido al gasto energético y mantenimiento suponen el factor más importante a considerar de cara a optimizar la productividad. Con este objetivo, han surgido diferentes técnicas enfocadas a mejorar la eficiencia de estos intercambiadores. Las técnicas de mejora de la transferencia de calor son soluciones tecnológicas alternativas a los diseños convencionales que, aplicadas correctamente, permiten una mayor eficiencia en la transmisión de calor. Éstas técnicas han proliferado en variedad y aplicación, y han sido ampliamente estudiadas por numerosos autores, principalmente en su aplicación a procesos que utilizan fluidos newtonianos.

Sin embargo, no todos los fluidos que se procesan en la industria tienen un comportamiento newtoniano, siendo especialmente notable la presencia de fluidos con comportamiento no newtoniano en las industrias química, petroquímica, alimenticia, bioquímica y farmacéutica. Todos estos fluidos deben pasar por un proceso de intercambio de calor en alguna fase de su preparación o aplicación. Aunque existen diferentes tipos de fluidos no newtonianos, generalmente son altamente viscosos y el flujo en los intercambiadores de calor suele ser laminar, lo que implica bajos coeficientes de transferencia de calor además de una mayor tendencia al ensuciamiento. De entre todos los tipos de comportamientos no newtonianos existentes, sin duda el más común es el seudoplástico, que presenta una viscosidad que disminuye al aumentar el esfuerzo cortante al que está sometido el fluido. 
Así, los flujos de fluidos no newtonianos, y en concreto los de fluidos seudoplásticos, presentan diferencias importantes de comportamiento en comparación con los de fluidos newtonianos: campos de velocidad distintos, degradación del fluido, mayor ensuciamiento, flujos muy laminares, alta viscosidad, etc.

Por lo tanto, para un mismo diseño de intercambiador de calor, el comportamiento del flujo será distinto dependiendo de si se utiliza en el proceso un fluido newtoniano o uno no newtoniano. De este modo, es de esperar que los resultados en cuanto a transferencia de calor, pérdida de presión y potencia de accionamiento sean así mismo diferentes.

A causa de los motivos expuestos, se considera conveniente realizar la presente tesis doctoral sobre el "Comportamiento de fluidos no Newtonianos en intercambiadores de calor tubulares con rascador alternativo", donde se estudia la influencia del comportamiento seudoplástico en este tipo concreto de intercambiador de calor mejorado.

El presente capítulo está estructurado como se describe a continuación. En primer lugar se define el concepto de fluido no newtoniano, donde además se describen los tipos de comportamiento no newtoniano más significativos y se hace hincapié en el comportamiento más común, el seudoplástico. A continuación se introduce el concepto de transferencia de calor mejorada y se describen las técnicas de mejora de la transferencia de calor existentes. Se detalla el problema del ensuciamiento, su eliminación y la interacción existente entre el ensuciamiento y la eficiencia en la transmisión de calor. Seguidamente se detalla la aplicabilidad de las técnicas de transferencia de calor al trabajo con fluidos no newtonianos. Posteriormente se presentan los intercambiadores de calor de superficie rascada como solución tecnológica para el trabajo con fluidos no newtonianos. Así mismo, a lo largo del capítulo se describe el estado del arte, resaltando las publicaciones más significativas y novedosas en los ámbitos de interés para la presente investigación. Finalmente, en base al estudio del arte realizado, se identifica el campo de investigación de la tesis doctoral y se establecen los objetivos de la misma.

\subsection{Fluidos no newtonianos. Fundamentos}

En este primer apartado se definen los conceptos de fluido newtoniano y de no newtoniano y se analizan los distintos tipos de comportamientos no newtonianos existentes.

La definición de fluido se realiza en base a la diferenciación de este respecto a un sólido. Un cuerpo sólido tiene una forma definida que solo cambia cuando cambian las condiciones exteriores, mientras que un líquido no tiene una forma dada para unas mismas condiciones 
exteriores. La diferencia principal entre sólidos y líquidos viene dada por su reacción ante un esfuerzo. Al aplicar una fuerza en un sólido se produce un cambio en su forma, una deformación finita, y si la fuerza es pequeña el cambio también lo será. En un fluido, si las fuerzas se aplican apropiadamente, por ejemplo como cortadura, el cambio de forma nunca será pequeño, por pequeñas que sean las fuerzas, si estas actúan durante suficiente tiempo, pues lo que se genera es una velocidad de deformación y no una deformación finita. El líquido presenta por lo tanto resistencia a la cizalladura, pues es necesario que se le apliquen esfuerzos cortantes para que se genere una velocidad de deformación, y así si la fuerza es pequeña, la velocidad de deformación también lo será.

Según la respuesta que tengan los fluidos ante un esfuerzo cortante, estos pueden clasificarse en newtonianos o no newtonianos. La relación entre esfuerzos cortantes y velocidades de deformación se puede expresar del siguiente modo:

$$
\tau=\mu \dot{\gamma}
$$

Chhabra y Richardson (2008) definen el fluido newtoniano como aquel en el cual la relación entre esfuerzos cortantes y velocidades de deformación es lineal. Por lo tanto, en un fluido newtoniano, la viscosidad dinámica $\mu$ es una constante, independiente del valor del esfuerzo cortante $\tau$ y de la velocidad de deformación $\dot{\gamma}$. Únicamente depende del material, la temperatura y la presión. Para este tipo de fluidos, si se representa en una gráfica el esfuerzo frente a la velocidad de deformación (lo que se conoce como diagrama de flujo o reograma), se tienen representaciones de una recta cuya pendiente es la viscosidad dinámica.

Todo aquel fluido que no cumple las condiciones para ser un fluido newtoniano, es no newtoniano. Lo será aquel fluido cuyo reograma es no-lineal o no pasa por el origen, es decir, aquel cuya viscosidad aparente no es constante para unas condiciones dadas de presión y temperatura, y depende de condiciones de flujo tales como su geometría y el valor del esfuerzo cortante e incluso de la historia cinemática del fluido.

\subsubsection{Comportamientos no newtonianos de los fluidos}

En los fluidos existen diferentes tipos de comportamientos no newtonianos que se pueden presentar. Chhabra y Richardson (2008) realizan una primera clasificación de los comportamientos no newtonianos a partir de su respuesta temporal, distinguiendo tres categorías:

1. Fluidos independientes del tiempo, puramente viscosos o inelásticos. Para los fluidos 
clasificados en esta categoría, la viscosidad en cualquier instante depende únicamente del esfuerzo cortante en dicho instante.

2. Fluidos dependientes del tiempo. La viscosidad del fluido depende, además del valor del esfuerzo aplicado, de la duración del mismo y su historia cinemática.

3. Fluidos viscoelásticos. Estos fluidos muestran una recuperación parcial elástica tras el cese del esfuerzo cortante.

A su vez, en cada una de las tres categorías se engloban diferentes comportamientos. Para la presente investigación únicamente resultan de interés los comportamientos independientes del tiempo, los cuales se describen en el siguiente apartado.

Es importante constatar que los fluidos reales tendrán a menudo un comportamiento que será combinación de dos o tres comportamientos no newtonianos, aunque siempre se podrá identificar uno de ellos como dominante.

\subsubsection{Fluidos independientes del tiempo o inelásticos}

El comportamiento de este tipo de fluidos ante esfuerzos cortantes puede describirse por una relación simple del tipo:

$$
\dot{\gamma}=\Psi(\tau)
$$

Así, dependiendo de la forma que adopte la función $\Psi$ los fluidos independientes del tiempo se puede clasificar en tres subgrupos:

1. Dilatantes (Shear-thickening). La viscosidad aparente del fluido aumenta al hacerlo el esfuerzo cortante. Es el comportamiento opuesto al de los fluidos seudoplásticos.

2. Seudoplásticos (Shear-thinning). La viscosidad aparente del fluido disminuye al aumentar el esfuerzo cortante al que están sometidos.

3. Visco-plásticos o fluidos tipo Bingham. Estos fluidos se caracterizan por un valor límite de esfuerzo tangencial (yield stress), que debe excederse para que el material comience a fluir.

En la Fig. 1.1 se muestran los reogramas para distintos tipos de fluidos independientes del tiempo. En el reograma, la viscosidad es la pendiente de la recta que une el origen con cada 


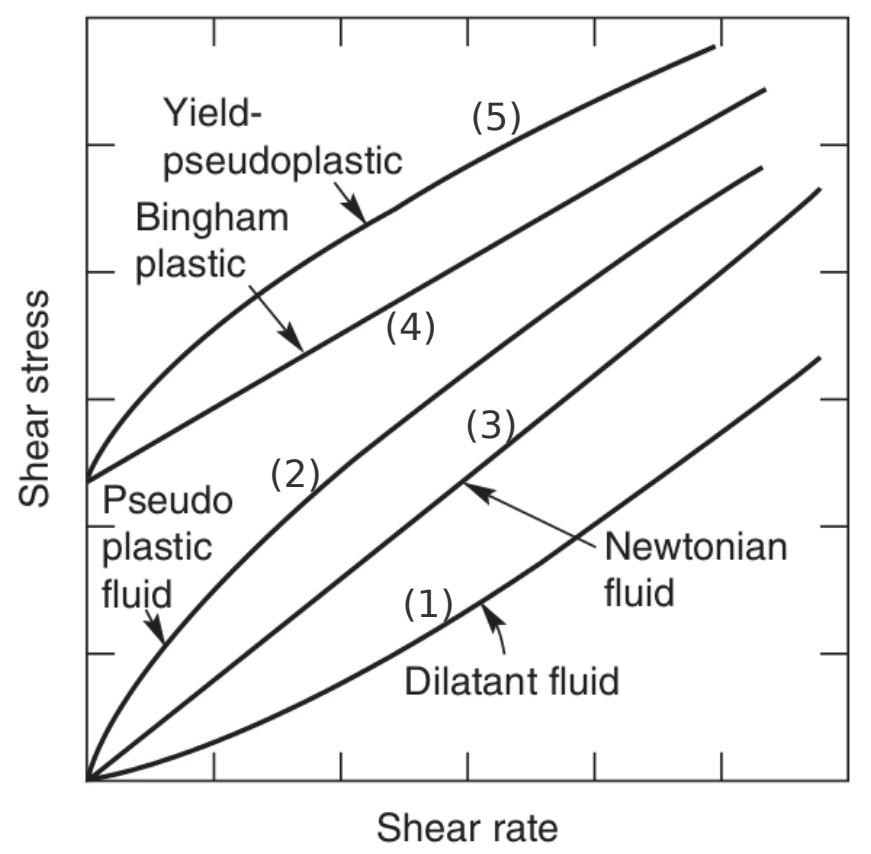

Figura 1.1: Tipos de comportamiento de flujos independientes del tiempo (Chhabra y Richardson, 2008).

punto de la curva de esfuerzo-velocidad de deformación. Se observa como en los comportamientos dilatante (1) la viscosidad aumenta con el esfuerzo cortante y en el seudoplástico (2) disminuye con $\tau$, mientras que en un fluido newtoniano (3), la viscosidad no es función del esfuerzo cortante y por tanto su reograma es una línea recta. En el comportamiento púramente visco-plástico (4), también llamado fluido Bingham, debe existir un esfuerzo mínimo para que el material fluya, de modo que para valores inferiores a dicho esfuerzo la viscosidad no está definida o sería infinita y para esfuerzos mayores la viscosidad es constante. Ahora bien, estos diferentes comportamientos pueden aparecer combinados en los fluidos reales, por ejemplo en la gráfica se presenta un fluido con comportamiento combinado visco-plástico y seudoplástico (5).

\section{Comportamiento Seudoplástico}

Tal y como se ha comentado, la seudoplasticidad es el tipo más común de comportamiento no newtoniano. Se traduce en una viscosidad aparente que disminuye al aumentar el esfuerzo cortante (véase Fig. 1.1). No obstante, para esfuerzos de cortadura muy bajos o muy elevados, la mayoría de los fluidos seudoplásticos exhiben un comportamiento newtoniano: a valores altos o bajos del esfuerzo cortante, la curva del reograma se hace una recta que pasa por el 


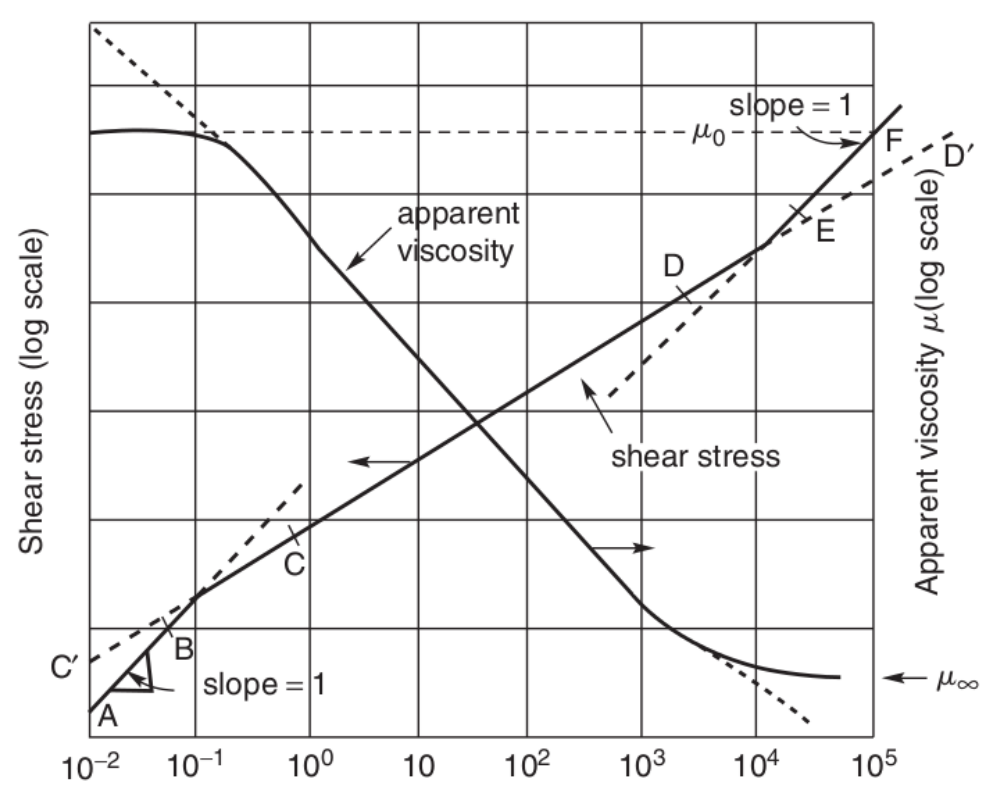

Figura 1.2: Representación esquemática del comportamiento seudoplástico (Chhabra y Richardson, 2008).

origen. Ante dichos esfuerzos extremos, se obtienen dos valores de viscosidad: $\mu_{0}$ a esfuerzos muy pequeños y $\mu_{\infty}$ a esfuerzos muy grandes. Estos valores dependen de diversos factores, tales como el tipo y concentración del polímero, su distribución de pesos moleculares y la naturaleza del disolvente. Aunque es difícil generalizar, muchos materiales muestran las viscosidades límite inferior y superior para esfuerzos por debajo de $10^{-2} s^{-1}$ y por encima de $10^{5} s^{-1}$, respectivamente.

En la literatura existente (Holdsworth, 1993; Lagarrigue y Alvarez, 2001) se han propuesto una gran variedad de modelos matemáticos para fluidos seudoplásticos, pero los 3 más comúnmente utilizados son los siguientes: Power Law Model, Ecuación de viscosidad de Carreau y el Modelo de Ellis (Bird et al., 1987; Carreu et al., 1997; Bird, 1976). El más interesante de cara a la investigación es, sin duda, el primero:

\section{- Modelo Power Law}

Es el método más empleado en la literatura relacionada con ingeniería de procesos. Al representar el esfuerzo de cortadura frente a la velocidad de deformación en coordenadas logarítmicas, se obtienen líneas rectas para un rango limitado de esfuerzos (o velocidades de deformación). En este rango, se puede utilizar una ley del tipo: 


$$
\tau=m(\dot{\gamma})^{n}
$$

De este modo, la viscosidad para un fluido Power Law se puede expresar como:

$$
\mu=\frac{\tau}{\dot{\gamma}}=m(\dot{\gamma})^{n-1}
$$

donde

- $0<n<1$ en fluidos seudoplásticos.

- $n=1$ en fluidos newtonianos.

- $n>1$ en fluidos dilatantes.

Los coeficientes $m$ (coeficiente de consistencia del fluido, fluid consistency index) y $n$ (índice de comportamiento del flujo, flow behaviour index) resultan de un ajuste estadístico de datos empíricos. En general $m$ es fuertemente dependiente de la temperatura, y $n$ es poco dependiente.

La caracterización de un fluido como Power Law es muy sencilla, sin embargo tiene algunas desventajas:

- El ajuste lineal se puede aplicar en un rango limitado, y por tanto los coeficientes $m$ y $n$ son solamente válidos en ese rango.

- El modelo no predice los valores de las viscosidades límite $\mu_{0}$ y $\mu_{\infty}$.

- Las dimensiones del coeficiente $m$ dependen del valor numérico del coeficiente $n$. Por tanto, no pueden compararse los valores de distintos coeficientes de consistencia $m$, cuando los valores $n$ difieren.

El modelo Power law es válido para explicar el comportamiento del fluido en un rango limitado de velocidades de deformación. Sobre dicho modelo, Capobianchi (2008) ha realizado un desarrollo posterior creando el llamado Extended Modified Power Law Model. El autor ha obtenido la expresión de la viscosidad aparente del fluido ante cualquier esfuerzo aplicado, incluyendo en dicha definición los valores de $m, n, \mu_{0}$ y $\mu_{\infty}$. Este modelo es más preciso, pero también más complejo. Además la obtención de los valores de $\mu_{0} \mathrm{y} \mu_{\infty}$ no resulta en absoluto sencilla, ya que varian según la preparación o la degradación del fluido (entre otros muchos factores) y se requieren procedimientos de ensayo complicados y costosos. 


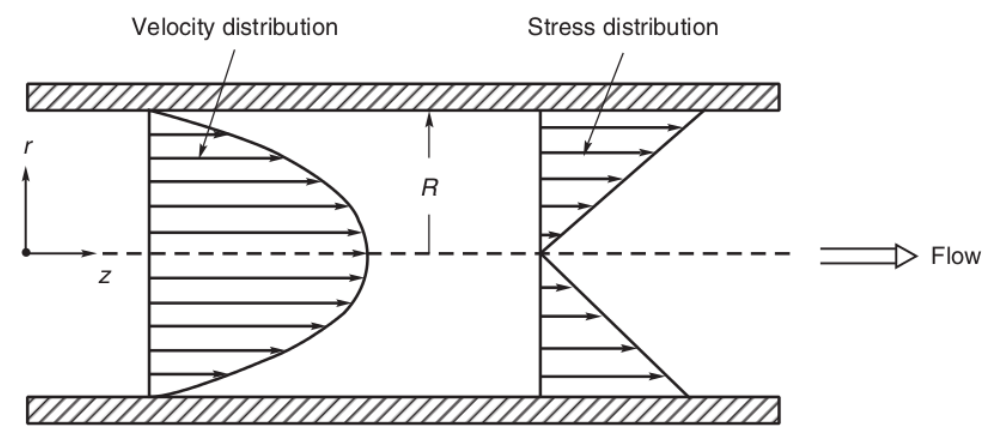

Figura 1.3: Representación esquemática de los esfuerzos tangenciales y distribución de velocidad en un flujo laminar plenamente desarrollado en un tubo (Chhabra y Richardson, 2008).

\subsubsection{Flujo laminar en tubos de fluidos seudoplásticos}

Tal y como se ha mencionado con anterioridad, debido a sus generalmente altas viscosidades, en la práctica, el flujo laminar es más común en fluidos no newtonianos, siempre que no se utilicen técnicas de mejora para adelantar la transición a la turbulencia a números de Reynolds menores (Apartado 1.2.1).

Para un flujo laminar a través de un tubo los esfuerzos cortantes que aparecen tienen una distribución como la mostrada en la Fig. 1.3. En el caso de un fluido no newtoniano del tipo Power Law el perfil de velocidades varía en función del parámetro $n$, siendo más plano para un fluido dilatante $(n>1)$ y más afilado para un fluido seudoplástico $(n<1)$, como se muestra en la Fig. 1.4.

El perfil de velocidades de un fluido Power Law en un tubo liso, en caso de que el fluido tenga propiedades homogéneas en toda la sección depende únicamente de la propiedad reológica $n$. Obviamente el perfil de velocidades será distinto para cada geometría y determinará además la viscosidad aparente del fluido. En el flujo en tubos, el perfil de velocidades viene dado por la siguiente ecuación (la obtención de la misma se detalla en el Apartado 5.1.1):

$$
u_{z}^{*}=\frac{u_{z}}{u_{b}}=\left(\frac{3 n+1}{n+1}\right)\left[1-\left(\frac{r}{R}\right)^{(n+1) / n}\right]
$$

donde $u_{z}$ es la velocidad axial del fluido en un punto, situado a una distancia $r$ del eje, siendo $R$ el radio del tubo y $u_{b}$ la velocidad longitudinal media del flujo. 


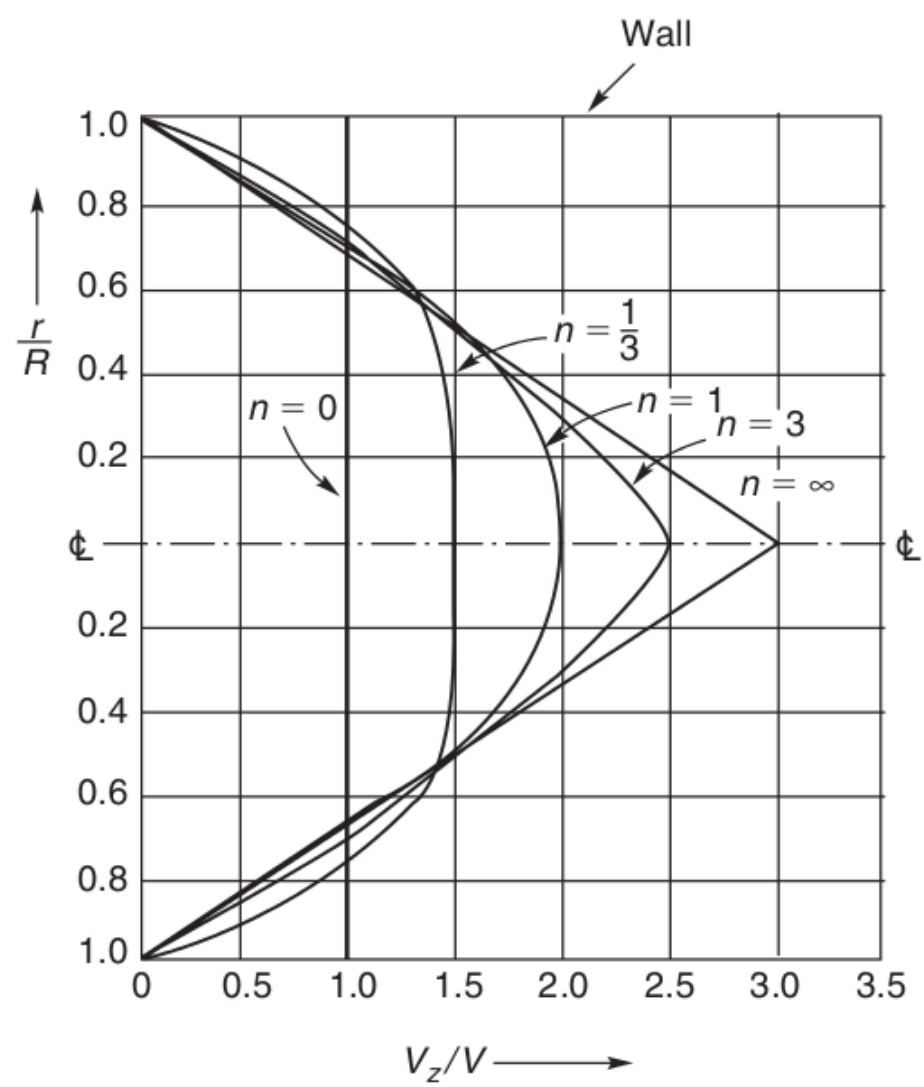

Figura 1.4: Distribución de velocidad en flujo laminar de fluidos Power Law en tubos, obtenido a partir de la Ec. 1.5 (Chhabra y Richardson, 2008). 


\subsubsection{Fluidos no newtonianos en la industria}

No todos los comportamientos no newtonianos de los fluidos tienen un interés práctico en los procesos de intercambio de calor en la industria. La experiencia demuestra que la dependencia de la viscosidad respecto a la velocidad de desplazamiento es el factor más importante en la mayoría de las aplicaciones en ingeniería, que normalmente operan de forma estacionaria. El comportamiento viscoelástico no influye de forma significativa en el flujo en tubos, aunque comienza a manifestarse para flujos en conductos no circulares y en elementos auxiliares de las líneas de conducción (Chhabra y Richardson, 2008). Es por ello que la mayoría de las investigaciones de la bibliografía versa sobre fluidos inelásticos. En algunos de los últimos estudios de importancia realizados Ternik (2010) realiza una recopilación de las nuevas contribuciones en flujo laminar de fluidos inelásticos, Guo y Guo (2009) realizan un estudio analítico sobre la convergencia de fluidos no newtonianos a las ecuaciones de Navier-Stokes, Capobianchi (2008) realiza un desarrollo avanzado del modelo Power Law que incluye viscosidades límite y Capobianchi y Wagner (2010) analizan el comportamiento de dicho modelo.

Por otro lado, los fluidos no newtonianos con más presencia en el mercado son los seudoplásticos, en los que la viscosidad aparente disminuye al aumentar el esfuerzo cortante (Cancela et al., 2005 y Chhabra y Richardson, 2008). Tradicionalmente, no ha habido en la industria tantos fluidos dilatantes (suspensiones concentradas, pastas de almidón) como seudoplásticos, aunque están aumentando debido al creciente interés en el manejo y procesado de sistemas con altas cargas sólidas (Barnes, 1989; Goddard y Bashir, 1990). No hay muchas investigaciones sobre fluidos dilatantes, pero las existentes indican que se ajustan al modelo Power Law con el índice $n>1$ (Vincent-Vela et al., 2010; Marn y Ternik, 2006; Ternik et al., 2006; Dhiman, 2009) aunque otros autores proponen modelos más complejos (Galindo-Rosales et al., 2011). La existencia de un yield stress (Barnes, 1999), un umbral de esfuerzo cortante que se debe superar para que el fluido comience a fluir, se da en los fluidos viscoplásticos tales como suspensiones de partículas, emulsiones, productos alimenticios o en la sangre. Hasta hace unos años existían muy pocas publicaciones experimentales sobre fluidos viscoplásticos, aunque han aumentado en los últimos años (Papanastasiou y Boudouvis, 1997; Balmforth et al., 2007; Tokpavi et al., 2008; Afonso et al., 2008).

Uno de los fluidos no newtonianos seudoplásticos que se ajusta de forma adecuada a los requisitos experimentales de la presente investigación (disoluble en agua caliente y fría, transparente en disolución, no tóxica y biodegradable) es la disolución en agua de Carboximetil 
celulosa (CMC). Pertenece a la familia de los hidrocoloides, que se utilizan para mejorar la consistencia y la textura de productos alimenticios líquidos, semilíquidos y semisólidos. En la industria alimenticia se usa como estabilizador, medio de unión, relleno y para retener agua en galletas, pasteles, helados, zumos, salsas, sopas deshidratadas y productos dietéticos (Pilizota et al., 1996).

El diseño de procesos industriales con fluidos no newtonianos, requiere de datos precisos sobre la reología de los mismos, ya que las características del flujo dependen de la reología y densidad del fluido. Es por ello que existen gran cantidad de publicaciones al respecto. Abdelrahim y Ramaswamy (1995), Ghannam y Esmail (1996), Abu-Jdayil (2003), Cancela et al. (2005), Yang y Zhu (2007) y Benchabane y Bekkour (2008) estudian las propiedades reológicas de las disoluciones de CMC en agua a diferentes concentraciones y temperaturas. Los resultados muestran importantes variaciones en el comportamiento que dependen de la concentración. En concentraciones cercanas al 1\% tiene un comportamiento casi newtoniano que evoluciona a seudoplástico al incrementar la concentración. Además, a concentraciones altas (mayores del $4 \%$ ) aparecen efectos viscoelásticos y tixotrópicos. A valores muy bajos velocidades de deformación Benchabane y Bekkour (2008) detectan una zona newtoniana de viscosidad $\mu_{0}$ en disoluciones de concentraciones altas. En la mayor parte del rango de esfuerzos, de acuerdo a los autores el fluido se ajusta correctamente al modelo Power Law.

\subsection{Transferencia de calor mejorada y ensuciamiento}

El concepto de transferencia de calor mejorada se conoce en la literatura anglosajona como heat transfer enhancement, augmentation o intensification. En general, implica el aumento del coeficiente de transferencia de calor teniendo presente los aumentos de pérdida de presión y potencia de bombeo derivados.

Si se considera un intercambiador de calor de dos fluidos a contracorriente, la ecuación básica para el calor transferido se escribe como sigue:

$$
\dot{q}=U S \Delta \bar{T}
$$

donde

- $\dot{q}$ : calor total intercambiado. 
- $U$ : coeficiente global de transferencia de calor.

- $S$ : área de intercambio.

- $\Delta T_{m}$ : diferencia de temperatura logarítmica media entre las dos corrientes.

Bergles (1997) define la finalidad de la transferencia de calor mejorada como el deseo de estimular o adaptar flujos altos de calor. Así, se busca aumentar el coeficiente de transferencia $U$ de la ecuación anterior para conseguir alguno de estos objetivos:

- Aumentar el calor total intercambiado.

- Reducir el tamaño del intercambiador.

- Reducir la diferencia de temperatura entre las corrientes de proceso.

- Reducir la potencia de bombeo.

- Reducir la temperatura de la pared interior del tubo (muy interesante en la industria alimentaria).

El aumento en el coeficiente de transferencia de calor que producen las técnicas de mejora, en principio, conlleva un aumento en la pérdida de presión en el interior del intercambiador. Sin embargo, gracias al aumento de este coeficiente, sería posible reducir las dimensiones del intercambiador o el gasto másico y, de esta forma, reducir la potencia de bombeo.

La literatura en este campo ha crecido más que la de ciencias de la ingeniería en conjunto, y al menos un $10 \%$ de los trabajos publicados sobre transferencia de calor están relacionados con la mejora (Bergles, 2002). Desde 1993 existe una publicación dedicada en exclusiva al tema: Journal of Enhanced Heat Transfer. Además, multitud de trabajos sobre transferencia de calor mejorada se publican en revistas de primer orden, como el International Journal of Heat and Mass Transfer, el ASME Journal of Heat Transfer o el International Journal of Heat and Fluid Flow. Existen incluso libros específicos como el de Webb (2005) y Thome (1990) y en libros genéricos sobre transferencia de calor como el de Hewitt et al. (1994) se dedica un capítulo a las técnicas de mejora.

\subsubsection{Técnicas de mejora de la transferencia de calor}

Una vez justificada la utilidad de conseguir mejoras en los procesos de transferencia de calor (Apartado 1.2), se pasa a enumerar las diferentes técnicas existentes. Las técnicas de 
mejora de la transmisión de calor fueron clasificadas por Webb (2005) en dos grupos, las técnicas activas y las pasivas, dependiendo de si requieren o no de potencia externa. En una segunda clasificación, se describieron 15 tipos de técnicas de mejora:

- Técnicas pasivas: superficies extendidas, recubrimientos superficiales, superficies rugosas, elementos desplazados, sistemas generadores de rotación, tubos en espiral, sistemas basados en la tensión superficial y aditivos para líquidos y gases.

- Técnicas activas: accionamiento mecánico, vibración de la superficie, vibración del fluido, fuentes electrohidrodinámicas, inyección, succión e impacto de chorros.

Las técnicas de mejora de la transferencia de calor orientadas a aumentar el coeficiente de película interior de los tubos pueden estar basadas en la variación de la geometría del propio tubo, o en la inserción de elementos en tubos. Además, los dispositivos insertados pueden estar estáticos o en movimiento. De entre las distintas técnicas no existe ninguna universal, sino que la técnica a aplicar deberá ser seleccionada teniendo en cuenta las características propias de cada proceso en particular. Por lo tanto, los estudios experimentales de mayor utilidad son aquellos que permiten comparar diferentes técnicas de mejora en amplios rangos de funcionamiento. Entre ellos destacan las tesis doctorales realizadas por el grupo de investigación "Mecánica de Fluidos e Ingeniería Térmica" de la Universidad Politécnica de Cartagena (Vicente, 2002; García, 2006; Illán, 2008; Solano, 2009a) al cual pertenece el autor del presente documento. Vicente (2002) caracterizó y comparó dos técnicas de mejora pasivas, los tubos deformados mecánicamente mediante abolladuras tipo dimpled y los tubos corrugados de una espira hard, García (2006) presentó un análisis del comportamiento termohidráulico de muelles en espiral insertados en tubos lisos, Illán (2008) analizó los intercambiadores de calor de superficie rascada (ICSR) como herramienta para el control de la cristalización en fluidos bifásicos y Solano (2009a) estudió el funcionamiento de los ICSR con movimiento alternativo en procesos que utilizan fluidos newtonianos (los ICSR y su aplicación se describen en el Apartado 1.3).

Las últimas publicaciones del grupo están orientadas en la misma dirección. Solano et al. (2011a) y Solano et al. (2011b) estudian el comportamiento del flujo en un ICSR con movimiento alternativo.García et al. (2012) realizan un estudio comparativo entre tres técnicas de mejora de la transferencia de calor (tubos corrugados, tubos deformados y muelles insertados), Crespí-Llorens et al. (2013) analizan el patrón de flujo turbulento en un ICSR mediante PIV. 
Debido al alto interés de esta rama científica, en la actualidad se publican numerosos trabajos sobre técnicas de mejora de la transferencia de calor. A modo de ejemplo se destacan algunas de las últimas publicaciones más relevantes en este ámbito. Muñoz-Esparza y Sanmiguel Rojas (2011) realizan un estudio sobre la simulación numérica en intercambiadores de calor con muelles insertados. Pethkool et al. (2011) realizan un estudio experimental sobre el flujo turbulento en tubos corrugados. Han et al. (2012) publican los resultados de simulaciones numéricas también en tubos corrugados. Rozzi et al. (2007) realizan un estudio comparativo sobre el comportamiento de intercambiadores de calor de tubo liso y de tubos deformados para su uso en el procesado de alimentos. Rainieri et al. (2013) realizan un compendio sobre la transferencia de calor mejorada en tubos corrugados helicoidales. Addio et al. (2012) escriben sobre nuevos diseños de ICSR que buscan la alta eficiencia térmica en flujos de fluidos áltamente viscosos.

\subsubsection{Ensuciamiento o fouling}

El ensuciamiento o fouling ha sido un problema desde la invención de los intercambiadores de calor. Webb (2005) lo definió como la deposición de material no deseado en la superficie donde se produce la transferencia de calor. Esta deposición de material, degrada el comportamiento tanto hidráulico como térmico del dispositivo, lo cual deriva en pérdidas energéticas. Otras consecuencias no deseables del ensuciamiento son la necesidad de realizar un mantenimiento periódico para eliminarlo y el deterioro que produce en la calidad del producto final.

Aunque es posible minimizar el ensuciamiento mediante modificaciones en el diseño y condiciones de operación de los intercambiadores, estos cambios entran frecuentemente en conflicto con las condiciones requeridas en los procesos.

En concreto, el ensuciamiento de los intercambiadores de calor en la industria petroquímica, química y alimenticia tiene un impacto muy alto en la recuperación energética que se lleva a cabo en estos equipos (oil, 2001; Muller-Steinhagen, 2000). Además, los procesos de intercambio de calor en los que intervienen fluidos no newtonianos son significativamente sensibles al efecto del ensuciamiento, ya que se trata de fluidos con viscosidades muy elevadas y que suelen trabajar en regímenes de flujo muy laminares.

Adicionalmente, la necesidad de realizar mantenimiento para eliminar el ensuciamiento se convierte en un problema especialmente grave en las industrias alimenticias. En ellas el ensuciamiento puede derivar en una pérdida de calidad del producto y la limpieza del 
intercambiador debe ser muy frecuente. Esto lleva a paradas continuas del proceso productivo, que además derivan en pérdidas del producto que se está procesando en ese momento y en general a un aumento de costes significativo.

En la Fig. 1.5 se muestra un estudio del ensuciamiento en el proceso de calentamiento de productos de la leche (Beuf et al., 2003). Como se puede observar, en un proceso de este tipo con intercambiadores tradicionales, es necesaria la interrupción de la producción cada cierto tiempo para realizar la limpieza del intercambiador, manteniendo así niveles de higiene adecuados. Este funcionamiento intermitente puede ser muy problemático para el resto de la cadena de producción, que debe amoldarse al mismo.

A modo de resumen, la prevención del ensuciamiento en un intercambiador de calor consigue evitar:

1. Pérdida de eficiencia del intercambiador. Se producen ineficiencias en la transmisión de calor y un aumento de la pérdida de presión en el intercambiador.

2. Descenso de la calidad y la higiene en la industria alimentaria.

3. Pérdida de productividad por las paradas de mantenimiento. Las paradas se producen de forma periódica para eliminar el ensuciamiento mediante productos de limpieza.

Desde un punto de vista macroscópico, la presencia de ensuciamiento en los intercambiadores de calor tiene importantes consecuencias tanto económicas como medioambientales. Las estimaciones del coste debido al ensuciamiento de los intercambiadores, como consecuencia del exceso de energía que hay que consumir para conseguir el calentamiento deseado, de las operaciones extraordinarias de mantenimiento, el sobredimensionamiento de los intercambiadores y las paradas de producción, son del orden del 0,25\% del Producto Interior Bruto (PIB) en países industrializados (Steinhagen et al., 1992). De acuerdo con Pritchard (1987), en torno al $15 \%$ de los costes de mantenimiento de una planta de proceso se pueden atribuir a calderas e intercambiadores de calor, y de esta cantidad, la mitad aproximadamente se debe al ensuciamiento o fouling. Estos costes pueden incluir mayores gastos de mantenimiento (típicamente $8 \%$ del mantenimiento de una planta de proceso se emplea en la limpieza), costes por paradas (que pueden ser del orden de 1,5 millones de dólares al día) y un aumento del consumo de energía y sobredimensionamiento de la capacidad de transmisión de calor.

A pesar del enorme coste asociado al ensuciamiento, y las numerosas investigaciones llevadas a cabo en este ámbito, son escasas las soluciones tecnológicas capaces de evitar el ensuciamiento para fluidos a alta temperatura, baja velocidad y en presencia de reacciones 


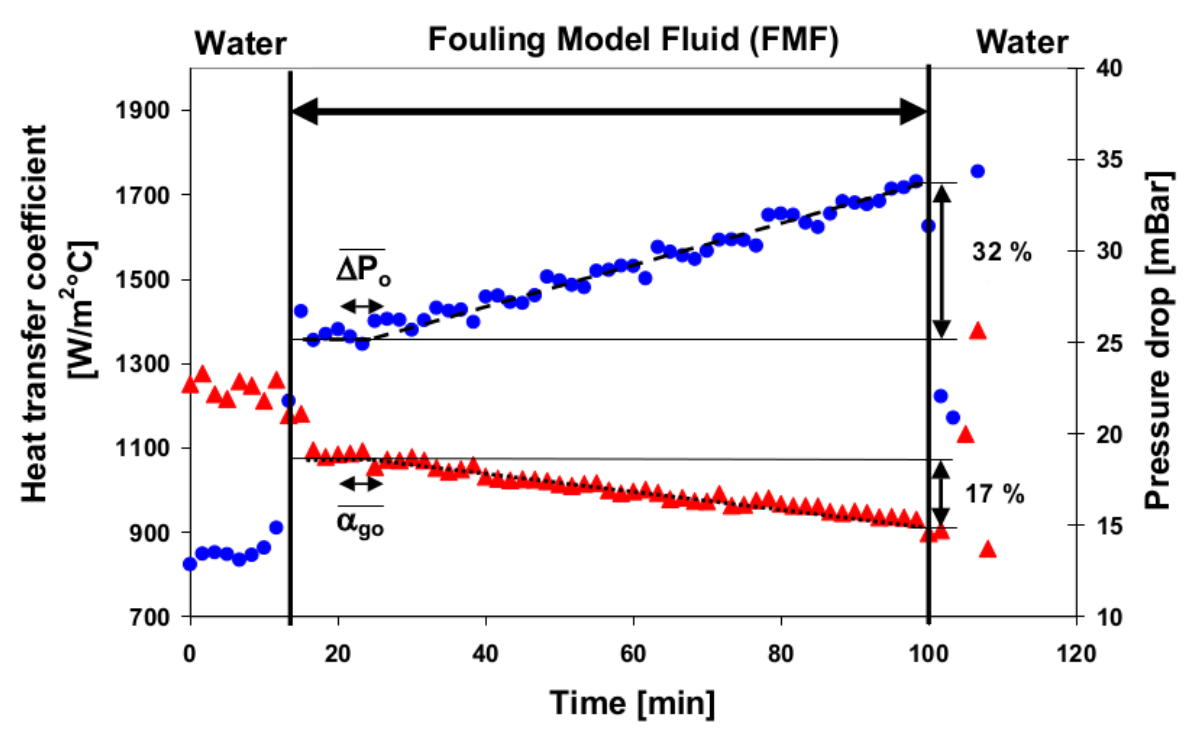

(a) Evolución del coeficiente de transmisión de calor promedio $(\triangle)$ y caída de presión (o) con el tiempo.

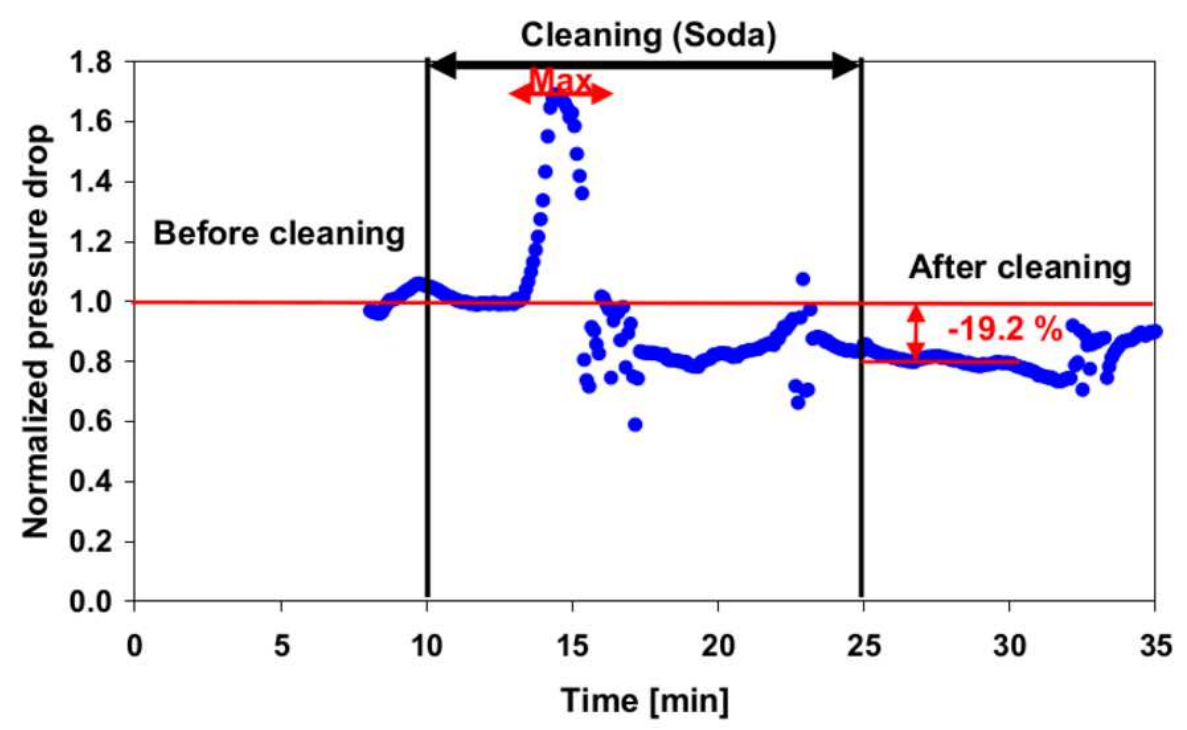

(b) Caída de presión de referencia en proceso de limpieza.

Figura 1.5: Efecto del ensuciamiento y proceso de limpieza en intercambiador de calor de placas durante el procesado de productos de la leche (Beuf et al., 2003). 
químicas. Por ejemplo, Blel et al. (2013) realiza un estudio sobre el ensuciamiento en ICSR, descritos en el Apartado 1.3.

\subsubsection{Transferencia de calor mejorada en fluidos no newtonianos}

De acuerdo a los apartados anteriores, la aplicación de técnicas de mejora de la transferencia de calor está ampliamente justificada y ha sido investigada en profundidad en procesos en los que intervienen fluidos newtonianos (véase revisión bibliográfica al respecto en el Apartado 1.2.1). Sin embargo, según lo expuesto en el Apartado 1.1, en los flujos de fluidos no newtonianos se producen situaciones significativamente diferentes a las que se dan con fluidos no newtonianos, lo cual justifica investigaciones específicas sobre el comportamiento de dichos fluidos en los intercambiadores de calor.

De hecho son numerosas las publicaciones que estudian el flujo de fluidos no newtonianos en conductos de geometrías sencillas. Son ya clásicas las primeras investigaciones en la materia realizadas por Metzner (1965) y Skelland (1967). Posteriormente son significativos los estudios de Joshi y Bergles (1979), Cho y Harnett (1982) y Liu et al. (1992) que analizan la transferencia de calor en flujos laminares en el interior de tubos. Esta línea de investigación sigue de actualidad y se ha ramificado en numerosas vertientes. Patnana et al. (2010) investigan sobre la transferencia de calor en un cilindro en la zona inestable del flujo. Sahu et al. (2009) también estudian la transferencia de calor en la zona inestable del flujo pero en un conducto de sección cuadrada, mientras que Rao et al. (2011) estudian el flujo en un conducto inclinado también de sección cuadrada. Nirmalkar y Chhabra (2012) analizan la convección forzada desde un conducto circular calentado confinado asimétrico. Chandra y Chhabra (2011, 2012) centran sus investigaciones en la transferencia de calor desde un cilindro semicircular en situaciones de convección forzada y convección libre respectivamente. Sasmal y Chhabra (2012) analizan el efecto de la orientación en la convección libre que se produce en un conducto de sección cuadrada que está siendo calentado. Cui et al. (2009) elaboran un estudio numérico sobre el comportamiento de un fluido Power Law en un conducto anular donde el cilíndro interior realiza un movimiento planetario.

A causa de la normalmente alta viscosidad de los mismos, los flujos de los fluidos no newtonianos suelen ser muy laminares con bajos números de Reynolds. Bergles. y Joshi (1983) realizaron un estudio sobre los métodos de mejora de la transferencia de calor más apropiados para bajos números de Reynolds. Uno de los primeros estudios en técnicas de transferencia de calor mejorada en intercambiadores (Gluck, 1959) examinaba las mejoras en un intercambia- 
dor de calor con láminas insertadas en espiral funcionando con un fluido seudoplástico. Los resultados presentaban unos aumentos en los coeficientes de transferencia de calor comparables a los que se producen con fluidos newtonianos. Desde entonces se vienen investigando los efectos de aplicar diferentes técnicas de mejora en intercambiadores de calor con fluidos no newtonianos. Los resultados arrojan significativas mejoras en el comportamiento termohidráulico de los fluidos. A continuación se detallan las investigaciones más relevantes en cuanto a técnicas de mejora pasivas:

- Láminas insertadas en espiral: Manglik et al. (1988) y Patil (2000).

- Muelles insertados: Rajasekaran et al. (1970), Igumentsev y Nazmeev (1978) y Nazmeev (1979).

- Tubos deformados: Rajasekaran et al. (1966), Igumentsev y Nazmeev (1978), Nazmeev (1979), Gupta y Rao (1979), Rainieri y Pagliarini (1997, 2002), Withers (1980).

- Tornillos insertados: Nazmeev (1981).

Debido a las características específicas de los fluidos no newtonianos, no todas las técnicas de mejora son igualmente interesantes para trabajar con ellos. Al ser corrientes los problemas de ensuciamiento al trabajar con este tipo de fluidos, aquellas técnicas de mejora que presenten superficies donde se pueda acumular ensuciamiento no serán convenientes en la mayoría de los casos. Por el contrario, las técnicas de mejora basadas en dispositivos insertados en movimiento, que realizan además una función de eliminación del ensuciamiento, son especialmente indicadas en estos casos. A pesar de la importancia de este tipo de soluciones tecnológicas, todavía son escasas las investigaciones experimentales al respecto. En el Apartado 1.3 se detalla una solución tecnológica a los problemas propuestos.

\subsection{Intercambiadores de calor de superficie rascada co- mo solución tecnológica}

Los intercambiadores de calor de superficie rascada, o ICSR, son un tipo de las llamadas técnicas de mejora de la transferencia de calor (Apartado 1.2.1). Existen diferentes diseños de ICSR, pero todos se basan en el mismo principio de funcionamiento: los intercambiadores están provistos de un dispositivo interno que mediante un mecanismo rasca la superficie interior del intercambiador. 
El uso de los ICSR en los procesos de intercambio de calor industriales tiene un doble objetivo. Por un lado, la presencia del dispositivo rascador modifica el flujo y aumenta el mezclado, mejorando el proceso de transmisión de calor (Apartado 1.2). Por otro lado, el elemento rascador insertado en movimiento limpia la superficie interior del tubo, eliminando así el ensuciamiento que se pudiese acumular en la misma (Apartado 1.2.2).

Los beneficios derivados del uso de este tipo de dispositivos todavía son mayores. Muchos de los fluidos no newtonianos sufren degradación cuando son sometidos a tratamientos térmicos, siendo el nivel de degradación mayor al aumentar la temperatura y pudiendo llegar a quemarse, lo que aumentaría el ensuciamiento (véase el caso del fluido de trabajo utilizado en el Capítulo 5). Esto provoca que el salto térmico con el que se puede trabajar en un intercambiador esté limitado. De nuevo el uso de ICSR resulta muy beneficioso en este punto, ya que el mezclado del flujo que producen, no sólo mejora la transferencia de calor, sino que además uniformiza la temperatura del fluido. De este modo, el fluido en la pared tiene menor temperatura de la que tendría en un simple intercambiador de tubos y por lo tanto se degrada menos y aumenta el límite de $\Delta T$ con el que se puede trabajar sin deteriorar la calidad del producto.

Las virtudes del ICSR anteriormente descritas se retroalimentan, ya que la mejora de transmisión de calor, el mezclado y el proceso de rascado llevan a una disminución de la degradación del fluido y del ensuciamiento, que a su vez redunda en una mejora de la transmisión de calor en un círculo virtuoso. Esto hace que los ICSR sean soluciones tecnológicas muy apropiadas, para su uso en procesos que trabajen con fluidos no newtonianos, especialmente en las industrias química (cosméticos, fármacos, ...) y alimenticia donde la calidad del producto es esencial.

Las investigaciones en este campo son recientes. Yataghene et al. (2009) estudian el efecto en la temperatura del fluido debido a la disipación viscosa que se produce en un ICSR de cuchillas rotativo, posteriormente Yataghene et al. (2011) ensayan experimentalmente el mismo dispositivo para obtener el patrón de flujo estacionario mediante PIV y Yataghene y Legrand (2013) vuelven a estudiar el mismo ICSR mediante métodos numéricos de simulación. Saraceno et al. (2011) obtienen correlaciones experimentales de transferencia de calor en un ICSR en una máquina de helado.

Una de las características de algunos fluidos no newtonianos, interesantes desde el punto de vista de la transmisión de calor en intercambiadores, es la dependencia que tiene la viscosidad de la temperatura. Ditchfield et al. (2007) y Wichterle (2004) estudian este efecto en los intercambiadores de calor y Rainieri y Pagliarini (2002) y Rennie y Raghavan (2007) 

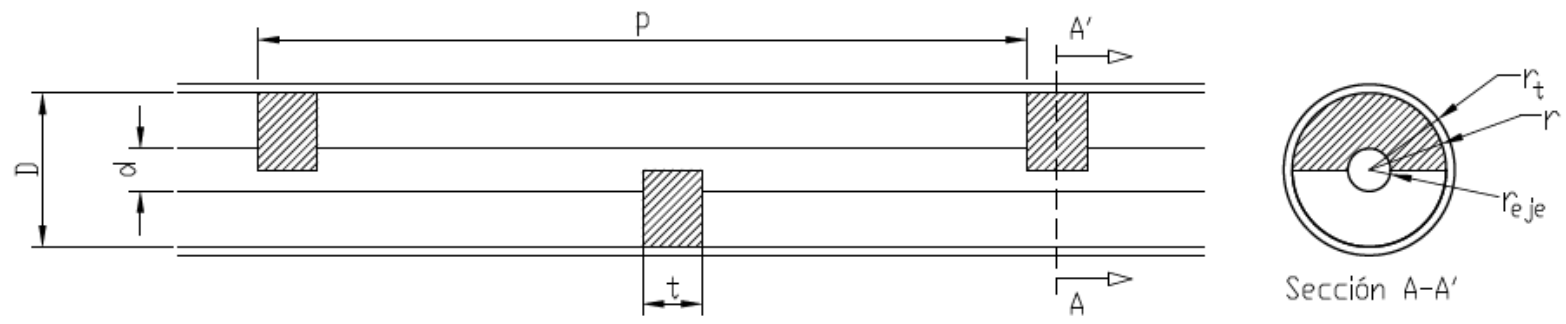

Figura 1.6: Detalle del dispositivo de mejora insertado.

en intercambiadores de calor mejorados. Mabit et al. (2008) desarrollan un integrador de temperatura-tiempo para cuantificar el intercambio térmico en intercambiadores de calor de superficie rascada.

\subsection{Objetivos de la tesis.}

A lo largo del capítulo se ha visto, que en los procesos de intercambio de calor en los que intervienen fluidos no newtonianos, los flujos existentes son fuertemente laminares. En esta situación, las técnicas de mejora pasivas no producen una mejora de la transferencia de calor significativa y pueden incrementar el ensuciamiento. Por lo tanto, si se quiere mejorar la eficiencia energética del proceso, es necesario utilizar técnicas activas, que tienen efecto a números de Reynolds menores. Además, en fluidos newtonianos el ensuciamiento puede llegar a ser muy significativo, siendo esencial el uso de un sistema que lo evite.

Las razones expuestas llevan a la selección de un intercambiador de calor de superficie rascada con movimiento lineal alternativo.

En consecuencia, el objetivo de la tesis doctoral es el de analizar el comportamiento del tipo más común de fluido no newtoniano en el $\operatorname{ICSR}^{1}$ de la Fig. 1.6, que está provisto de un movimiento lineal alternativo. En concreto, el fluido a analizar (disolución en agua de $\mathrm{CMC}$ ) tiene un comportamiento eminentemente seudoplástico, modelizable mediante el modelo Power Law.

El funcionamiento de dicho dispositivo con fluidos newtonianos es conocido (Solano, 2009a), de modo que el objetivo de la presente investigación es el de centrarse en la dife-

\footnotetext{
${ }^{1}$ Intercambiador de calor de superficie rascada
} 
rencia que supone el uso de fluidos no newtonianos.

La investigación está formada por cinco bloques de trabajo, cuyos objetivos serán los siguientes:

1. Implementación de un código CFD de simulación del flujo no newtoniano en geometrías de tubo liso y tubo con eje. Mediante dicho código se estudian los problemas fluidomecánico y térmico en dichas geometrías. Éstos sirven como punto de partida para el estudio del flujo en el tubo con rascador, así como de base comparativa, de modo que sea posible identificar el efecto del dispositivo rascador en el campo fluido y en el proceso de transferencia de calor.

2. Obtención experimental del campo de velocidades en el intercambiador de calor, utilizando disoluciones de CMC en agua. Se estudian los casos con rascador estático y dinámico, a diferentes regímenes de rascado $(\beta=[-2 ; 4])$. El estudio se centra en el rango de números de Reynolds entre 5 y 48. En este estudio se obtiene el patrón y las estructuras del flujo en el rascador, lo cual permite un mayor entendimiento sobre el funcionamiento del mismo.

3. Obtención experimental de la pérdida de presión en el tubo con rascador y de la potencia de accionamiento de dicho rascador. Se estudian los casos con rascador estático y dinámico, a velocidades de rascado bajas $(\beta=[0,5 ; 2,5])$. Se barre un rango de números de Reynolds entre 0,2 y 600. El estudio de la pérdida de presión por metro lineal es esencial para poder determinar el aumento de requisito de potencia de bombeo necesaria. Además permite identificar el régimen de flujo en función del número de Reynolds. Por último permite establecer una relación entre la viscosidad efectiva del fluido y los parámetros reológicos $m$ y $n$.

4. Obtención experimental de la transferencia de calor con el mismo fluido. Se estudian los casos con rascador estático y dinámico, a velocidades de rascado bajas $(\omega=[0,1 ; 1])$. Se barre un rango de números de Reynolds entre 0,2 y 400, y de números de Prandtal entre 100 y 5000. El aumento de la transferencia de calor es uno de los objetivos del uso de un ICSR y se cuantifica en este bloque de trabajo. Además en los resultados se pueden observar así mismo los regímenes de flujo en función de las variables del problema.

5. Extracción de conclusiones y comparación, en la medida de lo posible, con el comportamiento de un fluido newtoniano trabajando en las mismas condiciones. Los datos referentes a fluidos newtonianos se extraen de la bibliografía. 
Para lograr los objetivos marcados, el grupo de investigación cuenta con una dilatada experiencia en el estudio de técnicas de mejora de la transferencia de calor (Vicente, 2002; García, 2006; Illán, 2008; Solano, 2009a; Crespí-Llorens et al., 2013). En concreto la presente investigación ha sido financiada por el proyecto del Plan Nacional de I+D+I titulado Título Transferencia de calor y generación de hielo en intercambiadores tubulares con referencia DPI2007-66551-C02-01 y por una beca de Formación de Profesorado Universitario con referencia AP2007-03429.

\subsection{Desarrollo de la tesis doctoral}

El presente documento se estructura en dos partes diferenciadas. La primera parte la introducción, el planteamiento de la investigación y la descripción de las herramientas utilizadas en la investigación. En la segunda parte se presentan los resultados obtenidos y las conclusiones extraídas a partir de éstos.

\section{Parte I: Introducción y metodología experimental}

En el Capítulo 2 se realiza el análisis dimensional de los problemas fluidomecánico y térmico. Dicho estudio reduce los grados de libertad del problema, a partir de la definición de monomios adimensionales.

En el Capítulo 3 se describe la implementación en Matlab de códigos CFD para la simulación del flujo de fluidos seudoplásticos en geometrías de tubo liso y tubo con eje. Los códigos desarrollados son válidos tanto para el estudio del problema fluidomecánico como térmico, incluyendo el estudio de la región de entrada.

Por un lado, en el Capítulo 4 se describen las instalaciones experimentales utilizadas en la presente investigación. Por otro lado, la metodología empleada en la realización de los ensayos se describe más adelante en el Capítulo 6. En dichas instalaciones se realizarán ensayos de visualización del flujo y ensayos termohidráulicos.

En el Capítulo 5 se lleva a cabo la caracterización del fluido de trabajo, Así mismo, se describe la metodología empleada para la caracterización de las propiedades fisico-químicas del mismo. Concretamente las propiedades reológicas del fluido se miden en cada experimento debido a la alta variabilidad de las mismas. En este capítulo se incluye el desarrollo del método de generalización de la viscosidad, que depende de la geometría estudiada y resulta un novedad en su aplicación a geometrías de sección transversal no uniforme. La utilización de la viscosidad generalizada en las definiciones de los números adimensionales de Prandtl 
y Reynolds, dando lugar a los números de Prandtl y Reynolds generalizados, lleva a una simplificación significativa de los problemas fluidomecánico y térmico.

\section{Parte II: Resultados y conclusiones}

El Capítulo 7 versa sobre la visualización del campo fluido en el interior del tubo. En él se muestran los resultados de los ensayos de visualización y se relacionan con el comportamiento del flujo en cuanto a la caída de presión que se produce y a la transferencia de calor.

En el Capítulo 8 se estudia la caída de presión que se produce en el tubo a causa del dispositivo insertado en diferentes condiciones de flujo y utilizando fluidos con diferentes propiedades reológicas. En los resultados de caída de presión, se pueden observar las regiones del flujo en función del número de Reynolds generalizado. Además se observa la influencia que tiene la variación de los parámetros reológicos en la caída de presión en el tubo.

En el Capítulo 9 se estudia la eficiencia de la transmisión de calor al fluido. El dispositivo insertado mejora dicha transferencia de calor, principalmente por el adelanto de la transición a flujo turbulento. En este capítulo se cuantifica esta mejora en la eficiencia de intercambio y se evalúa la influencia del comportamiento no newtoniano del fluido en este proceso. Así mismo se realiza una evaluación de las prestaciones frente a geometrías de intercambiador de tubos estándar. Finalmente se estudia el interés energético del accionamiento del rascador al margen de la eliminación de ensuciamiento.

En el Capítulo 10 se presenta un esquema de la aplicación industrial de los resultados de la investigación. En él se detallan los pasos a seguir a la hora de valorar la conveniencia del uso del intercambiador de calor estudiado en función de las condiciones de trabajo. Así mismo se presenta un resumen de la consecución de los objetivos planteados y se detallan las conclusiones extraídas de la tesis doctoral. 


\section{Capítulo 2}

\section{Análisis dimensional}

Como parte de los objetivos de la tesis, se ha establecido tanto el estudio de la caída de presión en el intercambiador de calor (problema fluidomecánico), como el estudio de la transmisión de calor (problema termodinámico) en diferentes situaciones de funcionamiento.

Es común en mecánica de fluidos realizar un análisis dimensional del problema, lo que reduce los grados de libertad del mismo, simplificando significativamente su complejidad. Los orígenes del análisis dimensional se remontan al siglo XVIII (discusiones de Euler) y XIX (Fourier (1822)) y cobra una nueva dimensión gracias a los trabajos de Vaschy (1892) y Buckingham (1914), padres del Teorema de П de Vaschy-Buckingham.

El análisis dimensional (AD) correspondiente al problema fluidomecánico se detalla en el Apartado 2.1 y el correspondiente al problema térmico en el Apartado 2.2.

Tal y como se detalla en dichos apartados, mediante el análisis dimensional del problema se obtienen, entre otros, el número de Reynolds y el número de Prandtl (problema térmico), los cuales incluyen una viscosidad. Así, dependiendo de la definición utilizada para la viscosidad, se pueden obtener diferentes números de Reynolds y Prandtl. En la bibliografía se expone el método de generalización de la viscosidad, mediante el cual es posible reducir un grado de libertad del problema fluidomecánico. En el Apartado 2.4 se estudian las diferentes opciones propuestas en la bibliografía para aplicar el citado método de generalización, buscando la más adecuada al estudio de este problema concreto. Para obtener la definición más apropiada de la viscosidad generalizada, se debe recurrir a la experimentación. Esta tarea se lleva a cabo en el Apartado 5.3. 


\subsection{Problema fluidomecánico}

En este apartado el realiza el AD del problema fluidomecánico. En él, se desea estudiar la caída de presión que se produce en el intercambiador de calor (Fig. 4.2) en función del caudal de fluido circulante y las características del fluido. Así, para simplificar los grados de libertad del problema, se recurre al análisis dimensional del mismo.

Para realizar el análisis dimensional es conveniente simplificar la geometría del intercambiador a una geometría de tubo con eje concéntrico. Esta simplificación geométrica es equivalente a despreciar el efecto de las velocidades del fluido en direcciones radial y angular. Así, la geometría considerada es la de tubo de sección anular o de corona concéntrica como la representada en la Fig. 2.1.

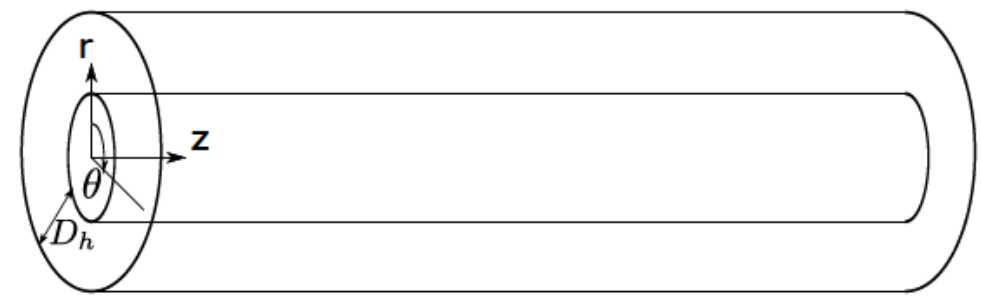

Figura 2.1: Geometría tubo de sección anular a la cual se puede simplificar la geometría estudiada de cara al análisis dimensional.

Las ecuaciones que rigen el movimiento del fluido en el conducto son las ecuaciones de Continuidad y de Cantidad de Movimiento. Por lo tanto, en primer lugar, se plantean ambas ecuaciones en su forma general (Ec. 2.1 y Ec.2.3) y se especifican las condiciones de contorno. A continuación se simplifican para el caso particular del flujo incompresible y estacionario en el tubo de sección anular (Ec. 2.2 y Ec.2.4) y por último se concretan para el modelo Power Law (Ec.2.2 y Ec.2.6).

Ec. continuidad

$$
\frac{\partial \rho}{\partial t}+\vec{\nabla} \cdot(\rho \vec{v})=0
$$

Para flujo estacionario incompresible:

$$
\begin{gathered}
\vec{\nabla} \cdot \vec{v}=0 \\
\rho\left[\frac{1}{r} \frac{\partial}{\partial r}\left(r u_{r}\right)+\frac{1}{r} \frac{\partial}{\partial \theta}\left(u_{\theta}\right)+\frac{\partial u_{z}}{\partial z}\right]=0
\end{gathered}
$$


En el flujo en el tubo de sección anular se consideran despreciables las componentes radial y angular de la velocidad, $u_{r}=u_{\theta}=0$.

$$
\frac{\partial u_{z}}{\partial z}=0
$$

Ec. Cantidad de Movimiento

$$
\rho \frac{\partial \vec{v}}{\partial t}+\rho(\vec{v} \cdot \vec{\nabla}) \vec{v}=-\vec{\nabla} p+\vec{\nabla} \overline{\bar{\tau}}^{\prime}+\rho \vec{f}_{m}
$$

Para flujo estacionario incompresible, la ecuación en el eje z queda:

$$
\rho\left(u_{r} \frac{\partial u_{z}}{\partial r}+u_{\theta} \frac{1}{r} \frac{\partial u_{z}}{\partial \theta}+u_{z} \frac{\partial u_{z}}{\partial z}\right)=-\frac{\partial p}{\partial z}+\frac{1}{r} \frac{\partial\left(r \tau_{r z}\right)}{\partial r}+\frac{1}{r} \frac{\partial \tau_{\theta z}}{\partial \theta}+\frac{\partial \tau_{z z}}{\partial z}
$$

Donde $u_{r}=u_{\theta}=0$, además por continuidad $\partial u_{z} / \partial z=0$ y debido a la simetría cilíndrica que presenta el flujo $\partial u_{z} / \partial \theta=0$ con lo que la ecuación se simplifica a la siguiente expresión:

$$
\frac{\partial p}{\partial z}=\frac{1}{r} \frac{\partial\left(r \tau_{r z}\right)}{\partial r}
$$

Por otro lado, el fluido de trabajo tiene un comportamiento no newtoniano que se ajusta al modelo Power Law y, consecuentemente, la expresión del esfuerzo en la dirección principal de flujo queda del siguiente modo:

$$
\begin{gathered}
\tau_{r z}=m \dot{\gamma}^{n}=m\left(\frac{\partial u_{z}}{\partial r}\right)^{n} \\
\frac{\partial p}{\partial z}=\frac{1}{r} \frac{\partial}{\partial r}\left(r m\left(\frac{\partial u_{z}}{\partial r}\right)^{n}\right)
\end{gathered}
$$

La ecuación general se simplifica bajo las siguientes consideraciones: el fluido es incompresible, el régimen de flujo es estacionario y la fuerza de la gravedad no tiene efectos significativos en el flujo en tubos.

$\underline{\text { Condiciones de contorno }}$

$$
\begin{aligned}
r=d / 2 & \rightarrow u_{z}=0 \\
r=D / 2 & \rightarrow u_{z}=0
\end{aligned}
$$


De las ecuaciones del problema se deduce que, en principio, si se conoce la caída de presión por metro lineal $p_{L}$, se puede obtener la velocidad axial del flujo $u_{z}(r)$ y su promedio en la sección $u_{b}$. Si no se conoce $p_{L}$, se necesita conocer la condición de caudal $Q$ para obtener el perfil de velocidades y $p_{L}$.

\subsubsection{Análisis dimensional del problema}

Una vez descritas las ecuaciones que explican el comportamiento del fluido, el análisis dimensional exige la aplicación del Teorema de $\Pi$.

La lista de variables relevantes del problema se obtiene del análisis realizado en el apartado anterior. Se debe tener en cuenta que el objetivo del problema es calcular la caída de presión por metro lineal de conducto, $p_{L}$. La geometría del conducto viene representada por dos variables, el diámetro del tubo, $D$, y el diámetro del eje, d. No obstante uno de dichos parámetros puede ser sustituido por el diámetro hidráulico, $D_{h}$, que puede ser más representativo

$$
D_{h}=4 r_{h}=\frac{\text { área }}{\text { perímetro }}=D-d
$$

Se elige además como velocidad longitudinal representativa, la velocidad media del fluido en la sección $u_{b}$. Además se incluyen las propiedades del fluido $\rho, m$ y $n$. Por lo tanto siguiente lista de 7 variables relevantes es la siguiente $\left\{p_{L}, D_{h}, d, u_{b}, \rho, m, n\right\}$. Donde sabemos que $p_{L}=$ $\Psi_{1}\left(D_{h}, d, u_{b}, \rho, m, n\right)$.

Además, el AD en mecánica del fluidos utiliza la siguiente base dimensional: $\{M, L, T\}$.

De acuerdo al Teorema de $\Pi$, el número de parámetros adimensionales será $j=N_{v}-$ $k_{d}$, siendo $N_{v}$ el número de variables relevantes y $k_{d}$ el número de dimensiones de la base dimensional. Se tendrán por lo tanto $j=7-3=4$ monomios adimensionales.

$$
\begin{gathered}
\pi_{1}=R e_{b}=\frac{\rho u_{b}^{2-n} D_{h}^{n}}{m}=\frac{\rho u_{b} D_{h}}{\mu_{b}} \\
\pi_{2}=\frac{p_{L} D_{h}}{\rho u_{b}^{2}} \\
\pi_{3}=n
\end{gathered}
$$


y un cuarto parámetro que sería $\pi_{4}=D_{h} / d$, aunque se suele utilizar la relación de diámetros, $\alpha$

$$
\pi_{4}=\alpha=d / D
$$

Los monomios adimensionales $\pi_{1}$ y $\pi_{2}$ se corresponden con dos números clásicos de la mecánica de fluidos como son el número de Reynolds (definido para fluidos no newtonianos) y el factor de fricción.

Tal y como se explica al inicio del capítulo, el número de Reynolds obtenido mediante el análisis dimensional incluye una viscosidad. En la ecuación anterior, la viscosidad viene dada por la siguiente expresión

$$
\mu_{b}=m\left(\frac{u_{b}}{D_{h}}\right)^{n-1}
$$

a la cuál llamaremos "definición básica de la viscosidad" y se denotará con el subíndice b. Esta viscosidad no tiene, en principio, ningún significado físico, símplemente es un parámetro con dimensiones de viscosidad obtenido como resultado directo de la aplicación del teorema de П. Igualmente, se denotará al número de Reynolds resultante (Ec. 2.7), número de Reynolds básico, $R e_{b}$. En el Apartado 2.4 siguiente se introducen expresiones de la viscosidad obtenidas por otros autores, en función de la geometría bajo estudio.

En cuanto al factor de fricción, existen dos definiciones extendidas del mismo, el factor de fricción de Darcy y el de Fanning, que son dimensionalmente iguales. En concreto, en el presente estudio se opta por utilizar la definición de Fanning (Ec.2.8), múltiplo del monomio $\pi_{2}$.

$$
f=\frac{p_{L} D_{h}}{2 \rho u_{b}^{2}}
$$

Por lo tanto el análisis dimensional ha reducido el número de variables que intervienen en el problema, quedando que:

$$
f=\Psi\left(R e_{b}, n, \alpha\right)
$$

donde si la relación de diámetros permanece constante, queda

$$
f=\Psi\left(R e_{b}, n\right)
$$




\subsection{Problema térmico}

En el presente apartado se lleva a cabo el análisis dimensional del problema térmico. La geometría considerada es la misma que en el apartado anterior (Fig. 2.1) . Al tubo se le aplica un flujo de calor constante (condición H) en la pared exterior y se mide la temperatura interior del tubo. La superficie del eje interior se considera adiabática.

El flujo de calor constante se transmite del tubo al fluido mediante los fenómenos combinados de conducción y convección forzada. Este último efecto depende, entre otros factores, del patrón de flujo en el interior del tubo, el cuál se pretende modificar mediante la inserción del rascador. En consecuencia, para evaluar la transferencia de calor mejorada mediante el uso de rascadores se debe evaluar $h_{i}$, que será por tanto la incógnita del problema.

Se plantea en primer lugar la Ecuación de la Energía en forma general:

$$
\rho c_{p} \frac{D T}{D t}=-p(\vec{\nabla} \cdot \vec{v})+\phi_{v}-\nabla \vec{q}+\dot{q}_{q}+\dot{q}_{r}
$$

En particular para un flujo incompresible estacionario, la geometría de tubos concéntricos, suponiendo nulos los flujos de calor por radiación y reacciones químicas y despreciando el término de disipación viscosa y la conducción longitudinal, se tienen las siguientes condiciones: $\partial / \partial t=\partial / \partial \theta=u_{r}=u_{\theta}=\dot{q}_{q}=\dot{q}_{r}=\vec{\nabla} \cdot \vec{v}=\phi_{v}=\frac{\partial}{\partial z}\left(k \frac{\partial T}{\partial z}\right)=0$. De modo que la ecuación de la energía en coordenadas cilíndricas se simplifica a la siguiente expresión.

$$
\rho c_{p} u_{z} \frac{\partial T}{\partial z}=\frac{1}{r} \frac{\partial}{\partial r}\left(r k \frac{\partial T}{\partial r}\right)
$$

Donde las condiciones de contorno son la temperatura en la pared, el flujo de calor en la pared del tubo y en la superficie del eje interior (adiabática) y la temperatura de entrada:

$$
\begin{aligned}
r=D / 2 & \rightarrow \quad \dot{q}_{i}^{\prime \prime}=\left.k \frac{\partial T}{\partial r}\right|_{r=R}=h_{i}\left(T_{p}-\bar{T}_{f}\right) \\
r=d / 2 & \rightarrow \dot{q}^{\prime \prime}=0 \\
z=0 & \rightarrow T=T_{o}
\end{aligned}
$$

siendo $T_{o}$ la temperatura a la entrada del tubo.

Si se integra la ecuación de la energía, resulta

$$
\dot{m} c_{p} \frac{d \bar{T}}{d z}=S \dot{q}_{i}^{\prime \prime}=S h_{i}\left(T_{p}-\bar{T}_{f}\right)
$$


Además también rigen la ecuación de momentos y de continuidad y las condiciones de contorno expuestas en el problema fluidomecánico.

\subsubsection{Análisis dimensional}

En el problema térmico se desea determinar el flujo de calor transferido al fluido, $q_{i}$. Éste, tal y como se analiza en el apartado anterior, dependerá de las siguientes variables:

$$
\dot{q}_{i}^{\prime \prime}=\Psi\left(\rho, c_{p}, k, m, n, D_{h}, d, \Delta T, u_{b}\right)
$$

Con lo que la lista de variables tiene 10 miembros: $<\dot{q}_{i}^{\prime \prime}, \rho, c_{p}, k, m, n, D_{h}, d, \Delta T, u_{b}>$. Por otro lado, la base dimensional está formada por 4 dimensiones $\{M, L, T, \Theta\}$, con lo que se deben obtener 6 monomios adimensionales.

$$
\begin{gathered}
\pi_{1}=R e_{b}=\frac{\rho u_{b}^{2-n} D_{h}^{n}}{m}=\frac{\rho u_{b} D_{h}}{\mu_{b}} \\
\pi_{2}=N u=\frac{\dot{q}_{i}^{\prime \prime} D_{h}}{k \Delta T}=\frac{h_{i} D_{h}}{k} \\
\pi_{3}=P r_{b}=\frac{c_{p} m}{k}\left(\frac{u_{b}}{D_{h}}\right)^{n-1}=\frac{c_{p} \mu_{b}}{k} \\
\pi_{4}=n \\
\pi_{5}=E c=\frac{u_{b}^{2}}{c_{p} \Delta T} \\
\pi_{6}=\alpha=d / D
\end{gathered}
$$

aunque el número de Eckert, Ec, únicamente tiene influencia a velocidades muy elevadas, así que su influencia en este problema se puede despreciar. Por tanto,

$$
N u=\Psi\left(\operatorname{Re}_{b}, \operatorname{Pr}_{b}, n, \alpha\right)
$$


y si de nuevo se fija la geometría del problema,

$$
N u=\Psi\left(R e_{b}, \operatorname{Pr}_{b}, n\right)
$$

\subsection{Problemas fluidomecánico y térmico con rascador en movimiento}

En el caso de que el dispositivo insertado se encuentra en movimiento a una velocidad constante, es necesario añadir una variable más a la lista de variables relevantes de los respectivos problemas. La opción más inmediata sería añadir la velocidad a la que se desplaza el rascador $v_{s}$, aunque estudios anteriores en intercambiadores de calor de superficie rascada con movimiento lineal alternativo (Crespí-Llorens et al., 2013; Solano et al., 2011b), demuestran que la variable significativa es $\left(u_{b}-v_{s}\right)$.

Como consecuencia de tener una variable adicional en los problemas tanto fluidomecánico como térmico, aparece un nuevo número adimensional, al que se conoce como factor de bloqueo.

$$
\pi=\frac{u_{b}-v_{s}}{u_{b}}=1-\frac{v_{s}}{u_{b}}=\beta
$$

El factor de bloqueo ha sido descrito por Solano et al. (2011b) y por Crespí-Llorens et al. (2013), en flujos laminares y turbulentos de fluidos newtonianos respectivamente, como el parámetro más apropiado para describir la influencia del movimiento del rascador en el flujo, en geometrías de tubo con rascador en las que existe un movimiento de rascado alternativo.

Por lo tanto para el problema fluidomecánico con el rascador en movimiento, la caída de presión adimensional será función de otros tres números adimensionales:

$$
f=\Psi\left(R e_{b}, n, \beta\right)
$$

mientras que en el problema térmico, el número de Nusselt depende de cuatro números adimensionales:

$$
N u=\Psi\left(\operatorname{Re}_{b}, \operatorname{Pr}_{b}, n, \beta\right)
$$




\subsection{Viscosidad generalizada y método de generaliza- ción}

Como se ha visto en los Apartados 2.1.1 y 2.2, el análisis dimensional aplicado a un flujo de un fluido newtoniano que circula por un conducto anular, arroja las siguientes expresiones del número de Reynolds y el número de Prandtl en función de la definición básica de la viscosidad.

$$
\begin{gathered}
R e_{b}=\frac{\rho u_{b} D_{h}}{\mu_{b}} \\
\operatorname{Pr}_{b}=\frac{c_{p} \mu_{b}}{k} \\
\mu_{b}=m\left(\frac{u_{b}}{D_{h}}\right)^{n-1}
\end{gathered}
$$

Esta definición del número de Reynolds, lleva a relaciones entre éste y el factor de fricción de Fanning fuertemente dependientes del índice de comportamiento de flujo, $n$, tal y como prevé el análisis dimensional (Ec. 2.9). Utilizarla puede ser útil para constatar dicha dependencia: véase la Fig. 2.2(a) en la que se muestra dicha relación para la geometría de tubo liso. Sin embargo, a la hora de analizar problemas más complejos puede ser conveniente definir la viscosidad de forma que se pueda reducir un grado de libertad del problema.

Tal y como se ha deducido con anterioridad, el estudio de la caída de presión en un tubo con eje, manteniendo $\alpha$ invariable, viene descrita por la siguiente función

$$
f=\Psi\left(R e_{b}, n\right)
$$

en geometrías cuya sección transversal es constante ${ }^{1}$, la relación existente viene dada por

$$
f=\text { cte } \times R e_{b}^{-1} \times \phi(n)
$$

donde la dependencia de $n$ viene dada por la función $\phi(n)$.

Así, si se conoce o se obtiene la función $\phi(n)$, se podría definir una viscosidad $\mu_{g}$ que incluya a dicha función $\phi(n)$. Obviamente, si dicha viscosidad se incluye en la lista de variables relevantes, también aparecería en los números de Reynolds $R e_{g}$ y Prandtl $P r_{g}$. De este modo

\footnotetext{
${ }^{1}$ Nótese que para geometrías con sección transversal no constante el exponente del número de Reynolds puede ser distinto de 1.
} 
se consigue reducir un grado de libertad del problema, que quedaría

$$
f=\Psi\left(R e_{g}\right)
$$

donde

$$
R e_{g}=R e_{b} / \phi(n)
$$

Es decir, que se habría conseguido eliminar una de las variables del problema reduciendo notablemente su complejidad. La utilidad de la simplificación se hace especialmente manifiesta a medida que el problema se complica y aparecen nuevas variables, por ejemplo en el problema térmico o en situaciones de rascador en movimiento.

A este procedimiento se le conoce en la bibliografía como el Método de generalización de la viscosidad y a las variables resultantes como viscosidad generalizada, número de Reynolds generalizado y número de Prandtl generalizado. A continuación se procede a realizar un análisis bibliográfico respecto a este método, teniendo siempre como objetivo aplicarlo a la geometría estudiada. En el apartado siguiente se analizan los estudios existentes para geometrías de sección transversal constante. Se verá que las soluciones aportadas por estos autores no son aplicables a la geometría de tubo con rascador, por ser ésta una geometría compleja de sección de paso variable. Sin embargo, dichas soluciones suponen una base sólida para el desarrollo de un método equivalente válido para la geometría de tubo con rascador. La metodología desarrollada para obtener viscosidad generalizada del flujo requiere de experimentación en las instalaciones y se aborda en el Apartado 5.3 del Capítulo 5.

\subsubsection{Geometrías con sección transversal constante}

Metzner y Reed (1955) fueron los primeros en aplicar el Método de generalización de la viscosidad en la geometría de tubo liso. En primer lugar, constataron analíticamente que, en dicha geometría, se cumple la siguiente relación:

$$
f \times R e_{b}=2 \times 8^{n} \times\left(\frac{3 n+1}{4 n}\right)^{n}
$$

Así que definieron la viscosidad generalizada del siguiente modo,

$$
\mu_{g, M R}=8^{n-1} \times\left(\frac{3 n+1}{4 n}\right)^{n} m\left(\frac{u_{b}}{D_{h}}\right)^{n-1}
$$




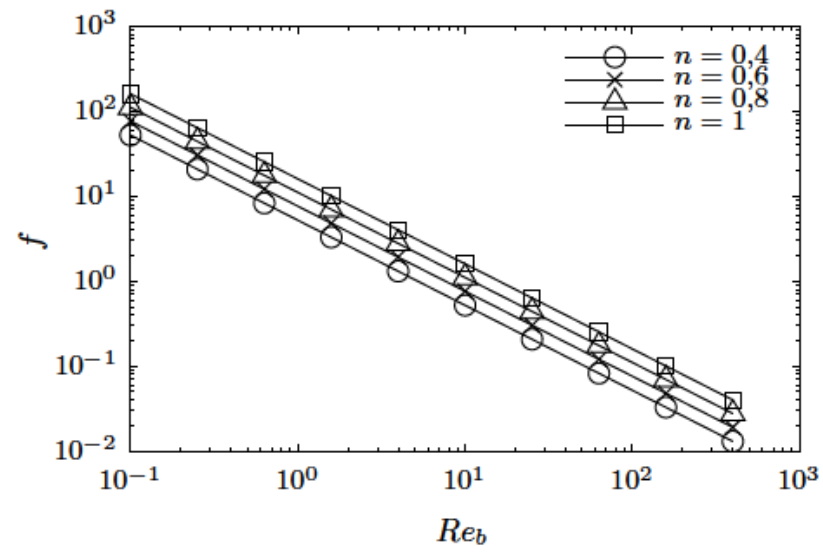

(a) Representación con $\operatorname{Re}_{b}$.

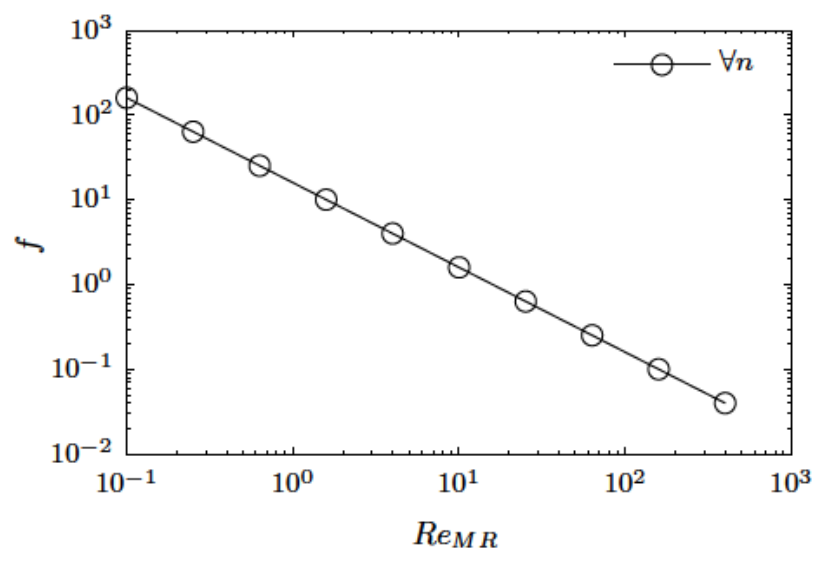

(b) Representación con $\operatorname{Re}_{M R}$.

Figura 2.2: Caída de presión en geometría de tubo liso en régimen de flujo laminar.

un nuevo número de Reynolds en función de la viscosidad generalizada del fluido $\mu_{g, M R}$ :

$$
R e_{M R}=\frac{\rho u_{b} D_{h}}{\mu_{g, M R}}=\frac{\rho u_{b}^{2-n} D_{h}^{n}}{m 8^{n-1}\left(\frac{3 n+1}{4 n}\right)^{n}}
$$

de forma que se cumpla que el producto del factor de fricción por el nuevo número de Reynolds sea constante e igual al producto que se obtiene en fluidos newtonianos ${ }^{2}$ :

$$
f \times R e_{M R}=16
$$

donde $\mu_{g, M R}$ es la viscosidad generalizada según Metzner y Reed (1955), que depende (para esta geometría) de $u_{b}$, el $D_{h}, m$ y $n$.

Así, mediante esta definición de la viscosidad y, consecuentemente, del número de Reynolds (Fig. 2.2(b)) se consiguen tres objetivos:

- El producto de $f \times R e_{M R}$ en régimen laminar es totalmente independiente de $n$.

- En consecuencia, la curva de $f=f\left(R e_{M R}\right)$ en régimen laminar es única para la geometría de tubo liso.

- El número de Reynolds se reduce al newtoniano cuando $n=1$.

\footnotetext{
${ }^{2}$ De esta forma se mantiene la universalidad de los resultados, ya que para $n=1$ tanto el número de Reynolds $\operatorname{Re}_{M R}$ como $f \times R e_{M R}$ se reducen a los valores obtenidos para fluidos newtonianos.
} 
Posteriormente, Kozicki et al. (1966) y Delplace y Leuliet (1995) introdujeron sucesivos desarrollos sobre el método: el primer autor obtuvo la relación entre $f \times R e_{b}$ para algunas geometrías simples (placas paralelas, tubos concéntricos y tubos de sección transversal de formas triangular, cuadrangular y rectangular) en función de dos parámetros $A$ y $B$ de carácter geométrico:

$$
f \times R e_{b}=2 \times 8^{n}\left(\frac{A+B n}{n}\right)^{n}
$$

Es debido a las conclusiones de Kozicky que algunos autores definen el número de Reynolds del siguiente modo:

$$
R e_{k o}=\frac{\rho u_{b}^{2-n} D_{h}^{n}}{m \times 8^{n-1}\left(\frac{A+B n}{n}\right)^{n}}
$$

De modo que se cumpla: $f \times R e_{k o}=16$. Sin embargo esta definición puede llevar a situaciones en las que el número de Reynolds $\left(R e_{k o}\right)$ no se reduzca al número de Reynolds newtoniano para $n=1$. Situación que se produce precisamente en geometrías en las que $f \times \operatorname{Re}(n=1) \neq 16$ (fluidos newtonianos).

Son Delplace y Leuliet (1995) quienes resuelven hábilmente dicha situación y al mismo tiempo reducen el número de parámetros geométricos a uno, que además tiene un significado físico. Los autores definen el parámetro $\xi$ como la mitad del producto de $f \times R e$ que tendría un fluido newtoniano en una geometría dada:

$$
f \times R e=2 \xi
$$

De este modo obtienen que en fluidos no newtonianos, el producto del factor de Fanning por el número de Reynolds que hemos llamado básico, tiene la siguiente forma:

$$
f \times R e_{b}=2 \times \xi^{n}\left(\frac{24 n+\xi}{(24+\xi) n}\right)^{n}
$$

Con lo que para todas las geometrías estudiadas por Kozicky, se podría definir la viscosidad generalizada de forma que para $n=1$ el número de Reynolds siempre se redujese a su definición para fluidos newtonianos

$$
\mu_{g, D L}=m\left(\frac{u_{b}}{D_{h}}\right)^{n-1} \xi^{n-1}\left(\frac{24 n+\xi}{(24+\xi) n}\right)^{n}
$$




\begin{tabular}{|c|c|c|c|}
\hline Subíndice & Denominación & Aplicabilidad & Viscosidad \\
\hline$b$ & Básica/o & Universal & $\mu_{b}=m\left(\frac{u_{b}}{D_{h}}\right)^{n-1}$ \\
\hline$M R$ & Metzner y Reed & Tubo liso & $\mu_{g, M R}=\mu_{e f, b} \times 8^{n-1}\left(\frac{3 n+1}{4 n}\right)^{n}$ \\
\hline ko & Kozicki & $\begin{array}{l}\text { Geometrías símples con } \\
\text { sección de paso constante }\end{array}$ & $\mu_{g, k o}=\mu_{e f, b} \times 8^{n-1}\left(\frac{A+B n}{n}\right)^{n}$ \\
\hline$D L$ & Delplace-Leuliet & Mismas que $k o$ & $\mu_{g, D L}=\mu_{e f, b} \times \xi^{n-1}\left(\frac{24 n+\xi}{(24+\xi) n}\right)^{n}$ \\
\hline$g$ & experimental & Tubo con rascador & $\mu_{g}=m n^{d} c^{n-1}\left(\frac{u_{b}}{D_{h}}\right)^{r}$ \\
\hline
\end{tabular}

Cuadro 2.1: Posibles definiciones de la viscosidad generalizada expuestas a lo largo del capítulo. Dichas definiciones se utilizan en las definiciones de $\operatorname{Pr}$ y $R e$, las cuales se denotan también con los subíndices de la primera columna. . La definición experimental se obtiene posteriormente en el Apartado 5.3 y los valores de las constantes de la misma se encuentran en la Tabla 5.8.

de modo que

$$
R e_{D L}=\frac{\rho u_{b}^{2-n} D_{h}^{n}}{m \times \xi^{n-1}\left(\frac{24 n+\xi}{(24+\xi) n}\right)^{n}}
$$

y que cumple

$$
f \times R e_{D L}=2 \xi
$$

siendo $\xi$ un parámetro geométrico con significado medible.

A modo de resumen, en la Tabla 2.1 se detallan las distintas definiciones del número de la viscosidad y el número de Reynolds y su utilidad.

\section{Obtención de $\xi$ para las geometrías estudiadas por Kozicki et al. (1966)}

Para obtener el valor de $\xi$ de geometrías simples, se recurre a los estudios de Kozicki et al. (1966). El autor obtuvo las correlación dada por la Ec. 2.12 entre la caída de presión adimensional y el número de Reynolds, para diferentes geometrías simples de tubos. Donde $A$ y $B$ son parámetros geométricos.

Para establecer la analogía entre la formulación de Kozicky (Ec. 2.12) y Delplace (Ec. 2.14), basta con considerar un comportamiento newtoniano, $n=1$. De forma que se obtiene la fórmula que relaciona $\xi \operatorname{con} A$ y $B$ :

$$
\xi=8(A+B)
$$




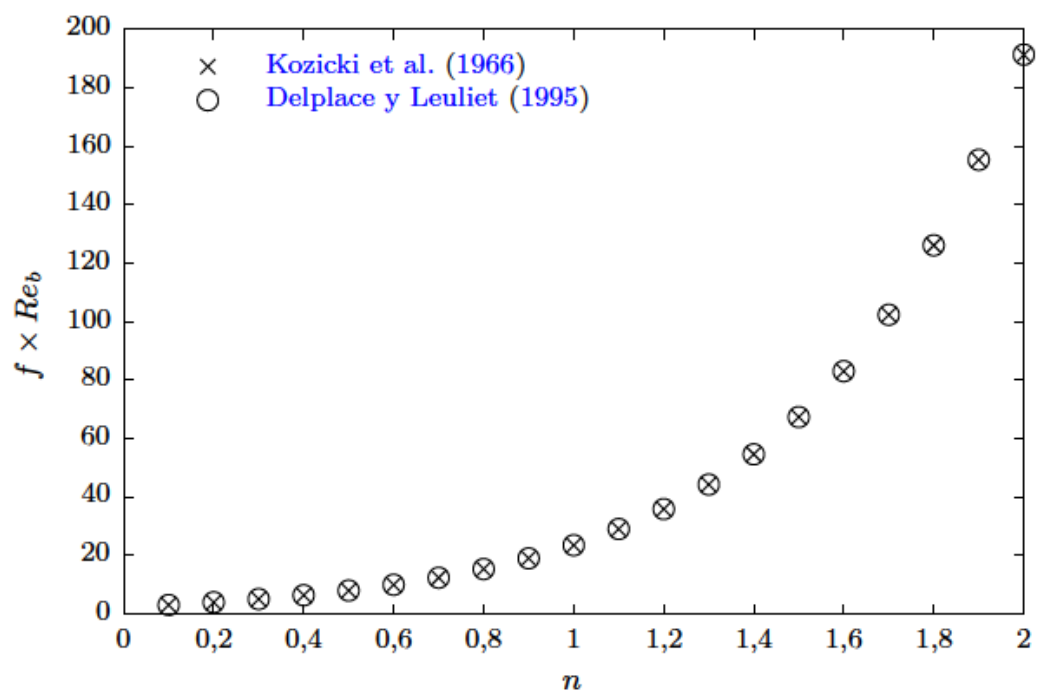

Figura 2.3: Precisión de la aproximación de Delplace-Leuliet. Se representa el producto $f \times R e$ en geometría de ejes concéntricos para $0,1 \leq n \leq 2$ y $\alpha=5 / 18$, donde se utiliza la definición del número de Reynolds dada por la Ec. 2.14 (formulación de Delplace-Leuliet) o por la Ec. 2.12 (formulación de Kozicky).

\begin{tabular}{cccc}
\hline & $A$ & $B$ & $\xi$ \\
\hline Tubo liso & 0,75 & 0,25 & 8 \\
Tubo sección anular & 0,4789 & 0,9823 & 11,69 \\
\hline
\end{tabular}

Cuadro 2.2: Valores de los parámetros geométricos $A, B$ y $\xi$ para las geometrías de interés para la investigación: tubo liso y conducto anular con $\alpha=5 / 18$.

En la Fig. 2.3 se comprueba que las expresiones de Delplace-Leuliet y Kozicky son totalmente equivalentes. En ella se representa el producto $f \times R e$ según la formulación del número de Reynolds de ambos autores (Ec. 2.15 y Ec. 2.13 respectivamente), en geometría de ejes concéntricos.

En la Tabla 2.2, se muestran los valores de $A, B$ y $\xi$ para las geometrías simples que pueden resultar de interés en la presente investigación.

La obtención de los parámetros $A$ y $B$ para geometría anular, vienen dados en función de la relación de diámetros $\alpha=d / D$ :

$$
A=\frac{(1-\alpha)^{2}}{4\left[1-\frac{1-\alpha^{2}}{2 \ln (1 / \alpha)}\left(1-\ln \frac{1-\alpha^{2}}{2 \ln (1 / \alpha)}\right)\right]}
$$




$$
B=\frac{(1-\alpha)^{2}}{1+\alpha^{2}-\frac{1-\alpha^{2}}{\ln (1 / \alpha)}}-A
$$

\subsection{Conclusiones}

A continuación se detallan las conclusiones obtenidas en los diferentes apartados del capítulo:

- Del análisis del problema fluidomecánico se extraen las siguientes conclusiones:

1. El estudio de la caída de presión en régimen laminar en el intercambiador de calor mejorado, donde el ratio de diámetros se mantiene constante, se reduce a obtener la relación existente entre $R e_{b}, n$ y $f$.

2. La caída de presión en tubos de flujos de fluidos Power Law, para caudal y dimensiones del tubo conocidas, depende tanto de $m$ como de $n^{3}$.

- La solución del problema térmico en régimen laminar en el intercambiador de calor mejorado se reduce a obtener la relación existente entre $R_{b}, n, \operatorname{Pr}_{b}$ y $N u$ para un valor constante de $d / D$.

- En situaciones en las que el rascador esté dotado de movimiento (ŕegimen de rascador dinámico), la solución del problema tanto fluidomecánico como término depende de un número adimensional adicional, el factor de bloqueo, $\beta$.

- En cuanto al estudio realizado sobre la definición de la viscosidad más apropiada a utilizar en los números de Reynolds y Prandtl (método de generalización) se extraen las siguientes conclusiones:

1. Gracias a las investigaciones de Metzner y Reed (1955), de Kozicki et al. (1966) y de Delplace y Leuliet (1995), se puede redefinir el número de Reynolds generalizado en función de la viscosidad generalizada del fluido, de forma que la relación entre éste y el factor de fricción de Fanning sea independiente del Índice de comportamiento de flujo, $n$, en régimen laminar. Reduciendo así los grados de libertad del problema.

\footnotetext{
${ }^{3}$ Entre otros factores
} 
2. A partir de los estudios existentes hasta el momento, ésta viscosidad generalizada únicamente se puede definir para geometrías de sección transversal constante.

3. La definición de este número de Reynolds depende de $\xi$, que toma un valor diferente para cada geometría tubo. El parámetro $\xi$ se puede obtener:

a) en geometrías simples: de los estudios de Kozicky.

b) en geometrías con sección transversal constante más compleja: determinando experimentalmente el valor de $\xi$ a partir de la Ec. 2.16.

4. En el siguiente capítulo (Apartado 5.3) se desarrollará un método experimental para la generalización de la viscosidad del flujo, tal que el la dependencia de $n$ desaparezca de la relación entre $f$ y $R e_{g}$ en geometrías con sección de paso variable. En el caso estudiado del tubo con rascador $f=\Psi\left(R e_{g}, \alpha\right)$

5. Con el uso de las definiciones de $R e_{g}, \mu_{g}$ y $P r_{g}$, cuya definición se concreta en el Capítulo 5, se consigue reducir la complejidad de los problemas fluidomecánico y térmico. 


\section{Capítulo 3}

\section{Modelo numérico}

En capítulos anteriores se ha descrito la geometría del intercambiador de calor analizado, la cual guarda una gran similitud con una geometría de tubos concéntricos, diferenciándose ambas únicamente en la presencia de los tacos. Tanto es así, que el estudio del flujo en la geometría de tubos concéntricos, puede servir de base para analizar el comportamiento del flujo en el tubo con rascador y explicar, en parte, las características del mismo. Además, en los capítulos sucesivos, el comportamiento del flujo en tubo liso se utilizará como base comparativa para los resultados obtenidos en el intercambiador de superficie rascada estudiado.

Para el caso de flujo incompresible estacionario de fluidos newtonianos, tanto el perfil de velocidades, como la caída de presión en el tubo con eje se pueden obtener de forma analítica. No obstante, si el fluido presenta un comportamiento representado por el modelo Power Law, el perfil de velocidades no tiene solución analítica para cualquier valor de $n$, con lo que es necesario recurrir a métodos numéricos, bien mediante la resolución de las integrales resultantes en el problema o bien mediante métodos de simulación del flujo.

En el presente capítulo se opta por la segunda de las opciones, desarrollando un método de simulación del flujo CFD, en el cual las ecuaciones de continuidad, cantidad de movimiento y energía son discretizadas mediante diferentes métodos.

El planteamiento general del problema es el siguiente: geometría de tubos concéntricos, donde el tubo interior puede estar dotado de movimiento axial, el flujo es incompresible, estacionario, laminar, el fluido es seudoplástico y sigue el modelo Power Law. Se estudia tanto el caso isotermo como el llamado problema de condición H (flujo de calor constante en la pared del tubo exterior). En el mismo código se implementa la opción de que no exista eje (tubo liso). 
Por motivos de optimización de los recursos de computación, en el presente capítulo se elaboran dos códigos de simulación en Matlab con objetivos distintos:

- Modelo 1 (problema isotermo): obtención del perfil de velocidades y caída de presión del flujo desarrollado en condiciones isotermas.

- Modelo 2 (problema de condición H): desarrollo fluidomecánico y térmico del flujo.

Ambos códigos serán válidos tanto para la geometría de tubo liso como la de tubo con eje.

\subsection{Flujo en tubos concéntricos. Fundamentos}

Tal y como se ha mencionado, el problema planteado tiene solución analítica si el fluido de trabajo es newtoniano. Pero si el fluido es no newtoniano $(n \neq 1)$ el problema no se puede resolver más que para algunos valores de $n$. En el presente apartado se detallan las soluciones analíticas existentes y se concretan los casos en los que no se dispone de solución. Lo expuesto en el presente apartado justifica el uso de modelos numéricos para la simulación del flujo.

Fluido newtoniano. En este caso, la expresión del perfil de velocidades en un conducto de sección anular con el eje interior en movimiento, con velocidad adimensional $\omega=v_{s} / u_{b}$, viene dado por la siguiente expresión:

$$
\begin{gathered}
u_{z}^{*}=\frac{1-\omega\left(\frac{1}{2 \ln (1 / \alpha)}-\frac{\alpha^{2}}{1-\alpha^{2}}\right)}{0,5\left(\frac{1-\alpha^{4}}{1-\alpha^{2}}-\frac{1-\alpha^{2}}{\ln (1 / \alpha)}\right)}\left[1-\left(\frac{r}{R}\right)^{2}-\frac{1-\alpha^{2}}{\ln (1 / \alpha)} \ln \left(\frac{R}{r}\right)\right]+\omega \frac{\ln (R / r)}{\ln (1 / \alpha)} \\
\alpha=R_{\text {eje }} / R
\end{gathered}
$$

Fluido no newtoniano. Para fluidos no newtonianos, si el eje se encuentra estático $\omega=0$, el perfil de velocidades viene dado por la expresión:

$$
\begin{aligned}
& u_{z, i}=R\left(-p_{L} \frac{R}{2 m}\right)^{1 / n} \int_{\alpha}^{r^{*}}\left(\frac{\lambda^{2}}{x}-x\right)^{1 / n} d x ; \quad \alpha<r^{*}<\lambda \\
& u_{z, e}=R\left(-p_{L} \frac{R}{2 m}\right)^{1 / n} \int_{r^{*}}^{1}\left(x-\frac{\lambda^{2}}{x}\right)^{1 / n} d x ; \lambda<r^{*}<1
\end{aligned}
$$


, donde $\lambda$ es la posición radial adimensional a la que se encuentra el punto de máxima velocidad, mientras que $u_{z, i}$ y $u_{z, e}$ representan la velocidad en las regiones interior y exterior en las que se divide el flujo. Por lo tanto si el valor de $\lambda$ es conocido, también lo sería el perfil de velocidades. Además a partir del mismo se pueden obtener tanto el caudal como la velocidad media del flujo.

La expresión analítica del caudal fue obtenida por Hanks y Larsen (1979).

$$
Q=\frac{n \pi R^{3}}{(3 n+1)}\left(-p_{L} \frac{R}{2 m}\right)^{1 / n} F(\lambda)
$$

, donde

$$
F(\lambda)=\left\{\left(1-\lambda^{2}\right)^{(n+1) / n}-\alpha^{(n-1) / n}\left(\lambda^{2}-\alpha^{2}\right)^{(n+1) / n}\right\}
$$

La expresión velocidad media se obtiene a partir de las Ecs. 3.2 y 3.3:

$$
u_{b}=\frac{n}{(3 n+1)} \frac{R}{\left(1-\alpha^{2}\right)}\left(\frac{-\Delta p \times R}{L \times 2 m}\right)^{1 / n} F(\lambda)
$$

La clave por lo tanto reside en obtener el valor de $\lambda$. Éste se evalúa igualando las velocidades de las regiones interior y exterior (Ec. 3.2) en el punto de máxima velocidad: $\left.u_{z, i}\right|_{r^{*}=\lambda}=\left.u_{z, e}\right|_{r^{*}=\lambda}$.

$$
\int_{\alpha}^{\lambda}\left(\frac{\lambda^{2}}{x}-x\right)^{1 / n} d x=\int_{\lambda}^{1}\left(x-\frac{\lambda^{2}}{x}\right)^{1 / n} d x
$$

Las integrales que aparecen en las expresiones de la velocidad (y de $\lambda$ ) únicamente son evaluables analíticamente en valores enteros de 1/n. Es por ello que Hanks y Larsen (1979) evaluaron la expresión anterior de forma numérica, y obtuvieron los valores de $\lambda$ en función de $\alpha$ y $n$, dando como resultado la Tabla 3.1.

A partir del valor de $\lambda$ obtenido en dicha tabla, mediante interpolación para los valores de $\alpha=0,25$ y de $n$ correspondiente a cada ensayo en particular, se puede obtener el perfil de velocidades no newtoniano en geometría de tubos concéntricos donde el eje interior se encuentra en reposo, evaluando numéricamente las integrales que aparecen en las ecuaciones de la velocidad. Esta solución es válida únicamente si el eje central se encuentra estático. 


\begin{tabular}{cccccccccc}
\hline \multirow{2}{*}{$n$} & \multicolumn{10}{c}{$\alpha$} \\
\cline { 2 - 10 } & 0,1 & 0,2 & 0,3 & 0,4 & 0,5 & 0,6 & 0,7 & 0,8 & 0,9 \\
\hline 0,1 & 0,3442 & 0,4687 & 0,5632 & 0,6431 & 0,7140 & 0,7788 & 0,8389 & 0,8954 & 0,9489 \\
0,2 & 0,3682 & 0,4856 & 0,5749 & 0,6509 & 0,7191 & 0,7818 & 0,8404 & 0,8960 & 0,9491 \\
0,3 & 0,3884 & 0,4991 & 0,5840 & 0,6570 & 0,7229 & 0,7840 & 0,8416 & 0,8965 & 0,9492 \\
0,4 & 0,4052 & 0,5100 & 0,5912 & 0,6617 & 0,7259 & 0,7858 & 0,8426 & 0,8969 & 0,9493 \\
0,5 & 0,4193 & 0,5189 & 0,5970 & 0,6655 & 0,7283 & 0,7872 & 0,8433 & 0,8972 & 0,9493 \\
0,6 & 0,4312 & 0,5262 & 0,6018 & 0,6686 & 0,7303 & 0,7884 & 0,8439 & 0,8975 & 0,9494 \\
0,7 & 0,4412 & 0,5324 & 0,6059 & 0,6713 & 0,7319 & 0,7893 & 0,8444 & 0,8977 & 0,9495 \\
0,8 & 0,4498 & 0,5377 & 0,6093 & 0,6735 & 0,7333 & 0,7902 & 0,8449 & 0,8979 & 0,9495 \\
0,9 & 0,4572 & 0,5422 & 0,6122 & 0,6754 & 0,7345 & 0,7909 & 0,8452 & 0,8980 & 0,9495 \\
1 & 0,4637 & 0,5461 & 0,6147 & 0,6770 & 0,7355 & 0,7915 & 0,8455 & 0,8981 & 0,9496 \\
\hline
\end{tabular}

Cuadro 3.1: Valores de $\lambda$ para geometría de tubos concéntricos. Hanks y Larsen (1979).

\subsection{Modelo numérico del flujo desarrollado}

Al no existir una solución analítica para el flujo de fluidos no newtonianos en la geometría, se opta por resolver el problema mediante métodos de discretización. Así, se desarrolla un modelo de simulación numérica para resolver el problema fluidomecánico del flujo desarrollado. Mediante este modelo se puede obtener tanto el perfil de velocidades como la caída de presión en dicho flujo. Además se pueden simular situaciones (de flujo estacionario) en las que el eje interior tenga una velocidad en dirección longitudinal.

Este modelo únicamente es válido para la resolución del problema fluidomecánico, es decir en condiciones isotermas.

Para ello se parte de las ecuaciones de Navier-Stokes, que en el Capítulo 2 se simplifican para la geometría de ejes concéntricos y flujo incompresible y estacionario, quedando las ecuaciones de continuidad y de cantidad de movimiento como siguen:

$$
\begin{gathered}
\frac{\partial u_{z}}{\partial z}=0 \\
\frac{\partial p}{\partial z}=\frac{1}{r} \frac{\partial}{\partial r}\left(r m\left(\frac{\partial u_{z}}{\partial r}\right)^{n}\right)
\end{gathered}
$$

El método de discretización elegido es el de volúmenes finitos. Así, se divide el dominio transversal al flujo en $N N$ volúmenes finitos, donde cada uno tiene un espesor $\Delta r$ y una dimensión en dirección longitudinal $\Delta x$. El mallado realizado se encuentra representado de 


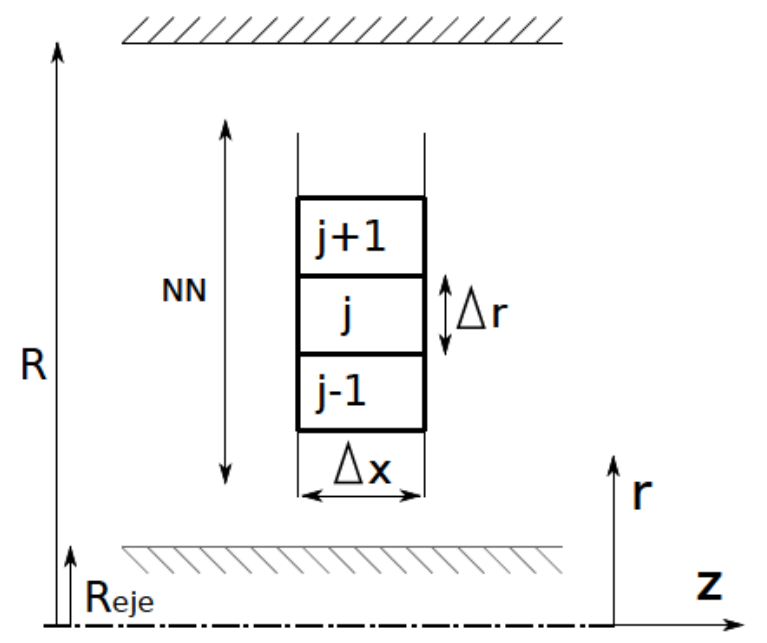

(a) Mallado.

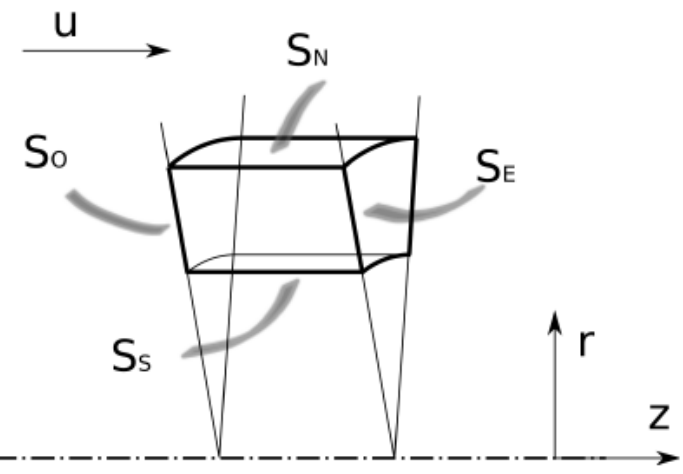

(b) Superficies de celda.

Figura 3.1: Esquema del mallado correspondiente al modelo numérico del flujo desarrollado.

forma esquemática en la Fig. 3.1, donde en el caso de simular el flujo en tubo liso: $R_{e j e}=0$ y las celdas llegarían hasta el eje del tubo.

La ecuación de continuidad se aplica simplemente con la condición de que el caudal se conserva en cada volumen finito. En cuanto a la ecuación de cantidad de movimiento, el método de discretización por volúmenes finitos seguido se detalla en el Apartado B.1 del Apéndice. La ecuación discretizada tiene la siguiente forma:

$$
0=p_{L} V-\mu_{S}\left(\frac{u_{j}-u_{j-1}}{\Delta r_{S}}\right) S_{S}+\mu_{N}\left(\frac{u_{j+1}-u_{j}}{\Delta r_{N}}\right) S_{N}
$$

, donde se define la viscosidad en las caras norte y sur de cada volumen finito respectivamente como

$$
\begin{aligned}
& \mu_{S}=m\left(\frac{u_{j}-u_{j-1}}{\Delta r_{S}}\right)^{n-1} \\
& \mu_{N}=m\left(\frac{u_{j+1}-u_{j}}{\Delta r_{N}}\right)^{n-1}
\end{aligned}
$$

\section{Condiciones de contorno}

Las condiciones de contorno del problema son las siguientes:

1. Para $r=R_{e j e}$, la velocidad del flujo es la del eje $u_{z}=v_{s}$. Que planteada de forma adimensional sería: $r^{*}=\alpha, u_{z}^{*}=\omega$. 
2. Para $r=R$, la velocidad del flujo es la del tubo exterior $u_{z}=0$. Que en forma adimensional sería $r^{*}=1, u_{z}^{*}=0$.

\section{Sistema de ecuaciones discretas}

Llegados a este punto se procede a plantear el sistema de ecuaciones discretas que conforma el método numérico. La primera ecuación y la última son las referidas a los nodos en la pared del eje y en la pared del tubo $(1 \mathrm{y} N N+2)$. En ellas se establecen las condiciones de contorno. Las ecuaciones desde 2 hasta $N N+1$, corresponden al planteamiento de la ecuación de cantidad de movimiento en cada volumen finito (Ec. 3.7). La última ecuación corresponde a la Ec. de continuidad, por la cual se cumple que el caudal indicado como condición de entrada se debe repartir entre los volúmenes finitos considerados (se considera que los volúmenes finitos son coronas circulares).

$$
\begin{aligned}
E: 1) & u_{1}=v_{s} \\
E: j=2, N N+1) & \mu_{S} u_{j-1} \frac{S_{S}}{\Delta r_{S}}-\left(\mu_{S} \frac{S_{S}}{\Delta r_{S}}+\mu_{N} \frac{S_{N}}{\Delta r_{N}}\right) u_{j}+\mu_{N} u_{j+1} \frac{S_{N}}{\Delta r_{N}}+p_{L} V=0 \\
E: N N+2) & u_{N N+2}=0 \\
E: N N+3) & \sum u_{j} S_{E}=Q
\end{aligned}
$$

En caso de que el fluido fuese newtoniano, $n=1$ y $\mu_{S}=\mu_{N}=m$, el cálculo se realiza en una sola iteración en la que se resuelven las ecuaciones en los nodos. En este caso la precisión de la solución viene dada por el número de volúmenes finitos considerados $N N$.

En caso de que el fluido tenga un comportamiento no newtoniano, $n \neq 1$, la viscosidad depende del perfil de velocidades y este a su vez de la viscosidad. En consecuencia, es necesario seguir el proceso iterativo siguiente:

1. Se resuelve el campo de velocidades para $n=1$.

2. Con el perfil de velocidades obtenido se calculan las viscosidades en las caras norte y sur de cada volumen finito.

3. Se calcula el nuevo campo de velocidades con las viscosidades calculadas.

4. Vuelta al paso 2 hasta que la solución converge. Se establece como criterio de convergencia la reducción de los residuos de la viscosidad por debajo de un valor configurable. 


\subsubsection{Validación del modelo}

La validación del modelo numérico presentado se realiza en 3 etapas:

1. Validación del caso newtoniano. Se compara el perfil newtoniano $(n=1)$ obtenido de forma numérica con la solución analítica (Ec. 3.1) para diferentes valores de omega, incluyendo el caso estático.

2. Validación del caso no newtoniano. Se compara la solución obtenida para fluidos seudoplásticos $(n<1)$ mediante el método numérico descrito y mediante el método desarrollado por Hanks y Larsen (1979), que obtiene el valor de $\lambda$ de forma numérica (resolviendo la Ec. 3.4) y a partir de este valor resuelve las integrales que expresan el perfil de velocidades (ecuaciones 3.2 y 3.3 ).

3. Validación de la caída de presión. Para diferentes valores de $n$, se comprueba que la caída de presión cumple la ecuación de Delplace y Leuliet (1995) del producto $f \times R e_{D L}$, basada en las relaciones aproximadas obtenidas por Kozicki et al. (1966).

$$
f \times R e_{D L}=f \times R e_{b} /\left(\xi^{n}\left(\frac{24 n+\xi}{(24+\xi) n}\right)^{n}\right)=2 \times \xi
$$

, donde $\xi$ es un factor geométrico que se obtiene a partir de las Ecs. 2.17, 2.18 y 2.19.

Los resultados de la validación se muestran gráficamente en la Fig 3.2 y las desviaciones de los resultados de la simulación respecto a los valores de referencia en la Tabla 3.2. En ellos se observa que las desviaciones se reducen al aumentar el número de celdas en la sección. En cuanto al perfil de velocidades obtenido mediante simulación, las desviaciones del modelo para $N N=100$ son menores del $0,3 \%$ de $u_{b}$ en todos los casos. En el caso de la caída de presión las desviaciones de $f \times R e_{D P}$ son menores menores del $1 \%$, aunque en este último caso se debe considerar que la solución de Kozicky para dicho producto en geometría de tubos concéntricos es exacta cuando $n=1$, pero para valores distintos de $n$ la solución es una aproximación. 


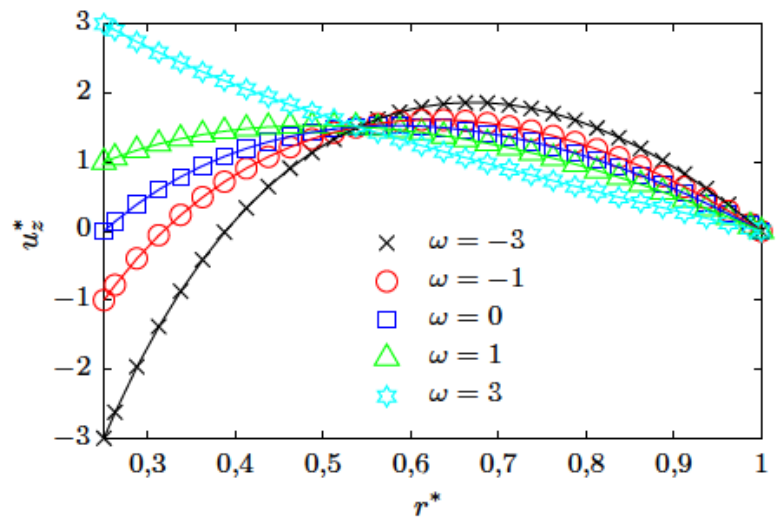

(a) Fluido newtoniano a diferentes velocidades de rascado.

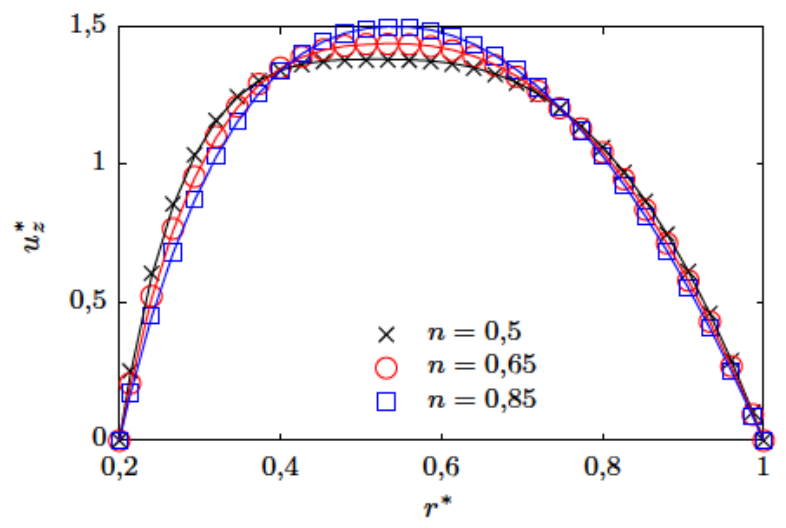

(b) Fluido no newtoniano con diferentes valores de $n$.

Figura 3.2: Perfiles de velocidad en geometría de tubos concéntricos donde el tubo interior se mueve con una velocidad adimensional $\omega=v_{s} / u_{b}$. Mediante símbolos varios se representan los valores obtenidos mediante el método numérico descrito. Mediante línea continua se representa, en la Fig. 3.2(a), la solución analítica del perfil de velocidades (Ec. 3.1) y en la Fig 3.2(b) la solución numérica obtenida por Hanks y Larsen (1979). 
(a) Perfil de velocidad del flujo newtoniano $(n=1) \cdot \alpha=0,25$.

\begin{tabular}{cccccc}
\hline \multirow{2}{*}{$N$} & \multicolumn{5}{c}{$\omega$} \\
\cline { 2 - 6 } & -3 & -1 & 0 & 1 & 3 \\
\hline 30 & $1,11 \%$ & $0,67 \%$ & $0,46 \%$ & $0,24 \%$ & $0,19 \%$ \\
50 & $0,42 \%$ & $0,26 \%$ & $0,17 \%$ & $0,09 \%$ & $0,07 \%$ \\
100 & $0,11 \%$ & $0,07 \%$ & $0,05 \%$ & $0,02 \%$ & $0,02 \%$ \\
200 & $0,03 \%$ & $0,02 \%$ & $0,01 \%$ & $<0,01 \%$ & $<0,01 \%$ \\
\hline
\end{tabular}

(b) Perfil de velocidad para $0,5 \leq n<$

1. $\alpha=0,2$.

\begin{tabular}{cccc}
\hline \multirow{2}{*}{$N N$} & \multicolumn{3}{c}{$n$} \\
\cline { 2 - 4 } & 0,5 & 0,65 & 0,85 \\
\hline 30 & $1,85 \%$ & $1,24 \%$ & $0,78 \%$ \\
50 & $0,73 \%$ & $0,49 \%$ & $0,30 \%$ \\
100 & $0,19 \%$ & $0,30 \%$ & $0,10 \%$ \\
200 & $0,05 \%$ & $0,32 \%$ & $0,12 \%$ \\
\hline
\end{tabular}

(c) Caída de presión adimensional, $f_{\infty} \cdot \alpha=0,25$.

\begin{tabular}{ccccc}
\hline \multirow{2}{*}{$N N$} & \multicolumn{4}{c}{$n$} \\
\cline { 2 - 5 } & 0,5 & 0,65 & 0,85 & 1 \\
\hline 30 & $1,29 \%$ & $1,00 \%$ & $0,58 \%$ & $0,26 \%$ \\
50 & $1,12 \%$ & $0,84 \%$ & $0,42 \%$ & $0,09 \%$ \\
100 & $1,04 \%$ & $0,76 \%$ & $0,35 \%$ & $0,02 \%$ \\
200 & $1,02 \%$ & $0,75 \%$ & $0,33 \%$ & $<0,01 \%$ \\
\hline
\end{tabular}

Cuadro 3.2: Desviaciones de los resultados de simulación respecto a la solución de referencia en función del caso simulado y de la malla. Los errores de velocidad se definen como tanto por cien de $u_{b}$.

\subsection{Modelo numérico del desarrollo del flujo}

El modelo numérico que se describe en el presente apartado, se puede utilizar para simular diferentes problemas, los cuales tienen en común las características de flujo estacionario, incompresible y cuyo comportamiento seudoplástico sigue el modelo Power Law. A continuación se detallan las situaciones que se pueden simular con el código implementado:

- Desarrollo del flujo isotermo. El código permite estudiar la región de entrada del flujo isotermo en determinadas circunstancias.

- Problema térmico con flujo de calor constante (condición H).

- Desarrollo térmico y fluidomecánico a partir de la sección de entrada.

- Desarrollo térmico cuando el perfil de velocidades ya se encuentra desarrollado. Este caso es el más parecido a lo que ocurre en la instalación de ensayos termohidráulicos y por tanto el que se estudia en mayor profundidad.

Además de simular el flujo de fluidos no newtonianos, el comportamiento newtoniano se puede obtener haciendo $n=1$. 


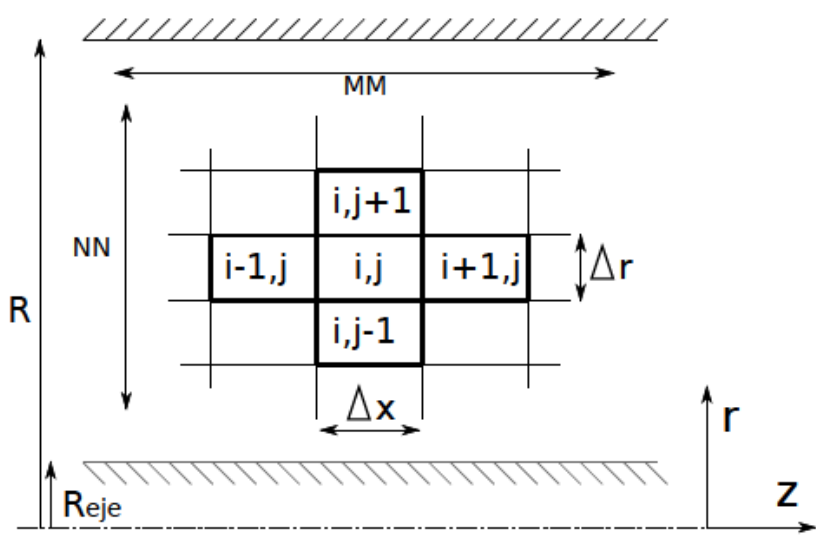

(a) Mallado.

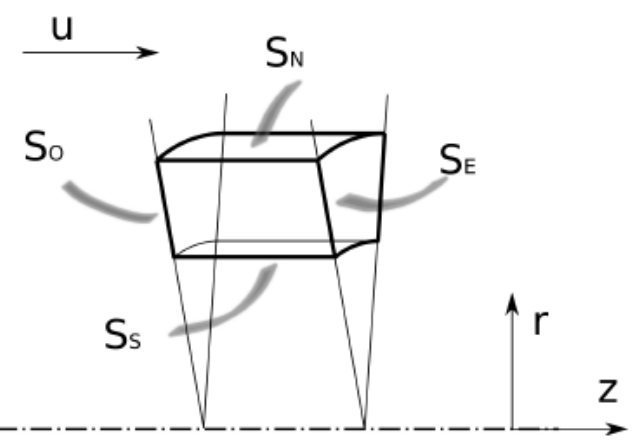

(b) Superficies de celda.

Figura 3.3: Esquema del mallado correspondiente al modelo numérico del desarrollo del flujo.

El mallado del espacio fluido es similar al del modelo anterior, pero considerando ahora una longitud del tubo dividida en $M M$ secciones. En la Fig. 3.3 se muestra un esquema representativo del mismo, donde en el caso de simular el flujo en tubo liso: $R_{e j e}=0$.

\subsubsection{Ecuaciones del modelo}

Al igual que en el modelo anterior, en éste se desprecian las diferencias de presión en dirección radial, las cuales pueden ser significativas en la región de entrada. Los errores cometidos a raíz de esta simplificación se cuantifican en el apartado de validación.

\section{Ecuación de Cantidad de Movimiento}

Comenzando con la ecuación de cantidad de movimiento en dirección axial, respecto al modelo anterior, al encontrarse el flujo en desarrollo, se deben añadir los términos convectivos. Además, el término de la difusión axial puede ser importante en la región de entrada. La importancia de este último término se estudia en el Apartado 3.3.3.1 (validación de longitud de desarrollo), donde se observa que tiene influencia significativa en la solución del problema en tubo liso para números de Reynolds $R e_{M R}<20$. La inclusión de dicho término en las ecuaciones del modelo implica aumentar el gasto computacional necesario significativamente. Además, en capítulos posteriores no se hace uso específico del simulador para estudiar la región de entrada en el problema fluidomecánico, así que este término no ha sido incluido finalmente en el código ${ }^{1}$. Finalmente la ecuación de cantidad de movimiento queda del

\footnotetext{
${ }^{1}$ Se han realizado pruebas con un modelo incluyendo la disipación viscosa en dirección axial, obteniendo resultados similares a los de Poole y Ridley.
} 
siguiente modo:

$$
\rho\left(u_{z} \frac{\partial u_{z}}{\partial z}+u_{r} \frac{\partial u_{z}}{\partial r}\right)=-\frac{\partial p}{\partial z}+\frac{1}{r} \frac{\partial}{\partial r}\left(r m\left(\frac{\partial u_{z}}{\partial r}\right)^{n}\right)
$$

La discretización de la ecuación se realiza combinando diferentes métodos: sobre el término convectivo en $z$ se aplica un método upwind de primer orden y sobre el término convectivo en $r$ un método de diferencias centrales. El resto de términos no varían respecto al modelo anterior (volúmenes finitos).

$$
\begin{array}{r}
-\rho u_{i-1, j} u_{i-1, j} S_{E}-\rho u_{i-1, j} u_{i, j} S_{E}+\rho v_{i, j-1} \frac{u_{i, j}-u_{i, j-1}}{2} S_{S}-\rho v_{i, j} \frac{u_{i, j}-u_{i, j+1}}{2} S_{N}= \\
p_{L} V-\mu_{S}\left(\frac{u_{i, j}-u_{i, j-1}}{\Delta r_{S}}\right) S_{S}+\mu_{N}\left(\frac{u_{i, j+1}-u_{i, j}}{\Delta r_{N}}\right) S_{N}
\end{array}
$$

Las condiciones de contorno del problema fluidomecánico son las mismas que en el modelo anterior:

1. Para $r=R_{e j e}$, la velocidad del flujo es la del eje $u_{z}=v_{s}$. Que planteada de forma adimensional sería: $r^{*}=\alpha, u_{z}^{*}=\omega$.

2. Para $r=R$, la velocidad del flujo es la del tubo exterior $u_{z}=0$. Que en forma adimensional sería $r^{*}=1, u_{z}^{*}=0$.

\section{Sistema de ecuaciones del problema fluidomecánico.}

Las ecuaciones de continuidad y cantidad de movimiento en dirección radial no varían respecto al modelo anterior, quedando el siguiente sistema de ecuaciones, donde ECM es la Ec. 3.10:

$$
\begin{aligned}
E: 1) & u_{1}=v_{s} \\
E: j=2, N N+1) & E C M \\
E: N N+2) & u_{N N+2}=0 \\
E: N N+3) & \sum u_{j} S_{E}=Q
\end{aligned}
$$

\section{Ecuación de la energía}

En cuanto a la ecuación de la energía, de nuevo aparecen los términos convectivos al incluir 
el caso del flujo en desarrollo. El término de disipación viscosa puede ser significativo en estos flujos tan viscosos, el cual para fluidos Power Law tiene la siguiente forma:

$$
\tau_{r z}\left(\frac{\partial u_{z}}{\partial r}\right)=m\left(\frac{\partial u_{z}}{\partial r}\right)^{n+1}=m\left(\frac{\partial u_{z}}{\partial r}\right)^{n-1}\left(\frac{\partial u_{z}}{\partial r}\right)^{2}
$$

, de modo que la ecuación de la energía queda:

$$
\rho c_{p}\left(u_{z} \frac{\partial T}{\partial z}+u_{r} \frac{\partial T}{\partial r}\right)=\frac{1}{r} \frac{\partial}{\partial r}\left(r k \frac{\partial T}{\partial r}\right)+m\left(\frac{\partial u_{z}}{\partial r}\right)^{n+1}
$$

La importancia del término de disipación viscosa se discute en el Apartado B.2 y se decide no considerarlo.

Así, la ecuación de la energía en el flujo en desarrollo (Ec. 3.11) discretizada, tiene la misma forma que la de cantidad de movimiento, donde $\phi$ es ahora la temperatura, el término fuente ha desaparecido y donde aparecía la viscosidad ahora aparece $k / \rho c_{p}$.

$$
\begin{array}{r}
-u_{i-1, j} \phi_{i-1, j} S_{E}+-u_{i-1, j} \phi_{i, j} S_{E}+v_{i, j-1} \frac{\phi_{i, j}-\phi_{i, j-1}}{2} S_{S}-v_{i, j} \frac{\phi_{i, j}-\phi_{i, j+1}}{2} S_{N}= \\
\frac{k}{\rho c_{p}}\left(\frac{\phi_{i, j}-\phi_{i, j-1}}{\Delta r_{S}}\right) S_{S}+\frac{k}{\rho c_{p}}\left(\frac{\phi_{i, j+1}-\phi_{i, j}}{\Delta r_{N}}\right) S_{N}
\end{array}
$$

Además las condiciones de contorno son:

- En la pared $r=R,-k \partial T / \partial r=\dot{q}$

- En el eje $r=R_{e j e}$, se considera una superficie adiabática: $-k \partial T / \partial r=0$

Por lo tanto el sistema de ecuaciones del problema térmico se construye del siguiente modo: la primera ecuación representa la condición de contorno en la pared del tubo exterior y la ecuación $N N+2$ representa la condición de pared adiabática en el eje. Las ecuaciones de la 2 a la $N N+1$ son la ecuación de la energía (E.E.) anteriormente expuesta, aplicada a la celda $i, j$.

$$
\begin{aligned}
E: 1) & T_{1}-T_{2}=\frac{\dot{q} \times \Delta r_{S}}{k} \\
E: j=2, N N+1) & \text { E.E. } \\
E: N N+2) & T_{N N+1}-T_{N N+2}=0
\end{aligned}
$$




\subsubsection{Método de resolución de las ecuaciones}

El método de resolución de las ecuaciones es más complejo que en el modelo anterior ya que se desea simular la región de entrada fluidomecánica y/o la región de entrada térmica. Debido a las condiciones impuestas, el problema fluidomecánico y térmico están desacoplados, de forma que una vez resuelto el primero, se puede resolver el segundo.

Así, el código funciona de manera distinta dependiendo del perfil de velocidades considerado a la entrada:

1. Perfil de velocidades desarrollado.

2. Velocidad de entrada uniforme (Slug flow).

Perfil de velocidades desarrollado a la entrada. En el primer caso, el método de resolución es más simple:

1. El perfil de velocidad del flujo desarrollado se mantiene a lo largo del tubo. Se obtiene del modelo numérico del Apartado 3.2.

2. La temperatura de entrada es uniforme.

3. Barrido de las secciones desde la entrada a la salida

a) Obtención de la viscosidad del fluido en función del perfil de velocidades.

b) Resolución del sistema de ecuaciones del campo térmico.

Llegados a este punto la simulación ha finalizado.

Velocidad de entrada uniforme (Slug flow). Para resolver el problema se hace un barrido de la malla de izquierda a derecha (partiendo de la entrada y avanzando aguas abajo), resolviendo cada una de las secciones. En cada sección se debe iterar para obtener el perfil de velocidades y a partir de él la viscosidad hasta que la solución converja. El procedimiento completo sería el siguiente:

1. En la sección 1 se impone el perfil de velocidad de entrada.

2. Comienza el cálculo para el resto de secciones empezando por la 2: 
a) Bucle de iteración para obtener el perfil de velocidades (termina cuando el residuo de la caída de presión es menor de $10^{-7}$ ).

1) Se resuelve el sistema de ecuaciones del problema fluidomecánico.

2) Se calcula la viscosidad en función del perfil de velocidades obtenido.

b) Se resuelve el sistema de ecuaciones del campo térmico.

3. Una vez llegados a la última sección, la simulación ha terminado.

Los criterios de convergencia de los residuos son configurables mediante variables en el programa. En este modelo no se ha considerado la influencia de la temperatura en los parámetros reológicos u otras propiedades del fluido.

\subsubsection{Validación del modelo}

Para validar el modelo numérico es necesario comparar los resultados obtenidos por este con soluciones analíticas o aproximadas de la bibliografía. Aunque el propósito del modelo es simular la geometría de tubos concéntricos, hay más estudios sobre el flujo en tubo liso disponibles en la bibliografía. De este modo el modelo numérico se valida en primer lugar con las soluciones para tubo liso y en segundo lugar con las soluciones existentes para sección anular o tubos concéntricos.

\subsubsection{Tubo liso}

Para el problema analizado en tubo liso se pueden obtener:

- De forma analítica:

- Flujo desarrollado.

- Perfil de velocidades.

- Caída de presión por unidad de longitud.

- El perfil de temperaturas y número de Nusselt para $n=1$ y $n=0,5$, dentro del rango de propiedades ensayado (deducción en Apéndice B.3)

- La longitud de desarrollo a partir de un valor del número de Reynolds.

- Mediante una solución aproximada: 
(a) Perfil de velocidades. Desviaciones en tanto por cien de $u_{b}$.

\begin{tabular}{ccccc}
\hline$n=$ & 0,5 & 0,65 & 0,85 & 1 \\
\hline$N N=16 ; M M=50$ & $6,91 \%$ & $6,52 \%$ & $6,62 \%$ & $7,1 \%$ \\
$N N=30 ; M M=50$ & $1,98 \%$ & $1,86 \%$ & $1,88 \%$ & $2,03 \%$ \\
$N N=50 ; M M=100$ & $0,71 \%$ & $0,67 \%$ & $0,66 \%$ & $0,73 \%$ \\
$N N=100 ; M M=150$ & $0,18 \%$ & $0,17 \%$ & $0,17 \%$ & $0,18 \%$ \\
$N N=100 ; M M=200$ & $0,18 \%$ & $0,17 \%$ & $0,17 \%$ & $0,18 \%$ \\
\hline
\end{tabular}

(b) Caída de presión adimensional, $f_{\infty}$.

\begin{tabular}{ccccc}
\hline$n=$ & 0,5 & 0,65 & 0,85 & 1 \\
\hline$N N=16 ; M M=50$ & $0,3238 \%$ & $0,3383 \%$ & $0,3656 \%$ & $0,3891 \%$ \\
$N N=30 ; M M=50$ & $0,0925 \%$ & $0,0962 \%$ & $0,1043 \%$ & $0,1110 \%$ \\
$N N=50 ; M M=100$ & $0,0333 \%$ & $0,0348 \%$ & $0,0376 \%$ & $0,0400 \%$ \\
$N N=100 ; M M=150$ & $0,0083 \%$ & $0,0087 \%$ & $0,0094 \%$ & $0,0099 \%$ \\
$N N=100 ; M M=200$ & $0,0083 \%$ & $0,0087 \%$ & $0,0094 \%$ & $0,0099 \%$ \\
\hline
\end{tabular}

Cuadro 3.3: Validación del modelo en tubo liso. Desviaciones de los resultados numéricos respecto a la solución de referencia en función del caso simulado y de la malla.

- Para obtener el número de Nusselt para valores no enteros de $1 / n$ se puede utilizar la expresión obtenida por Bird (1959) utilizando la aproximación de Leveque.

\section{Perfil de velocidades desarrollado.}

El perfil de velocidades desarrollado en tubo liso, tiene la siguiente expresión:

$$
\frac{u_{z}}{u_{b}}=\left(\frac{3 n+1}{n+1}\right)\left(1-\left(\frac{r}{R}\right)^{(n+1) / n}\right)
$$

En la Fig. 3.4 se representan el perfil de velocidades numérico frente al analítico. Los errores en la solución obtenida por el simulador en cuanto al perfil de velocidades se muestran en la Tabla 3.3.

\section{Caída de presión.}

La caída de presión se compara con la obtenida a partir de la relación entre el número de Reynolds y el factor de fricción, obtenida (para fluidos Power Law) por Metzner y Reed (1955), cuya solución es exacta:

$$
f \times R e_{M R}=16
$$




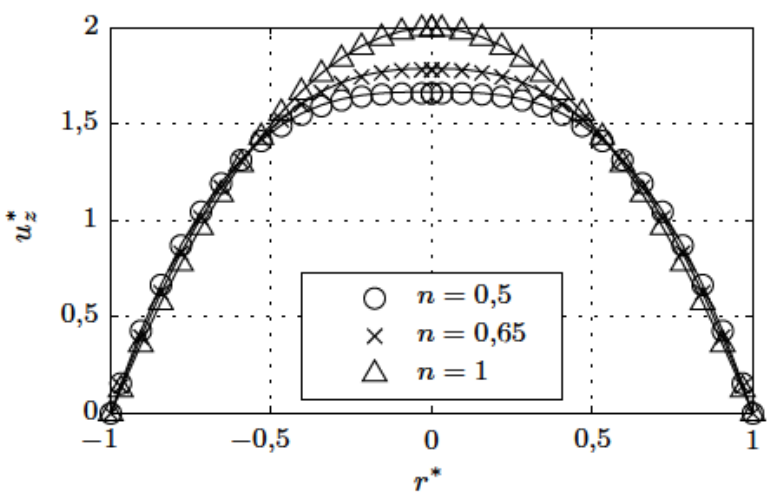

Figura 3.4: Perfil de velocidades desarrollado en tubo liso. Mallado: $N N=16 ; M M=50$.

donde el número de Reynolds de Metzner y Reed (1955) es:

$$
R e_{M R}=\frac{\rho u_{b}^{2-n} D^{n}}{8^{n-1} m\left(\frac{3 n+1}{4}\right)^{n}}
$$

\section{Longitud de desarrollo.}

La longitud de desarrollo se define como la distancia $L_{e}$ necesaria para que la velocidad axial en el eje $u_{z}(r=0)$ alcance el $99 \%$ de su valor para el flujo desarrollado.

La expresión obtenida numéricamente por Poole y Ridley (2007) para la longitud de entrada es la siguiente:

$$
\frac{L_{e}}{D}=\left[\left(0,25 n^{2}-0,675 n+1,03\right)^{\varsigma}+\left(0,0567 R e_{M R}\right)^{\varsigma}\right]^{1 / \varsigma}
$$

donde $\varsigma=1,6$.

En la Ec. 3.13, el primer término a la derecha de la ecuación es debido a la difusividad y el segundo término a la convección. Para números de Reynolds muy pequeños, la difusividad será dominante y por lo tanto $L_{e} / D \approx 0,25 n^{2}-0,675 n+1,03$, mientras que para números de Reynolds altos, la difusividad pierde importancia siendo $L_{e} / D \approx 0,0567 R e_{M R}$.

En la Fig. 3.5, se muestra la comparativa entre la solución del modelo numérico y la obtenida por Poole y Ridley (2007). Como se puede observar, las diferencias respecto a la solución de referencia para $R e_{M R}>20$ son mínimas. Para $R e_{M R}<20$, la solución del modelo y la de Poole y Ridley difieren debido al término de difusividad. Este término no ha sido incluido en el modelo, ya que no se han utilizado simulaciones en dichas condiciones en la 


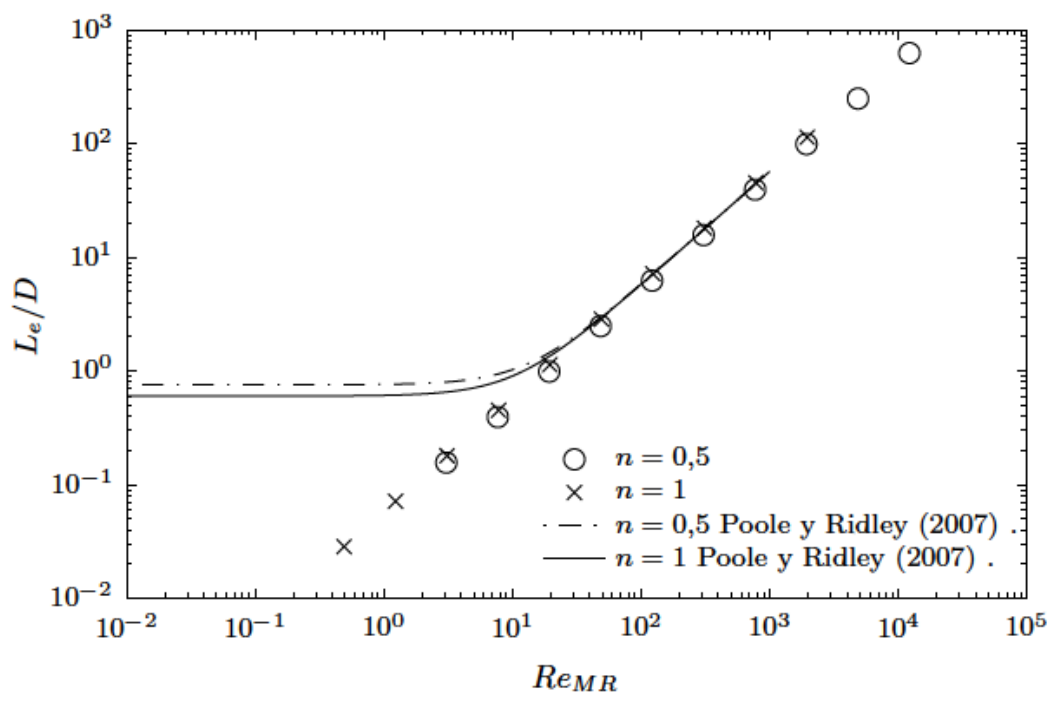

Figura 3.5: Validación del modelo CFD problema fluidomecánico en tubo liso. Longitud de entrada. Mallado utilizado: $N N=50 ; M M=100$. La solución de Poole y Ridley (2007) viene dada por la Ec. 3.13 .

presente investigación e implica un gasto computacional mayor.

En principio el objetivo del modelo numérico está más centrado en la región de entrada térmica que en la fluidomecánica, lo que justifica el uso de un modelo más simple y rápido.

\section{Perfil temperaturas y número de Nusselt.}

En la Fig. 3.6 se muestra la comparativa del perfil de velocidades obtenido mediante simulación con el perfil analítico. Las temperaturas se representan en forma adimensional:

$$
\theta=\frac{T-T_{e j e}}{\frac{q^{\prime \prime} D}{k}}
$$

donde $T_{\text {eje }}$ es la temperatura en el eje del tubo.

En la Fig. 3.6, se muestra el perfil de velocidades obtenido mediante simulación y comparado con las soluciones analíticas obtenidas en el Apéndice B.3 para $n=0,5 ; 1$. Por la definición de la temperatura adimensional, las mayores desviaciones entre ambas soluciones se dan en la pared. En la Tabla 3.3(b) se detallan las desviaciones de la solución numérica en función del mallado.

La solución analítica del número de Nusselt del flujo desarrollado para $n=0,5 ; 1$, se 
(a) Número de Nusselt teórico del flujo desarrollado.

\begin{tabular}{ccc}
\hline$n$ & $N u_{\infty}$ (analítico) & $N u_{\infty}$ (aprox.) \\
\hline 0,5 & $48 / 11$ & 4,7458 \\
0,65 & - & 4,5804 \\
0,85 & - & 4,4378 \\
1 & $280 / 59$ & 4,3636 \\
\hline
\end{tabular}

(b) Desviaciones de temperatura del flujo desarrollado.

\begin{tabular}{ccc}
\hline$n=$ & 0,5 & 1 \\
\hline$N N=16 ; M M=50$ & $0,3862 \%$ & $0,2601 \%$ \\
$N N=30 ; M M=50$ & $0,1101 \%$ & $0,0740 \%$ \\
$N N=50 ; M M=100$ & $0,0397 \%$ & $0,0267 \%$ \\
$N N=100 ; M M=10$ & $0,0099 \%$ & $0,0067 \%$ \\
$N N=100 ; M M=150$ & $0,0099 \%$ & $0,0067 \%$ \\
$N N=100 ; M M=200$ & $0,0099 \%$ & $0,0067 \%$ \\
\hline
\end{tabular}

(c) Desviaciones en el número de Nusselt del flujo desarrollado.

\begin{tabular}{ccccc}
\hline$n=$ & 0,5 & 0,65 & 0,85 & 1 \\
\hline$N N=16 ; M M=50$ & $0,4930 \%$ & $0,3954 \%$ & $0,3195 \%$ & $0,2827 \%$ \\
$N N=30 ; M M=50$ & $0,1409 \%$ & $0,1129 \%$ & $0,0912 \%$ & $0,0807 \%$ \\
$N N=50 ; M M=100$ & $0,0508 \%$ & $0,0407 \%$ & $0,0329 \%$ & $0,0290 \%$ \\
$N N=100 ; M M=10$ & $0,0127 \%$ & $0,0102 \%$ & $0,0082 \%$ & $0,0073 \%$ \\
$N N=100 ; M M=150$ & $0,0127 \%$ & $0,0102 \%$ & $0,0082 \%$ & $0,0073 \%$ \\
$N N=100 ; M M=200$ & $0,0127 \%$ & $0,0102 \%$ & $0,0082 \%$ & $0,0073 \%$ \\
\hline
\end{tabular}

Cuadro 3.4: Problema térmico del flujo desarrollado en tubo liso. 


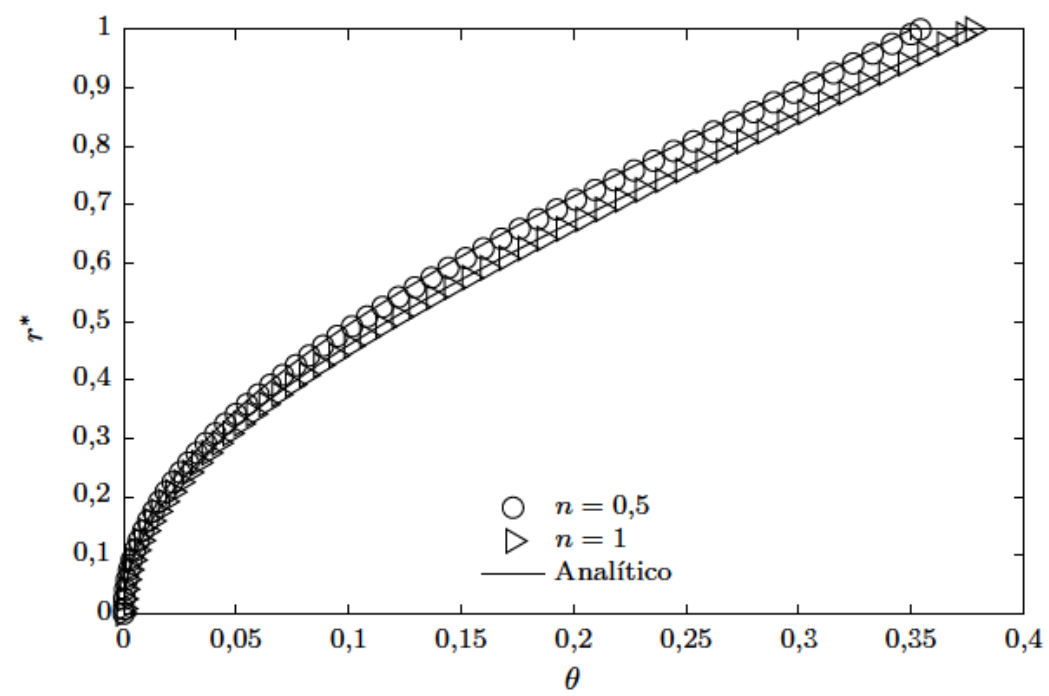

Figura 3.6: Validación del perfil de temperaturas adimensionales en tubo liso.

deduce igualmente en el Apéndice B.3. Además, para $0,5<n<1$, los resultados de la simulación se comparan con la solución aproximada obtenida mediante la aproximación de Leveque:

$$
N u_{\infty}=\frac{8(3 n+1)(5 n+1)}{\left(31 n^{2}+12 n+1\right)}
$$

En la Tabla 3.4 se muestran los valores de Nusselt obtenidos mediante sendos métodos y en la Tabla 3.3(c) se observa la precisión del código numérico en función del mallado. Tal y como se puede observar, el número de secciones $M M$ en las que se divida la longitud del tubo no es tan determinante para este problema como el número de celdas en sentido radial $N N$. Es suficiente con un valor de $M M>10$.

\section{Longitud de desarrollo térmica.}

La longitud de desarrollo térmica se define como la distancia a la entrada de la sección en la cual se cumple que $1,05 \times N u_{\infty}$. Como referencia, se hace uso de la solución obtenida recientemente por Barletta y Magyari (2007).

$$
\frac{L_{t h}}{D_{h} P e}=0,0430527
$$



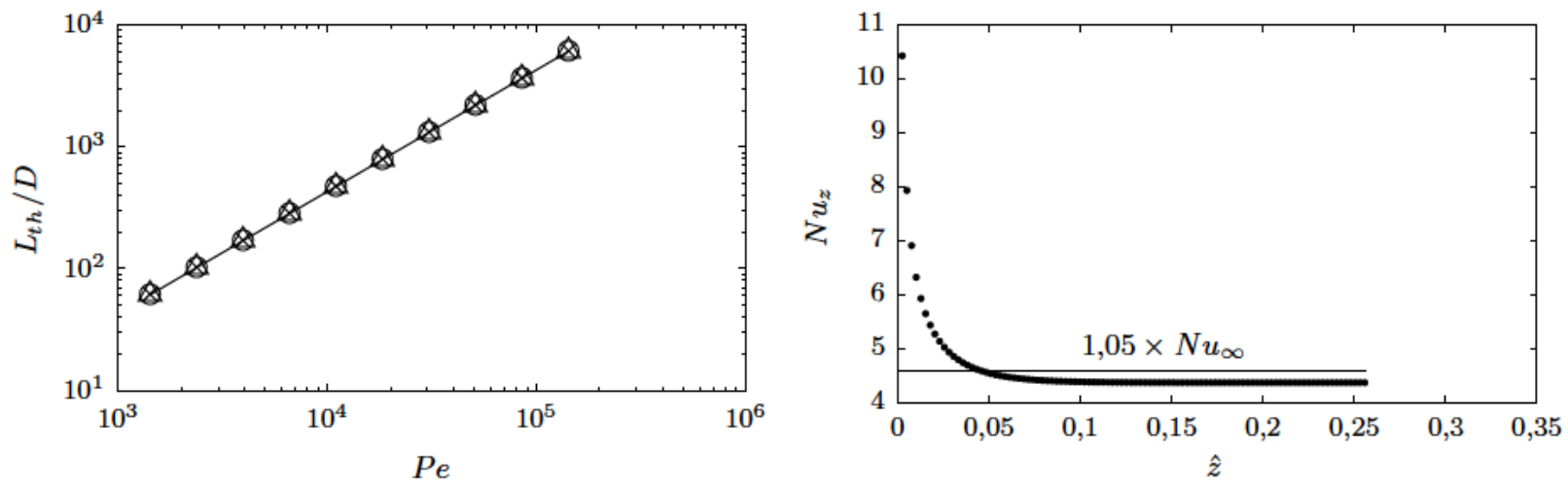

(a) Longitud de entrada.

(b) $n=1$.
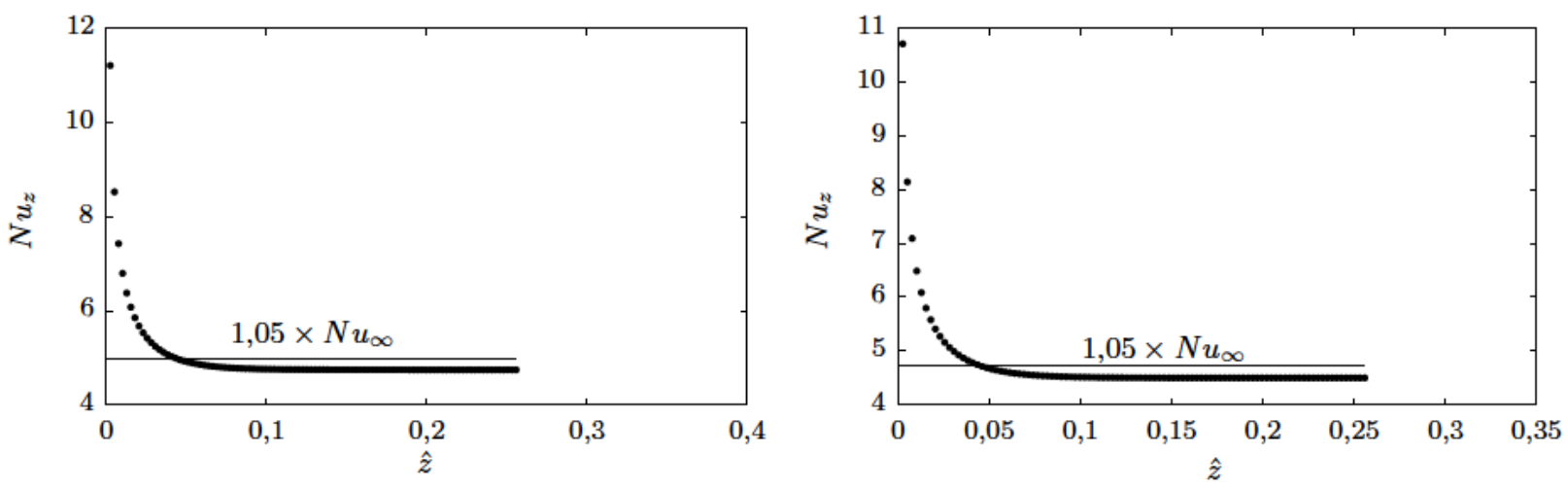

(c) $n=0,5$.

(d) $n=0,75$.

Figura 3.7: Longitud de entrada térmica en tubo liso. Representación Fig. (a): solución de Barletta y Magyari (2007) en línea continua y simulación mediante símbolos ( $n=$ $0,5 ; 0,75 ; 1)$. Mallado: $N N=50 ; M M=100$. 
la cual es válida siempre que el número de Peclet sea superior a 1000; en caso contrario no se podría despreciar la conducción axial. La definición del número de Peclet en fluidos seudoplásticos es idéntica a la de fluidos newtonianos:

$$
P e=\frac{u_{b} D_{h} \rho c_{p}}{k}=\operatorname{Re} \times \operatorname{Pr}>1000
$$

A pesar de los bajos números de Reynolds ensayados, no se han realizado experimentos con $P e<1000$. Por ejemplo, para tubo de $18 \mathrm{~mm}$, Pe podría estar por debajo de $1000 \mathrm{si}$ $Q<7 \mathrm{l} / \mathrm{h}$ (considerando las propiedades físicas del agua), o en el caso de un tubo concéntrico como el de la instalación de la UPCT $Q<5 \mathrm{l} / \mathrm{h}$. Tal y como se ha descrito en la metodología experimental, no se ha ensayado con caudales tan bajos, de modo que esta posibilidad se descarta y por lo tanto la solución de Barletta y Magyari (2007) es válida en todo el rango de ensayos.

En la Fig. 3.7(a) se muestra la representación de la longitud de entrada térmica mediante simulación y la obtenida a partir de la ecuación de Barletta y Magyari (2007). Tal y como se puede observar, la longitud de entrada no depende de $n$. Además en la Fig. 3.7 se muestra la evolución del número de Nusselt en la región de entrada para tres simulaciones distintas, donde se representa el valor de $1,05 \times N u_{\infty}$, al cuál debe llegar el valor de $N u_{z}$ para cumplir la condición que define la longitud de entrada.

Resulta significativo que aunque $N u_{\infty}$ varíe en función de $n$, la longitud de entrada no lo haga.

\subsubsection{Geometría de tubos concéntricos o sección anular}

A la hora de validar el flujo en tubos concéntricos, la tarea resulta más complicada, ya que no existen soluciones analíticas para la mayoría de los problemas. Las soluciones existentes se encuentran descritas en el Apartado 3.1.

En primer lugar, para este modelo de simulación se realiza la validación del perfil de velocidades y de la caída de presión del flujo desarrollado, de forma similar a la validación realizada en el primer modelo. La comparativa de los perfiles de velocidad se muestra en la Fig. 3.8, mientras que las desviaciones de los resultados de simulación respecto a los de referencia se muestran en la Tabla 3.5. En dicha tabla se observa cómo a la hora de obtener buenos resultados para el perfil desarrollado basta con dividir el dominio en $M M=10$ secciones, y sin embargo a mayor valor de $N N$ se obtienen resultados de mayor precisión.

En cuanto a la longitud de desarrollo del flujo isotermo, el problema viene dado por la 
(a) Perfil de velocidades desarrollado para factores de bloqueo $\beta=4,2,1,-2$.

\begin{tabular}{cccccc}
\hline$\omega$ & -3 & -1 & 0 & 1 & 3 \\
\hline$N N=16 ; M M=50$ & $0,0348 \%$ & $0,0211 \%$ & $0,0143 \%$ & $0,0074 \%$ & $0,0063 \%$ \\
$N N=30 ; M M=50$ & $0,0111 \%$ & $0,0067 \%$ & $0,0046 \%$ & $0,0024 \%$ & $0,0019 \%$ \\
$N N=50 ; M M=100$ & $0,0042 \%$ & $0,0026 \%$ & $0,0017 \%$ & $0,0009 \%$ & $0,0007 \%$ \\
$N N=100 ; M M=10$ & $0,0011 \%$ & $0,0007 \%$ & $0,0005 \%$ & $0,0002 \%$ & $0,0002 \%$ \\
$N N=100 ; M M=150$ & $0,0011 \%$ & $0,0007 \%$ & $0,0005 \%$ & $0,0002 \%$ & $0,0002 \%$ \\
\hline
\end{tabular}

(b) Perfil de velocidades desarrollado para diferentes propiedades de flujo $n$.

\begin{tabular}{cccc}
\hline$n=$ & 0,5 & 0,65 & 0,85 \\
\hline$N N=16 ; M M=10$ & 0,0524 & 0,0362 & 0,0237 \\
$N N=16 ; M M=50$ & 0,0524 & 0,0362 & 0,0237 \\
$N N=30 ; M M=50$ & 0,0185 & 0,0123 & 0,0078 \\
$N N=50 ; M M=100$ & 0,0073 & 0,0049 & 0,0030 \\
$N N=100 ; M M=10$ & 0,0019 & 0,0030 & 0,0010 \\
$N N=100 ; M M=150$ & 0,0019 & 0,0030 & 0,0010 \\
\hline
\end{tabular}

(c) Pérdida de presión adimensional, $f$.

\begin{tabular}{ccccc}
\hline$n=$ & 0,5 & 0,65 & 0,85 & 1 \\
\hline$N N=16 ; M M=10$ & $1,98 \%$ & $1,66 \%$ & $1,23 \%$ & $0,91 \%$ \\
$N N=16 ; M M=50$ & $1,98 \%$ & $1,66 \%$ & $1,23 \%$ & $0,91 \%$ \\
$N N=30 ; M M=50$ & $1,29 \%$ & $1,00 \%$ & $0,58 \%$ & $0,26 \%$ \\
$N N=50 ; M M=100$ & $1,12 \%$ & $0,84 \%$ & $0,42 \%$ & $0,09 \%$ \\
$N N=100 ; M M=10$ & $1,04 \%$ & $0,76 \%$ & $0,35 \%$ & $0,02 \%$ \\
$N N=100 ; M M=150$ & $1,04 \%$ & $0,76 \%$ & $0,35 \%$ & $0,02 \%$ \\
\hline
\end{tabular}

Cuadro 3.5: Validación del problema fluidodinámico del flujo desarrollado en tubos concéntricos. Desviaciones del método numérico respecto a la solución de referencia.

\begin{tabular}{ccc}
\hline$N N$ & $M M$ & $E\left(N u_{\infty}\right)$ \\
\hline 16 & 10 & $0,1437 \%$ \\
16 & 50 & $0,1437 \%$ \\
30 & 50 & $0,0521 \%$ \\
50 & 100 & $0,0286 \%$ \\
100 & 10 & $0,0186 \%$ \\
100 & 150 & $0,0186 \%$ \\
150 & 200 & $0,0168 \%$ \\
\hline
\end{tabular}

Cuadro 3.6: Desviaciones en la estimación de $N u_{\infty}$ para fluidos newtonianos partiendo de flujo hidrodinámicamente desarrollado y temperatura constante. Condición de flujo de calor constante en el tubo exterior e interior adiabático. 


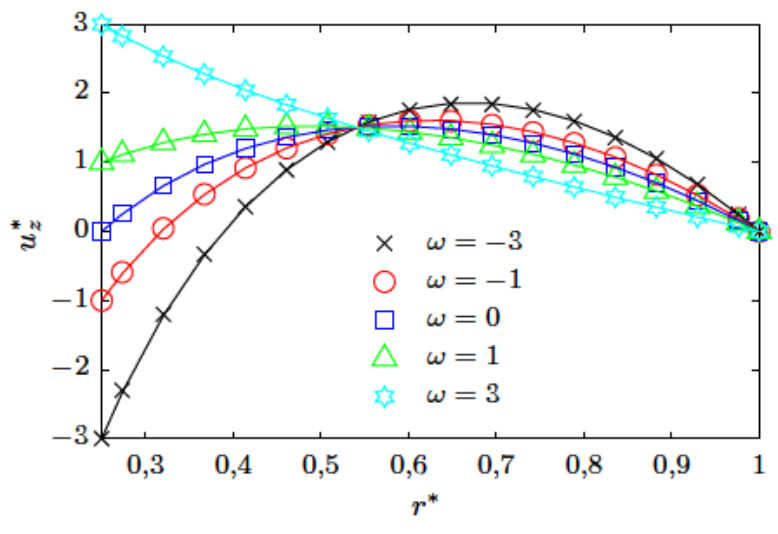

(a) $n=1$.

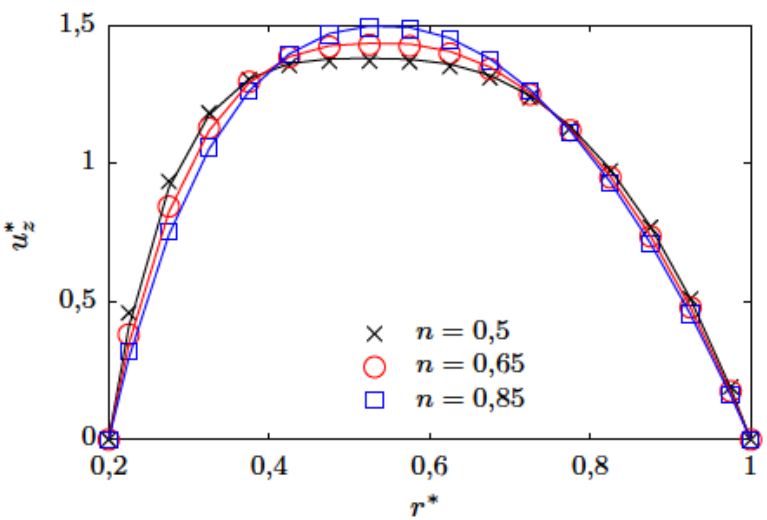

(b) Eje central estático.

Figura 3.8: Validación del perfil de velocidades desarrollado en geometría de tubos concéntricos, con de entrada de flujo uniforme.

forma de definir dicha longitud de desarrollo, ya que ésta se define para la posición radial en la cual la velocidad es máxima, mientras que en otras posiciones radiales el flujo alcanza el desarrollo considerablemente antes. Así, los diferentes autores consultados dan soluciones significativamente distintas (Poole (2010); Nouar et al. (1995)). Tal disparidad de soluciones las hace inválidas para un proceso de validación, por lo que se descarta este paso en el proceso, que sí se ha realizado en tubo liso para el mismo modelo numérico.

En lo que respecta al problema térmico se aborda tanto la validación de $N u_{\infty}$ como la validación del número de Nusselt local, ambos para un fluido newtoniano. Por un lado, los resultados de validación de $N u_{\infty}$ se muestran en la Tabla 3.6. En ellos se observa que la precisión del resultado numérico mejora principalmente al aumentar $N N$, siempre y cuando $M M \geq 10$. El error para $N N=100$ es menor del $0,02 \%$ de la solución. Por otro lado, los resultados de la validación del $N u$ local en la región de entrada térmica, con perfil de velocidades desarrollado a la entrada, se muestran en la Tabla 3.7. Para dichas simulaciones es necesario simular una longitud de conducto tal que permita al flujo desarrollarse. Se simula a lo largo de una longitud $\hat{L}=4$. Además es necesario un gran número de celdas en dirección longitudinal para que el mallado sea denso a lo largo de $L_{t h}$ y para poder obtener valores en las posiciones $\hat{z}=4 z / D h P e$ conocidas $(0,004-0,4)$. De este modo se opta por un mallado de $M M=5000$ y otro de $M M=10000$. Se debe notar que la primera posición de comparación $\hat{z}=0,004$ se encuentra en la sección número 5 ó 10 según el valor de $M M$, por lo que los errores en el correspondiente Nusselt local son mayores. Los resultados mostrados en la 
(a) Nusselt lo-

cal.

\begin{tabular}{cc}
\hline$\hat{z}$ & $N u_{z}$ \\
\hline 0,004 & 13,8 \\
0,02 & 8,28 \\
0,04 & 6,80 \\
0,2 & 5,04 \\
0,4 & 4,91 \\
\hline
\end{tabular}

(b) Desviaciones Nusselt local.

\begin{tabular}{ccccccc}
\hline & & \multicolumn{5}{c}{$\hat{z}$} \\
\cline { 3 - 7 }$N N$ & $M M$ & 0,004 & 0,02 & 0,04 & 0,2 & 0,4 \\
\hline 16 & 5000 & $4,27 \%$ & $1,25 \%$ & $0,865 \%$ & $0,302 \%$ & $0,202 \%$ \\
30 & 5000 & $2,87 \%$ & $0,669 \%$ & $0,462 \%$ & $0,151 \%$ & $0,104 \%$ \\
50 & 5000 & $2,46 \%$ & $0,520 \%$ & $0,358 \%$ & $0,112 \%$ & $0,079 \%$ \\
100 & 5000 & $2,30 \%$ & $0,457 \%$ & $0,315 \%$ & $0,096 \%$ & $0,068 \%$ \\
16 & 10000 & $3,00 \%$ & $0,994 \%$ & $0,731 \%$ & $0,279 \%$ & $0,200 \%$ \\
30 & 10000 & $1,62 \%$ & $0,418 \%$ & $0,330 \%$ & $0,128 \%$ & $0,101 \%$ \\
50 & 10000 & $1,26 \%$ & $0,270 \%$ & $0,227 \%$ & $0,089 \%$ & $0,076 \%$ \\
100 & 10000 & $1,11 \%$ & $0,208 \%$ & $0,183 \%$ & $0,073 \%$ & $0,065 \%$ \\
\hline
\end{tabular}

Cuadro 3.7: Desviaciones en la estimación de $N u_{z}$ para fluidos newtonianos en tubos concéntricos, partiendo de flujo hidrodinámicamente desarrollado y temperatura constante. Condición de flujo de calor constante en el tubo exterior e interior adiabático. $\alpha=0,25$. El número de Nusselt en función de la distancia adimensional a la entrada fue obtenido por Reynolds et al. (1963).

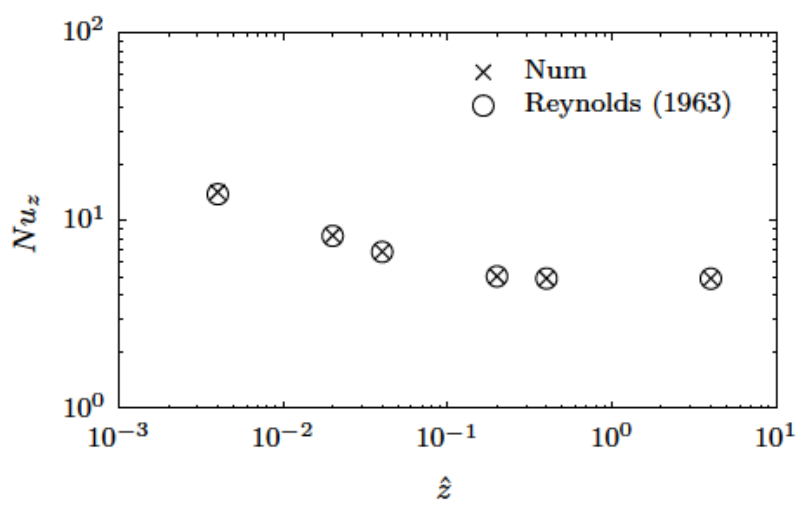

Figura 3.9: Nusselt local del modelo y de referencia para fluidos newtonianos. A la entrada el flujo está hidrodinámicamente desarrollado y la temperatura es constante. Condición $\mathrm{H}$, de flujo de calor constante en el tubo exterior e interior adiabático. $\alpha=0,25$. 
Tabla 3.6(a) indican que los errores del modelo numérico disminuyen al aumentar la densidad de la malla.

\subsection{Conclusiones}

En el presente capitulo se ha presentado el desarrollo y validación de dos modelos numéricos de simulación del flujo en tubo liso y en tubos concéntricos. Los modelos permiten simular el comportamiento del flujo incompresible y estacionario en las siguientes situaciones:

- Perfil de velocidad a la entrada desarrollado o perfil uniforme para simular el comportamiento del flujo en la región de entrada. Este último válido únicamente para $R e_{M R}>20$.

- Flujo isotermo o con condición de flujo de calor constante en la pared exterior y adiabático en la pared interior.

- Fluido newtoniano o no newtoniano (implementación del modelo Power Law para la viscosidad).

El código se ha implantado buscando la eficiencia en el tiempo de procesado, de modo que las simulaciones realizadas pueden ser llevadas a cabo por cualquier ordenador de forma holgada.

Los resultados de la simulación numérica han sido correctamente validados en todas las condiciones de trabajo contempladas en el presente documento.

La simulación del flujo en tubo liso y en tubos concéntricos se utiliza en capítulos posteriores con diferentes objetivos: para complementar el estudio de visualización del flujo en el intercambiador con el rascador insertado o como base comparativa para el estudio de la transmisión de calor. 


\section{Capítulo 4}

\section{Instalaciones experimentales}

En el presente capítulo se realiza una descripción detallada del intercambiador de superficie rascada estudiado y de las instalaciones experimentales utilizadas a lo largo de la investigación. Con el objetivo de realizar ensayos de visualización del campo fluido, de pérdida de presión, de potencia de accionamiento del rascador y de transmisión de calor en el tubo del intercambiador estudiado, se han montado dos instalaciones experimentales:

1. Instalación de ensayos termohidráulicos (Apartado 4.2). La instalación se encuentra ubicada en la Universidad Politécnica de Cartagena. Está preparada para el ensayo de la pérdida de presión, la potencia de accionamiento del rascador y la transmisión de calor en el tubo del intercambiador de superficie rascada.

2. Instalación de visualización (Apartado 4.3). Se encuentra situada en la Universidad Miguel Hernández de Elche. Está preparada para la visualización del campo fluido en el tubo con el rascador insertado mediante la técnica de velocimetría por imágenes de partículas, conocida por sus siglas en inglés PIV (Particle Image Velocimetry).

La metodología experimental de los diferentes ensayos que se llevan a cabo en estas instalaciones se describe en el Capítulo 6.

\subsection{Descripción de la geometría}

Tal y como se menciona en el primer capítulo, el dispositivo estudiado está clasificado como un intercambiador de calor de tubos de superficie rascada con movimiento lineal alternativo. En concreto se trata del modelo UNICUS diseñado por la empresa HRS-Spiratube. Este 


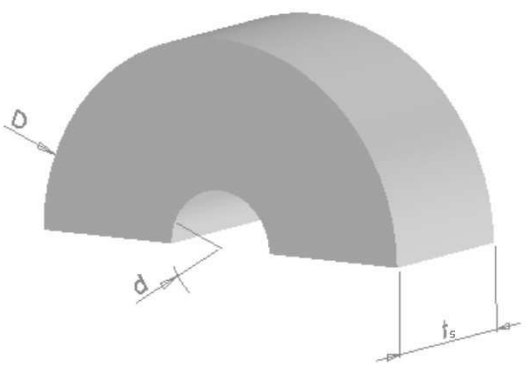

(a) Taco semicircular acoplado al eje del dispositivo insertado.

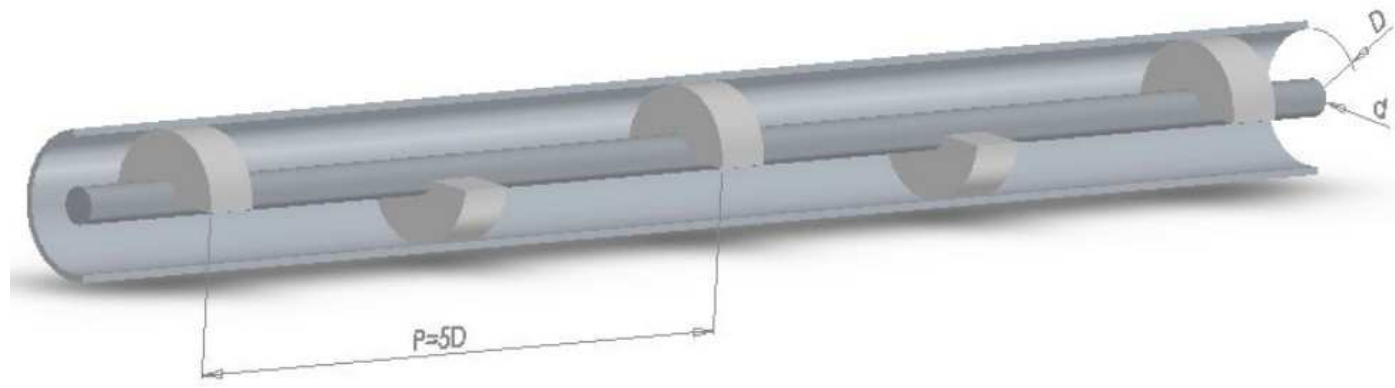

(b) Vista en 3D del tubo con el rascador.

Figura 4.1: Geometría de rascador estudiada.

\begin{tabular}{ccccc}
\hline & $D$ & $d$ & $t_{s}$ & $P$ \\
\hline I. de ensayos termohidráulicos & 18 & 5 & 7,3 & 90 \\
I. de visualización & 32 & 8 & 13 & 160 \\
\hline
\end{tabular}

Cuadro 4.1: Dimensiones en mm del rascador ensayado en las instalaciones descritas.

diseño consiste en un eje insertado en el tubo y concéntrico con éste. Sobre el eje se montan tacos rascadores en forma de media luna de diámetro exterior igual al diámetro del tubo, diámetro interior igual al del eje y de espesor $t_{s}$ (véase Fig. 4.1(a)).

Los tacos se encuentran montados en el eje al tresbolillo, con un paso $P=5 D$, tal y como se muestra en la Fig. 4.1(b).

En la instalación de visualización se ha construido una réplica del tubo del intercambiador comercial del mismo tamaño, mientras que en la instalación de ensayos termohidráulicos se ha diseñado un prototipo a escala. En la Tabla 4.1 se detallan las dimensiones del rascador en cada una de las instalaciones. 

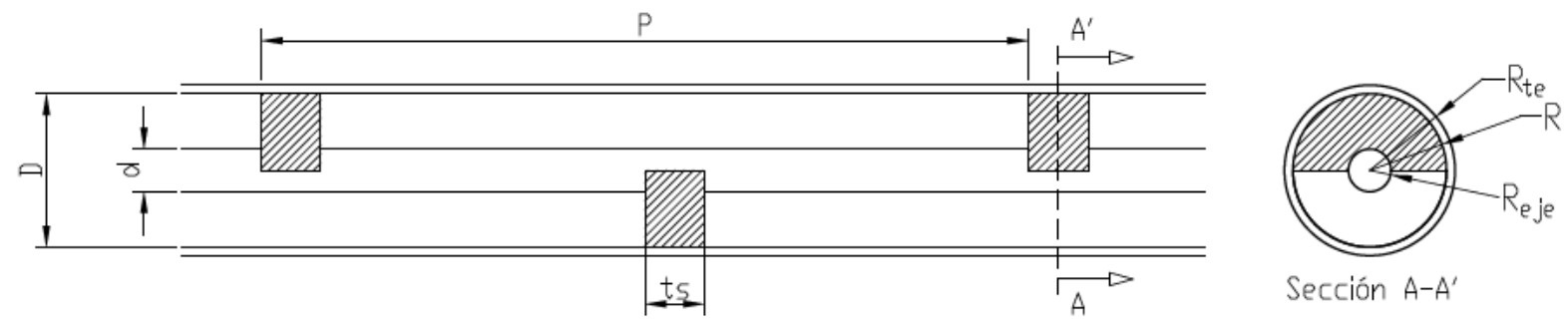

Figura 4.2: Detalle del dispositivo de mejora insertado.

\subsection{Instalación de ensayos termohidráulicos}

La instalación de ensayos termohidráulicos ha sido diseñada para el ensayo de tubos de intercambiadores mejorados. En ella se puede realizar pruebas de transferencia de calor bajo condiciones de flujo de calor uniforme y de pérdida de presión en condiciones isotermas y no isotermas. Las características más importantes de la instalación son las siguientes:

- Obtención de datos de pérdida de presión en un amplio rango de números de Reynolds.

- Obtención de datos de transferencia de calor en regímenes laminar y turbulento en un amplio rango de números de Reynolds y de Prandtl.

- Posibilidad de realizar análisis en un rango continuo de números de Reynolds, lo que permite estudiar la región de transición de régimen laminar a régimen turbulento.

- Medición de propiedades reológicas del fluido a partir de medidas de caída de presión en el viscosímetro de tubo liso.

- Posibilidad de medir la potencia de accionamiento de rascadores con movimiento lineal alternativo.

La instalación está diseñada de modo que el fluido de trabajo, mediante la correcta configuración de las válvulas de paso, puede fluir por dos circuitos alternativos:

1. Circuito de ensayo. En él se encuentra la sección de ensayo del tubo con rascador y se utilizará para realizar las medidas de los experimentos de transmisión de calor o de pérdida de presión. 
2. Circuito de medición de propiedades reológicas. El circuito es prácticamente el mismo, pero el fluido se hace circular por el viscosímetro en lugar de la sección de ensayo, para poder medir las propiedades reológicas del mismo.

En la Figura 4.3 se muestra el esquema de la instalación, que está compuesta por tres lazos cerrados:

- Lazo principal. Por él circula el fluido de trabajo. Contiene, en paralelo, el tubo de ensayo (1) y el tubo liso utilizado como viscosímetro (2).

- Lazo de refrigeración del fluido de trabajo. Una bomba de engranajes (5b) hace circular el fluido de trabajo por un intercambiador de placas (13) con el objetivo de refrigerarlo.

- Lazo de agua fría. Una enfriadora (17) mantiene una temperatura en torno a $5^{\circ} \mathrm{C}$ del depósito de agua (16). El agua fría se utiliza para refrigerar el fluido de trabajo al circular por el intercambiador de calor (13).

Viscosímetro. En el Circuito principal, en paralelo con la sección de ensayo (1 - Fig. 4.3), se encuentra instalado un tubo liso de acero inoxidable de $D_{v}=16 \mathrm{~mm}$ de diámetro, $e_{v}=2 \mathrm{~mm}$ de espesor y $3 \mathrm{~m}$ de longitud (2 - Fig. 4.3). Dicho tubo se utiliza para medir las propiedades reológicas del fluido no newtoniano a partir de la caída de presión que en él se produce. Para ello dispone de dos tomas de presión separadas $L_{v m}=1,885 \mathrm{~m}$ entre sí consistentes en sendas perforaciones de $d_{p}=2 \mathrm{~mm}$. El tramo entre ambas tomas constituye la sección de medida del viscosímetro. Aguas arriba se encuentra la sección de entrada, de $L_{v e}=0,8 \mathrm{~m}$ de longitud y aguas abajo la sección de salida, de $L_{v s}=0,315 \mathrm{~m}$. La salida está conectada a un tubo flexible que proporciona en torno a $0,5 \mathrm{~m}$ adicionales de salida suave.

La metodología para la medición de las propiedades reológicas del fluido se detalla en la Sección 5.1.1.

Tubo de ensayo. La sección de ensayo (1 - Fig. 4.3) consta de un tubo de acero inoxidable de $D=18 \mathrm{~mm}$ de diámetro interior y $3 \mathrm{~m}$ de longitud. En él se encuentra insertado el dispositivo de mejora de la transferencia de calor a estudiar, cuyas características geométricas se han descrito en el Apartado 4.1.

Las tomas de presión del tubo de ensayo están separadas 1,85 m entre sí. Son precisamente dichas tomas las que delimitan la sección de ensayo en los experimentos de pérdida de presión. Una descripción más detallada del sistema de medida de la caída de presión se encuentra en el siguiente apartado. 


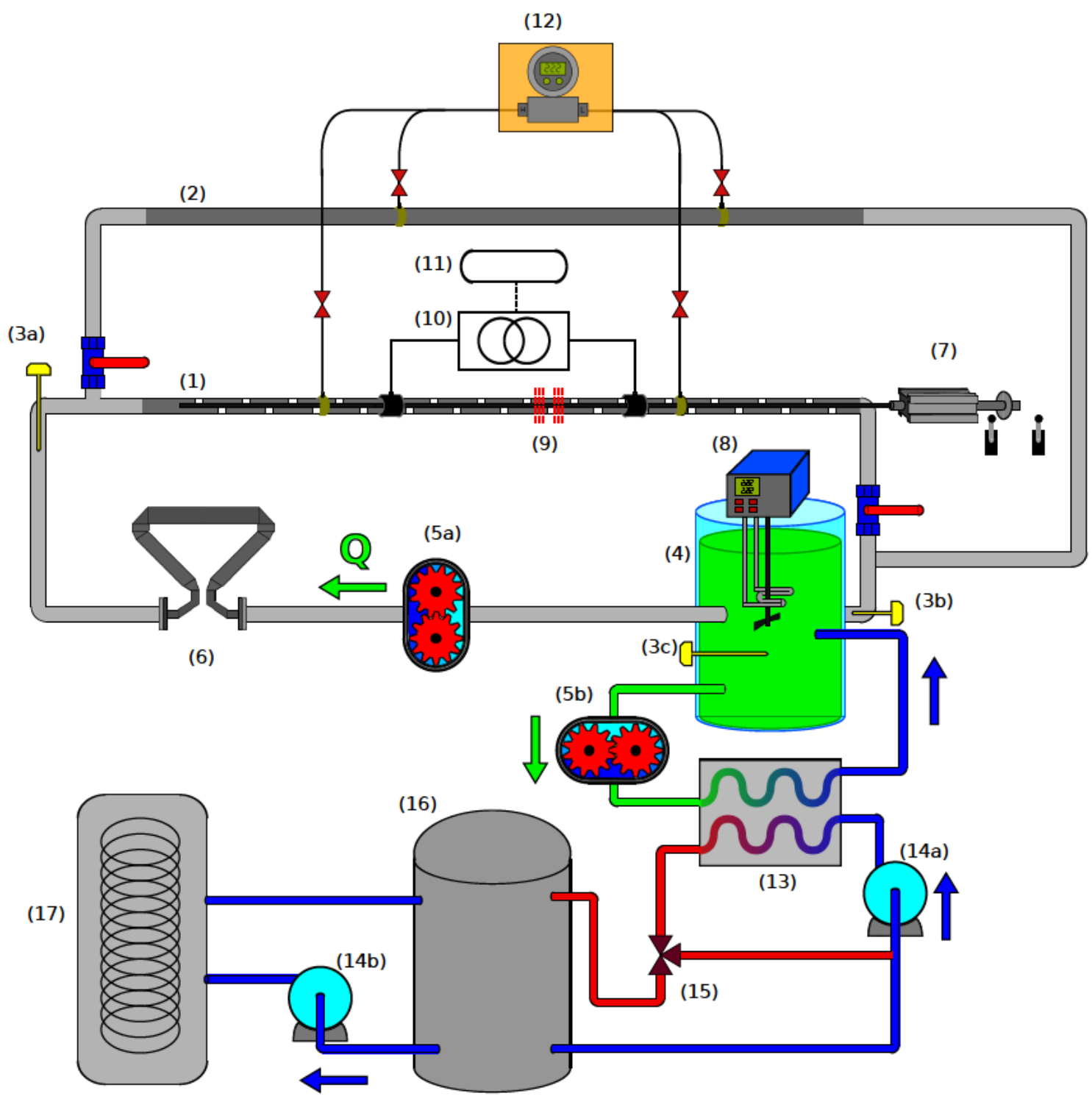

Figura 4.3: Esquema de la instalación experimental de ensayos termohidráulicos: (1) tubo de ensayo, (2) viscosímetro, (3a, 3b, 3c) Sondas RTD de temperatura a la entrada y salida del tubo de ensayo-viscosímetro y en el depósito, (4) depósito con el fluido de trabajo, $(5 \mathrm{a}, \mathrm{b})$ bombas de engranajes controladas mediante variador de frecuencia, (6) caudalímetro de efecto Coriolis, (7) pistón hidráulico que imprime movimiento al rascador, (8) sistema de calentamiento del fluido, (9) secciones de termopares que miden la temperatura de la pared exterior, (10) transformador eléctrico, (11) autotransformador, (12) sistema de medida de la caída de presión en viscosímetro y tubo de ensayo (detallado en Fig. 4.9), (13) intercambiador de calor de placas a contracorriente, (14a,b) bombas centrífugas, (15) válvula de 3 vías regulada mediante PID a partir de la temperatura del depósito, (16) depósito de agua fría, (14) enfriadora de agua. 


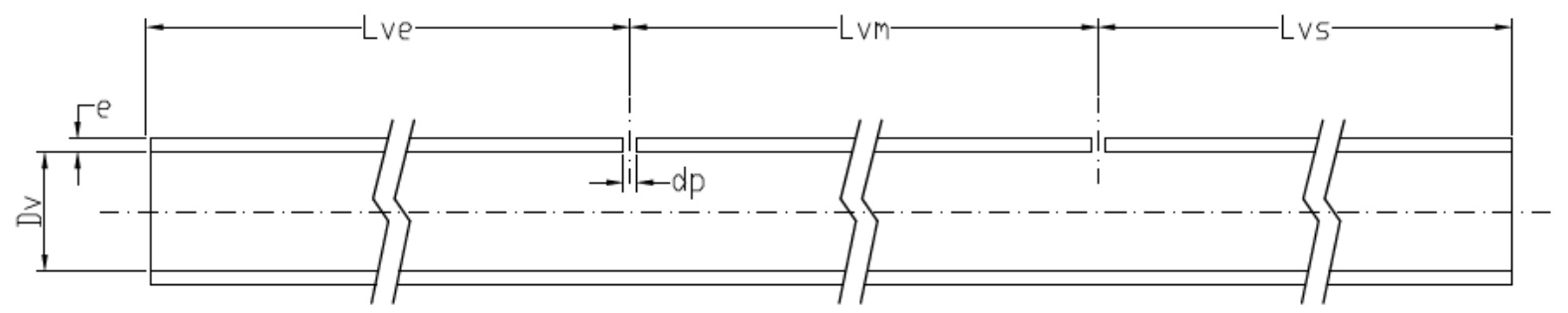

Figura 4.4: Esquema del viscosímetro.

Control de la temperatura del depósito. La temperatura del fluido en el depósito (4) de la Figura 4.3 se regula mediante aportes simultáneos de frío y de calor. Por un lado, el aporte de frío se realiza a través de un intercambiador de placas (13), mediante una máquina frigorífica (17). Por otro lado se dispone de un sistema de calentamiento doble (8), formado por una resistencia eléctrica y, aparte, por un serpentín por el que circula agua caliente. El agua que circula por el serpentín se calienta en un depósito exclusivo utilizando una segunda resistencia. De este modo el fluido se puede calentar directamente mediante la resistencia instalada en el depósito o indirectamente a través del serpentín. Este último sistema permite una mejor conservación de las propiedades del sistema (véase Apartado 5.1.2), pero no posee control alguno y es más lento. Un sistema de control PID regulará el aporte de frío mediante el actuador de la válvula de tres vías (15) a partir de la temperatura medida en el depósito (3c) por una sonda RTD.

Sistema de medición de la temperatura del fluido. La temperatura del fluido a la entrada (3a) y a la salida (3b) de la sección de ensayo se mide mediante sondas RTD de inmersión de alta precisión (clase B 1/10 DIN). Una sonda RTD de inmersión clase B (3c) situada en el depósito principal se encarga de medir su temperatura para el control de la misma. La temperatura ambiente se mide con una sonda RTD clase B.

Sistema de aporte de calor en el tubo de ensayo. Con el objetivo de aportar un flujo de calor constante al fluido de trabajo a través del tubo, se hace circular una intensidad de corriente por el mismo. Para ello se conectan dos electrodos con forma de abrazadera separados entre sí $1001 \mathrm{~mm}$ (midiendo desde las caras interiores). Mediante un autotransformador motorizado (11) se puede regular la tensión que se aplica al tubo, de entre $0 \mathrm{~V}$ y $15 \mathrm{~V}$. La 

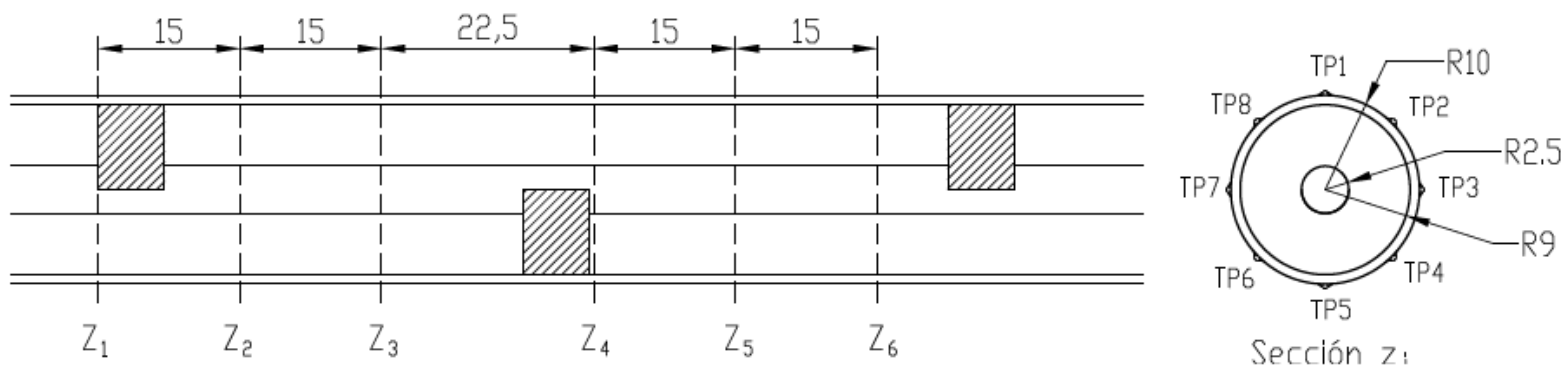

Figura 4.5: Distribución de las secciones de termopares en el tubo con rascador.

intensidad máxima es de $600 \mathrm{~A}$ y la potencia máxima tanto del transformador (10) como del autotransformador es de $6 \mathrm{kVA}$. El autotransformador está controlado por un autómata programable Field Point 2000 de National Intruments.

Aislando convenientemente el tubo se consigue que la práctica totalidad del calor generado por efecto Joule pase al fluido.

La intensidad circulante se mide mediante un anillo amperimétrico cuyo principio de funcionamiento se basa en la medición del campo eléctrico creado por la corriente circulante por los cables. La tensión se mide directamente mediante el sistema de adquisición de datos descrito más adelante.

Sistema de medición de la temperatura de la pared exterior del tubo de ensayo. En la pared exterior del tubo de ensayo, en la zona entre los electrodos, hay situados 48 termopares, dispuestos en 6 secciones con 8 termopares cada una (9). Los termopares están fijados a la pared del tubo y se ha utilizado pasta conductora para mejorar la conductividad térmica entre estos y el tubo. Cada grupo de ocho termopares mide la temperatura de la pared en una misma sección en ocho puntos equidistantes unos de otros. En la Fig. 4.5 se muestra la disposición de las secciones de termopares en el tubo con rascador, diseñada de forma que para cualquier posición del rascador, los tacos cubran como máximo la mitad de los termopares de 1 sección. La posición de la primera sección de termopares que se encuentra el flujo es de $717 \mathrm{~mm}$ respecto a la cara interna del primer electrodo y a $284 \mathrm{~mm}$ del otro. Esto permite que el desarrollo térmico del flujo sea completo en las secciones de medida.

Sistema de bombeo. Se han seleccionado bombas de engranajes de gran tamaño para el sistema de impulsión, el cual consta de dos bombas idénticas modelo AL-3 de hasta 10 bar, 

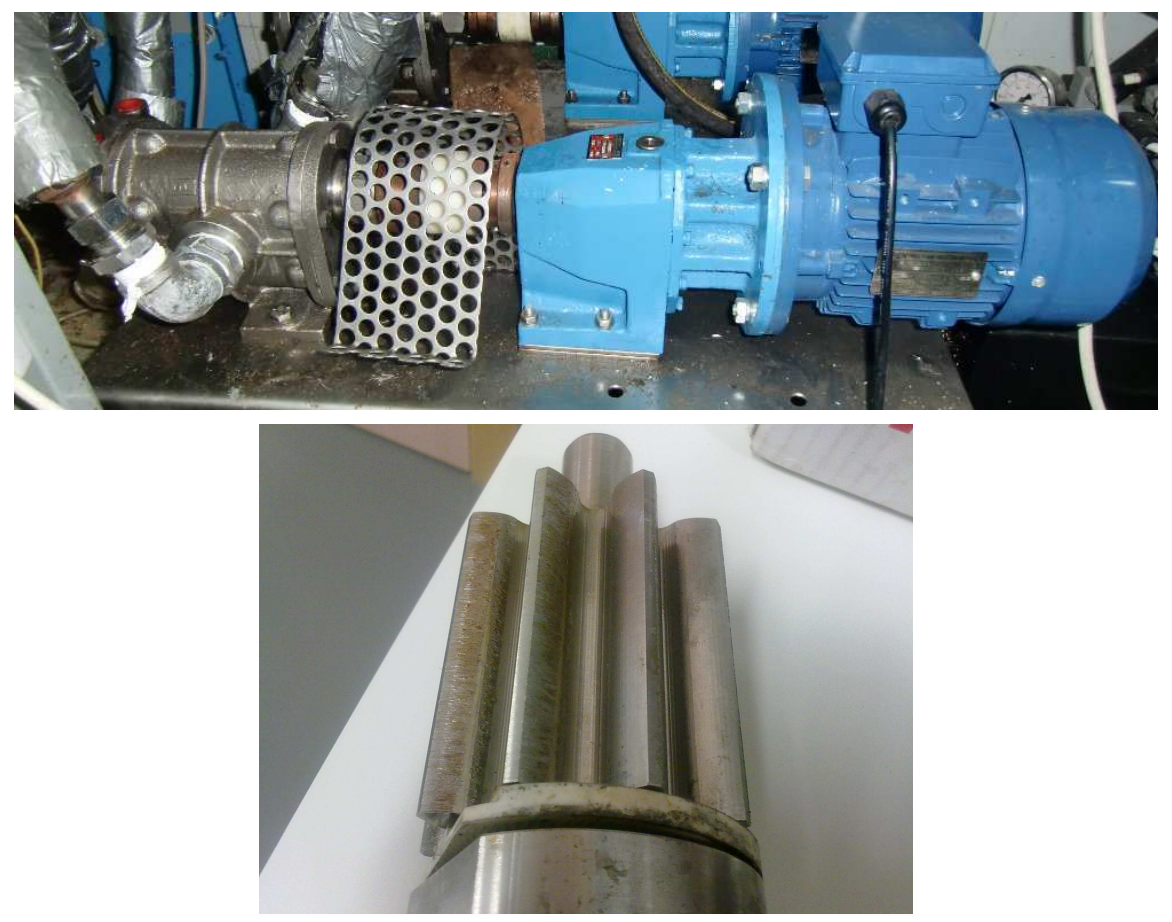

Figura 4.6: Engranajes de las bombas utilizadas en la instalación.

una para el circuito secundario de refrigeración (5b) y otra para el circuito principal (5a). El motor asíncrono que ambas tienen instaladas posee una potencia nominal de 1,5 kW y su velocidad nominal de giro es de $2840 \mathrm{rpm}$ a $50 \mathrm{~Hz}$. El engranaje de acero inoxidable está acoplado al motor mediante una reductora 1:9 con lo que la velocidad nominal de giro será de 316 rpm. Los engranajes de esta bomba se muestran en la Fig. 4.6.

Además, debido a la problemática tratada en el Capítulo 8, en determinados ensayos la bomba del circuito principal ha sido sustituida por una con motor de potencia similar, pero con engranajes de tamaño significativamente menor y tolerancias más ajustadas. En la Fig. 4.7 se muestran detalles constructivos de la misma.

Medición de caudal. El caudal se mide utilizando un caudalímetro de efecto Coriolis (6) de excelentes prestaciones: estos dispositivos presentan un error muy bajo en la medida y siempre proporcional a ésta. El modelo utilizado es el ELITE CMF025, que además es capaz de medir otras propiedades del fluido como la temperatura y la densidad. El dispositivo tiene las siguientes prestaciones en la toma de datos:

- Rango de medida caudal: $[0,027$ - 2180] kg/h. 

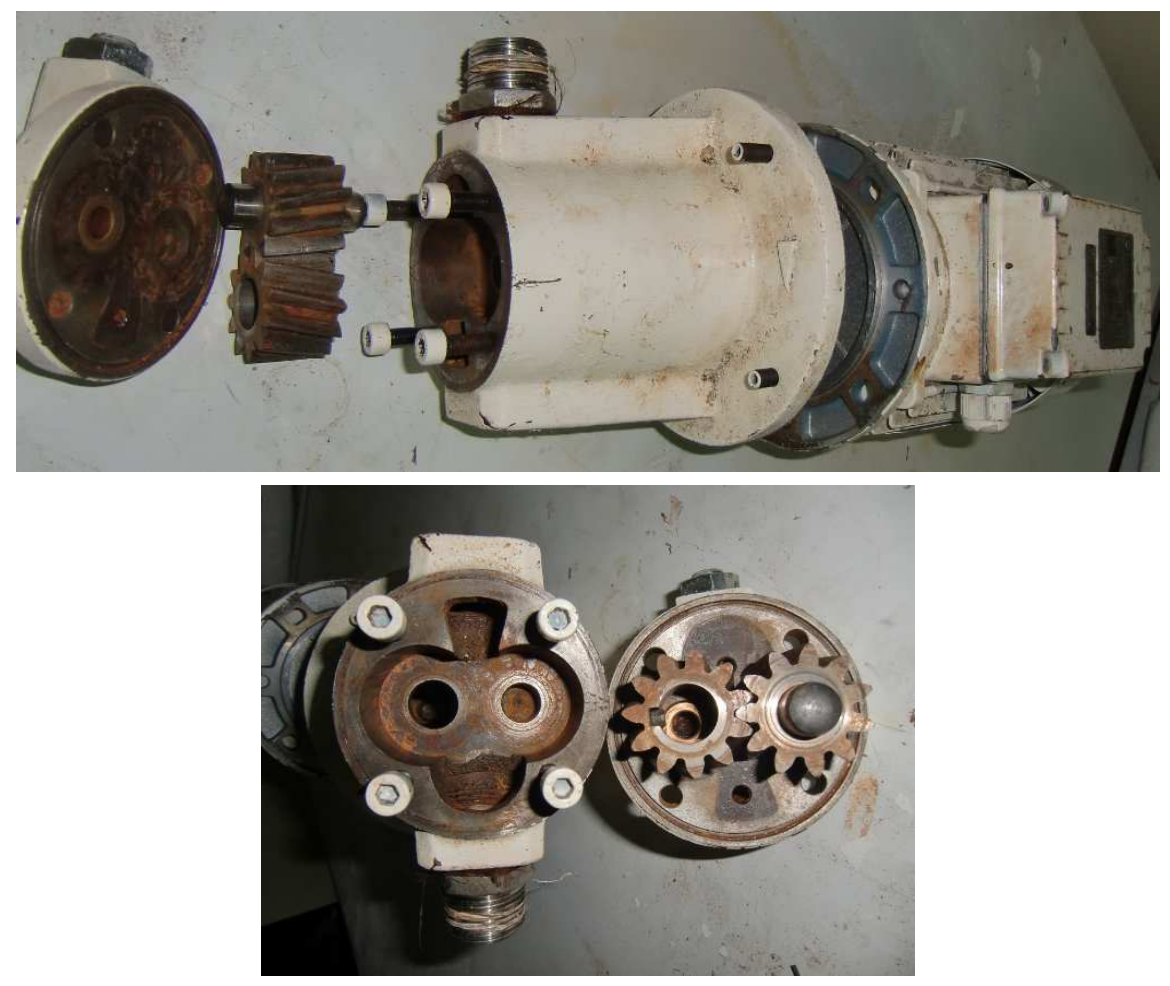

Figura 4.7: Bomba de engranajes utilizada en los ensayos a caudal constante.

- Precisión caudal: $\pm 0,1 \%$ de la medida.

- Repetibilidad caudal: $\pm 0,05 \%$ de la medida.

- Precisión densidad: $\pm 0,5 \mathrm{~kg} / \mathrm{m}^{3}$.

- Repetibilidad densidad: $\pm 0,2 \mathrm{~kg} / \mathrm{m}^{3}$.

Sistema de impulsión del eje. El dispositivo rascador insertado en el tubo, tendrá un movimiento lineal alternativo. Para impulsarlo se utiliza un pistón hidráulico (7) cuyo esquema de funcionamiento se detalla en la Fig. 4.8. En los extremos del recorrido del pistón, dos finales de carrera limitan el movimiento del mismo, de manera que la recolocación de éstos supondrá variar la amplitud del rascado. En los experimentos, los finales de carrera se posicionan de forma que la amplitud de rascado sea de $180 \mathrm{~mm}$. El pistón es impulsado por una centralita hidráulica formada por: una bomba, un depósito de aceite, un limitador de presión, sistema de filtrado, un manómetro y dos manguitos que la conectan con el pistón.

La velocidad del rascador, $v_{s}$, se regula mediante un variador de frecuencia que controla la 


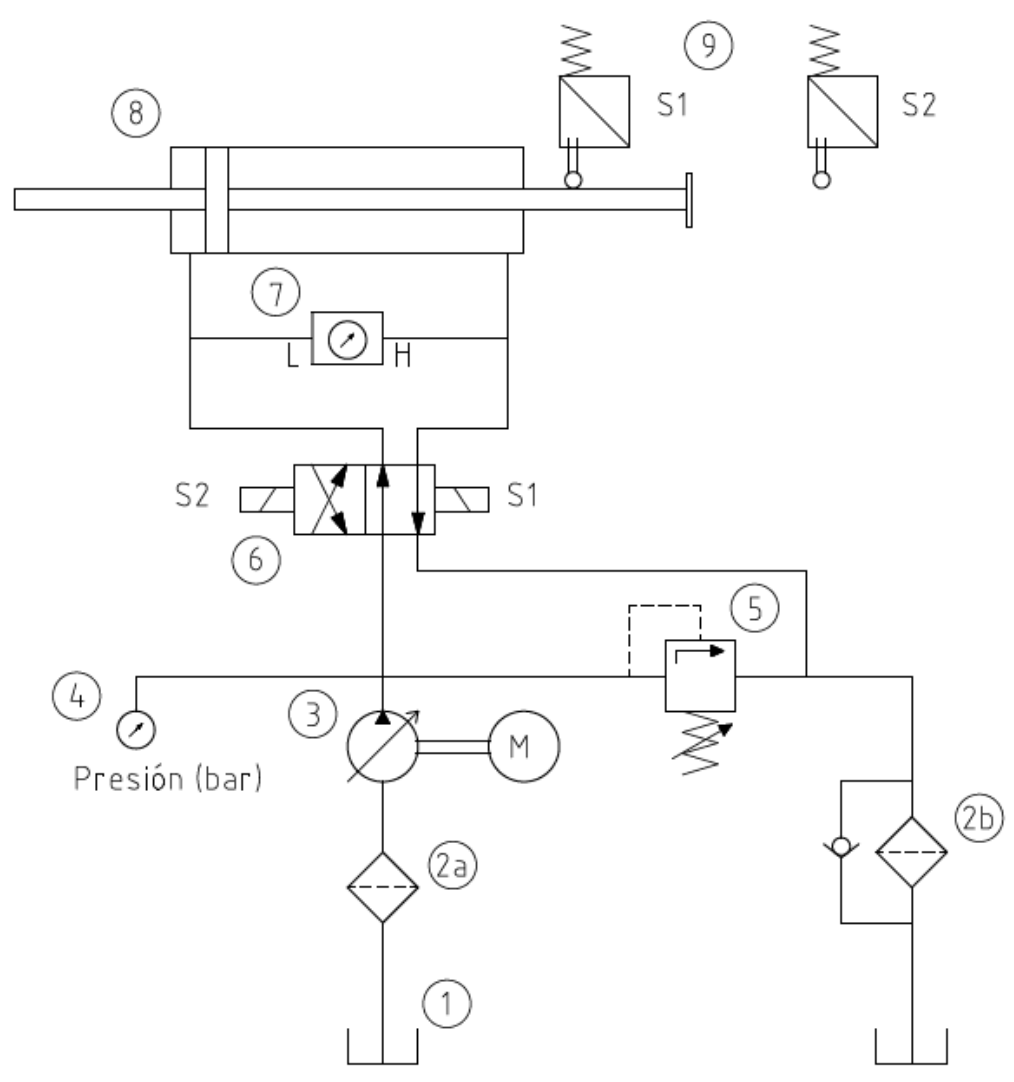

Figura 4.8: Esquema del sistema de potencia fluida utilizado para accionar el pistón hidráulico que da movimiento al rascador. 1) Depósito de aceite, 2) Sistema de filtrado, 3) BDP accionada por motor de velocidad regulable mediante variador de frecuencia, 4) manómetro, 5) válvula limitadora de presión, 6) válvula distribuidora accionada mediante finales de carrera, 7) sensor de presión diferencial dinámica $[-15,20]$ bar, 8) cilindro de doble efecto, 9) finales de carrera. 
velocidad de giro de la bomba centrífuga de la centralita hidráulica. Para medir $v_{s}$ se dispone de un sistema de medición del periodo de rascado, mediante el uso de un tacómetro digital modelo TA 202. En cada cámara del pistón hay una toma de presión, conectadas ambas a un sensor piezoeléctrico de presión diferencial bidireccional (Fig. 4.8, elemento 7). Estas medidas se utilizan para evaluar el gasto energético que supone el movimiento de rascado.

Características del sensor de presión bidireccional:

- Marca: GE measurement and control.

- Modelo: Unik 5000.

- Rango de medida: $[-15,20]$ bar.

- Precisión: $\pm 0,04 \%$ F.E.

Medición de la caída de presión en los tubos. Para medir la caída de presión tanto en el viscosímetro como en el tubo de ensayo, se dispone de cuatro sensores diferenciales de presión. Dos de ellos miden presiones diferenciales estacionarias y otros dos miden presión diferencial no estacionaria. Los sensores de presión diferencial estacionaria se usan tanto en el viscosímetro como en los ensayos con el rascador en régimen estático, mientras que los sensores de presión diferencial no estacionaria se utilizan en los ensayos donde el rascador se encuentra en movimiento. El motivo de tener dos sensores de cada tipo es conseguir precisión para valores pequeños de presión diferencial y al mismo tiempo poder medir en un rango amplio de diferencias de presión. A continuación se detallan las características de cada uno de los sensores.

- Sensores diferenciales de presión estacionaria PE1 y PE2.

- Marca: SMAR.

- Modelo: LD 301.

- Alimentación: 12 VCC.

- Señal de Salida: $[4-20] \mathrm{mA}$.

- Precisión: 0,04\% del F.E

- Rango de medida del Sensor PE1: [5 - 500] $\mathrm{mmH}_{2} \mathrm{O}$.

- Rango de medida del Sensor PE2: [20 - 2500] $\mathrm{mmH}_{2} \mathrm{O}$. 


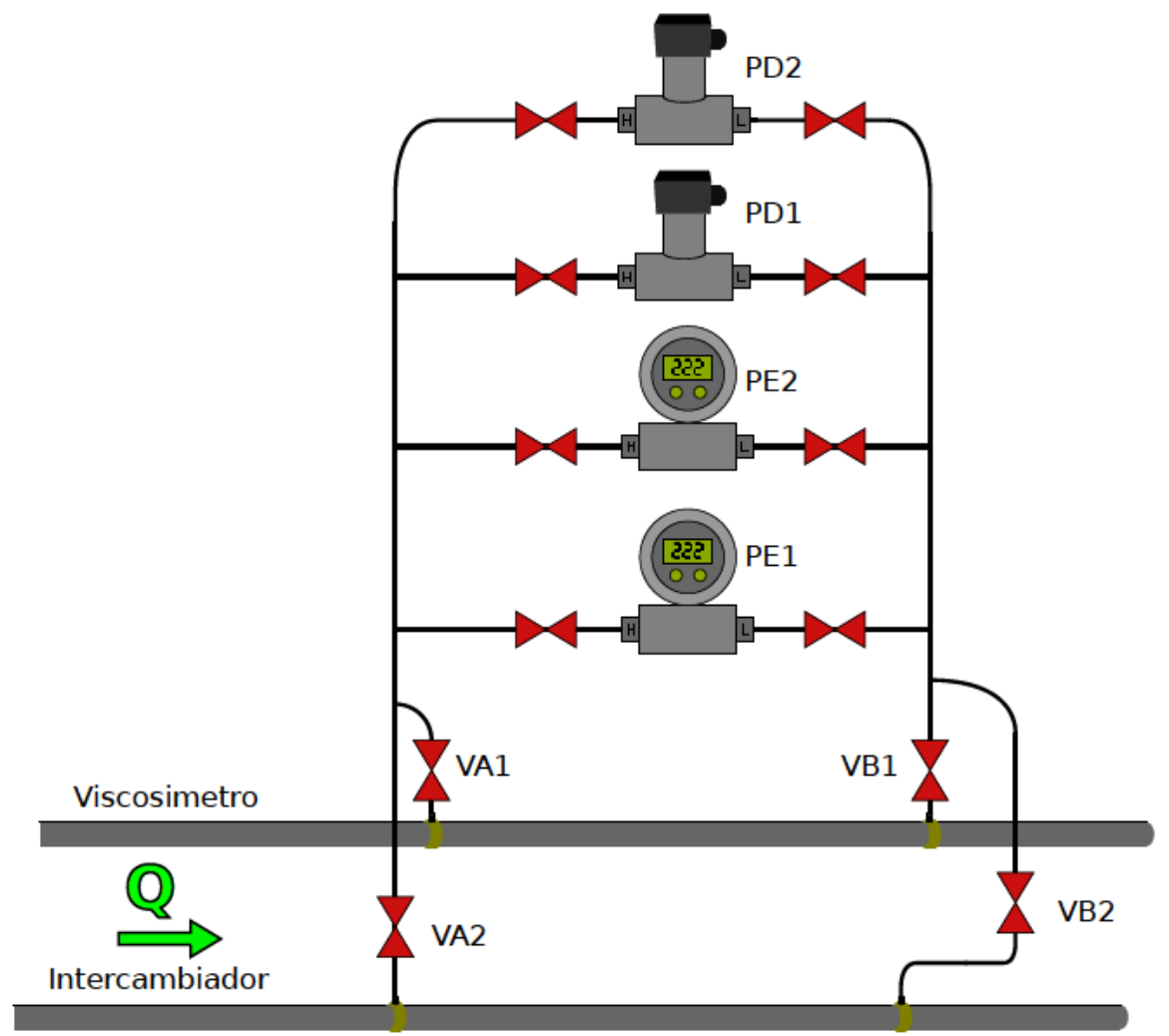

Figura 4.9: Detalle del circuito de medida de la caída de presión del tubo de ensayo o del viscosímetro.

- Sensores de presión diferencial no estacionaria PD1 y PD2

- Marca: Kistler.

- Tipo: 4264A.

- Rango PD1: $0-2$ bar.

- Rango PD2: 0 - 5 bar.

- No-linearidad, histéresis y precisión: $\pm 0,1 \%$ del F.E.

- Desviación debida a la temperatura entre $-15^{\circ} \mathrm{C}$ y $50^{\circ} \mathrm{C}: \pm 1 \%$.

Tal y como se ha descrito con anterioridad, el circuito principal por el que circula el fluido seudoplástico consta de dos tubos en paralelo: el viscosímetro y el tubo de ensayo. Mediante un sistema de válvulas de paso (véase Fig. 4.3), se puede dirigir el flujo hacia uno $\mathrm{u}$ otro tubo, dependiendo de si se quieren medir las propiedades del fluido o ensayar el tubo con el rascador. A consecuencia de esto, los sensores de presión se conectan a ambos tubos, 
tal y como muestra la Fig. 4.9. De este modo, si se desea medir la caída de presión en el viscosímetro, se deben abrir las válvulas VA1 y VB1 y cerrar VA2 y VB2. En cambio, si se desea medir en el tubo de ensayo, dichas válvulas deberían estar a la inversa. Además cada sensor de presión tendrá a la entrada y a la salida válvulas de paso, que permiten aislarlos del resto del circuito si no se van a utilizar o para protegerlos de presiones por encima de su rango de medida.

Las conexiones entre los tubos y los sensores se realizan mediante tubos de silicona. Existen válvulas de purga de aire tanto en los sensores de presión como en las tomas de presión en viscosímetro y tubo de ensayo.

Por un lado, cada toma de presión del tubo de ensayo consiste en un brida de 4 tomas que abraza el tubo, donde se han practicado 4 orificios de $2 \mathrm{~mm}$ de diámetro equiespaciados $90^{\circ}$. La distancia entre las bridas de presión en el tubo de ensayo define la sección de ensayo en las pruebas de fricción. Por otro lado, las tomas de presión del viscosímetro, en el que se supone un perfil de flujo de simetría radial, consisten en 1 sola perforación de $2 \mathrm{~mm}$ de diámetro.

Sistema de adquisición de datos. Se dispone de tres dispositivos para realizar la adquisición de las medidas de los diferentes sensores de los que dispone la instalación:

1. Primer dispositivo Agilent HP-34970A con 3 tarjetas. Cada tarjeta dispone de 20 entradas de tensión continua 0V - 10V y 2 entradas de señales analógicas. Esta dispositivo se utiliza para registrar las señales correspondientes a los siguientes dispositivos:

- 48 termopares del tubo de ensayo.

- Sondas RTD de temperatura: dos sondas en los extremos del paralelo entre el tubo de ensayo y el viscosímetro, una más en el depósito y otra midiendo la temperatura ambiente.

- Medidores de periodo de rascado. Señales $4-20 \mathrm{~mA}$.

- Señal de caudal. Esta señal también será leída por el segundo dispositivo Agilent HP-34970A. En los ensayos en los que el caudal no oscile, se utiliza la lectura de este primer dispositivo. Señal $4-20 \mathrm{~mA}$.

- Tensión aplicada al tubo de ensayo por el sistema de aporte de calor (señal analógica de tensión).

- Intensidad de corriente circulante por el tubo de ensayo. Señal 4-20 mA. 
2. Segundo dispositivo Agilent HP-34970A conectado a un PC adicional. Este dispositivo estará dedicado única y exclusivamente a la lectura de caudal en los ensayos dinámicos. Esto permite un registro detallado del mismo, ya que éste oscila en este tipo de ensayos.

3. Tarjeta de adquisición. Esta tarjeta se utiliza para la adquisición de las señales producidas por los sensores de presión no estacionaria, todas ellas $0-10 \mathrm{~V}$ :

- Sensor de presión diferencial no estacionaria del tubo de ensayo de rango [0,2] bar.

- Sensor de presión diferencial no estacionaria del tubo de ensayo de rango [0,5] bar.

- Sensor de presión no estacionaria diferencial bidireccional conectado al pistón que impulsa el rascador, de rango $[-15,20]$ bar.

Sistema de control de la instalación. Un programa en LabVIEW se encarga de controlar la instalación (temperatura de consigna, aporte de calor en la sección de ensayo, caudal, configuración del PID) y la adquisición de datos de las tarjetas. El llamado segundo dispositivo de adquisición de datos Agilent HP-34970A será controlado en mediante un PC dedicado que utiliza el Software del fabricante del propio dispositivo.

Mediante el programa de LabVIEW se pueden controlar los siguientes parámetros de la instalación: la temperatura del depósito, el caudal entregado por cada una de las bombas y el calor aportado mediante el sistema de aporte de calor.

\subsection{Instalación de visualización}

En el Departamento de Ingeniería Mecánica y Energía de la Universidad Miguel Hernández de Elche se encuentra la instalación de visualización del flujo en tubos, cuya fotografía aparece en la Fig. 4.10. Construida en 2004 para la visualización de flujos de fluidos como el agua o el propilenglicol en un rango de números de Reynolds alto, ha sido modificada para trabajar con fluidos no newtonianos de gran viscosidad. La geometría de rascador instalada es idéntica a la ensayada en la instalación de ensayos termohidráulicos pero a distinta escala (véanse las dimensiones en la Tabla 4.1).

La instalación, montada sobre una estructura de aluminio, consiste en el circuito detallado en la Figura 4.11, el cual consta de los siguientes elementos:

1. Depósito de remanso. Situado en la parte superior del circuito, se utiliza para proceder al llenado de la instalación. Además dispone de un sensor de temperatura tipo RTD 


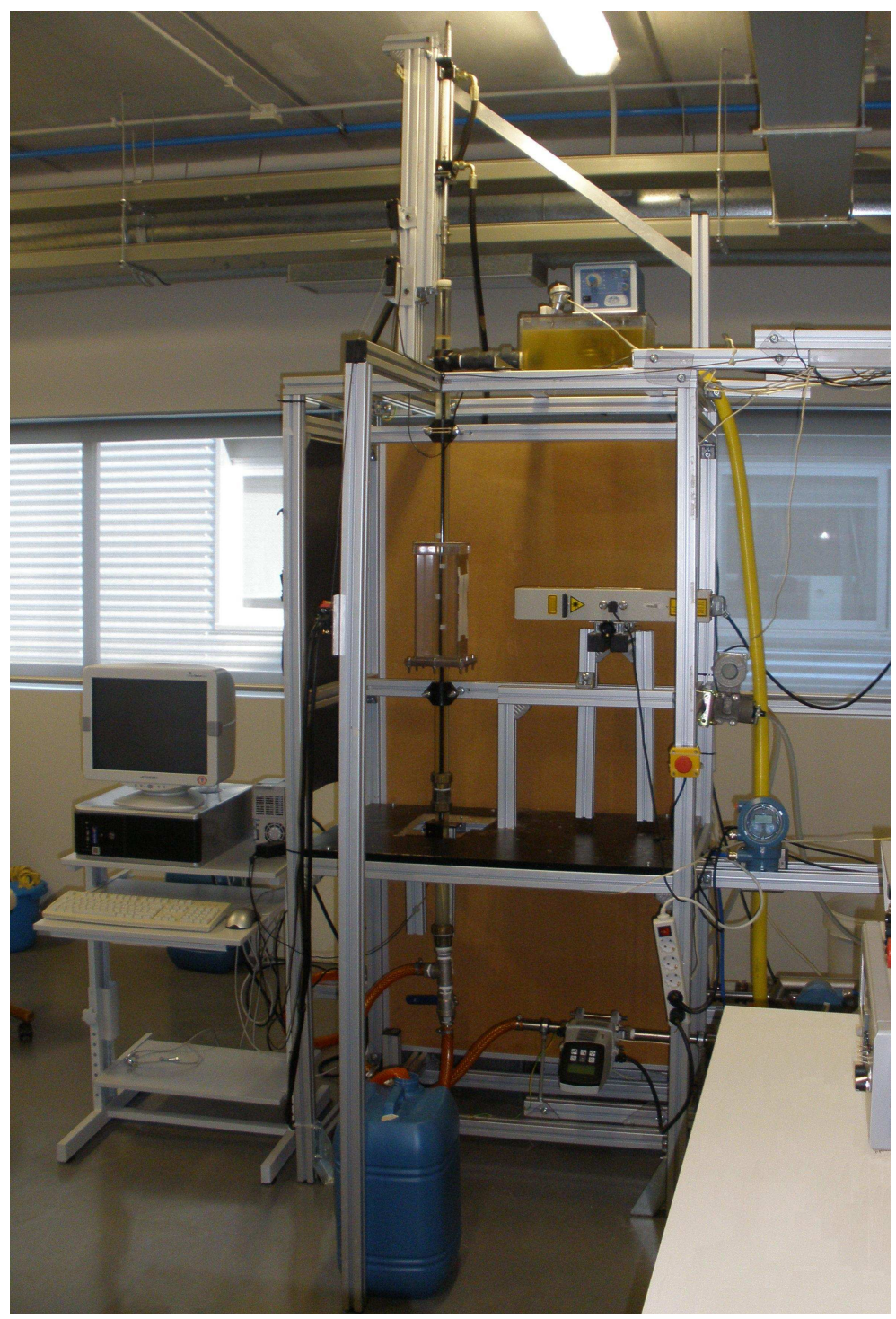

Figura 4.10: Fotografía de la instalación experimental para la visualización del flujo. Vista de conjunto del sistema. 


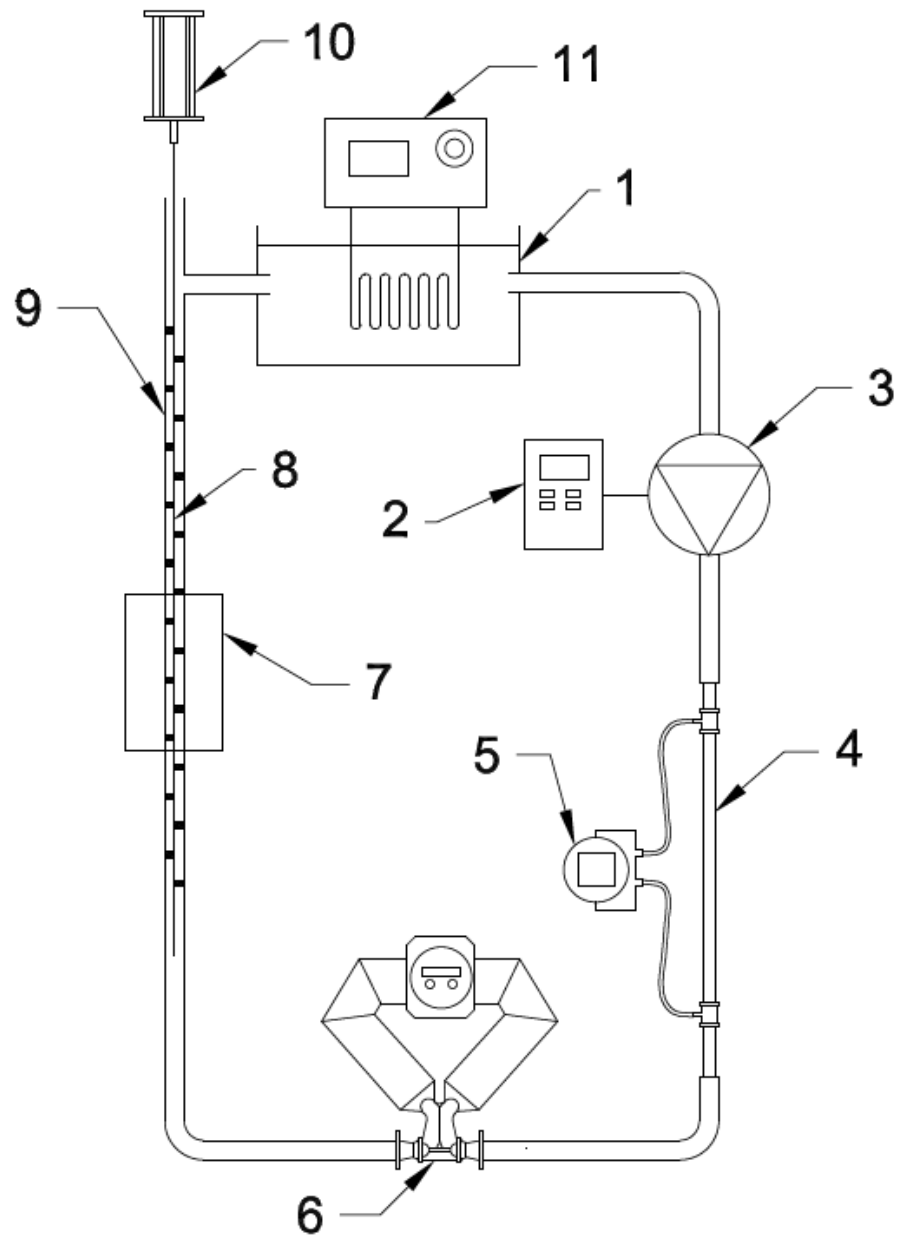

Figura 4.11: Esquema de la instalación de visualización. (1) Depósito de remanso, (2) variador de frecuencia, (3) bomba de engranajes, (4) viscosímetro, (5) sensor de presión diferencial estacionaria, (6) caudalímetro de efecto Coriolis, (7) sección de visualización, (8) rascador, (9) tubo acrílico, (10) sistema de impulsión del rascador, (11) baño térmico. 
(a la entrada del fluido al depósito) y de un baño térmico, utilizados para controlar la temperatura del fluido en la instalación.

2. Variador de frecuencia de la bomba. Regula las revoluciones de la bomba.

3. Bomba de engranajes. Modelo AL-3 de hasta 10 bar acoplada a un motor de $1,5 \mathrm{~kW}$ y $2840 \mathrm{rpm}$ mediante una reductora 1:9. Es un modelo idéntico al utilizado en la instalación del Apartado 4.2.

4. Viscosímetro. El viscosímetro, es constructivamente similar al utilizado en la instalación de ensayos termohidráulicos. Para hacerlo se ha utilizado el mismo tipo de tubo de $18 \mathrm{~mm}$ de diámetro interior y $2 \mathrm{~mm}$ de espesor. Sin embargo el tubo es un poco más corto por motivos de espacio, 2,5 m de longitud total, donde la sección de entrada mide $0,8 \mathrm{~m}$, la de ensayo $1,38 \mathrm{~m}$ y la de salida $0,5 \mathrm{~m}$, con un tubo flexible que provoca una salida suave del tubo. Se encuentra instalado en serie con la sección de ensayo, de modo que es posible medir las propiedades reológicas del fluido en cualquier momento.

5. Transmisor diferencial de presión. Modelo SMAR-LD301 de rango 0 - $500 \mathrm{mmH}_{2} \mathrm{O}$, mide la caída de presión en el viscosímetro mediante 2 tomas de presión consistentes en sendos orificios en el tubo liso cubiertos con bridas y conectadas al sensor mediante tubos de silicona.

6. Caudalímetro de efecto Coriolis. Modelo ELITE CMF025. Rango 2 - 2000 l/h. Capacidad para medir caudales, flujo másico, densidad y temperatura. El dispositivo tiene una precisión del $\pm 1 \%$ de la medida y una repetibilidad del $\pm 0,05 \%$ en las medidas de caudal. Es idéntico al utilizado en la instalación de ensayos termohidráulicos.

7. Sección de visualización. Sección del tubo acrílico donde se realizará la visualización del flujo. Está situado a una distancia de 15 diámetros desde la entrada al tubo para asegurar condiciones de flujo plenamente desarrollado. La sección del tubo se encuentra en el interior de un depósito de forma de prisma cuadrangular, que se rellena con el fluido de trabajo para evitar distorsiones en la imagen.

8. Elemento insertado. El rascador insertado realizará un movimiento alternativo, impulsado por el cilindro neumático (10). La geometría del elemento insertado se muestra en la Fig 1.6. La geometría del mismo se encuentra descrita en el Apartado 4.1. 
9. Tubo acrílico. Diseñado con un diámetro interior igual al de los intercambiadores comerciales, de $32 \mathrm{~mm}$.

10. Cilindro neumático de doble efecto. Conectado a una centralita hidráulica de aceite y sujeto al elemento rascador por la parte superior del último, provee a éste de movimiento. Al igual que en la instalación de ensayos termohidráulicos (Apartado 4.2), la amplitud del movimiento se limita mediante finales de carrera (véase Fig. 4.8), la velocidad de desplazamiento del rascador se controla a partir del régimen de giro de la bomba de la centralita hidráulica, regulado por un variador de frecuencia y el periodo de rascado se mide mediante un tacómetro digital TA-202.

11. Baño térmico. Se encuentra instalado en el depósito de reserva y regula la temperatura del fluido de trabajo.

Para la adquisición de imágenes se aplica la técnica de Velocimetría por Imágenes de Partículas (Particles Image Velocimetry - PIV), descrita en el Apartado 4.3.1. A continuación se detallan las características de los elementos principales del sistema de PIV:

1. Sección de visualización (elemento 7 de la Fig. 4.11). Se trata de una caja acrílica con forma de prisma cuadrangular instalada alrededor del tubo acrílico. Ésta se encuentra rellena con el fluido de ensayo. Su función es la de evitar la distorsión de la imagen.

2. Sistema de iluminación láser. Se trata de un láser de diodos pulsado OXFORD LASER HSI 5000 que, gracias al sistema de lentes que incorpora, emite un plano de luz infrarroja. Las características del láser son las siguientes:

- Fuente de luz: láser de diodos.

- Clase del láser: IV.

- Longitud de onda:808 nm.

- Potencia pico: $200 \mathrm{~W}$.

- Duración del pulso individual: $1 \mu \mathrm{s}$ a $80 \mu \mathrm{s}$.

- Energía del pulso: 0,15 mJ a 15 mJ.

3. Cámara. Modelo MotionScope M3. Se trata de una cámara de tecnología CMOS de alta velocidad: 


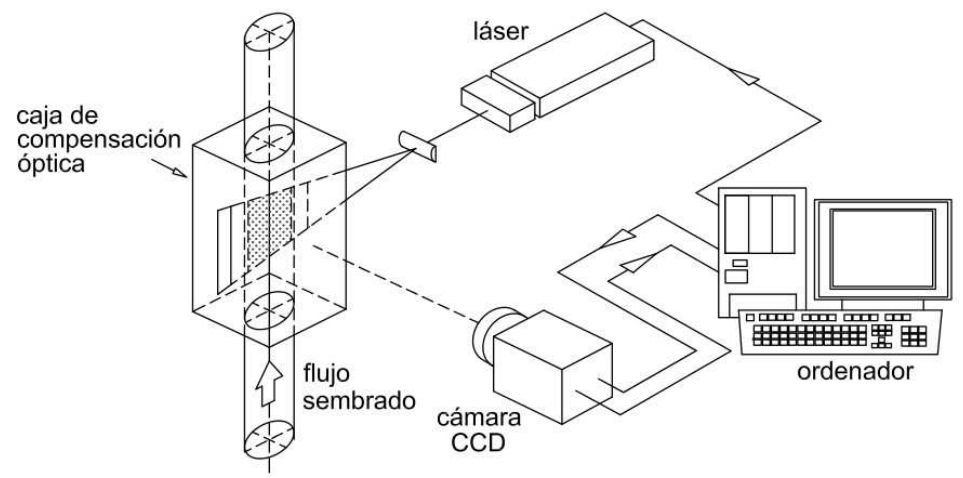

Figura 4.12: Principio de funcionamiento de la técnica PIV.

a) Velocidad de captura de imágenes: 10 - 1000 fps a máxima resolución. Puede llegar hasta velocidades de adquisición de 31000 fps con pérdida de resolución vertical.

b) Resolución máxima 1280x1024 píxeles. Resolución a 31000 fps: 1280x10 píxeles.

c) Capacidad de disparo mediante disparador externo. Esta capacidad será utilizada en los ensayos dinámicos.

d) Óptica de la cámara. Se ha acoplado a la cámara una óptica de 20 aumentos. El número focal del objetivo empleado es 4, lo que permite capturar la máxima cantidad de luz.

4. Disparador fotoeléctrico. Modelo E3JM-R4M4T-G. Emite una señal de disparo al paso del rascador por un determinado punto. Además incorpora temporizador interno que puede retrasar la señal de disparo a partir del paso del rascador.

\subsubsection{Técnica de Velocimetría por Imágenes de Partículas (PIV)}

La Velocimetría por Imágenes de Partículas (PIV) es una potente técnica de medida no intrusiva, que permite obtener dos componentes de la velocidad del flujo en un plano del campo fluido. Generalmente, proporciona una alta resolución espacial, suficiente para capturar las estructuras del flujo más importantes. Los principales componentes de un sistema típico de PIV son los siguientes:

- Flujo sembrado de partículas trazadoras.

- Un cabezal láser. 
- Una cámara de alta velocidad.

- Un sistema de sincronización entre el láser y la cámara.

- Un sistema de disparo.

- Un software de procesado de datos.

El principio de trabajo de esta técnica, ilustrado en la Figura 4.12, se basa en el sembrado del flujo con pequeñas partículas trazadoras, que deben tener una densidad muy similar a éste. Un haz de luz producido por un láser ilumina dos veces las partículas contenidas en un plano del campo fluido. El intervalo de tiempo entre los dos pulsos generados por el láser depende de la velocidad del flujo y de las dimensiones de la superficie bajo estudio. Una cámara de alta velocidad captura la luz desprendida por las partículas, y almacena los dos fotogramas sucesivos en formato digital.

Para procesar las imágenes grabadas, los dos fotogramas se dividen en pequeñas regiones llamadas ventanas o áreas de interrogación, tal y como se muestra en el ejemplo de la Fig. 4.13(a). Asumiendo que todas las partículas contenidas en una ventana de interrogación se desplazan homogéneamente durante el intervalo entre pulsos, se puede emplear un método estadístico de correlación cruzada para determinar el vector desplazamiento más probable asociado a cada ventana de interrogación. Finalmente, la velocidad se calcula tomando el tiempo de separación entre los dos pulsos de luz.

Los técnicas más avanzadas de PIV aplican con posterioridad al algoritmo de Correlación Cruzada, sucesivos algoritmos de Correlación Cruzada Adaptativa, que utilizan información de la primera correlación para desplazar las ventanas/áreas de interrogación en la dirección del flujo, aumentando la precisión y resolución de los resultados (Scarano y Reithmuller, 2000). En la Fig. 4.13(b) se muestra un ejemplo del desplazamiento de las Áreas de Interrogación que utiliza la Correlación Cruzada Adaptativa.

De éste modo, la Velocimetría por Imágenes de Partículas permite obtener la velocidad del flujo indirectamente, midiendo la velocidad de las partículas trazadoras.

La elección del tiempo de separación entre dos imágenes consecutivas está condicionada por dos factores: por un lado, el desplazamiento máximo de las partículas debe ser igual a 1/4 del tamaño de la ventana de interrogación, y por otro lado, es necesario comprobar que el producto del tiempo de separación entre fotogramas y la componente máxima de velocidad fuera del plano de estudio es menor que $1 / 4$ del espesor de la lámina de luz. 


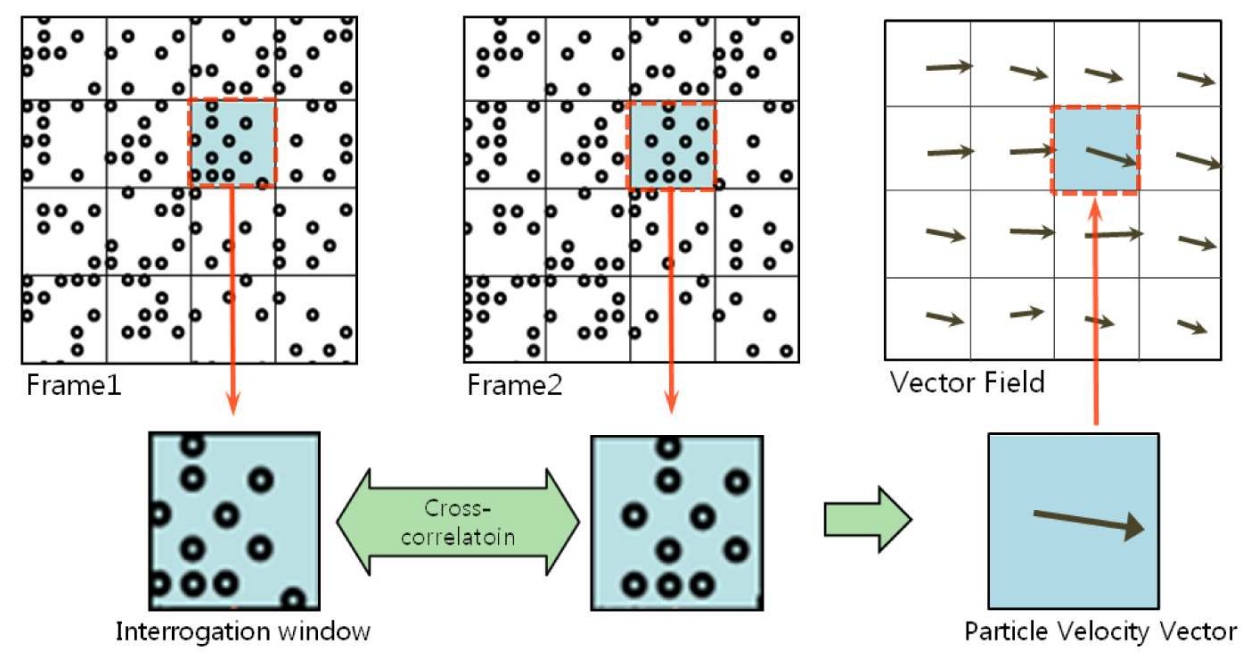

(a) Principio de Correlación Cruzada. Imagen creada por Choi et al. (2011)

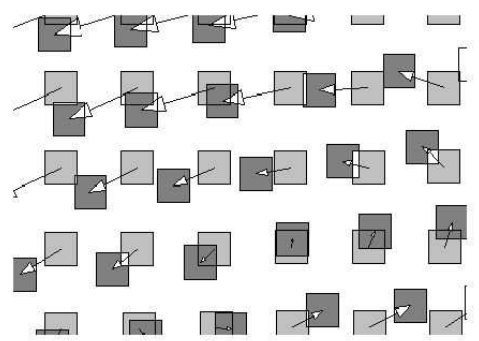

(b) Desplazamiento de las Áreas de Interrogación al aplicar la Correlación Cruzada Adaptativa.

Figura 4.13: Esquemas sobre los principios de los algoritmos de Correlación Cruzada y Correlación Cruzada Adaptativa 
En la bibliografía (Raffel et al., 2000; Doorne y Westerweel, 2007) se encuentran descripciones más detalladas sobre los principios en los que se basa la técnica de Velocimetría por Imágenes de Partículas, así como otras variantes de la técnica basadas en PIV. 


\section{Capítulo 5}

\section{Caracterización del fluido de trabajo}

Para la presente investigación se ha seleccionado como fluido de trabajo una disolución en agua de carboximetil celulosa (CMC) con una concentración del $1 \%$ en peso. La selección de dicho fluido se justifica en base a los siguientes factores:

- Es un fluido de comportamiento seudoplástico cuyo comportamiento reológico se ajusta correctamente al modelo Power Law.

- La preparación del fluido es sencilla: consiste en disolver en agua ligeramente ácida (adición de $\mathrm{HCl}$ ), a temperatura ambiente, la correspondiente cantidad de $\mathrm{CMC}$ mediante agitación.

- El fluido es incoloro y transparente: apto para la visualización del flujo mediante la técnica de Velocimetría por Imágenes de Partículas (PIV).

- Seguridad en la manipulación: compuesto no tóxico y biodegradable.

La carboximetil celulosa pertenece a la familia de los hidrocoloides y se utiliza habitualmente para mejorar la consistencia y la textura de productos alimenticios líquidos, semilíquidos y semisólidos. La CMC tiene gran cantidad de aplicaciones en numerosas industrias (Yang y Zhu (2007)): alimentación, cosmética, farmacéutica y en la industria del petróleo. Su propiedad más interesante para dichas aplicaciones es su alta viscosidad a bajas concentraciones. Por ejemplo, en la industria alimenticia se usa como estabilizador, medio de unión, relleno y para retener agua en galletas, pasteles, helados, zumos, salsas, sopas deshidratadas y productos dietéticos (Pilizota et al. (1996)). 
Para el estudio de los procesos de transferencia de calor en intercambiadores, las propiedades termofísicas del fluido de trabajo que se deben conocer, son: las propiedades reológicas, la conductividad térmica, la densidad, el calor específico y el coeficiente de expansión térmica. Donde ésta última propiedad será necesaria únicamente para el estudio de la transferencia de calor por convección libre.

Se establece por tanto como primer objetivo del presente capítulo, el de caracterizar el comportamiento del fluido de trabajo en el tubo con rascador en cuanto a sus propiedades termofísicas.

Por otro lado, en el Capítulo 2 se establece la conveniencia de utilizar un método de generalización de la viscosidad y consecuentemente de los números de Reynolds y Prandtl, de modo que el problema fluidomecánico se reduce en un grado de libertad, siendo $f=\Psi\left(R e_{g}\right)$. No obstante, las alternativas valoradas en dicho capítulo no son de aplicación en la geometría de tubo con rascador analizada. Así, se establece como segundo objetivo del capítulo la obtención de la definición apropiada para la viscosidad generalizada (Apartado 5.3).

En resumen, los objetivos del capítulo son:

1. Caracterización del fluido de trabajo.

2. Generalización de la viscosidad.

\subsection{Reología}

La reología se define como la parte de la física que estudia la relación entre el esfuerzo y la deformación en los materiales que son capaces de fluir. Así, el diseño de procesos industriales con fluidos no newtonianos, requiere de datos precisos sobre la reología de los mismos, ya que las características del flujo dependen de la reología y densidad del fluido. Es por ello que existen numerosas publicaciones al respecto: Cancela et al. (2005), Yang y Zhu (2007), Abdelrahim y Ramaswamy (1995), Ghannam y Esmail (1996) y Abu-Jdayil (2003) estudian las propiedades reológicas de las disoluciones de CMC en agua a diferentes concentraciones y temperaturas. Los resultados muestran importantes variaciones en el comportamiento que dependen de la concentración. En concentraciones bajas, el fluido tiene un comportamiento casi newtoniano que evoluciona a seudoplástico al incrementar la concentración. Además, a concentraciones muy altas aparecen significativos efectos viscoelásticos y tixotrópicos.

Según todos los estudios mencionados, el modelo Power Law es el que mejor describe el comportamiento reológico del fluido (Ghannam y Esmail (1996); Abdelrahim y Ramaswamy 
(1995); Cancela et al. (2005); Joshi y Bergles (1980b)). Tal y como se ha comentado en el Apartado 1.1.1.1, el modelo Power Law relaciona los esfuerzos tangenciales aplicados a un fluido $(\tau)$ con la velocidad de deformación resultante de dichos esfuerzos $(\gamma)$, mediante la Ec. 5.1 .

$$
\tau=m \gamma^{n}
$$

Así, para conocer el comportamiento reológico del fluido, únicamente es necesario conocer el valor de los parámetros $m$ (Índice/Coeficiente de consistencia del fluido) y $n$ (Índice de comportamiento de flujo). La metodología que se ha utilizado para hallar los valores de $m$ y $n$ se describe en el Apartado 5.1.1.

En la Fig. 5.1 se representa el esfuerzo tangencial frente a la velocidad de deformación en escala logarítmica para diferentes preparaciones de CMC. Como se puede observar, los datos experimentales de las mezclas se pueden ajustar a una recta, lo cual justifica la modelización del fluido mediante la Ec. 5.1.

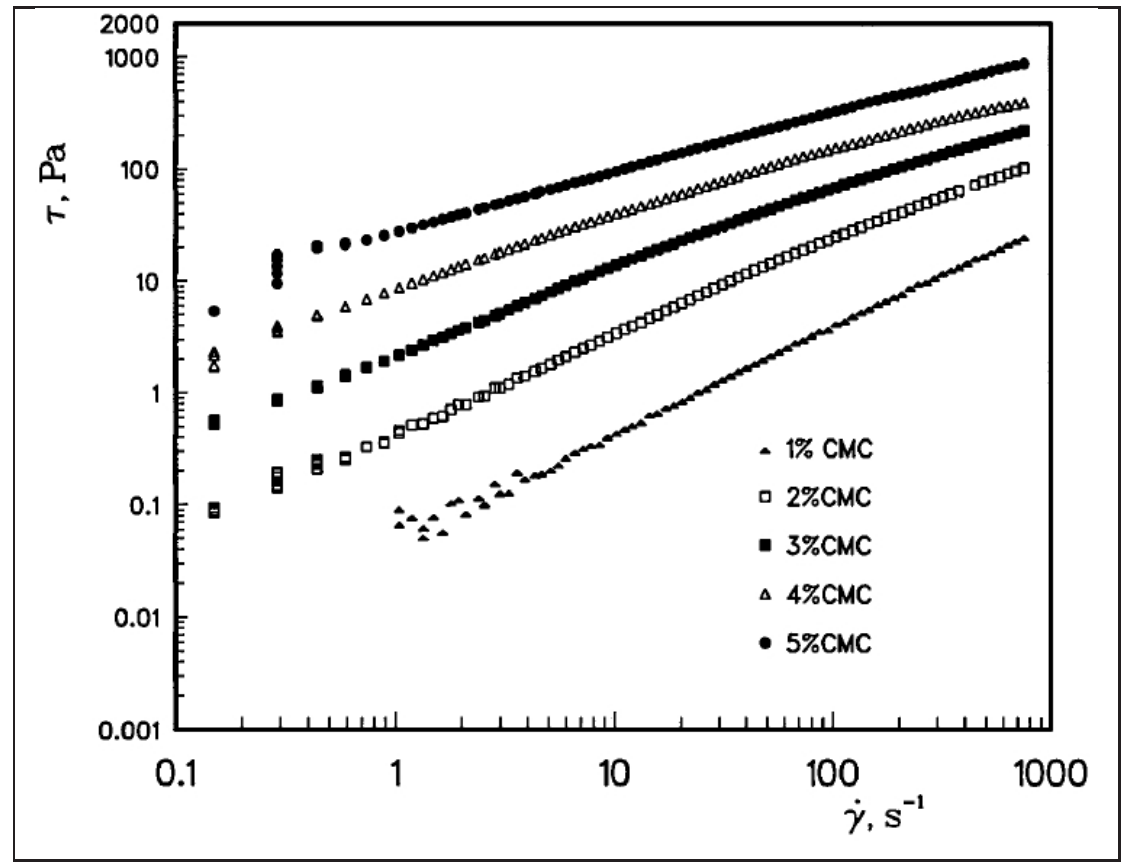

Figura 5.1: Representación del esfuerzo tangencial frente a la velocidad de deformación en disoluciones de diferentes concentraciones de CMC en agua. La representación es original de Ghannam y Esmail (1996).

El objetivo que se persigue será poder medir o estimar el valor de $m$ y $n$ durante el transcurso de los ensayos realizados en el laboratorio. 
La mayoría de las propiedades que tenga la disolución de CMC en agua dependerán, tanto del tipo de CMC utilizado (longitud de cadena y pureza), como del proceso de preparación del fluido. Es por ello que las conclusiones extraídas de los análisis que se realizan en la bibliografía, son válidos únicamente de forma general. Por ejemplo, no es apropiado utilizar las correlaciones obtenidas por otros autores para modelar la dependencia de las propiedades reológicas del fluido $(m$ y $n$ ) con la temperatura, ya que el fluido preparado mediante otros métodos y con CMC de otras características puede tener propiedades distintas. Sin embargo, sí sería apropiado utilizar expresiones sugeridas por otros autores, pero obteniendo nuevos coeficientes, válidos únicamente para el fluido de trabajo utilizado en la presente investigación. Por lo tanto, se impone la medición de las propiedades reológicas en el laboratorio. El método utilizado para realizar dicha medición se describe en el Apartado 5.1.1.

Por último, las propiedades del fluido durante los ensayos pueden diferir de las medidas dependiendo de diferentes factores, tales como la degradación del fluido (tixotropía) o la variación de la temperatura del mismo. En consecuencia, se estimará la desviación sobre las propiedades medidas producida por ambos efectos, mediante sendos métodos descritos en el Apartado 5.1.2.

\subsubsection{Metodología de medición de las propiedades reológicas}

Antes de realizar un experimento se deben conocer las propiedades reológicas del fluido utilizado, o lo que es lo mismo, los valores de $m$ y $n$. Éstos varían: con el tipo y la concentración de CMC, la temperatura y la degradación del fluido.

Así, interesa determinar dichos parámetros antes y después de cada serie de ensayos, controlando que la variación de las propiedades reológicas del fluido debidas a la degradación del fluido durante el ensayo sea pequeña, para así minimizar el error que se pueda cometer.

La medición de las propiedades reológicas se basa en la relación existente entre el caudal circulante por un tubo liso en régimen de flujo laminar y la caída de presión que se debe producir en el mismo.

A partir de la caída de presión $\Delta p$ se puede obtener el esfuerzo cortante en la pared:

$$
\tau_{w}=\frac{\Delta p D_{v}}{4 L}
$$

El perfil de velocidad de un fluido Power Law en un tubo liso se puede deducir del planteamiento de la ecuación de conservación de cantidad de movimiento en el eje $x$, considerando 
que el flujo es axisimétrico y aplicando las condiciones de contorno pertinentes:

- flujo estacionario,

- $u_{r}=0$ y $\partial u_{z} / \partial z=0$,

- condición de no deslizamiento en la pared (en $r=0 \rightarrow u_{z}=0$ )

- condición de simetría en el eje (en $\left.r=R \rightarrow \partial u_{z} / \partial r=0\right)$.

Aplicando las condiciones mencionadas y desarrollando la ecuación, se obtiene que el perfil de velocidades viene dado por la siguiente expresión:

$$
\frac{u_{z}(r)}{u_{b}}=\left(\frac{3 n+1}{n+1}\right)\left[1-\left(\frac{r}{R}\right)^{(n+1) / n}\right]
$$

Por otro lado de la ecuación constitutiva del modelo, se obtiene una relación entre $\tau_{w}$, $d u_{z} / d r$ en la pared, $n$ y $m$ (Ec. 5.3). Resolviendo la derivada y tomando logaritmos neperianos a ambos lados de la ecuación, se obtiene una relación lineal entre la velocidad media del fluido, $u_{b}$, y el esfuerzo cortante en la pared, $\tau_{w}$.

Por último, los coeficientes de la ecuación lineal (Ec. 5.4) se pueden obtener, a partir de una serie de medidas experimentales de $\tau_{w}$ y $u_{b}$, mediante un ajuste por mínimos cuadrados, obteniendo los valores de $m$ y $n$.

$$
\begin{gathered}
\tau_{w}=m\left(\left.\frac{\partial u_{z}}{\partial r}\right|_{w}\right)^{n} \\
\tau_{w}=m\left[\frac{8 u_{b}}{D_{v}}\left(\frac{3 n+1}{4 n}\right)\right]^{n} \\
\ln \left(\tau_{w}\right)=n \times \ln \left(u_{b}\right)+\ln (m)+n \times \ln \left[\frac{8}{D_{v}}\left(\frac{3 n+1}{4 n}\right)\right]
\end{gathered}
$$

Por lo tanto, para medir $m$ y $n$, se necesita un dispositivo capaz de medir la caída de presión y el caudal circulante en un tubo liso en condiciones de flujo laminar.

Para ello se utiliza un viscosímetro diseñado ex profeso. En la Fig. 5.2 se muestra un esquema del diseño del mismo. Existe un viscosímetro instalado en cada una de las instalaciones experimentales utilizadas y cuyas dimensiones concretas se detallan en la descripción de dichas instalaciones (Capítulo 4). El viscosímetro consiste de un tubo liso, que a su vez 


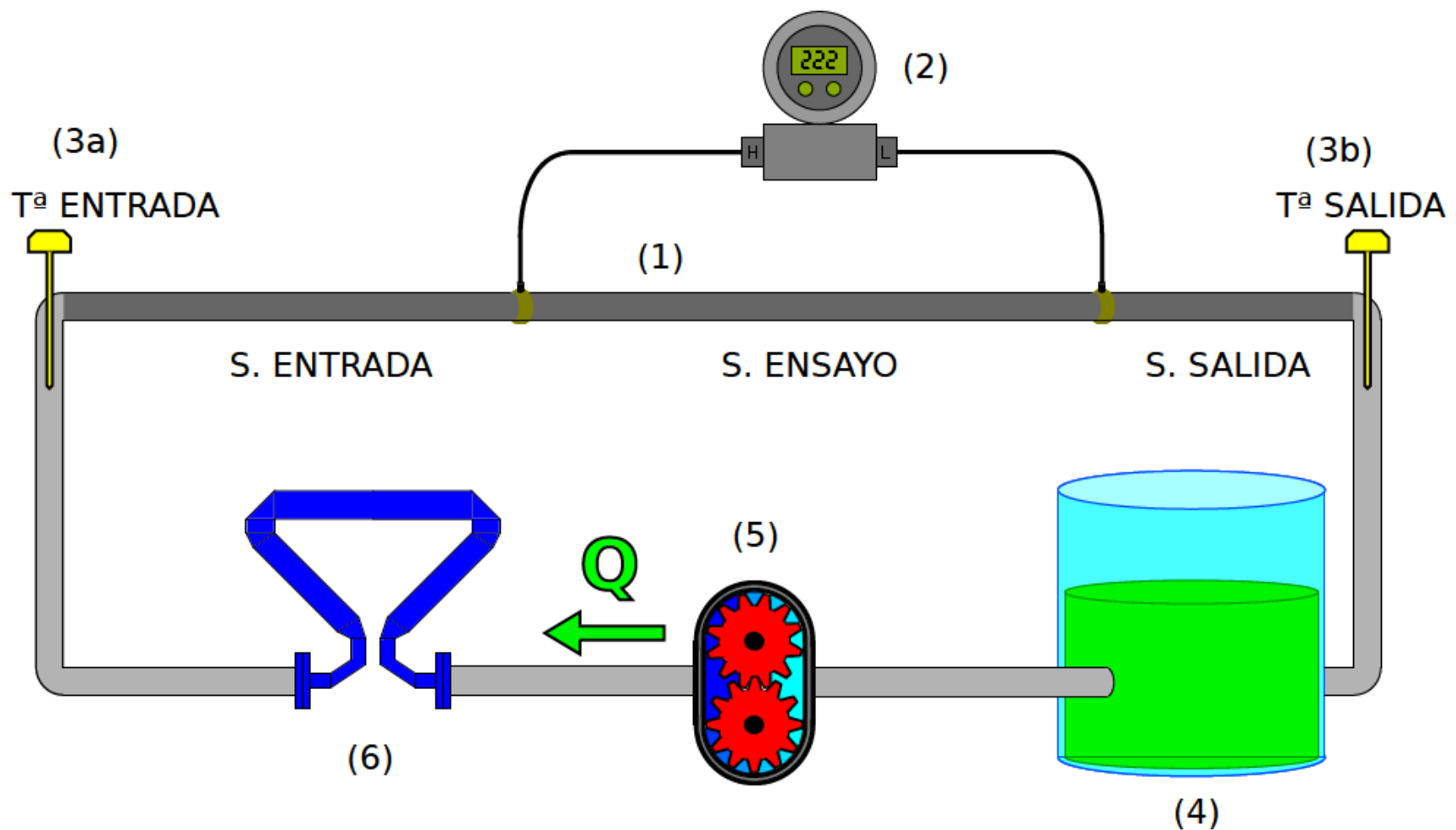

Figura 5.2: Esquema del viscosímetro utilizado para medir las propiedades reológicas en las instalaciones experimentales. Longitud de las diferentes secciones del tubo liso: sección entrada de $1 \mathrm{~m}$, sección de ensayo de $1,8 \mathrm{~m}$ y sección de salida de $0,8 \mathrm{~m}$. Elementos de la instalación: (1) Tubo liso de $18 \mathrm{~mm}$ de diámetro interior -viscosímetro-, (2) Sensor de presión diferencial estacionario ( 5 - 500 mbar), (3) Sondas de temperatura RTD de alta precisión, (4) Depósito de fluido con sistema de regulación de temperatura incorporado, (5) Bomba de engranajes, (6) Caudalímetro de efecto Coriolis. 
consta de 3 tramos distintos: 1) región de entrada para asegurar el desarrollo completo del flujo laminar, 2) a continuación, entre las tomas de presión de un sensor diferencial, se encuentra el tramo de ensayo, 3) y por último una región de salida, cuya función consiste en que el flujo laminar en el tramo de ensayo no se vea perturbado.

El procedimiento de ensayo consiste en hacer circular fluido por el viscosímetro, se toman entre 10 y 20 medidas de caída de presión para cada caudal (20 medidas para los caudales más pequeños y 10 para los más grandes), ensayando un total de 5 caudales en el rango que proporciona el sistema de bombeo; típicamente desde $100 \mathrm{l} / \mathrm{h}$ hasta $1500 \mathrm{l} / \mathrm{h}$.

Por último, los resultados obtenidos en el experimento se ajustan mediante la Ec. 5.4, para obtener los valores de $m$ y $n$. En la Fig. 5.3 se muestra a modo de ejemplo el ajuste realizado en uno de los ensayos.

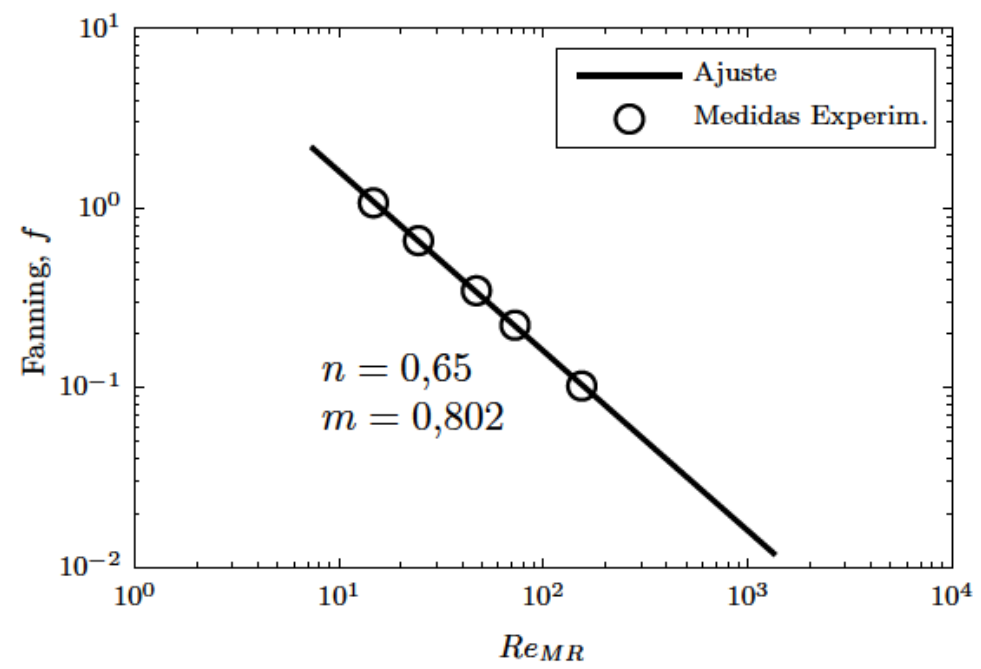

Figura 5.3: Medición de las propiedades reológicas del fluido de trabajo durante el transcurso de uno de los ensayos.

\subsubsection{Variación de las propiedades reológicas del fluido}

Tal y como se ha comentado con anterioridad, en el modelo Power Law el comportamiento de un fluido en concreto viene dado por las propiedades $m$ (índice de consistencia de fluido) y $n$ (índice de comportamiento de flujo). Éstas se mantienen constantes mientras también lo hagan las condiciones en las que se encuentra el fluido. Ahora bien, según indican numerosos estudios, las propiedades reológicas del fluido, $m$ y $n$, pueden variar significativamente debido 
a: una degradación del fluido (tixotropía) o a una variación en la temperatura del fluido.

- Degradación o tixotropía. La tixotropía del fluido tiene que ver con una degradación de la estructura de las macromoléculas que conforman el fluido. La consecuencia dicha degradación es una disminución de la viscosidad del fluido y, en el caso concreto de las disoluciones de CMC en agua, una disminución del comportamiento seudoplástico. Es decir, una disminución de $m$ y un aumento de $n$. Las causas de la degradación durante los ensayos pueden ser: someter el fluido a esfuerzos cortantes o/y a tratamientos térmicos.

- Variación de la temperatura del fluido. Al igual que ocurre en los fluidos newtonianos, los fluidos no newtonianos varían sus propiedades en función de la temperatura a la que se encuentren. En concreto, las propiedades reológicas $m$ y $n$ de un fluido seudoplástico sufrirán una variación al variar la temperatura del fluido.

Resulta significativo que un tratamiento térmico del fluido para calentarlo, provocará ambos efectos, ya que las propiedades del fluido cambiarán al hacerlo la temperatura del mismo, pero a su vez, se producirá una degradación del fluido debida al tratamiento térmico al que es sometido.

Teniendo en cuenta estos efectos, en el presente apartado se busca el método para determinar o estimar con suficiente exactitud, las propiedades reológicas del fluido en las condiciones en que se realiza el ensayo. Para ello, es necesario conocer cómo varían las propiedades reológicas al hacerlo la temperatura y con la degradación del fluido. A continuación se analizan ambos efectos por separado.

\subsubsection{Degradación del fluido o tixotropía}

La degradación del fluido es un proceso complejo y difícil de cuantificar, ya que dependerá de los esfuerzos y tratamientos térmicos a los que se someta el fluido. Por ejemplo, Rao et al. (1981), realizando ensayos a $30^{\circ} \mathrm{C}$ antes y después del procesado, demostraron que el procesado térmico tiene efectos negativos sobre la estructura del CMC.

Ante la dificultad de cuantificar la degradación del fluido en procesos complejos en los que existen diferentes causas para la misma, se impone medir las propiedades $n$ y $m$ antes y después de cada ensayo o de cada grupo de ensayos. De este modo, se puede suponer una degradación lineal del fluido y conocer sus propiedades reológicas en cada momento. Para utilizar esta técnica, minimizando el error que se comete al obtener $m$ y $n$ durante uno de los 
ensayos, se debe perseguir y comprobar que la variación de las propiedades del fluido entre una medida y la siguiente no sea excesivamente importante.

Para reducir la degradación del fluido durante los ensayos, se toman las siguientes medidas en el diseño de la instalación:

1. Sistema de bombeo: se trabaja con bombas de engranajes sobredimensionadas, de modo que trabajen a bajas revoluciones.

2. Sistema de regulación de temperatura: ha sido diseñado con el objetivo de minimizar la degradación del fluido, ya que mal diseñado, puede ser una de las mayores fuentes de degradación. Esto se ha conseguido utilizando, para el calentamiento del fluido de trabajo, un circuito secundario de agua caliente que circula por un serpentín instalado en el depósito de regulación. Se ha comprobado además, que el uso de una resistencia eléctrica para este menester degrada significativamente el fluido. En el Apartado 4.2 se describe con más detalle el sistema de regulación de la temperatura.

3. Simplificación del circuito hidráulico: se ha simplificado al máximo el circuito hidráulico, eliminando elementos susceptibles de degradar el fluido (filtros, codos, bifurcaciones, y otros elementos prescindibles).

\begin{tabular}{ccc}
\hline & Ensayo anterior & Ensayo posterior \\
\hline$n$ & 0,693 & 0,71 \\
$m\left[\right.$ Pa.s $\left.{ }^{\mathrm{n}}\right]$ & 0,643 & 0,579 \\
$T\left[{ }^{\circ} \mathrm{C}\right]$ & 16,5 & 16,5 \\
\hline
\end{tabular}

Cuadro 5.1: Degradación de las propiedades reológicas durante la realización de una serie de ensayos de transferencia de calor. Entre ambas medidas se han realizado 52 ensayos dinámicos de transferencia de calor de forma consecutiva. Nota: los resultados reológicos, medidos a temperaturas de $16,9^{\circ} \mathrm{C}$ y $16^{\circ} \mathrm{C}$, han sido corregidos mediante las correlaciones obtenidas en el Apartado 5.1.2.2, y trasladadas a una temperatura intermedia para que sean comparables.

Para poder cuantificar la degradación que se produce en una serie de ensayos, se toma como ejemplo una de las series en las que la degradación es mayor: se trata de una serie correspondiente a los ensayos dinámicos de transferencia de calor. En la Tabla. 5.1 se muestran las mediciones de propiedades realizadas antes y después de la serie de ensayos en cuestión. Como se puede observar, la variación que se ha producido en las propiedades es aceptable. 
Así, para cada ensayo de la serie se puede hacer una estimación del valor de las propiedades reológicas suponiendo que la degradación del fluido es lineal en el tiempo.

\subsubsection{Variación de las propiedades con la temperatura}

En la bibliografía existente, queda constancia de la variación que sufren las propiedades $m$ y $n$ del modelo Power Law al variar la temperatura de un fluido seudoplástico. Sin embargo, cada autor utiliza modelos distintos para caracterizar dicha variación y a menudo discrepan en la relación existente entre $n$ y $T$.

Por un lado Joshi y Bergles (1980b), utilizan una relación exponencial para la relación entre $m$ y $T$ (Ec. 5.5) y suponen $n$ invariable con la temperatura.

$$
m=A e^{B T}
$$

Cancela et al. (2005), demuestran que la dependencia de las propiedades con la temperatura es lineal para ambas $(n, m)$ y basan sus deducciones en 4 puntos de temperatura $\left(25,30,35 \mathrm{y} 40^{\circ} \mathrm{C}\right)$ y diferentes concentraciones.

Abdelrahim y Ramaswamy (1995) evalúan la variación de las propiedades de las disoluciones de CMC en agua con diferentes modelos, donde varían la temperatura y la concentración. Los autores concluyen que tanto $m$ como $n$ de las disoluciones de CMC en agua son sensibles a cambios en la temperatura y la concentración. Además proponen una modificación de la aproximación de Turian (1964) para describir la influencia combinada de la temperatura y la concentración, el cual podría ser incorporado al modelo Power Law para realizar cálculos de ingeniería (Awuah et al. (1993)). Por otro lado confirman que el modelo desarrollado por Weltmann (1943) describe de forma adecuada la dependencia temporal de las propiedades de las disoluciones de CMC con el tiempo. De este modo, proponen las relaciones expresadas por la Ec. 5.6 y la Ec. 5.7 para cuantificar las propiedades del fluido, $m$ (con $\left.R^{2}=0.97\right)$ y $n$ (con $\left.R^{2}=0.94\right)$ respectivamente, a partir de la concentración de CMC, $C$, y la temperatura $T$.

$$
\begin{gathered}
\log _{10}(m)=-0,66-0,013 T+0,159 / C+0,005 T C+59,5 C / T \\
n=0,329+0,007 T-0,002 T C-4,088 C / T
\end{gathered}
$$


Los diferentes autores coinciden en que el coeficiente de consistencia del fluido, $m$, aumenta con la concentración de CMC en la disolución y disminuye con la temperatura y con la degradación. Por otro lado, el índice de comportamiento de flujo, $n$, presenta el comportamiento contrario. Este comportamiento general de las propiedades ante variaciones de la temperatura y ante una degradación del fluido, se confirma mediante mediciones realizadas en el laboratorio. La metodología de estos ensayos y los resultados de los mismos se exponen a continuación.

\section{Ensayos de variación de las propiedades reológicas con la $\mathrm{T}^{\mathrm{a}}$}

En la instalación de ensayos termohidráulicos, se ha procedido a la medición de las propiedades reológicas de una preparación del fluido de trabajo a diferentes temperaturas. La mezcla utilizada para crear el fluido ha sido de CMC de alta viscosidad al $1 \%$ en agua destilada. Para abarcar una mayor rango de valores de $m$ y $n$, se ensaya el fluido con diferentes grados de degradación.

El procedimiento consiste en regular la temperatura del depósito principal mediante el aporte bien de frío, de calor o de ambos. Para la refrigeración del fluido de trabajo, éste se hace pasar por un intercambiador de placas. El agua circulante por el circuito secundario se enfría hasta $7^{\circ} \mathrm{C}$ haciendo uso de una enfriadora. El aporte de calor es el que controla la temperatura del fluido. Como aporte de calor se utilizan dos sistemas distintos. El primero de ellos consiste en un circuito secundario de agua caliente $\left(50^{\circ} \mathrm{C}\right)$, la cual circula por un serpentín instalado en el depósito principal. Este primer sistema, que minimiza la degradación del fluido, se utiliza exclusivamente para calentar el fluido no newtoniano hasta la temperatura de ensayo. El segundo de los sistemas de calentamiento consiste en una resistencia eléctrica instalada en el depósito principal. Este segundo sistema no se utiliza para variar significativamente la temperatura del fluido, ya que su alta potencia lo degrada fuertemente, sino para mantener la temperatura de consigna.

Para medir la temperatura del fluido, se utilizan las sondas de temperatura RTD dispuestas a la entrada y la salida del viscosímetro.

El procedimiento para medir las propiedades del fluido a cada temperatura será el descrito en el Apartado 5.1.1.

Para caracterizar la variación que sufren las propiedades reológicas del fluido al variar la temperatura del mismo, se han realizado un total de 4 ensayos. Cada uno de ellos abarcando un rango de valores de las propiedades distinto.

En cada uno de dichos ensayos se ha seguido el mismo procedimiento. En primer lugar 
se enfría el fluido circulante hasta la temperatura menor a la que se desea ensayar y se realiza la primera medida de las propiedades. A partir de ahí se aumenta progresivamente la temperatura del fluido, midiendo las propiedades del mismo a intervalos regulares, hasta llegar a la temperatura máxima. Aquí finaliza el tramo del ensayo de temperatura ascendente. A continuación se realiza el proceso inverso, de reducción progresiva de la temperatura, de forma que el fluido termina el procedimiento a una temperatura similar a la que tenía cuando comenzó. El objetivo de empezar y terminar en el mismo punto, es el de eliminar el efecto de la degradación del fluido en la medición.

A continuación se detallan los resultados obtenidos en los ensayos realizados para ambas propiedades reológicas ( $m$ y $n$ ) y las conclusiones que de ellos se obtienen.

\section{Coeficiente de consistencia de flujo, $m$. Resultados.}

En la Fig. 5.4 se muestran las mediciones realizadas en los ensayos a diferentes temperaturas y con el fluido en diferentes estados de degradación.

Ante las diferentes propuestas de los autores de la bibliografía, los resultados obtenidos se han ajustado mediante relaciones lineales y exponenciales:

$$
\begin{gathered}
\log (m)=B_{m, e} T+c t e \\
m=B_{m, l} T+c t e
\end{gathered}
$$

Los resultados de ambos ajustes para cada ensayo, se resumen en la Tabla 5.2.

\begin{tabular}{cccccc}
\hline \multirow{2}{*}{ Ensayo } & \multirow{2}{*}{ Rango $m\left[\right.$ Pa.s $\left.{ }^{\mathrm{n}}\right]$} & \multicolumn{2}{c}{ Rel. exponencial } & \multicolumn{2}{c}{ Rel. lineal } \\
\cline { 3 - 6 } & & $B_{m, e}$, asc. & $B_{m, e}$, desc. & $B_{m, l}$, asc. & $B_{m, l}$, desc. \\
\hline 1 & $0,4-0,8$ & $-0,0452$ & $-0,0435$ & $-0,0256$ & $-0,0253$ \\
2 & $0,4-1,8$ & $-0,0451$ & $-0,0416$ & $-0,0454$ & $-0,0361$ \\
3 & $0,05-0,25$ & $-0,0457$ & $-0,0426$ & $-0,00578$ & $-0,00497$ \\
4 & $2,8-4,1$ & $-0,0421$ & $-0,0306$ & $-0,144$ & $-0,11$ \\
\hline
\end{tabular}

Cuadro 5.2: Variación de la propiedad $m$ con la temperatura. Valores de la constante $B_{m}$ en las relaciones lineales (Ec. 5.8) o exponenciales (Ec. 5.9).

Como se puede observar en la Fig. 5.4, y especialmente en los ensayos que abarcan un mayor rango de temperaturas, los resultados confirman una variación exponencial de $m$ con la temperatura. 

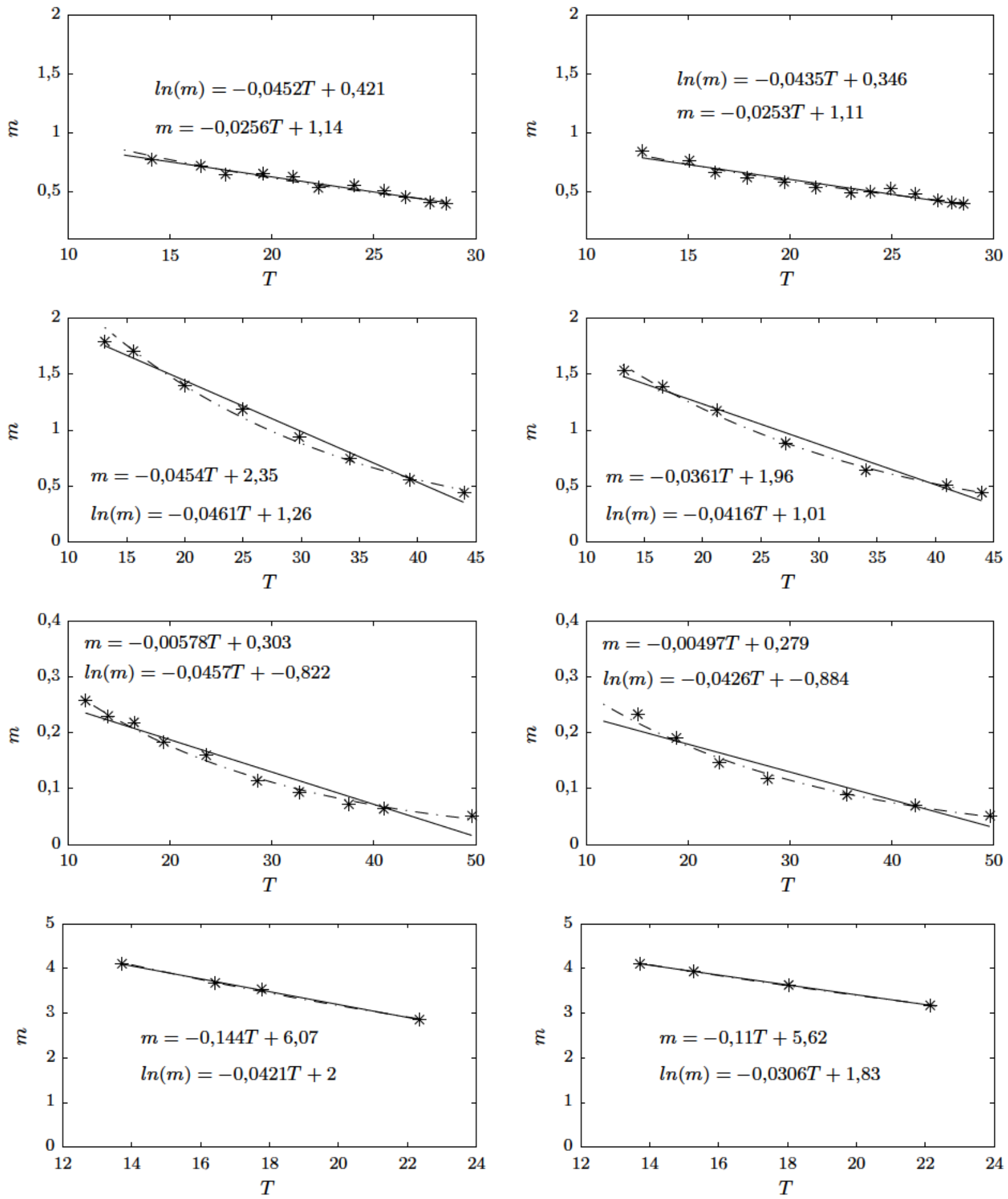

Figura 5.4: Variación de $m$ con la temperatura en fluidos con distinta degradación. Simbología: "asteriscos" medidas experimentales, "línea continua" ajuste lineal, "línea punto-raya" ajuste exponencial. 
Para obtener el valor de $B_{m, e}$, se promedian los resultados de los experimentos realizados, dando más importancia a los ensayos en los que se ha realizado un mayor número de medidas:

$$
B_{m, e}=-0,0433
$$

De los experimentos, se extraen las siguientes conclusiones referentes a la relación entre la temperatura del fluido y el Coeficiente de consistencia del fluido, $m$ :

- La variación de $m$ con la temperatura es significativa.

- El valor de $m$ disminuye al aumentar la temperatura.

- La relación medida entre la variación de temperatura y $m$, tiene forma exponencial.

- El valor de $B_{m, e}$ no varía al trabajar con fluidos con diferentes grados de degradación.

- Ante variaciones no demasiado grandes de la temperatura se podría utilizar una relación lineal, sin cometer un error demasiado grande. Aunque la pendiente de la recta $\left(B_{m, l}\right)$ varía al trabajar con un fluido en diferentes estados de degradación.

\section{Índice de comportamiento de flujo, $n$. Resultados.}

En las Fig. 5.5 se muestran las mediciones realizadas en los ensayos a diferentes temperaturas y con el fluido en diferentes estados de degradación.

Los resultados se han ajustado mediante la siguiente expresión lineal:

$$
n=B_{n, l} T+c t e
$$

Los resultados del ajustes para cada ensayo, se resumen en la Tabla 5.3 para el índice de comportamiento de flujo $(n)$.

\begin{tabular}{cccc}
\hline Ensayo & Rango $n$ & $n$, asc. & $n$, desc. \\
\hline 1 & $0,64-0,7$ & $3,54 e^{-3}$ & $3,49 e^{-3}$ \\
2 & $0,58-0,7$ & $4,20 e^{-3}$ & $3,68 e^{-3}$ \\
3 & $0,81-0,9$ & $2,75 e^{-3}$ & $2,33 e^{-3}$ \\
4 & $0,43-0,47$ & $4,15 e^{-3}$ & $2,66 e^{-3}$ \\
\hline
\end{tabular}

Cuadro 5.3: Variación de la propiedad $n$ con la temperatura. Valores de las constantes del término de la temperatura en las relaciones lineales o exponenciales. 



Figura 5.5: Variación de $n$ con la temperatura en fluidos con distinta degradación. Simbología: "asteriscos" medidas experimentales, "línea continua" ajuste lineal. 
A tenor de los resultados obtenidos en los 4 experimentos y haciendo un promedio de los resultados, se obtiene el siguiente valor de proporcionalidad entre $n$ y la temperatura:

$$
B_{n, l}=3,34 \times 10^{-3}
$$

Si se desea cuantificar la variación de $n$ por cada grado de temperatura, tomando un valor medio de $n=0,6$, se obtiene:

$$
\left.\frac{\Delta n}{n}\right|_{(\Delta T=1)}=\frac{3,34 \times 10^{-3}}{0,6}=0,56 \%
$$

Lo que significa que para saltos de temperatura menores de $10^{\circ} \mathrm{C}$, la variación de $n$ estará en torno al $6 \%$ de su valor, lo que explica porque algunos autores (Joshi y Bergles (1980b)) consideran $n$ invariable bajo determinados supuestos.

De los resultados experimentales se podrían extraer las siguientes conclusiones en cuanto al Índice de comportamiento de flujo, $n$ :

- La variación de $n$ con la temperatura es poco significativa $(<6 \%)$ para saltos de temperatura $\Delta T<10^{\circ} \mathrm{C}$.

- El valor de $n$ aumenta al hacerlo la temperatura.

- La relación medida entre la variación de temperatura y $n$, tiene forma lineal.

- Ante variaciones pequeñas de temperatura, la variación de $n$ es despreciable.

\subsection{Otras propiedades termofísicas del fluido}

Cho y Hartnett (1982) e Irvine y Karni (1987) estudiaron la variación de la conductividad térmica, la densidad, el calor específico y el coeficiente de expansión térmica en disoluciones de los polímeros más comunes, incluyendo la CMC, concluyendo que las propiedades de la disolución difieren de las del agua en no más de un 5-10\%. Por otro lado, se podría esperar que la conductividad térmica fuese dependiente del esfuerzo cortante, ya que tanto ésta como la viscosidad dependen de la estructura del fluido. Sin embargo, Loulou et al. (1992) confirmaron que el efecto es mínimo. Consecuentemente, Chhabra y Richardson (1999) estiman, que para aplicaciones de ingeniería, utilizar el valor de las propiedades del agua para los cálculos conllevará un error pequeño. 


\begin{tabular}{ccc}
\hline Muestra & $m\left[\right.$ Pa. $\left.\mathrm{s}^{\mathrm{n}}\right]$ & $n$ \\
\hline 1 & \multicolumn{2}{c}{ agua destilada } \\
2 & 2,54 & 0,52 \\
3 & 0,87 & 0,65 \\
4 & 0,309 & 0,76 \\
\hline
\end{tabular}

Cuadro 5.4: Propiedades reológicas de las muestras de fluido a $20^{\circ} \mathrm{C}$. Las muestras se corresponden con preparaciones de CMC al $1 \%$ en agua destilada en diferentes estados de degradación. Nota: Las medidas de las muestras 3 y 4 se han realizado a temperaturas de $17,5^{\circ} \mathrm{C}$ y $26,2^{\circ} \mathrm{C}$ respectivamente y se han trasladado a $20^{\circ} \mathrm{C}$ mediante el método descrito en el Apartado 5.1.2.

\begin{tabular}{ccccccccc}
\hline & \multicolumn{1}{c}{1} & \multicolumn{3}{c}{2} & \multicolumn{3}{c}{4} \\
\hline$T$ & $c_{p}$ & $\mathrm{SD}$ & $c_{p}$ & $\mathrm{SD}$ & $c_{p}$ & $\mathrm{SD}$ & $c_{p}$ & $\mathrm{SD}$ \\
\hline 10 & 3,97 & 0,05 & 3,85 & 0,08 & 3,88 & 0,16 & 3,94 & 0,06 \\
20 & 4,00 & 0,02 & 3,87 & 0,08 & 3,89 & 0,15 & 3,96 & 0,05 \\
30 & 4,03 & 0,02 & 3,89 & 0,08 & 3,92 & 0,15 & 3,99 & 0,05 \\
40 & 4,07 & 0,02 & 3,90 & 0,09 & 3,92 & 0,16 & 4,01 & 0,04 \\
50 & 4,07 & 0,03 & 3,92 & 0,09 & 3,94 & 0,17 & 4,04 & 0,04 \\
60 & 4,13 & 0,02 & 3,97 & 0,09 & 3,99 & 0,16 & 4,09 & 0,03 \\
70 & 4,12 & 0,07 & 4,02 & 0,10 & 4,04 & 0,17 & 4,14 & 0,04 \\
\hline
\end{tabular}

Cuadro 5.5: Resultados de las medidas experimentales de calor específico. Cada valor de calor específico en $\mathrm{kJ} / \mathrm{kgK}$ se obtiene de promediar los resultados de 3 medidas.

Para confirmar las deducciones de Chhabra y Richardson, se realizarán experimentos en el laboratorio para medir la conductividad térmica del fluido. Se ensayan 5 muestras de fluido en distintos estados de degradación y con propiedades reológicas distintas. El procedimiento seguido para realizar las medidas es el IsoStep 0-80 52 con blanco (Toledo, 1998), que barre el rango de temperaturas entre $0^{\circ} \mathrm{C} \mathrm{y} 80^{\circ} \mathrm{C}$ aumentando la temperatura por pasos: durante 5 minutos mantiene la temperatura estable y durante los siguientes 5 minutos incrementa la temperatura a una velocidad de $2^{\circ} \mathrm{C} / \mathrm{min}$, continuando de forma cíclica. La medida de $c_{p}$ se obtiene por comparación con las propiedades del zafiro, el cual se somete al mismo tratamiento térmico. Según el fabricante este método ofrece alta precisión, siendo el error del $2 \%$.

En la Tabla 5.4 se detallan las características de las muestras de fluido ensayadas. Los resultados se muestran en la Tabla 5.5 y en la Figura 5.6 donde se observa que las diferencias entre los calores específicos de los distintos fluidos son mínimas (por debajo del 2,5\%) y que por tanto se pueden considerar como iguales a los del agua. 


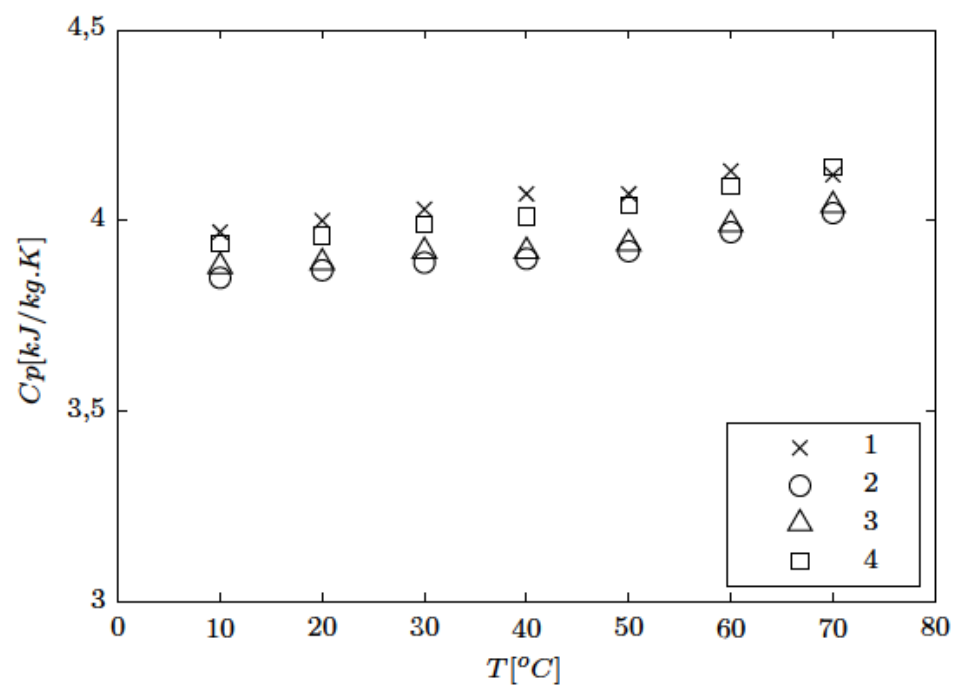

Figura 5.6: Mediciones experimentales del calor específico de cuatro fluidos distintos. Las propiedades de cada fluido se encuentran detalladas en la Tabla 5.4 y las mediciones realizadas en la Tabla 5.5.

No se dispone de equipos para ensayar la conductividad térmica del fluido de trabajo, pero vistos los resultados obtenidos con la densidad y el calor específico y las bajas concentraciones de CMC con las que se trabaja, se seguirán las indicaciones de Cho y Hartnett (1982), Irvine y Karni (1987) y Loulou et al. (1992) y se supondrá la conductividad térmica igual a la del agua.

\subsubsection{Densidad}

Cancela et al. (2005) midieron experimentalmente la densidad del fluido (disoluciones de $\mathrm{CMC}$ en agua) en función de la concentración de CMC y obtuvieron una correlación entre la densidad de la muestra preparada, la concentración de CMC y la densidad del agua. En el estudio se demuestra que la densidad es dependiente de la temperatura y de la concentración de la mezcla. Para correlar los datos utilizaron la expresión desarrollada por Choudary y Jasra (1994):

$$
\rho=\rho_{H_{2} O}+\sum_{i=2}^{4} A_{i}(C)^{i / 2}
$$

donde $\rho_{\mathrm{H}_{2} \mathrm{O}}$ es la densidad del agua y $C$ la concentración de CMC. 
A partir de los datos experimentales obtuvieron los valores de los coeficientes $A_{i}$ (a diferentes temperaturas) para la preparación que realizaron (véase Fig. 5.7).

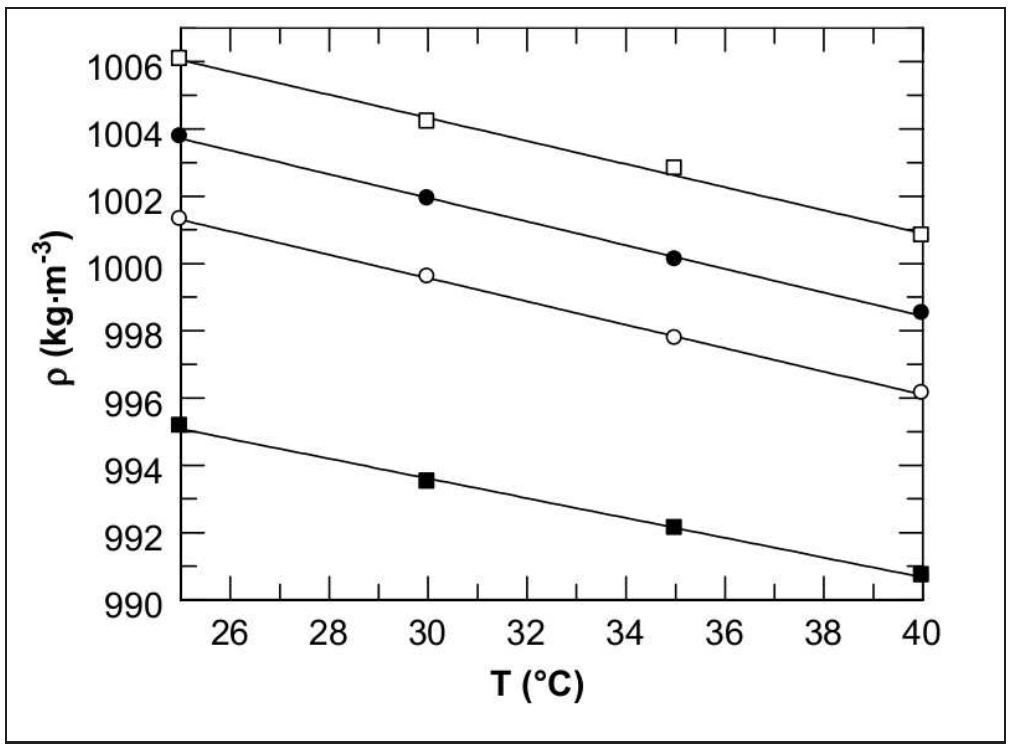

Figura 5.7: Densidad de las disoluciones de CMC en agua, variando las concentraciones de CMC ( cuadrados negros $0,5 \%$; círculos blancos $1 \%$; círculos negros $1,5 \%$ y cuadrados blancos $2 \%)$ y la temperatura del fluido. La representación es original de Cancela et al. (2005).

De dicho estudio se extrae la conclusión de que la densidad de las disoluciones con concentraciones de CMC al $1 \%$ es, dependiendo de la temperatura, entre un $0,28 \%$ (a $40^{\circ} \mathrm{C}$ ) y un $0,33 \%$ (a $\left.25^{\circ} \mathrm{C}\right)$ superior a la del agua.

Para poder valorar estas afirmaciones, se han realizado 3 mediciones a $26^{\circ} \mathrm{C}$ con muestras de fluidos de propiedades reológicas diferentes, detalladas en la Tabla 5.4. Para las pruebas se ha utilizado un estereopicnómetro de helio de Quantachrome modelo SPY-6DC, que usa celdas de pequeño volumen $(10$ cc). En los resultados mostrados en la Tabla 5.6 no se observan diferencias significativas en la densidad.

En consecuencia, para cuantificar de forma precisa la densidad del fluido, ésta se medirá durante la realización de los ensayos haciendo uso del caudalímetro de efecto Coriolis. 


\begin{tabular}{ccc}
\hline Muestra & $\bar{\rho}[\mathrm{g} / \mathrm{cc}]$ & $\mathrm{SD}$ \\
\hline 1 & 1,026 & 0,003 \\
2 & 1,032 & 0,016 \\
3 & 1,019 & 0,003 \\
4 & 1,028 & 0,004 \\
\hline
\end{tabular}

Cuadro 5.6: Medida de la densidad de las muestras a $26^{\circ} \mathrm{C}$. Sobre cada una de las 4 muestras de fluido se han realizado 3 medidas, donde la primera muestra corresponde a agua destilada.

\subsection{Método de generalización de la viscosidad para la geometría bajo estudio}

Del análisis dimensional realizado en el Apartado 2.1.1 se obtiene la definición del número de Reynolds $R e_{b}$ dada por la Ecuación 2.7, la cual utiliza la definición básica de la viscosidad $\mu_{b}$ (véase Tabla 2.1). Dicha definición de la viscosidad, obtenida directamente del análisis dimensional es utilizada por numerosos autores de la bibliografía (Sun et al. (2003); Chebbi (2002); Kakaç et al. (1987)). Sin embargo, en estudios más complejos, como pueden ser los experimentales, es más apropiado utilizar la viscosidad generalizada del flujo, definida de forma análoga a las propuestas en el Apartado 2.4.1.

Tal y como se introduce en el Capítulo 2, el llamado método de generalización de la viscosidad permite reducir un grado de libertad del problema fluidomecánico en el dispositivo. Así la relación entre el número de Reynolds generalizado $R e_{g}$ y el factor de fricción no dependería del índice de comportamiento de flujo $n$, en contraposición a lo que ocurre si se utiliza la definición básica del mismo, $R e_{b}$. Con lo que se podría escribir ${ }^{1}$

$$
\begin{gathered}
f=\Psi\left(R e_{g}\right) \\
f=\Psi\left(R e_{b}, n\right)
\end{gathered}
$$

Por claridad, se repiten a continuación las siguientes definiciones:

$$
\mu_{b}=m\left(\frac{u_{b}}{D_{h}}\right)^{n-1}
$$

\footnotetext{
${ }^{1}$ El símbolo $\Psi$ se utiliza para indicar que diversos parámetros están relacionados por una función desconocida.
} 


$$
R e_{b}=\frac{\rho u_{b} D_{h}}{\mu_{b}}
$$

Por un lado, el uso de la viscosidad generalizada está plenamente justificada por los motivos que se detallan a continuación. En los ensayos experimentales, donde se trabaja con fluidos reales, éstos se degradan de forma progresiva con el transcurso de cada ensayo, lo cual hace que las propiedades $m$ y $n$ del fluido varíen constantemente y sean distintas para cada una de las medidas realizadas. Esto hace inviable en la práctica que se puedan realizar varios ensayos manteniendo constantes los valores de $m$ y $n$. Además, si se quieren estudiar efectos más allá de la caída de presión, como pueden ser el efecto del movimiento del rascador o la transferencia de calor, el uso de un número de Reynolds generalizado que incluya la dependencia de $n$ en su definición resulta mucho más conveniente.

Por otro lado, los métodos de generalización descritos por Metzner y Reed (1955) y por Delplace y Leuliet (1995) (Ecuaciones 2.11 y 2.15 respectivamente) únicamente son apropiados para tubos cuya sección transversal al flujo es constante. En concreto, en la geometría de tubo con rascador estudiada (véase Fig. 1.6) la presencia de los tacos rascadores provoca una sección de paso variable. Por lo tanto, en principio, los métodos descritos en la Sección 2.4.1 no son de aplicación en el presente caso.

El problema que se presenta en este punto, es que el comportamiento del fluido depende fuertemente de $n$ y que, consecuentemente, el estudio de la caída de presión y transmisión de calor en el tubo resultan de gran dificultad sin una correcta generalización de la viscosidad del flujo (especialmente si el rascador se encuentra en movimiento). Así, el objetivo es encontrar una definición de la viscosidad generalizada del flujo $\mu_{g}$, tal que la relación entre $f$ y $R e_{g}$ en régimen laminar sea independiente del índice de comportamiento de flujo, $n$ (véase Ec. 5.13).

Ante la complejidad de la geometría y la dificultad de realizar un estudio analítico del problema, se impone la realización de un estudio experimental. De este modo, se realizan ensayos de pérdida de presión en el tubo con rascador estático en los que se varía el caudal y el fluido de ensayo. Los detalles sobre el método experimental empleado se detallan en el Apartado 6.1. En los ensayos realizados el Índice de comportamiento de flujo varía entre valores que van desde $n=0,45$ hasta $n=1$. Los valores más bajos de $n$ se corresponden con un comportamiento fuertemente seudoplástico y los valores de $n \approx 1$ con un comportamiento totalmente newtoniano.

En la Fig. 5.8, se muestran los resultados de los ensayos realizados, utilizando para la representación la definición básica del número de Reynolds (Ec. 2.7), que incluye a $\mu_{b}$ en su definición. Como se puede observar, de forma análoga a lo que ocurre en geometría de tubo 


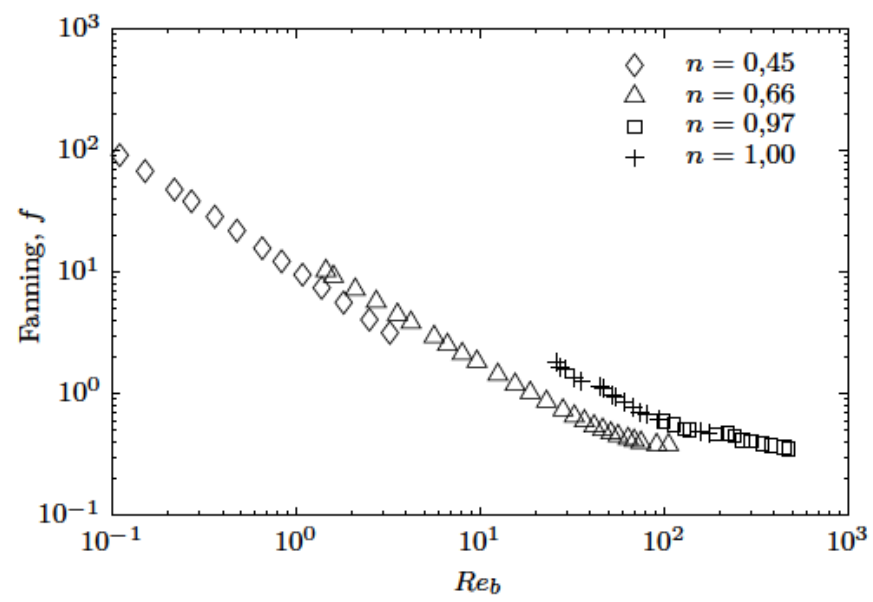

Figura 5.8: Factor de fricción frente $a e_{b}$ en régimen estático, dada por la Ec. 2.7. Únicamente se muestran los resultados más significativos.

liso (véase Fig. 2.2(a)), $f=\Psi\left(R e_{b}, n\right)$.

Para generalizar correctamente la viscosidad del flujo se exploran diferentes alternativas:

1. Utilizar la viscosidad generalizada basada en la caída de presión en geometría de tubo de sección anular, obtenida a partir de las expresión de Delplace y Leuliet (1995) y los estudios de Kozicki et al. (1966).

2. Evaluar la propuesta de Delplace y Leuliet (1995) de obtener el valor de $\xi$ de la Ec. 5.16 a partir de los datos experimentales.

3. Obtener una correlación experimental en régimen laminar distinta de las propuestas en la bibliografía y definir la viscosidad generalizada a partir de la misma.

En los Apartados 5.3.1, 5.3.2 y 5.3.3 respectivamente se evalúan dichas posibilidades.

\subsubsection{Generalización basada en la geometría de tubo de sección anular}

Como se ha comentado con anterioridad, en la bibliografía únicamente existen expresiones de la viscosidad efectiva aplicables a tubos de sección de paso constante (Delplace y Leuliet, 1995; Metzner y Reed, 1955; Kozicki et al., 1966), las cuales, en principio, no se pueden utilizar para estudiar la caída de presión en el tubo con el rascador insertado, dada la geometría de paso variable del mismo (véase Fig. 1.6). Sin embargo, dicha geometría tiene una alta 


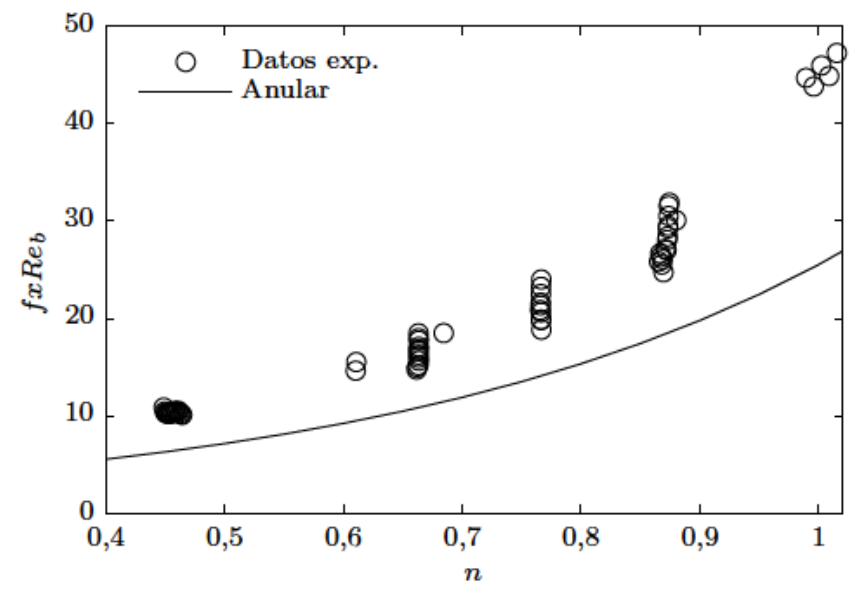

Figura 5.9: Comparación de $f \times R e_{b}$ frente a $n$ entre los resultados experimentales y el valor teórico en tubo de sección anular.

similitud con la geometría anular o de corona circular. De éste modo, se podría aplicar la definición de la viscosidad y del número de Reynolds dados por las Ecuaciones 5.14 y 5.15, donde se utilizaría el valor de $\xi$ correspondiente a la geometría de tubos concéntricos ( para el valor de $\alpha=5 / 18$ ensayado, $\left.\xi_{a n}=11,69\right)$.

$$
\begin{gathered}
\mu_{g, D L}=m\left(\frac{u_{b}}{D_{h}}\right)^{n-1} \xi^{n-1}\left(\frac{24 n+\xi}{(24+\xi) n}\right)^{n} \\
R e_{D L}=\frac{\rho u_{b}^{2-n} D_{h}^{n}}{m \times \xi^{n-1}\left(\frac{24 n+\xi}{(24+\xi) n}\right)^{n}}
\end{gathered}
$$

donde para la geometría para la que se obtiene el valor de $\xi$, se debe cumplir que

$$
f \times R e_{D L}=2 \xi
$$

Para comprobar si es válido aplicar esta definición de la viscosidad generalizada con $\xi=\xi_{a n}$, se representa en la Fig. 5.9 el valor de $f \times R e_{b}$ frente a $n$ para la geometría de tubo anular frente a los datos experimentales obtenidos en el tubo con rascador. Tal y como se observa en los resultados, el uso de $\xi=\xi_{a n}$ lleva a una infravaloración del $34 \%$ del valor de $f \times R e_{b}$.

Por otro lado, la representación del factor de fricción de Fanning frente al número de Reynolds generalizado mediante $\xi=\xi_{a n}$ (Ec. 5.15) se encuentra en la Fig. 5.10. De los 
resultados se deduce que dicha definición del número de Reynolds funciona mejor la definición básica (Ec. 2.7). Sin embargo, tal y como se observa la Fig. 5.10(b), ampliada para destacar los detalles, los resultados del factor de fricción todavía son ligeramente dependientes del parámetro $n$. Se llega de este modo a la conclusión de que, si bien la definición del número de Reynolds en base a la geometría de conducto anular reduce significativamente la dependencia de $n$ del producto $f \times R e_{b}$, ésta no desaparece completamente y es, por lo tanto, susceptible de ser mejorada.

\subsubsection{Valor experimental de $\xi$}

En esta sección, se evalúa la propuesta de Delplace y Leuliet (1995) de obtener el valor de $\xi$ en 5.16 de forma experimental en geometrías complejas. Aunque los autores no detallan de qué modo debería llevarse a cabo dicho proceso.

Cuadro 5.7: Correlación experimental de $\xi$ en la Ec. 5.17

\begin{tabular}{ccc}
\hline$b$ & $\xi$ & Error \\
\hline$-0,974$ & 19,38 & $17,0 \%$ \\
\hline
\end{tabular}

En el presente trabajo, la ecuación propuesta por los autores se modifica para incluir un exponente al número de Reynolds,

$$
f \times R e_{b}^{-b}=2 \xi^{n}\left(\frac{24 n+\xi}{(24+\xi) n}\right)^{n}
$$

Tal y como se observa en la Fig. 5.10, los resultados experimentales en la región laminar no son paralelos a la solución del tubo anular, lo que implica que el exponente del número de Reynolds es distinto de 1.

La Ec. 5.17 se ha correlado mediante los datos experimentales. Para ello, únicamente se han considerado números de Reynolds por debajo de 40 (región claramente laminar). El valor experimental de $\xi$ y su correspondiente error para un nivel de confianza del $95 \%$ se indica en la Tabla 5.7. Tanto los datos experimentales, como la Ec. 5.17 con $\xi=\xi_{\text {exp }}$, se encuentran representados en la Fig. 5.11. Además, en la Fig. 5.12 se representa el factor de fricción frente al número de Reynolds generalizado de este modo.

Los resultados en la Fig. 5.11 muestran una infravaloración del producto $f \times R e_{b}^{-b}$ para $n \approx 0,45$ y $n \approx 1$. Además, en la Fig. 5.12 se observa que los resultados experimentales para 


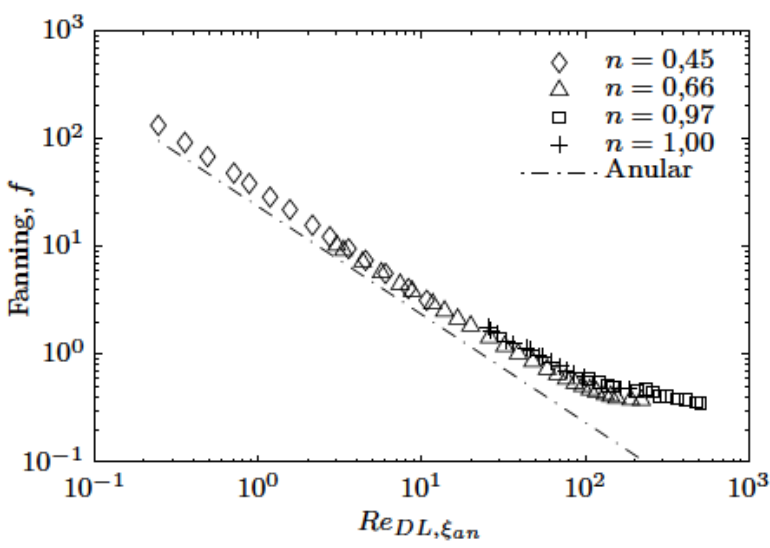

(a) Rango de Reynolds completo

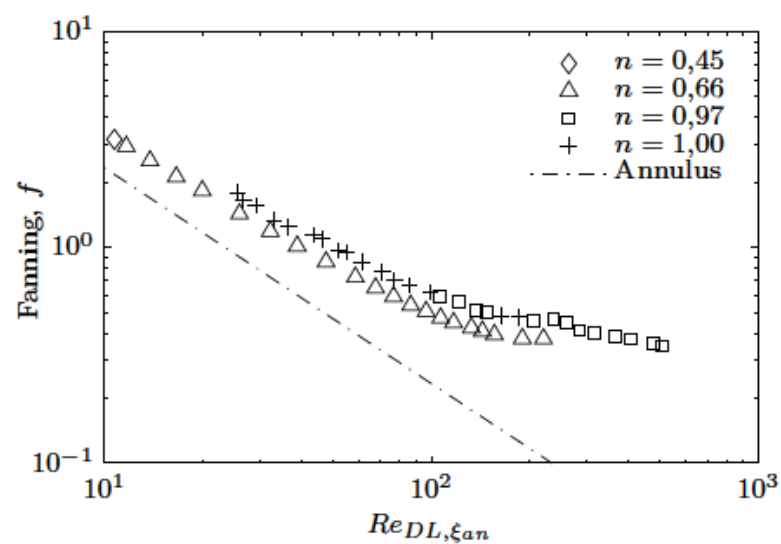

(b) Rango de Reynolds reducido

Figura 5.10: Pérdida de presión en régimen de rascador estático, haciendo uso del número de Reynolds generalizado definido para geometría anular. (Ec. 5.15 para $\xi=11,69$ ).

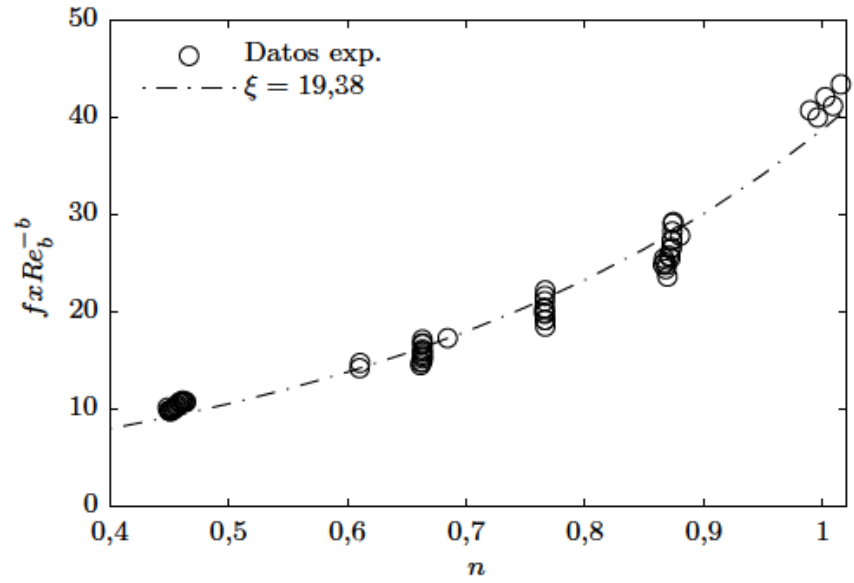

Figura 5.11: Comparación entre los ensayos experimentales y la Ec. 5.17 para el valor experimental de $\xi$ (ver Tabla 5.7) 


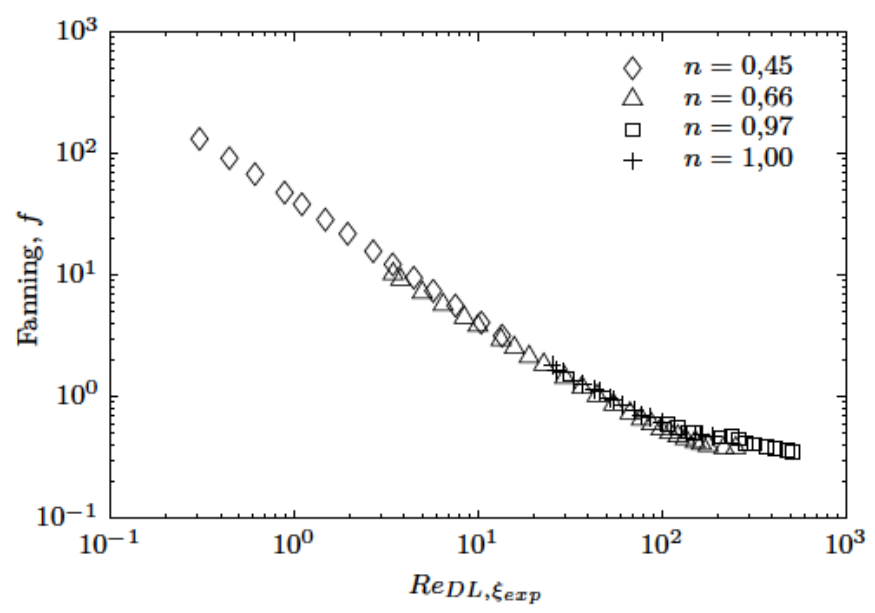

Figura 5.12: Número de Reynolds generalizado con la Ec. 5.17 para el valor experimental de $\xi$ (ver Tabla 5.7)

diferentes valores de $n$ no quedan representados por una única curva. Se concluye por tanto, que los resultados de este método de generalización todavía no son plenamente satisfactorios.

\subsubsection{Generalización a partir de correlaciones experimentales}

Llegados a este punto, es necesario realizar una correlación experimental de $f \times R e_{b}^{-b}$ para poder aplicar un método de generalización de la viscosidad adecuado. Con dicho objetivo, se proponen diferentes expresiones:

1. Expresión con dos parámetros,

$$
f \times R e_{b}^{-b}=a c^{n-1}
$$

2. Expresión con tres parámetros,

$$
f \times R e_{b}^{-b}=a c^{n-1} n^{d}
$$

3. Expresión con cuatro parámetros,

$$
f \times R e_{b}^{-b}=a\left(\frac{c n^{2}+d n+e}{(c+d+e) n^{2}}\right)^{n}
$$




\begin{tabular}{crrr}
\hline & Ec. 5.18 & Ec. 5.19 & Ec. 5.20 \\
\hline$a$ & 39.742 & 41.403 & 41.729 \\
$b$ & -0.974 & -0.974 & -0.974 \\
$c$ & 15.536 & 262.27 & 212.8 \\
$d$ & & -2.1177 & -319.16 \\
$e$ & & & 158.93 \\
Error (\%) & 15.9 & 11.4 & 9.6 \\
\hline
\end{tabular}

Cuadro 5.8: Resultados de las correlaciones experimentales.

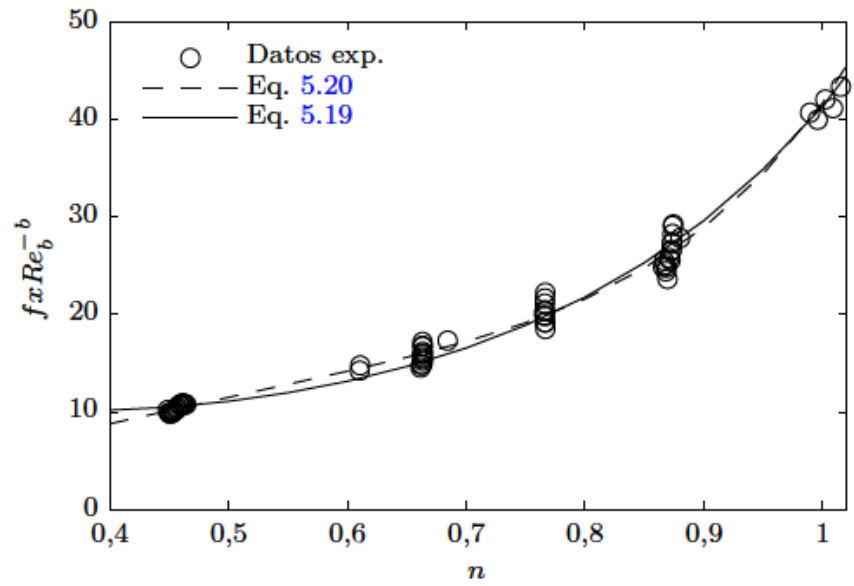

Figura 5.13: Comparativa entre los datos experimentales y los ajustes realizados (ecuaciones $5.18,5.19$ y 5.20$)$.

donde $a, c, d \mathrm{y} e$ son las constantes de las correlaciones. Al igual que en el apartado anterior, el exponente del número de Reynolds, $b$, se ha incluido debido a la naturaleza peculiar del tubo, donde el flujo no se comporta exactamente igual que en una geometría de sección de paso constante.

Los resultados de las diferentes correlaciones se muestran en la Tabla 5.8. Las tres correlaciones propuestas se ajustan mejor a los resultados experimentales que la propuesta por Delplace y Leuliet (1995). El menor error se corresponde con la Ec. 5.20 seguida de la Ec. 5.19, presentando ambas un buen ajuste a los datos experimentales. Ambas correlaciones se muestran en la Fig. 5.13 frente a los resultados experimentales.

A nuestro entender, la Ec. 5.19 ofrece una buena aproximación a los resultados experimentales, únicamente con tres parámetros, dos de los cuales aparecerán en la definición de la viscosidad generalizada. 

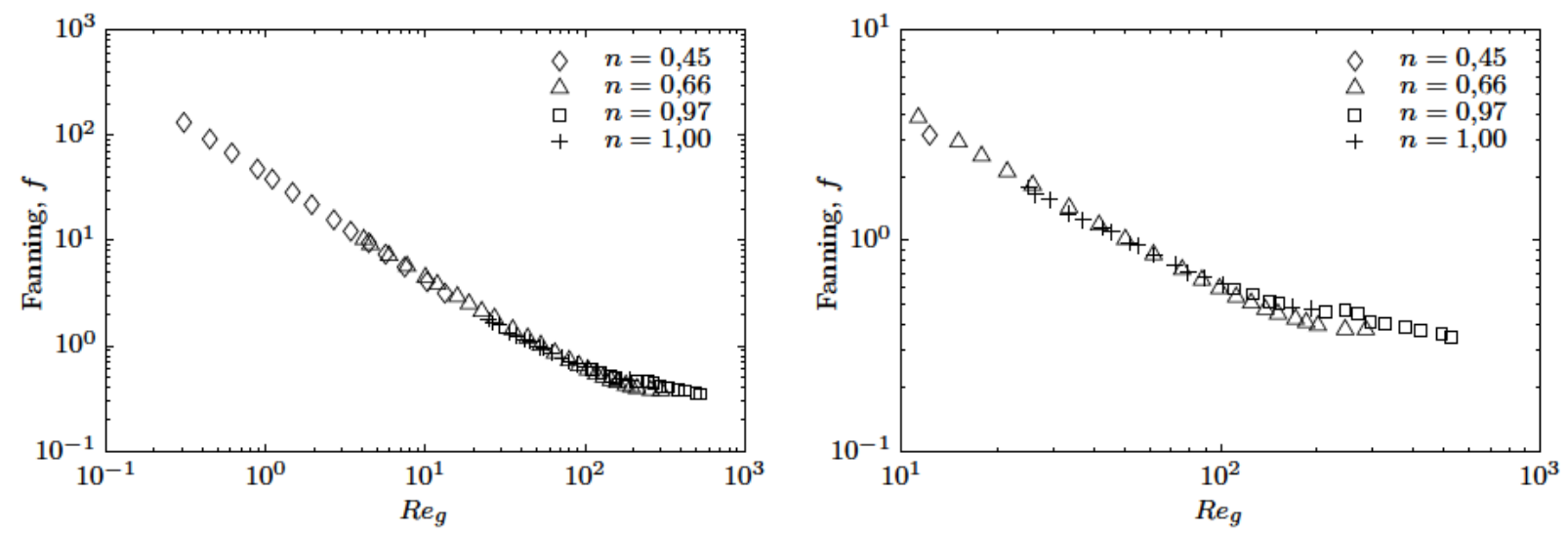

Figura 5.14: Número de Reynolds generalizado $R e_{g}$ (Ec. 5.22) frente al factor de fricción de Fanning.

Para definir la viscosidad generalizada $\mu_{g}$ y el Reynolds generalizado $R e_{g}$ de acuerdo con Delplace y Leuliet (1995), se debe cumplir que $\phi(n)=1$ en la Ec. 5.21.

$$
R e_{g}=\frac{R e_{b}}{\phi(n)}
$$

por lo tanto

$$
\begin{gathered}
\phi(n)=c^{n-1} n^{d} \\
R e_{g}=\frac{R e_{b}}{n^{d} c^{n-1}}=\frac{\rho u_{b}^{2-n} D_{h}^{n}}{m n^{d} c^{n-1}} \\
\mu_{g}=m n^{d} c^{n-1}\left(\frac{u_{b}}{D_{h}}\right)^{n-1}
\end{gathered}
$$

Los resultados de pérdida de presión se muestran en la Fig. 5.14, donde se utiliza el número de Reynolds generalizado definido por la Ec. 5.22. La figura muestra como los datos experimentales para fluidos con diferente comportamiento seudoplástico (diferente $n$ ) se pueden representar con una sola curva en el régimen laminar, mientras que aparecen ciertas diferencias en la región de transición.

El método de generalización propuesto tiene más parámetros que la ecuación de Delplace y Leuliet, pero se ajusta mejor a los datos experimentales, siendo también muy simple. Este método de generalización permite reducir la complejidad de los problemas fluidomecánico y térmico en esta y en otras geometrías complejas. Debe quedar presente que el método de 
generalización, únicamente está justificado en el régimen laminar.

Al utilizar el número de Reynolds generalizado, en la Fig. 5.14 se observa que en la región de transición aparecen diferentes curvas para diferentes valores de $n$. No obstante, en la región turbulenta, los resultados muestran que en la práctica se obtiene de nuevo una sola curva prácticamente independiente del valor de $n$, a pesar de que no existe justificación teórica para este hecho.

El uso de dicha definición de la viscosidad generalizada también implica la siguiente definición del número de Prandtl generalizado.

$$
\operatorname{Pr}_{g}=\frac{c_{p} \mu_{g}}{k}
$$

\subsection{Conclusiones}

Del estudio llevado a cabo en el presente capítulo, se extraen las siguientes conclusiones respecto a las propiedades termofísicas del fluido de trabajo:

1. Las propiedades del fluido de trabajo tales como la conductividad térmica, la densidad y el calor específico no difieren significativamente de las propiedades del agua y se comete un error pequeño al suponerlas iguales.

2. La conductividad térmica y el calor específico se supondrán iguales a los del agua.

3. La densidad del fluido preparado será medida en la instalación al realizar los ensayos.

4. La determinación de las propiedades reológicas $m$ y $n$ es un paso previo necesario para el estudio del comportamiento del flujo en el tubo con el rascador insertado.

5. Se ha diseñado e instalado un sistema de medida de las propiedades reológicas que permite una caracterización reológica del fluido anterior y posteriormente a la realización de los ensayos.

6. Se ha comprobado que el valor del Coeficiente de consistencia del fluido, $m$, disminuye al aumentar la temperatura y con la degradación del fluido, mientras que el Índice de comportamiento de flujo, $n$, tiene el comportamiento contrario. 
7. Se ha comprobado experimentalmente que la relación medida entre la temperatura y $m$ tiene forma exponencial. Además, se ha medido el valor de la constante de proporcionalidad entre la temperatura, $T$, y el logaritmo de $m$ (Ec. 5.10).

8. Se ha comprobado experimentalmente, que la relación medida entre la temperatura y $n$, tiene forma lineal y se ha medido el valor de la constante de proporcionalidad entre la temperatura, $T$, y $n$ (Ec. 5.12).

Además, en cuanto al método de generalización de la viscosidad, se concluye:

1. A lo largo de la tesis, para estudiar el comportamiento del tubo con rascador, se utilizarán los números adimensionales $R e_{g}$ y $P r_{g}$, obtenidos mediante el método de generalización propuesto.

2. La utilización de la viscosidad generalizada definida según la Ec. 5.23, $\mu_{g}$, permite un grado de simplificación mayor de los problemas fluidomecánico y térmico, incluyendo la dependencia de $n$ de las medidas de pérdida de presión en dicha definición.

3. En régimen laminar, la relación $f=\Psi\left(R e_{g}\right)$ es totalmente independiente del índice de comportamiento de flujo, $n$.

4. En régimen turbulento, en la relación $f=\Psi\left(R e_{g}, n\right)$, la dependencia de $n$ es despreciable. 


\section{Capítulo 6}

\section{Procedimiento de ensayo y cálculo de incertidumbres}

En el presente capítulo se realiza una descripción detallada de la metodología empleada en la realización de los ensayos experimentales de la investigación. A lo largo de la misma se han realizado tres tipos de ensayos destinados a caracterizar el funcionamiento del tubo del intercambiador de calor mejorado funcionando con fluidos no newtonianos:

1. Ensayos de visualización. Visualización del campo fluido y obtención del campo de velocidades.

2. Ensayos de pérdida de presión. Evaluación de las pérdidas de presión en el tubo y la potencia de accionamiento del rascador.

3. Ensayos de transmisión de calor. Determinación del coeficiente de transmisión de calor del lado tubo.

Para realizar cada uno de los ensayos enumerados, tal y como se comenta en el Capítulo 5, es necesario medir en línea las propiedades reológicas del fluido. La metodología de medición de las mismas se describe en el Apartado 5.1.1.

Los ensayos de visualización se realizan en la instalación de visualización de flujos. En ella se utiliza la técnica de Velocimetría por Imágenes de Partículas a la que corresponden las siglas PIV (Particle Image Velocimetry). En el Apartado 4.3 se realiza una descripción detallada de esta instalación y de la técnica de PIV y en el Apartado 6.4 se detalla la metodología experimental utilizada en la realización de los ensayos correspondientes. 
Tanto para realizar los ensayos de pérdida de presión, como los de transmisión de calor se hace uso de la instalación de ensayos termohidráulicos, la cual se describe en el Apartado 4.2. Por un lado, la metodología utilizada en los ensayos de pérdida de presión se describe en el Apartado 6.1, mientras que en los ensayos de transmisión de calor se sigue el procedimiento de ensayo detallado en el Apartado 6.3.

\subsection{Ensayos de pérdida de presión}

Los ensayos de pérdida de presión se realizan en la instalación experimental de ensayos termohidráulicos descrita en el Apartado 4.2. El objetivo perseguido es el de obtener la caída de presión en el tubo de ensayo en función del resto de parámetros que influyen en el problema. Para ello, a parte de los parámetros geométricos que se mantienen constantes, en cada experimento será necesario medir: $u_{b}, \rho, m, n, p_{L}$. Además en los ensayos dinámicos es necesario medir la velocidad del rascador a partir del periodo de rascado y la diferencia de presión existente entre las cámaras del pistón que lo impulsa, para así determinar la potencia de accionamiento consumida.

En el Capítulo 2 se realizará el análisis dimensional del problema para reducir los grados de libertad del mismo, donde se deduce que, una vez fijada la geometría, la caída de presión adimensionalizada mediante el factor de fricción de Fanning dependerá del número de Reynolds y del Índice de comportamiento de flujo, $n$.

El fluido se hace circular por el tubo con el elemento insertado en su interior. Los ensayos realizados se clasifican en estáticos o dinámicos, en referencia a si el rascador se encuentra estático o en movimiento. Los dos sensores diferenciales de presión estacionaria y los dos sensores de presión diferencial no estacionaria se encargan de medir la caída de presión en los ensayos estáticos y dinámicos respectivamente (véase Fig. 4.9).

Ambos tipos de ensayos se agrupan en tandas de experimentos, de modo que antes y después de cada tanda se miden las propiedades del fluido. Las tandas se han realizado de modo que contengan una cantidad adecuada de experimentos, para que la degradación del fluido a lo largo de la tanda completa no sea excesiva. El procedimiento de medición de propiedades reológicas se describe en el Apartado 5.1, donde también se cuantifica la degradación del fluido en una tanda.

En una tanda de ensayos de tipo estático, se ensaya un mismo fluido haciendo un barrido de caudales entre $15 \mathrm{l} / \mathrm{h}$ y $800 \mathrm{l} / \mathrm{h}$, tomando medidas en aproximadamente 15 caudales. Además a cada caudal se toman entre 10 y 20 medidas, que se promedian con objeto de reducir 


\begin{tabular}{ccccccc}
\hline$Q[\mathrm{l} / \mathrm{h}]$ & $|\omega|=0,1$ & $|\omega|=0,2$ & $|\omega|=0,3$ & $|\omega|=0,5$ & $|\omega|=1$ & $|\omega|=1,5$ \\
\hline 35 & 43 & 21,5 & 14,41 & 8,70 & 4,40 & 2,93 \\
50 & 30,1 & 15,13 & 10,09 & 6,14 & 3,08 & \\
70 & 21,5 & 10,81 & 7,25 & 4,40 & & \\
100 & 15,13 & 7,61 & 5,13 & 3,08 & & \\
125 & & & 4,10 & & & \\
170 & 8,95 & 4,53 & 3,02 & & & \\
250 & 6,14 & 3,08 & & & & \\
350 & 4,40 & & & & & \\
500 & 3,08 & & & & & \\
\hline
\end{tabular}

Cuadro 6.1: Semiperiodo de rascado (s) correspondiente a los caudales y relaciones de velocidades en los ensayos.

el error de los datos. En los ensayos a caudales pequeños se toman más medidas, de modo que el número de medidas compense la pérdida de precisión del sensor de presión.

En los ensayos dinámicos, se busca evaluar el efecto de la velocidad de rascado en la caída de presión, para ello se define el parámetro adimensional $\omega$ como la velocidad relativa entre el rascador y el fluido:

$$
\omega=\frac{v_{s}}{u_{b}}
$$

Así, un valor de $\omega=0$ supone que el rascador está parado, $\omega>0$ corresponde al movimiento de rascado en sentido equicorriente y $\omega<0$ en caso de que sea en sentido contracorriente. Nótese que para $\omega=+1$ el rascador se mueve a la velocidad media del fluido. Además, para un mismo régimen de rascado, se debe cumplir lo siguiente:

$$
\omega_{e c}=-\omega_{c c} \rightarrow|\omega|=c t e
$$

Se ha ensayado un rango de velocidades de rascado entre $0<\omega<1,5$, teniendo mayor interés los régimen es de rascado más lentos, debido al alto gasto energético que supone el rascado a alta velocidad Solano (2009a). Por consiguiente, las velocidades de rascado seleccionadas para los ensayos son $\omega=-0,5 ;-0,3 ;-0,2 ;-0,1 ; 0 ; 0,1 ; 0,2 ; 0,3 ; 0,5 ; 1 ; 1,5$.

Para cada valor de $\omega$, se determinan los caudales máximo y mínimo a los que es posible ensayar, siendo los factores limitantes por capacidad de la instalación y por precisión en las medidas: la velocidad máxima del rascador $\left(v_{s, \max } \approx 67 \mathrm{~mm} / \mathrm{s}\right)$, tiempo máximo del semiciclo 
de rascado $(\sim 50 \mathrm{~s})$, caudal mínimo $(15 \mathrm{l} / \mathrm{h})$ y una caída de presión superior a 10 mbar. Las tandas de ensayos se pueden diseñar de dos modos:

- Fijando $\omega$ y barriendo el rango de caudales.

- Barrer el rango de caudales, fijando el caudal y ensayando todas los valores de $\omega$ posibles a dicho caudal.

Se han utilizado ambos métodos de forma satisfactoria, aunque ha resultado más eficaz el segundo. En la Tabla 6.1 se muestran los ensayos programados a cada velocidad de rascado.

Se han realizado experimentos con fluidos en diferentes estados de degradación, de forma que las propiedades reológicas de los mismos varían en los siguientes rangos: $n \in[0,45 ; 1] \mathrm{y}$ $m \in[4 ; 0,01]$ Pa.s ${ }^{\mathrm{n}}$.

\subsubsection{Oscilación del caudal}

En los ensayos dinámicos, la presión que tiene que vencer el sistema de bombeo sufre grandes variaciones entre ciclos de rascado. Esto, unido al tipo de bomba utilizado (sobredimensionada y con grandes tolerancias) y a la baja viscosidad del fluido a grandes esfuerzos cortantes, provoca altas oscilaciones del caudal circulante por la instalación. En consecuencia, para leer los datos de caudal se utiliza un dispositivo de adquisición de datos Agilent 34790A y un PC en exclusiva, de modo que la velocidad de toma de datos de caudal sea suficientemente alta para captar dichas oscilaciones. La tarjeta de adquisición utilizada para capturar la caída de presión dinámica es capaz de leer a gran velocidad los datos de todos los sensores de presión dinámicos simultáneamente. En la Fig. 6.1 se representa un ejemplo de las oscilaciones de caudal que se producen.

Ante esta circunstancia de oscilación de caudal se decide realizar 2 tipos de procesado distintos:

1. Procesado 1. Se considera un único ciclo de rascado, equicorriente o contracorriente, y únicamente una vez que el caudal se ha estabilizado.

2. Procesado 2. Se considera el ciclo completo de rascado, obteniéndose valores medios de caída de presión y caudal.

El primer tipo de procesado es útil para evaluar el comportamiento del dispositivo en cada ciclo de rascado por separado, mientras el segundo evaluará el comportamiento del dispositivo 


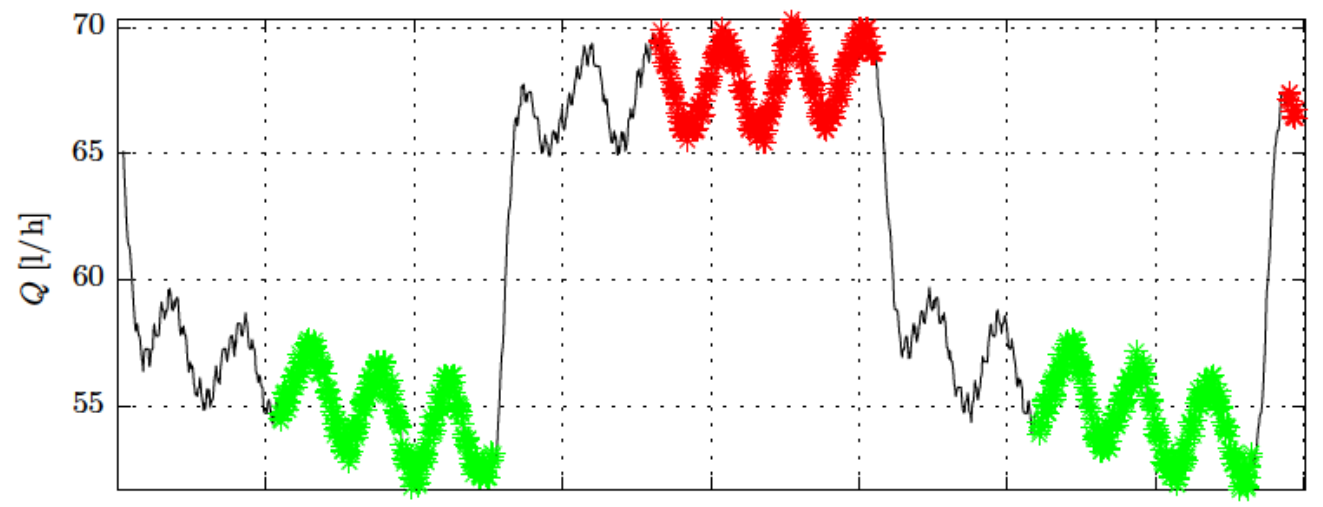

(a) $Q_{e c}=68 \mathrm{l} / \mathrm{h}$ y $\omega=0,1$

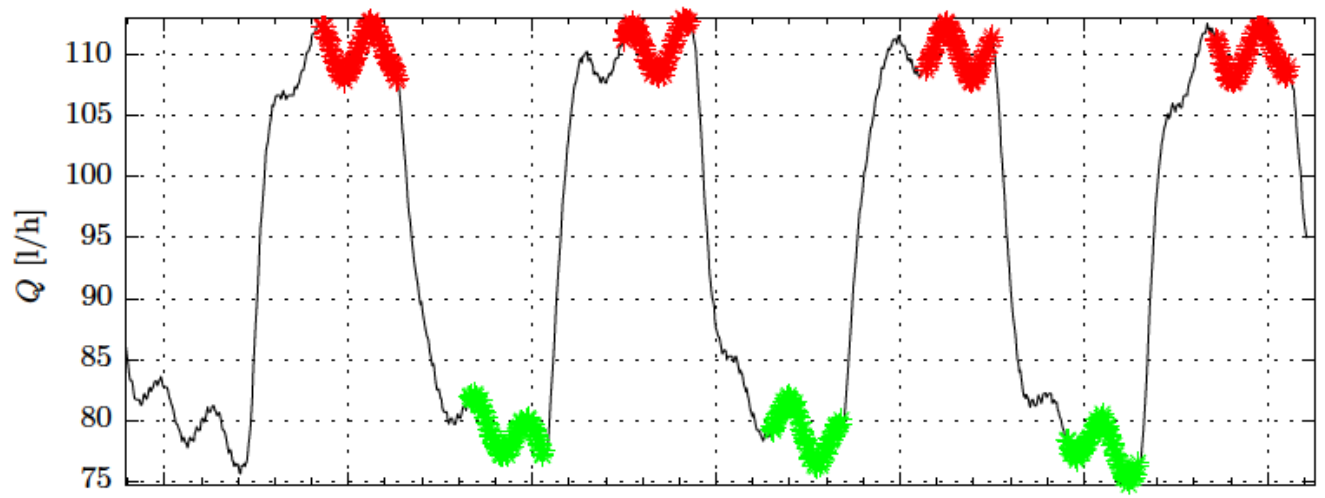

(b) $Q_{e c}=110 \mathrm{l} / \mathrm{h} \mathrm{y} \omega=0,2$

Figura 6.1: Oscilación de caudal en ensayos en equicorriente. En rojo se marcan los datos válidos en sentido equicorriente y en verde los de contracorriente utilizados para el Procesado 1 . 
en el ciclo completo. Cada tipo de procesado requiere que el ensayo se diseñe de forma diferente:

\section{Procesado 1.}

a) Equicorriente. Se ajusta el régimen de funcionamiento para que la relación de velocidades sea la buscada en el ciclo equicorriente

b) Contracorriente. Se diseña el ensayo para ajustar $\omega$ en el ciclo contracorriente.

2. Procesado 2. Se realiza el diseño del ensayo para ajustar $\omega$ de acuerdo al caudal medio circulante durante el ciclo completo de rascado.

Nótese, que el ensayo de la Fig. 6.1 se ha diseñado para que $\omega=0,1$ en el semiciclo de rascado equicorriente $y$, sin embargo, en el semiciclo contracorriente $\omega \neq-0,1$, ya que el caudal varía pero no así la velocidad del rascador. Por lo tanto, para cada ensayo, en el Procesado 1 se obtienen datos en equicorriente a la $\omega$ de diseño y datos en contracorriente a una relación de velocidades mayor. El caso contrario se da en los ensayos diseñados en contracorriente. Por lo tanto, de cara a la obtención de las correlaciones experimentales se dispondrá también de datos a valores de $\omega$ distintos a los de diseño. Sin embargo, estos datos no se incluyen en las representaciones gráficas, ya que se encuentran a valores de $\omega$ dispersos.

\subsection{Ensayo de calibración de los termopares}

Tal y como se describe en el Apartado 4.2, en la instalación de ensayos termohidráulicos, sobre la superficie del tubo de ensayo se han colocado 48 termopares dispuestos en 6 secciones tal y como se indica en la Fig. 4.5.

Los termopares tipo $\mathrm{k}$ utilizados necesitan ser calibrados para poder obtener medidas precisas. La calibración se ha realizado una vez que los termopares se han instalado sobre la superficie del tubo de ensayo. En el proceso de calibración se necesita fijar la temperatura de la superficie exterior del tubo a un valor conocido, que podamos comparar con la medida de los termopares. El fluido utilizado será agua. El procedimiento a seguir es el siguiente:

1. Se fija la temperatura del fluido de la instalación mientras se le hace circular a un caudal suficientemente alto por el interior del tubo de ensayo sin la presencia del rascador.

2. Se espera al equilibrio térmico del sistema. 


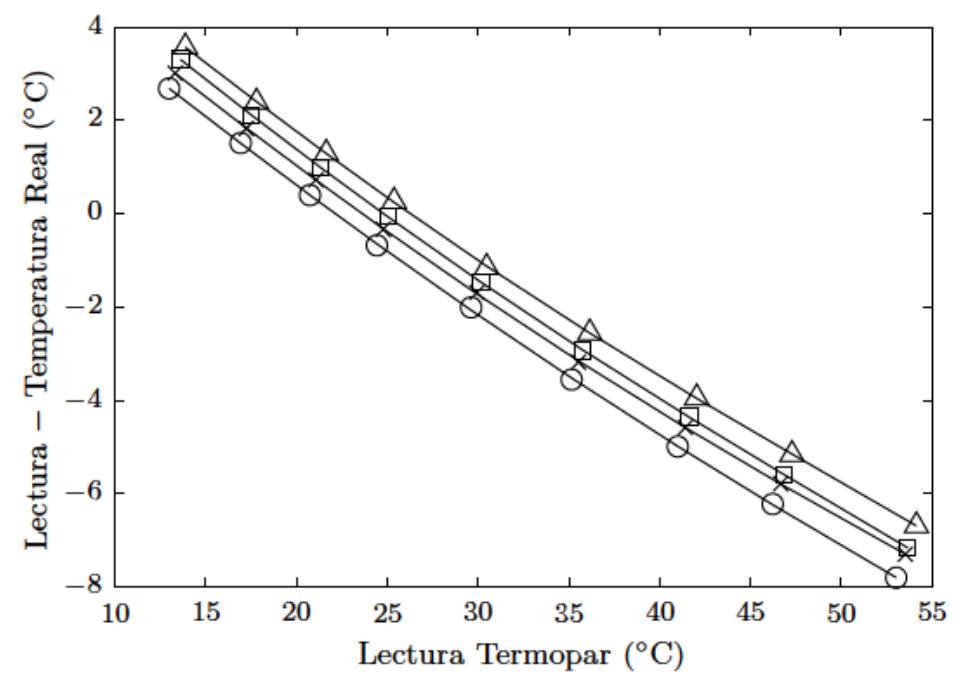

Figura 6.2: Ensayo de calibración de termopares. A modo de ejemplo se muestran las desviaciones de las medidas (símbolos) y el ajuste correspondiente (línea continua) para 4 termopares de la primera sección.

3. Se procede a la adquisición de las temperaturas de los termopares, así como las de las sondas RTD de entrada y salida. Se toman 12 medidas.

4. Vuelta al paso 1 cambiando de temperatura. El procedimiento se repite barriendo temperaturas en el rango $[10,60]^{\circ} \mathrm{C}$. Se ensayan un total de 9 temperaturas en dicho rango.

Una vez se han obtenido los datos, se pasa al procesado de los mismos detallado en el Apartado A.4. El error de las lecturas de cada termopar se ajusta por mínimos cuadrados a una función polinómica de grado 4. En la Fig. 6.2 se representan los resultados de la calibración para algunos termopares.

\subsection{Ensayos de transmisión de calor}

Los ensayos de transmisión de calor se realizan en la instalación descrita en el Apartado 4.2. El objetivo de estos consiste en determinar experimentalmente el coeficiente de transmisión de calor local del lado tubo $h_{i}$ (Ec. 6.1) en función del resto de variables intervinientes en el problema para una geometría dada: $u_{b}, \rho, m, n, p_{L}, k, c_{p}$. Según el análisis dimensional realizado en el Capítulo 2, el problema se reduce a determinar el número de Nusselt en función de los monomios de Prandtl y de Reynolds y de la propiedad reológica $n$. 


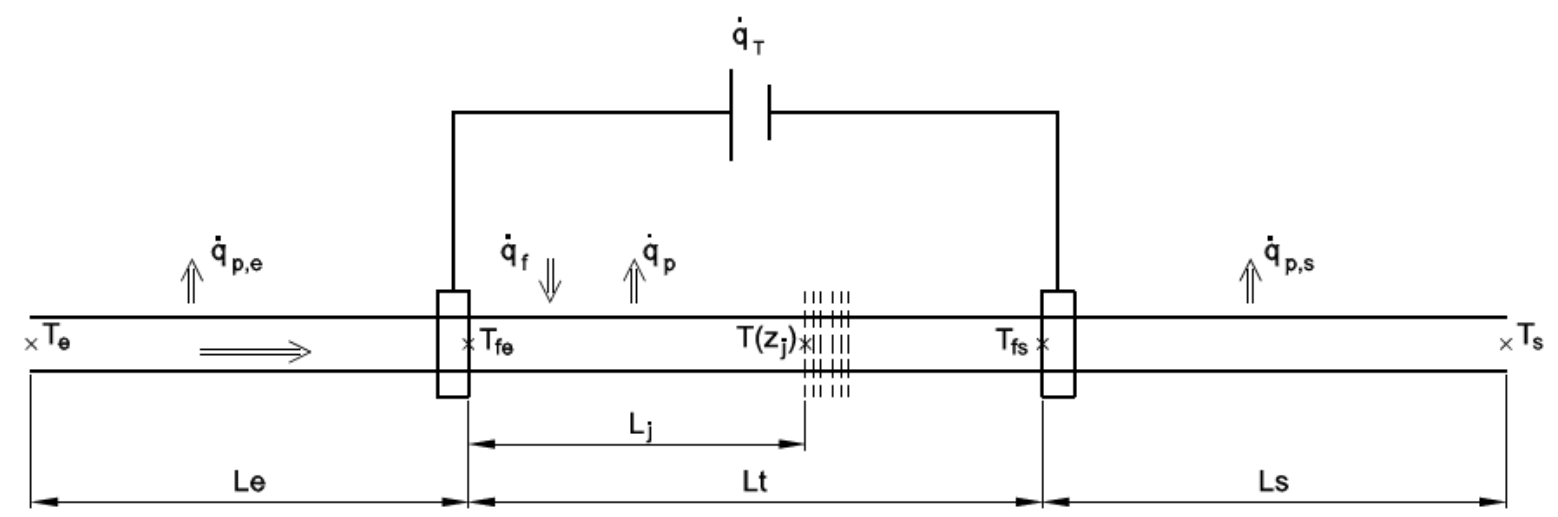

Figura 6.3: Balance de flujos de calor en ensayo de transmisión de calor.

Si se utiliza la definición de la viscosidad obtenida experimentalmente en el Apartado 5.3.3 la dependencia de $n$ debería ser mínima.

$$
N u=\frac{D_{h} h_{i}}{k}=F_{1}\left(\operatorname{Re}_{b}, P r_{b}, n\right)=F_{2}\left(R e_{c}, P r_{c}\right)
$$

En la geometría estudiada, el coeficiente de transmisión de calor local del lado tubo, para una sección situada a una distancia $L_{x}$ del primer electrodo, se define a partir de la siguiente ecuación:

$$
h_{i}\left(z_{j}\right)=\frac{\dot{q}_{F}^{\prime \prime}}{\bar{T}_{p i}\left(z_{j}\right)-\bar{T}\left(z_{j}\right)}
$$

El procedimiento de medida para los experimentos de transmisión de calor, consiste en calentar por efecto Joule el tramo del tubo de ensayo entre los electrodos. Por lo tanto, la distancia entre los electrodos, define la sección de ensayo. En la Fig. 6.3 se muestra el esquema del balance energético en los experimentos. Tal y como se observa en la misma, el tubo de ensayo queda dividido en tres tramos:

1. Tramo de entrada. Comprende desde el punto de medida de la temperatura por la sonda RTD de entrada $\left(T_{e}\right)$ hasta la cara interna del primer electrodo. Su longitud es de $L_{E}=2,05 \mathrm{~m}$. En este tramo existe un intercambio de calor entre el fluido y el ambiente $\dot{q}_{p, e}$, debido a la diferencia de temperaturas entre ambos fluidos y que dependerá también de la conductividad del aislante. La dirección del flujo dependerá de las temperaturas del fluido y del ambiente. 
2. Tramo con aporte de calor. Comprende la distancia entre las caras internas de los electrodos, de $L_{T}=1,001 \mathrm{~m}$. El calor aportado mediante efecto Joule $q_{T}$ viene dado por la tensión entre bornes y la intensidad circulante por el tubo. Debido a este aporte de calor la temperatura del tubo sube considerablemente. La diferencia generada entre la temperatura del tubo y el ambiente provoca un flujo de calor perdido $\dot{q}_{p}$. Por lo tanto, el flujo de calor realmente aportado al fluido será igual a la diferencia de ambos $\dot{q}_{f}=\dot{q}_{T}-\dot{q}_{p}$.

3. Tramo de salida. $L_{S}=2,33 \mathrm{~m}$. Comprende la distancia entre la cara interna del segundo electrodo y la sonda RTD a la salida $T_{s}$. En este tramo, donde el fluido circulante tendrá una temperatura alta, existirá un flujo de calor perdido desde el fluido al ambiente $\dot{q}_{p, s}$.

Para resolver la Ec. 6.1, es necesario conocer la temperatura media de la pared interior del tubo $\bar{T}_{p i}\left(z_{j}\right)$ y la temperatura media del fluido en la sección de ensayo $\bar{T}\left(z_{j}\right)$. Estas se pueden estimar a partir de las mediciones realizadas. Por un lado, el aumento de temperatura del fluido que se produce a causa del aporte de calor, se mide mediante las sondas RTD situadas a la entrada $T_{e}$ y salida del tubo de ensayo $T_{s}$. Por otro lado, los 48 termopares colocados en 6 secciones se encargan de medir la temperatura de la pared exterior del tubo en cada una de las secciones $\bar{T}_{p e}\left(z_{j}\right)$.

La temperatura media del fluido en las secciones de ensayo se calcula a partir del flujo de calor aportado al fluido, donde para conocerlo es necesario estimar la temperatura de entrada a la sección de entrada y el calor perdido en la sección de ensayo.

$$
\begin{gathered}
\dot{q}_{f}^{\prime \prime}=\frac{\dot{q}_{f}}{S}=\frac{\dot{q}_{T}-\dot{q}_{p}}{S} \\
\bar{T}\left(z_{j}\right)=T_{f e}+\frac{\dot{q}_{f} L_{j}}{\dot{m} c_{p} L_{T}}
\end{gathered}
$$

En este punto, no sería descabellado despreciar $\dot{q}_{p}$ frente a $\dot{q}_{T}$, de modo que $\dot{q}_{f} \approx \dot{q}_{T}$ a causa del aislamiento instalado, de $25 \mathrm{~mm}$. Sin embargo, se ha preferido cuantificar dichas pérdidas. El procedimiento de cálculo seguido para la obtención de $\bar{T}\left(z_{j}\right)$ y $\bar{T}_{p i}\left(z_{j}\right)$ se detalla en el Capítulo A del Apéndice.

En los ensayos realizados, el aporte de calor se encuentra en un rango de $\dot{q}_{T}=[500,5000] \mathrm{W}$. Además, con el objetivo de conseguir precisión en las medidas, para cada ensayo se elige el aporte de calor de forma que los saltos de temperatura entre la pared exterior del tubo y el fluido se encuentren siempre entre $10^{\circ} \mathrm{C}$ y $20^{\circ} \mathrm{C}$. 
La planificación de ensayos es igual a la realizada para los ensayos de pérdida de presión. Los experimentos se agrupan en tandas. Antes y después de cada tanda de experimentos se miden las propiedades reológicas del fluido, de modo que para cada experimento éstas se obtienen por interpolación y en función de la temperatura media en cada experimento en concreto. En cada tanda de ensayos, se recorre el rango de caudales más amplio posible.

Se trabajará con una temperatura del depósito constante. Además es deseable que la temperatura del fluido sea lo más fría posible, ya que esto evita su degradación. Sin embargo, para obtener resultados en un mayor rango de números de Reynolds y Prandtl es necesario ensayar con fluidos a distintas temperaturas. El rango de temperaturas del fluido en el depósito es de $[10,40]^{\circ} \mathrm{C}$. Obviamente al calentar el fluido en el tubo de ensayo, éste puede alcanzar temperaturas mayores.

El procedimiento de ensayos concreto es el siguiente:

1. Se posicionan las válvulas del circuito de modo que el fluido circule tanto por el viscosímetro como por el tubo de ensayo.

2. Se espera al equilibrio térmico a la temperatura de consigna indicada en el programa de Labview. El controlador PID se encarga de esta tarea mediante la regulación de la apertura de la válvula de tres vías del circuito frigorífico. Como aporte de calor se utiliza un serpentín situado en el interior del depósito.

3. Se circula el fluido únicamente por el viscosímetro.

4. Se miden las propiedades reológicas del fluido mediante la metodología descrita para tal efecto.

5. Se hace circular el fluido por el tubo de ensayo.

6. Se circula el caudal a ensayar.

7. Se conecta el transformador a baja potencia.

8. Regulación del flujo de calor aportado. Se regula la tensión del transformador de modo que el salto de temperaturas entre la temperatura media del fluido y la temperatura de la pared exterior $T_{p, e}$ se encuentre entre $10^{\circ} \mathrm{C}$ y $20^{\circ} \mathrm{C}$.

9. Esperar equilibrio del sistema.

10. Adquisición de datos. 
11. Se varía el caudal y se vuelve al paso 6 .

12. Una vez realizadas las medidas, volver a circular por el viscosímetro.

13. En equilibrio, medir propiedades.

Es importante destacar que, debido a la definición de los números adimensionales, variar el caudal implica variar los números de Reynolds y Prandtl simultáneamente. Esto implica que mediante el método descrito no se obtendrán series de resultados a números de Prandtl constantes, sino una nube de puntos en los que varían tanto el número de Reynolds, como el de Prandtl y para los que se obtienen los números de Nusselt local.

En los ensayos dinámicos, a parte de los números adimensionales de Reynolds y de Prandtl, también interviene la velocidad de rascado adimensionalizada con la velocidad media del fluido.

La metodología experimental es similar a la utilizada en los ensayos estáticos. Únicamente se introducen modificaciones en el diseño de los experimentos. Este proceso es totalmente equivalente al realizado para los ensayos dinámicos de pérdida de presión.

\subsubsection{Factor de corrección del número de Nusselt}

En los ensayos de transferencia calor, el fluido que atraviesa una sección del tubo tiene temperaturas distintas en la zona en contacto con la pared, en la capa límite o en la zona central del flujo. Sin embargo, el método utilizado para la obtención del número de Nusselt presupone, para una sección del tubo, una temperatura del fluido constante e igual a la temperatura media del fluido. Y no sólo eso, sino que además las propiedades del fluido dependientes de la temperatura se han considerado constantes. La viscosidad es la propiedad que más significativamente varía con la temperatura del fluido, o en el caso de un fluido Power Law, las propiedades reológicas $m$ y $n$. Estas variaciones de viscosidad, provocan cambios en los perfiles de velocidad y temperatura que llevan a diferentes coeficientes de fricción y transmisión de calor de los que se obtendrían si las propiedades fueran constantes.

Kays et al., (2005, cap. 15) propone un método de corrección por viscosidades, mediante el cual los métodos que consideran propiedades del fluido constantes pueden ser corregidos de forma sencilla para tener el mencionado efecto en consideración. Este método, definido para fluidos newtonianos consiste en aplicar las correlaciones de la Ec.6.2 y la Ec.6.3 a los coeficientes de Nusselt y de fricción respectivamente. 


$$
\begin{gathered}
\frac{N u_{p i}}{N u_{m}}=\left(\frac{\mu_{p i}}{\mu_{m}}\right)^{p_{1}} \\
\frac{f_{f}}{f_{f, m}}=\left(\frac{\mu_{p i}}{\mu_{m}}\right)^{p_{2}}
\end{gathered}
$$

Los valores de $p_{1}$ y $p_{2}$ fueron medidos por Deissler, 1951 para flujos de líquidos en tubos en los cuales la viscosidad del fluido depende de la temperatura. El factor para el coeficiente de transferencia de calor es $p_{1}=-0,14$. Para corregir el coeficiente de fricción, $p_{2}=0,5$ en casos de enfriamiento y $p_{2}=0,58$ para calentamiento.

De modo que el número de Nusselt se definiría del siguiente modo a partir del coeficiente convectivo medido en los ensayos $h_{i}$ :

$$
\begin{gathered}
N u=\frac{h_{i} D_{h}}{k} \times\left(\frac{\mu_{m}}{\mu_{p i}}\right)^{p_{1}} \\
N u=\frac{h_{i} D_{h}}{k} \times\left(\frac{\mu_{m}}{\mu_{p i}}\right)^{-0,14}
\end{gathered}
$$

En el caso de un fluido Power Law, la viscosidad puede tener diferentes expresiones, todas ellas dependientes de: $m, n$, diámetro, y la velocidad media del fluido:

$$
\mu=m\left(\frac{d u}{d r}\right)^{n-1}
$$

o bien la viscosidad efectiva que se ha definido de dos formas distintas,

$$
\begin{gathered}
\mu_{e f, b}=m\left(\frac{u_{b}}{D}\right)^{n-1} \\
\mu_{e f, c}=m\left(\frac{u_{b}}{D}\right)^{n-1} \phi(n)^{n}
\end{gathered}
$$

Si bien es cierto que $n$ varia con la temperatura, ante variaciones pequeñas de la temperatura, la variación de $n$ se puede despreciar, de modo que la variación de la viscosidad (en todas sus posibles definiciones), sería debida únicamente a la variación de $m$. Así, en cualquier caso el cociente de viscosidades vendría dado por $m_{m} / m_{p i}$ en todos los casos. 


\begin{tabular}{lccc}
\hline & $n$ & $m\left[\mathrm{~Pa} . \mathrm{s}^{\mathrm{n}}\right]$ & $T\left[{ }^{\mathrm{o}} \mathrm{C}\right]$ \\
\hline fluido 1 & 0,9 & 0,087 & 17,5 \\
fluido 2 & 0,44 & 3,85 & 19,7 \\
\hline
\end{tabular}

Tabla 6.2: Propiedades reológicas de los dos fluidos utilizados en los experimentos.

Joshi (1978) y Joshi y Bergles (1980a) propusieron la siguiente correlación para fluidos no newtonianos

$$
N u=\frac{h_{i} D_{h}}{k} \times\left(\frac{m_{m}}{m_{p i}}\right)^{0,44 n-0,58}
$$

Donde $m_{m}$ sería el índice de consistencia de flujo a la temperatura media del fluido, y $m_{p i}$ sería el mismo parámetro evaluado en el caso de que el fluido estuviese a la temperatura de la pared.

\section{Ensayos}

Para corroborar la validez del método descrito, en cuanto a la insignificancia de la influencia de $n$ en la variación de la viscosidad y la validez del valor de $p_{1}$ medido por Joshi (1978) y Joshi y Bergles (1980a), se decide realizar una comprobación experimental.

Se han utilizado dos fluidos de trabajo seudoplásticos con propiedades reológicas distintas, las cuales se muestran en la Tabla 6.2. Se mide el coeficiente de convección en el lado tubo $h_{i}$ en 4 ensayos donde se utilizan saltos de temperatura entre la pared del tubo y el fluido entre $10^{\circ} \mathrm{C}$ y $30^{\circ} \mathrm{C}$. El rango de números de Reynolds ensayado abarca tanto la región laminar como la transición y la turbulenta.

En las Figuras 6.4(a) y 6.4(a) se muestran los resultados de los ensayos en los que no se ha aplicado corrección alguna al número de Nusselt, mientras que en la Fig. 6.4(b) se utiliza la corrección dada por la Ec. 6.4.

Los resultados muestran la variación que sufre el número de Nusselt medido en ensayos de transferencia de calor (sin corregir), al hacerlo el salto térmico del ensayo. Tal y como se observa en las Figuras 6.4(b) y 6.4(d), la correlación aplicada corrige de forma adecuada el número de Nusselt, de forma que éste sea independiente del salto térmico aplicado al tubo.

De los resultados, se deducen las siguientes conclusiones:

1. Si se consideran las propiedades del fluido a la temperatura media del mismo, al aplicar diferentes saltos de temperatura en los ensayos, se producen desviaciones importantes en las mediciones del número de Nusselt. 


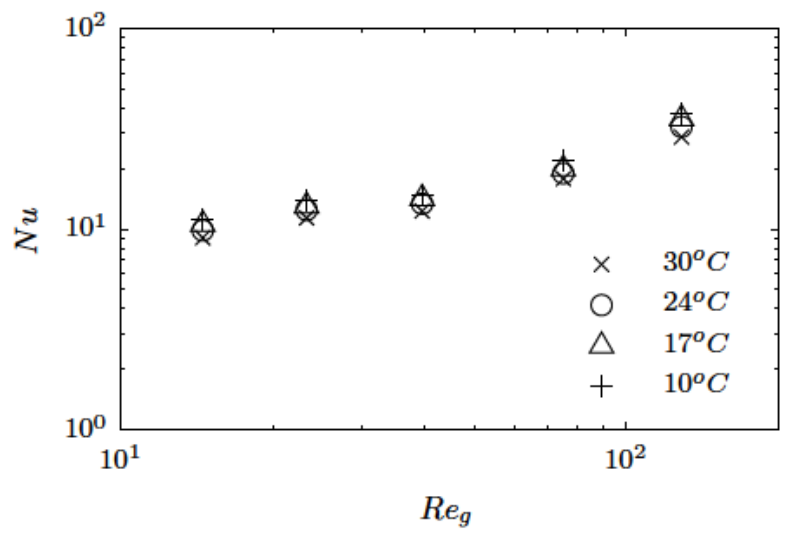

(a) Nusselt no corregido. Fluido 1.

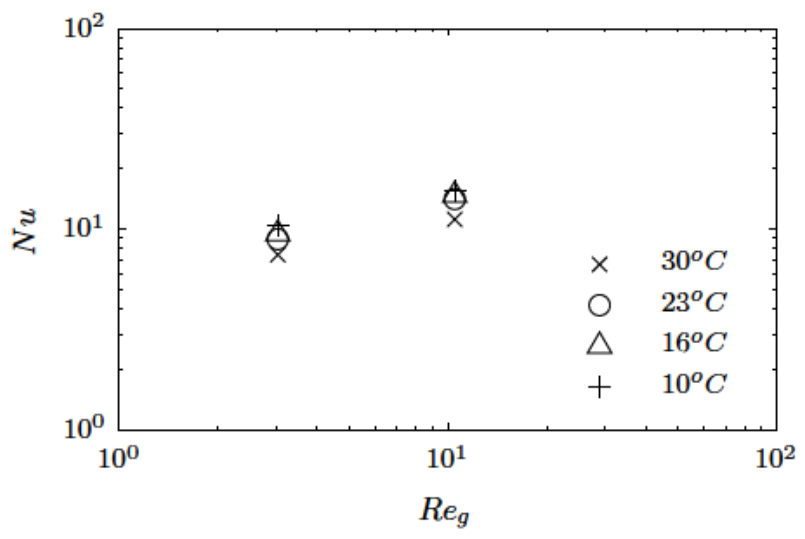

(c) Nusselt no corregido. Fluido 2.

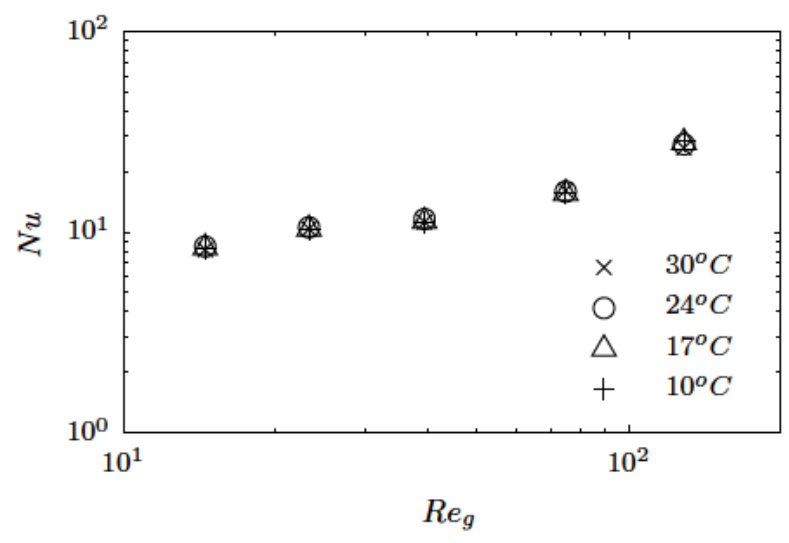

(b) Nusselt corregido según Ec. 6.4. Fluido 1.

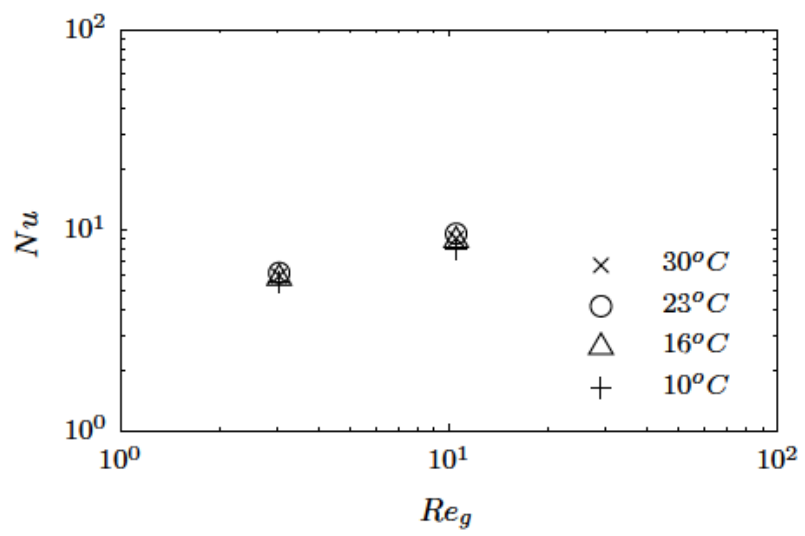

(d) Nusselt corregido según Ec. 6.4. Fluido 2.

Figura 6.4: Medidas del número de Nusselt en ensayos con saltos de temperatura distintos (indicados en la leyenda). Ensayos realizados con dos disoluciones de CMC en agua con propiedades reológicas distintas (Tabla 6.2). 


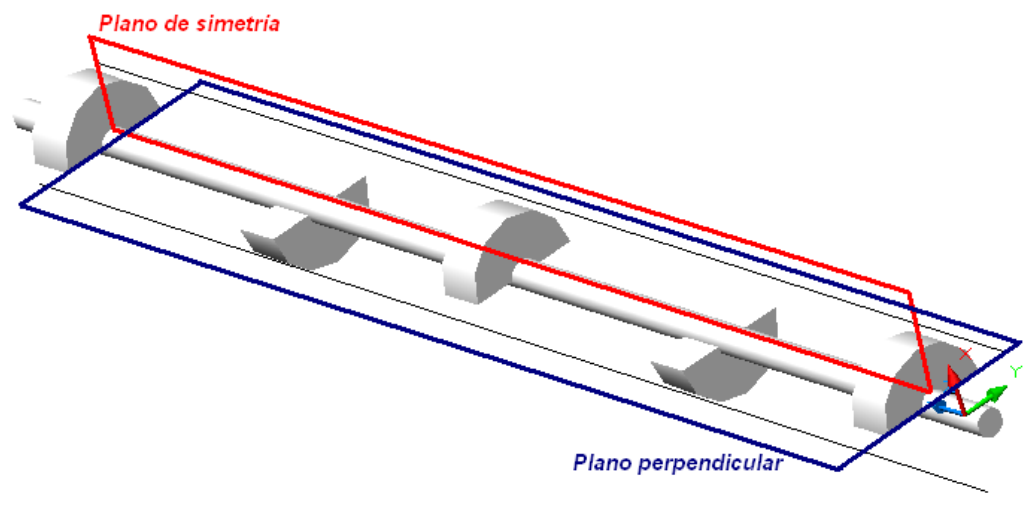

Figura 6.5: Plano de simetría del rascador.

2. Dichas variaciones se deben a la disminución del índice de consistencia de flujo, $m$, que se produce en la región externa del tubo, donde las temperaturas son mayores.

3. En consecuencia, el número de Nusselt calculado a partir de las propiedades medias del fluido, debe ser corregido según la Ec. 6.4.

\subsection{Ensayos de visualización}

Tal y como se ha mencionado en la descripción de la instalación de visualización, para obtener el patrón de flujo se hace uso de la técnica de Velocimetría por Imágenes de Partículas (PIV). Los principios en que se basa esta técnica se describen en el Apartado 4.3.1.

\subsubsection{Metodología empleada}

El flujo circulante por la geometría de rascador ensayada, tiene un plano de simetría (véase Fig. 6.5). En dicho plano se cumple que la velocidad perpendicular a este es nula, lo cual hace de éste el plano ideal para realizar los ensayos de PIV, ya que la velocidad perpendicular al plano iluminado debe ser pequeña. Por lo tanto, en los ensayos realizados se obtiene el campo de velocidades en el plano de simetría del flujo. En consecuencia, el sistema de láser se debe posicionar de manera que la lámina de luz se sitúe exactamente en el plano longitudinal que contiene al eje del tubo. Este ajuste es especialmente importante para evitar el movimiento de las partículas fuera del plano de medida. 


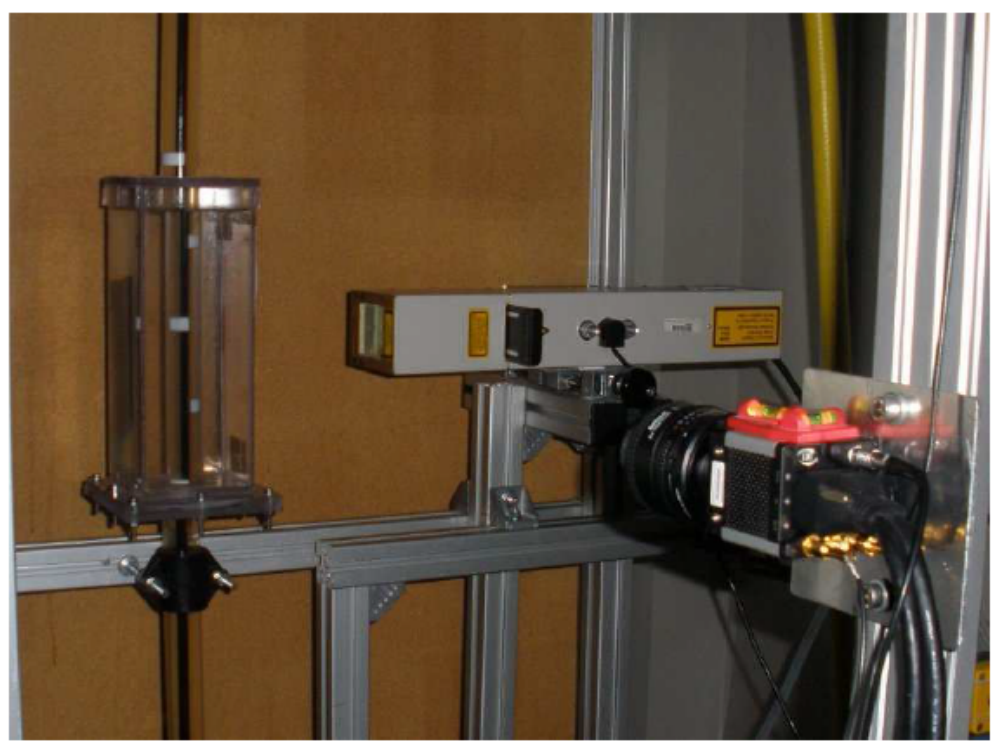

(a) Vista de la sección de ensayo.
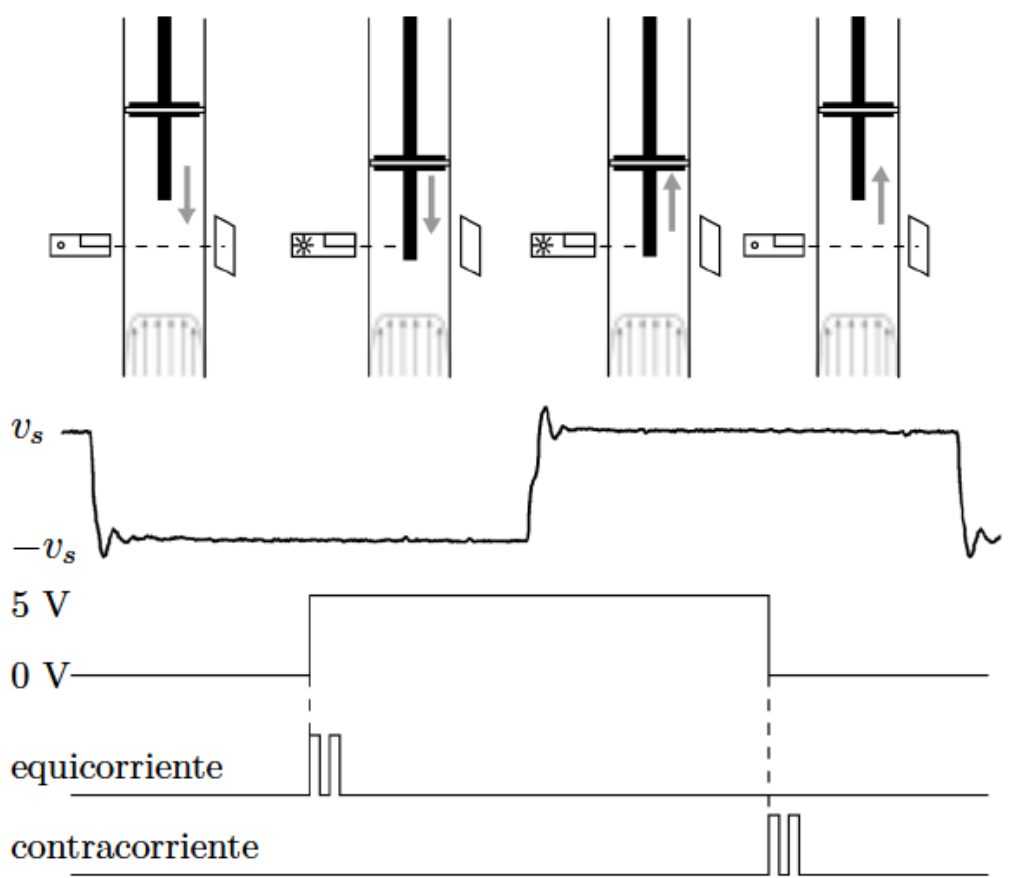

(b) Funcionamiento del sensor óptico. De arriba a abajo: disposición del sensor óptico, velocidad del rascador en el ciclo completo, señal de salida del sensor (TTL) y señal de disparo de la cámara en los dos modos de operación (equicorriente y contracorriente).

Figura 6.6: Componentes del sistema de PIV. 
Siendo el fluido de trabajo una disolución en agua de CMC al $1 \%$ de densidad similar a la del agua (tal y como se ha justificado en el Apartado 5), se utilizan, como partículas trazadoras, esferas de poliamida de $1016 \mathrm{~kg} / \mathrm{m}^{3}$ de densidad y $57 \mu \mathrm{m}$ de diámetro medio.

La instalación experimental permite ensayar en un rango de caudales de $100-1500 \mathrm{l} / \mathrm{h}$ aunque este rango se reduce por dos motivos. El primero, la posible entrada de aire al fluido del sistema en el tramo de aspiración de la bomba. Es difícil establecer un límite de caudal para que esto no ocurra, ya que este fenómeno varía de forma importante en función de la viscosidad del fluido ensayado. Se ha observado que entra aire al sistema cuando en el viscosímetro se mide una caída de presión mayor de 1,5 m.c.a. El segundo motivo es la frecuencia máxima de muestreo por parte de la cámara, relacionada directamente con la velocidad máxima de las partículas en el plano de visualización. Este parámetro viene condicionado por la capacidad de iluminación del láser en las imágenes, que se reduce a velocidades de adquisición por encima de $500 \mathrm{~Hz}$. En los ensayos realizados se utilizan frecuencias de disparo entre $100 \mathrm{~Hz}$ y $500 \mathrm{~Hz}$.

El fabricante proporciona el software que controla la cámara, en el cual se pueden configurar los parámetros de captura: frecuencia de muestreo, tiempo de exposición y número de imágenes a grabar. El láser se configura para que reciba la señal de disparo desde la cámara, de modo que la apertura del obturador de la cámara y el disparo del haz de luz están sincronizados. Se ajustará el tiempo apertura del obturador para establecer la cantidad de luz captada por la cámara, configurando la duración del pulso del láser para que sea superior. En las imágenes de PIV capturadas, la relación de dimensiones entre los píxeles de las imágenes y el tamaño real del plano es de $e_{c}=0,191 \mathrm{~mm} /$ pix.

El fluido de trabajo se puede bombear a temperaturas entre $20^{\circ} \mathrm{C}$ y $60^{\circ} \mathrm{C}$ y de este modo conseguir rangos amplios de condiciones de flujo. Sin embargo, tal y como se ha descrito en el Apartado 5.1.2, el tratamiento térmico degrada rápidamente el fluido y para evitarlo, el rango de temperaturas en los ensayos se reduce a $T \in[20,30]^{\circ} \mathrm{C}$. Las propiedades físicas del fluido se calculan mediante el método descrito en el Apartado 5.1, a partir de la Temperatura medida en el depósito superior y las mediciones en el viscosímetro.

Para seleccionar los parámetros apropiados del procesado, se llevó a cabo un estudio paramétrico, utilizando como criterio para la obtención de valores razonables de Signal To Noise Ratio $(\mathrm{SNR}<4)$ y vectores no válidos tras el filtrado inferiores al $10 \%$ del número total de valores obtenidos. El SNR se define en cada ventana de interrogación como la relación entre el valor máximo (pico) de correlación cruzada, y el ruido promediado de la ventana.

Como resultado de este estudio, el tamaño inicial de las ventanas de interrogación uti- 


\begin{tabular}{ccc}
\hline Ensayos & Imágenes & Pares de img. consecutivas \\
\hline dinámicos & $2 \times 100$ & 100 \\
estáticos & $2 \times 100$ & 199 \\
\hline
\end{tabular}

Tabla 6.3: Pares de imágenes capturadas en los experimentos realizados.

lizadas para el procesado de PIV es de $32 \times 32$ píxeles. Posteriormente, se aplican 2 veces el algoritmo de correlación cruzada adaptativa, reduciendo el tamaño de las ventanas a $16 \times 16$ píxeles, con un solapamiento del $50 \%$.

Para obtener un campo de velocidades libre de puntos erróneos (outliers), tras cada aplicación de un algoritmo de correlación cruzada o de correlación cruzada adaptativa, se aplica un filtrado global y un filtrado local, tras lo cual, los vectores eliminados se sustituyen mediante interpolación bidimensional.

La técnica de PIV implementada solamente ofrece buenos resultados en una región de $80 \mathrm{~mm}$ en dirección axial, donde la calidad de la iluminación es óptima. Los resultados se procesan en tres regiones como se muestra en la Fig. 6.7(a): Región A, aguas abajo del rascador, Región C, aguas arriba del rascador, Región B, entre las regiones A y C. Tal y como se puede apreciar en la figura, hay un solapamiento de $20 \mathrm{~mm}$ entre las regiones A y B, así como entre B y C. La posición de cada región se referencia al rascador. El resultado final se obtiene al recomponer el campo fluido a partir de las mediciones en las tres regiones.

El campo de velocidades final es el promediado de la solución obtenida al procesar $N$ pares de imágenes. El número de pares de imágenes necesarias $N$, dependerá del tipo de experimento a llevar a cabo, siendo necesarias más imágenes para mediciones en ensayos dinámicos. En la Tabla se detallan el número de pares de imágenes capturadas en cada tipo de ensayo. 6.3

En cuanto a los programas utilizados para llevar a cabo el procesado (VidPIV y PIVlab), ambos aplican de forma similar el procedimiento descrito. Sin embargo, presentan características diferentes que hacen interesante el uso de ambos. Por un lado, VidPIV es más detallado en la información, por ejemplo ofrece datos de SNR, y por otro lado PIVlab ofrece una mejor presentación de los resultados intermedios y finales, lo cual permite un mejor diseño del procesado de PIV.

En los ensayos dinámicos, la velocidad del rascador se controla mediante la regulación de la velocidad de giro de la bomba de la instalación oleohidráulica. Una vez purgado el aire de la misma correctamente, la relación entre la velocidad de giro del motor de la bomba y la velocidad del rascador se mantiene constante. La velocidad del rascador ha sido medida 
utilizando la cámara CMOS para todo el rango de velocidades de giro del motor, mediante la adquisición de dos imágenes del rascador en movimiento separadas un tiempo determinado. Midiendo el desplazamiento del rascador entre ambas se obtiene la velocidad del mismo. Los resultados se muestran en la Fig. 6.8. En los ensayos se ha establecido en $5 \mathrm{~Hz}$ la velocidad de giro mínimo de la bomba de la instalación oleohidráulica para que la velocidad del rascador sea estable. Esto proporciona el siguiente rango de velocidades de rascado: $v_{s} \in[0,034,0,275] \mathrm{m} / \mathrm{s}$.

En el movimiento del rascador se produce una distorsión en la velocidad del mismo al cambiar de dirección, la cual no afecta significativamente a la velocidad media de cada ciclo, siendo ambas velocidades prácticamente iguales en módulo pero de signo contrario: $v_{s, e c} \approx$ $-v_{s, c c}$. La velocidad del rascador ha sido medida off-line por un sistema de rastreo por imagen (ver representación en Fig. 6.6(b)), obteniendo como resultado que una vez superada la distorsión en los extremos $\left|v_{s, e c}-v_{s, c c}\right|<2 \%$. Por otro lado, la amplitud del rascado es de $200 \mathrm{~mm}(6,25 D)$.

El sistema de captura actuará ante la señal de disparo de un sensor óptico, tal y como se describe en el Fig. 6.6(b). El sensor óptico se encuentra localizado en el extremo inferior del rascador, de forma que el estado TTL de su señal de salida cambiará de $0 \mathrm{~V}$ a $5 \mathrm{~V}$ cuando el eje del rascador sea detectado y pasará de nuevo a $0 \mathrm{~V}$ cuando retroceda. No obstante la señal también puede ser configurada con el comportamiento contrario. Mediante el uso de ambos modos de funcionamiento y del temporizador incorporado en el sensor, la señal se puede retrasar para que el disparo de la cámara se produzca exactamente cuando las regiones $\mathrm{A}, \mathrm{B}$ o $\mathrm{C}$ del rascador se encuentren en posición para la adquisición y el rascador se mueva en la dirección correcta. Cuando la cámara recibe la señal de disparo, ésta captura dos imágenes consecutivas, las descarga al ordenador y espera hasta la siguiente señal. Este procedimiento se repite $N$ veces y los resultados serán promediados. A esta técnica se la conoce como promediado en fase o phase average. Además cada experimento se ha repetido un mínimo de 3 veces para asegurar que los resultados son correctos.

En los ensayos las constantes reológicas del fluido varían en los rangos 3,5>m>0,070 y $0,52<n<0,94$. El caudal circulado entre $280 \mathrm{l} / \mathrm{h}$ y $355 \mathrm{l} / \mathrm{h}$ a temperaturas entre $25^{\circ} \mathrm{C}$ y $30^{\circ} \mathrm{C}$, resultando en números de Reynolds en el rango $R e_{c}=[3,8,55]$ o $R e_{c, d}=[0,56,8,32]$. En los ensayos dinámicos se han analizado velocidades de rascado entre $0<v_{s} / \bar{u}_{z}<3$.

Para validar la metodología de ensayo, se realizan ensayos de visualización del flujo en el tubo sin el rascador insertado. Los resultados se comparan con el perfil adimensional de velocidades en tubo liso para un fluido que responde al modelo Power Law viene dado por la Ec. 5.2. 


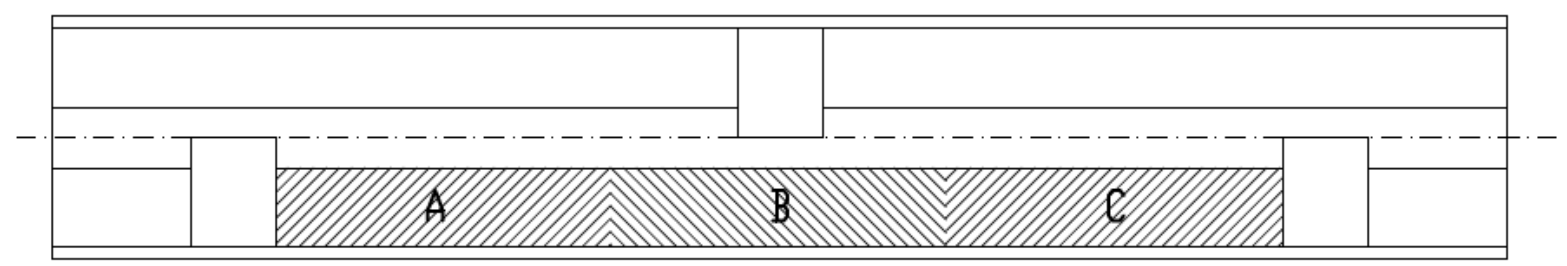

(a) Disposición de las regiones A, B y C en el rascador.
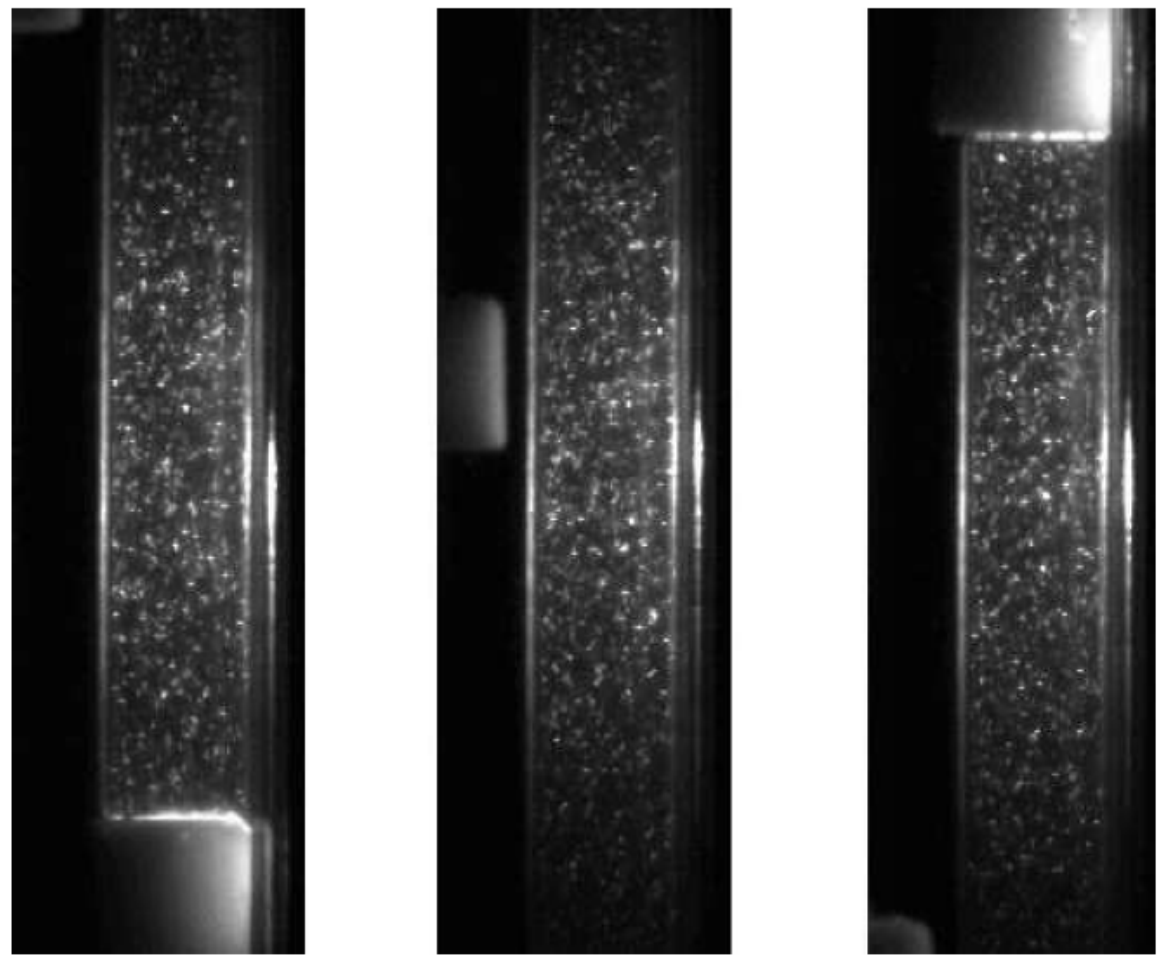

(b) De izquierda a derecha: Región A, Región B y Región C

Figura 6.7: Regiones de captura de las imágenes de PIV. 


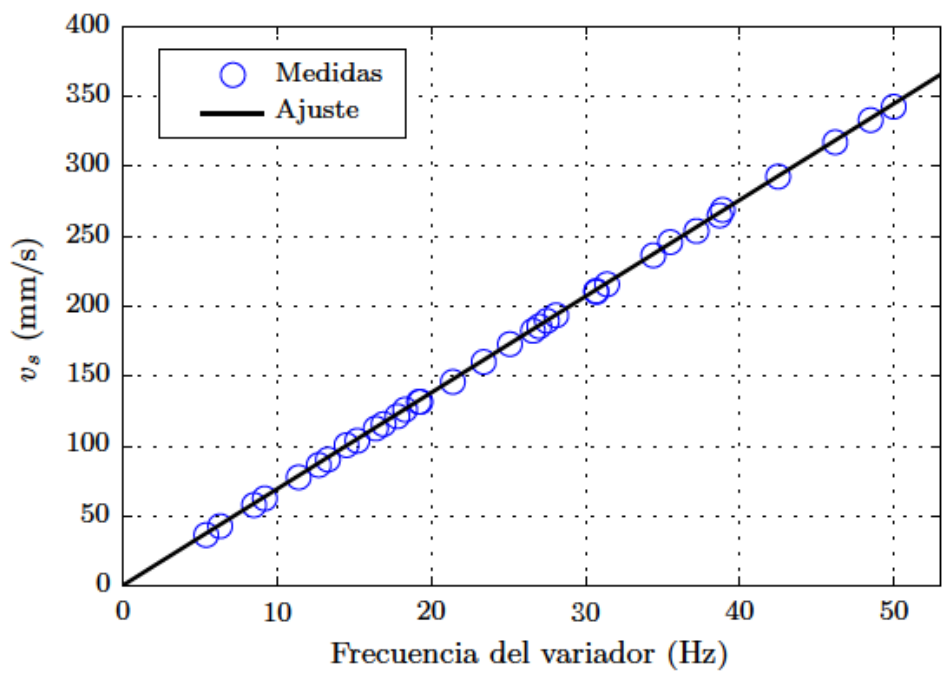

Figura 6.8: Relación entre velocidad de giro del motor de la bomba de la instalación oleohidráulica y la velocidad del rascador.

Por otro lado, con el objetivo de observar el efecto del rascado aislado, se realizan ensayos a caudal cero con diferentes velocidades de rascado. En estos experimentos, para conseguir la temperatura deseada, se necesita que el fluido esté en circulación. Una vez calentado, se detiene la bomba y se acciona el rascador para proceder con el ensayo. La temperatura exacta durante el transcurso del ensayo no se puede medir, sin embargo, ésta se mide antes y después del mismo, comprobando que la diferencia es despreciable.

\subsubsection{Validación de la metodología}

Para validar la metodología de visualización descrita se visualiza el flujo en geometría de tubo liso. El diámetro interior del tubo es de $16 \mathrm{~mm}$. En dicha geometría la solución analítica para fluidos seudoplásticos que siguen el modelo Power law es conocida y viene dado por la siguiente expresión.

$$
u_{z}^{*}=\frac{u_{z}}{u_{b}}=\left(\frac{3 n+1}{n+1}\right)\left[1-\left(\frac{r}{R}\right)^{(n+1) / n}\right]
$$

Tal y como se observa en la solución analítica, el perfil de velocidades es función de $n$. Se realizan tres ensayos variando las condiciones de ensayo y utilizando fluidos de trabajo con parámetros reológicos distintos. En concreto se ensayan fluidos con índice de comportamiento 


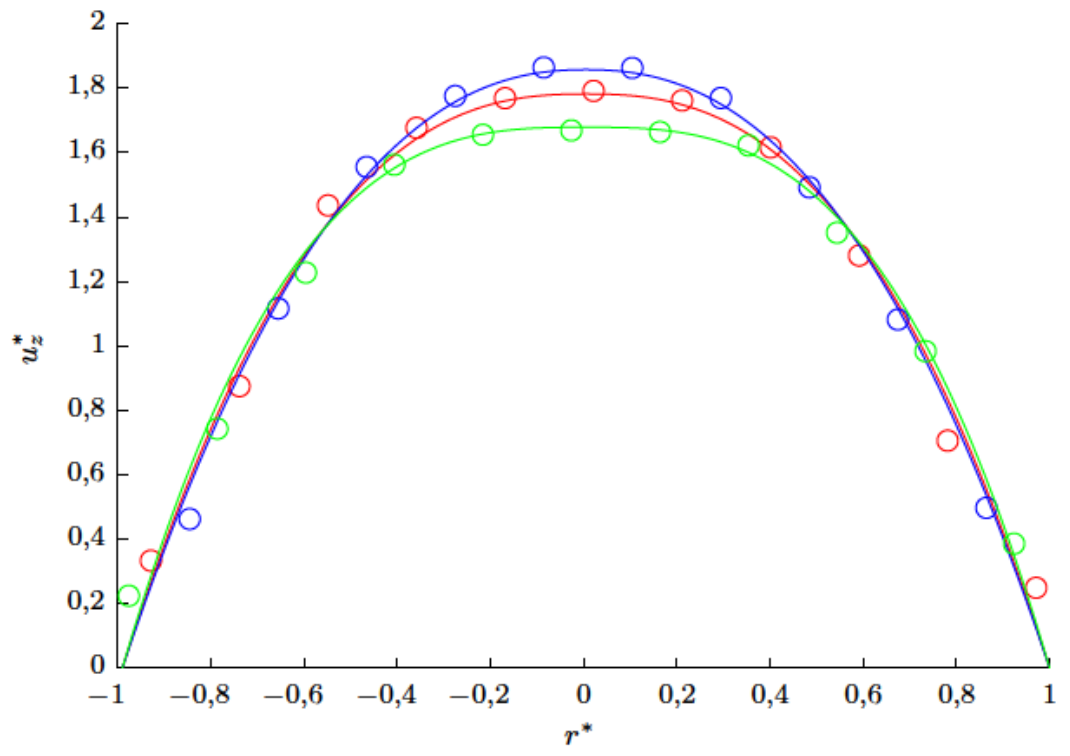

(a) Resultados. Verde: $n=0,51$; Rojo $n=0,64$; azul: $n=0,75$

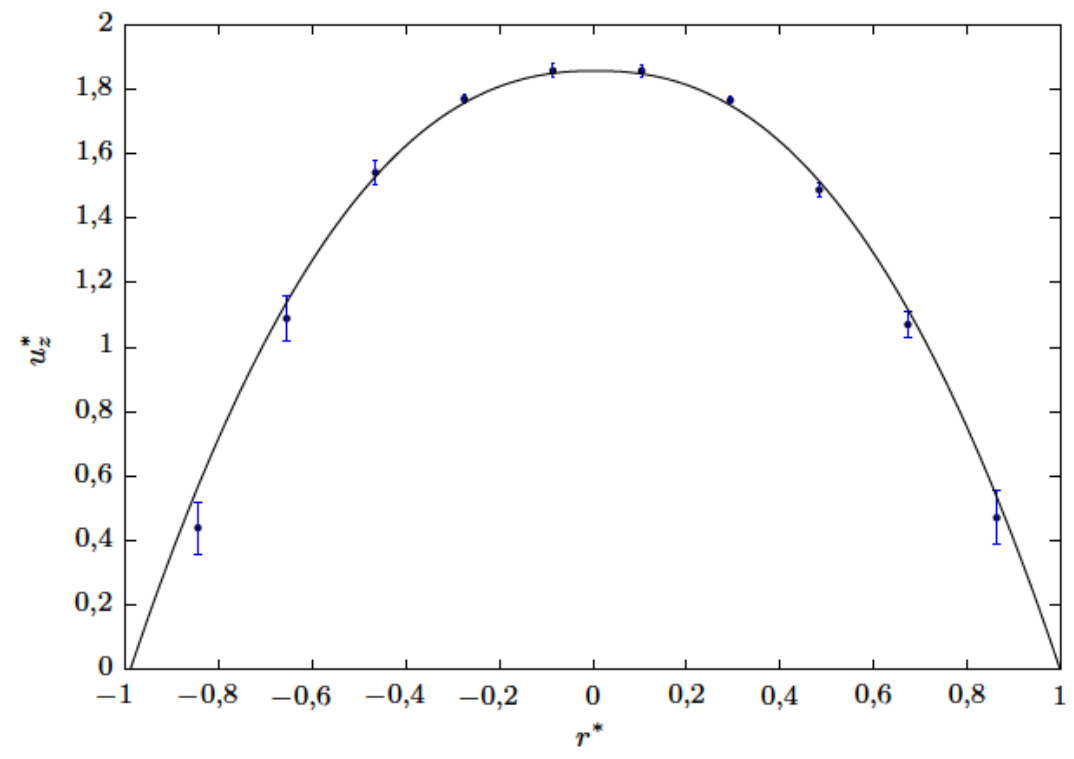

(b) Incertidumbre de la medida. Ensayo $n=0,64$

Figura 6.9: La solución analítica se representa mediante línea continua. 
(a) Configuración de PIV.

\begin{tabular}{ccc}
\hline Parámetro & Valor & Unidades \\
\hline escala & 0,19 & $\mathrm{~mm} / \mathrm{pix}$ \\
Imágenes & 100 (PIV: 99 pares $)$ & \\
Área de interrogación & 32 & píx \\
Separación entre A.I. & 16 & píx \\
Radio interior tubo & 0,016 & $\mathrm{~m}$ \\
\hline
\end{tabular}

(b) Condiciones de ensayo.

\begin{tabular}{cccccc}
\hline & $n$ & $m$ & $Q(\mathrm{l} / \mathrm{h})$ & $T^{\circ} \mathrm{C}$ & $f(\mathrm{~Hz})$ \\
\hline Exp 1 & 0,51 & 3,29 & 162 & 30 & 60 \\
Exp 2 & 0,64 & 0,512 & 300 & 20 & 110 \\
Exp 3 & 0,75 & 0,273 & 230 & 30 & 90 \\
\hline
\end{tabular}

Tabla 6.4: Ensayos de visualización en tubo liso. Nota: las 100 imágenes consecutivas adquiridas son emparejadas por el software por pares $1-2,2-3, \ldots, 99-100$ procesando un total de 99 pares.

de flujo $n=0,51 ; 0,64 ; 0,75$.

La configuración utilizada para el procesado de PIV se detalla en la Tabla 6.3(a). Además, en la Tabla 6.3(b) se especifican las condiciones de cada ensayo.

En la Fig. 6.9(a) se muestran los resultados de los tres experimentos realizados. Como se puede observar, los perfiles de velocidad obtenidos se ajustan con precisión a las soluciones analíticas. 


\subsection{Cálculo de incertidumbres}

Los instrumentos de medida utilizados en la instalación han sido seleccionados para realizar mediciones de alta precisión. El objetivo es obtener resultados con baja incertidumbre, de modo que se asegure la validez de los mismos.

En el presente apartado se procede a determinar la incertidumbre con la que se determinan los valores de los resultados adimensionales. El procedimiento utilizado es el descrito por la norma ISO 98, 1995, "Guide to the expression of uncertainty in measuremet".

En las Tablas 6.5 y 6.6 se detallan las variables utilizadas en la investigación y sus incertidumbres correspondientes.

\subsubsection{Instalación de ensayos termohidráulicos}

\subsubsection{Dimensiones de la geometría}

En primer lugar se detallan las incertidumbres correspondientes a las distintas dimensiones de la instalación.

Para medir $D_{v}, D, d, e_{t}$ se ha hecho uso de un calibre cuyo rango de precisión es de $\pm 0,05 \mathrm{~mm}$. Consecuentemente,

$$
i\left(D_{v}, D, d, e_{t}\right)=\frac{0,05}{\sqrt{3}} \mathrm{~mm}
$$

Para medir las diferentes longitudes del tubo $L_{v m}$ y $L_{p}$, se ha utilizado una cinta métrica cuya precisión es de $\pm 0,5 \mathrm{~mm}$.

$$
i\left(L_{v m}, L_{p}\right)=\frac{0,5}{\sqrt{3}} \mathrm{~mm}
$$

Para las longitudes medidas en los ensayos térmicos se consideran incertidumbres mayores debido a la imprecisión para establecer el punto del electrodo en el que comienza a circular la corriente por el tubo. Por lo tanto, para las medidas de $L_{E}, L_{s}, L_{i}$ y $L_{T}$, considera una precisión de $\pm 5 \mathrm{~mm}$.

$$
i\left(L_{E}, L_{S}, L_{T}, L_{i}\right)=\frac{5}{\sqrt{3}} \mathrm{~mm}
$$

A partir de los diámetros del tubo de ensayo y del viscosímetro se calculan las áreas de 
sus respectivas secciones de paso $A$ y $A_{v}$ y el diámetro hidráulico del tubo de ensayo $D_{h}$. Las incertidumbres correspondientes a dichos parámetros se obtienen como sigue.

$$
\begin{gathered}
i(A)=\frac{\pi}{2} \sqrt{(D \times i(D))^{2}+(d \times i(d))^{2}} \\
i\left(A_{v}\right)=\frac{\pi}{2} D_{v} i\left(D_{v}\right) \\
i\left(D_{h}\right)=\sqrt{i(D)^{2}+i(d)^{2}}
\end{gathered}
$$

En la Tabla 6.4(a) se detallan el valor de las dimensiones analizadas y sus incertidumbres.

\subsubsection{Medidas de los sensores}

En la Tabla 6.4(b) se detallan los valores típicos de las medidas o el rango de valores y las incertidumbres correspondientes. A continuación se describe el cálculo de los mismos.

\section{Sensores de presiones diferencial estacionaria/no estacionaria}

En la instalación se utilizan hasta 5 sensores de presión diferencial: 2 sensores de presión diferencial estacionaria y 2 de presión diferencial no estacionaria conectados al viscosímetro y al tubo de ensayo y 1 sensor de presión diferencial conectado a las cámaras del pistón que impulsa el rascador.

Sensores de presión diferencial estacionaria. Estos sensores tienen una repetibilidad del 0,075\% F.E. del rango de medida en al que estén configurados. Tal y como se detalló anteriormente, los rangos de medida para los sensores PE1 y PE2 son 5 - 405 mbar y 20 - 2500 mbar respectivamente. En los ensayos se toman $N$ medidas donde $N \in[10,20]$ dependiendo del ensayo. En los ensayos a caudales pequeños donde las caídas de presión son pequeñas se aumenta el número de medidas para compensar la pérdida de precisión.

$$
i\left(p_{E 1}\right)=\frac{0,075 \times 400 \mathrm{mbar}}{100 \times \sqrt{N}}
$$




$$
i\left(p_{E 2}\right)=\frac{0,075 \times 2480 \mathrm{mbar}}{100 \times \sqrt{N}}
$$

Sensores de presión diferencial no estacionaria en el tubo de ensayo. Se dispone de dos sensores de este tipo para medir la caída de presión en el tubo, cuyos fondos de escala son 2 bar y 5 bar. Estos sensores presentan tanto una precisión como una repetibilidad de 0,1\% F.E.. En régimen de funcionamiento dinámico, la presión diferencial medida por los sensores oscila de forma cíclica (véase Fig. 6.1). Durante los ensayos se mide la presión diferencial durante al menos 3 ciclos completos. Únicamente se tienen en cuenta los valores de presión cuando ésta se estabiliza y mientras el rascador se mueve en una sola dirección. La velocidad de adquisición de los datos es muy rápida, y en se puede asegurar que en todos los ensayos, se realiza un número de medidas $N \geq 1000$, siendo mucho mayor de forma que la incertidumbre del valor promediado es:

$$
\begin{gathered}
i\left(p_{D 1}\right)=\frac{0,1 \times 2 \mathrm{bar}}{100 \times \sqrt{N}} \\
i\left(p_{D 2}\right)=\frac{0,1 \times 5 \mathrm{mbar}}{100 \times \sqrt{N}}
\end{gathered}
$$

Sensor de presión diferencial en el pistón de impulsión. El sensor de alta precisión presenta una precisión y repetibilidad del 0,04\% F.E, siendo su rango de medición de $[-10,20]$ bar. Las medidas de presión dinámica en el pistón $p_{a c c}$ se realizan de forma similar a $p_{D 1}$ y $P_{D 2}$, con lo que $N \geq 1000$ :

$$
i\left(p_{a c c}\right)=\frac{0,04 \times 35 \mathrm{bar}}{100 \times \sqrt{N}}
$$

\section{Caudalímetro de efecto Coriolis}

El dispositivo se utiliza para medir el caudal y para medir la densidad del fluido.

Medida de caudal. El caudalímetro presenta una repetibilidad del 0,025\% de la medida. Al igual que ocurre con la caída de presión estática en el tubo, en los ensayos se adquieren entre $N \in[10,20]$ medidas, correspondiente un mayor número de medidas a los ensayos a caudales bajos. 


$$
i^{\prime}(Q)=\frac{i(Q)}{Q}=\frac{0,025}{100 \times \sqrt{N}}
$$

Medida de densidad. El dispositivo presenta una precisión del $0,2 \mathrm{~kg} / \mathrm{m}^{3}$. En los ensayos se realiza únicamente una medición de la densidad, cuya incertidumbre viene dada por la siguiente expresión:

$$
i(\rho)=\frac{0,2}{\sqrt{3}} \mathrm{~kg} / \mathrm{m}^{3}
$$

\section{Termopares}

Una vez que se ha realizado la calibración de los termopares anteriormente descrita, se observan diferencias en los mismos de hasta $0,2^{\circ} \mathrm{C}$ como máximo. En consecuencia se considera una incertidumbre de $0,1^{\circ} \mathrm{C}$ para las medidas de estos.

En los ensayos térmicos se toman $N=15$ medidas, con lo que la incertidumbre de la medida de un termopar viene dada por:

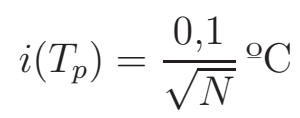

La Temperatura promedio de la pared se obtiene promediando la lectura de $N_{T P}=8$ termopares. Por lo tanto la incertidumbre de la media será

$$
i\left(\bar{T}_{p}\right)=\frac{i\left(T_{p}\right)}{\sqrt{N_{T P}}}=\frac{0,1}{\sqrt{N \times N_{T P}}} \stackrel{\circ}{ } \mathrm{C}
$$

\section{Sondas de temperatura RTD}

Hay sondas de temperatura RTD instaladas para medir la temperatura del fluido a la entrada y a la salida del tubo de ensayo/viscosímetro y en el depósito. La medida de temperatura del depósito se utiliza únicamente para el control de la temperatura del fluido. Las sondas que miden $T_{e}$ y $T_{s}$ son de Clase B 1/10 DIN las cuales tienen una precisión/repetibilidad de $\pm \frac{1}{10}\left(0,3+0,005 T\left({ }^{\circ} \mathrm{C}\right)\right)$.

En los ensayos $10^{\circ} \mathrm{C}<T<70^{\circ} \mathrm{C}$, donde la situación más desfavorable es el límite inferior del rango. La incertidumbre del promedio de $N$ medidas con el fluido a $10^{\circ} \mathrm{C}$ es

$$
i\left(T_{e}, T_{s}\right)=\frac{0,035}{\sqrt{N}} \stackrel{\circ}{ } \mathrm{C}
$$


y en tanto por uno de la medida:

$$
i_{\text {max }}^{\prime}\left(T_{e}, T_{s}\right)=\frac{i\left(T_{e}, T_{s}\right)}{T}=\frac{0,035}{10 \sqrt{N}}
$$

Se ha instalado además una sonda para medir la temperatura ambiente de Clase B, con precisión/repetibilidad a una temperatura ambiente máxima de $40^{\circ} \mathrm{C}$ igual a $\pm(0,3+$ $\left.0,005 T\left({ }^{\circ} \mathrm{C}\right)\right)= \pm(0,5)$.

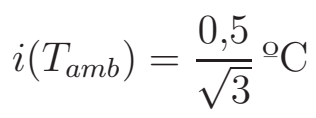

\section{Medidas de tensión e intensidad.}

La tensión alterna se mide directamente utilizando la tarjeta de adquisición Agilent. Esta medida tiene una precisión muy alta y no merece la pena ser considerada en los cálculos de incertidumbres para el número de Nusselt, ya que existen incertidumbres con mucho más peso.

En cuanto a la medida de la Intensidad, se utiliza un sensor que produce una señal $4-20 \mathrm{~mA}$, el cual es capaz de medir con una repetibilidad de $0,1 \% \mathrm{~F}$.E, teniendo un rango de medida $0-600 \mathrm{~A}$. Se toman entre $N \in[10,20]$ medidas.

$$
u(I)=\frac{0,6}{\sqrt{N}}
$$

\subsubsection{Variables medidas indirectamente}

\section{Velocidad media del flujo}

La velocidad media del flujo se obtiene como el cociente entre el caudal y la sección de paso. Por lo tanto,

$$
\begin{gathered}
i\left(u_{b}\right)=\frac{Q}{A} \sqrt{i^{\prime}(Q)^{2}+\left(\frac{i(A)}{A}\right)^{2}} \\
i^{\prime}\left(u_{b}\right)=\frac{i\left(u_{b}\right)}{u_{b}}=\sqrt{i^{\prime}(Q)^{2}+\left(\frac{i(A)}{A}\right)^{2}}
\end{gathered}
$$

en la instalación $i^{\prime}(Q)^{2}<<i^{\prime}(A)^{2}$, con lo que

$$
i^{\prime}\left(u_{b}\right) \approx i^{\prime}(A)
$$




\section{Viscosidad efectiva del fluido}

A lo largo de la tesis se han definido diferentes viscosidades efectivas o aparentes a incluir en los números adimensionales de Reynolds y Prandtl:

$$
\mu_{b}=m\left(\frac{u_{b}}{D_{h}}\right)^{n-1}
$$

cuyas incertidumbres respectivas son

$$
\begin{aligned}
\frac{i^{2}\left(\mu_{b}\right)}{\mu_{b}^{2}}= & \left(\frac{(n-1) \times i\left(u_{b}\right)}{u_{b}}\right)^{2}+\left(\ln \left(\frac{u_{b}}{D_{h}}\right) i(n)\right)^{2}+\left(\frac{i(m)}{m}\right)^{2} \\
& +\left(\frac{(n-1) \times i\left(D_{h}\right)}{D_{h}}\right)^{2}
\end{aligned}
$$

\subsubsection{Propiedades del fluido}

\section{Propiedades reológicas}

Las propiedades reológicas se obtienen a partir de un ajuste por mínimos cuadrados compensado $^{1}$ a una correlación lineal del tipo:

$$
y=c_{1} x+c_{0}
$$

donde la ecuación es

$$
\ln \left(\tau_{w}\right)=n \times \ln \left(\bar{u}_{z}\right)+\ln (m)+n \times \ln \left[\frac{8 u_{b}}{D_{v}}\left(\frac{3 n+1}{4 n}\right)\right]
$$

de modo que $c_{1}=n$ y $m=e^{c_{2}} /\left[\frac{8 u_{b}}{D_{v}}\left(\frac{3 n+1}{4 n}\right)\right]^{n}$. La incertidumbre en las medidas de las propiedades se obtiene a partir de la desviación estándar en el proceso de ajuste por mínimos cuadrados. Dicho ajuste nos proporciona la incertidumbre de $c_{1}=n$ y la de $c_{2}$. La incertidumbre de $m$ se obtiene a partir de la de $c_{2}$ :

$$
i(m)=m \times\left[u\left(c_{2}\right)^{2}+\left(1+\frac{1}{n(3 n+1)}\right)^{2} u(n)^{2}+\left(\frac{1}{D_{v}}\right)^{2} u\left(D_{v}\right)^{2}\right]
$$

\footnotetext{
${ }^{1}$ Considera que pueden existir incertidumbres en las medidas tanto de $x_{i}$ como $y_{i}$.
} 


$$
i^{\prime}(m)=\frac{i(m)}{m}=\left[u\left(c_{2}\right)^{2}+\left(1+\frac{1}{n(3 n+1)}\right)^{2} u(n)^{2}+\left(\frac{1}{D_{v}}\right)^{2} u\left(D_{v}\right)^{2}\right]
$$

Por lo tanto, para obtener los valores de $i(n)$ e $i(m)$ se debe realizar primero el ajuste por mínimos cuadrados para obtener las incertidumbres derivadas del mismo. Esto implica que estas incertidumbres se deben calcular para cada experimento. A modo ilustrativo se toman 3 experimentos y se muestran las incertidumbres obtenidas (véase Fig. 6.10). En adelante se considerará la mayor de las tres.

\section{Otras propiedades termofísicas}

Para las propiedades del fluido que no se miden directamente en la instalación, $k$ y $c_{p}$, se supone una incertidumbre del $1 \%$. La incertidumbre de la densidad, medida con el caudalímetro de efecto coriolis, ya se ha obtenido anteriormente.

\subsubsection{Resultados de pérdida de presión y potencia de accionamiento}

En los ensayos de pérdida de presión, los resultados se presentan como relaciones entre el número de Reynolds y el factor de fricción de Fanning, calculados a partir de las distintas variables medidas. El factor de fricción de Fanning se obtiene de la siguiente expresión

$$
f=\frac{p D_{h}}{L_{p} \rho u_{b}^{2}}
$$

y la incertidumbre correspondiente

$$
i(f)=f\left[\left(\frac{i(\rho)}{\rho}\right)^{2}+\left(\frac{i(p)}{p}\right)^{2}+\left(\frac{i\left(D_{h}\right)}{D_{h}}\right)^{2}+\left(\frac{2 i\left(u_{b}\right)}{u_{b}}\right)^{2}+\left(\frac{i\left(L_{p}\right)}{L_{p}}\right)^{2}\right]^{1 / 2}
$$

El número de Reynolds básico tiene la siguiente expresión

$$
R e_{b}=\frac{\rho u_{b} D_{h}}{\mu_{b}}
$$




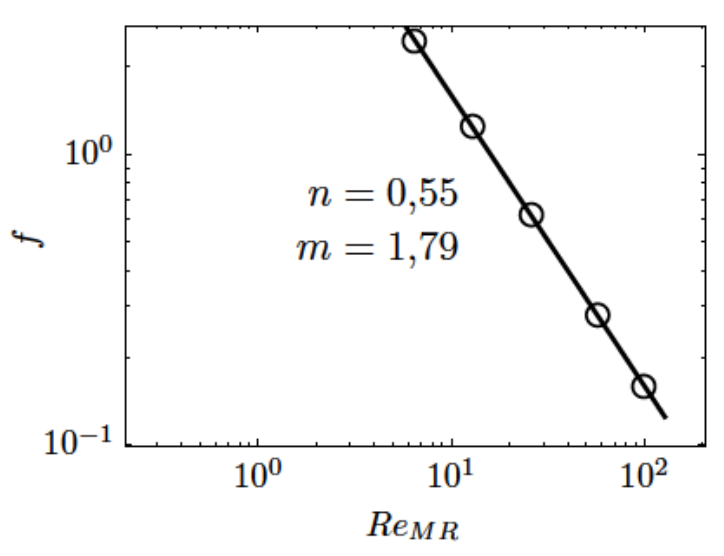

(a) Ensayo 1

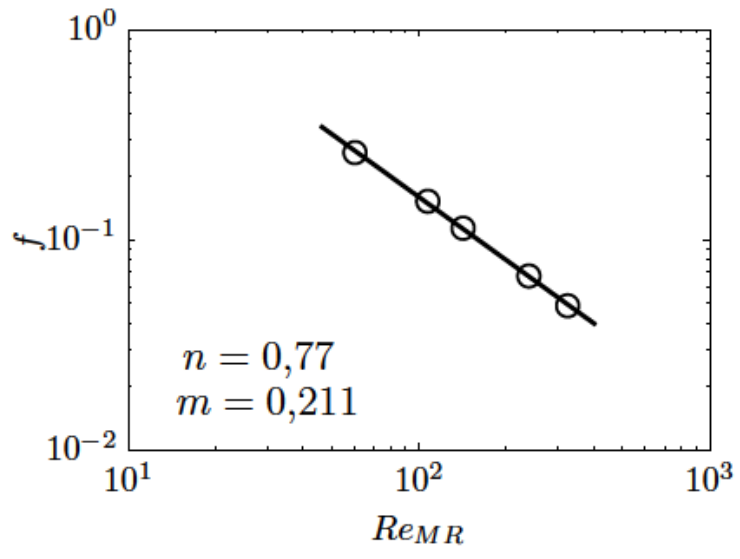

(b) Ensayo 2.

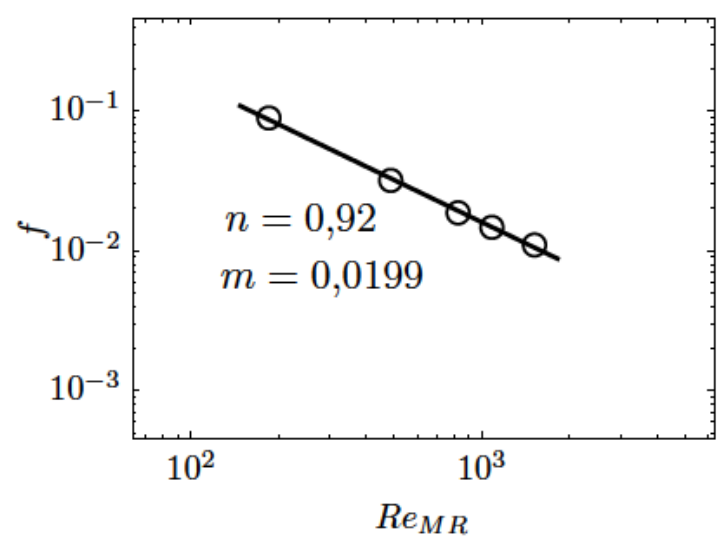

(c) Ensayo 3.

Figura 6.10: A estos ensayos corresponden las siguientes incertidumbres respectivamente: $i(n)=3 \times 10^{-6} ; 10^{-5} ; 3 \times 10^{-5}$, siendo $i(n) / n=6 \times 10^{-6} ; 10^{-5} ; 4 \times 10^{-5}$ y $i(m)=3 \times$ $10^{-3} ; 410^{-4} ; 4 \times 10^{-5}$, siendo $i(m) / m=0,0018$ en los 3 casos. 
en consecuencia, la incertidumbre asociada es

$$
i\left(\operatorname{Re}_{b}\right)=R e_{b}\left[\left(\frac{i(\rho)}{\rho}\right)^{2}+\left(\frac{i\left(\mu_{b}\right)}{\mu_{b}}\right)^{2}+\left(\frac{i\left(D_{h}\right)}{D_{h}}\right)^{2}+\left(\frac{i\left(u_{b}\right)}{u_{b}}\right)^{2}\right]^{1 / 2}
$$

La incertidumbre de $R e_{g}$, viene dada por la precisión de la correlación obtenida, del $9 \%$.

En los ensayos dinámicos además se evalúa la velocidad de rascado adimensional, ya sea mediante $\omega$ o mediante $\beta$.

$$
\begin{gathered}
\omega=\frac{v_{s}}{u_{b}} ; \beta=1-\frac{v_{s}}{u_{b}} \\
i(\omega)=i(\beta)=\omega \sqrt{\left(\frac{i\left(v_{s}\right)}{v_{s}}\right)^{2}+\left(\frac{i\left(u_{b}\right)}{u_{b}}\right)^{2}} \\
i^{\prime}(\beta)=\frac{\omega}{\beta} \sqrt{\left(\frac{i\left(v_{s}\right)}{u_{b}}\right)^{2}+\left(\frac{v_{s} i\left(u_{b}\right)}{u_{b}^{2}}\right)^{2}}
\end{gathered}
$$

La potencia requerida para accionar el rascador se mide a partir de la diferencia de presión en las cámaras del pistón. Teniendo el pistón ambas cámaras iguales,

$$
\begin{gathered}
\dot{W}=\frac{W}{t}=\frac{A_{r} \Delta p_{p} \times S_{p}}{T_{r} / 2} \\
i(\dot{W})=\dot{W} \sqrt{\left(\frac{i\left(A_{r}\right)}{A_{r}}\right)^{2}+\left(\frac{i\left(\Delta p_{p}\right)}{\Delta p_{p}}\right)^{2}+\left(\frac{i\left(S_{p}\right)}{S_{p}}\right)^{2}+\left(\frac{i\left(T_{r}\right)}{T_{r}}\right)^{2}}
\end{gathered}
$$

\subsubsection{Resultados de transmisión de calor}

Los resultados de los ensayos de transmisión de calor se presentan en forma de correlaciones entre los números adimensionales de Nusselt, Prandtl y Reynolds.

$$
\operatorname{Pr}_{b}=\frac{c_{p} \mu_{b}}{k}
$$




$$
i\left(\operatorname{Pr}_{b}\right)=P r_{b} \sqrt{\left(\frac{i\left(c_{p}\right)}{c_{p}}\right)^{2}+\left(\frac{i(\mu)}{\mu}\right)^{2}+\left(\frac{i(k)}{k}\right)^{2}}
$$

La incertidumbre de $P r_{g}$, está relacionada con la de la viscosidad generalizada, del $9 \%$.

$$
\begin{gathered}
N u=\frac{h_{i} D_{h}}{k}=\frac{V I D_{h}}{\pi D L_{T}\left(\bar{T}_{p}-\bar{T}_{f}\right)} \\
h_{i}=\frac{V I}{\pi D L_{T}\left(\bar{T}_{p}-\bar{T}_{f}\right)} \\
\frac{i^{2}(N u)}{N u^{2}}=\left(\frac{i(V)}{V}\right)^{2}+\left(\frac{i(I)}{I}\right)^{2}+\left(\frac{i\left(D_{h}\right)}{D_{h}}\right)^{2}+\left(\frac{i(D)}{D}\right)^{2}+\left(\frac{i\left(L_{T}\right)}{L_{T}}\right)^{2}+ \\
+\left(\frac{i\left(\bar{T}_{p}\right)}{\left(\bar{T}_{p}-\bar{T}_{f}\right)}\right)^{2}+\left(\frac{i\left(\bar{T}_{f}\right)}{\left(\bar{T}_{p}-\bar{T}_{f}\right)}\right)^{2}
\end{gathered}
$$


(a) Dimensiones.

\begin{tabular}{ccccc}
\hline Variable & Valor & Incertidumbre & Unidades & Inc. \% \\
\hline$D$ & 18 & $0,05 / \sqrt{3}$ & $\mathrm{~mm}$ & 0,2 \\
$d$ & 5 & $0,05 / \sqrt{3}$ & $\mathrm{~mm}$ & 0,6 \\
$D_{h}$ & 13 & 0,04 & $\mathrm{~mm}$ & 0,3 \\
$A$ & 234,8 & 0,7 & $\mathrm{~mm}^{2}$ & 0,4 \\
$D_{v}$ & 16 & $0,05 / \sqrt{3}$ & $\mathrm{~mm}$ & 0,2 \\
$A_{v}$ & 201,1 & 0,8 & $\mathrm{~mm}^{2}$ & 0,4 \\
$e_{v}$ & 2 & $0,05 / \sqrt{3}$ & $\mathrm{~mm}$ & 1 \\
$L_{v m}$ & 1885 & $0,5 / \sqrt{3}$ & $\mathrm{~mm}$ & 0,02 \\
$L_{p}$ & 1850 & $0,5 / \sqrt{3}$ & $\mathrm{~mm}$ & 0,02 \\
$L_{E}$ & 2050 & $5 / \sqrt{3}$ & $\mathrm{~mm}$ & 0,1 \\
$L_{T}$ & 1001 & $5 / \sqrt{3}$ & $\mathrm{~mm}$ & 0,3 \\
$L_{S}$ & 2330 & $5 / \sqrt{3}$ & $\mathrm{~mm}$ & 0,1 \\
$L_{i}$ & $717-800$ & $5 / \sqrt{3}$ & $\mathrm{~mm}$ & 0,4 \\
\hline
\end{tabular}

(b) Medidas de los sensores.

\begin{tabular}{cccccc}
\hline Variable & Valor & $N$ & Incert. & Uds. & Incert. máx. en \% \\
\hline$p_{E 1}$ & $10-405$ & $20-10$ & $0,07-0,09$ & mbar & 0,7 \\
$p_{E 2}$ & $400-2500$ & 10 & 0,6 & mbar & 0,1 \\
$p_{D 1}$ & $0,05-2$ & $\geq 1000$ & $0,06 \times 10^{-3}$ & bar & 0,1 \\
$p_{D 2}$ & $2-5$ & $\geq 1000$ & $0,2 \times 10^{-3}$ & bar & 0,008 \\
$p_{a c c}$ & -15 a 20 & $\geq 1000$ & $0,44 \times 10^{-3}$ & bar & 0,04 \\
$Q$ & $30-2000$ & $10-20$ & $\%$ medida & $\mathrm{kg} / \mathrm{h}$ & $7,9 \times 10^{-3}$ \\
$\rho$ & 1000 & 1 & 0,1 & $\mathrm{~kg} / \mathrm{m}^{3}$ & 0,01 \\
$\bar{T}_{P}$ & $10-70$ & $15 \times 8$ & 0,03 & ${ }^{\circ} \mathrm{C}$ & 0,2 \\
$T_{e}, T_{s}$ & $10-70$ & 15 & $0,009-0,02$ & ${ }^{\circ} \mathrm{C}$ & 0,09 \\
$T_{a m b}$ & $20-35$ & 15 & 0,1 & ${ }^{\circ} \mathrm{C}$ & 0,2 \\
$I$ & $50-600$ & $20-10$ & 0,2 & $\mathrm{~A}$ & 0,2 \\
\hline
\end{tabular}

Tabla 6.5: Incertidumbre en las dimensiones y en las medidas realizadas por los sensores. 
(a) Variables calculadas a partir de las medidas.

\begin{tabular}{ccccc}
\hline Variable & Valor / rango & Incert. & Uds. & Incert. máx. en $\%$ \\
\hline$u_{b}$ (t.ensayo) & $0,09-1,4$ & $3 \times 10^{-4}-5 \times 10^{-3}$ & $\mathrm{~m} / \mathrm{s}$ & 0,4 \\
$u_{b}$ (vicosim) & $0,1-1,7$ & $4 \times 10^{-4}-6 \times 10^{-3}$ & $\mathrm{~m} / \mathrm{s}$ & 0,4 \\
$\mu_{b}$ & $1,483-0,01$ & $0,0044-1,8 \times 10^{-5}$ & $\mathrm{~Pa} . \mathrm{s}$ & $0,3-0,18$ \\
$m$ & $4-0,01$ & $0,0072-1,8 \times 10^{-5}$ & $\mathrm{~Pa} \cdot \mathrm{s}^{n}$ & 0,18 \\
$n$ & $0,5-1$ & $3 \times 10^{-6}-3 \times 10^{-5}$ & - & $4 \times 10^{-3}$ \\
$k$ & 0,58 & 0,01 & $\mathrm{~W} / \mathrm{mK}$ & $1,7 \%$ \\
$c_{p}$ & 4,18 & 0,04 & $\mathrm{~kJ} / \mathrm{kg} \mathrm{K}$ & $1 \%$ \\
\hline
\end{tabular}

(b) Resultados adimensionales.

\begin{tabular}{cccc}
\hline Variable & Valor & Incert. & Incert. máx. en \% \\
\hline$R e_{b}$ & $0,1-1000$ & $6 \times 10^{-4}-5$ & 0,6 \\
$R e_{g}$ & $1-600$ & - & 9 \\
$f$ & $0,3-100$ & $0,003-1$ & 1 \\
$N u$ & $5-80$ & $0,02-0,4$ & 0,5 \\
$P r_{b}$ & $100-2000$ & $0,3-6$ & 0,3 \\
$\operatorname{Pr}_{g}$ & $200-3500$ & - & 9 \\
\hline
\end{tabular}

Tabla 6.6: Incertidumbre en los datos adquiridos mediante los sensores instalados.

\subsubsection{Ensayos de visualización}

Al obtener el campo de velocidades a partir de un par de imágenes, el algoritmo de PIV calcula la velocidad en cada punto como sigue:

$$
u_{i}=\frac{\Delta z_{i}}{e_{c} \Delta t}
$$

donde $e_{c}$ es el

$$
e_{c}=\frac{N_{p i x}}{D_{h}}
$$

de modo que la incertidumbre asociada a la velocidad instantánea se puede obtener a partir de la Ec. 6.5 y la Ec. 6.6, donde se ha despreciado $u(\Delta t)$ debido a la alta precisión en la temporización de los disparos por parte de la cámara (Westerweel, 2000).

$$
i\left(e_{c}\right)=e_{c}\left[\left(\frac{i\left(N_{p i x}\right)}{N_{p i x}}\right)^{2}+\left(\frac{i\left(D_{h}\right)}{D_{h}}\right)^{2}\right]^{1 / 2}
$$




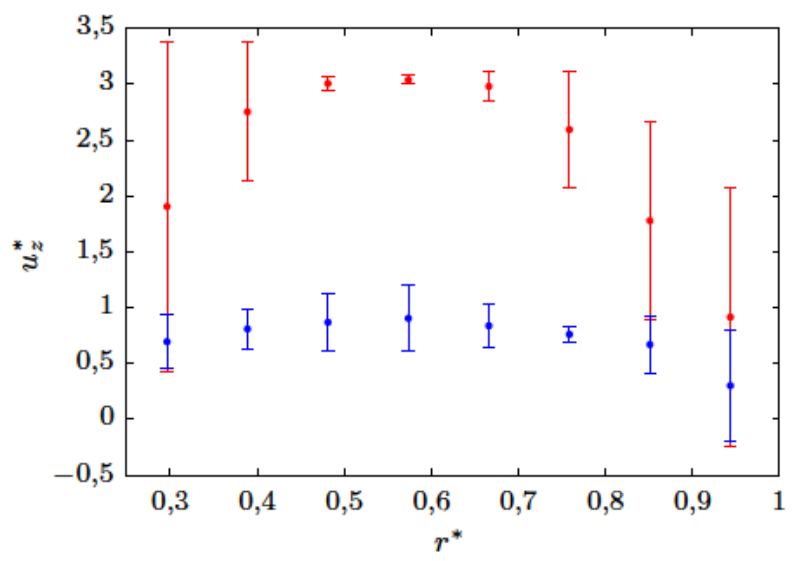

(a) Ensayo estático: $n=0,52$ y $R e_{g}=5$.

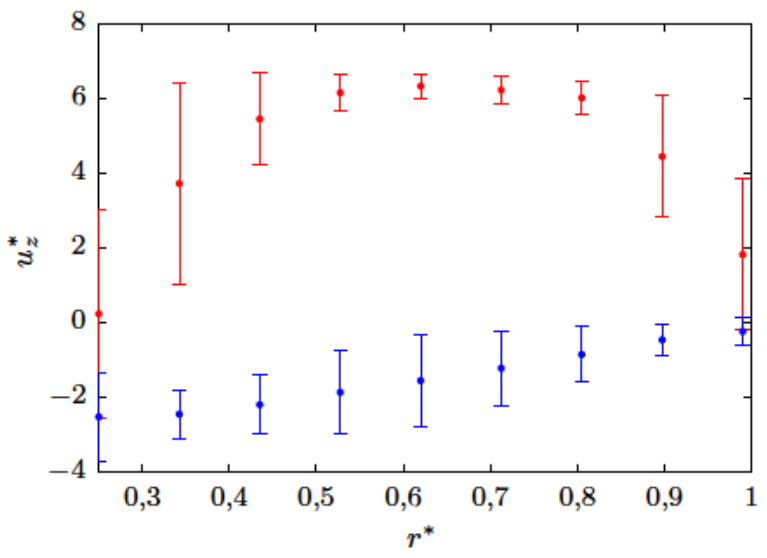

(b) Ensayo dinámico: $v_{s} / u_{b}=-3, n=0,66$ y $R e_{g}=$ 14.

Figura 6.11: Intervalo confianza de la medida de velocidad obtenida de aplicar PIV en un sólo par de imágenes. Perfiles de velocidades en $z=4 P / 5-t_{s}$ y en $z=\left(P-t_{s}\right) / 2$, representados en rojo y azul respectivamente.

$$
i\left(u_{i}\right)=u_{i}\left[\left(\frac{i\left(\Delta z_{i}\right)}{\Delta z_{i}}\right)^{2}+\left(\frac{u\left(e_{c}\right)}{e_{c}}\right)^{2}\right]^{1 / 2}
$$

Si se mide el mismo parámetro $N$ veces y el resultado se promedia, la incertidumbre el valor promediado viene dado por la Ec. 6.7, donde $s\left(u_{i}\right)$ es la desviación típica de las muestras.

$$
s\left(U_{i}\right)=\sqrt{\frac{s\left(u_{i}\right)}{N}}
$$

Si el número de muestras es suficientemente alto, la incertidumbre de la estimación de la velocidad en cada punto se reduce de forma significativa.

De acuerdo con Scarano y Reithmuller (2000), si no hay gradiente de velocidades, el algoritmo de PIV estima el desplazamiento de las partículas con una precisión de $i(\Delta x)=$ 0,005 píxel. Sin embargo, en caso de que sí exista un gradiente de velocidades, aparece un error adicional, cuyo valor máximo viene dado por la diferencia máxima de velocidades en un área de interrogación $\left(16 \times 16\right.$ píxel $\left.^{2}\right)$.

En todos los ensayos realizados el flujo es laminar y por lo tanto el campo de velocidades instantáneas en régimen estacionario es constante. De modo que al capturar $N$ pares de imágenes se realiza $N$ veces la misma medida y por lo tanto, la incertidumbre de dicho 


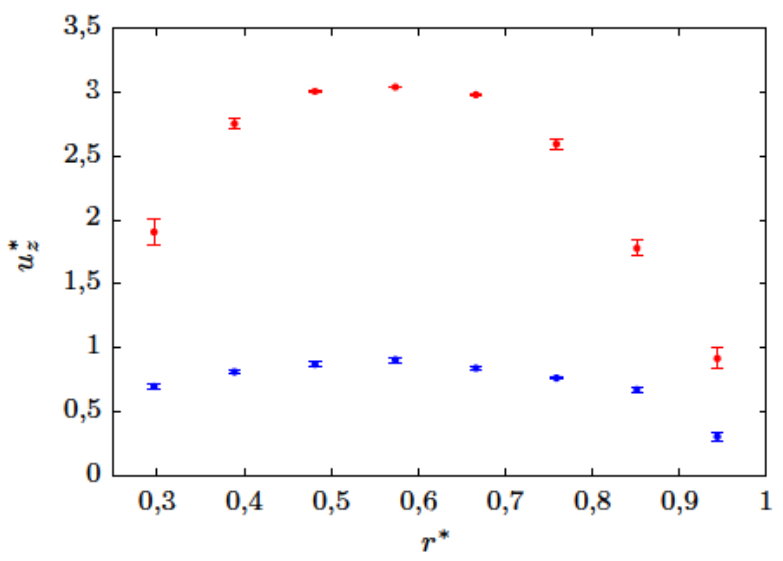

(a) Ensayo estático: $n=0,52$ y $R e_{g}=5$.

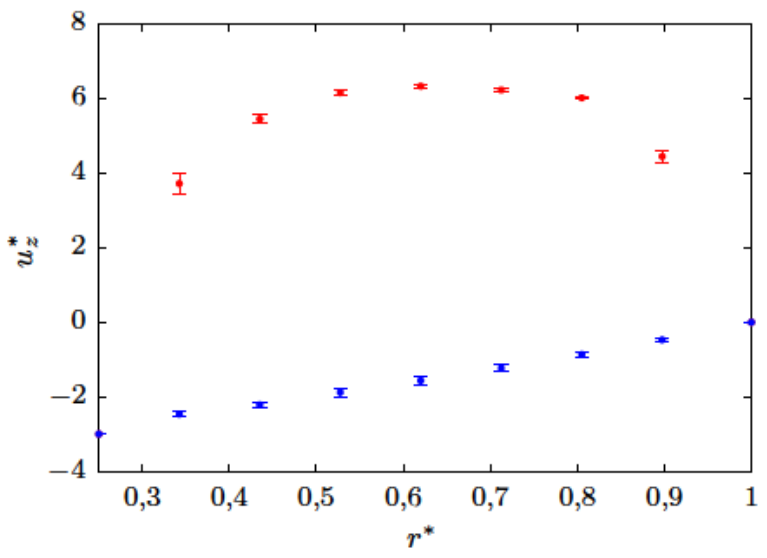

(b) Ensayo dinámico: $v_{s} / u_{b}=-3, n=0,66$ y $R e_{g}=$ 14 .

Figura 6.12: Intervalo confianza del campo de velocidades promedio obtenido mediante PIV. Perfiles de velocidades en $z=4 P / 5-t_{s}$ y en $z=\left(P-t_{s}\right) / 2$, representados en rojo y azul respectivamente.

campo de velocidades viene dado por la Ec. 6.7. La incertidumbre de las medidas del campo de velocidades en régimen dinámico viene igualmente dada por la Ec. 6.7, ya que las $N$ capturas de los diferentes pares de imágenes se realizan en el mismo punto del recorrido del rascador, con lo que el campo de velocidades medido es siempre el mismo. En la etapa de validación del método mediante ensayos en tubo liso, se representó la incertidumbre de las medidas en dicho ensayo (véase Fig. 6.9(b)). Para los ensayos con rascador, en la Fig. 6.11 se muestran los intervalos de confianza correspondientes a un nivel de confianza del $95 \%$ al realizar una sola medida (un par de imágenes). En la figura, donde se representan un caso estático y otro dinámico, se observa como la incertidumbre crece en las zonas donde el gradiente de velocidades es alto, tal y como indican Scarano y Reithmuller (2000). Además, las velocidades medidas en las áreas de interrogación cuyo centro se encuentra en las paredes del eje y del tubo, se desvían significativamente de su valor real. Este efecto se observa mejor en el ensayo dinámico, donde la velocidad del eje es $v_{s}=-3 u_{b}$ y la de la pared del tubo debe ser cero. Esta incertidumbre en las medidas de velocidad en las paredes es propia de la técnica de visualización: por un lado, hasta la mitad del área de interrogación puede estar fuera del flujo, donde no hay partículas, por otro lado las paredes provocan reflejos que distorsionan la medida. Es por ello que de ahora en adelante, la velocidad en las paredes representada en los perfiles de velocidad se fija al valor dado por la condición de contorno correspondiente.

Por último, en la Fig. 6.12 se representa la incertidumbre del campo de velocidades ob- 
tenido del promedio de los resultados de aplicar PIV a los $N$ pares de imágenes capturados $s\left(U_{i}\right)$. En las gráficas se observa que el número de imágenes capturado es suficiente para asegurar una alta precisión en las medidas. 


\section{Parte II}

\section{Resultados y conclusiones}





\section{Capítulo 7}

\section{Visualización del flujo}

Tal y como se indicó en el Capítulo 1, para poder evaluar la eficiencia de un sistema de mejora de la transferencia de calor en intercambiadores, se deben ponderar los beneficios y los costes derivados de su utilización. Para ello se deben cuantificar los siguientes efectos: la mejora en la transferencia de calor, el aumento de pérdida de presión y, de haberla, la potencia adicional consumida por el sistema. La evaluación de estos tres factores permitirá realizar un balance energético, que servirá para determinar la eficiencia del método de mejora y el rango en el que se deben encontrar los distintos parámetros de funcionamiento para optimizar el proceso. Estas tareas se llevan a cabo en los capítulos sucesivos, sin embargo, la medición de los citados parámetros no es suficiente. Además, resulta de suma importancia conocer la estructura del flujo en las diversas situaciones de funcionamiento. El conocimiento de las estructuras del flujo ayuda a identificar el comportamiento del fluido en el intercambiador en cuanto a: variaciones en la caída de presión por metro lineal, incrementos en la transmisión de calor, transiciones entre regímenes de flujo, etc. Así mismo, a la hora de establecer correlaciones experimentales para cuantificar los diferentes efectos, éstas deben estar apoyadas en el conocimiento del patrón de flujo en cada situación de funcionamiento. Por lo tanto, se establece como objetivo del presente apartado, la determinación del patrón de flujo en el tubo con rascador en las diferentes situaciones de funcionamiento.

En primer lugar cabe destacar que el intercambiador puede funcionar con el rascador en movimiento o estático. En régimen de rascador estático puede ser muy interesante, porque se evita el consumo de potencia de accionamiento en el rascador y sin embargo existe un mezclado del fluido que puede producir mejoras en la transferencia de calor, aumentando también las pérdidas de presión. Por otro lado, el régimen dinámico aumentará aún más la 
eficiencia en el intercambio de calor y evitará el ensuciamiento, eso sí, a costa de un consumo energético adicional. Se espera igualmente que la velocidad de rascado tenga una influencia significativa en los efectos mencionados.

Con el objetivo mencionado se han realizado los ensayos de visualización del patrón de flujo mediante el uso de la técnica de Velocimetría por Imágenes de Partículas (PIV). En el siguiente apartado se define el plano de visualización del flujo y se detallan las características del flujo en dicho plano. En los Apartados 7.2 y 7.3 se estudian el régimen de rascado estático y régimen de rascador dinámico respectivamente, donde además se analiza la influencia de la velocidad de rascado en el campo fluido.

En aras del cumplimiento del objetivo marcado, a lo largo del capítulo se persigue identificar el efecto del comportamiento no newtoniano del fluido en el campo de velocidades resultante. En principio, del análisis dimensional realizado en el Capítulo 2, se deduce que el campo de velocidades depende de dos números adimensionales, el número de Reynolds y el índice de comportamiento de flujo $(n)$. Por lo tanto, para caracterizar completamente el patrón de flujo, se deben conocer los efectos que producen la variación de dichos parámetros de forma independiente. Sin embargo, esto no es del todo posible debido debido a las limitaciones propias del método experimental, ya que resulta prácticamente imposible planificar ensayos en los que se mantenga el número de Reynolds fijo variando $n$. La razón es que, tal y como se estudia en el Apartado 5.1.2, las propiedades reológicas del fluido varían de forma continua, incluso a lo largo de un sólo ensayo y se debe esperar a que dicho ensayo haya finalizado para conocer los valores de $m$ y $n$ que permitan calcular el número de Reynolds del ensayo.

Para tratar de paliar este inconveniente se toman dos medidas que reducen la incertidumbre al respecto. Por un lado, se hace uso del número de Reynolds $R e_{g}$, definido de tal modo que la caída de presión en el intercambiador únicamente depende del número de Reynolds (véase Apartado 5.3.3). Es de esperar por tanto, que dicha definición del número de Reynolds incluya parte de la influencia de $n$ en el patrón de flujo. Por otro lado, los perfiles de velocidades medidos en el intercambiador se comparan con los que se obtendrían en una geometría de ejes concéntricos de las mismas dimensiones, en las que además el tubo interior se mueve igual que el eje del rascador. En dicha geometría, si el flujo es laminar, el perfil de velocidades únicamente varía si lo hace $n$. De este modo se puede observar el efecto de variar $n$ en una geometría similar a la estudiada. Además, realizar esta comparativa también es útil para observar el efecto de la presencia de los tacos. Los resultados obtenidos se comparan con los perfiles de velocidad que se dan en la geometría de tubos concéntricos, donde 
el eje interior puede tener un movimiento longitudinal. Para obtener el campo de velocidades en esta geometría se recurre al método numérico mediante volúmenes finitos descrito en el Apartado 3.2.

\subsection{Plano de visualización del flujo}

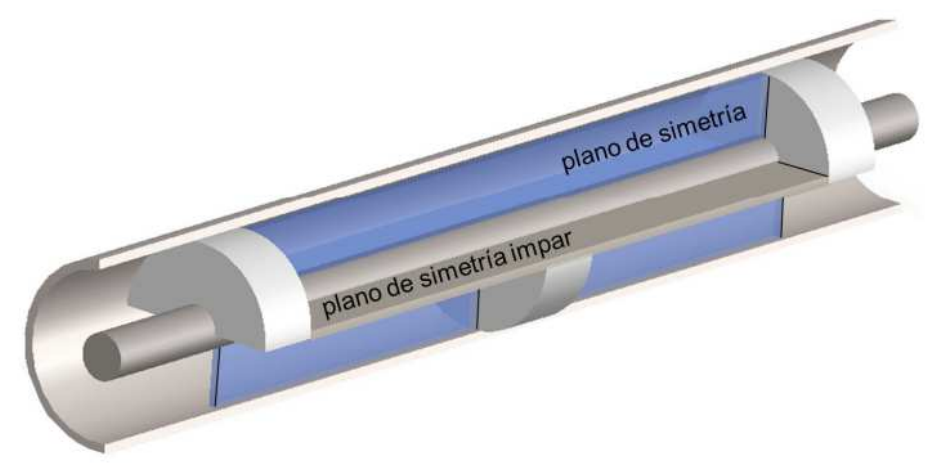

Figura 7.1: Plano de simetría y plano de simetría impar.

Aunque existen variantes tridimensionales de PIV, la técnica utilizada en esta investigación, detallada en el Capítulo 4, únicamente permite la visualización del patrón de flujo en 2 dimensiones. Este procedimiento tiene una limitación importante: las velocidades transversales al plano de visualización deberán ser mínimas, de modo que las partículas trazadoras que aparecen en imágenes sucesivas sean prácticamente las mismas. En la práctica esta condición obliga a visualizar en un plano de simetría del flujo.

El diseño del rascador, con un eje concéntrico y la geometría simétrica de los tacos, colocados al tresbolillo, aseguran la existencia de dicho plano de simetría y de un plano de simetría impar. En la Fig. 7.1 se muestras sendos planos. La importancia del plano de simetría impar radica en que a ambos lados del mismo se producirán idénticas estructuras de flujo, simétricas pero desfasadas una distancia $P / 2$.

Se puede afirmar por tanto, que en el plano de simetría se cumplen las siguientes relaciones:

$$
\begin{aligned}
& u_{z}(y, z+P / 2)=u_{z}(-y, z) \\
& u_{r}(y, z+P / 2)=u_{r}(-y, z)
\end{aligned}
$$




$$
u_{\theta}(y, z)=0
$$

donde para las velocidades se ha considerado un sistema de referencia cilíndrico y para las posiciones, uno cartesiano. Ambos sistemas se eligen de modo que el eje $z$ coincide en ambos y el origen de coordenadas es el mismo, el cual se representa en la Fig. 7.2(a).

En consecuencia, únicamente será necesario realizar la visualización en una de las mitades en las que el eje divide el plano de simetría, ya que en la otra mitad el flujo será idéntico pero desfasado. Una descripción más detallada del método experimental se encuentra en el Capítulo 6.

\subsection{Régimen de rascador estático}

En el presente apartado se analizan los resultados obtenidos en los ensayos en régimen del rascador estático. Tal y como se ha mencionado con anterioridad, el objetivo de los ensayos realizados consiste en observar la estructura general del flujo y las variaciones que ésta sufre al variar los diferentes parámetros del mismo, en este caso, $R e_{g}$ y $n$. Para ello, en los subsiguientes apartados, en primer lugar se analiza la estructura general del flujo en uno de los ensayos y a continuación se estudia el efecto que la variación de $R e_{g}$ y $n$ tienen en tal estructura.

\subsubsection{Estructura general del flujo}

Tal y como se representa en la Fig. 7.2(a), la disposición en tresbolillo de los tacos rascadores, que bloquean el paso del flujo por un $50 \%$ del área de paso, obliga al flujo a realizar un recorrido sinuoso para pasar a través de la sección libre opuesta al taco. Tal y como veremos a continuación, este trazado determina completamente el comportamiento del flujo en este régimen de funcionamiento.

En la Fig. 7.2(b) se muestra el campo de velocidades, adimensionalizado con $u_{b}$, obtenido en la el plano de simetría del rascador. Los resultados se corresponden con el ensayo a $R e_{g}=15$, cuyas características se detallan en Tabla 7.1. Tal y como se puede observar en la figura, en la zona donde la presencia del taco reduce la sección de paso aparecen altas velocidades. En esta zona la velocidad máxima del flujo aumenta de forma considerable, alcanzando valores de hasta 3 veces la velocidad media del flujo $u_{b}$. Además, aguas arriba y 


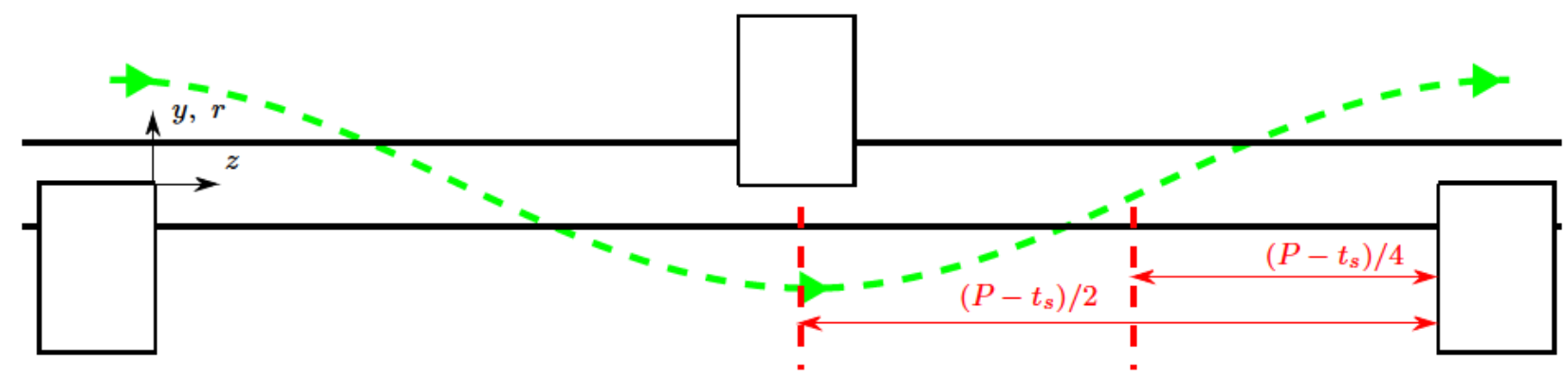

(a) En verde: trayectoria general del flujo. En rojo: posiciones de perfiles a representar. $S_{A}$ : situada en $z=$ $\left(P-t_{s}\right) / 2 . S_{B}$ : situada en $z=3\left(P-t_{s}\right) / 4$

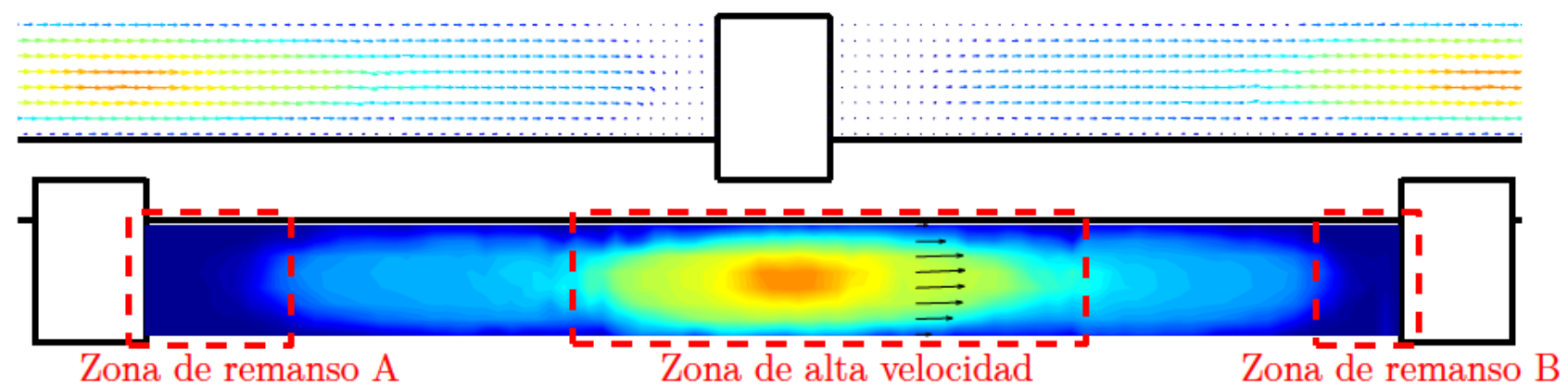

(b) Estructura del flujo. Ensayo a $R e_{g}=15, n=0,62$.

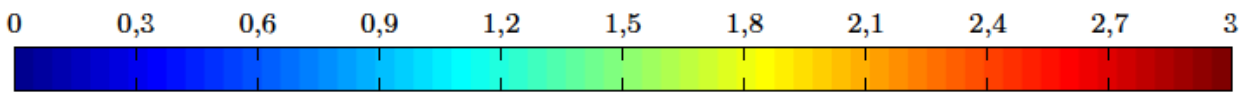

Figura 7.2: Estructura del flujo en régimen de rascador estático. 


\begin{tabular}{cccccccc}
\hline Núm. & $n$ & $m\left(\mathrm{~Pa}_{\mathrm{s}}{ }^{n}\right)$ & $Q(\mathrm{l} / \mathrm{h})$ & $R e_{b}$ & $R e_{g}$ & $\mu_{b}\left(\mathrm{~Pa} . \mathrm{s}^{n}\right)$ & $\mu_{g}\left(\mathrm{~Pa}_{\mathrm{s}}{ }^{n}\right)$ \\
\hline 1 & 0,52 & 3,50 & 280 & 1,4 & 5 & 0,575 & 2,014 \\
2 & 0,621 & 0,987 & 318 & 5,2 & 15 & 1,848 & 5,416 \\
3 & 0,682 & 0,576 & 347 & 9,0 & 23 & 2,954 & 7,551 \\
4 & 0,944 & 0,0695 & 355 & 49 & 60 & 15,835 & 19,109 \\
\hline
\end{tabular}

Tabla 7.1: Ensayos en régimen de rascador estático. Las imágenes capturadas en los ensayos tienen una escala de $0,191 \mathrm{~mm} /$ pix.

aguas abajo de cada taco aparecen zonas de remanso, provocadas por la presencia de éste, donde la velocidad del flujo en el plano de visualización es muy baja.

Por lo tanto la presencia del rascador estático provoca un mezclado adicional del flujo, debido al recorrido sinuoso que realiza el fluido. Por otro lado, las altas velocidades que aparecen en las reducciones de sección, acarrearán altos coeficientes de transferencia de calor. Sin embargo, la presencia de los tacos también conlleva la aparición de zonas de remanso, donde el coeficiente de transmisión de calor local será bajo. Además las bajas velocidades que se dan en dichas zonas pueden acarrear problemas de ensuciamiento, que se podrían evitar con ciclos de rascado esporádicos.

\subsubsection{Influencia del número de Reynolds y de $n$ en el flujo}

Con el objetivo de determinar la influencia conjunta del número de Reynolds $R e_{g}$ y de $n$ en el flujo, se han llevado a cabo cuatro experimentos. En ellos se abarca un rango de números de Reynolds $R e_{g} \in[5 ; 60]$ para valores del índice de comportamiento del flujo en un rango de $n \in[0,52 ; 0,95]$, presentando el flujo una viscosidad efectiva $\mu_{g} \in[2 ; 19,1]$ Pa.s ${ }^{n}$. En la Tabla 7.1 se detallan los parámetros correspondientes a los ensayos realizados.

En la Fig. 7.3 se muestran los resultados de los ensayos realizados, en los cuales se pueden observar los siguientes efectos, ante el aumento de $n$ y el número de Reynolds $R e_{g}$ :

1. La zona de alta velocidad aumenta de tamaño en dirección aguas abajo.

2. La región de remanso A (ver imagen 7.2(b)), situada aguas abajo del taco, aumenta de tamaño.

3. Por el contrario, la región de remanso B, situada aguas arriba del taco, disminuye su tamaño. 


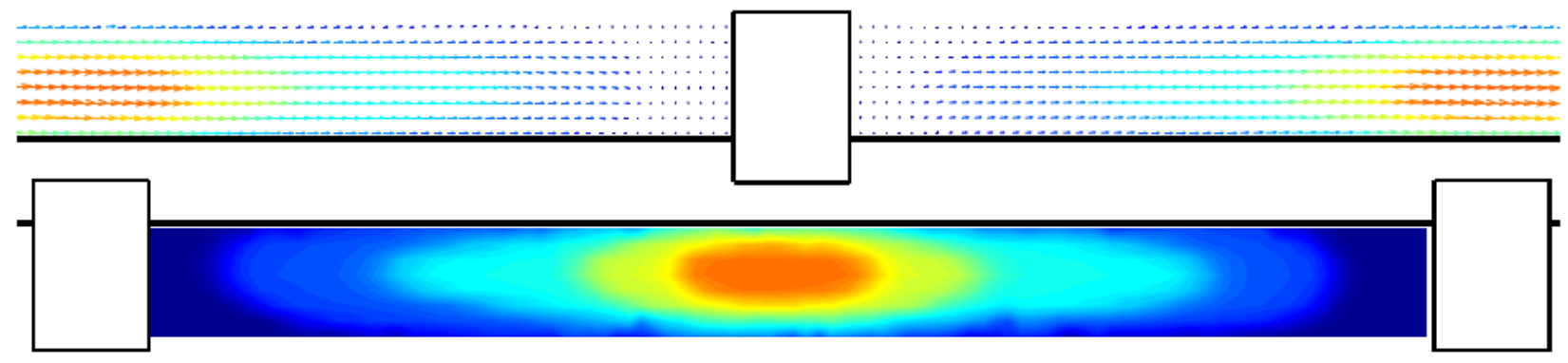

(a) $R e_{g}=5, n=0,52$.

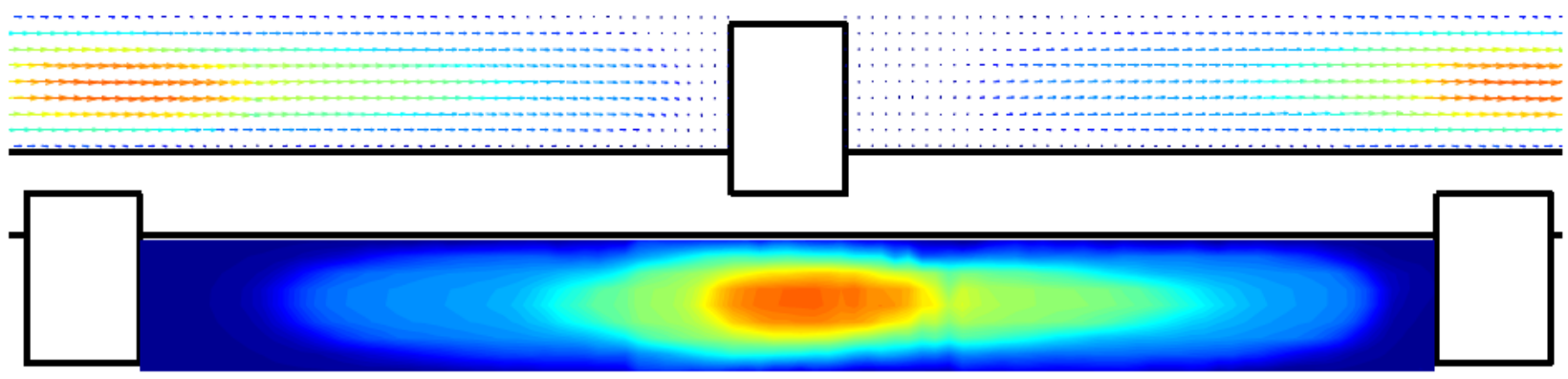

(b) $R e_{g}=23, n=0,68$

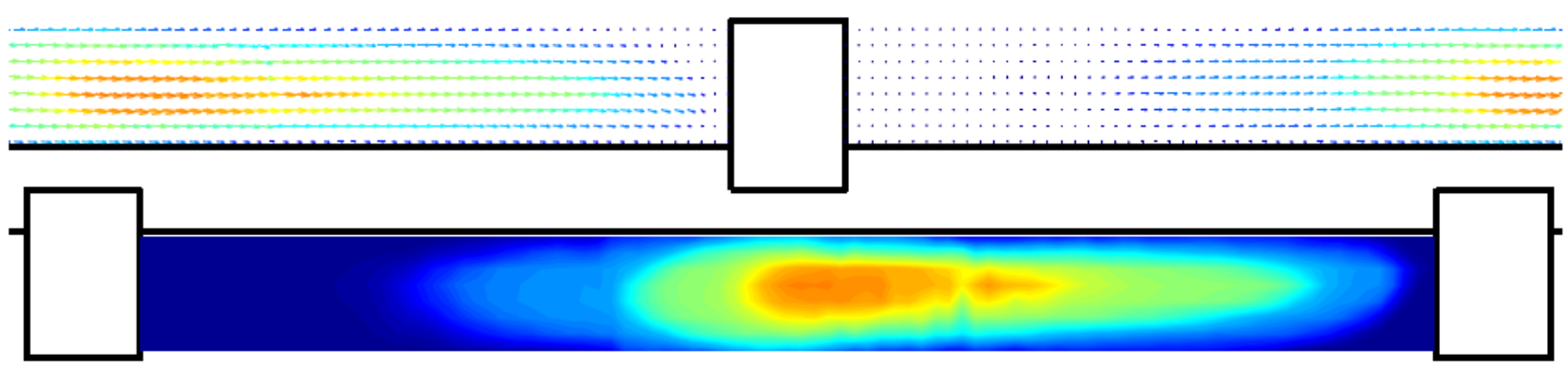

(c) $R e_{g}=60, n=0,94$

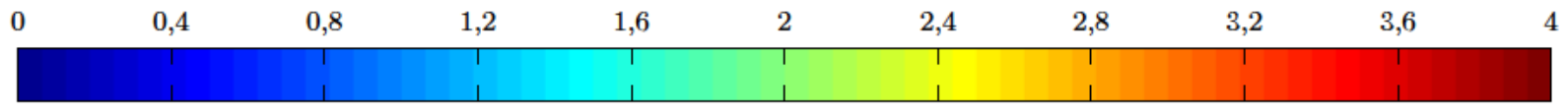

Figura 7.3: Campo de velocidades en régimen estático. 
4. En ninguno de los ensayos se han detectado recirculaciones o indicios de turbulencia. El flujo en el rango de números de Reynolds ensayado es totalmente laminar.

Ahora bien, a partir de los resultados obtenidos hasta el momento, es difícil establecer si el origen de estos efectos es la variación del índice de comportamiento de flujo o la del número de Reynolds. Con tal objetivo se realizan las representaciones de los perfiles de velocidad en las dos secciones del intercambiador situadas en las posiciones indicadas en la Fig. 7.2(a). Dichas secciones están situadas en el centro de la sección libre junto a uno de los tacos $\left(z=\left(P-t_{S}\right) / 2\right)$ y en la posición $z=3\left(P-t_{S}\right) / 4$ respectivamente y en adelante se denotarán como $S_{A}$ y $S_{B}$ respectivamente.

En la Fig. 7.4 se representan los perfiles de velocidades en sendas secciones para los ensayos estáticos. En ambas gráficas los perfiles obtenidos se comparan con el perfil de velocidades teórico que se obtendría en una geometría de tubos concéntricos con un fluido de las mismas propiedades reológicas. La obtención de dichos perfiles se realiza mediante el método analíticonumérico descrito en el Apartado 3.1. En una geometría de sección anular, siempre que se den las condiciones de flujo laminar, el único parámetro que puede variar el perfil de velocidades adimensional, es $n$.

En las figuras 7.4(a) y 7.4(b) se observa que en la geometría de ejes concéntricos el perfil de velocidades depende $n$. Para valores de $n$ mayores, el perfil se hace más afilado, mientras que al disminuir $n$ éste se achata, reduciéndose la velocidad máxima.

En la Fig. 7.4(b) los resultados experimentales muestran un perfil de velocidades en la sección $S_{B}$ del intercambiador que varía con $R e_{g}$ y $n$. Se comprueba que al aumentar ambos números adimensionales, la velocidad máxima aumenta considerablemente y los perfiles son más afilados. Estas diferencias se deben en parte al valor distinto de $n$ en cada ensayo, tal y como ocurre en la geometría de tubos concéntricos, y en parte al distinto número de Reynolds de cada ensayo. Aunque en base a los resultados en el conducto de sección anular, y estimando que en el rascador la influencia de $n$ es del mismo orden de magnitud, se concluye que en los ensayos realizados, el número de Reynolds tiene más peso a la hora de modificar los perfiles de velocidad en la sección $S_{B}$.

Por otro lado, en la sección $S_{A}$ (Fig. 7.4(a) ), situada en la zona de alta velocidad, se observan diferencias entre los perfiles de velocidad mucho menores y no siguen un patrón fijo. En este caso, con las herramientas de que se disponen, no es posible discernir el efecto de variar el número de Reynolds del de variar $n$.

Por último, se estudia la variación del tamaño de las zonas de baja velocidad A y B al variar Re y $n$. Este efecto se puede apreciar en la Fig. 7.5. En ella se representa el contorno 


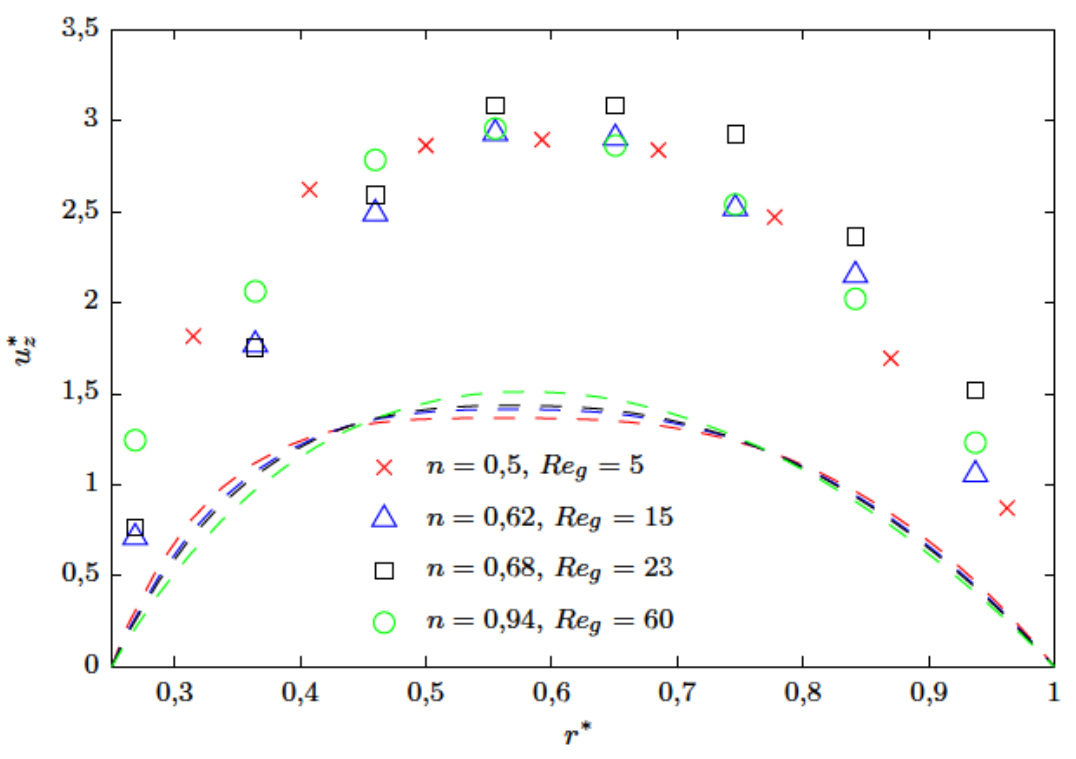

(a) $z=\left(P-t_{s}\right) / 2$.

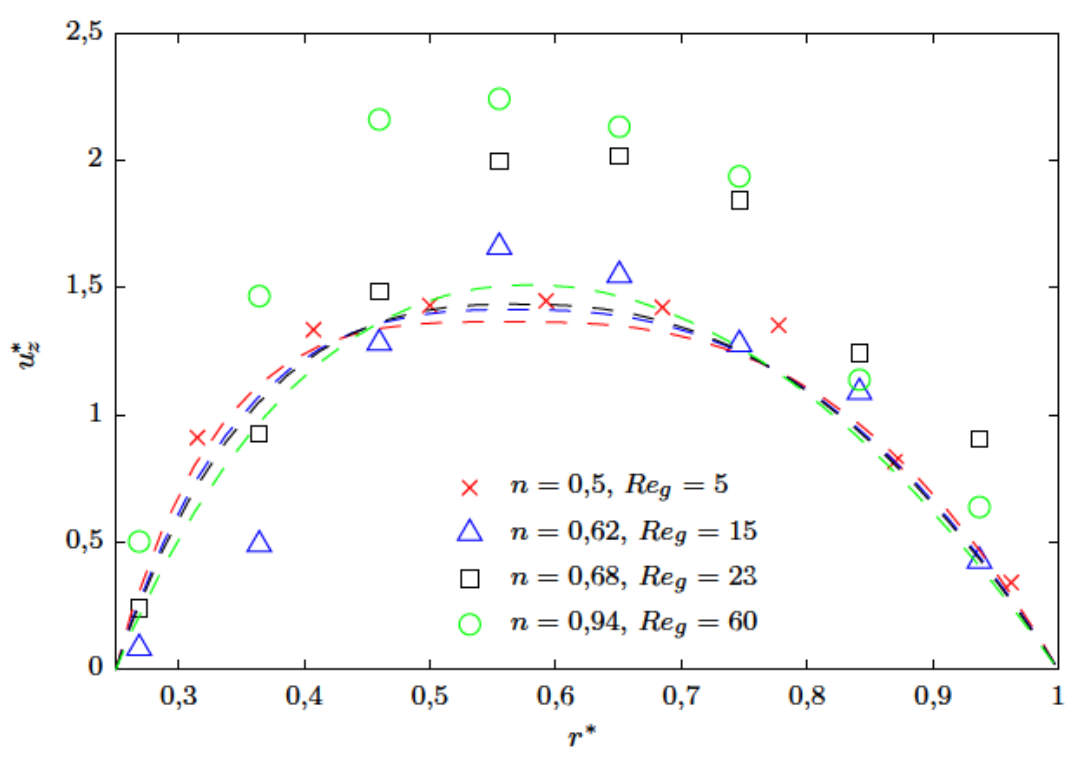

(b) $z=3\left(P-t_{s}\right) / 4$.

Figura 7.4: Perfiles de velocidades en los ensayos estáticos (representados mediante símbolos). En línea discontinua se representan los perfiles de velocidades en geometría de ejes concéntricos correspondientes a un fluido seudoplástico cuya propiedad $n$ sea igual a la del fluido utilizado en el ensayo experimental representado con el mismo color. 


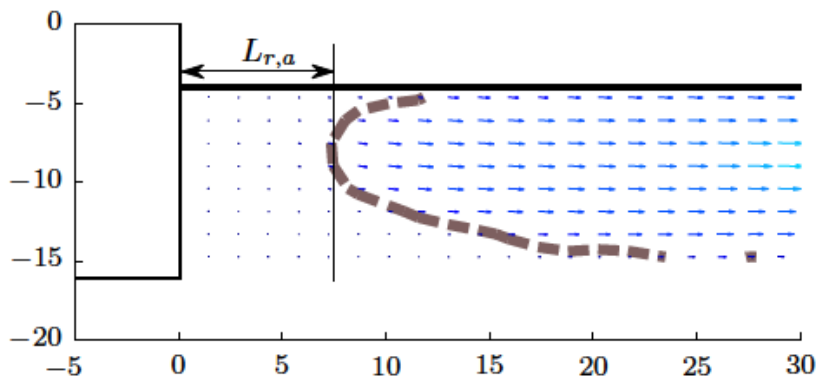

(a) $R e_{g}=5, n=0,52$. Zona A.

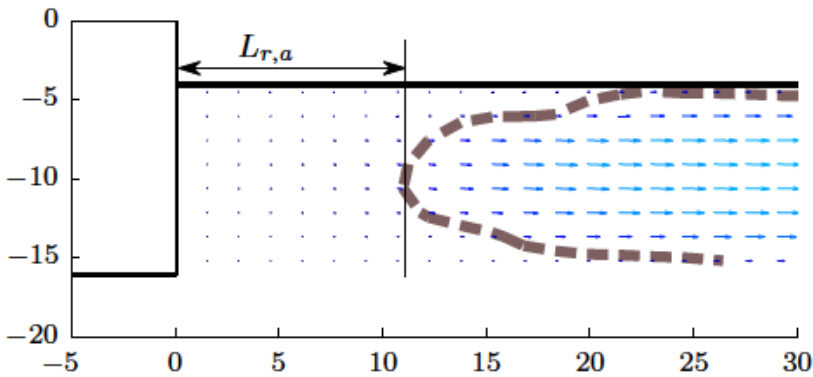

(c) $R e_{g}=15, n=0,62$. Zona A.

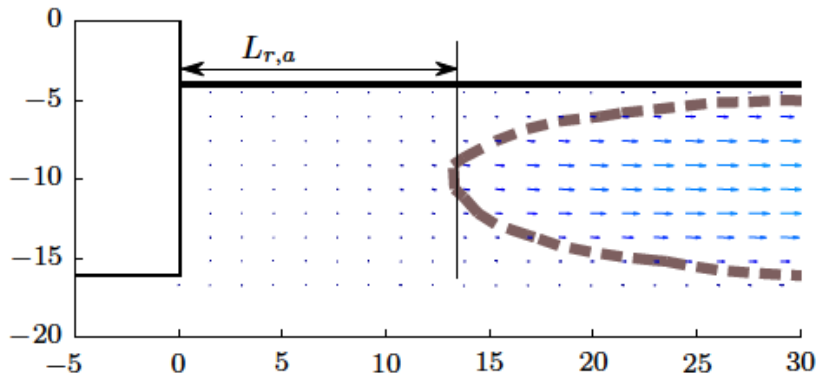

(e) $R e_{g}=23, n=0,68$. Zona A.

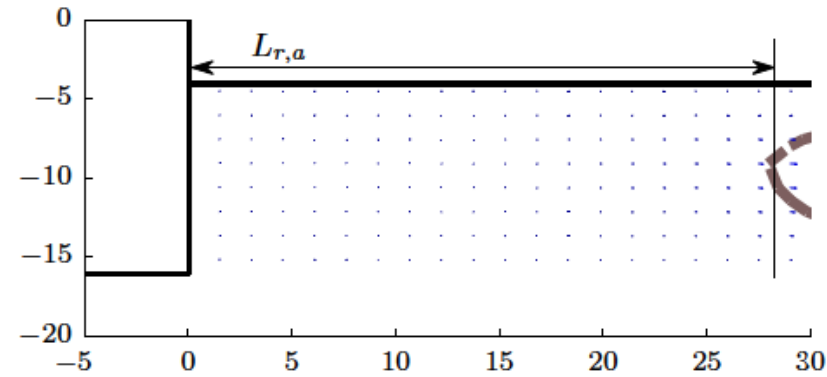

(g) $R e_{g}=60, n=0,94$. Zona A.

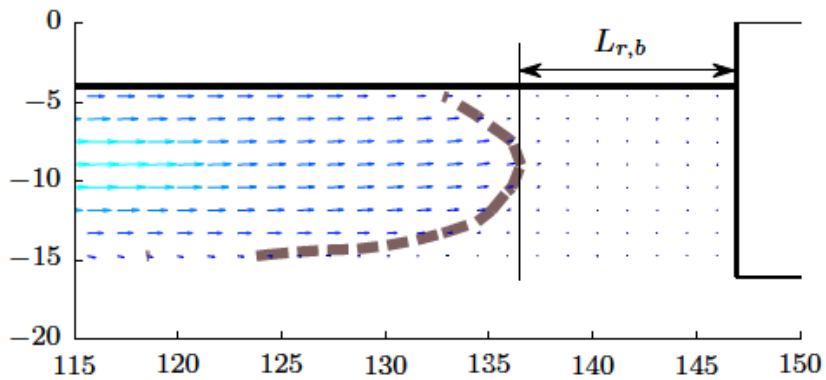

(b) $R e_{g}=5, n=0,52$. Zona B.

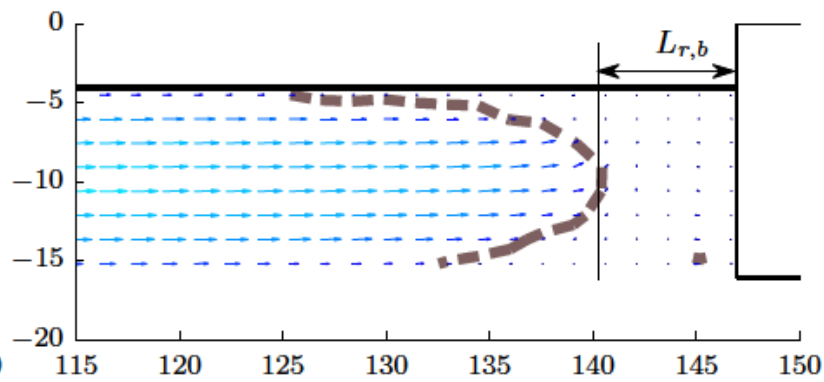

(d) $R e_{g}=15, n=0,62$. Zona B.

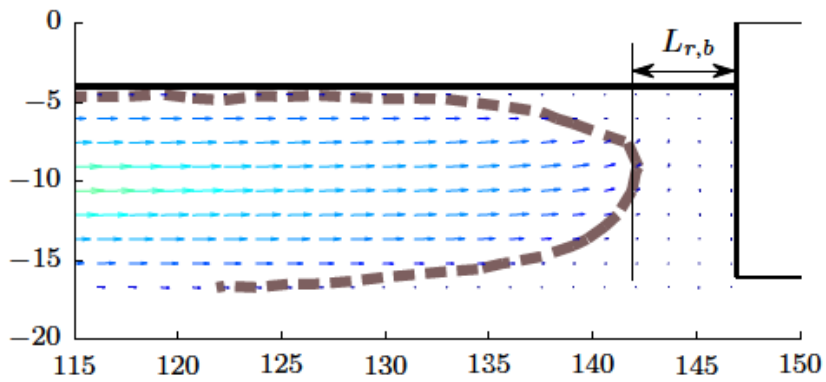

(f) $R e_{g}=23, n=0,68$. Zona B.

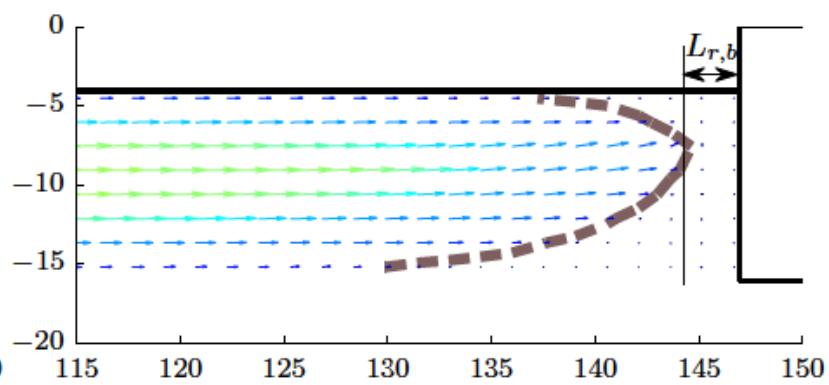

(h) $R e_{g}=60, n=0,94$. Zona B.

Figura 7.5: Tamaño de la zonas de remanso aguas arriba (A) y aguas abajo (B) del taco. $L_{r, a}=10,5 ; 6,6 ; 4,8 ; 2,4 \mathrm{~mm}, L_{r, b}=7,4 ; 11,1 ; 13,3 ; 28 \mathrm{~mm}$. respectivamente. 


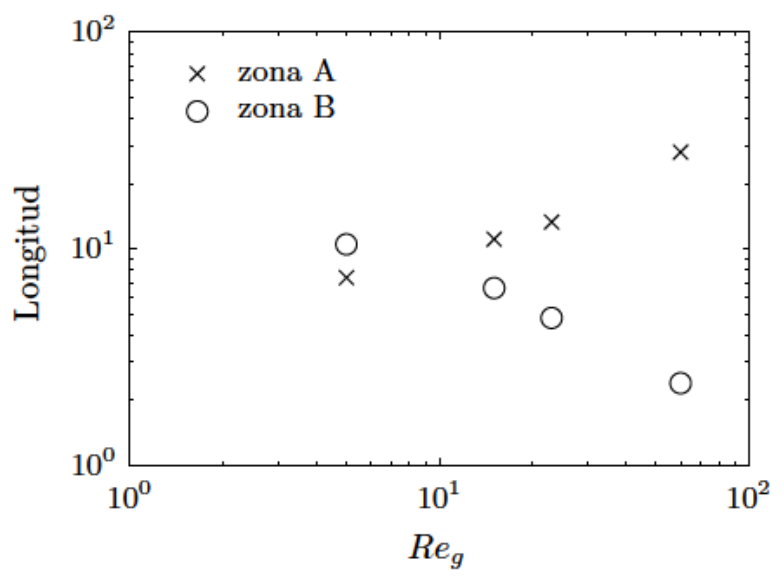

Figura 7.6: Evolución tamaño zonas de remanso A y B, aguas abajo y aguas arriba del taco respectivamente.

para un módulo de la velocidad adimensional de $u^{*}=0,25$. De este modo se considera como zona de remanso aquella en la que la velocidad es menor de dicho valor. La elección del valor umbral de $u^{*}$ no es excesivamente relevante, ya que el gradiente de velocidad en dicho contorno es alto. En la figura se observa la variación del tamaño de dichas zonas en los tres experimentos realizados. El tamaño de la zona de remanso aguas abajo del taco aumenta al hacerlo el número de Reynolds, mientras que ocurre el efecto contrario con la situada aguas arriba del mismo. En la Fig. 7.6 se representa la variación de las longitudes de sendas zonas de remanso. Los resultados indican por un lado, que para el tamaño de la zona de remanso A existe una asíntota horizontal con un valor mínimo de longitud para Reynolds muy pequeños y muestra un crecimiento no lineal al aumentar el Reynolds. Además, es imperativo que debido a las dimensiones del rascador, exista una asíntota horizontal superior a la cual tiendan las longitudes de la zona de remanso A a altos números de Reynolds. Por otro lado, el tamaño zona de remanso B se reduce y alcanzará un valor mínimo de longitud de forma asintótica al aumentar el número de Reynolds.

\subsubsection{Comparación con comportamiento newtoniano en la misma geometría}

En flujos de fluidos newtonianos en la misma geometría, en rangos de Reynolds $R e \in$ $[36,80]$, se encuentran estructuras de flujo muy similares a las observadas en la presente investigación utilizando los fluidos no newtonianos. Sin embargo, a partir de $R e>80$ se 

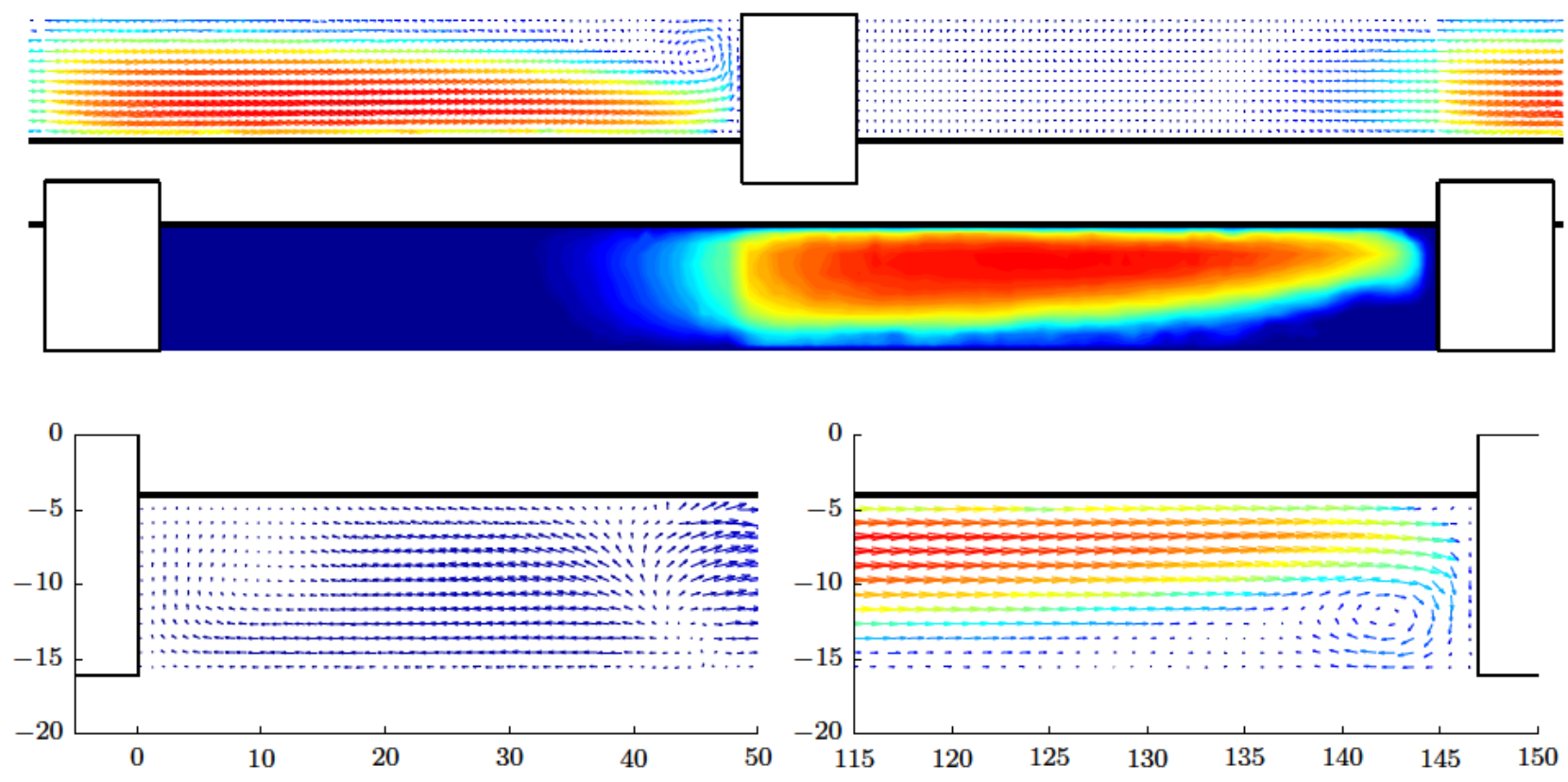

(a) Zona A.

(b) Zona B.

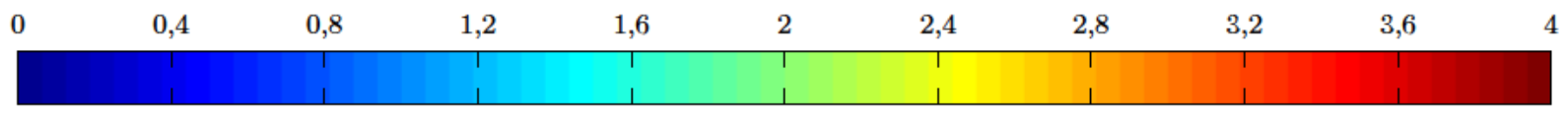

Figura 7.7: Campo de velocidades del flujo newtoniano a $R e=164$. Resultados obtenidos por Solano (2009a).

observa la aparición de recirculaciones en las zonas de remanso cercanas al taco, tanto aguas arriba como aguas abajo del mismo. En la Fig. 7.7 se muestran los resultados de uno de sus ensayos donde se pueden observar las recirculaciones mencionadas, las cuales, además varían en forma y tamaño al hacerlo el número Reynolds. La aparición de dichas recirculaciones indica el comienzo de una región de transición hacia una turbulencia inducida por la presencia del rascador. Debido a las limitaciones que presenta la instalación, no ha sido posible visualizar flujos con fluidos no newtonianos en régimen de transición o turbulento, ya que el Reynolds máximo conseguido es de $R e_{g}=60$ y además éste se ha dado en un ensayo con un fluido de comportamiento prácticamente newtoniano $(n=0,94)$. De hecho el ensayo de mayor Reynolds con un fluido de marcado comportamiento seudoplástico, $n \approx 0,7$, presenta un $R e_{g}=23$, significativamente alejado de la presumible zona de transición $\left(R e_{g}>70-80\right)$. Aunque en los ensayos de PIV con fluidos no newtonianos no ha sido posible visualizar el flujo en dicha región de funcionamiento, ésta sí se detecta en los ensayos de caída de presión 
y transmisión de calor analizados en capítulos posteriores.

Por lo tanto, los patrones de flujo observados en unos y otros fluidos son hasta cierto punto similares. La diferencia más significativa viene dada por el rango de Reynolds en el que trabaja uno y otro tipo de fluidos. Los fluidos no newtonianos y en concreto, el fluido de trabajo utilizado en la presente investigación, suelen presentar viscosidades aparentes significativamente mayores que los fluidos newtonianos y por lo tanto tienen más tendencia a trabajar en regímenes laminares. En este fluido en concreto, por la relación existente entre $m$ y $n$, la viscosidad aparente aumenta al disminuir el valor de $n$.

\subsection{Régimen de rascador dinámico}

En el presente apartado se busca obtener el patrón de flujo en las situaciones en las que el rascador se encuentra en movimiento. Como se ha mencionado anteriormente, el movimiento del rascador se produce en dirección longitudinal, en ciclos alternos en dirección equicorriente y contracorriente. En concreto, el objetivo es el de determinar la influencia que tiene el movimiento del rascador en el flujo, así como el efecto de la velocidad a la que se produzca el rascado. Además, no se debe perder de vista el objetivo general de identificar la influencia de $n$ en el flujo.

Para conseguir el objetivo marcado, se realizan en primer lugar ensayos de visualización del flujo en los cuales el caudal circulante es nulo. De esta forma es posible observar de forma aislada el efecto del rascador.

A continuación se realizan ensayos en los que sí se circula caudal. Por un lado se realizan ensayos variando la velocidad de rascado, lo que permite analizar las variaciones del patrón de flujo en función de ésta. Además, a cada velocidad de rascado se realizan tres ensayos utilizando fluidos de distintas propiedades reológicas en los que, en consecuencia, se darán diferentes números de Reynolds.

\subsubsection{Ensayos a caudal cero}

Estos experimentos tienen la particularidad de que, al ser la velocidad media del flujo nula $u_{b}=0$, las velocidades del campo fluido se adimensionalizan utilizando la velocidad del rascador, de modo que la velocidad adimensional representada será $u^{\prime *}=u / v_{s}$. Además, resulta obvio que el sentido de rascado es indiferente.

Se realizan ensayos a tres velocidades de rascado $v_{s}=50 ; 100 ; 200 \mathrm{~mm} / \mathrm{s}$, cubriendo el 


\begin{tabular}{ccccc}
\hline$v_{s}(\mathrm{~mm} / \mathrm{s})$ & $f_{a d}(\mathrm{~Hz})$ & $n$ & $m\left(\mathrm{~Pa}_{\mathrm{s}} \mathrm{s}^{\mathrm{n}}\right)$ & $T\left({ }^{\circ} \mathrm{C}\right)$ \\
\hline 50 & 203 & 0,47 & 4,82 & 19,4 \\
100 & 405 & 0,48 & 4,79 & 19,7 \\
200 & 405 & 0,48 & 4,68 & 19,7 \\
\hline
\end{tabular}

Tabla 7.2: Ensayos de movimiento del rascador a caudal cero.

rango de velocidades que posteriormente se ensaya con caudales no nulos. Los parámetros de los ensayos realizados se detallan en la Tabla 7.2.

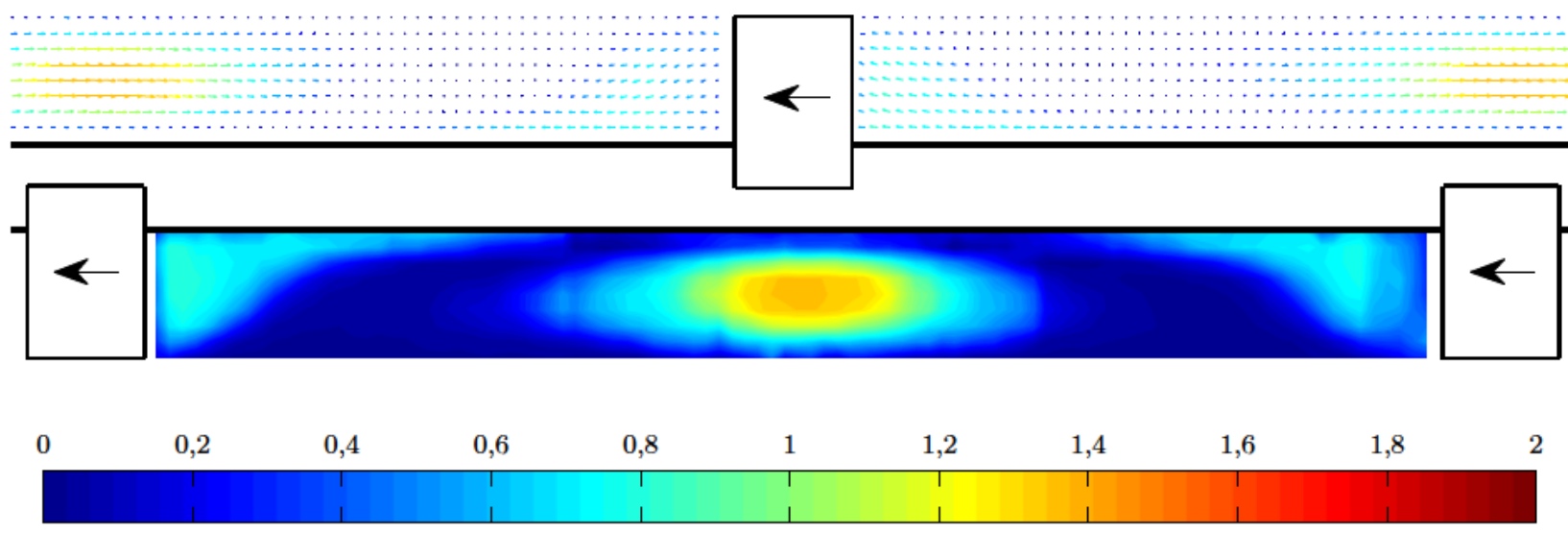

Figura 7.8: Campo de velocidades del flujo en ensayo a caudal cero con $v_{s}=200 \mathrm{~mm} / \mathrm{s}$. Se representan las velocidades adimensionalizadas con la velocidad de rascado, $u^{\prime *}=u / v_{s}$.

En los resultados de los tres ensayos no se aprecian diferencias significativas al variar la velocidad del rascador. De modo que en la Fig. 7.8 se muestra el campo de velocidades adimensional obtenido en el ensayo a $v_{s}=200 \mathrm{~mm} / \mathrm{s}$. Tal y como se observa en la figura, el movimiento del rascador hacia la izquierda provoca una zona de velocidades en ese sentido del flujo, donde el módulo de la velocidad alcanza valores en torno a $u^{\prime *}=0,8$. Como se puede apreciar en la imagen, dicha zona de velocidades en sentido del desplazamiento del rascador se extiende desde una cara del taco hasta la cara opuesta del siguiente. Por otro lado, en el tramo del tubo donde la sección de paso se ve reducida por la presencia del taco, se produce una zona de altas velocidades en sentido contrario al movimiento del rascador, donde se alcanzan velocidades máximas de $u^{* *}=1,36$. Dicha zona tiene dimensiones similares a las observadas en el caso de rascador estático estudiado en el Apartado 7.2. 


\begin{tabular}{lccc}
\hline \multicolumn{4}{c}{ Ensayos dinámicos } \\
\hline$Q(\mathrm{l} / \mathrm{h})$ & 210 & 226 & 236 \\
$n$ & 0,64 & 0,78 & 0,88 \\
$m\left(\mathrm{~Pa} . \mathrm{s}^{\mathrm{n}}\right)$ & 0,68 & 0,21 & 0,08 \\
$R e_{g}$ & 13 & 25 & 45 \\
\hline
\end{tabular}

Tabla 7.3: Características de los ensayos dinámicos. Los valores de las propiedades reológicas son valores intermedios de una misma serie de ensayos, ya que éstas varían en cada ensayo. Las características detalladas de cada ensayo se muestran en la Tabla C.1. En consecuencia también lo es el valor de $R e_{g}$.

\subsubsection{Ensayos dinámicos}

En régimen dinámico, el rascador se mueve alternativamente en la dirección principal del flujo. Es de esperar que el flujo en el intercambiador sea parecido al visualizado en régimen estático a velocidades de rascado bajas y que la influencia del movimiento de rascado, estudiado de forma aislada en el apartado anterior, sea más marcada a medida que aumenta $v_{s}$.

Se han planificado ensayos en un rango de velocidades de rascado $\left|v_{s} / u_{b}\right|=0,5 ; 1 ; 2 ; 3$. Para cada velocidad de rascado, se han realizado tres ensayos, abarcando un rango de números de Reynolds entre $R e_{g} \in[13 ; 45]$. El valor del Índice de comportamiento del flujo varía entre $n=0,64$ para los ensayos a menor número de Reynolds y $n=0,88$ para los ensayos a mayor número de Reynolds. Los parámetros concretos de cada experimento vienen detallados en la Tabla 7.3.

El análisis dimensional realizado en el Apartado 2.3 para el problema en régimen dinámico, se obtiene un nuevo número adimensional del cual depende el problema. Se trata del factor de bloqueo:

$$
\beta=\frac{u_{b}-v_{s}}{u_{b}}=1-\frac{v_{s}}{u_{b}}
$$

El factor de bloqueo expresa si el movimiento del rascador supone una ayuda en la impulsión del fluido $(\beta<0)$ o si por el contrario obstaculiza el flujo $(\beta>0)$. De este modo, una situación con factor de bloqueo nulo $(\beta=0)$, implicaría una mínima influencia del rascador en el flujo. Tal y como se ha mencionado anteriormente, la utilidad de este número parámetro para la adimensionalización del movimiento del rascador, en geometrías de tubo con elemento insertado de movimiento lineal alternativo, ha sido demostrada en investigaciones anteriores (Crespí-Llorens et al. (2013); Solano et al. (2011b)). 


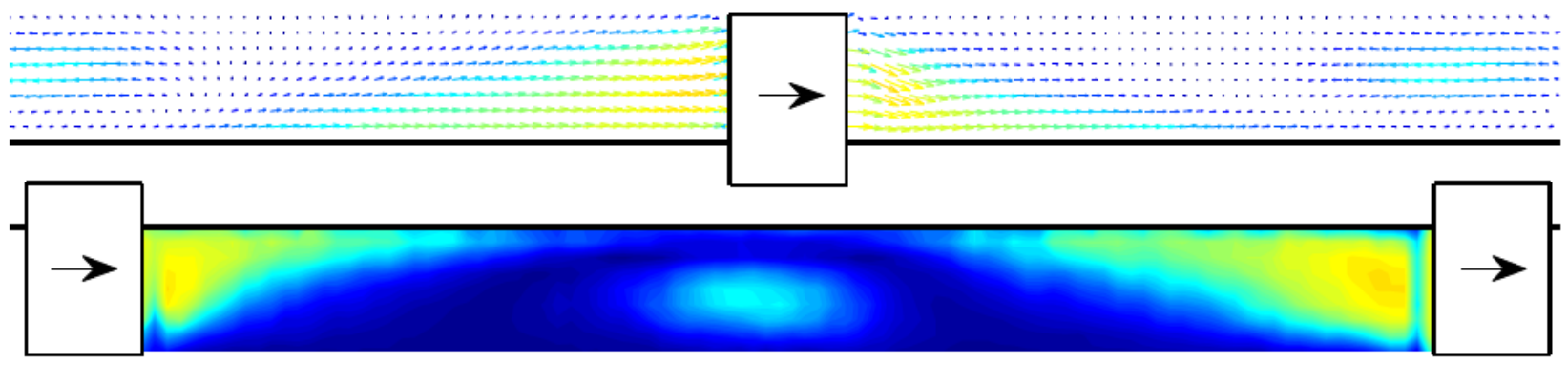

(a) $\beta=-2$.

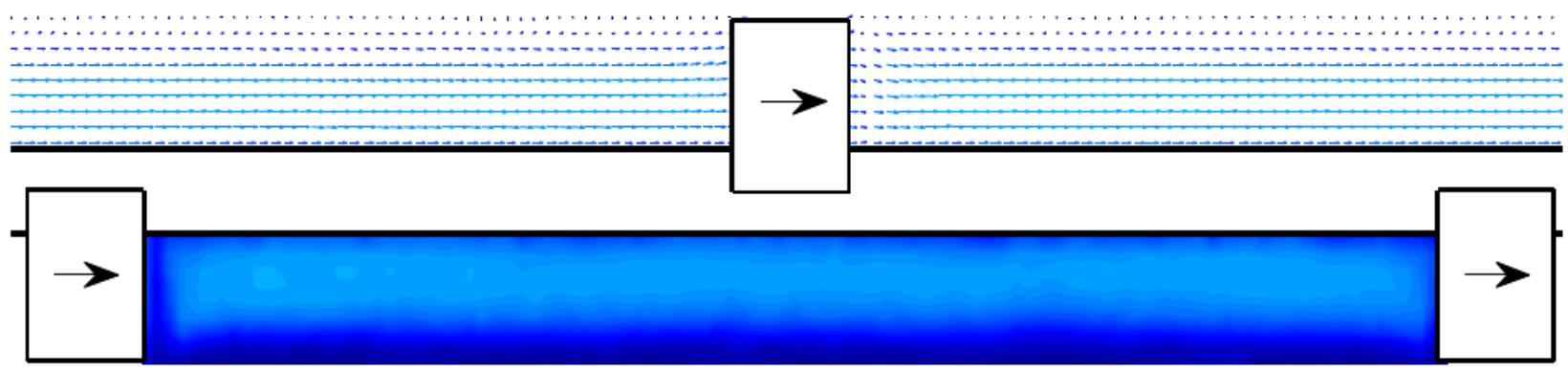

(b) $\beta=0$.

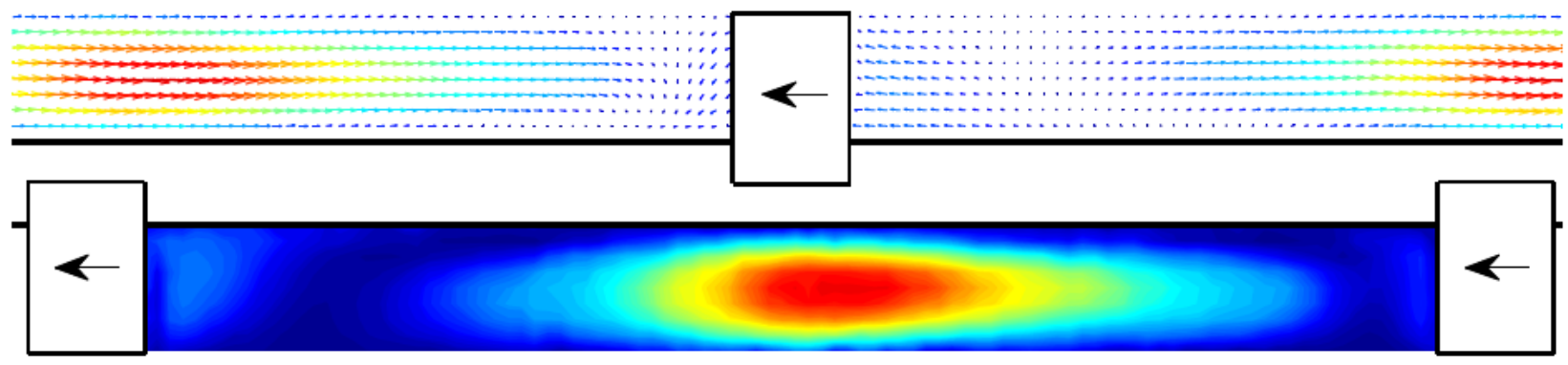

(c) $\beta=2$.

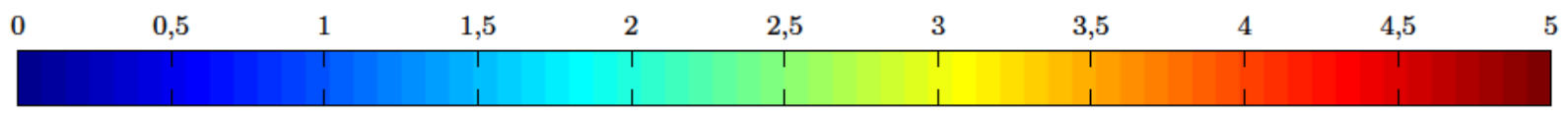

Figura 7.9: Efecto del factor de bloqueo en el flujo. Se representan ensayos con números de Reynolds e índice de comportamiento de flujo similares: $R e_{g}=13 ; n=0,64$. 
Tal y como se ha descrito en el Capítulo 6, el rascador trabajará a una velocidad constante. Esto implica que el factor de bloqueo en las direcciones equicorriente y contracorriente tomará valores distintos, y además, en sentido equicorriente existen diferentes posibilidades. En todo caso, los valores del factor de bloqueo en ambos sentidos están relacionados mediante la Ec. 7.6 .

$$
\begin{aligned}
& v_{s, e c}=-v_{s, c c} \\
& \beta_{e c}+\beta_{c c}=2
\end{aligned}
$$

El factor de bloqueo en los ensayos varía desde $\beta \in[-2,4]$. Cabe destacar que, siendo la velocidad media del flujo en la sección un número positivo $\left(u_{b}>0\right)$ si el sentido de rascado es contracorriente $\left(v_{s}<0\right)$, el factor de bloqueo siempre será positivo, $\beta>0$. Si el rascador se encuentra estático $\left(v_{s}=0\right)$, el factor de bloqueo toma un valor de $\beta=1$. Y por último, en sentido de rascado equicorriente $\left(v_{s}>0\right)$ el factor de bloqueo se puede encontrar en el rango $\beta \in(1,-2]$, para los ensayos realizados. Es decir, que en sentido equicorriente de rascado se presentan tres situaciones de factor de bloqueo: positivo, nulo o negativo.

En la Fig. 7.9 se muestra el campo adimensional de velocidades para $R e_{g}=13$ y $\beta=$ $-2 ; 0 ; 2$. Tal y como se puede observar, el campo de velocidades depende fuertemente del factor de bloqueo y es por ello que los resultados de los ensayos se agruparán en función de $\beta$.

\subsubsection{Factor de bloqueo nulo}

El caso particular en el que $\beta=0$, se da en la situación en la que la velocidad del rascador en el sentido equicorriente es la misma que la velocidad media del fluido $\left(v_{s}=u_{b}\right)$. Este es un caso peculiar en el que, al desplazarse el rascador en el sentido del flujo y a una velocidad igual a la velocidad media del mismo, la influencia del rascador en el flujo será muy pequeña. Es de esperar por lo tanto, que el campo de velocidades sea similar al que se presentaría en una geometría de sección anular uniforme.

En la Figura 7.10 se muestra el campo de velocidades obtenido en el caso mencionado, a 3 valores distintos del número de Reynolds $\left(R e_{g}=12,28,49\right)$. En las representaciones se observa un patrón de flujo en el que el perfil de velocidades a lo largo del tubo no sufre variaciones significativas más que en las proximidades de los tacos (donde la técnica de visualización no ofrece buenos resultados). 


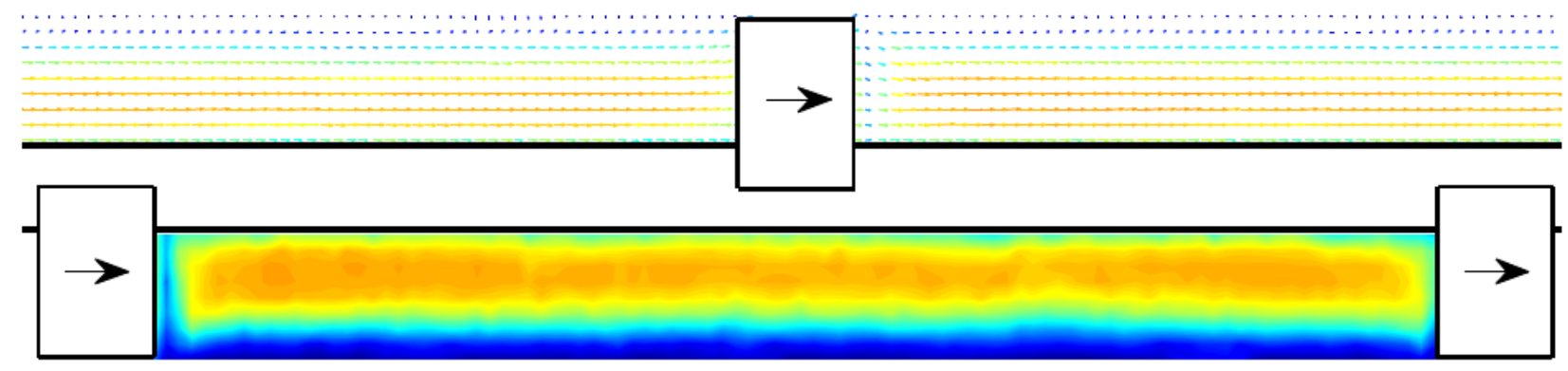

(a) $R e_{g}=12, n=0,61, m=0,74 \mathrm{~Pa} \cdot \mathrm{s}^{n}$.

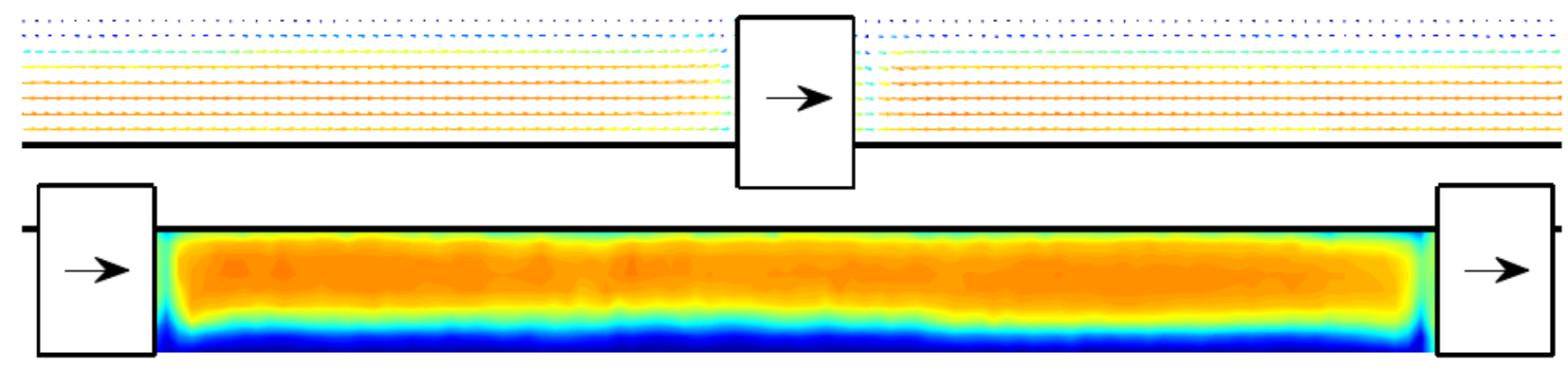

(b) $R e_{g}=27,8, n=0,79, m=0,18 \mathrm{~Pa} \cdot \mathrm{s}^{n}$.

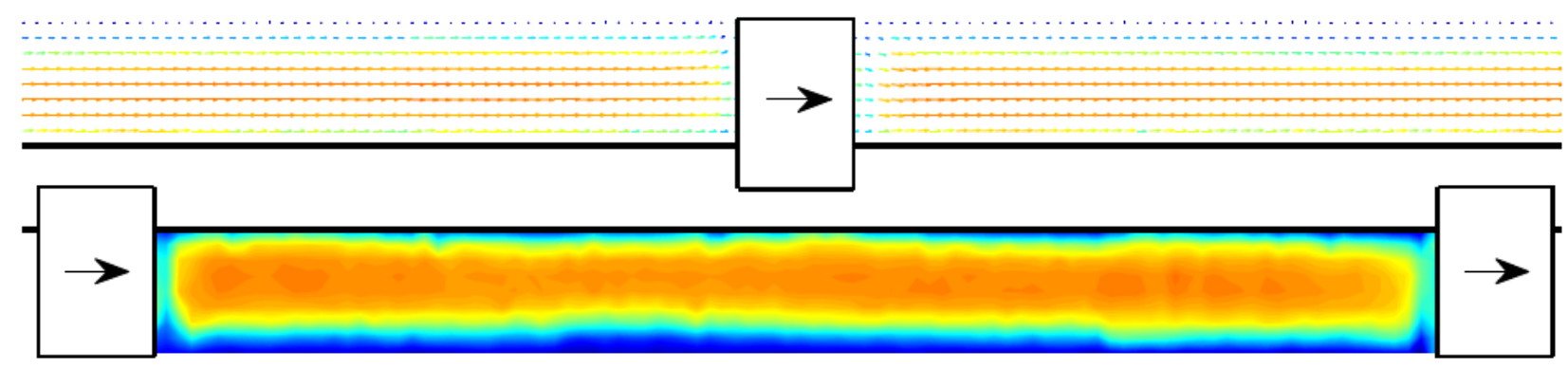

(c) $R e_{g}=49, n=0,86, m=0,08 \mathrm{~Pa}_{\mathrm{s}} \mathrm{s}^{n}$.

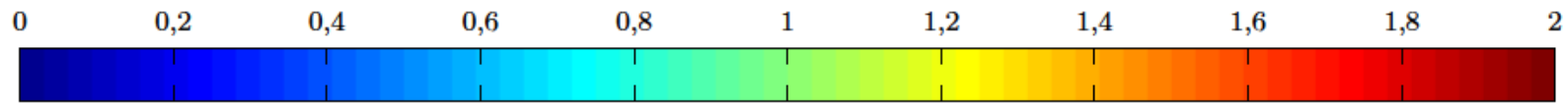

Figura 7.10: Sentido de rascado equicorriente con bloqueo nulo $\beta=0$, con $v_{s}=u_{b}$. 


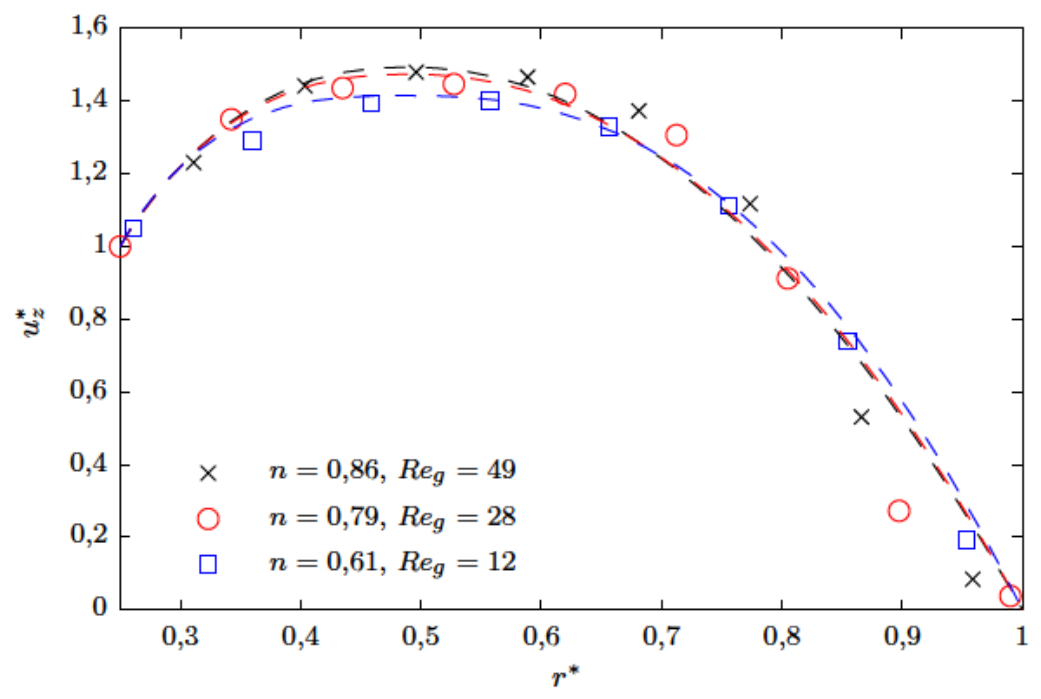

Figura 7.11: Perfil de velocidades en ensayos con bloqueo nulo $\beta=0$ en la sección situada en $z=3\left(P-t_{s}\right) / 4$.

Además, en la Fig. 7.11 se representa el perfil de velocidades en la posición $z=\left(P-t_{s}\right) / 2$ para cada uno de los tres ensayos realizados. Los perfiles obtenidos en los ensayos se comparan con el perfil de velocidades que tendría un fluido de idénticas propiedades reológicas en una geometría de tubos concéntricos. De dicha comparación se extrae la conclusión de que efectivamente, el perfil de velocidades en condiciones de bloqueo nulo es similar al que se obtendría en dicha geometría. Así mismo se observa que al disminuir el valor de $n$ la velocidad máxima del flujo se reduce y el perfil se hace más plano. 


\subsubsection{Factor de bloqueo positivo}

El factor de bloqueo será positivo en los ensayos en contracorriente, en régimen del rascador estático y en los ensayos en equicorriente en los cuales $v_{s}<u_{b}$. En régimen de funcionamiento con el rascador estático ya se ha estudiado en el Apartado 7.2, a continuación se procede al estudio de los otros dos casos.

$\beta>0$ en equicorriente Para que exista un bloqueo positivo en el caso de que el rascador se mueva en sentido equicorriente, la velocidad de rascado debe ser menor que la velocidad media del flujo $0<v_{s}<u_{b}$.

En los casos con rascado en dirección equicorriente con bloqueo positivo $(0<\beta<1)$, el campo de velocidades es similar al obtenido en ensayos estáticos, en los cuales el bloqueo también es positivo $(\beta=1)$. Sin embargo, en este caso la diferencia entre la velocidad del rascador y la velocidad media del fluido es menor que en el caso estático y, como consecuencia, también lo es la influencia del rascador en el flujo. Además dicha influencia disminuirá a medida que se $\beta$ acerque a 0 (caso de bloqueo nulo) y se parecerá más al caso estático al acercarse a 1. El margen de variación se puede observar en la Fig. 7.12, donde se representa un caso de $\beta>0$ en equicorriente junto a los casos limitantes de $\beta=1$ y $\beta=0$. En la Fig. 7.13 se realiza una representación de los perfiles de velocidades en los tres casos manteniendo $n \in[0,61 ; 0,62]$ y $R e_{g} \approx 13$. En ellos se pueden observar los siguientes resultados:

1. El perfil en $z=3\left(P-t_{s}\right) / 4$ es similar en los casos $\beta=0 ; 0,5 ; 1$.

2. En el perfil en $z=\left(P-t_{s}\right) / 2$ las diferencias son más significativas. Dándose en el caso $\beta=0,5$, una situación intermedia entre los otros dos:

a) Para $\beta=0$, la velocidad máxima del perfil es 1,4 veces $u_{b}$.

b) Para $\beta=0,5$, la velocidad máxima del perfil es 2,1 veces $u_{b}$.

c) Para $\beta=1$, la velocidad máxima del perfil es 2,9 veces $u_{b}$.

Se han realizado 3 ensayos en régimen dinámico con $\beta=0,5$ a distintos números de Reynolds: $R e_{g} \in[12 ; 51]$. El campo de velocidades obtenido en dicho ensayo se muestra en la Fig. 7.14 . De la observación de los resultados, se confirma que los efectos del rascador son menores que en el caso estático. Concretamente se observan los siguientes efectos:

1. La velocidad máxima se ha reducido a 2,3 veces la velocidad media del fluido. 


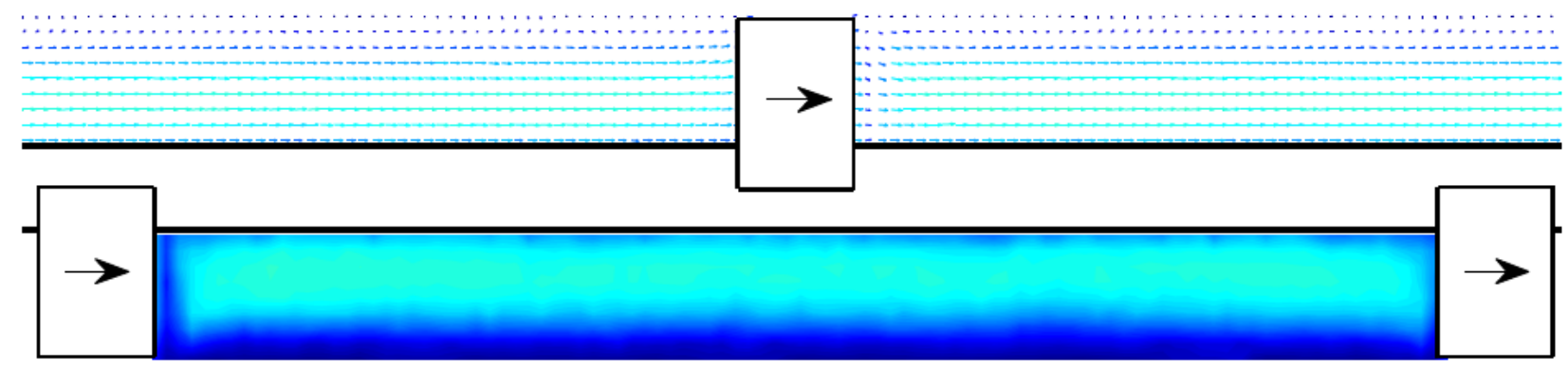

(a) Caso $\beta=0 . R e_{g}=12$ y $n=0,61$.

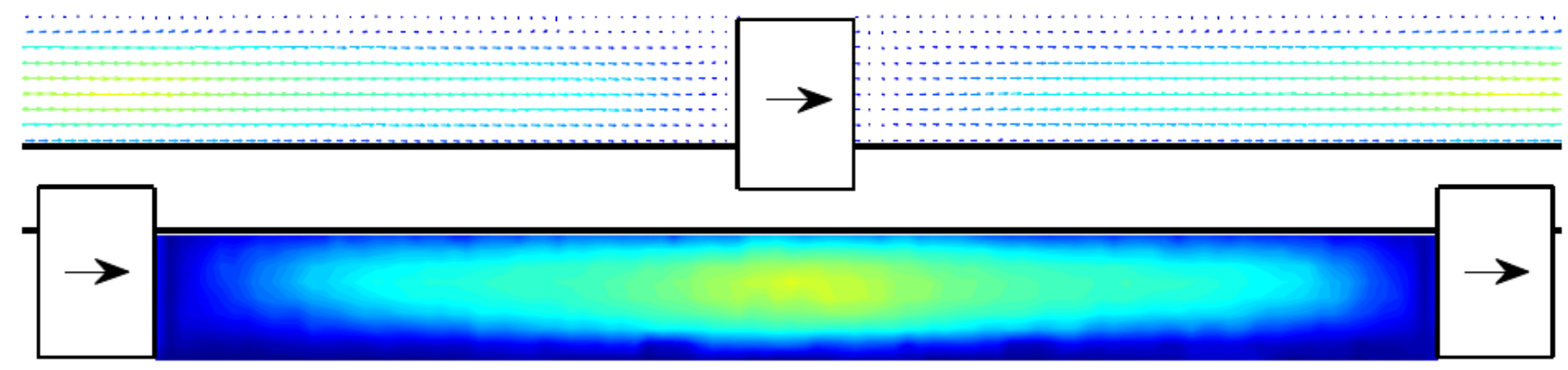

(b) Caso $\beta=0,5 \cdot R e_{g}=11,5, n=0,61$

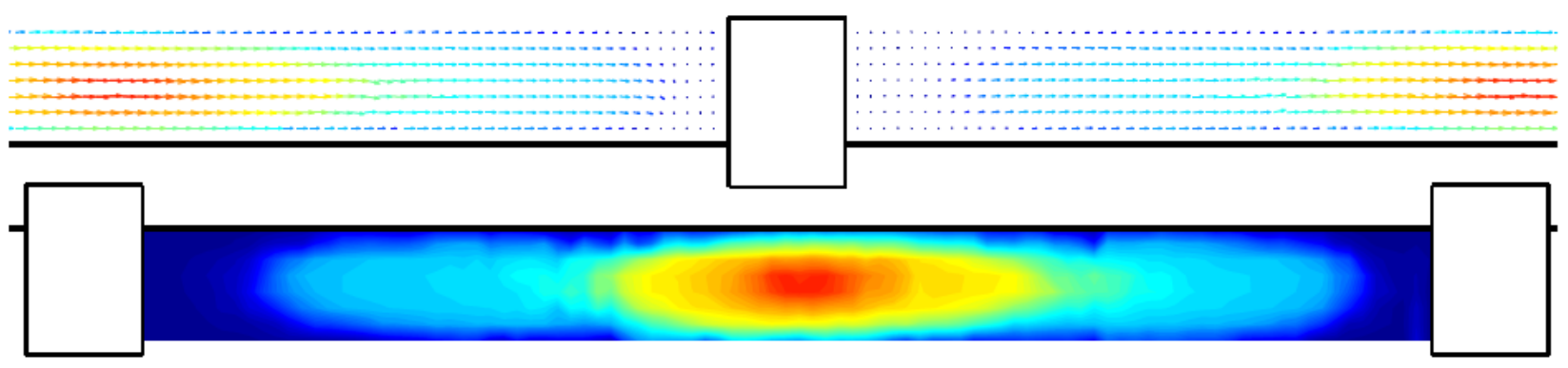

(c) Caso $\beta=1$ (estático). $R e_{g}=15, n=0,62$.

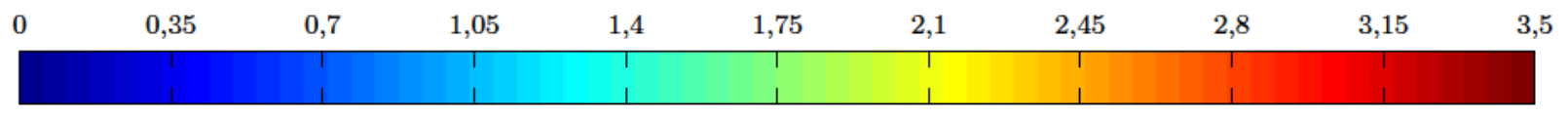

Figura 7.12: Comportamiento del rascador en el rango $0 \leq \beta \leq 1$. 

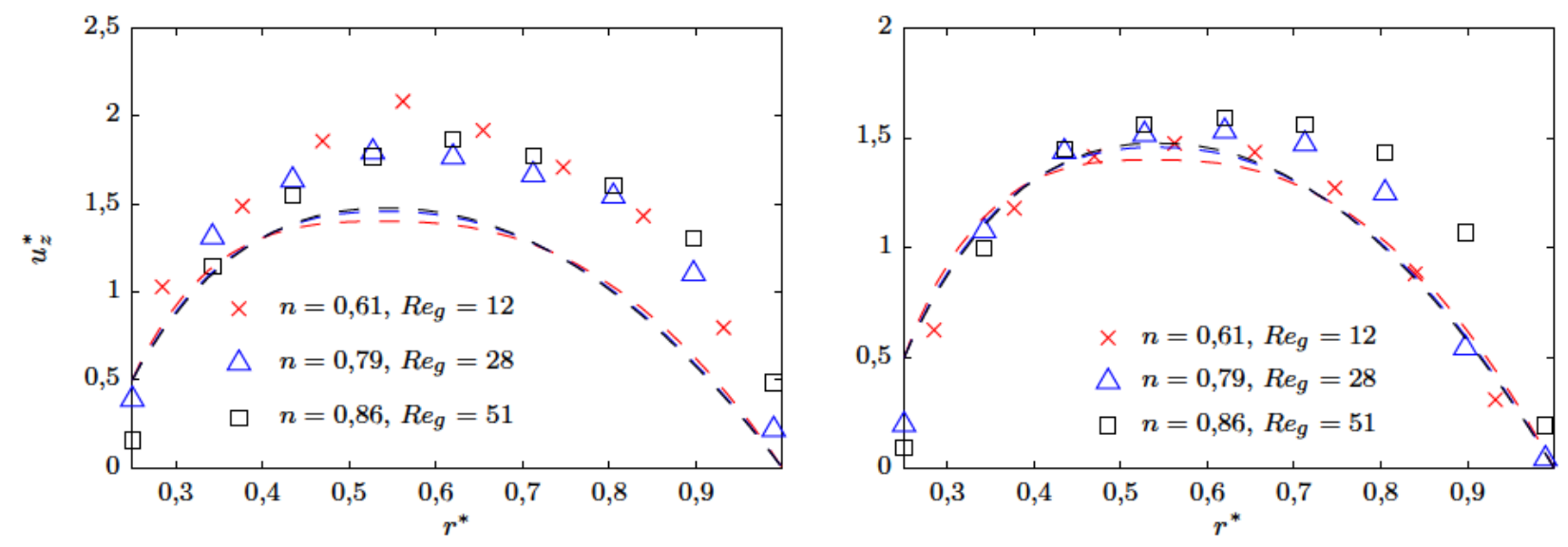

(a) $\beta=0,5$. Perfil central $z=\left(P-t_{s}\right) / 2$.

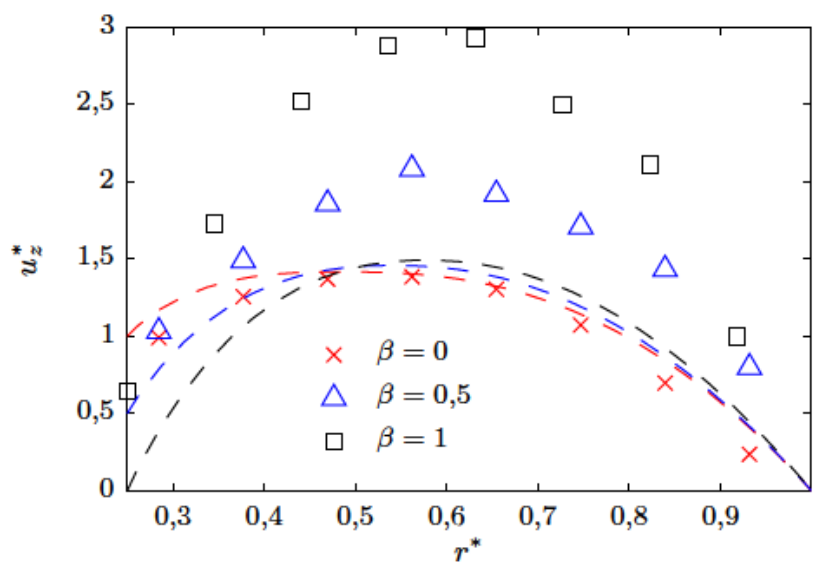

(b) $\beta=0,5$. Perfil en $z=3\left(P-t_{s}\right) / 4$.

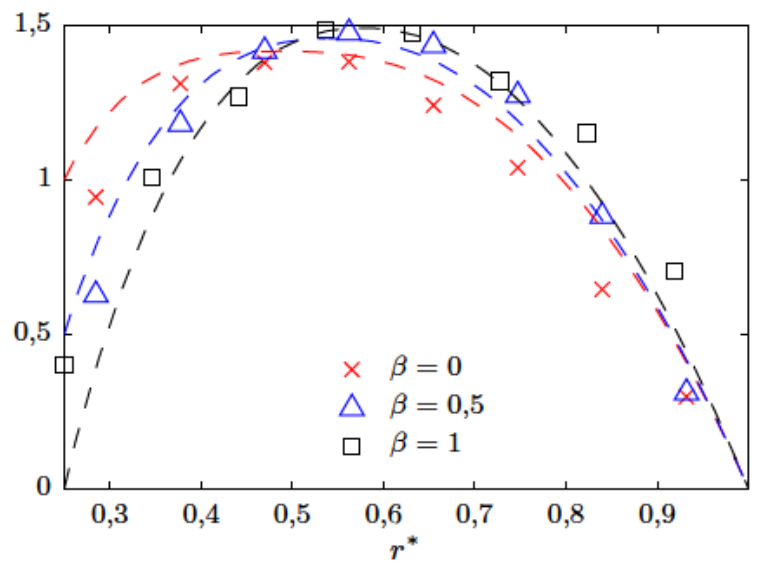

(c) $n=0,61-0,62$. Perfil en $z=\left(P-t_{s}\right) / 2$.

(d) $n=0,61-0,62$. Perfil en $z=3\left(P-t_{s}\right) / 4$.

Figura 7.13: Comportamiento del flujo en casos de rascado equicorriente con $\beta>0$, donde $\beta=0,5$. En las figuras $c$ ) y $d$ ) se compara este caso con los casos limitantes en los que $\beta=0 ; 1$. 


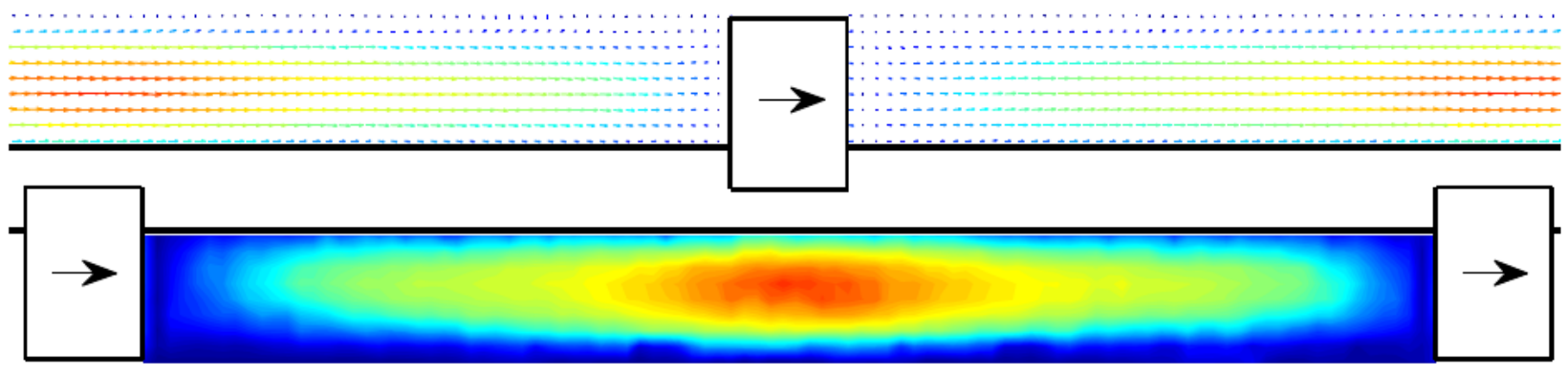

(a) $R e_{g}=12, n=0,61$.

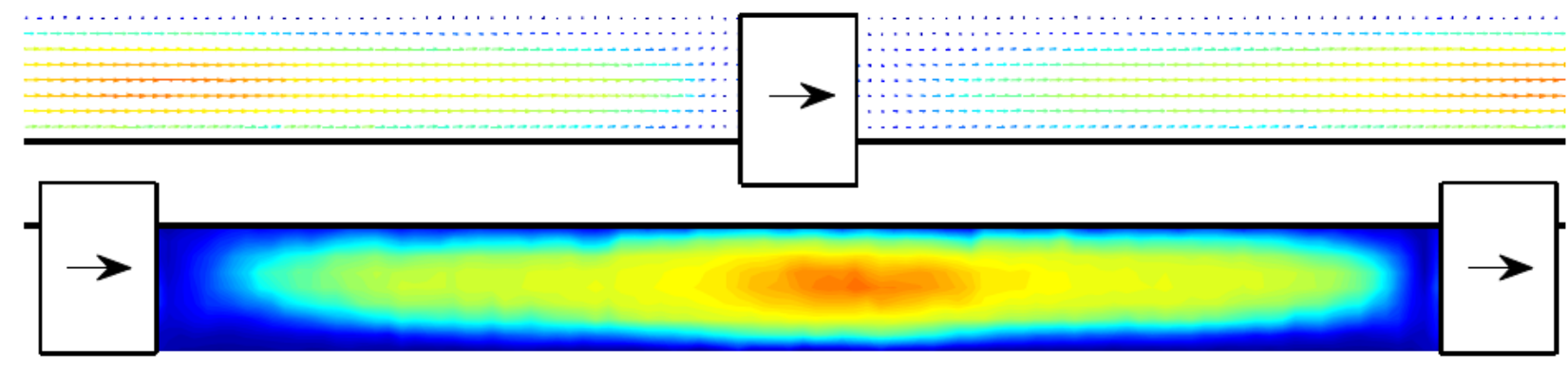

(b) $R e_{g}=27,5, n=0,79$.

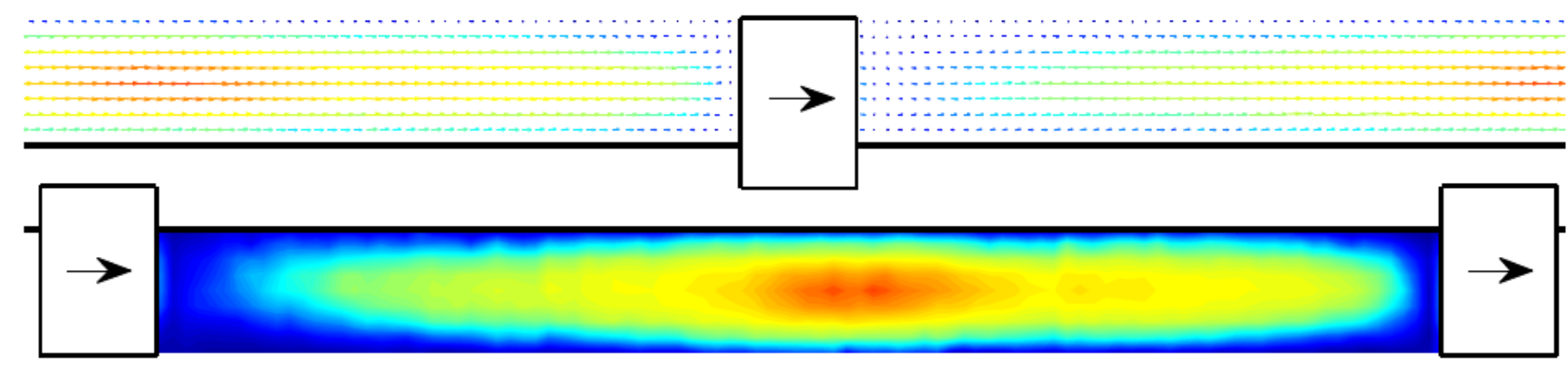

(c) $R e_{g}=51, n=0,86$.

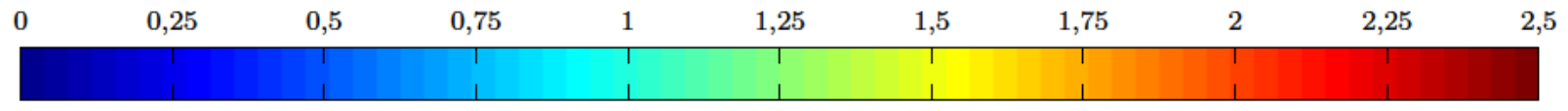

Figura 7.14: Rascador en régimen dinámico, sentido de rascado equicorriente con bloqueo positivo $\beta=0,5$. 
2. El tamaño de las zonas de baja velocidad se ha reducido considerablemente.

3. Al estar el rascador en movimiento, han desaparecido las zonas con velocidades casi nulas que, en el caso de rascador estático, aparecen en las cercanías de los tacos.

$\beta>0$ en contracorriente En los ensayos en los que el rascador se mueve en sentido contrario al flujo, el bloqueo siempre es positivo y es mayor a medida que aumenta la velocidad de rascado. Por lo tanto, las estructuras de flujo que aparecen son similares a las que aparecen en los ensayos estáticos o en los ensayos en equicorriente en los que el bloqueo también es positivo. Además el efecto del rascador, aumenta al hacerlo el factor de bloqueo.

En contracorriente se han realizado ensayos a $\beta=1,5 ; 2 ; 3$ y 4 cada uno a tres números de Reynolds diferentes en el rango $R e_{g} \in[13 ; 43]$, con $n \in[0,62 ; 0,89]$. En primer lugar, en las figuras 7.15 y 7.17(a) se comparan los resultados de los ensayos en los que varía la velocidad de rascado y en los que la variación de $n$ y el $R e_{g}$ es menor. Además en las figuras 7.16(c) y 7.16(d) se representan los perfiles de velocidades para los mismos ensayos. En los resultados se observan los siguientes efectos:

1. A medida que aumenta $\beta$, la zona de alta velocidad aumenta de tamaño.

2. Al aumentar $\beta$, aumenta la velocidad máxima del flujo. Se pasa de velocidades máximas en torno a $u_{z, \max }^{*}=3,3$ para $\beta=1,5$ a velocidades de más de 6 veces la velocidad media del flujo para $\beta=4$.

3. El movimiento contracorriente de los tacos provoca un efecto de arrastre con velocidades en contra del flujo, cuya estructura es similar a la observada en los ensayos a caudal cero.

4. La zona con flujo contracorriente aguas abajo de los tacos (zona A) aumenta su tamaño al hacerlo $\beta$. Mientras que esto no ocurre con la zona con flujo contracorriente situada aguas arriba de los tacos.

En segundo lugar, en las figuras 7.17, 7.16(a) y 7.16(b) se realiza la comparación manteniendo el factor de bloqueo constante y variando el número de Reynolds y $n$. En los resultados se aprecia que al aumentar el $R e_{g}$ y $n$ :

1. La zona de alta velocidad en dirección equicorriente aumenta de tamaño.

2. El perfil de velocidades en la zona intermedia (Fig. 7.16(a)) no varía significativamente. 


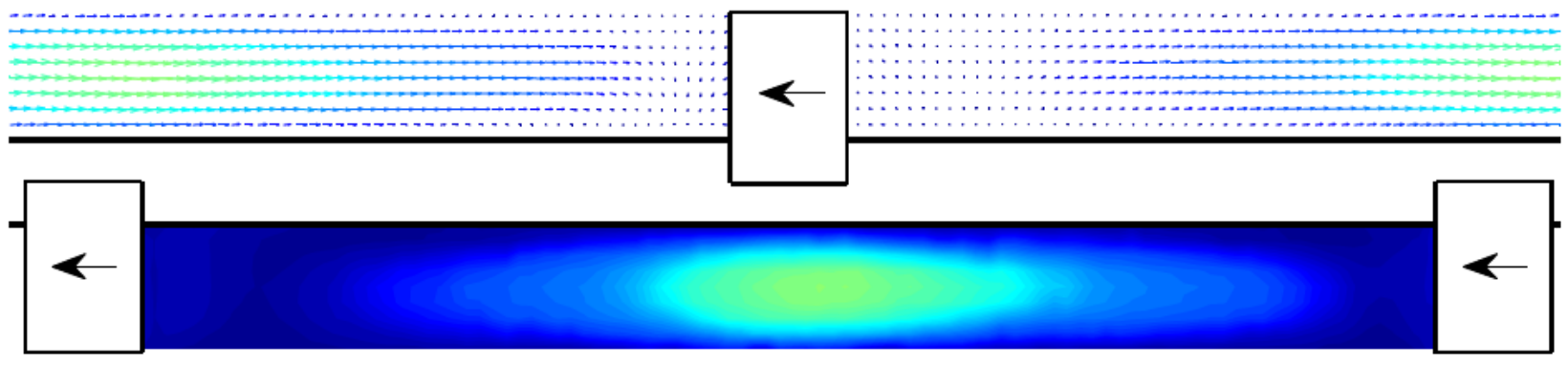

(a) $\beta=1,5$. $R e_{g}=12,4, n=0,62$.

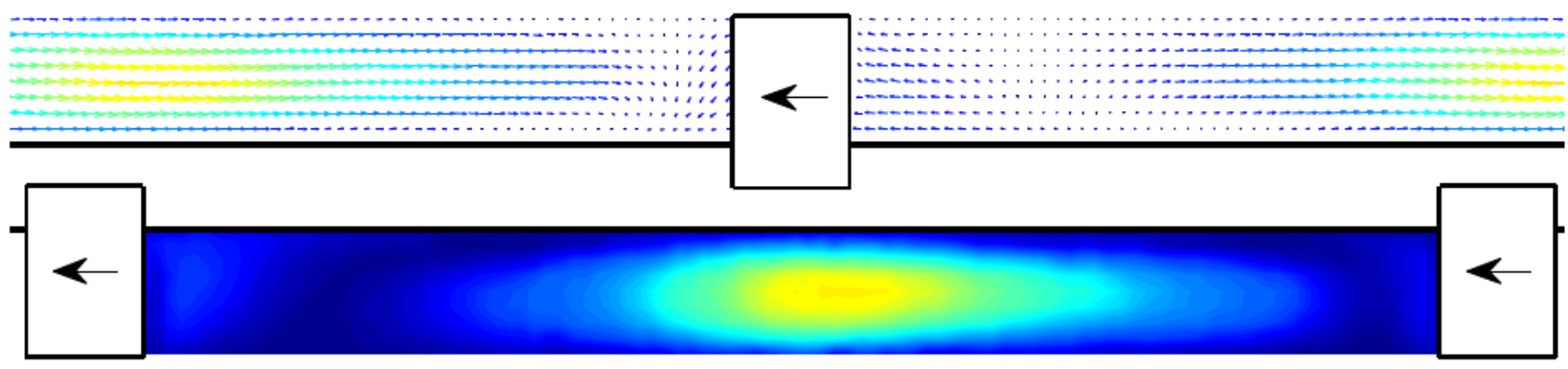

(b) $\beta=2$. $R e_{g}=12,5, n=0,64$.

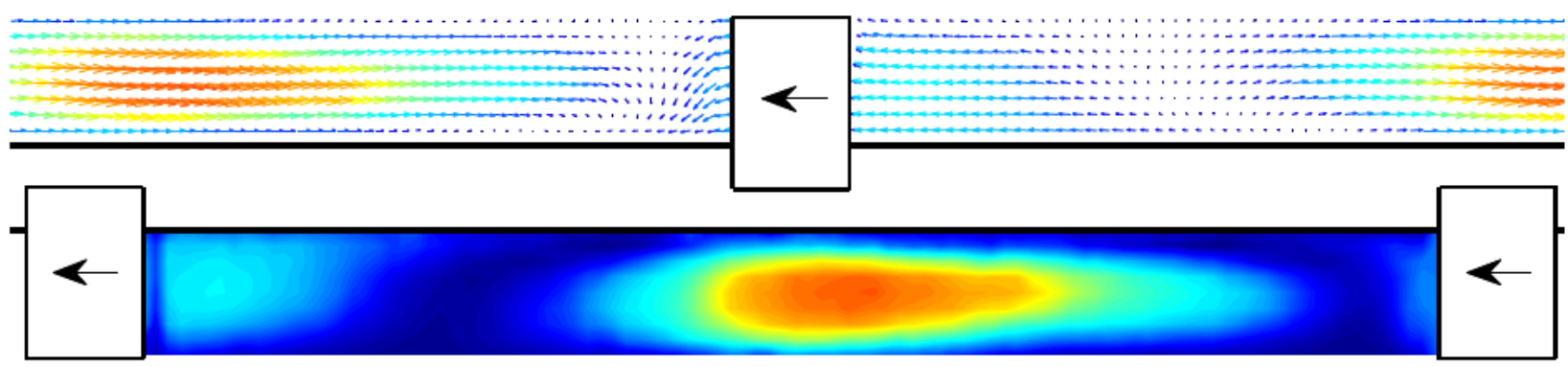

(c) $\beta=3$. $R e_{g}=13, n=0,65$.

\begin{tabular}{ccccccccccc}
0 & 0,7 & 1,4 & 2,1 & 2,8 & 3,5 & 4,2 & 4,9 & 5,6 & 6,3 & 7 \\
\hline & 1 & 1 & 1 & 1 & 1 & 1 & 1 & 1
\end{tabular}

Figura 7.15: Campo fluido en ensayos con factor de bloque positivo y sentido de rascado contracorriente. 


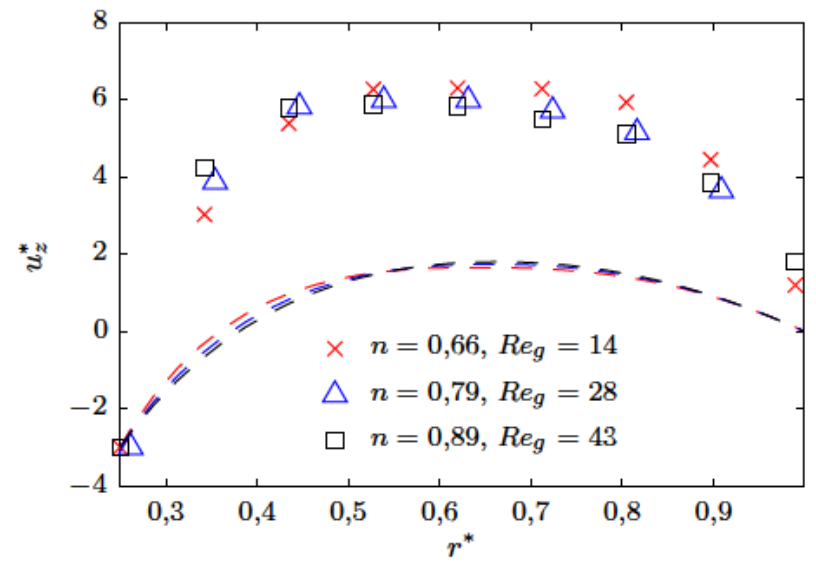

(a) $\beta=4$. Perfil central $z=\left(P-t_{s}\right) / 2$.

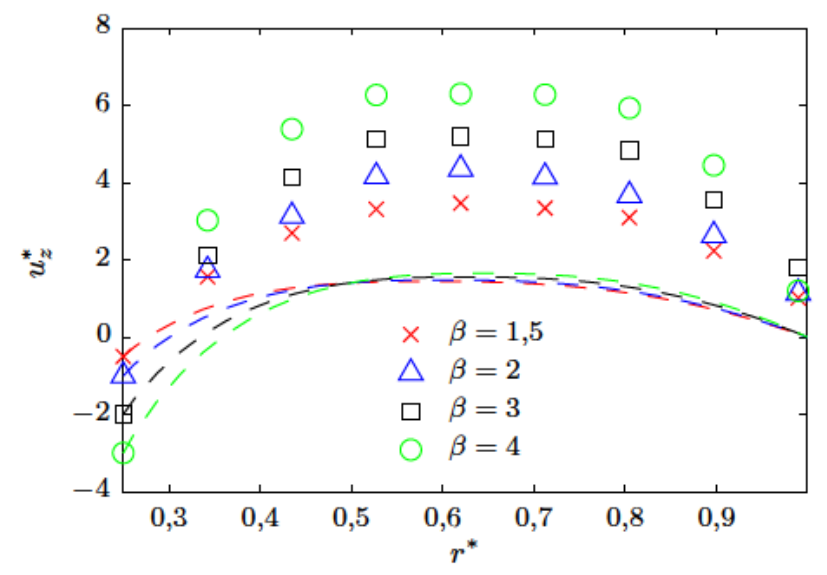

(c) $n=0,62-0,66$ Perfil en $z=\left(P-t_{s}\right) / 2$.

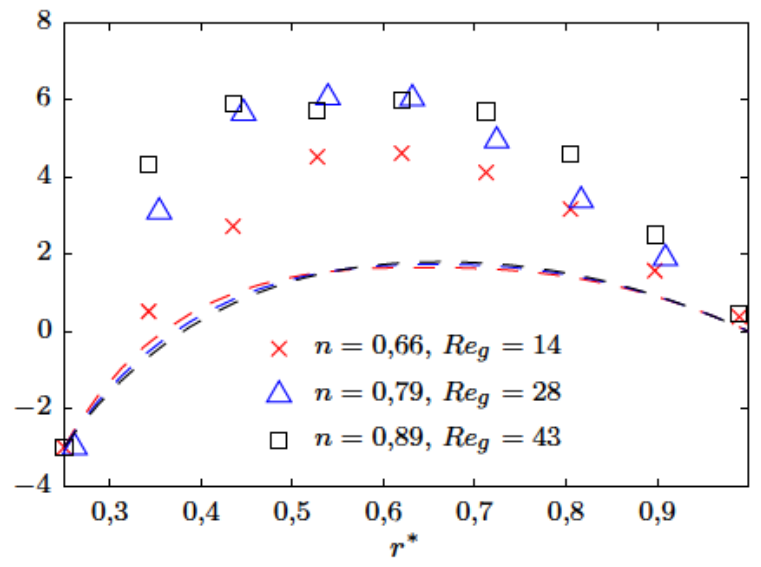

(b) $\beta=4$. Perfil en $z=3\left(P-t_{s}\right) / 4$.

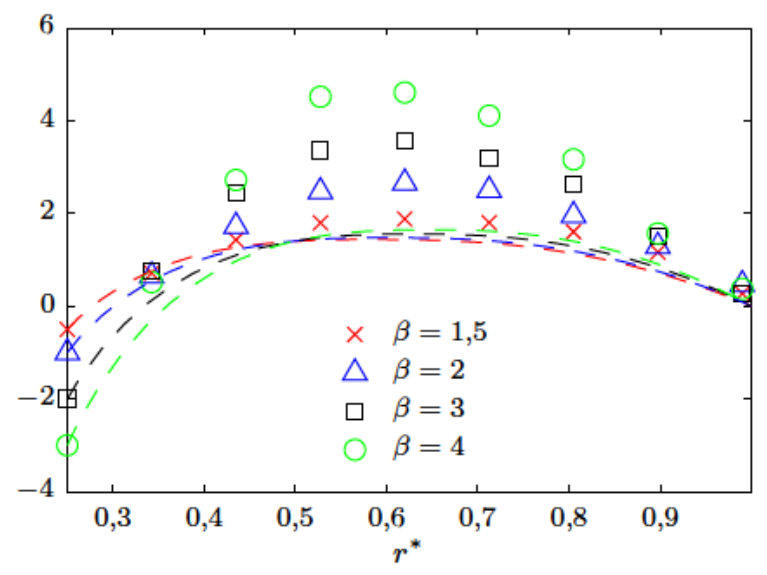

(d) $n=0,62-0,66$. Perfil en $z=3\left(P-t_{s}\right) / 4$.

Figura 7.16: Comportamiento del flujo en casos de rascado contracorriente con $\beta>0$. 


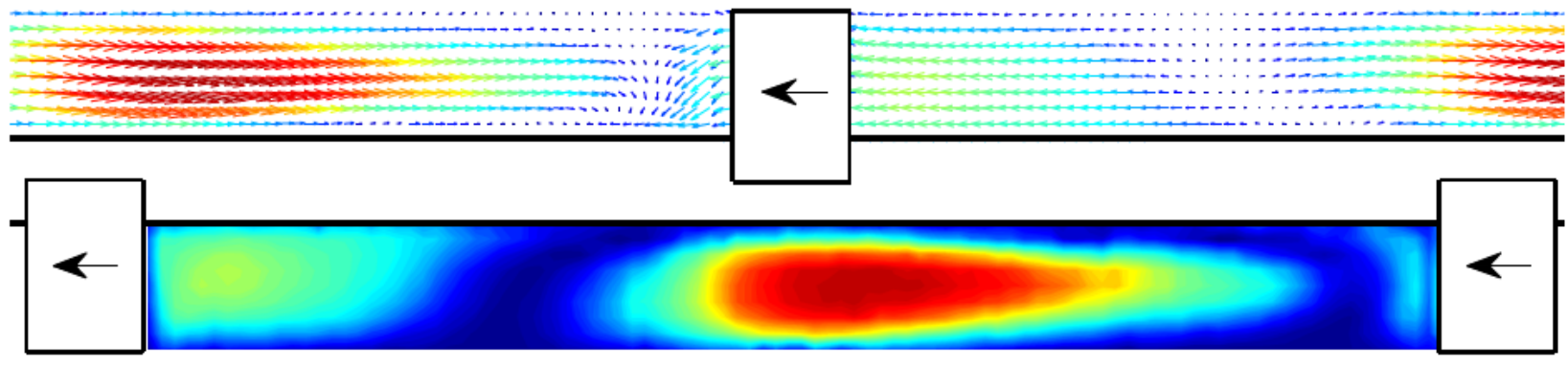

(a) $R e_{g}=14, n=0,66$.

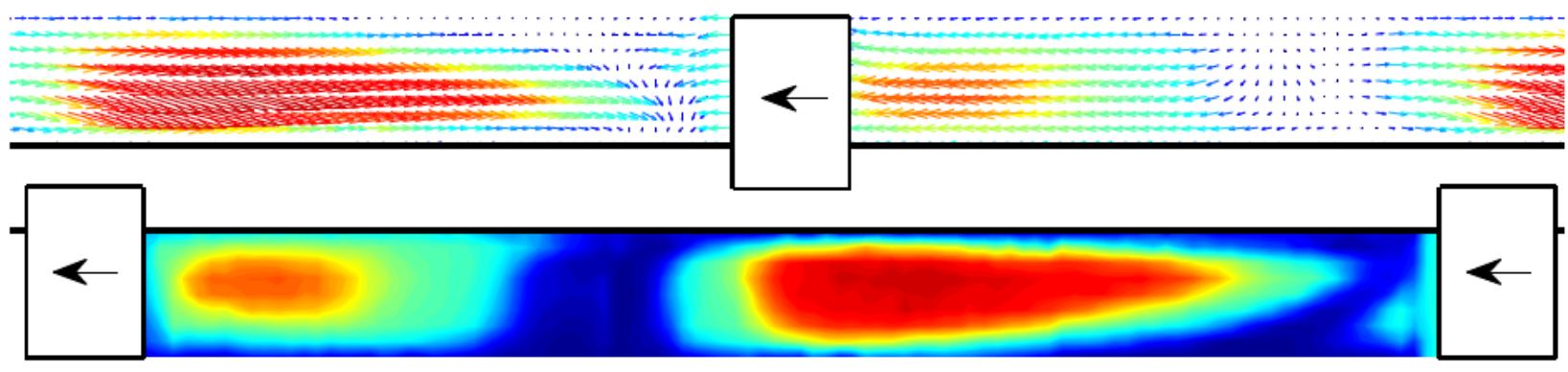

(b) $R e_{g}=28, n=0,79$.

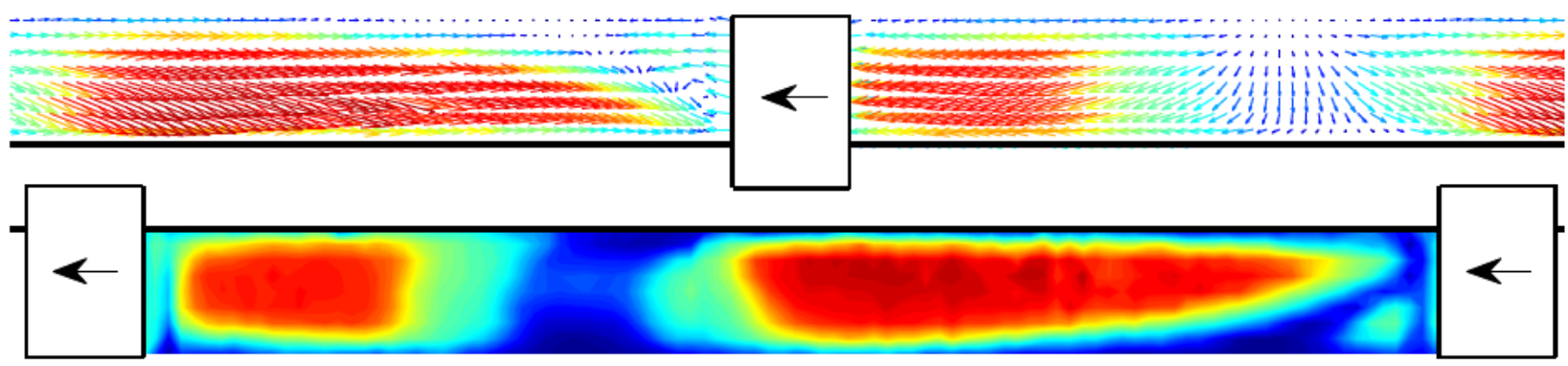

(c) $R e_{g}=43, n=0,89$.

\begin{tabular}{rrrrrrrrrrr}
0 & 0,7 & 1,4 & 2,1 & 2,8 & 3,5 & 4,2 & 4,9 & 5,6 & 6,3 & 7 \\
\hline & 1 & 1 & 1 & 1 & 1 & $\mid$ & 1 & & \\
\hline
\end{tabular}

Figura 7.17: Caso dinámico contracorriente con $\beta=4$. 
3. En el perfil de velocidades en $z=3\left(P-t_{s}\right) / 4$ se observa que para el ensayo a $R e_{g}=14$ las velocidades son menores porque la región de alta velocidad no cubre dicho perfil, mientras que en los otros dos ensayos el perfil es similar y las velocidades centrales alcanzan su valor máximo (en torno a 6).

4. La zona A (aguas abajo del taco) de flujo inverso aumenta ligeramente su tamaño.

5. Las velocidades en la zona A aumentan hasta alcanzar valores similares a las velocidades máximas en la dirección del flujo.

El resto de resultados obtenidos, a $\beta=1,5 ; 2$ y 3 , manteniendo $\beta$ constante y variando $R e_{g}$ y $n$, se muestran en el Apartado C del Apéndice. 


\subsubsection{Factor de bloqueo negativo}

Cuando el régimen de rascado implica valores de $\beta<0$, el movimiento del rascador facilita la circulación del fluido. Este únicamente ocurre en el sentido de rascado equicorriente siempre que $v_{s}>u_{b}$.

En este caso la velocidad del rascador es superior a la velocidad media del fluido y la estructura cambia de forma significativa respecto a los casos anteriores, tal y como se observa en la Fig. 7.18(c). El movimiento del rascador induce una depresión aguas arriba del mismo, lo cual a su vez provoca altas velocidades en esa zona. Además, por efecto de impulsión de rascador también se genera una pequeña zona de alta velocidad aguas abajo del mismo. Así, la región de mayor velocidad en sentido equicorriente se extiende desde la zona aguas abajo de un taco a la zona aguas arriba del siguiente taco, en posición opuesta. En condiciones de bloqueo negativo y sentido equicorriente, se han realizado ensayos a valores de $\beta=-1 \mathrm{y}$ $\beta=-2$, y a tres números de Reynolds en el rango entre $R e_{g}=12$ y $R e_{g}=48$.

El efecto del factor de bloqueo negativo, se puede observar en la Fig. 7.18. La estructura del flujo evoluciona desde la situación de $\beta=0$, donde el perfil de velocidad, similar al obtenido en geometría de tubos concéntricos, se mantiene prácticamente constante a lo largo del tubo. Al decrecer $\beta$, los efectos del rascado se acentúan y el patrón de flujo guarda alta similitud con el obtenido en los ensayos a caudal cero. Las velocidades máximas son del orden de la velocidad del rascador (para los valores de $R e_{c}$ y $n$ representados). Además, en el perfil central (Fig. 7.20(c)) se aprecia que el perfil de velocidades evoluciona desde el obtenido en los casos donde $\beta=0$, hacia perfiles con velocidades negativas de hasta 2 veces $u_{b}$ y en dirección opuesta al flujo, para $\beta=-2$. Se observa así mismo que los perfiles de velocidades situados a 3/4 de la separación entre tacos son muy similares a los obtenidos mediante simulación en la geometría de tubos concéntricos.

En la Fig. 7.19 se puede apreciar el efecto de variar $R e_{g}$ y $n$ simultáneamente. Al aumentar ambos números adimensionales:

- Las velocidades máximas se incrementan significativamente, pasando de valores cercanos a la velocidad del taco $u_{z}^{*}=3$ en el caso de menor $R e_{g}$ y $n$, hasta valores de $u_{z}^{*}=5$.

- Las velocidades en sentido aguas arriba en el perfil central disminuyen en módulo en el ensayo a mayor $R e_{g}$ y $n$, ya que la zona de altas velocidades en dicho sentido se desplaza ligeramente aguas arriba. 


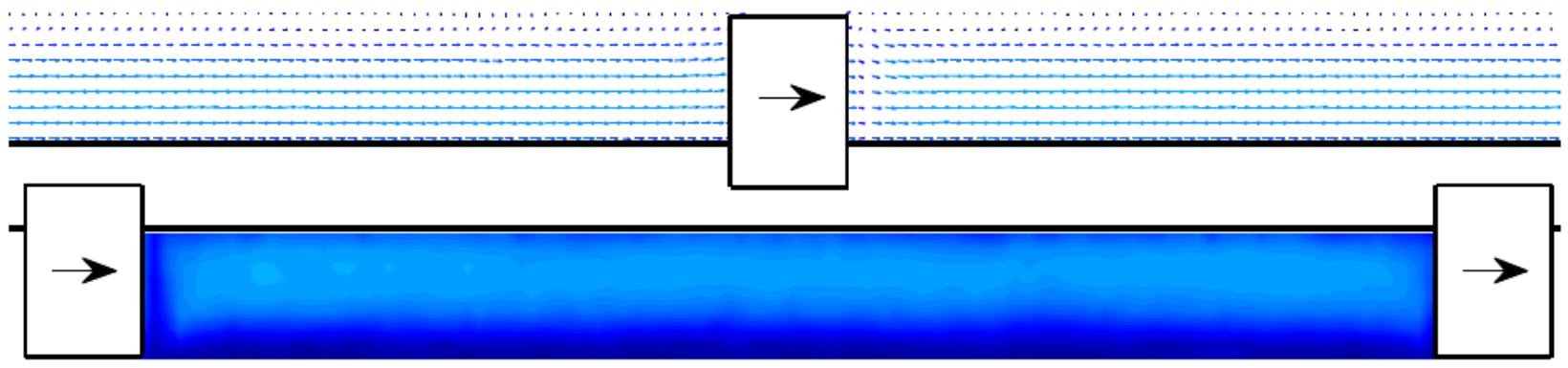

(a) $\beta=0 . R e_{g}=12, n=0,61$.

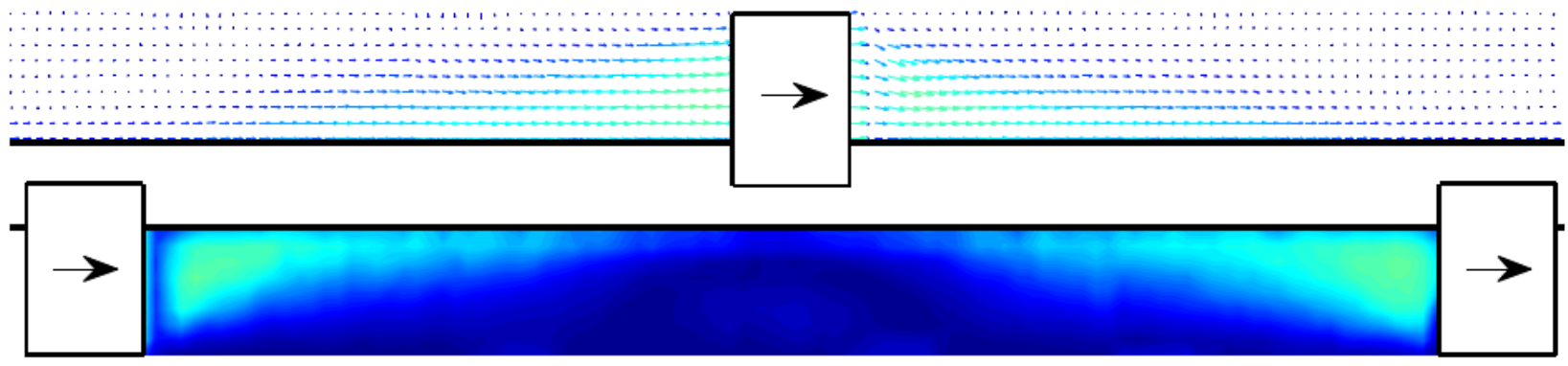

(b) $\beta=-1$. $R e_{g}=12, n=0,62$.

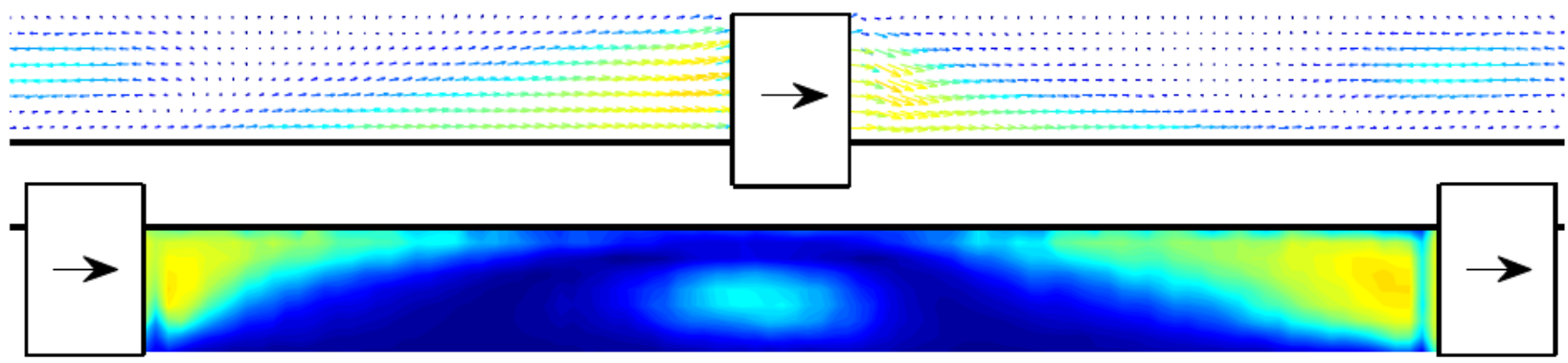

(c) $\beta=-2 . R e_{g}=12, n=0,63$.

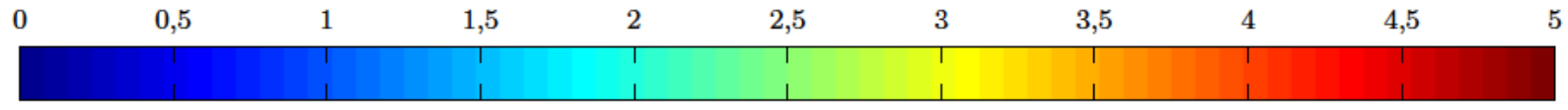

Figura 7.18: Campo de velocidades en ensayos con bloqueo negativo. 


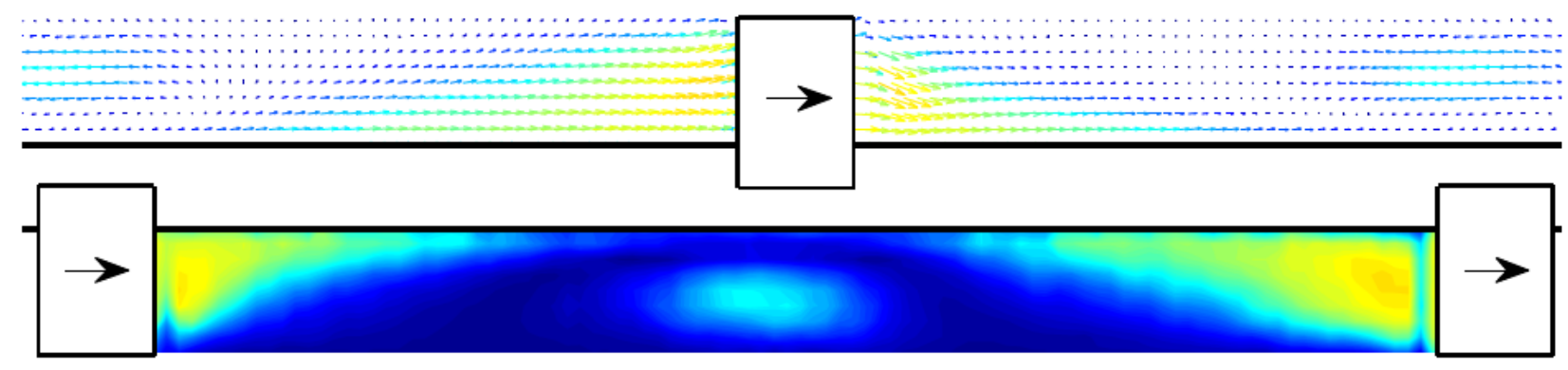

(a) $R e_{g}=12, n=0,63$.

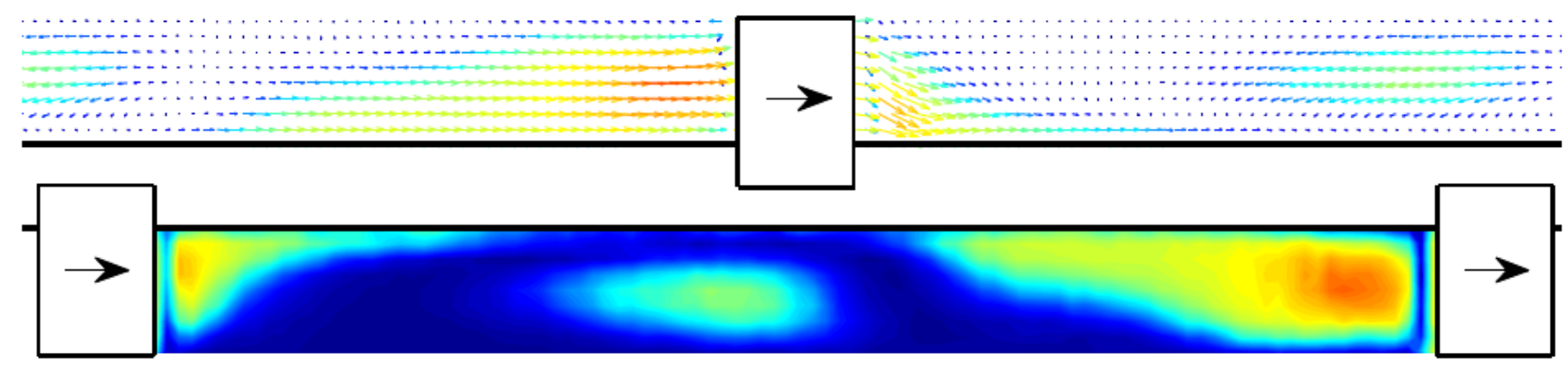

(b) $R e_{g}=28, n=0,79$.

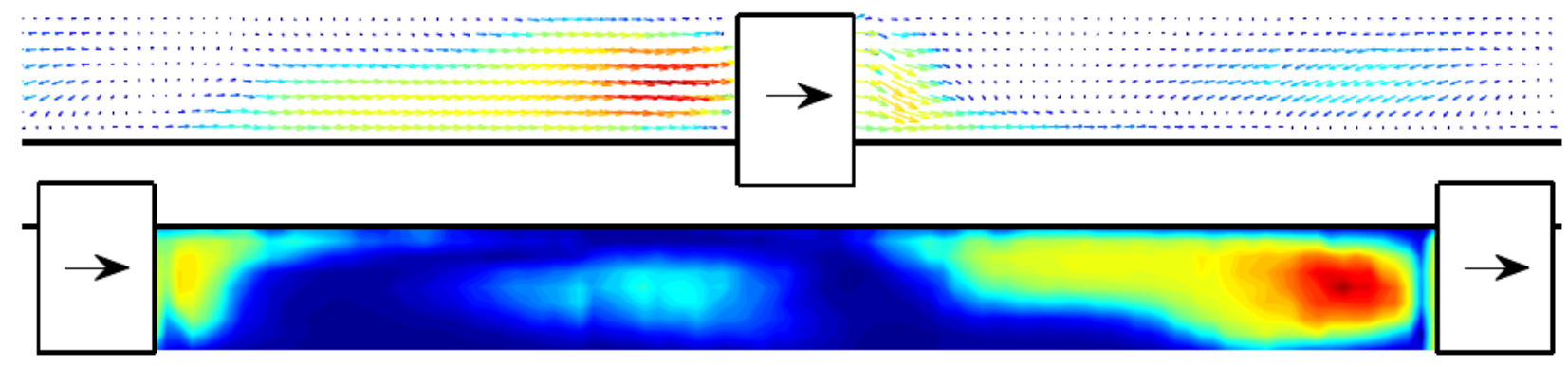

(c) $R e_{g}=48, n=0,87$.

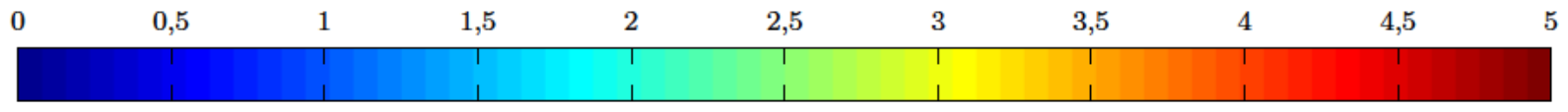

Figura 7.19: Caso dinámico equicorriente con bloqueo negativo $\beta=-2$ variando el número de Reynolds. 


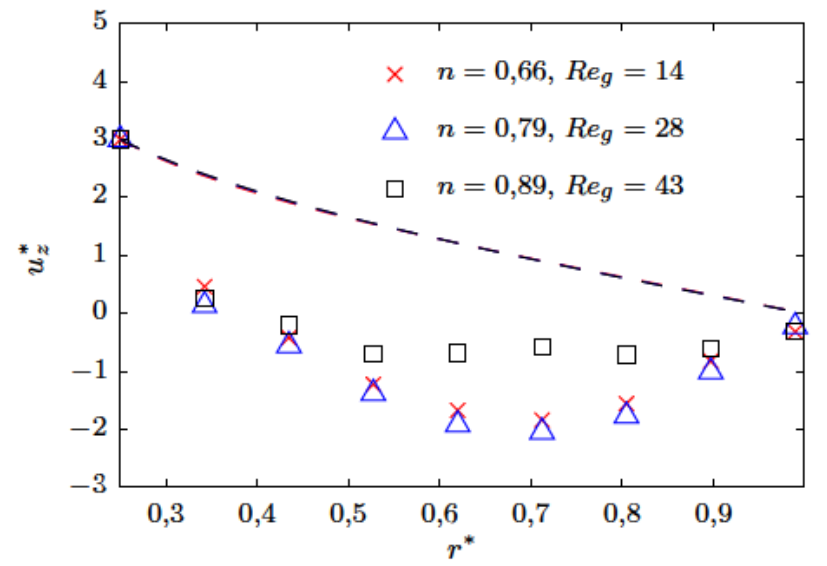

(a) $\beta=-2$. Perfil central $z=\left(P-t_{s}\right) / 2$.

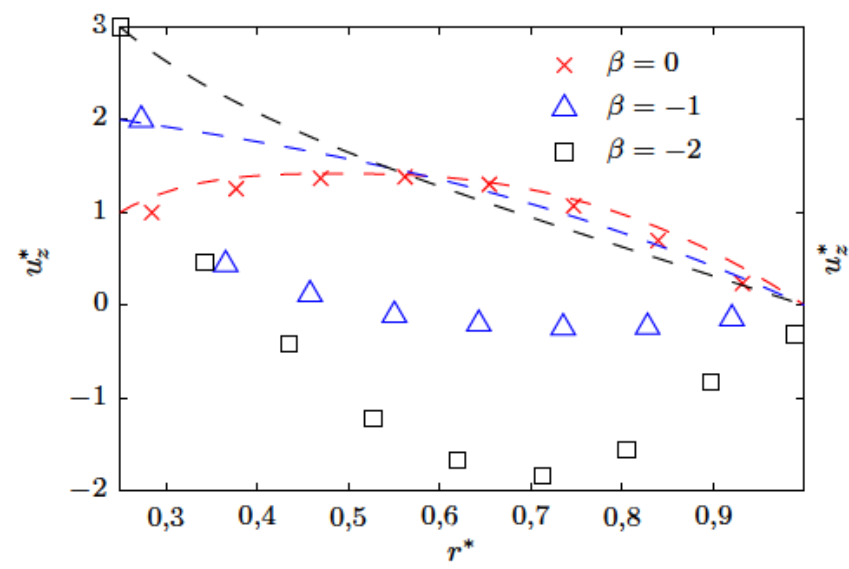

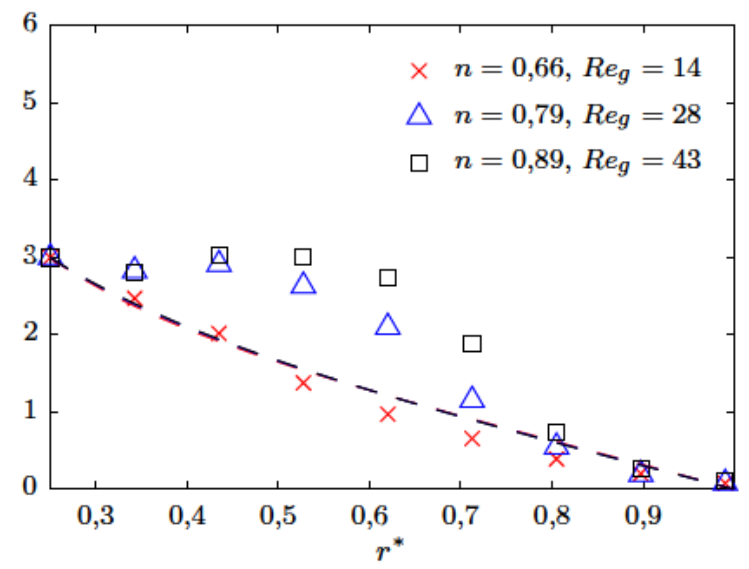

(b) $\beta=-2$. Perfil en $z=3\left(P-t_{s}\right) / 4$.

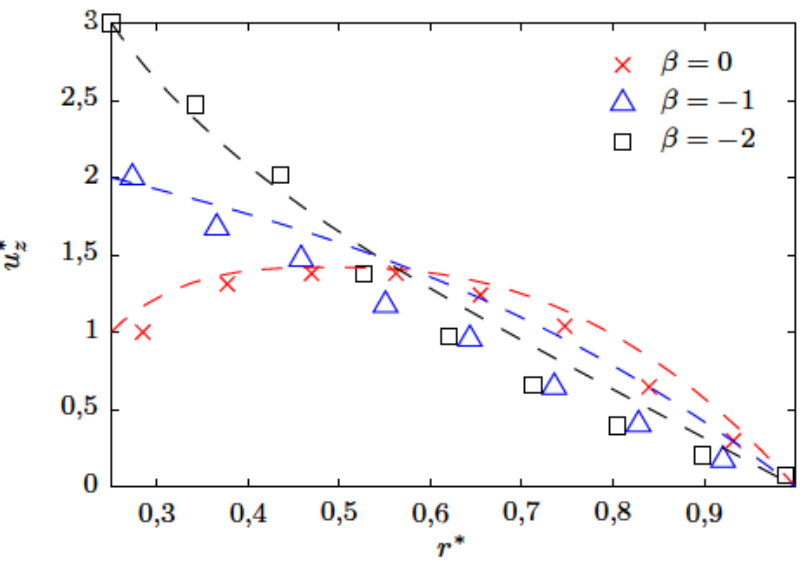

(c) $n=0,61-0,63$. Perfil en $z=\left(P-t_{s}\right) / 2$. $n \approx 0,62$. (d) $n=0,61-0,63$. Perfil en $z=3\left(P-t_{s}\right) / 4 . n \approx$ 0,62 .

Figura 7.20: Comportamiento del flujo en casos de rascado equicorriente con $\beta<0$. 
- En la sección situada a 3/4 de la separación entre tacos, el perfil de velocidades para el caso de menor Reynolds es similar al obtenido para la geometría de tubos concéntricos, pero al aumentar $R e_{g}$ las velocidades aumentan, ya que la influencia del taco se expande hasta dicha sección.

Los resultados de los ensayos a distintos números de Reynolds y $\beta=-1$ se encuentran en el Apartado C del apéndice.

\subsection{Conclusiones}

El capítulo presenta el campo de velocidades, de un fluido seudoplástico, que se produce a causa de un dispositivo insertado en un tubo para mejorar la transferencia de calor y eliminar el ensuciamiento.

1. Se ha obtenido el campo de velocidades en regímenes de rascado estático y dinámico, en direcciones equicorriente y contracorriente. Se ha estudiado el efecto de la velocidad de rascado, variando $\beta \in[-2,4]$. Para cada caso de estudio se han realizado ensayos en un rango de números de Reynolds en el rango $R e_{g} \in[5,48]$.

2. Se ha comprobado que la estructura del flujo depende fuertemente del factor de bloqueo, apareciendo tres estructuras distintas para los siguientes rangos de valores del parámetro: $\beta<0, \beta=0$ y $\beta>0$.

a) Las estructura del flujo para $\beta<0$ viene condicionada por el arrastre de los tacos, que se mueven a mayor velocidad que el flujo en su conjunto.

b) En condiciones de $\beta=0$, el flujo es muy similar al que se produce en la geometría de tubo con eje.

c) En condiciones de $\beta>0$, los tacos suponen un impedimento al paso del fluido, provocando estructuras de flujo zigzagueante.

3. Los regímenes de funcionamiento con valores altos de $|\beta|$ provocan mayores gradientes de velocidades, recirculaciones y en general un mayor mezclado del flujo. Se espera por tanto que a valores mayores de $|\beta|$ mejore la eficiencia de la transmisión de calor.

4. Mediante el ensayo a diferentes números de Reynolds y diferentes valores del Índice de comportamiento del flujo ( $n$ ), se ha comprobado la influencia que tiene sobre las 
estructuras de flujo el hecho de variar conjuntamente ambos parámetros. Al aumentar $R e_{g}$ y $n$ en general crecen las zonas de alta velocidad y aumentan las recirculaciones, en general mejoran las condiciones para un transferencia de calor más eficiente.

5. El efecto de variar $n$ y $R e_{g}$ por separado no se ha podido comprobar debido a las limitaciones experimentales existentes. La simulación del flujo en la geometría de tubos concéntricos ha servido para comprobar el efecto de variar $n$ en una geometría similar, sin embargo el efecto exacto en la geometría bajo estudio sigue siendo desconocido. Esta dificultad encontrada en los ensayos de visualización pone de manifiesto la utilidad de disponer de una definición del número de Reynolds que permita obtener la caída de presión a partir de una curva independientemente del valor de $n$ del fluido. 


\section{Capítulo 8}

\section{Evaluación experimental de la pérdida de presión y la potencia de accionamiento}

En los capítulos precedentes se ha puesto de manifiesto la necesidad de evaluar tanto la caída de presión en el tubo con el rascador insertado, como la potencia de accionamiento consumida por este último, al variar las condiciones de funcionamiento. Esto permitirá realizar un balance energético para evaluar la conveniencia del uso del sistema de mejora en función de las diferentes variables. El estudio de la caída de presión se realiza en base al análisis dimensional realizado en el Capítulo 2, donde se obtienen los monomios adimensionales que describen el problema.

Al igual que en el capítulo anterior, al ser conocido el funcionamiento del dispositivo con fluidos newtonianos (Solano, 2009b), el estudio se centra en la influencia del comportamiento no newtoniano del fluido. En consecuencia, el objetivo del capítulo es el de cuantificar los costes energéticos derivados de la pérdida de presión adicional producida por el elemento insertado y de la potencia de accionamiento requerida, utilizando un fluido seudoplástico como fluido de trabajo (véase una descripción más detallada del fluido de trabajo y su comportamiento en el Capítulo 5).

El dispositivo rascador estudiado, podrá trabajar tanto en régimen de rascado estático, como en régimen de rascado dinámico. De modo que ambas situaciones se analizan por separado.

Por un lado, en el funcionamiento del intercambiador de calor con el rascador estático se 
producirán los siguientes efectos, los cuales es necesario cuantificar:

- Existe una pérdida de presión adicional que deriva en un mayor gasto energético de bombeo.

- No se produce gasto energético en el sistema de impulsión del rascador.

- Al no haber movimiento de rascado, no se previene la acumulación de ensuciamiento.

La pérdida de presión que se produce en el tubo cuando el rascador insertado se encuentra estático se cuantifica en el Apartado 8.1.

Por otro lado, en el caso de trabajar con el dispositivo insertado en régimen dinámico, aparecen los siguientes efectos:

- Al igual que en el caso estático, existe una pérdida de presión adicional en el flujo que deriva en un mayor gasto energético de bombeo.

- Es necesario un gasto energético adicional para el sistema de impulsión del rascador.

- Se evita la formación de ensuciamiento.

- El sistema tiene dos ciclos de funcionamiento diferenciados correspondientes a los dos sentidos en los que se desplaza el rascador en su movimiento: equicorriente y contracorriente.

- Tal y como se demostró en el Capítulo 7, el factor de bloqueo es una variable fundamental, que influye fuertemente en los efectos provocados por el rascador.

En el Apartado 8.2, se estudia la pérdida de presión que se produce en situaciones de trabajo con el rascador en movimiento.

Por último, en el Apartado 8.3, se cuantifica la potencia necesaria para producir el movimiento del rascador mediante el sistema de impulsión hidráulico.

\subsection{Caída de presión en régimen estático}

En presente apartado, se estudia la caída de presión que se produce en el tubo en el que el rascador se encuentre estático. En esta situación el dispositivo insertado tiene el mismo principio de funcionamiento que otros dispositivos insertados estáticos (muelles, láminas en 
espiral, ...). Los dispositivos de este tipo promueven el mezclado del flujo entre las zonas interior y exterior del campo fluido, mejorando de este modo la transferencia de calor. Además, el diseño del rascador con tacos semicirculares reduce significativamente la sección de paso en cada taco, lo cual aumenta considerablemente la velocidad del fluido, produciendo un segundo efecto de mejora de la transferencia de calor. En el Capítulo 7 se han observado ambos fenómenos en el campo fluido adquirido mediante PIV.

La pérdida de presión que se produce en el intercambiador de calor mejorado tiene dos orígenes: el esfuerzo cortante en la pared y las fuerzas de arrastre en el flujo.

Por un lado, el esfuerzo cortante en la pared depende del gradiente de velocidad y de las propiedades reológicas $m$ y $n$, tal y como muestra la definición del mismo $\left(\tau=m \gamma^{n}\right)$. La presencia de los tacos rascadores, que bloquean el paso del fluido desviándolo y acelerándolo, provocan efectos locales de aumento de los esfuerzos cortantes.

Por otro lado, la expansión del flujo aguas abajo del rascador genera la separación del flujo de la pared, provocando, a números de Reynolds suficientemente altos, dos recirculaciones simétricas. Esto implica la aparición de grandes fuerzas de arrastre sobre el flujo, que también derivan en un aumento de la caída de presión.

El análisis adimensional del caso, realizado en el Apartado 2.1, reduce el problema al estudio de los siguientes números adimensionales: la relación de diámetros $\alpha$, el número de Reynolds, el factor de fricción $f$ y, dependiendo de la definición del número de Reynolds utilizada, el índice de comportamiento de flujo $n$. En los capítulos 2 y5 se introducen dos definiciones del número de Reynolds $R e_{b}$ y $R e_{g}$, definidos según las Ecuaciones 2.7 y 5.22 , cada una de las cuales ofrece una información diferente.

Por un lado, la definición del número de Reynolds básico $R e_{b}$ (Ec. 8.1) no incluye el término $\phi(n)$, que aparece únicamente en fluidos no newtonianos. Por lo tanto, la utilidad de este monomio reside en que, al representar $R e_{b}$ frente a $f$, se puede observar el efecto que tendría en la caída de presión, el hecho de variar la propiedad $n$ del fluido si $R e_{b}$ se mantiene constante. Es decir, que es posible visualizar el efecto de utilizar en las mismas condiciones dos fluidos con valor de $n$ distinto. Nótese no obstante que para que dos fluidos con distinto valor de $n$ circulen con el mismo valor de $R e_{b}$, el caudal circulante debe ser distinto.

Por otro lado, utilizando la definición del número de Reynolds generalizado $R_{g}$ (Ec. 8.2) se consigue el objetivo contrario. Esta definición del número de Reynolds incorpora completamente la información del comportamiento seudoplástico del fluido, o lo que es lo mismo, la función $\phi(n)$, en la definición de la viscosidad generalizada. La variación del comportamiento del fluido debida a la variación de $n$ queda oculta y todas las curvas de caída de presión 


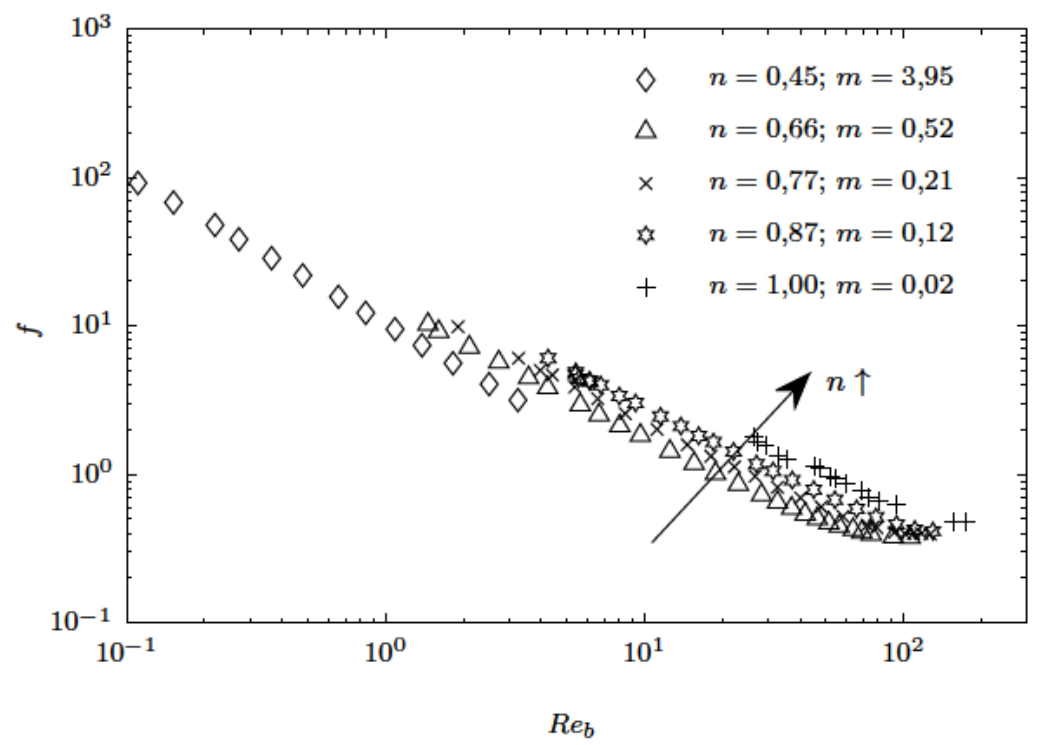

Figura 8.1: Representación de resultados de caída de presión utilizando la definición básica del número de Reynolds, $R e_{b}$. Únicamente se muestran los resultados más representativos.

en régimen laminar (para diferentes $n$ ) son la misma. Con ello se consigue simplificar al máximo el problema de cara al estudio de otras variables como son la velocidad de rascado (Apartado 8.2) o la transferencia de calor (Capítulo 9).

En cuanto al factor de fricción se utiliza la definición obtenida en el Capítulo 2 (Ec. 2.8).

Los ensayos se han realizado siguiendo el procedimiento descrito en el Apartado 6.1. Se han realizado experimentos utilizando disoluciones de CMC en agua al $1 \%$, abarcando números de Reynolds en el rango $0,2<R e_{g}<600$, donde el fluido ha variado desde un comportamiento fuertemente seudoplástico $(n=0,45$ y $m=3,95)$ a un comportamiento totalmente newtoniano newtoniano ( $n=1$ y $m=0,02) .0,2<R e_{g}<600$,

\subsubsection{Resultados de pérdida de presión}

En la Fig. 8.1 se representa la caída de presión en el tubo utilizando la definición básica del número de Reynolds (Ec. 2.7).

$$
R e_{b}=\frac{\rho u_{b} D_{h}}{m}\left(\frac{D_{h}}{u_{b}}\right)^{n-1}
$$

En dicha gráfica se puede observar el efecto de la variación de $\phi(n)$ provocada por la variación del índice de comportamiento de flujo $n$. Si se consideran dos fluidos circulando con el mismo 
número de Reynolds básico $R e_{b 1}=R e_{b 2}$, con valores de coeficiente de consistencia idénticos $m_{1}=m_{2}$ e índices de comportamiento distintos $0,45 \leq n_{1}<n_{2} \leq 1$, de los resultados de la Fig. 8.1 se deduce que $f_{1}<f_{2}$. Por lo tanto, se deduce que dentro del rango de $n$ estudiado, a mayor valor de $n$ y si no varía el número de Reynolds $R e_{b}$, la caída de presión será mayor. No obstante, se debe tener en cuenta que en un fluido real, una variación de $n$ suele comprender también variaciones de $m$. Además si $n_{1} \neq n_{2}, m_{1}=m_{2}$ y $R e_{b 1}=R e_{b 2}$, esto implica necesariamente que $u_{b, 1} \neq u_{b, 2}$ con lo que la comparativa resulta incompleta. En consecuencia, en el Apartado 8.1.2 se estudia más en profundidad la influencia del comportamiento seudoplástico en la caída de presión.

De cara a estudios posteriores, tal y como se ha comentado con anterioridad, la representación de los resultados utilizando $R e_{g}$ será más conveniente, debido a la simplificación que implica el uso de la viscosidad generalizada en el número de Reynolds.

$$
R e_{g}=\frac{\rho u_{b} D_{h}}{\mu_{g}} ; \quad \mu_{g}=m n^{d} c^{n-1}\left(\frac{u_{b}}{D_{h}}\right)^{n-1}
$$

donde los coeficientes $c$ y $d$ se obtuvieron en el Apartado 5.3.3 y están indicados en la Tabla 5.8 .

En la Fig. 8.2 se representan un total de 161 medidas, con fluido en diferentes estados de degradación y temperaturas distintas, con los siguientes rangos de variación de las propiedades reológicas: $0,45 \leq n \leq 1$ y $3,95 \geq m \geq 0,02$. En los resultados se observan tres regiones de funcionamiento:

1. Región laminar (I): $R e_{g}<100$.

2. Región de transición (II): $100<R e_{g}<300$.

3. Región turbulenta (III): $R e_{g}>300$.

Al tratarse de un fluido de gran viscosidad, la mayoría de ensayos se encuentran en la región laminar (I). Además en esta región, los resultados obtenidos con fluidos de diferentes propiedades reológicas caen sobre la misma curva. Es de destacar así mismo, lo extremadamente laminar que puede llegar a ser el flujo, habiéndose obtenido números de Reynolds tan bajos como el del siguiente ensayo: con el fluido recién preparado (sin degradación), a $26^{\circ} \mathrm{C}$ y caudal pequeño $(17 \mathrm{l} / \mathrm{h})$ se consigue un número de Reynolds $R e_{g}=0,2$.

Las regiones II y III se observan mejor en la Fig. 8.3 donde además se comparan los resultados con los obtenidos por Solano (2009b) para fluidos Newtonianos. En la región II 


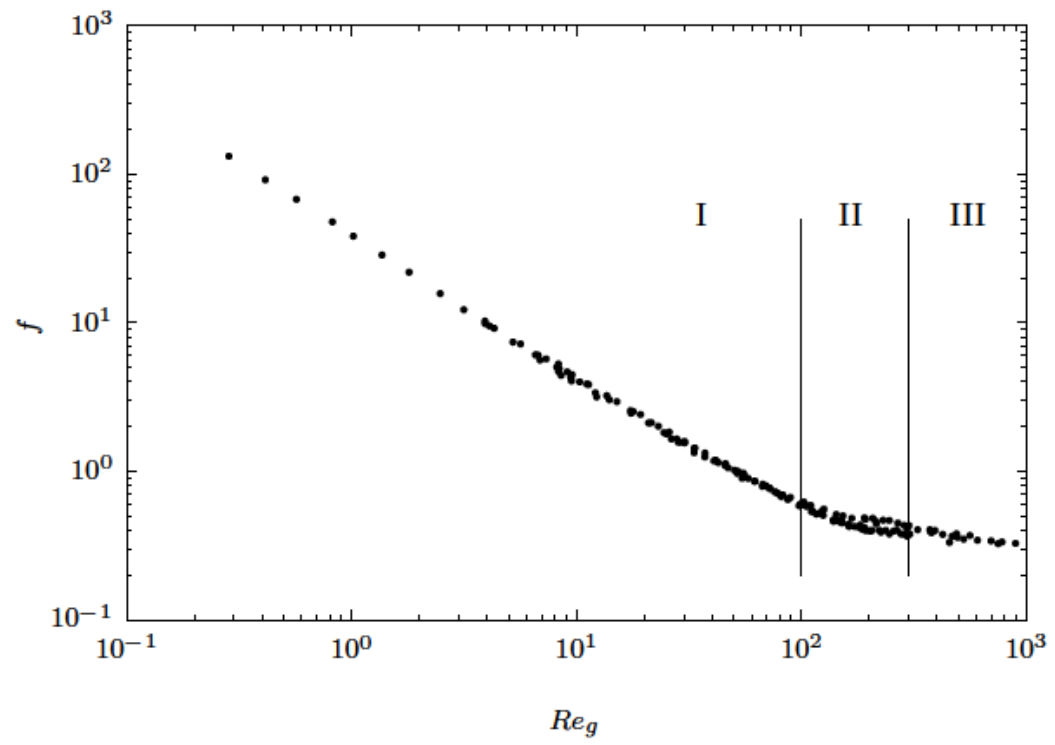

Figura 8.2: Representación de resultados de caída de presión utilizando la definición del número de Reynolds generalizado. Propiedades reológicas del fluido en los rangos $0,45 \leq n \leq$ 1 y $3,95 \geq m \geq 0,02$.

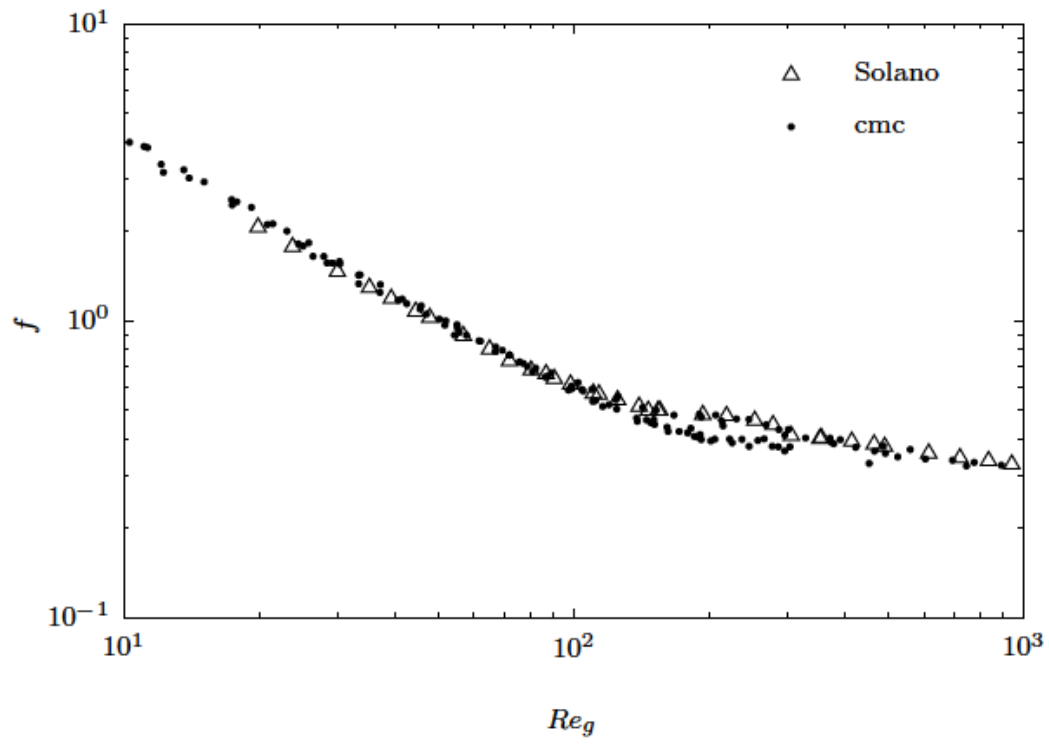

Figura 8.3: Comparación de la caída de presión en el tubo con rascador utilizando fluidos newtonianos (Solano, 2009) y no newtonianos. 
se produce la transición a la turbulencia. En dicha región se observa que las curvas de los fluidos con distintas propiedades reológicas se separan. Se puede apreciar que los resultados con $n \approx 1$ provocan valores de $f$ mayores para un mismo $R e_{g}$ que los fluidos con comportamiento más seudoplástico. El efecto comentado tiene su fundamento en la definición de viscosidad generalizada que da lugar al número de Reynolds generalizado. Dicha definición de la viscosidad sólo es válida en la región laminar (I), lo cual implica que al llegar a la región de transición la viscosidad efectiva cambie y las curvas de los distintos fluidos se separen.

En la región turbulenta (III) únicamente ha sido posible realizar medidas con fluidos degradados hasta un comportamiento newtoniano $(n \approx 1)$, cuyos resultados coinciden plénamente con los existentes en la bibliografía. Los estudios existentes en la bibliografía para régimen turbulento en tubo liso sugieren que las curvas en de Reynolds generalizado frente a $f$ son ligeramente distintas para cada valor de $n$ (Chhabra y Richardson, 2008). Es probable que esto también suceda en la geometría estudiada, aunque no se puede cuantificar este efecto a partir de los resultados obtenidos.

\subsubsection{Efecto del comportamiento seudoplástico en la caída de pre- sión}

En el presente apartado, se trata de discernir el efecto de la seudoplasticidad en la caída de presión en régimen laminar. Hasta ahora, por un lado, en la Fig. 8.1 se han representado los resultados utilizando el número de Reynolds básico $R e_{b}$, en los cuales se observa el efecto de la influencia de $\phi(n)$ en la caída de presión. Por otro lado, se ha definido un

número de Reynolds basado en la viscosidad generalizada $R e_{g}$, que presenta una curva de $f$ frente al $R e_{g}$ independiente del índice de comportamiento de flujo $n$ (Fig. 8.2). Sin embargo, queda por conocer la respuesta a la cuestión clave: ¿cuál es el efecto global que produce el comportamiento seudoplástico en la caída de presión de un flujo?.

Con el objetivo de responder a dicha pregunta se realiza la siguiente comparativa:

- Sean dos tubos con rascador idénticos, con unas dimensiones concretas: $D, L, d, P, \ldots$

- Por cada uno de los tubos circula un fluido:

1. Tubo N: fluido newtoniano $(n=1)$, de viscosidad $\mu$.

2. Tubo NN: fluido seudoplástico $(n<1)$, de propiedades reológicas $m$ y $n$, siendo la viscosidad $\mu_{g}$ definida en apartados anteriores. 
- El resto de propiedades termofísicas de los fluidos son idénticas $\left(\rho, c_{p}, \ldots\right)$.

- Además se establece una condición de similaridad entre las viscosidades de ambos flujos para un punto de funcionamiento de referencia. Para un valor de número de Reynolds de referencia en ambos tubos $R e_{g, 0}$, los caudales circulantes deben ser iguales. Esto implica además que las caídas de presión en sendos tubos los factores de fricción sean los mismos, así como las viscosidades de ambos flujos $\mu=\mu_{g, 0}$.

El objetivo es, partiendo de dicho valor de Reynolds de referencia en que ambos flujos tienen las mismas prestaciones, conocer la variación de las mismas en cada tubo al cambiar las condiciones de funcionamiento en ambos en la misma medida. Se considera por ejemplo, el caso de que el sistema de bombeo varíe la altura manométrica que entrega con respecto a la situación de referencia en la misma medida en ambos tubos. En este caso se produce una variación en el caudal circulante por sendos tubos, que no será la misma y se desea cuantificar dicha diferencia.

Para realizar este apartado, se puede suponer, cometiendo un mínimo error, que el exponente del número de Reynolds en la Ec.5.19 es $b \approx-1$. Así, para ambos fluidos se debe cumplir que

$$
f R e_{g}=a
$$

Si el caudal circulante por ambos tubos es el mismo, también lo es la velocidad media del flujo $u_{b, 0}$. De la Ec. 8.3, se deduce por tanto que el factor de fricción también debe ser el mismo, así como $P_{L, 0}$ (ya que $u_{b, 0}$ es la misma). Como los números de Reynolds en ambos casos deben ser iguales, se obtiene la relación que debe existir entre la viscosidad del fluido newtoniano y las propiedades reológicas del fluido seudoplástico:

$$
\begin{gathered}
R e_{N}=R e_{g, N N} \\
\frac{\rho u_{b, 0} D_{h}}{\mu}=\frac{\rho u_{b, 0}^{2-n} D_{h}^{n}}{m c^{n-1} n^{d}}
\end{gathered}
$$

de modo que la viscosidad del fluido newtoniano $\mu$, para que se cumplan las condiciones anteriormente descritas, debe ser

$$
\mu=m c^{n-1} n^{d}\left(\frac{u_{b, 0}}{D_{h}}\right)^{n-1}
$$


Una vez establecidas las condiciones en el punto de funcionamiento de referencia, se desea evaluar cómo varía el caudal impulsado (o la velocidad media $u_{b, N}$ y $u_{b, N N}$ ) en ambos tubos, cuando el sistema de bombeo entrega una altura distinta, es decir, permite que $p_{L}$ varíe en un determinado grado.

\section{Fluido newtoniano}

En primer lugar se obtiene la expresión de la velocidad media en el tubo $\mathrm{N}, u_{b, N}$, en función de las condiciones iniciales y de $p_{L}$.

Sustituyendo en la Ec. 8.3 las definiciones de los números adimensionales, se obtiene

$$
\begin{gathered}
\frac{p_{L} D_{h}}{2 \rho u_{b, N}^{2}} \frac{\rho u_{b, N} D_{h}}{\mu}=a \\
\frac{p_{L} D_{h}^{2}}{2 u_{b, N}} \frac{1}{\mu}=a
\end{gathered}
$$

Al ser un fluido newtoniano, su viscosidad no varía, de modo que ésta viene dada por la Ec. 8.4,

$$
\frac{p_{L} D_{h}^{2}}{2 u_{b, N}} \frac{1}{m c^{n-1} n^{d}\left(\frac{u_{b, 0}}{D_{h}}\right)^{n-1}}=a
$$

y despejando se obtiene el valor de la velocidad media del flujo en el tubo $\mathrm{N}$ en función de las condiciones iniciales, $p_{L} \mathrm{y}$ de las propiedades reológicas del otro fluido

$$
u_{b, N}=\frac{p_{L} D_{h}^{n+1} u_{b, 0}^{1-n}}{2 a m c^{n-1} n^{d}}
$$

\section{Fluido no newtoniano}

Realizando el procedimiento equivalente, con el fluido seudoplástico que circula por el tubo NN, de nuevo se parte de la Ec. 8.3, y se sustituyen $R e_{g}$ y $f$ por sus definiciones respectivas,

$$
\frac{p_{L} D_{h}}{2 \rho u_{b, N N}^{2}} \frac{\rho u_{b, N N}^{2-n} D_{h}^{n}}{m c^{n-1} n^{d}}=a
$$

y finalmente se obtiene el valor de la velocidad media del flujo en el tubo NN en función de las condiciones iniciales, $p_{L}$ y de las propiedades reológicas del fluido que circula por el tubo, 


$$
u_{b, N N}^{n}=\frac{p_{L} D_{h}^{n+1}}{2 a m c^{n-1} n^{d}}
$$

\section{Resultado}

De este modo, para conocer como ha variado el caudal si variamos la $p_{L}$ en la misma medida en ambos tubos, calculamos el cociente entre las ecuaciones:

$$
\frac{u_{b, N}}{u_{b, N N}^{n}}=\frac{\frac{p_{L} D_{h}^{n+1} u_{b, 0}^{1-n}}{2 a m c^{n-1} n^{d}}}{\frac{p_{L} D_{h}^{n+1}}{2 a m c^{n-1} n^{d}}}=u_{b, 0}^{1-n}
$$

y obtenemos la relación que guardan las nuevas velocidades medias en sendos tubos, para una misma variación de $p_{L}$, en función de la velocidad media en el punto de referencia:

$$
u_{b, N}=u_{b, 0}\left(\frac{u_{b, N N}}{u_{b, 0}}\right)^{n}
$$

con $0,45<n<1$.

Si la caída de presión aumenta $p_{L}>p_{L, 0}$ claramente $u_{b, N N}>u_{b, 0}$ y viceversa.

Por lo tanto, se puede concluir que el caudal de fluido no newtoniano será más sensible a los cambios de $p_{L}$ que el newtoniano, ya que:

1. Al aumentar $p_{L}$ en ambos tubos, el caudal aumenta más en el tubo NN (fluido no newtoniano) que en el tubo $\mathrm{N}$ (fluido newtoniano).

2. Al disminuir $p_{L}$ en ambos tubos, el caudal disminuye más en el tubo NN(fluido no newtoniano) que en el tubo $\mathrm{N}$ (fluido newtoniano).

De esto también se deduce, que si consideramos el caso en el que variamos $u_{b, N}$ y $u_{b, N N}$ en la misma medida en ambos tubos, la variación de $p_{L, N}$ será mayor que la de $p_{L, N N}$.

\subsubsection{Conclusiones}

De los resultados de caída de presión en régimen estático obtenidos y el análisis realizado, se llega a las siguientes conclusiones:

1. Se han realizado ensayos de pérdida de presión en régimen estático del rascador para números de Reynolds $0,2<R e_{g}<600$, utilizando para ello fluidos de distinto comportamiento reológico: desde un comportamiento fuertemente seudoplástico $(n=0,45 \mathrm{y}$ $m=3,95)$ a un comportamiento totalmente newtoniano $(n=1$ y $m=0,02)$. 
2. Mediante el uso del número de Reynolds generalizado, en régimen laminar, la curva de $f$ frente $R e_{g}$ es única para cualquier valor de $n$.

3. Para $R e_{g}<100$ el flujo es laminar. La región de transición se alcanza para valores de Reynolds entre $100<R e_{g}<200$, donde el valor de $R e_{g}$ en el que se alcanza dicha región varía con $n$. A partir de $R e_{g} \geq 300$ el flujo es completamente turbulento.

4. En el régimen de transición se observan diferentes comportamientos en función del índice de comportamiento de flujo $n$.

5. En régimen turbulento no se observa influencia de $n$ en las curvas de $f$ frente a $R e_{g}$, aunque los resultados obtenidos en dicho rango se corresponden con valores de $n$ cercanos a 1.

6. En base a las correlaciones obtenidas en régimen laminar, se ha estudiado la influencia del comportamiento seudoplástico en la relación entre el caudal circulante por el tubo con rascador y la caída de presión en el mismo. Para ello se ha estudiado el comportamiento de dos fluidos que circulan por el interior de dos tubos con rascador insertado estático de idénticas dimensiones, el tubo $\mathrm{N}$ con un fluido newtoniano y el tubo NN con un fluido no newtoniano, que para un mismo caudal de referencia, tengan un mismo valor del número de Reynolds $R e_{g}$ (únicamente para dicho caudal). De dicho estudio se ha concluido que:

a) Ante cambios en $p_{L}$, el caudal de fluido no newtoniano será más sensible que el newtoniano. Por un lado, al aumentar $p_{L}$ en ambos tubos en la misma proporción, el caudal aumenta más en el tubo NN (fluido no newtoniano) que en el tubo N (fluido newtoniano). Por otro lado, al disminuir $p_{L}$ en ambos tubos en la misma proporción, el caudal disminuye más en el tubo NN (fluido no newtoniano) que en el tubo N (fluido newtoniano).

b) Si se considera el caso en el que se varía el caudal circulante en la misma medida en ambos tubos, la variación de $p_{L, N}$ (fluido newtoniano) será mayor que la de $p_{L, N N}$ (fluido no newtoniano). 


\subsection{Caída de presión en régimen dinámico}

En este apartado se estudia el funcionamiento del intercambiador de calor cuando el rascador está en movimiento. El movimiento alternativo del dispositivo rascador busca obtener los siguientes beneficios en el proceso:

1. Limpieza de la superficie interior del tubo. Al trabajar con todo tipo de fluidos, pero especialmente en el caso de fluidos viscosos, se suele acumular ensuciamiento en la superficie interior del tubo, lo que puede generar ineficiencias en el proceso de intercambio de calor. El rascado continuo de la superficie evita que se forme ensuciamiento o, llegado el caso, lo elimina.

2. Mezclado del flujo. El movimiento del rascador fomentará el mezclado del flujo, obligando al flujo a desprenderse de la zona cercana a la pared del tubo y desplazarse hacia la zona interna del mismo, lo cual mejora significativamente la eficiencia de la transmisión de calor.

Las mencionadas son dos ventajas muy importantes en un proceso de transferencia de calor y además, constituyen la razón principal por la cual se utilizan los Intercambiadores de Calor de Superficie Rascada (ICSR). Sin embargo, este aumento de prestaciones tiene sus contrapartidas:

1. Gasto energético del sistema de impulsión del rascador. Resulta obvio que el elemento móvil necesita de un sistema que le proporcione dicho movimiento, el cual tendrá un consumo energético que disminuye la eficiencia global del sistema.

2. Caída de presión adicional en el tubo causada por el rascador. En el apartado anterior se ha estudiado la caída de presión producida por el rascador en régimen estático. Al estar el rascador en movimiento la caída de presión debe variar respecto a dicho régimen.

3. Oscilación de caudal. El movimiento de rascado alternativo puede provocar cambios bruscos en la presión que ha de vencer el sistema de bombeo; si éste no tiene una curva de caudal-presión plana, se producirán oscilaciones de caudal.

Por otro lado, todos los efectos mencionados dependen de la velocidad y el sentido de rascado. En el Capítulo 7, se ha comprobado que el parámetro más representativo del movimiento del rascador en el flujo es el factor de bloqueo $\beta=1-v_{s} / u_{b}$, y que el campo de velocidades del flujo depende fuertemente del mismo. Así, es de esperar que los efectos enumerados anteriormente también sean función de $\beta$. 


\subsubsection{Selección del sistema de bombeo}

En toda instalación en la cual se desea impulsar un fluido, el diseñador se verá en la tesitura de tener que seleccionar un sistema de bombeo. Además de la problemática habitual, si el fluido de trabajo es altamente viscoso, se hace necesario trabajar con bombas de desplazamiento positivo, no siendo funcionales las centrífugas. Las bombas de desplazamiento positivo sí cumplirán correctamente la función, pero tienen algunos inconvenientes como son las pérdidas que se producen por el rozamiento entre las piezas, o el flujo pulsado que puede aparecer dependiendo del diseño de las mismas.

Por un lado, para evitar los rozamientos entre las piezas existen tres posibles soluciones:

1. Uso de fluidos de trabajo con propiedades lubricantes

2. Mezclar lubricantes con el fluido de trabajo.

3. Aumentar las tolerancias en el diseño de la mecánica.

Por otro lado, para evitar tener un flujo pulsado se puede optar por:

1. Utilizar diseños de bombas volumétricas que por su sistema de funcionamiento no provoquen flujos pulsados.

2. Dimensionar correctamente la bomba, de forma que funcione siempre a revoluciones medias y altas disminuyendo el pulsado.

Éstas soluciones son válidas habitualmente, pero en el caso ensayado en la presente investigación la problemática se complica:

1. Falta de lubricación. Por una parte, el fluido creado mediante la disolución de CMC en agua no es un fluido con propiedades notablemente lubricantes, ya que su viscosidad disminuye fuertemente al aumentar el esfuerzo cortante, que es precisamente el caso en el que la lubricación es necesaria. Y por otra parte, este fluido se utiliza habitualmente en procesos industriales en los que no es aceptable el añadido de aceites (principalmente en la industria alimenticia), por lo que dicho sistema de lubricación no es viable.

2. Ante la baja lubricación, un sistema de bombeo diseñado con tolerancias muy ajustadas se deteriorará con rapidez, producirá muchas pérdidas y calor y probablemente deje de funcionar en un periodo corto. Si además las bombas trabajan a altas revoluciones este proceso se aceleraría. 
3. Aumentar las tolerancias entre los engranajes y de éstos con la carcasa, aumenta la oscilación del flujo producida por la oscilación de la presión, provocada a su vez por el movimiento del rascador. Este efecto es más acusado al utilizar un fluido seudoplástico que al utilizar otros fluidos viscosos, ya que el fluido seudoplástico disminuye considerablemente su viscosidad al aumentar el esfuerzo cortante, situación que se da precisamente en los engranajes de la bomba. Así, en el interior de la bomba, el fluido tiene una viscosidad mucho menor, lo que provoca mayores pérdidas volumétricas en la misma y que ésta funcione en una región, en la que su curva de caudal presión es considerablemente más inclinada que la curva teórica de una bomba volumétrica.

4. Trabajar con un sistema de bombeo girando a altas revoluciones provoca mayores efectos cortantes en su interior y, en consecuencia, una degradación acelerada del fluido. Por lo tanto debe evitarse en lo posible que las bombas trabajen a altas revoluciones.

Así, en la selección de un sistema de bombeo que deba trasegar el fluido de trabajo (disoluciones de CMC al $1 \%$ en agua) u otros de características similares, se debe llegar a un compromiso entre todos estos factores.

En principio parece razonable recurrir a un sistema de bombeo con tolerancias holgadas y sobredimensionado, de forma que trabaje a bajas revoluciones. Sin embargo esta elección, que implica un caudal de trabajo menos estable, puede acarrear variaciones en los resultados obtenidos: en la caída de presión media en el tubo (en régimen de funcionamiento dinámico del rascador), en la eficiencia en la transferencia de calor o en la energía requerida para mover el dispositivo insertado.

Para poder tomar una decisión respecto a la elección del sistema de bombeo en base a un conocimiento más profundo de la situación, se decide realizar ensayos de pérdida de presión y de transmisión de calor utilizando dos sistemas de bombeo diferentes: uno con bombas sobredimensionadas y tolerancias holgadas (BB), y otro con tolerancias más ajustadas y de menor tamaño que funcionará a más revoluciones por minuto (BA).

\subsubsection{Resultados}

Para el estudio de la caída de presión en régimen dinámico se utiliza la definición del número de Reynolds generalizado $R e_{g}$. Esto permite que los resultados en régimen laminar sean independientes de la variación en la viscosidad efectiva, introducida por variaciones en el índice de comportamiento de flujo $n$. 


\begin{tabular}{lcc}
\hline & EC & CC \\
\hline$a$ & 44.931 & 40.63 \\
$b$ & -0.9593 & -0.9307 \\
$c$ & 0.4624 & 0.54 \\
$i(f)(\%)$ & 7.9 & 7.3 \\
Error (\%) & 15.4 & 14.2 \\
\hline
\end{tabular}

Tabla 8.1: Coeficientes experimentales de los experimentos de caída de presión en régimen dinámico correspondientes a la Ec 8.7. Correlaciones obtenidas para el régimen laminar. Se muestran la incertidumbre típica y el error correspondiente a un nivel de confianza del $95 \%$ para la estimación del factor de fricción, $f$.

\subsubsection{Caída de presión en equi-/contracorriente}

La caída de presión que se produce en el tubo, depende del número de Reynolds $R e_{g}$, del sentido de rascado y de la relación de velocidades entre este y el fluido, que vienen representados por el factor de bloqueo $\beta$, tal y como se deduce en el Capítulo 7.

En los ensayos realizados, mediante la metodología descrita en el Apartado 6.1, se han analizado ambos sentidos de rascado por separado: equicorriente y contracorriente. Se han realizado experimentos para valores de $-1,5 \leq v_{s} / u_{b} \leq 0,8$, en los que $2,5>\beta>0,2$, donde los valores los ensayos con $\beta>1$ se corresponden con el sentido de rascado contracorriente y los valores de $\beta<1$ con el sentido de rascado equicorriente. Es de destacar que las limitaciones experimentales impiden ensayar a valores de $\beta<0,2$ asociadas a caídas de presión muy bajas o negativas y por ello imposibles de medir con la configuración de la instalación descrita en el Capítulo 6.

Los datos experimentales obtenidos se ajustan adecuadamente a la Ec. 8.7 y los coeficientes experimentales correspondientes se detallan en la Tabla 8.1.

$$
\begin{gathered}
f=a \times R e_{g}^{b} \times \beta^{c} \\
\beta=1-\frac{v_{s}}{u_{b}}
\end{gathered}
$$

Se han realizado ajustes distintos para los sentidos de rascado equicorriente y contracorriente y los resultados de los mismos se muestran en la Tabla 8.1. En sentido equicorriente, la caída de presión viene condicionada por el factor de bloqueo $\beta$ con un exponente de 0,36, que indica una menor influencia de la velocidad de rascado que en sentido contracorriente, 
donde el exponente es de 0,58. En la Fig. 8.8 se representa la caída de presión según las correlaciones obtenidas para diferentes valores de $\beta$.

Por un lado, en las Figuras 8.4 y 8.5 se muestran los resultados obtenidos en los experimentos equicorriente para valores de omega $\beta=0,9 ; 0,8 ; 0,7$ y 0,5 para los ensayos realizados utilizando las bombas BA (de grandes engranajes) y BB (de pequeños engranajes y mayor ajuste) respectivamente. Como es lógico, no hay diferencia entre las prestaciones de ambos tipos de bomba en este sentido. Por otro lado, en las Figuras 8.6 y 8.7 se representan los resultados en sentido contracorriente para las siguientes relaciones de velocidades: $\beta=1,1$; 1,$2 ; 1,3 ; 1,5 ; 2$ y 2,5 .

En cuanto a la transición, en la Fig 8.9 se muestran los números de Reynolds a los que se produce la transición en fluidos newtonianos en función de $\beta$. Únicamente se alcanzan Reynolds de transición a velocidades de rascado bajas ( $\beta$ cercanos a 1 ), para $\beta=$ 0,$9 ; 0,8 ; 1 ; 1,1 ; 1,2 ; 1,3$. De hecho únicamente se aprecia dicha transición en el caso de $\beta=$ 0,$9 ; 1 ; 1,1 ; 1,2$ donde no se observan diferencias significativas entre fluidos con distinto índice de comportamiento de flujo $n$, máxime cuando los ensayos en la región de transición se corresponden con fluidos con valores de $n>0,85$. 


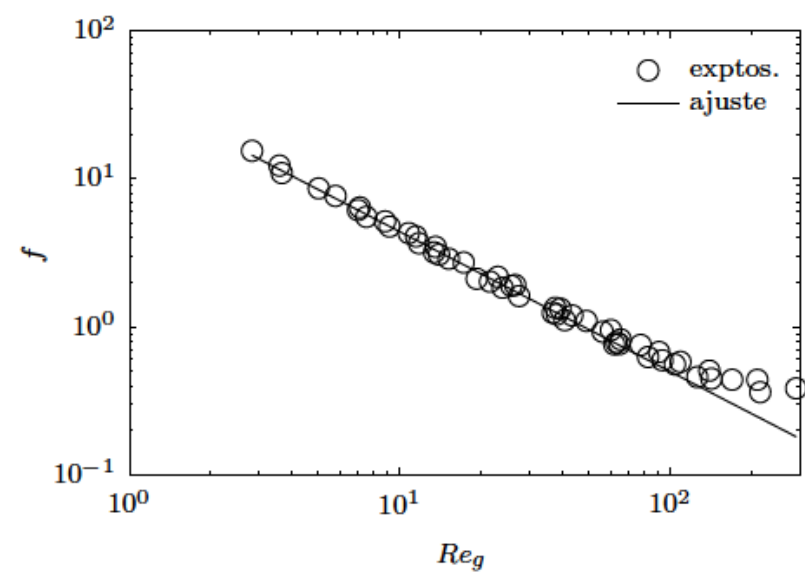

(a) $\beta=0,9$

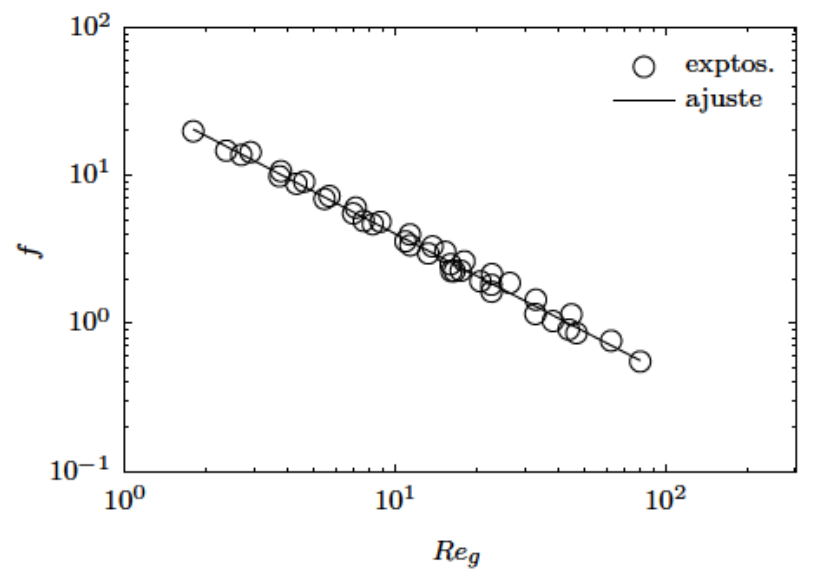

(c) $\beta=0,7$

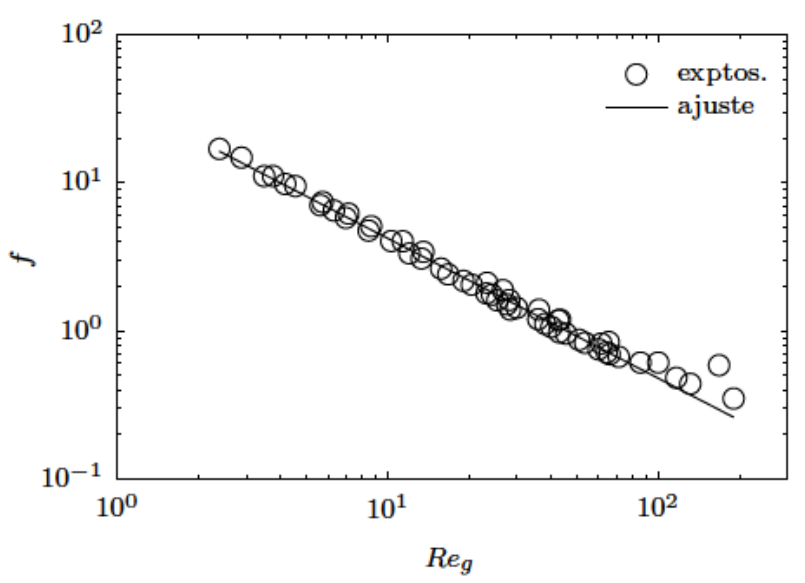

(b) $\beta=0,8$

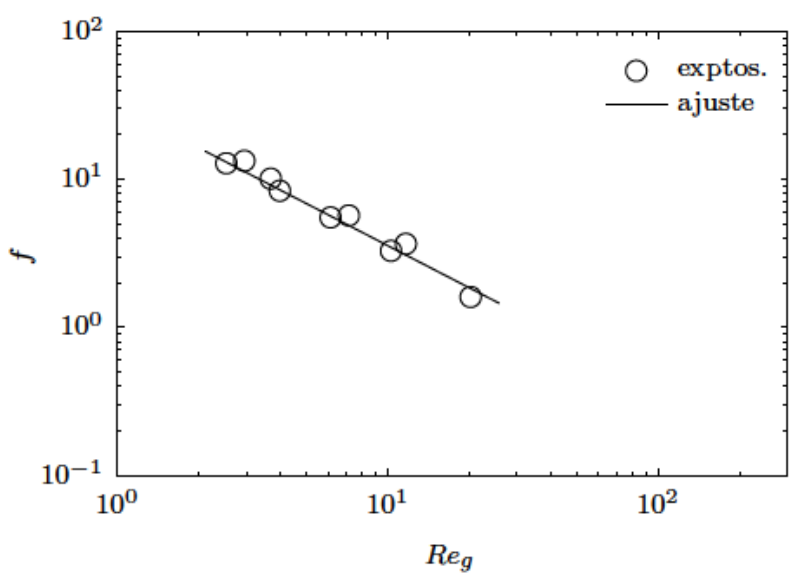

(d) $\beta=0,5$

Figura 8.4: Caída de presión en sentido de rascado equicorriente. Bomba BA. Ajuste según la Ec. 8.7 y la Tabla 8.1. 


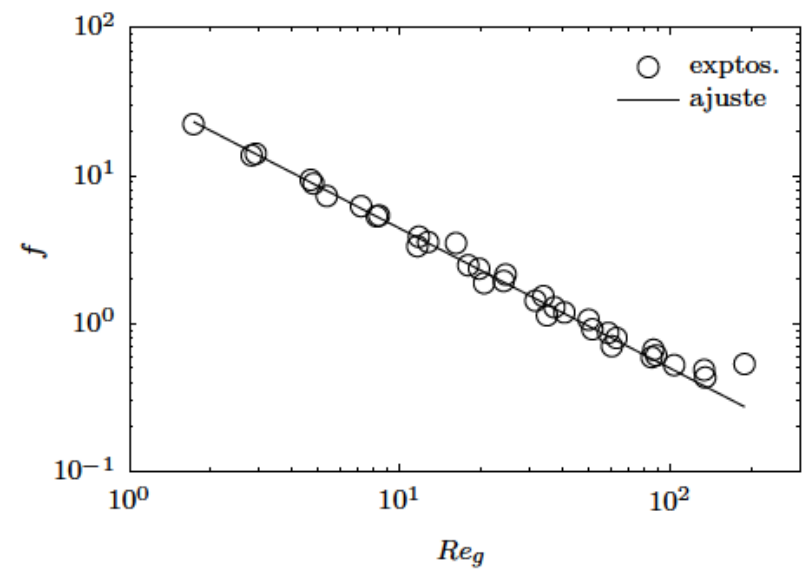

(a) $\beta=0,9$

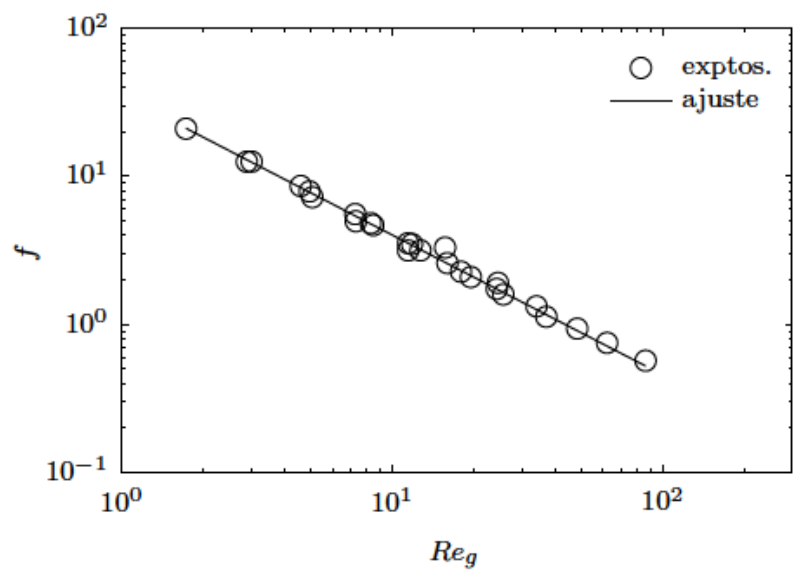

(c) $\beta=0,7$

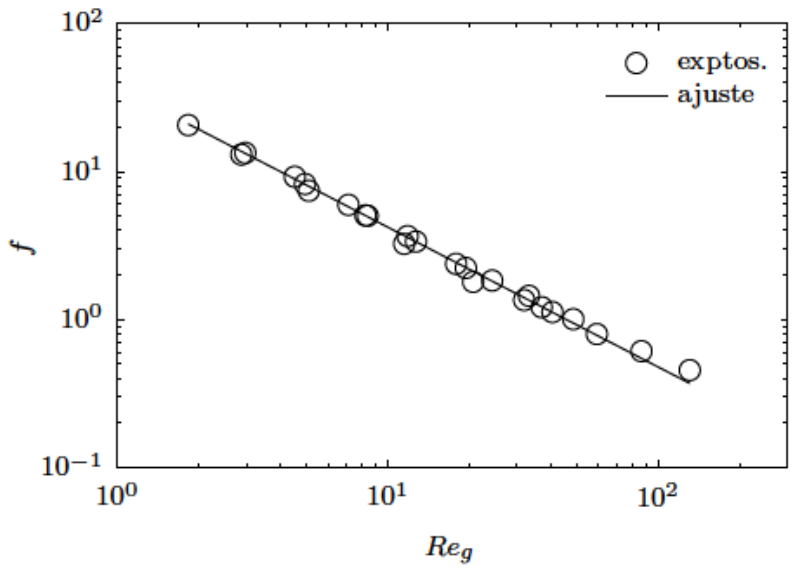

(b) $\beta=0,8$

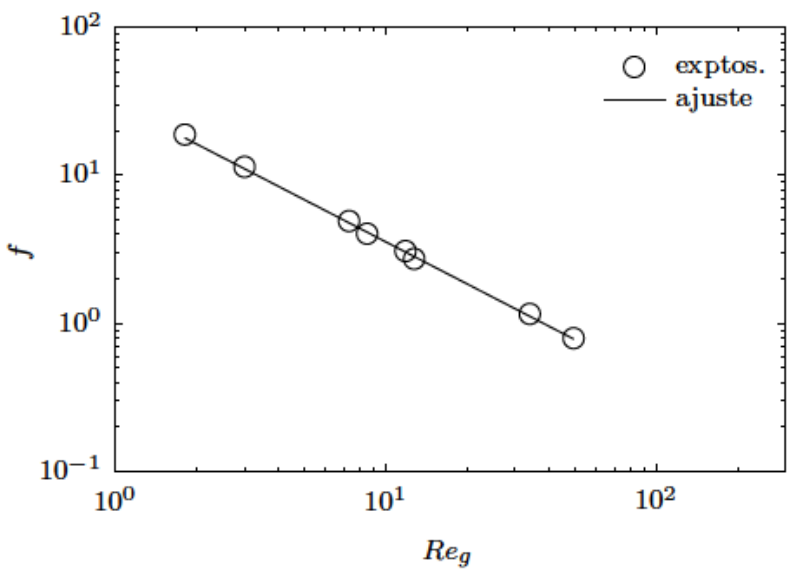

(d) $\beta=0,5$

Figura 8.5: Caída de presión en sentido equicorriente. Bomba BB. Ajuste según la Ec. 8.7 y la Tabla 8.1. 


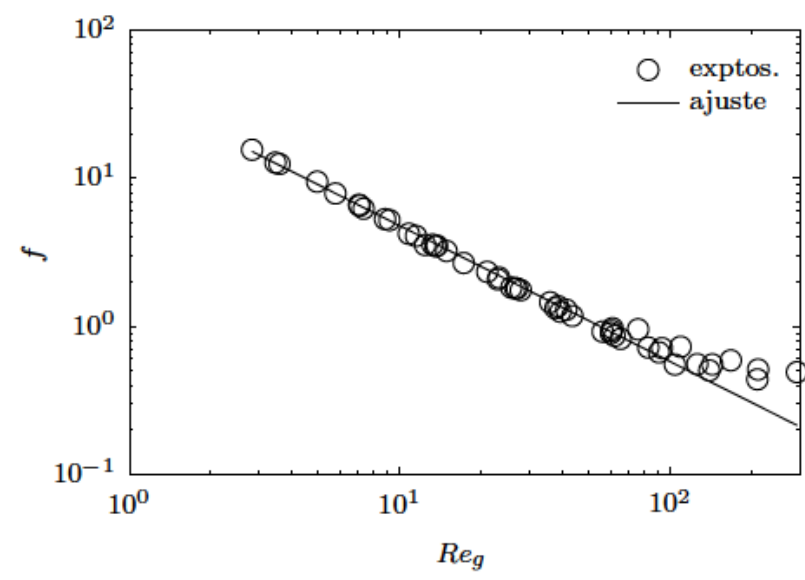

(a) $\beta=1,1$

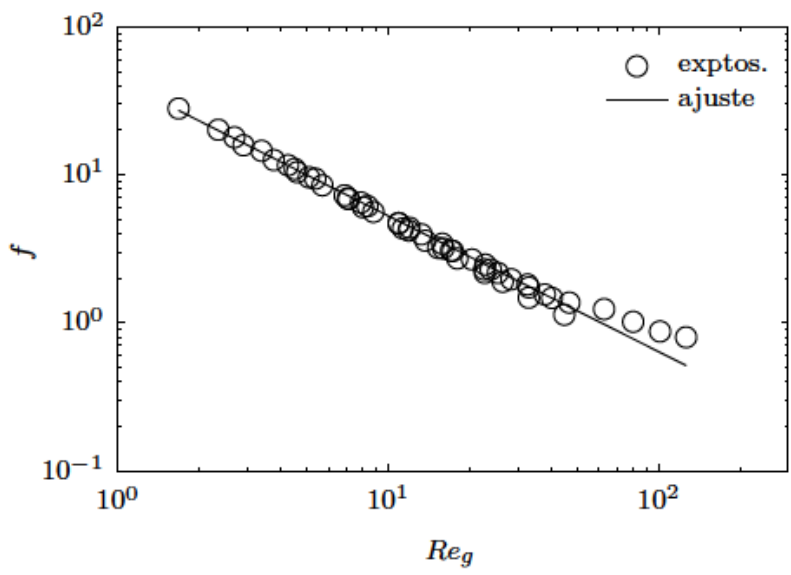

(c) $\beta=1,3$

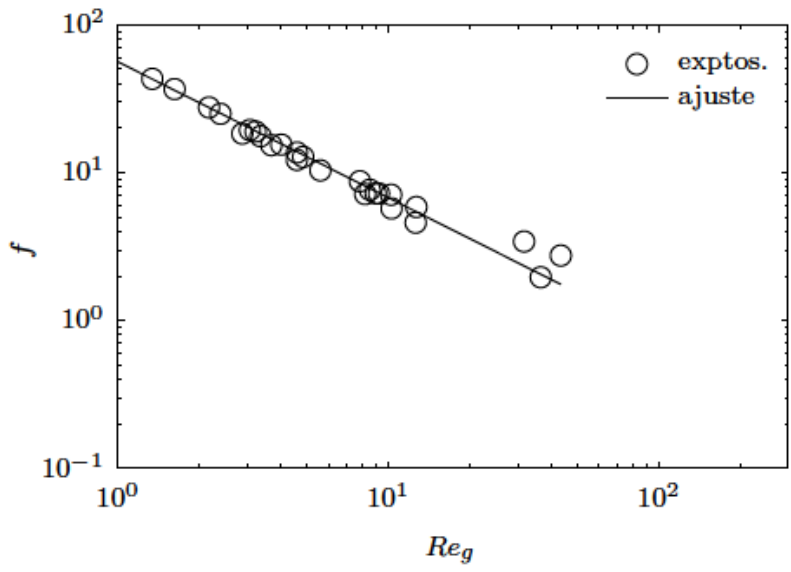

(e) $\beta=2$

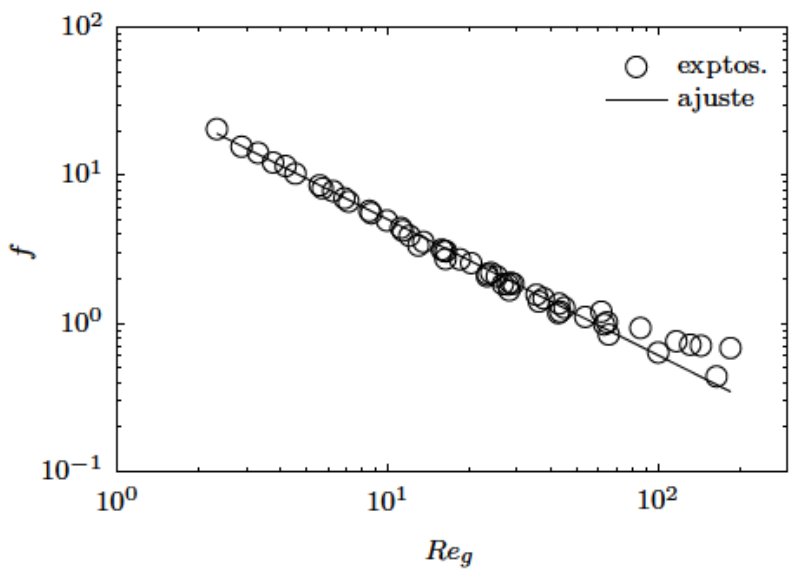

(b) $\beta=1,2$

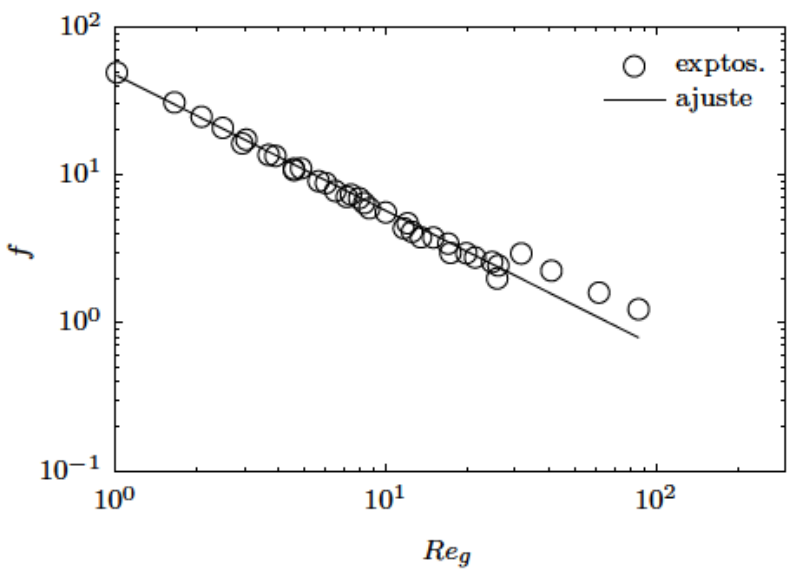

(d) $\beta=1,5$

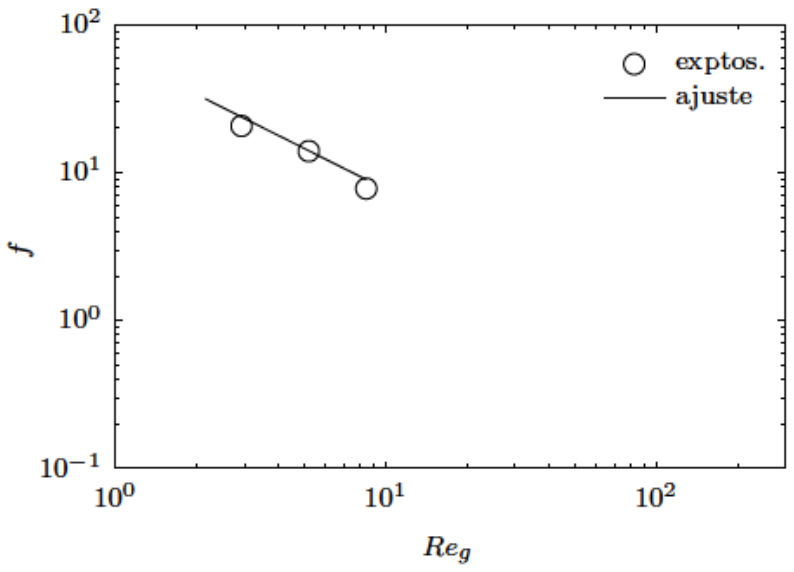

(f) $\beta=2,5$

Figura 8.6: Caída de presión en sentido contracorriente. Bomba BA. Ajuste según la Ec. 8.7 y la Tabla 8.1. 


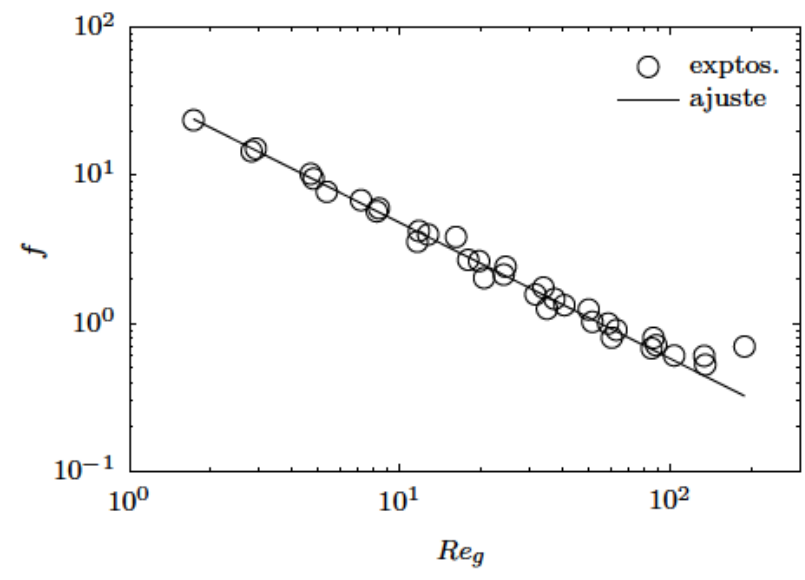

(a) $\beta=1,1$

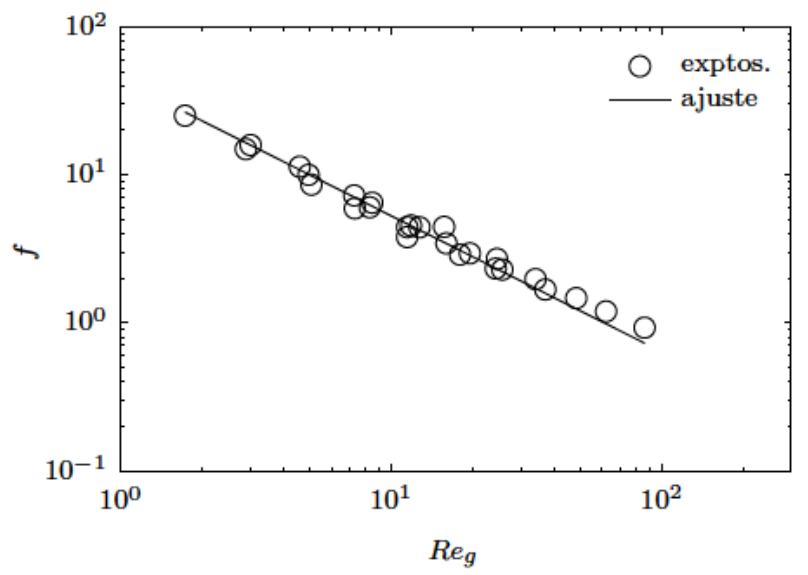

(c) $\beta=1,3$

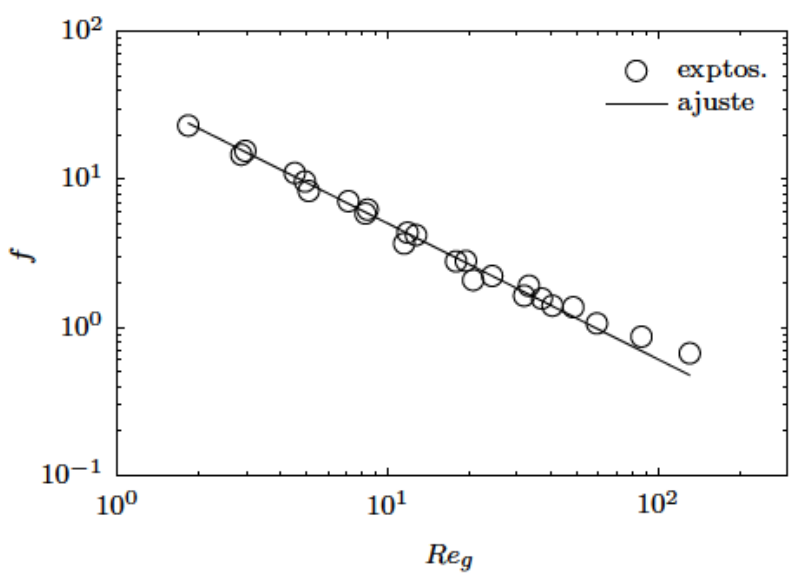

(b) $\beta=1,2$

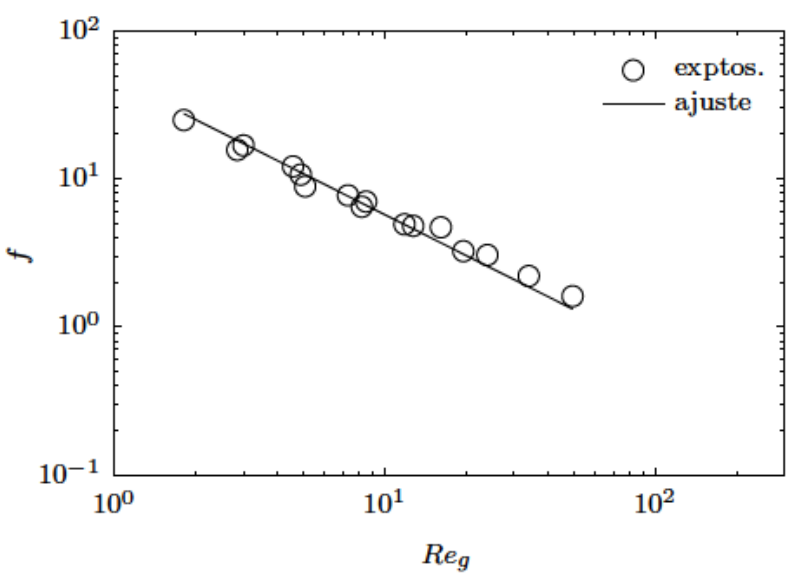

(d) $\beta=1,5$

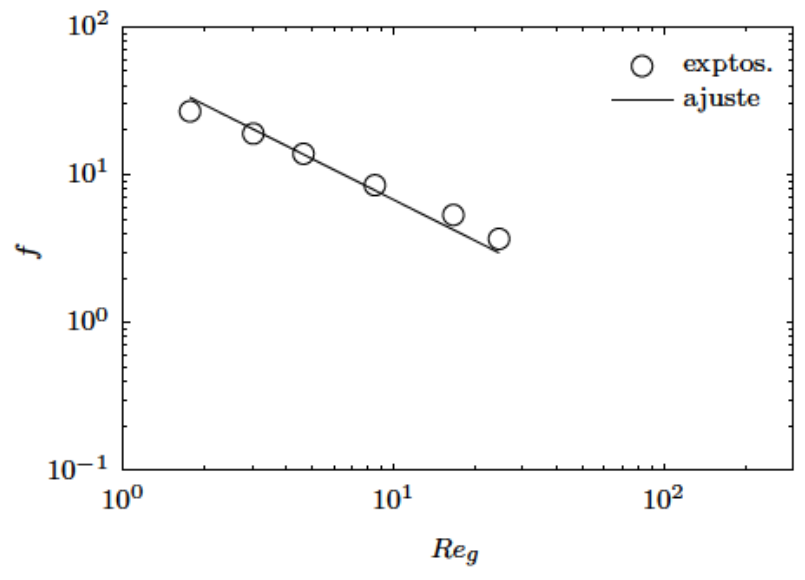

(e) $\beta=2$

Figura 8.7: caída de presión en sentido contracorriente. Bomba BB. Ajuste según la Ec. 8.7 y la Tabla 8.1. 


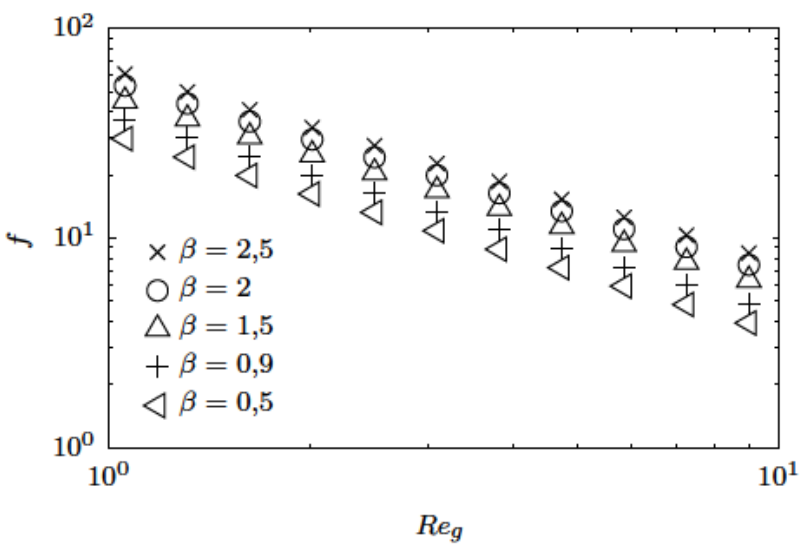

Figura 8.8: Representación de las correlaciones experimentales obtenidas (véase Tabla 8.1).

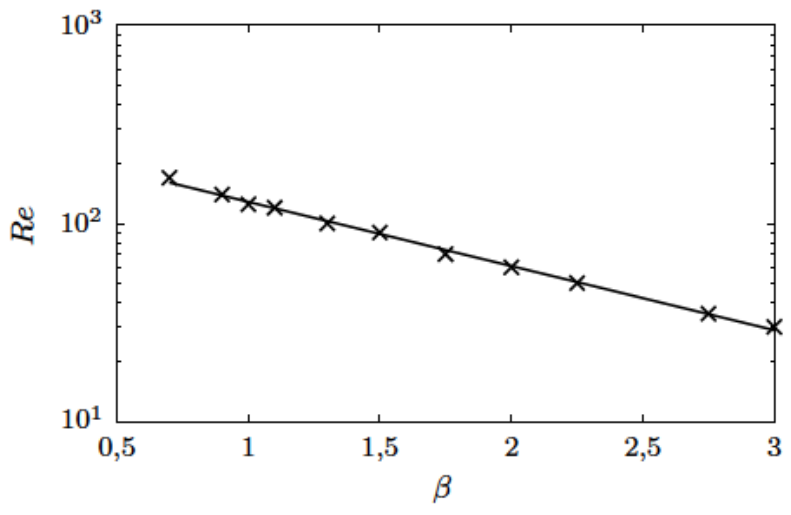

Figura 8.9: Número de Reynolds a partir del cual aparece la transicion en fluidos newtonianos. El ajuste se corresponde a la ecuación: $\ln (R e)=-0,746 \beta+5,6$. Datos extraidos de los estudios de Solano (2009b). 


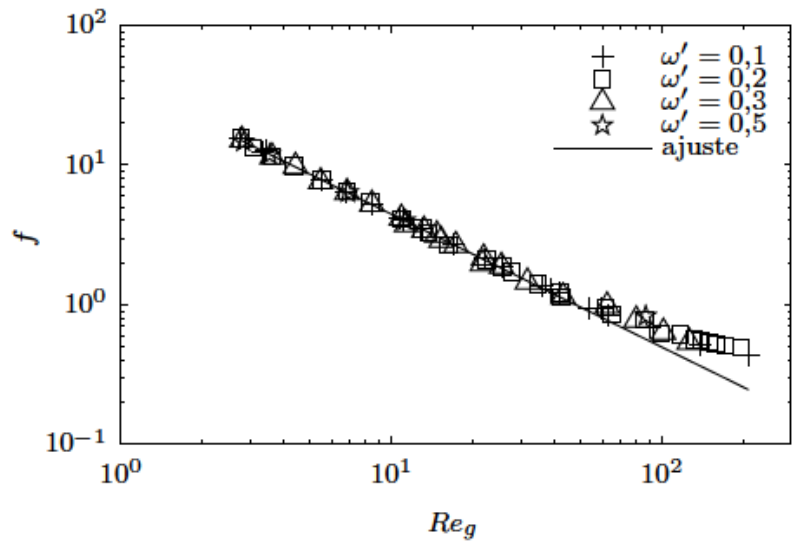

(a) Bomba BA

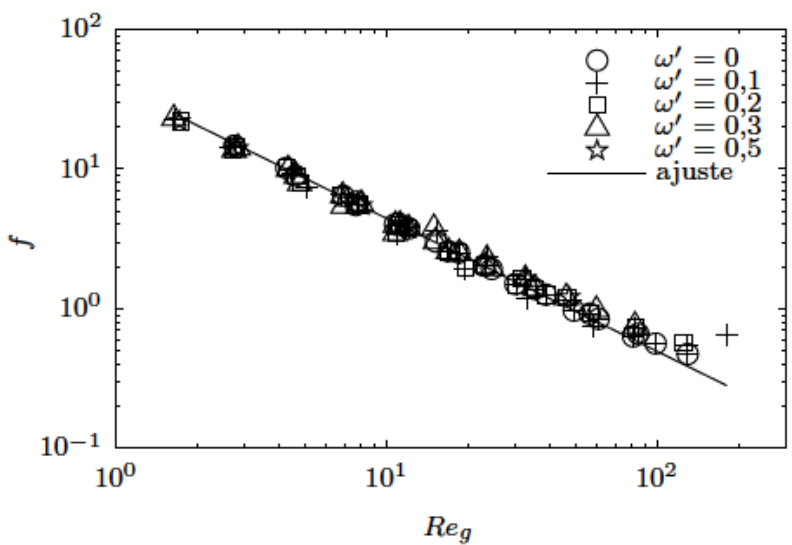

(b) Bomba BB

Figura 8.10: Caída de presión promedio en todo el ciclo de rascado. Ajustes correspondientes a la Ec. 8.10. Las constantes del ajuste se muestran en la Tabla 8.2.

\subsubsection{Caída de presión promedio}

En principio, la caída de presión promedio dependerá de los siguientes factores: la oscilación de caudal entre los ciclos equicorriente y contracorriente, el caudal promedio, la relación de velocidades, o las caídas de presión en cada ciclo. Mientras que las caídas de presión en los ciclos equicorriente y contracorriente se han evaluado en el Apartado 8.2.2.1, la oscilación del caudal depende, entre otros factores, del tipo de bomba utilizada.

Es por ello que, al igual que en el apartado anterior, se ha realizado el mismo estudio utilizando dos bombas distintas en cuanto al material constructivo y en cuanto a sus dimensiones. Son las Bombas BA y BB descritas en el Apartado 4.2. La primera, BA, está diseñada con engranajes sobredimensionados y con cierta holgura entre estos y la carcasa, mientras que la segunda, BB, posee unos engranajes más pequeños y tiene ajustes más finos entre estos y la carcasa. En el Apartado 6.1.1 se realiza una descripción de la oscilación que se produce en el caudal impulsado por la bomba BA en los ensayos en régimen dinámico.

Para un régimen de rascado dado, el valor promedio de $\beta$ siempre es $\bar{\beta}=1$, de modo que, a diferencia de los apartados anteriores, en el presente apartado se utiliza como variable representativa del rascado $\omega^{\prime}$, definida mediante la siguiente ecuación:

$$
\omega^{\prime}=\left|v_{s}\right| / \bar{u}_{b}
$$

siendo $\bar{u}_{b}$ el promedio temporal de $u_{b}$ en el ciclo completo de rascado. 


\begin{tabular}{lrr}
\hline & \multicolumn{1}{c}{ BA } & \multicolumn{1}{c}{ BB } \\
\hline$a$ & 39.52 & 38.495 \\
$b$ & -0.9558 & -0.9188 \\
$i(f)(\%)$ & 7.5 & 6.9 \\
Error $(\%)$ & 14.6 & 13.5 \\
\hline
\end{tabular}

Tabla 8.2: Coeficientes de las correlaciones experimentales para la caída de presión promedio (Ec. 8.10) utilizando sistemas de bombeo distintos: BA y BB. Se muestran la incertidumbre típica y el error correspondiente a un nivel de confianza del $95 \%$ para la estimación del factor de fricción, $f$.

Se han llevado a cabo ensayos dinámicos de pérdida de presión con ambas bombas, variando el rango de números de Reynolds entre $1<R e_{g}<300$, a distintas relaciones de velocidades $\omega^{\prime}=0,1 ; 0,2 ; 0,3 ; 0,5$ y con fluidos de características diferentes: $0,45 \leq n \leq 1 \mathrm{y}$ $4,53 \geq m \geq 0,1$. En los resultados de la Fig. 8.10, no se observa una dependencia significativa de la caída de presión promedio con la velocidad de rascado. Para corroborarlo, en primer lugar se ajustan los resultados en régimen laminar a la Ec. 8.9, que contiene la velocidad de rascado adimensional $\omega^{\prime}$. El valor del exponente $c$ obtenido es del orden de 0,01 con desviaciones típicas de un orden de magnitud inferior. El resultado indica que la relación entre el factor de fricción y $\omega^{\prime}$ en el rango ensayado es mínima y que, por lo tanto, la caída de presión se puede ajustar mediante la Ec. 8.10 sin cometer errores significativos. Los coeficientes obtenidos en este segundo ajuste se muestran en la Tabla 8.2.

$$
\begin{gathered}
f=a \times R e_{g}^{b} \times\left(0,1+\omega^{\prime}\right)^{c} \\
f=a \times R e_{g}^{b}
\end{gathered}
$$

Si se comparan los valores de los parámetros del ajuste de los ensayos realizados con una y otra bomba se observan ligeras diferencias. Al utilizar la bomba BB (con engranajes de menor tamaño), el valor de la constante $a$ es ligeramente inferior, al igual que el valor de -b. Sin embargo estas variaciones no se consideran suficientemente significativas y pueden deberse a los rangos de números de Reynolds de los ensayos realizados con una y otra, que son ligeramente diferentes.

Además, en la Fig. 8.10(b) también se incluyen resultados de los ensayos en régimen estático. Se observa en dicha gráfica que los resultados de pérdida de presión promedio en el ciclo de rascado completo son similares a los medidos en régimen de rascador estático. 
A pesar de no existir diferencias significativas entre las bombas BA y BB, se ha comprobado que la bomba BA, de engranajes más grandes, conserva mucho mejor las propiedades del fluido, lo que implica que durante los ensayos la variación que se produce en las propiedades reológicas del fluido sea menor y por lo tanto las estimaciones de dichas propiedades en los experimentos sean más precisas. En consecuencia, se estima que la bomba BA es más apropiada para el trabajo con este tipo de fluidos. En el Capítulo 9 se realizan ensayos de transmisión de calor con ambos sistemas para analizar las posibles diferencias existentes.

\subsubsection{Conclusiones}

A continuación se detallan las conclusiones obtenidas de los ensayos en pérdida de presión en régimen de rascador dinámico:

- Se han realizado ensayos para medir la caída de presión en función de la velocidad de rascado, obteniendose correlaciones experimentales en régimen laminar para las direcciones de rascado en dirección equicorriente y contracorriente. El número de Reynolds en los ensayos ha variado en el margen $1<R e_{g}<200$, mientras que $0,5<\beta<2,5$.

- Al igual que ocurre con el patrón del flujo, la caída de presión en el tubo es función del factor de bloqueo $\beta$.

- La caída de presión promediada en el ciclo completo de rascado no depende de la velocidad adimensional de rascado $\left|v_{s}\right| / \bar{u}_{b}$. Además dichas caídas muy similares a las que se producen en el caso con rascador estático.

- No se han obtenido medidas suficientes en la región de transición o turbulenta para poder determinar con seguridad a qué número de Reynolds se produce la transición.

En cuanto al sistema de bombeo a utilizar, del estudio realizado se concluye que

1. Al estudiar los ciclos de rascado por separado, una vez el flujo es estable, no hay diferencia entre utilizar la BA o la BB en cuanto a la caída de presión que se produce.

2. La caída de presión promedio ${ }^{1}$ en el ciclo de rascado completo no difiere significativamente de la caída de presión en régimen estático.

\footnotetext{
${ }^{1}$ Caída de presión promedio en el ciclo de rascado, en función del número de Reynolds $R e_{g}$ calculado con $\bar{u}_{b}$ (promedio en el ciclo de rascado de $u_{b}$ ).
} 
3. La caída de presión promedio no depende del tamaño y los ajustes de los engranajes del sistema de bombeo.

4. Hasta este punto, el uso de bombas de engranajes sobredimensionadas se considera más oportuno debido a la baja degradación que sufre el fluido. En capítulos sucesivos se realizan comparativas entre los dos sistemas de bombeo en cuando a la transferencia de calor.

\subsection{Potencia de accionamiento}

En el presente apartado se estudia la potencia necesaria para accionar el rascador. Para ello se estudia el mecanismo de consumo de potencia para tal fin, realizando una serie de medidas experimentales en la instalación.

En el Capítulo 6 se describe el sistema de accionamiento del rascador. Éste consiste en un pistón hidráulico de doble efecto de $50 \mathrm{~mm}$ de diámetro y doble vástago de $20 \mathrm{~mm}$ de diámetro. La diferencia de presión entre las cámaras del pistón se mide mediante un sensor de presión diferencial bidireccional, de modo que la potencia de accionamiento se calcula mediante la siguiente expresión:

$$
P=A v_{s} \Delta p=\frac{A_{p} S_{i} \Delta p}{T_{r} / 2}[W]
$$

donde $P$ es la potencia proporcionada al émbolo, $A_{p}$ es el área útil de una de las caras del émbolo, $S_{i}$ es la amplitud de rascado, $\Delta p$ es la diferencia de presión medida por el sensor, $T_{r}$ el periodo de rascado y $v_{s}=2 S_{i} / T_{r}$ es la velocidad de rascado.

En principio, la potencia total consumida por el sistema de accionamiento del rascador viene dada por la contribución de tres factores independientes:

1. La potencia de accionamiento únicamente del pistón, sin el rascador acoplado.

2. La potencia necesaria para mover el rascador sin flujo circulante (en condiciones de lubricación).

3. La influencia del flujo en la potencia de accionamiento del rascador.

A continuación se evalúa cada uno de dichos efectos por separado. 


\subsubsection{Potencia de accionamiento del pistón}

Para determinar la primera de las contribuciones, se trabaja con el pistón libre y se mide la potencia necesaria para accionarlo en función de la velocidad de rascado. El resultado mostrado en la Fig. 8.11 se puede ajustar mediante la siguiente expresión, cuyos coeficientes de ajuste se muestran en la Tabla 8.3.

$$
P_{p}=a_{p} \times v_{s}^{b_{p}}
$$

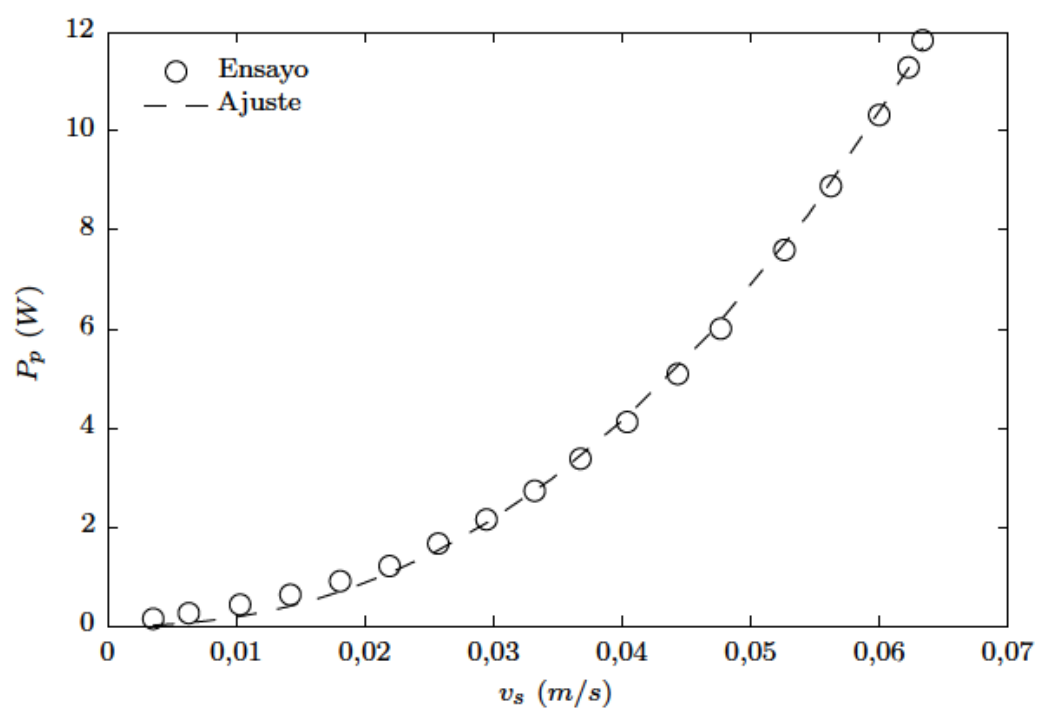

Figura 8.11: Potencia de accionamiento del pistón libre, $P_{p}$.

\subsubsection{Contribución del rascador}

En este punto, se rosca el rascador al pistón, de modo que la potencia medida será la suma de $P_{p}$, medida en el apartado anterior, más la contribución del rascador $P_{s}$.

Para que el rozamiento entre el rascador y el tubo tenga una lubricación similar a la existente cuando circula flujo por el tubo existen dos opciones, dando ambas el mismo resultado: se puede vaciar el tubo de fluido, pero dejando fluido suficiente a modo de lubricante, o se puede dejar el fluido en el interior sin circulación, ya que esto no variará el consumo de potencia de forma apreciable. En los ensayos se opta por la segunda opción, de más sencilla ejecución. Se han realizado dos ensayos variando la temperatura del fluido, a $20^{\circ} \mathrm{C}$ y a $40^{\circ} \mathrm{C}$. 


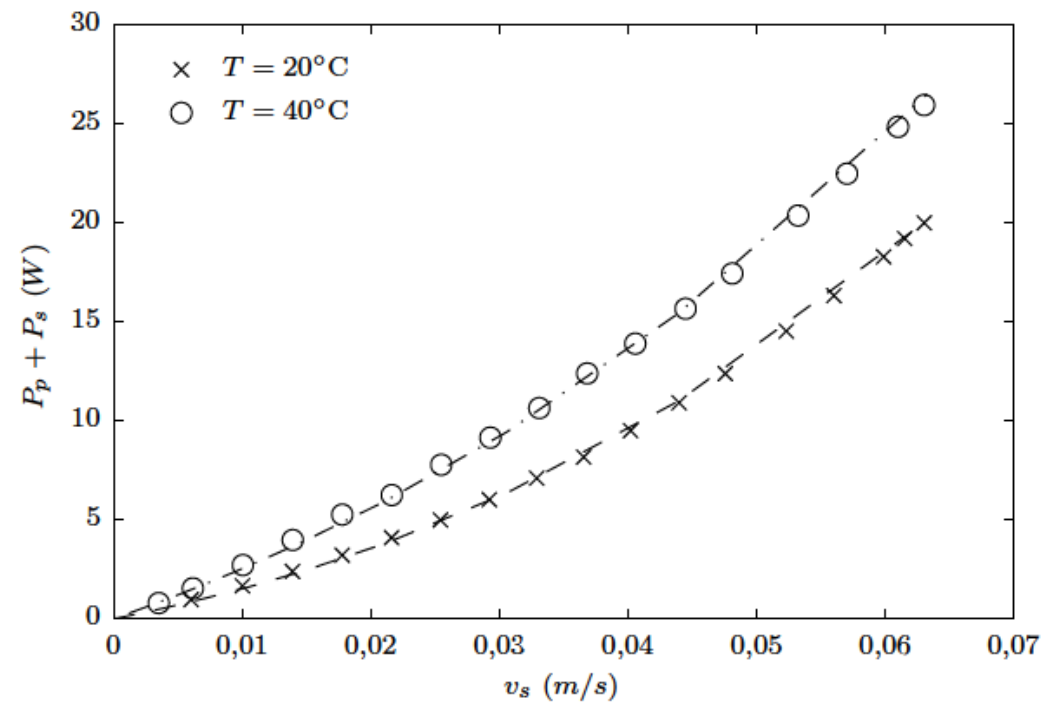

(a) Potencia de accionamiento del rascador sin flujo, $P_{p}+P_{s}$.

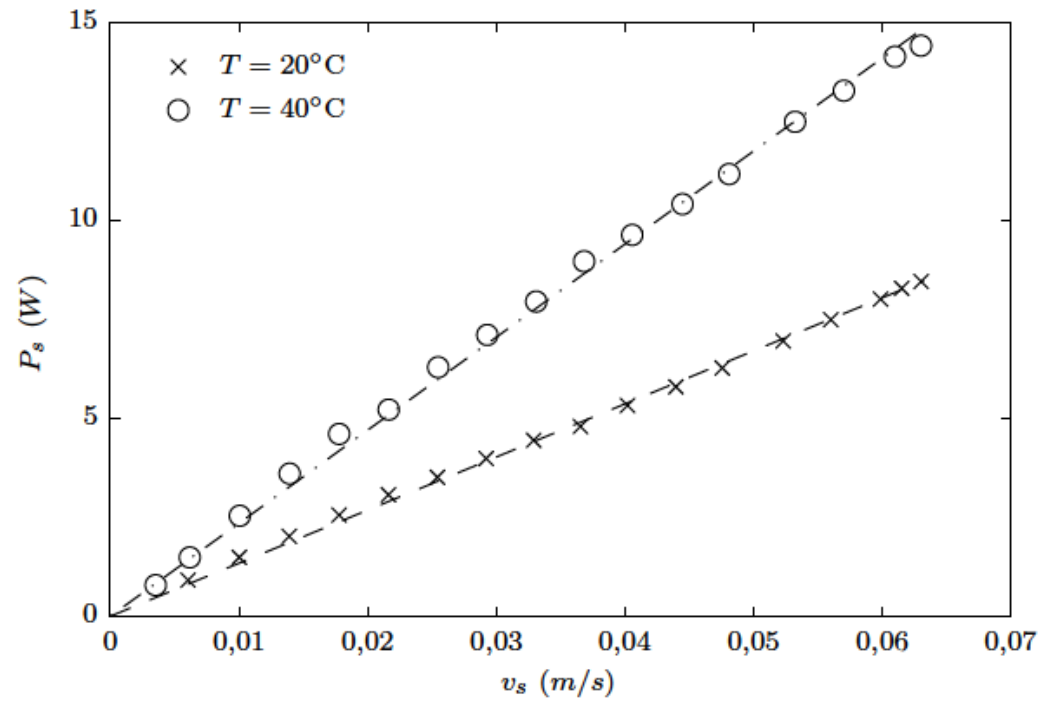

(b) Contribución del rascador a la potencia de accionamiento, $P_{s}$

Figura 8.12: Análisis de la potencia de accionamiento en situación de flujo circulante nulo. 


\begin{tabular}{ccccccc}
\hline & Ec. & $a$ & $s(a)$ & $b$ & $s(b)$ & $s(P)$ \\
\hline Pistón libre $\left(a_{p}, b_{p}\right)$ & 8.11 & 5876 & 776,76 & 2,255 & 0,0458 & 0,2748 \\
Aportación rascador $20^{\circ} \mathrm{C}\left(a_{s}\right)$ & 8.12 & 134 & 3 & - & - & 0,5 \\
Aportación rascador $40^{\circ} \mathrm{C}\left(a_{s}\right)$ & 8.12 & 235 & 1,5 & - & - & 0,2 \\
\hline
\end{tabular}

Tabla 8.3: Parámetros de ajuste de la potencia de accionamiento.

La potencia de accionamiento necesaria en este caso se muestra en la Fig. 8.12(a). El resultado muestra un aumento de la potencia de accionamiento necesaria con la temperatura del fluido. Este efecto se puede explicar por la dilatación de los distintos elementos en contacto con el fluido, principalmente: los tacos del rascador y el tubo.

A partir de los resultados se puede obtener la contribución del rascador a la potencia de accionamiento restando la contribución del pistón libre, de modo que la contribución del rascador queda representada en la Fig. 8.12(b) y se puede ajustar mediante la Ec. 8.12.

$$
P_{s}=a_{s}(T) \times v_{s}
$$

donde $a_{s}(T)$ es función de la temperatura $T$.

El consumo del conjunto pistón+rascador vendría dado por la siguiente expresión:

$$
P_{p}+P_{s}=a_{p} \times v_{s}^{b_{p}}+a_{s}(T) \times v_{s}
$$

\section{Dependencia con la temperatura}

$\mathrm{Al}$ ser $a_{s}(T)$ función de la temperatura, es necesario estudiar cómo se relacionan ambos parámetros. Con tal fin se realiza un ensayo a velocidad de rascado constante $v_{s}=0,03 \mathrm{~m} / \mathrm{s}$, variando la temperatura del fluido.

El resultado de la Fig. 8.13 muestra la importancia que tiene la temperatura en la potencia de accionamiento.

\subsubsection{Efecto del flujo en la velocidad de rascado}

Es de esperar que la potencia de accionamiento se vea afectada, en mayor o menor medida, por el flujo del fluido circulante y las características del propio fluido. Al estar el rascador en contacto con el flujo y existir una velocidad relativa entre ambos, aparece una fuerza de arrastre sobre el rascador, cuyo origen son los esfuerzos de fricción y el campo de presiones del flujo en la zona de contacto con los tacos. Además el fluido actuará de lubricante entre 


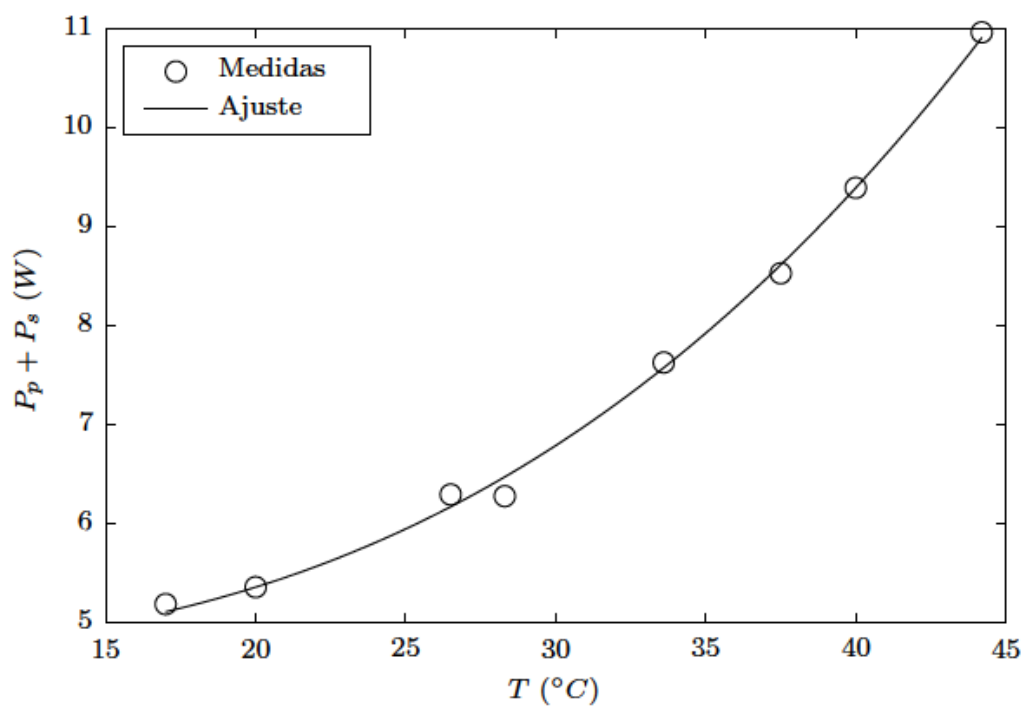

Figura 8.13: Potencia de accionamiento en función de la temperatura.

la pared del tubo y los tacos, por lo tanto la viscosidad de dicho fluido puede ser también un factor significativo.

Para poder determinar los efectos mencionados, se realizan 4 tandas de ensayos a $20^{\circ} \mathrm{C}$ con 4 fluidos de diferentes propiedades reológicas. En cada ensayo se barre un rango de velocidades de rascado hasta $v_{s}=0,06 \mathrm{~m} / \mathrm{s}$, variando la velocidad de rascado adimensional $\left|v_{s}\right| / u_{b}=0,1 ; 0,2 ; 0,3 ; 0,5 ; 1$. Para estos ensayos se utiliza la bomba de engranajes pequeños (BB), que proporciona un flujo estable a pesar de los fuertes cambios de presión provocados por el movimiento del rascador. El objetivo de los ensayos es observar el efecto del flujo en la potencia de accionamiento partiendo de las contribuciones ya conocidas del pistón y del rascador.

Por un lado, se representan en la Fig. 8.14 los resultados identificados según la tanda de ensayos a la que pertenecen y la velocidad de rascado. Como en cada tanda de ensayos las propiedades se mantienen relativamente constantes, esta representación muestra de manera cualitativa el efecto de trabajar con fluidos con distintas propiedades reológicas. En los resultados se puede observar claramente el efecto de la viscosidad: tanto en sentidos de rascado equicorriente como en contracorriente, un mayor índice de consistencia de fluido $m$ redunda en una mayor potencia de accionamiento. No obstante este efecto no es muy significativo.

Por otro lado, los resultados de los ensayos, representados en la Fig. 8.15 para los cuatro fluidos ensayados, permiten obtener las correlaciones experimentales para la potencia de 


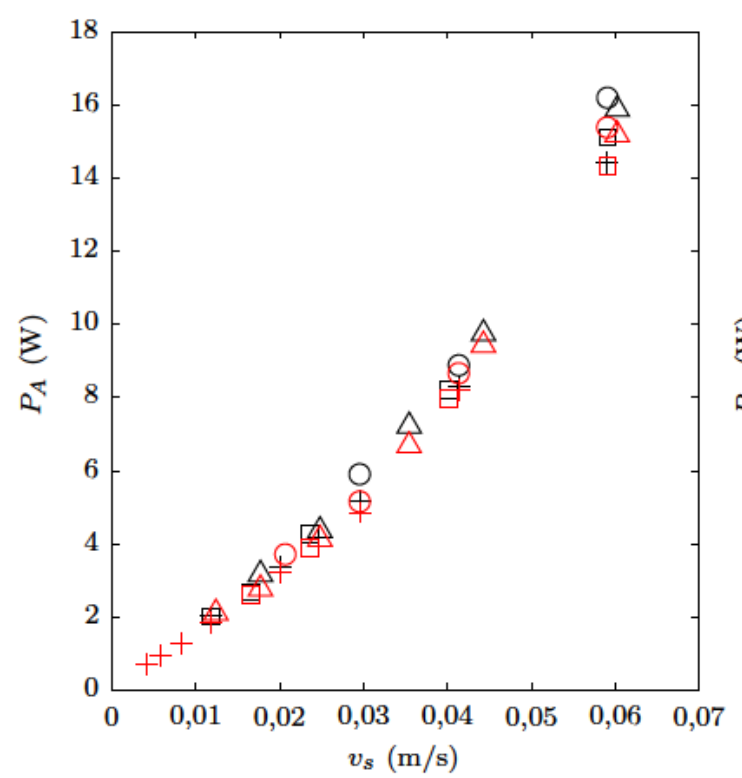

(a) Equicorriente.

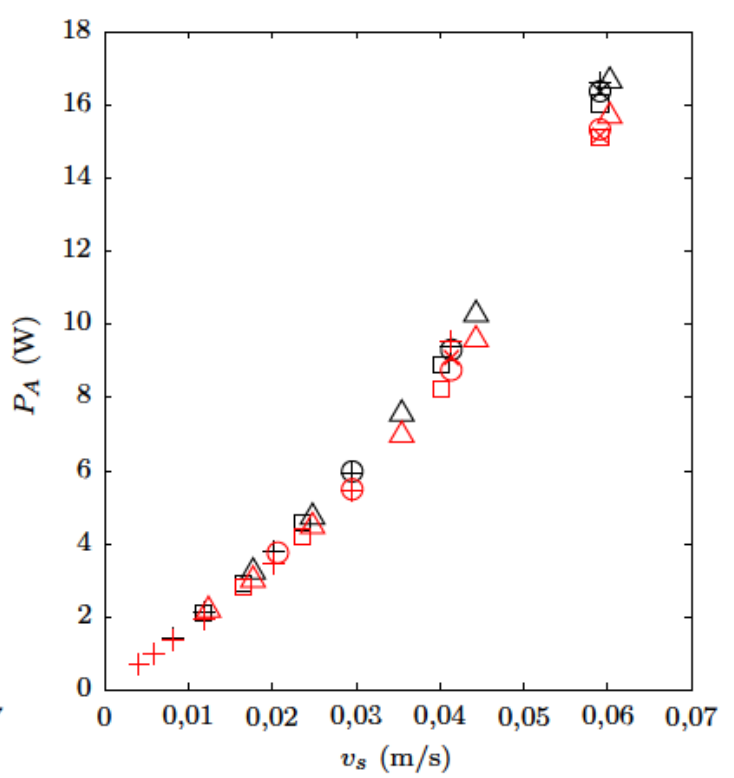

(b) Contracorriente

Figura 8.14: Potencia de accionamiento en ensayos con fluido circulante, $P_{A}$. Los colores azul y rojo corresponden a dos fluidos distintos. Cada símbolo corresponde a una relación de rascado $|\omega|=\left|v_{s} / u_{b}\right|$. Color rojo; $m=4,46 \mathrm{~Pa}_{\mathrm{s}} \mathrm{s}^{n}$ y $n=0,42$, color negro $m=0,054 \mathrm{~Pa} \cdot \mathrm{s}^{n}$ y $n=0,91$. Velocidades rascado: $\omega=0,1 ; 0,2 ; 0,3 ; 0,5 ; 1 \equiv+, \square, \triangle, \circ, \times$. 


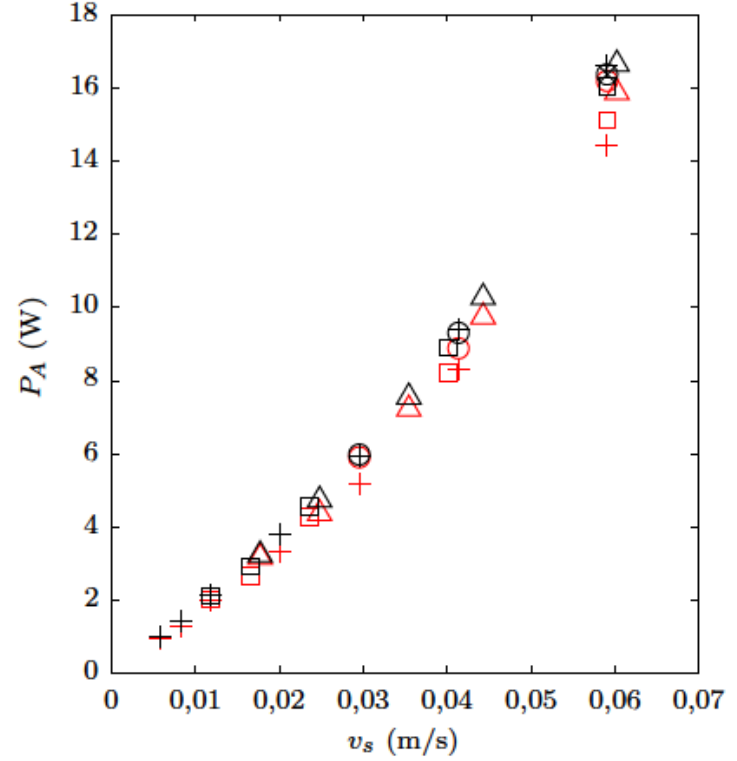

(a) $m=4,46 \mathrm{~Pa} \cdot \mathrm{s}^{n} ; n=0,42$.

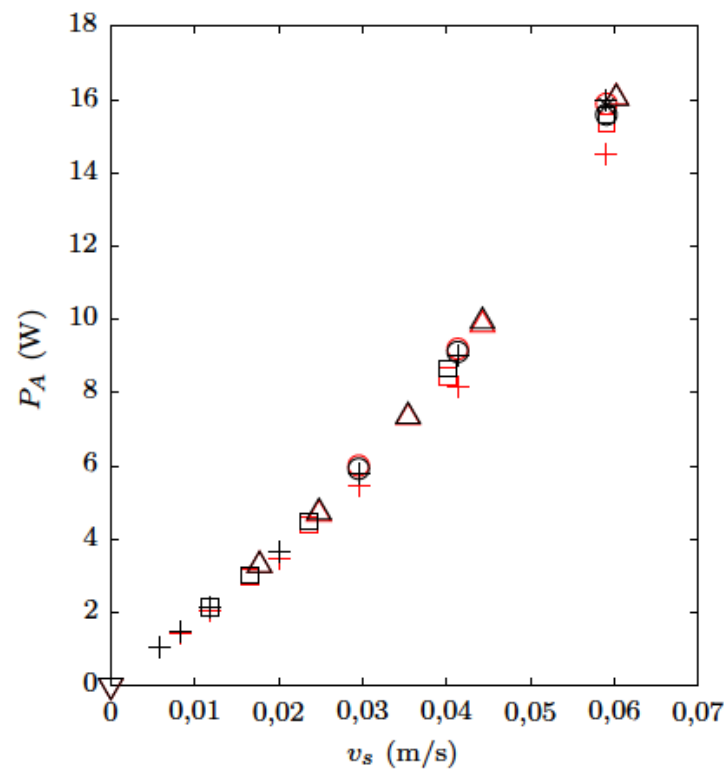

(c) $m=0,85 \mathrm{~Pa} . \mathrm{s}^{n} ; n=0,64$

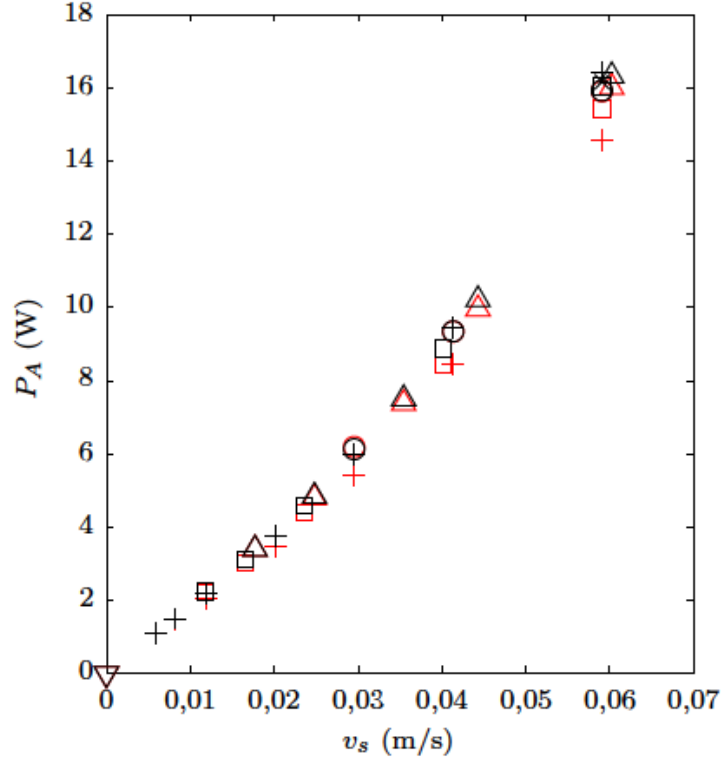

(b) $m=1,71 \mathrm{~Pa} . \mathrm{s}^{n} ; n=0,54$

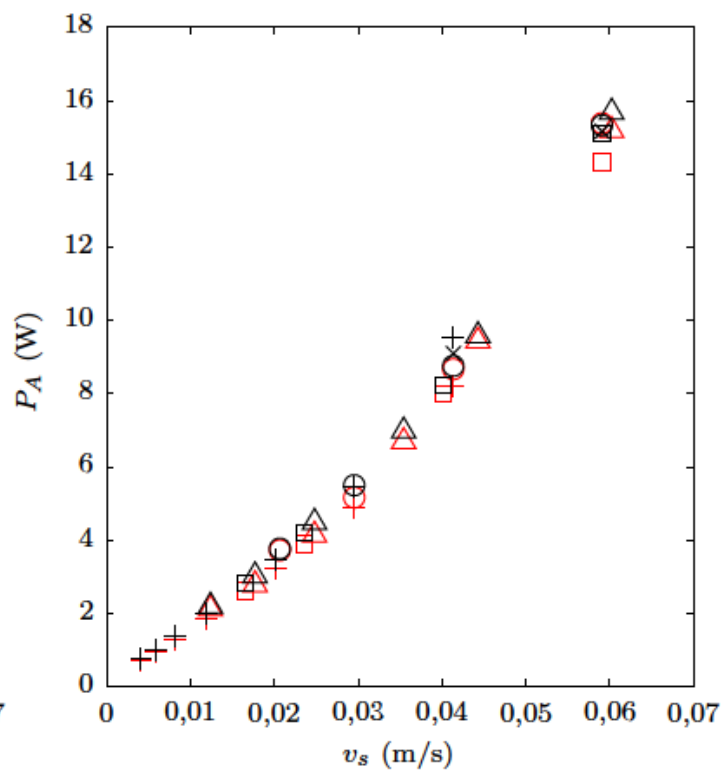

(d) $m=0,054$ Pa.s ${ }^{n} ; n=0,91$

Figura 8.15: Potencia de accionamiento en ensayos con fluido circulante, $P_{A}$, clasificados por tandas con propiedades del fluido aproximadamente constantes. Colores: negro para ensayos contracorriente y rojo para equicorriente. Velocidades rascado: $\left|v_{s}\right| / u_{b}=$ 0,$1 ; 0,2 ; 0,3 ; 0,5 ; 1 \equiv+, \square, \triangle, \circ, \times$. 


\begin{tabular}{cccccccccc}
\hline & $a_{f}$ & $b_{f}$ & $c_{f}$ & $d_{f}$ & $i\left(a_{f}\right)$ & $i\left(b_{f}\right)$ & $i\left(c_{f}\right)$ & $i\left(d_{f}\right)$ & $i\left(P_{A}\right)$ \\
\hline $\mathrm{EC}(\beta<1)$ & -33.895 & 4.2244 & 87.544 & 0.0438 & 3.3240 & 1.1110 & 2.3546 & 0.0050 & 0.2214 \\
$\mathrm{CC}(\beta>1)$ & 70.131 & 0.0307 & 15.596 & 0.3480 & 4.3269 & 0.0373 & 4.7127 & 0.1105 & 0.1861 \\
\hline
\end{tabular}

Tabla 8.4: Correlaciones experimentales para determinar la contribución del flujo $\left(P_{f^{-}}\right.$ Ec. 8.15) en la potencia de accionamiento del rascador, $P_{A}$ (Ec. 8.14).

accionamiento. Para ello los datos experimentales se ajustan a la Ec. 8.14, donde a las constantes $a_{p}, b_{p}$ y $a_{s}$ se les asigna el valor calculado en los apartados anteriores y los parámetros del ajuste son $a_{f}, b_{f}, c_{f} \mathrm{y} d_{f}$. Dicha ecuación representa la potencia de accionamiento total consumida:

$$
P_{A}=a_{p} \times v_{s}^{b_{p}}+\left(a_{s}(T)+a_{f} \beta^{b_{f}}+c_{f} m^{d_{f}}\right) v_{s}
$$

siendo la contribución del flujo a la potencia de accionamiento

$$
P_{f}=\left(a_{f} \beta^{b_{f}}+c_{f} m^{d_{f}}\right) v_{s}
$$

El valor de las constantes obtenidas en la correlación se muestra en la Tabla 8.4.

Las correlaciones obtenidas, corroboran las conclusiones cualitativas obtenidas en cuanto al efecto del índice de consistencia de fluido $m$ en la potencia de accionamiento. Por otro lado, se observa que el efecto de la velocidad de rascado es el siguiente:

- para $\beta>1: P_{A}$ aumenta al hacerse $\beta$ más grande, alejandose del valor $\beta=1$

- para $\beta<1: P_{A}$ aumenta al hacerse $\beta$ menor, alejandose del valor $\beta=1$

donde el factor de bloqueo se define como

$$
\beta=1-\frac{v_{s}}{u_{b}}=1-\omega
$$

Para corroborar la validez de las correlaciones, éstas se comparan con los resultados experimentales de las cuatro tandas de ensayos en la Fig. 8.16 y la Fig. 8.17.

Por último se muestra en la Fig. 8.18 el efecto de la viscosidad en el flujo, de acuerdo a las correlaciones obtenidas. 


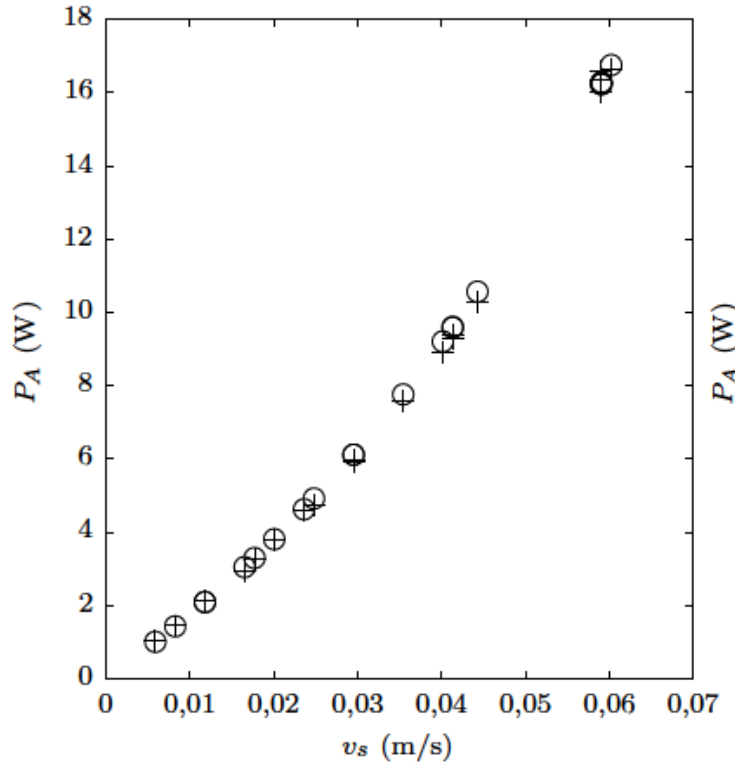

(a) $m=4,46$ Pa.s ${ }^{n} ; n=0,42$.

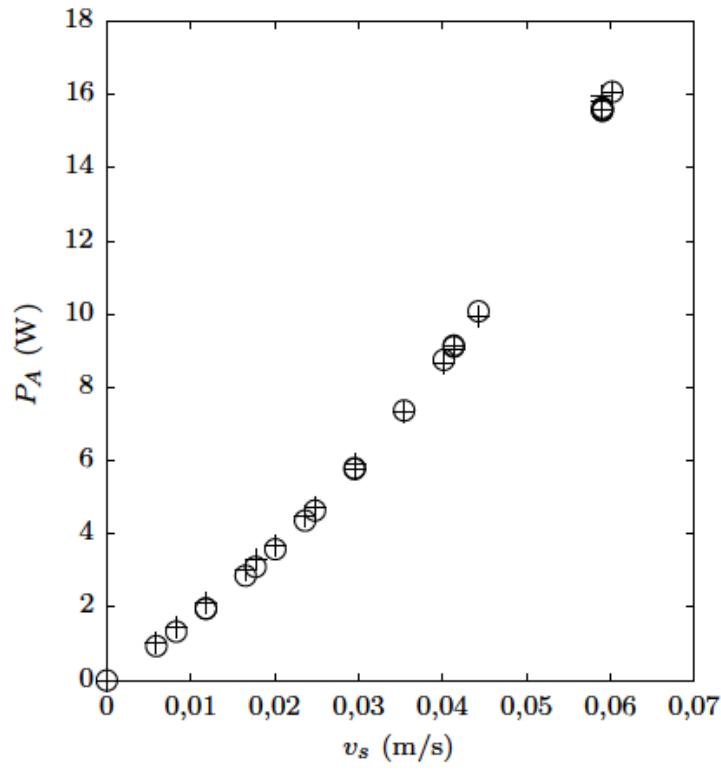

(c) $m=0,85$ Pa.s ${ }^{n} ; n=0,64$

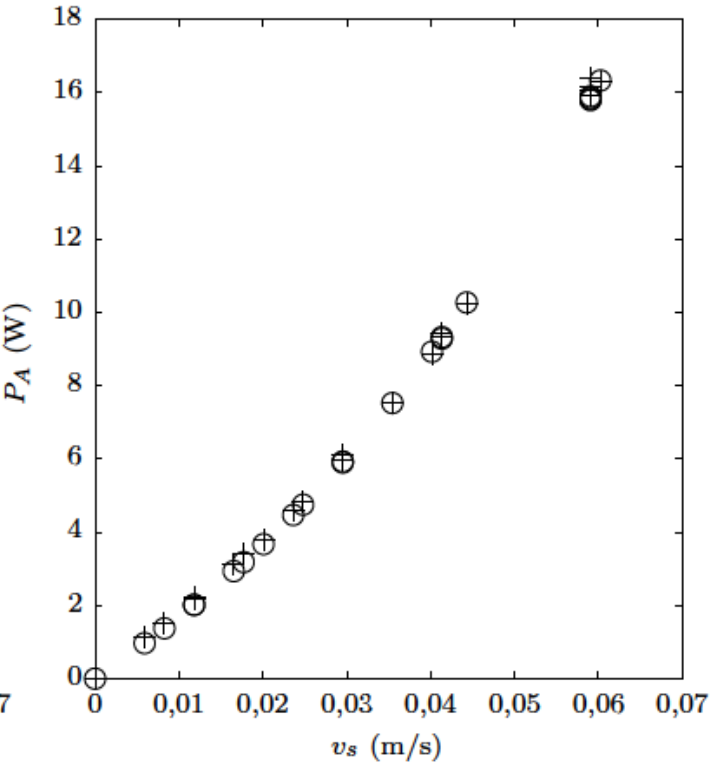

(b) $m=1,71 \mathrm{~Pa} . \mathrm{s}^{n} ; n=0,54$

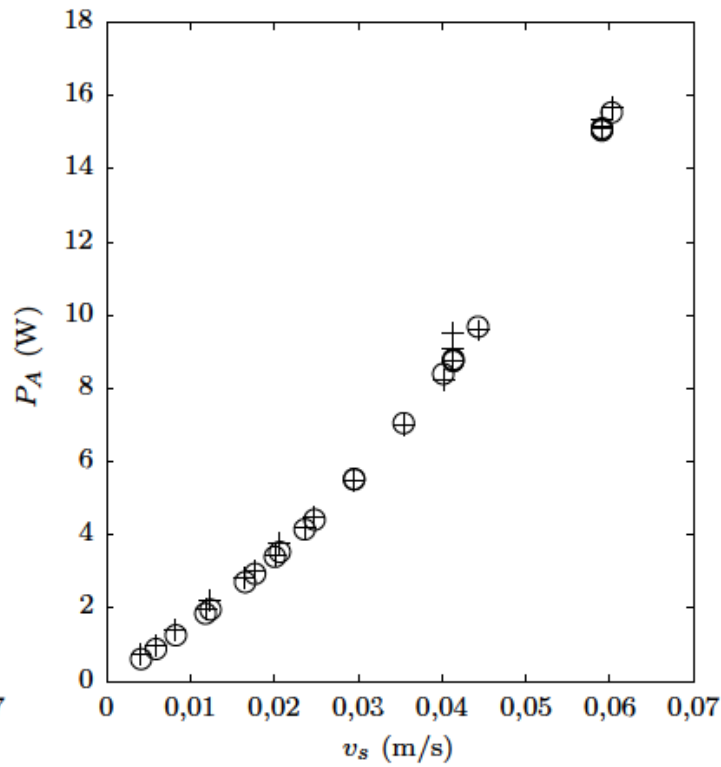

(d) $m=0,054$ Pa.s ${ }^{n} ; n=0,91$

Figura 8.16: Potencia de accionamiento $\left(P_{A}\right)$ en ensayos contracorriente: comparación entre correlaciones $(\circ)$ y experimentos $(+)$ para diferentes regímenes de rascado. 

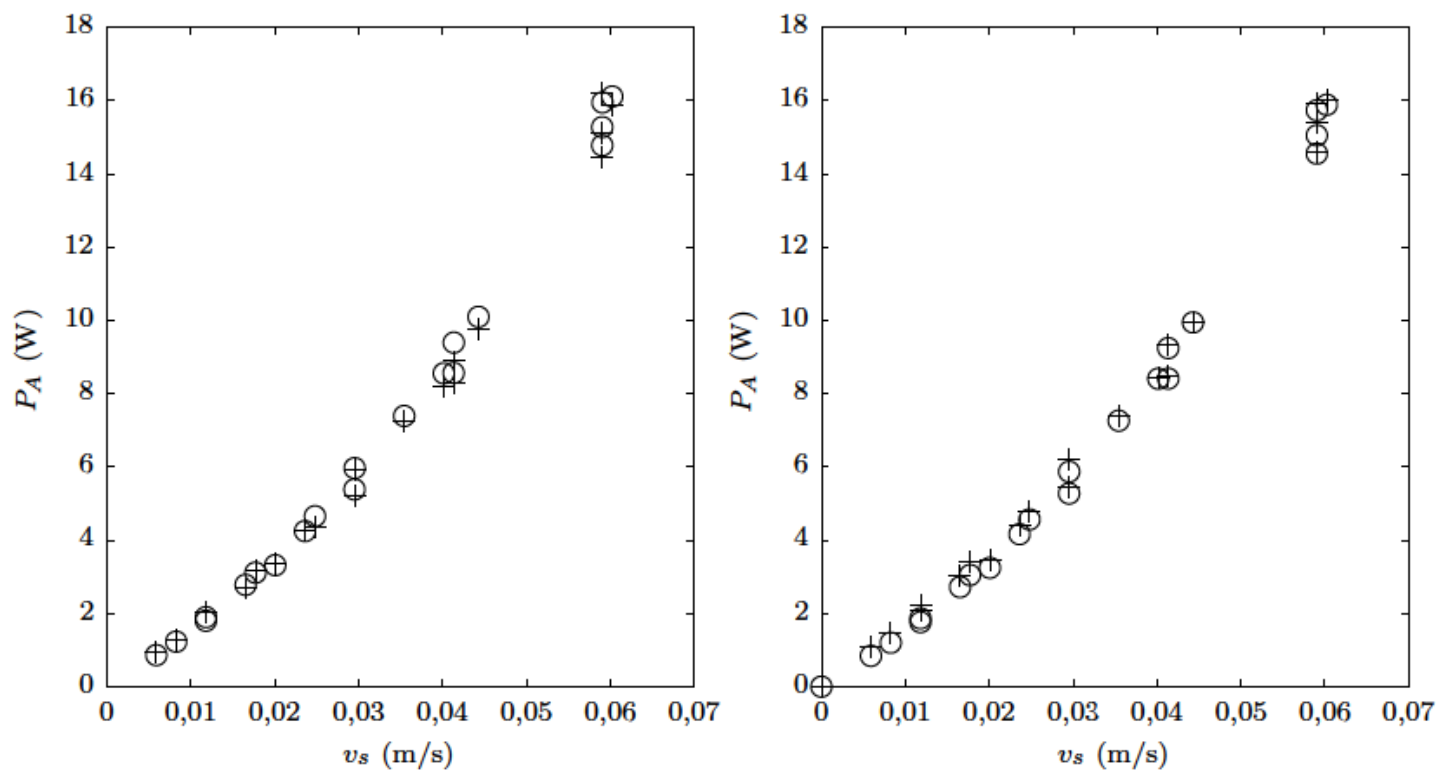

(a) $m=4,46$ Pa.s ${ }^{n} ; n=0,42$.

(b) $m=1,71 \mathrm{~Pa} . \mathrm{s}^{n} ; n=0,54$
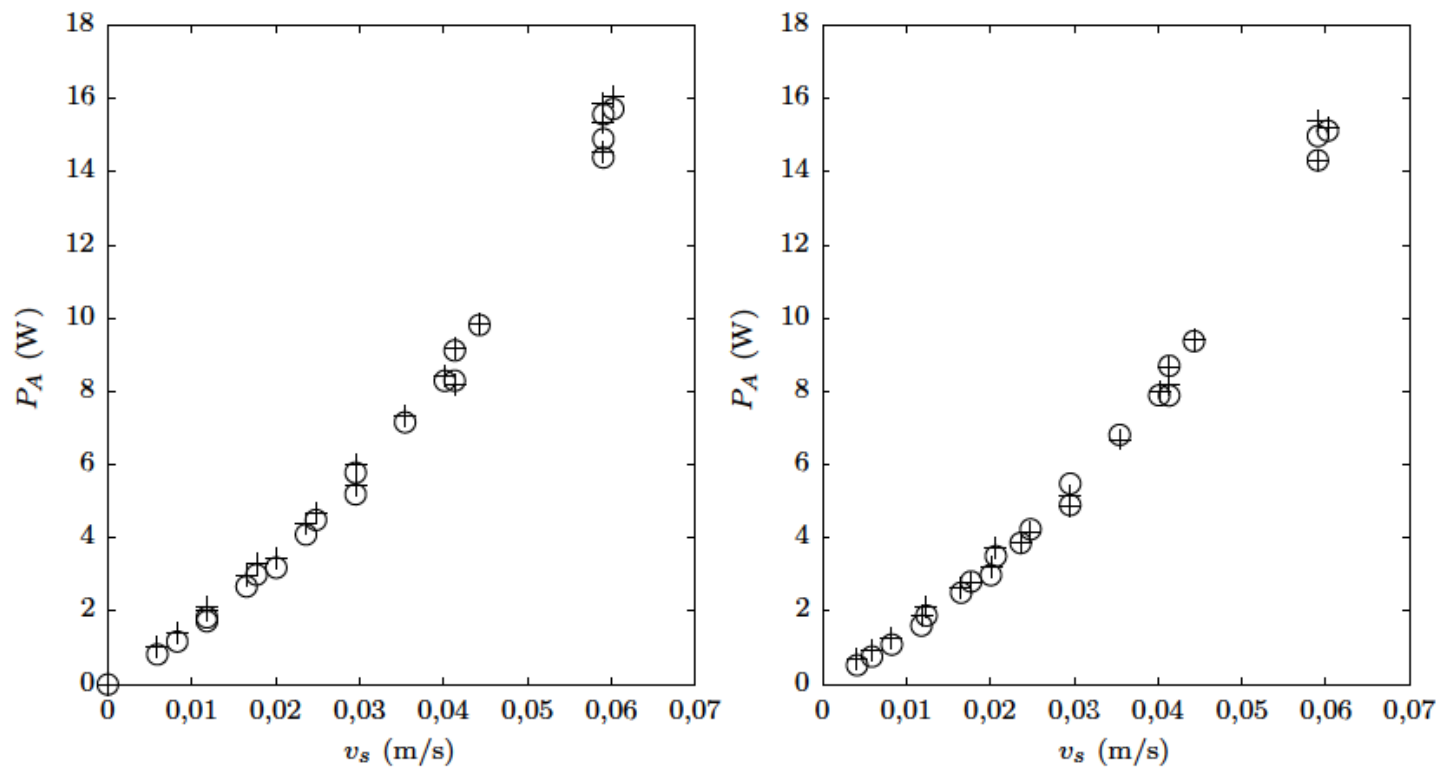

(c) $m=0,85$ Pa.s ${ }^{n} ; n=0,64$

(d) $m=0,054$ Pa.s ${ }^{n} ; n=0,91$

Figura 8.17: Potencia de accionamiento $\left(P_{A}\right)$ en ensayos equicorriente: comparación entre correlaciones $(\circ)$ y experimentos $(+)$ para diferentes regímenes de rascado. 


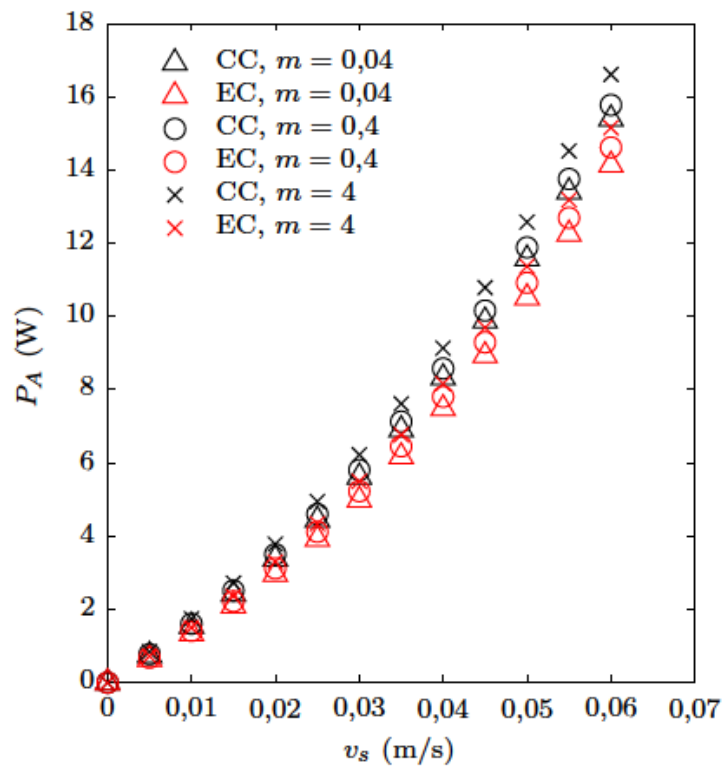

Figura 8.18: Potencia de accionamiento obtenida a partir de las correlaciones, en ensayos con fluido circulante, $P_{A}$.

\subsection{Conclusiones}

- Se han realizado ensayos de pérdida de presión en régimen estático del rascador para números de Reynolds $0,2<R e_{g}<600$, utilizando para ello fluidos con diferente comportamiento seudoplástico: desde fluidos con $n=0,45$ y $m=3,95$ hasta fluidos newtonianos con $n=1$ y $m=0,02$. Además, se han identificado las regiones del flujo en régimen estático, comprobando que en régimen laminar, los resultados de $f$ se pueden representar mediante una sóla curva. Se ha detectado que la transición se produce a valores de $100<R e_{g}<200$.

- En base a los resultados en régimen estático, se ha evaluado el efecto del comportamiento seudoplástico en la relación existente entre el caudal circulante y la caída de presión en el tubo con rascador.

- En régimen de rascador dinámico, se han realizado ensayos para medir la caída de presión en función de la velocidad de rascado. El número de Reynolds en los ensayos ha variado en el margen $1<R e_{g}<200$, mientras que $0,5<\beta<2,5$. Se han obtenido correlaciones experimentales en régimen laminar para las direcciones de rascado en dirección equicorriente y contracorriente para dichos ensayos, corroborando la influencia 
del factor de bloqueo $\beta$.

- Se ha comprobado la influencia del tamaño de los engranajes del sistema de bombeo al trabajar con el fluido de trabajo (CMC al 1\% en agua) en el tubo con el rascador en movimiento. Como resultado se ha concluido que, a pesar de la oscilación de caudal que se produce con el sistema de bombeo de engranajes grandes y tolerancias holgadas, éste resulta más conveniente de cara a evitar la degradación del fluido.

- Se ha obtenido la potencia consumida por el sistema de accionamiento del rascador en función de la temperatura, la velocidad de rascado, las propiedades reológicas y el factor de bloqueo. 


\section{Capítulo 9}

\section{Transmisión de calor}

De acuerdo con los objetivos definidos en el Capítulo 1, una vez evaluadas la pérdida de presión que se produce en el tubo y la potencia de accionamiento necesaria para impulsar el rascador, únicamente queda por estudiar el proceso de transmisión de calor en el dispositivo.

En primer lugar se realiza un estudio de la transferencia de calor a fluidos no newtonianos en geometrías de tubo liso y tubo de sección anular. En este estudio se puede observar cómo influye el comportamiento no newtoniano del fluido en la transmisión de calor en tubos. Además, se cuantifica dicha influencia en la geometría de tubo de sección anular, la cual se considera una buena aproximación a la situación de tubo con rascador insertado.

En una segunda fase, se realizan mediciones del coeficiente convección medio en función del régimen de funcionamiento. Tras el análisis dimensional realizado en el Capítulo 2 el problema queda reducido al estudio de los siguientes monomios adimensionales: los números de Prandtl, Nusselt y Reynolds, el coeficiente de comportamiento de flujo $n$ y además, en el caso dinámico, la velocidad de rascado adimensional. Además, en dicho capítulo se indicaba la influencia de la relación de diámetros en el problema, la cual se ha mantenido constante en los casos analizados.

Al igual que en capítulos anteriores, se estudia por un lado el régimen estático, cuyo principal atractivo radica en el nulo consumo de potencia de accionamiento. Y por otro lado se analiza el proceso de transmisión de calor en régimen de rascador dinámico. Cabe destacar que se estudian los mismos regímenes de funcionamiento que en el Capítulo 8, de modo que los resultados obtenidos se complementan. La instalación utilizada para realizar los ensayos se describe en el Capítulo 4, mientras que el procedimiento de ensayo se detalla en el Capítulo 6.

Además del estudio específico de la transferencia de calor, en el último apartado se analiza 
el comportamiento termohidráulico completo del rascador. Para ello se tienen en cuenta todas las variables estudiadas hasta el momento, de modo que, mediante los criterios clásicos de mejora propuestos por Bergles (1997), se puedan establecer los rangos de funcionamiento adecuados para conseguir los objetivos deseados.

\subsection{Transmisión de calor en geometrías de tubo liso y tubo de sección anular.}

En esta primera fase del análisis se estudia la transmisión de calor a fluidos seudoplásticos en dos geometrías sencillas: tubo liso y tubo con eje. Con ello se pretende, por un lado, obtener un patrón sobre la influencia de la seudoplasticidad en los procesos de intercambio de calor, y por otro, se busca una aproximación para cuantificar dicha influencia en el tubo con rascador.

Se estudia el caso de convección puramente forzada en el que se aplica un flujo de calor constante a través de la pared del tubo, circulando por el interior un fluido seudoplástico. El perfil de velocidades a la entrada se considera plenamente desarrollado. Para el problema en tubo liso, existen soluciones analíticas o/y aproximadas en la bibliografía, no así para la geometría de tubo con eje. Por consiguiente, en la geometría de tubo con eje, se hace uso del modelo de simulación numérica descrito en el Capítulo 3 para obtener el número de Nusselt.

En ambas geometrías, el número de Nusselt local en la región de entrada depende de la coordenada $\hat{z}$ adimensional, medida desde la entrada al tubo, definida del siguiente modo:

$$
\hat{z}=\frac{4 z}{D_{h} P e}
$$

\subsubsection{Tubo liso}

En el problema en tubo liso, el número de Nusselt en varía con la seudoplasticidad del fluido (Chhabra y Richardson, 1999). En el flujo en desarrollo, el número de Nusselt viene dado por la siguiente expresión obtenida de forma analítica por Bird (1959), quien parte de la aproximación de Leveque.

$$
N u=2,11 \Delta^{1 / 3} G z^{1 / 3}
$$




\begin{tabular}{ccccc}
\hline$n$ & $\Delta^{1 / 3}$ & Ec. 9.2 & Error & Simulación \\
\hline 0,45 & 1,093 & 1,104 & $1,1 \%$ & 1,105 \\
0,50 & 1,077 & 1,088 & $1,0 \%$ & 1,088 \\
0,60 & 1,053 & 1,061 & $0,7 \%$ & 1,061 \\
0,70 & 1,035 & 1,040 & $0,5 \%$ & 1,040 \\
0,80 & 1,020 & 1,024 & $0,3 \%$ & 1,024 \\
0,90 & 1,009 & 1,011 & $0,2 \%$ & 1,011 \\
1,00 & 1,000 & 1,000 & $0,0 \%$ & 1,000 \\
\hline
\end{tabular}

Tabla 9.1: Comparación entre el valor de $N u_{\infty} / N u_{\infty, n=1}$ obtenido a partir de las ecuaciones 9.2 y 9.3. En la última columna se adjunta el valor obtenido en las simulaciones con $N N=$ 100.

con $G z=\dot{m} c_{p} /(k L)$ y siendo

$$
\Delta=\frac{\left(\left.\frac{\partial u}{\partial r}\right|_{r=R}\right)_{n \neq 1}}{\left(\left.\frac{\partial u}{\partial r}\right|_{r=R}\right)_{n=1}}
$$

que en tubo liso toma un valor de $\Delta=(3 n+1) / 4 n$.

Bajo las mismas suposiciones (aproximación de Leveque) se puede llegar a la expresión del número de Nusselt del flujo desarrollado (Bird, 1959):

$$
N u_{\infty}=\frac{8(3 n+1)(5 n+1)}{\left(31 n^{2}+12 n+1\right)}
$$

para la que se podría obtener la siguiente relación:

$$
\frac{N u_{\infty}}{N u_{\infty, n=1}}=\frac{11(3 n+1)(5 n+1)}{6\left(31 n^{2}+12 n+1\right)}
$$

Por otro lado, Grigull (1956) estimó el valor de $N u$ a partir del número de Nusselt para fluidos newtonianos $N u_{n=1}$, aplicando un factor de $\Delta^{1 / 3}$, donde

$$
\frac{N u}{N u_{n=1}}=\Delta^{1 / 3}
$$

En la bibliografía (Manglik et al., 1988; Martínez et al., 2014) es habitual utilizar dicho factor para englobar el efecto del comportamiento no newtoniano del flujo en tubos, tanto en flujo en desarrollo como en flujo desarrollado. En apartados sucesivos se obtiene una relación similar válida para el tubo con rascador.

Los resultados para $N u_{\infty}$ de las Ec. 9.3 y 9.2 se comparan en la Tabla 9.1 , donde además 


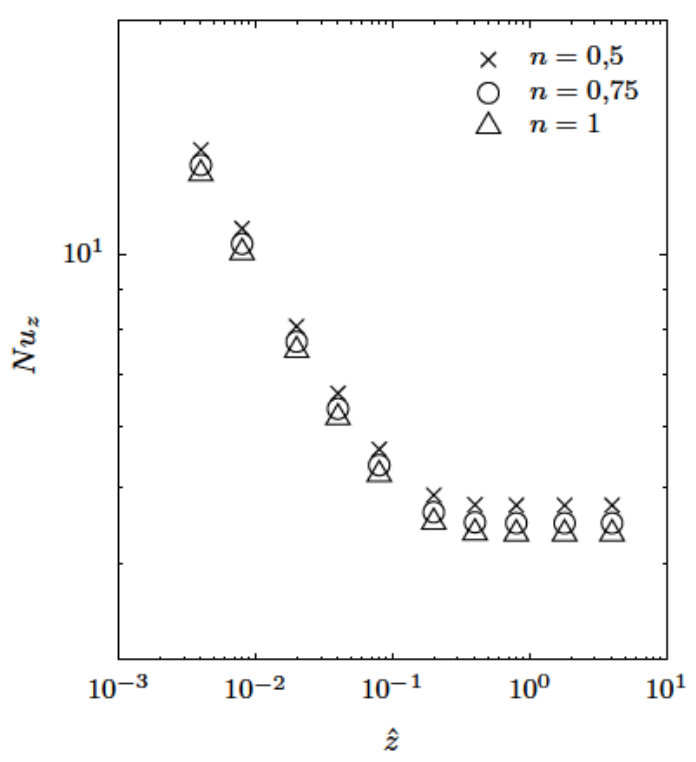

(a) Tubo liso.

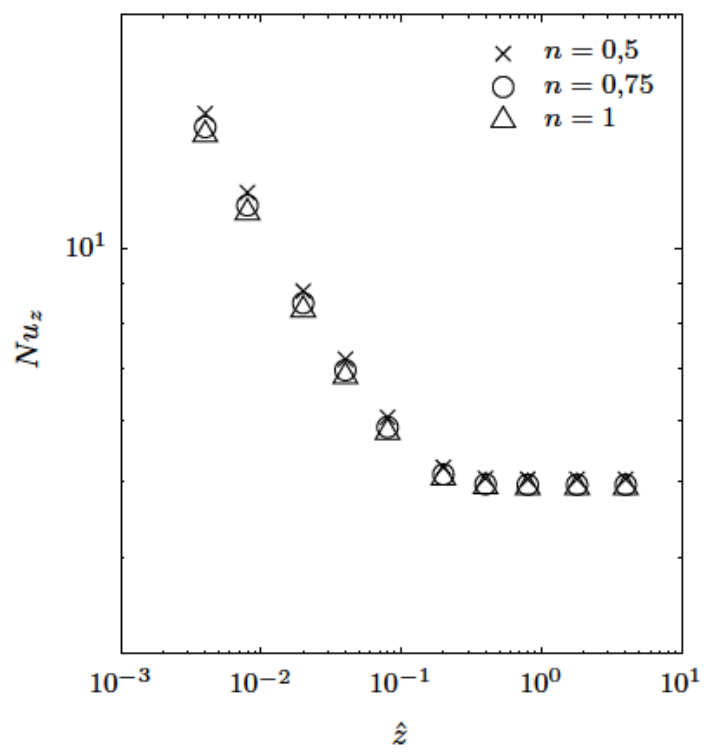

(b) Tubo con eje $(\alpha=0,277)$

Figura 9.1: Numero de Nusselt local en flujo de fluidos no newtonianos en situación de convección puramente forzada.

se adjuntan los resultados numéricos obtenidos mediante el modelo descrito en el Capítulo 3. Los datos muestran que los resultados numéricos y de la Ec. 9.2 prácticamente coinciden (desviaciones del 0,01\%), mientras que existen desviaciones de hasta el 1,1\% con respecto a la aproximación de la Ec. 9.3. Además en la Fig. 9.1(a) se encuentra representado el número de Nusselt local en la región de entrada para diferentes fluidos seudoplásticos, obtenido mediante el modelo numérico citado. Para el flujo completamente desarrollado en régimen de convección forzada en tubo liso, el número de Nusselt para $n=0,5$ es en torno a un $8 \%$ superior al obtenido con un fluido newtoniano $(n=1)$.

\subsubsection{Tubo con eje}

En el presente apartado se busca definir una expresión aproximada para tubo de sección anular o tubo con eje, similar a la definida en la Ec. 9.3 para tubo liso. El objetivo final es el de aplicar esta corrección al tubo con rascador, debido a la similitud existente entre las geometrías. Los detalles de cálculo correspondientes al presente apartado se han incluido en el Apartado A.5 del Apéndice.

El primer paso para lograr el objetivo marcado es el de obtener el valor de $\Delta$, definida 
según la Ec. 9.1. Para ello, de nuevo, se hace uso del simulador numérico descrito en el descrito en el Capítulo 3, validando el método previamente con la obtención de $\Delta$ en tubo liso. El procedimiento se realiza para la geometría de tubo con eje con una relación de radios igual a la del tubo con rascador de la instalación de ensayos térmicos, $\alpha=5 / 18$. Los valores obtenidos numéricamente para $\Delta$ se ajustan correctamente a la siguiente ecuación con una constante $h=7,532 \pm 0,001$ (intervalo de confianza del 95\%).

$$
\Delta=\frac{24 n+h}{(24+h) n}
$$

Así mismo se ha comprobado que en situación de movimiento alternativo del eje, el valor promedio de $\Delta$ viene dado por la ecuación anterior, es decir, que no varía respecto a la situación con rascador estático. Por lo tanto este valor de $\Delta$ es válido tanto para los regímenes estático como dinámico.

En la Fig. 9.1(b) se muestran los resultados del número de Nusselt local en la región de entrada en la geometría de tubo con eje. Se observa que para el flujo completamente desarrollado en régimen de convección forzada en tubo liso, el número de Nusselt para $n=0,5$ es en torno a un $2 \%$ superior al obtenido con un fluido con $n=1$.

A partir de los resultados numéricos se obtiene el valor de $N u_{\infty} / N u_{\infty, n=1}$. Así, de forma análoga al procedimiento de Grigull (1956), se obtiene el exponente de $\Delta$ para la geometría de tubo con eje, que se ajustan a

$$
N u_{\infty} / N u_{\infty, n=1}=\Delta^{1 / 9}
$$

con un error del $0,15 \%$, estando $\Delta$ definida por la Ec. 9.4.

La definición de $\Delta$ obtenida para la geometría de tubo con eje se utiliza posteriormente para caracterizar la influencia de la seudoplasticidad en la transmisión de calor en el tubo con rascador, tanto en régimen estático como dinámico.

\subsection{Transmisión de calor en régimen estático}

En el presente apartado se exponen y analizan los resultados de transmisión de calor en el tubo con rascador estático. Se espera que la inserción del rascador en reposo en el interior del tubo, produzca mejoras de la transferencia de calor debido al trayecto zigzagueante que debe realizar el flujo. Tal y como se estudia en el Capítulo 7, la disposición al tresbolillo de los 


\begin{tabular}{cccccc}
\hline Expto & $T\left({ }^{\circ} \mathrm{C}\right)$ & $n$ & $m\left({\left.\mathrm{~Pa} . \mathrm{S}^{\mathrm{n}}\right)}^{\mathrm{o}}\right.$ & $P r_{g}$ & $P r_{g, r e f}$ \\
\hline TC1 & 20 & $0,85-0,82$ & $0,1091-0,2297$ & $415-550$ & 500 \\
TC2 & 18 & $0,86-0,84$ & $0,1244-0,1836$ & $394-529$ & 470 \\
TC3 & 25 & $0,88-0,86$ & $0,1048-0,1115$ & $288-356$ & 330 \\
TC4 & 35 & $0,93-0,94$ & $0,0488-0,0433$ & $180-201$ & 190 \\
TC5 & 15 & $0,45-0,45$ & $4,571-4,527$ & $1240-4500$ & 2960 \\
TC6 & 25 & $0,46-0,47$ & $3,873-3,470$ & $967-4240$ & 2400 \\
TC7 & 35 & $0,52-0,52$ & $2,113-2,093$ & $637-2560$ & 1430 \\
TC8 & 15 & $0,60-0,60$ & $1,439-1,371$ & $712-2110$ & 1320 \\
TC9 & 25 & $0,62-0,64$ & $1,026-0,886$ & $556-1510$ & 940 \\
T10 & 35 & $0,69-0,70$ & $0,5510-0,4820$ & $413-865$ & 630 \\
T11 & 25 & $0,74-0,74$ & $0,3810-0,3622$ & $396-721$ & 550 \\
T12 & 35 & $0,79-0,8$ & $0,2216-0,1838$ & $307-481$ & 390 \\
\hline
\end{tabular}

Tabla 9.2: Parámetros correspondientes a las tandas de ensayos de transmisión de calor en régimen estático realizados.

tacos obliga al flujo a desprenderse de las paredes y a realizar un movimiento zigzagueante que resulta positivo para la transferencia de calor. Además, tal y como se ha visto en el Capítulo 8, la presencia del rascador adelanta la transición a la turbulencia, lo que puede provocar considerables incrementos en la transmisión de calor.

Con el objetivo de cuantificar la mejora en la transmisión de calor que produce la presencia del elemento insertado, se han realizado ensayos con fluidos en diferentes estados de degradación. Los valores de las propiedades reológicas del fluido han variado en los siguientes márgenes: $n \in[0,45 ; 0,94], m \in[4,6 ; 0,04]$ Pa.s ${ }^{n}$. Para dichas propiedades del fluido, variando el caudal circulante y manteniendo el salto térmico, en los ensayos realizados los números adimensionales de Prandtl y Reynolds generalizados han variado en los rangos $R e_{g} \in[0,4 ; 320]$ y $\operatorname{Pr}_{g} \in[180,4500]$. En la Tabla 9.2 se encuentran detallados los ensayos realizados.

Por conveniencia, se repiten a continuación las expresiones de los monomios adimensionales que describen el proceso:

$$
\begin{aligned}
& R e_{g}=\frac{\rho u_{b} D_{h}}{\mu_{g}} ; \quad \quad P r_{g}=\frac{c_{p} \mu_{g}}{k} ; \quad N u=\frac{h_{i} D_{h}}{k} \\
& \mu_{g}=m n^{d} c^{n-1}\left(\frac{u_{b}}{D_{h}}\right)^{n-1}
\end{aligned}
$$

Tal y como se detalla en el Apartado 6.3.1, el número de Nusselt se corrige para tener en cuenta la variación del coeficiente de consistencia del fluido $m$, al aplicar la condición de contorno de flujo en la pared. 
Nótese que debido a la definición del número de Prandtl, éste depende entre otros parámetros de la velocidad media del flujo, $u_{b}$ y de $n$. Esto implica que para un mismo fluido (suponiendo que no se degrada) es inviable realizar ensayos a diferentes números de Reynolds sin variar también el Prandtl. En consecuencia, para una misma tanda de ensayos, en la que se varía el caudal y donde también varían ligeramente las propiedades reológicas, el número de Prandtl varía en un rango amplio. Esto supone un inconveniente a la hora de analizar los resultados, ya que los efectos del Reynolds y el Prandtl se encuentran superpuestos en los resultados obtenidos. Para solventarlo, se recurre al siguiente procedimiento:

1. Identificar regiones: en primer lugar se identifican las regiones de comportamiento del flujo en función de $R e_{g}$.

2. Obtención de correlaciones: A partir de los datos experimentales obtenidos se obtienen correlaciones experimentales del número de Nusselt en cada región.

3. Traslado de resultados a un mismo $\operatorname{Pr}_{g}$ : utilizando las correlaciones obtenidas, se trasladan los resultados de $N u$ a un mismo $P r_{g}$, lo que permite observar el efecto de $R e_{g}$ de forma aislada.

4. Proceso iterativo: Por último se vuelve a comenzar el procedimiento en el paso 1 comprobando que la identificación de las regiones es correcta y finalizando en el paso 3.

\subsubsection{Resultados}

De acuerdo con el procedimiento descrito, en primer lugar se identifican las regiones del flujo. En la Fig. 9.2 se representan las medidas del número de Nusselt en función del Reynolds para una selección de las tandas de ensayos realizadas. En ella se puede observar la variación que se produce en el número de Prandtl a lo largo de un ensayo. En dicha representación, a pesar de la superposición del efecto del Prandtl se pueden observar al menos tres regiones de comportamiento del flujo en función del número de Reynolds (regímenes laminar, de transición y turbulento). Estas regiones se analizan en profundidad posteriormente, donde se detalla la separación de la región laminar en dos subregiones.

En el segundo paso se obtienen las correlaciones experimentales correspondientes a cada región. En este punto, partiendo del desarrollo del Apartado 9.1, se plantea la siguiente correlación

$$
N u=a \times \operatorname{Re}_{g}^{b} \times \operatorname{Pr}_{g}^{c} \times \Delta^{1 / 9}
$$



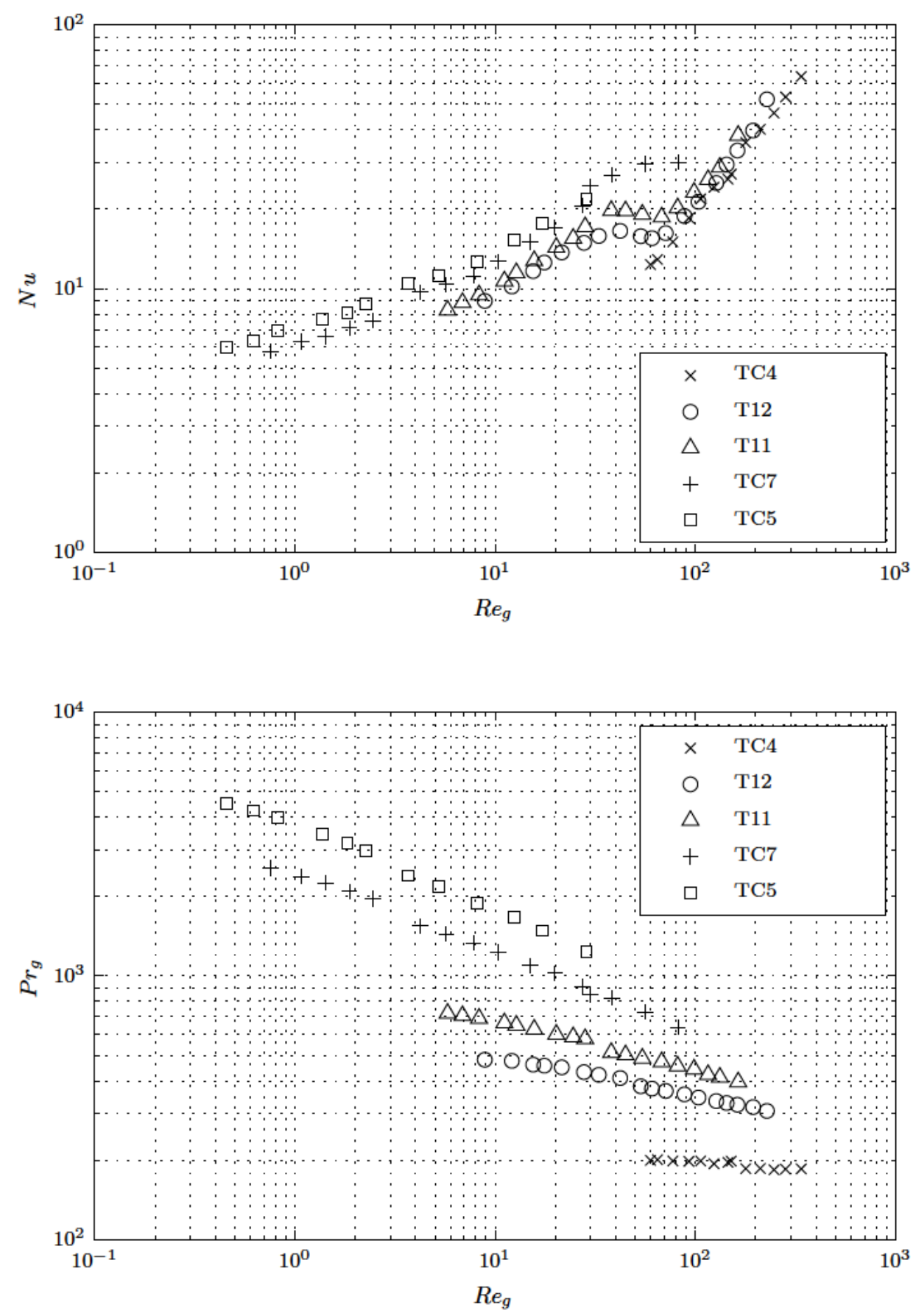

Figura 9.2: Resultados en los ensayos de transmisión de calor en régimen estático, donde se observa la variación simultánea de los números de Reynolds y Prandtl generalizados para una misma tanda de ensayos. 


\begin{tabular}{ccccc}
\hline Región & $a$ & $b$ & $c$ & Error \\
\hline I & 0,4037 & 0,3735 & 0,3002 & $4,8 \%$ \\
II & 0,4148 & 0,5921 & 0,2352 & $7,4 \%$ \\
IV & 0,0259 & 1,1107 & 0,2354 & $19,8 \%$ \\
\hline
\end{tabular}

Tabla 9.3: Correlaciones experimentales del número de Nusselt medio, en las diferentes regiones del flujo (véase Fig. 9.4).

donde se utiliza el valor de $\Delta$ definido para geometría de tubo con eje (Ec. 9.4), es decir, sin tacos. Mientras que $a, b$ y $c$ son las constantes de la correlación. Los valores obtenidos en los ajustes experimentales para dichos coeficientes se detallan en la Tabla 9.3 en función de la región del flujo.

En adelante se representa en las figuras el cociente $N u / \Delta^{1 / 9}$ para simplificar dicha representación.

En el tercer paso, los valores de Nusselt medidos $N u_{i}$, cada uno a un valor de $\operatorname{Pr}_{g, i}$ distinto, se pueden trasladar a un mismo $P r_{g, r e f}$ mediante la expresión siguiente

$$
N u=N u_{i}\left(\frac{P r_{g, r e f}}{P r_{g, i}}\right)^{c}
$$

de modo que se puede observar perfectamente la influencia aislada del número de Reynolds en el proceso de transmisión de calor. Nótese el uso del coeficiente $c$ obtenido en las correlaciones experimentales (Tabla 9.3).

Mediante el procedimiento descrito se pueden trasladar los resultados de una tanda de experimentos al número de Prandtl medio de la tanda. De este modo en los resultados del número de Nusselt, únicamente se observa el efecto del número de Reynolds. En la Fig. 9.3 se representan dichos resultados para una selección de las tandas de experimentos realizadas. El resto de resultados se muestran en el Apéndice D.1.

De forma análoga, se pueden trasladar todas las medidas del número de Nusselt de las diferentes tandas de ensayos a un Prandtl intermedio: $P r_{g, r e f}=1000$. La representación de dichos resultados se muestra en la Fig. 9.4, donde se pueden apreciar claramente las regiones en las que se divide el flujo:

- Región de flujo laminar: la región de flujo laminar se divide en dos subregiones donde la dependencia del Nusselt con el Reynolds en una y otra es distinta.

- Región I: región de flujo que aparece a valores del número de Reynolds muy bajos $R e_{g}<5$. En el gráfico se observa que la pendiente de la curva en esta región 


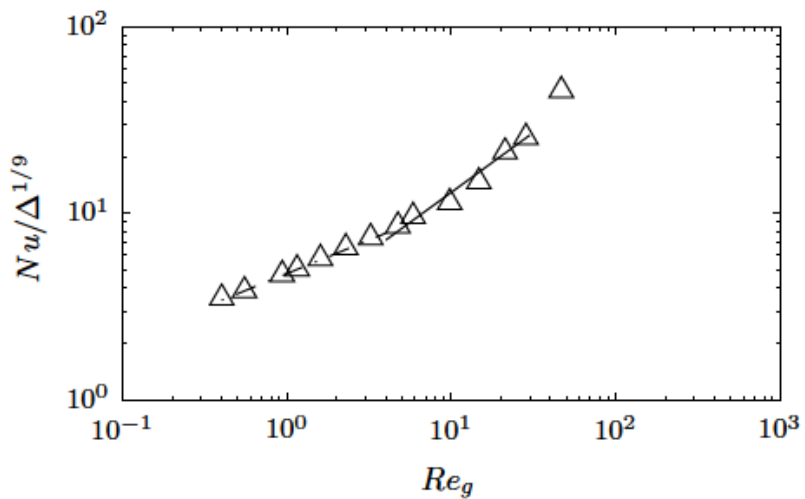

(a) TC6, $P r_{g, r e f}=2400$

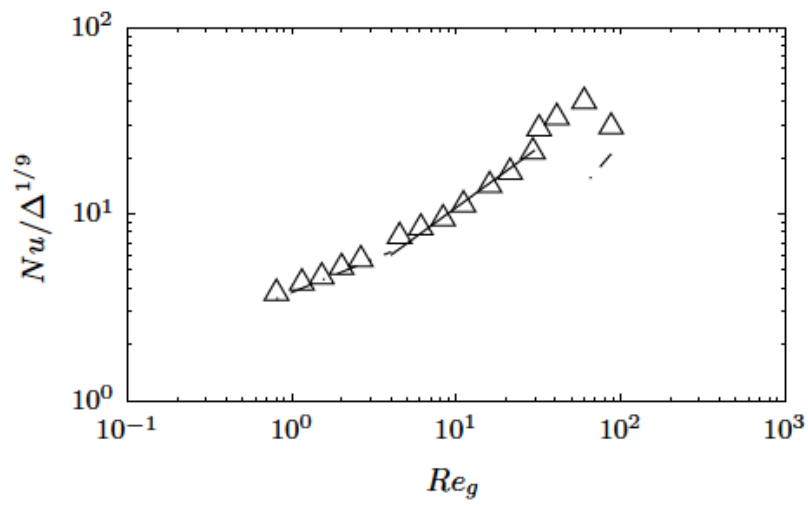

(c) TC7, $P r_{g, r e f}=1430$

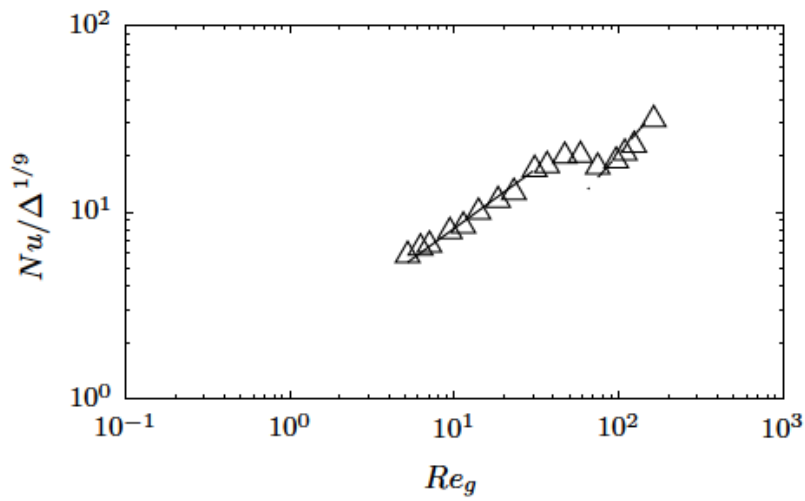

(e) $\mathrm{TC} 10, P r_{g, r e f}=630$

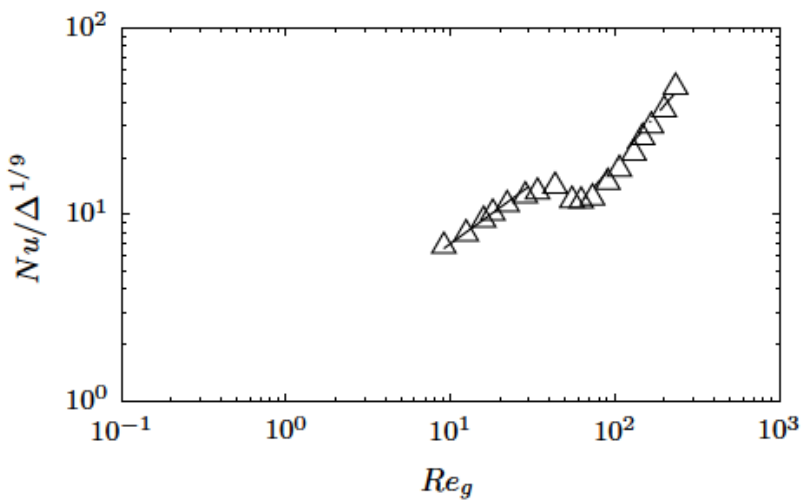

(b) $\mathrm{T} 12, P r_{g, r e f}=390$

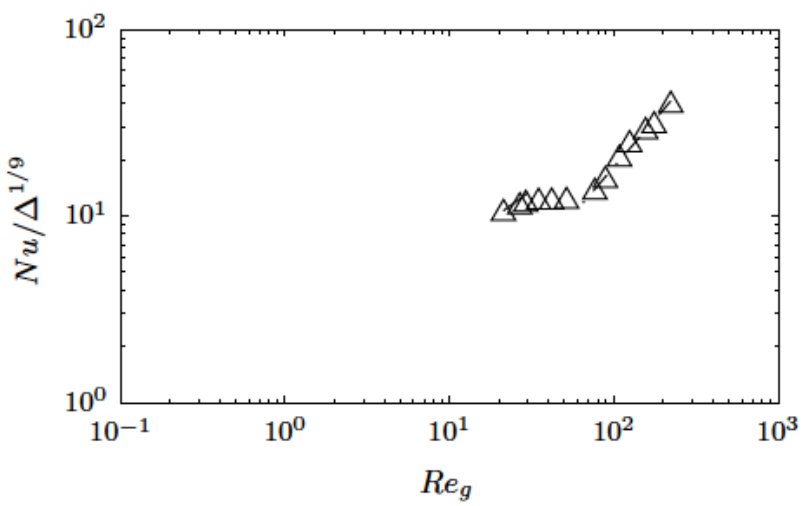

(d) TC3, $P r_{g, r e f}=330$

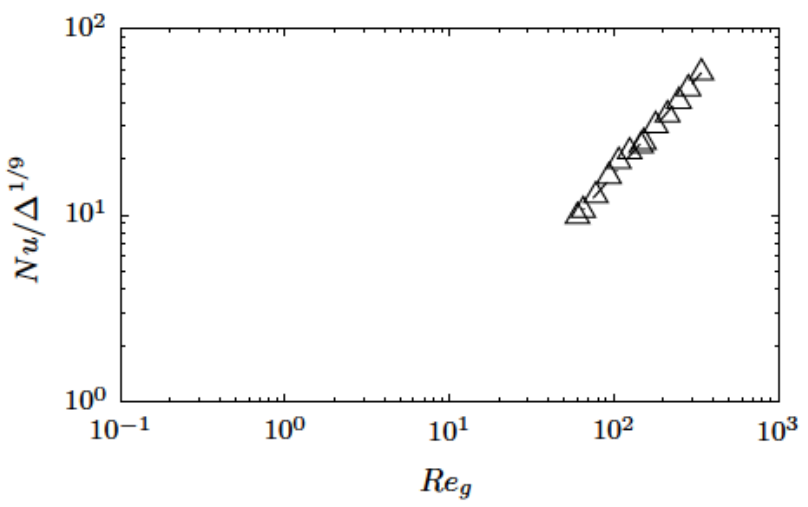

(f) $\mathrm{TC} 4, P r_{g, r e f}=190$

Figura 9.3: Selección de tandas de ensayos trasladados al número de Prandtl medio de la tanda. Las correlaciones se representan con una línea continua (región II) o discontinua (regiones I y IV). 


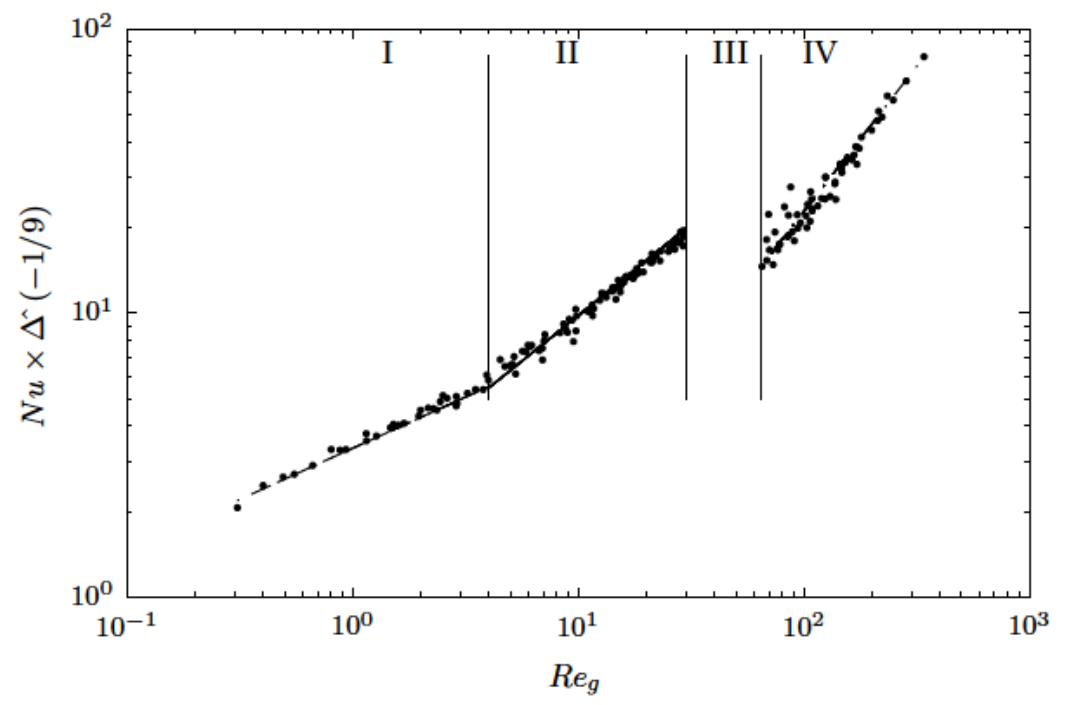

Figura 9.4: Regiones del flujo. Se representa el total de ensayos realizados trasladados a $\operatorname{Pr}_{g}=1000$. Los ensayos en la región turbulenta (III) no se pueden trasladar a $\operatorname{Pr}_{g}=1000$, ya que no se han obtenido correlaciones.

es comparativamente baja (ejes logarítmicos), indicando una menor influencia del número de Reynolds.

- Región II: región de flujo laminar que aparece a números de Reynolds entre $5>$ $R e_{g}<30$. Se observa una pendiente mayor de la curva en esta región, indicando que la influencia del número de Reynolds es más importante que en la región I.

- Región III: región de transición. Esta región abarca números de Reynolds entre $30<$ $R e_{g}<65$. En esta región el $N u$ tiene una baja dependencia de $R e_{g}$ y resulta más importante el efecto del Prandtl. En general es difícil predecir el comportamiento del flujo en esta zona, en la que la incertidumbre es alta, tal y como se puede observar en la Fig. 9.4.

- Región IV: región de flujo turbulento. El flujo turbulento aparece a números de Reynolds $R e_{g}>65$. En esta región la curva en ejes logarítmicos tiene una pendiente alta, ya que el número de Nusselt aumenta rápidamente con el número de Reynolds.

Por último, a modo de validación de la metodología, se comparan los resultados obtenidos con los existentes en la bibliografía. En ella existen medidas experimentales del número de Nusselt en la misma geometría para números de Prandtl entre 150 y 700. En la Fig. 9.5 se 


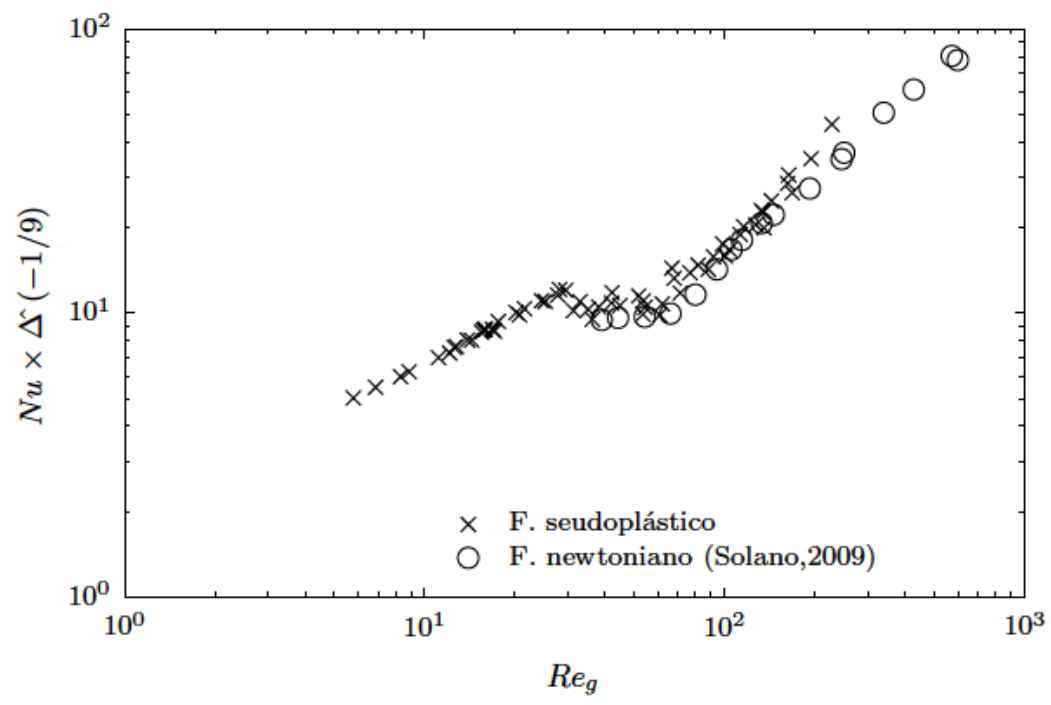

Figura 9.5: Comparación de los resultados de transmisión de calor en régimen estático, obtenidos en ensayos con flujos de comportamiento seudoplástico con los existentes en la bibliografía para la misma geometría y fluidos newtonianos. En ambos casos $P r_{g}=300$. Los resultados representados corresponden a los ensayos TC1, TC2, TC11 y TC12.

comparan las medidas experimentales a $P r=300$ para fluidos newtonianos existentes en la bibliografía, frente a los resultados de los ensayos con fluidos seudoplásticos realizados a números de Prandtl alrededor de $\operatorname{Pr}_{g}=300$ y cuyos resultados han sido trasladados a dicho Prandtl. En el gráfico se observa que los resultados coinciden. Esto confirma la validez de las definiciones de los números adimensionales, y por otro lado pone de manifiesto la utilidad de la definición de la viscosidad efectiva del fluido en los números de Prandtl y Nusselt.

\subsection{Transmisión de calor en régimen dinámico}

Se estudia en este apartado la transferencia de calor en regímenes de rascado dinámico. De este modo se pretende caracterizar el proceso de transmisión de calor con fluidos seudoplásticos en situaciones de rascador dinámico.

Tal y como se menciona anteriormente y como se ha estudiado en el Capítulo 2, el problema dinámico únicamente añade la variable $\left|v_{s}\right|$ al sistema. En el estudio de la pérdida de 
presión realizado en el Capítulo 8, la variable $v_{s}$ se adimensionaliza utilizando el factor de bloqueo, $\beta$, pero en este caso no es posible, ya que al estudiar ciclos de rascado completos el valor promedio del factor de bloqueo siempre sería 1, independientemente del valor de $\left|v_{s}\right|$, de modo que se opta por el uso de $|\omega|$ como variable adimensional.

$$
|\omega|=\frac{\left|v_{s}\right|}{u_{b}}
$$

Tal y como se introduce en el Capítulo 6, el proceso se estudia con dos sistemas de bombeo distintos, uno con mayores engranajes y funcionando a regímenes de giro menores (BA) y otro con engranajes más pequeños, tolerancias más ajustadas y funcionando a regímenes de giro mayores (BB). Dependiendo del sistema de bombeo utilizado, la diferencia entre el caudal impulsado entre el semiciclo de rascado equicorriente y el contracorriente difiere, siendo significativamente mayor al utilizar la bomba BA, mientras que con la bomba BB, la oscilación es muy pequeña. En el Capítulo 8 se estudia la caída de presión que producen ambos sistemas y se concluye que, a pesar de que los caudales en equicorriente y contracorriente son distintos, la caída de presión promedio es prácticamente la misma para el mismo caudal medio. Además en los ciclos equicorriente y contracorriente, para el mismo caudal circulante y la misma relación de velocidades $|\omega|$, la caída de presión también es la misma.

En el análisis del problema térmico en régimen dinámico, debido a las inercias térmicas, no tiene sentido el estudio de los sentidos de rascado equicorriente y contracorriente de forma diferenciada, sino que se debe estudiar el ciclo completo. De este modo se define la estabilidad térmica como aquella situación en la que los márgenes de variación de la temperatura de pared del tubo son estables en el tiempo. En concreto se establece que la estabilidad debe alcanzar una duración de al menos 3 minutos para que la medida sea válida. La amplitud de la oscilación de la temperatura depende del cada ensayo en concreto, así que se establece como condición que la temperatura media de la pared de 1 ciclo de rascado se encuentre en un margen de $0,05^{\circ} \mathrm{C}$.

En los ensayos, se utilizan fluidos con distintas propiedades reológicas, que varían en los mismos rangos que en apartados anteriores $n \in[0,45 ; 0,94], m \in[4,6 ; 0,04]$ Pa.s ${ }^{n}$. Para dichas propiedades del fluido, en los ensayos realizados los números adimensionales de Prandtl y Reynolds han variado en los rangos $R e_{g} \in[1,3 ; 216]$ y $\operatorname{Pr}_{g} \in[250,2600]$. Al igual que en el Capítulo 8, se han analizado ciclos de rascado a baja velocidad, que tendrán una potencia de accionamiento más pequeña. En concreto se ha estudiado el rango de $|\omega| \in[0,1,1,5]$. Los ensayos se han repartido en 9 tandas de experimentos, en cada una de las cuales se barre 


\begin{tabular}{cccccc}
\hline Expto & Bomba & $T\left(^{\circ} \mathrm{C}\right)$ & $n$ & $m\left(\right.$ Pa.s $\left.^{n}\right)$ & $\operatorname{Pr}_{g}$ \\
\hline TCD-1 & BB & $16,17-17,23$ & $0,48-0,53$ & $2,862-1,928$ & $215-2120$ \\
TCD-2 & BB & $16,9-17,61$ & $0,59-0,63$ & $1,292-0,8988$ & $632-1450$ \\
TCD-3 & BB & $16,7-16,3$ & $0,81-0,82$ & $0,2235-0,1907$ & $410-600$ \\
TCD-4 & BB & $17,2-18$ & $0,93-0,94$ & $0,0624-0,0551$ & $250-290$ \\
TCD-5 & BA & $25,8-25,5$ & $0,75-0,74$ & $0,3478-0,3588$ & $456-746$ \\
TCD-6 & BA & $16,9-16$ & $0,69-0,71$ & $0,6303-0,5910$ & $630-1070$ \\
TCD-7 & BA & $17,6-17,0$ & $0,52-0,52$ & $2,640-2,557$ & $790-2625$ \\
TCD-8 & BA & $18,0-17,7$ & $0,62-0,64$ & $1,164-0,9691$ & $700-1590$ \\
TCD-9 & BA & $15,8-15,8$ & $0,87-0,89$ & $0,1267-0,1118$ & $330-425$ \\
\hline
\end{tabular}

Tabla 9.4: Experimentos de transmisión de calor en régimen de rascador dinámico. BA: Bomba de engranajes grandes. BB: bomba engranajes pequeños.

el rango de caudales de ensayo completo y todas las velocidades de rascado. Los ensayos realizados se detallan en la tabla 9.4, donde se puede observar que con los dos sistemas de bombeo utilizados se ha abarcado todo el rango de propiedades del fluido.

\subsubsection{Resultados de transmisión de calor en régimen dinámico}

Al igual que ocurre con los ensayos estáticos, el valor del número de Prandtl varía a lo largo de una tanda de experimentos, debido principalmente a la dependencia de la viscosidad generalizada del fluido con la velocidad media del flujo. En la Fig. 9.6 se muestran los resultados para 3 de las 9 tandas de ensayos realizadas (el resto se adjuntan en el Apartado D.1 del Apéndice). Según la metodología de ensayo utilizada, en una misma tanda se realizan ensayos para una serie de caudales y diferentes velocidades de rascado, coincidiendo los caudales a los que se realizan los ensayos a diferente velocidad de rascado. Así, entre los ensayos a un mismo caudal y diferente $\omega$, el número de Prandtl sólo puede variar a causa de la variación de las propiedades reológicas o a la imprecisión a la hora de regular el caudal. De este modo se puede observar en las figuras mencionadas que el Nusselt aumenta para un mismo caudal al aumentar la velocidad de rascado. Sin embargo, este efecto es difícil de cuantificar, más aún al variar $R e_{g}$ ya que, como se observa en las figuras, $P r_{g}$ también varía.

Por otro lado, en la Fig. 9.7 y la Fig. 9.8 se muestran los resultados en régimen dinámico en los que se agrupan los resultados en función de la velocidad de rascado, de modo que los resultados de las diferentes tandas quedan representados en la misma gráfica. Sin embargo, y de nuevo debido a la variación del $P r_{g}$, no se puede apreciar el efecto de la velocidad de rascado o si existen diferencias entre fluidos con diferentes propiedades reológicas. 

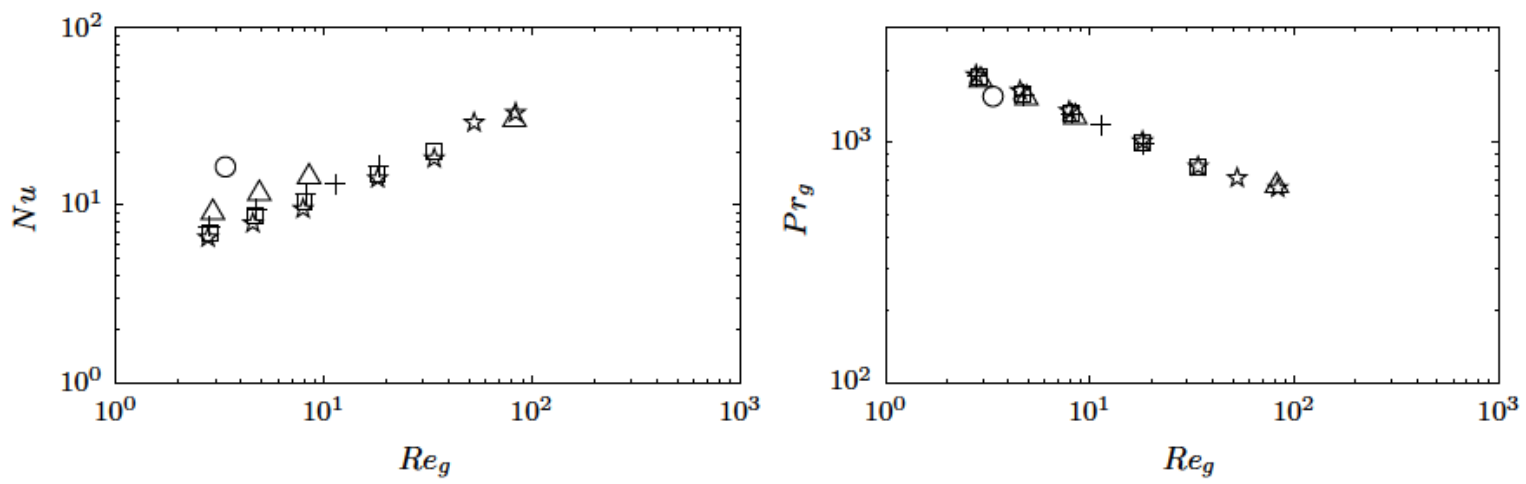

(a) $T C D-1(\mathrm{BB})$
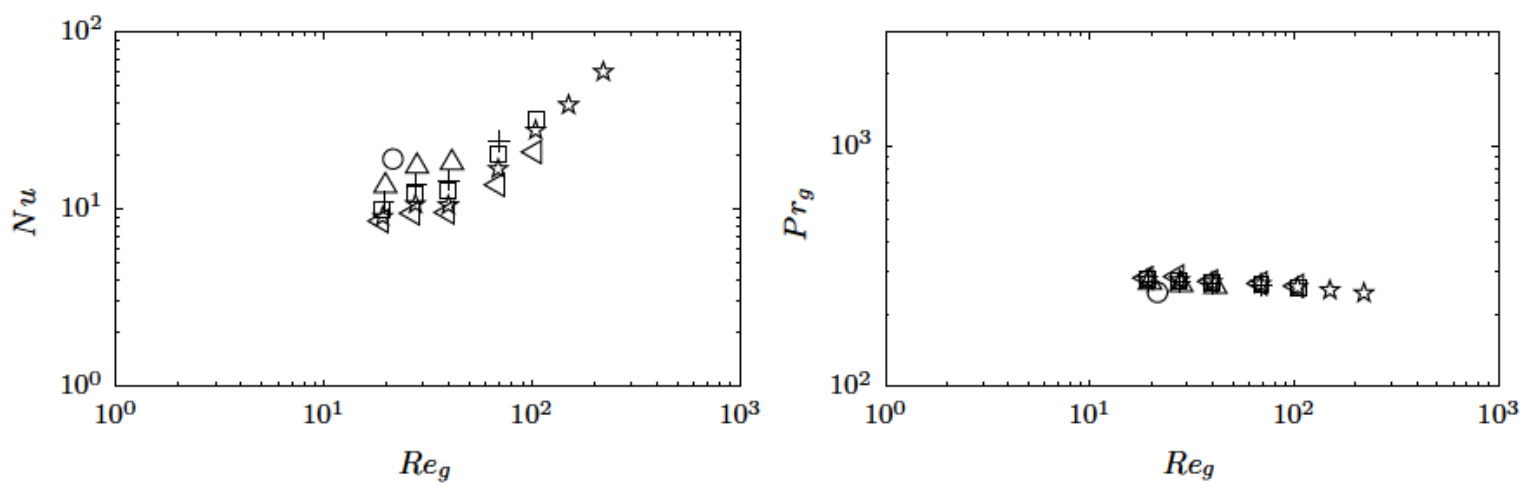

(b) $T C D-4(\mathrm{BB})$
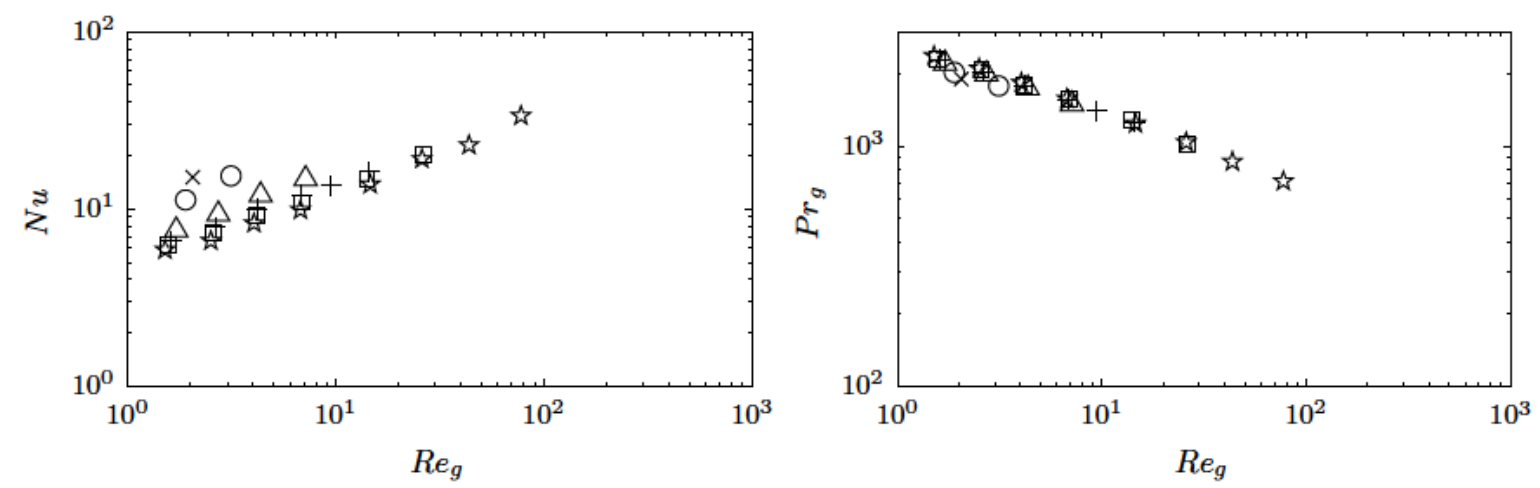

(c) $T C D-7$ (BA)

\begin{tabular}{|c|c|c|c|c|c|c|c|}
\hline$x$ & $\omega=1,5$ & $\triangle$ & $\omega=0,5$ & $\square$ & $\omega=0,2$ & $\triangleleft$ & $\omega=0$ \\
\hline O & $\omega=1$ & + & $\omega=0,3$ & t5 & $\omega=0,1$ & & \\
\hline
\end{tabular}

Figura 9.6: Medidas del número de Nusselt en las diferentes tandas de experimentos (I). 

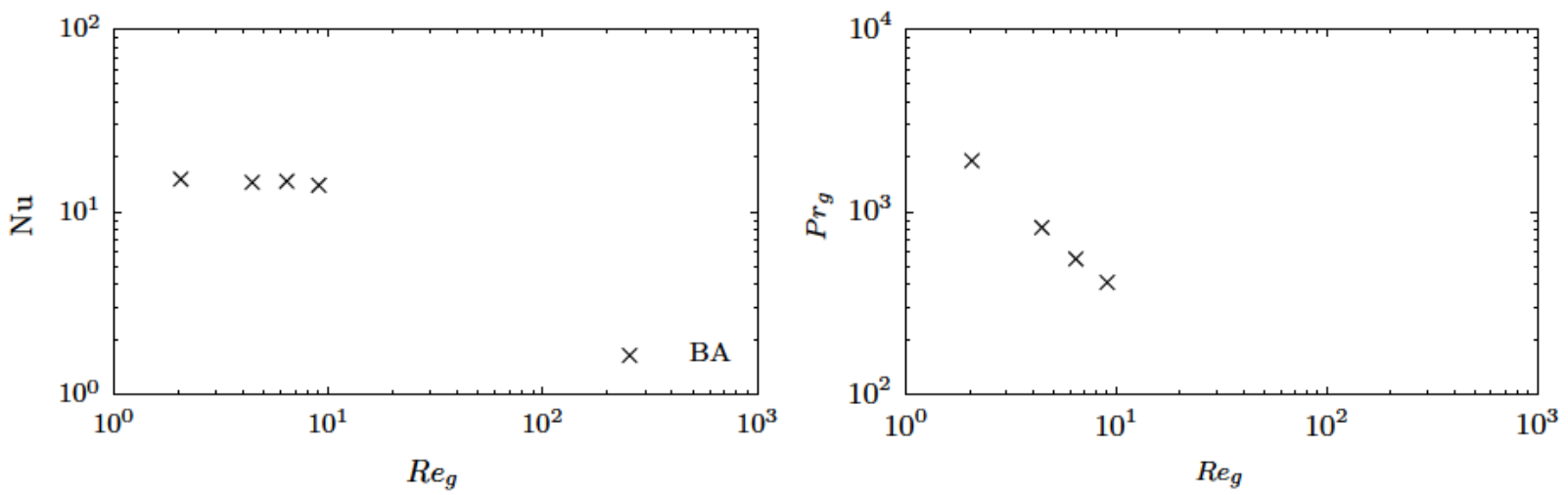

(a) $|\omega|=1,5$
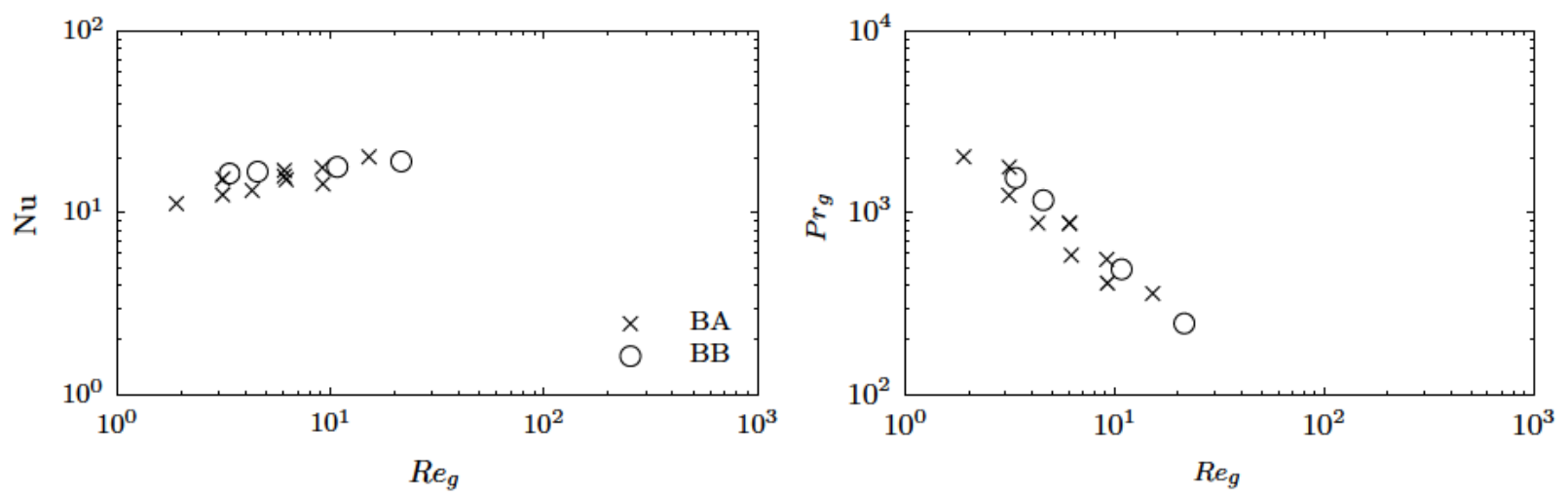

(b) $|\omega|=1$
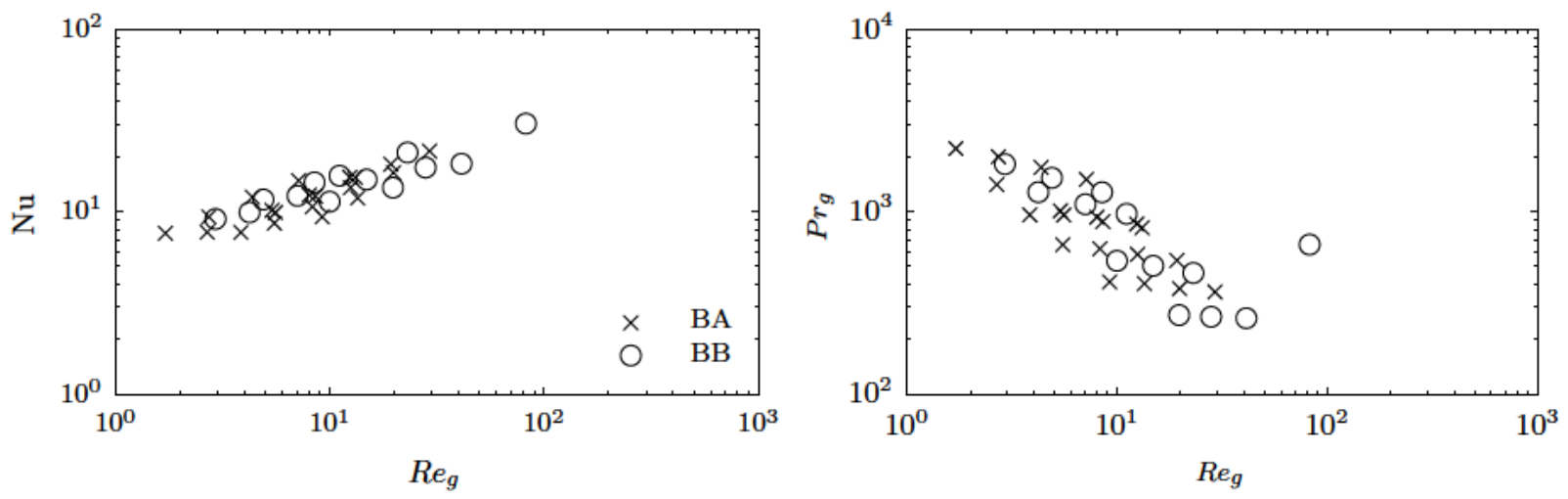

(c) $|\omega|=0,5$

Figura 9.7: Medidas del número de Nusselt agrupadas por velocidad de rascado (I). 

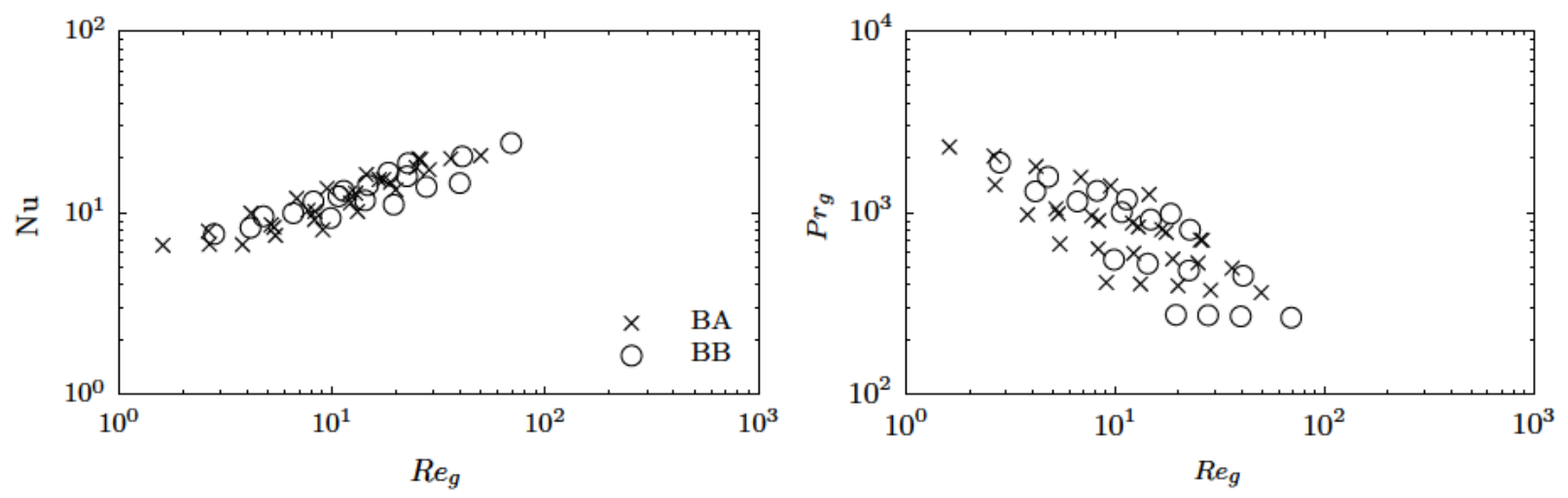

(a) $|\omega|=0,3$
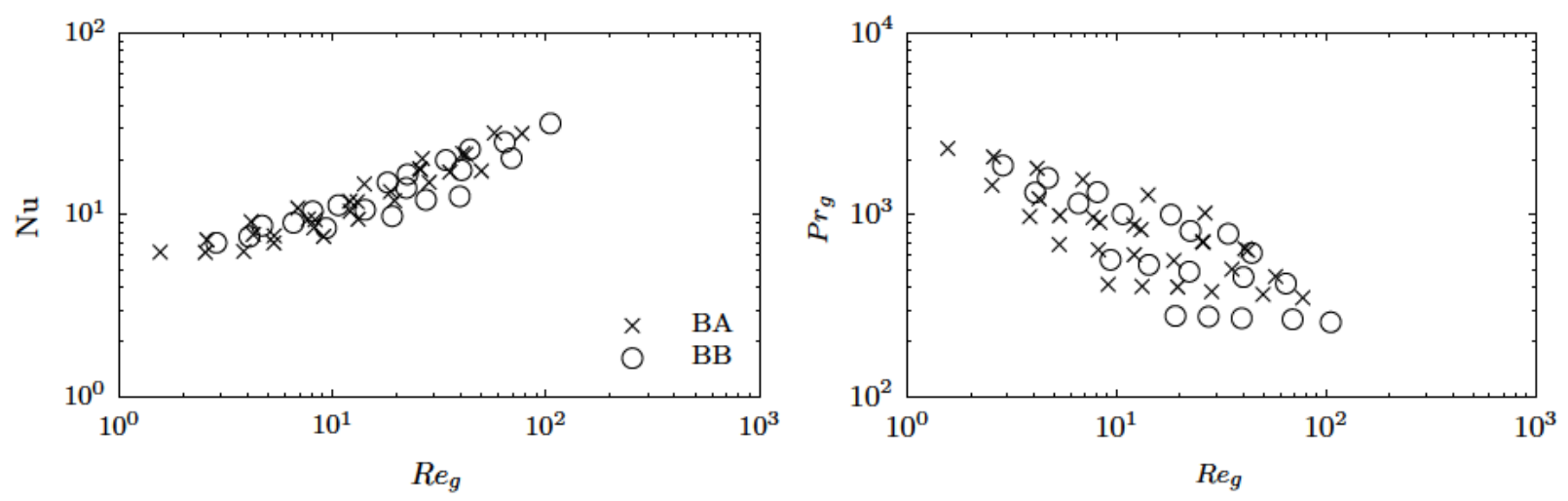

(b) $|\omega|=0,2$
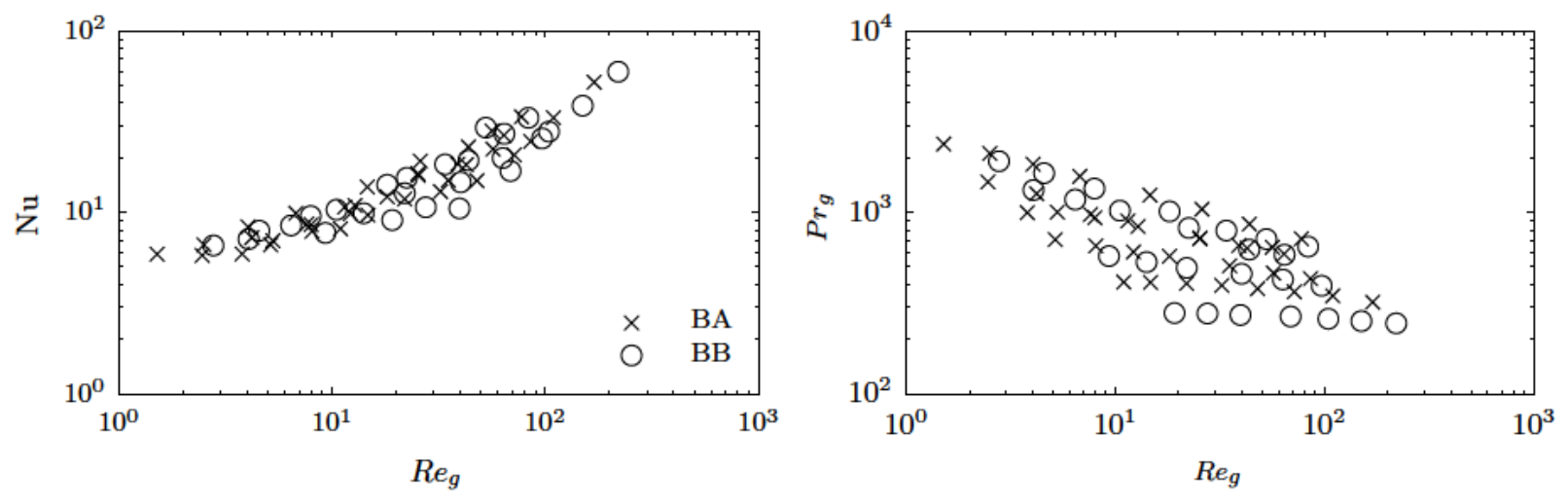

(c) $|\omega|=0,1$

Figura 9.8: Medidas del número de Nusselt agrupadas por velocidad de rascado (II). 

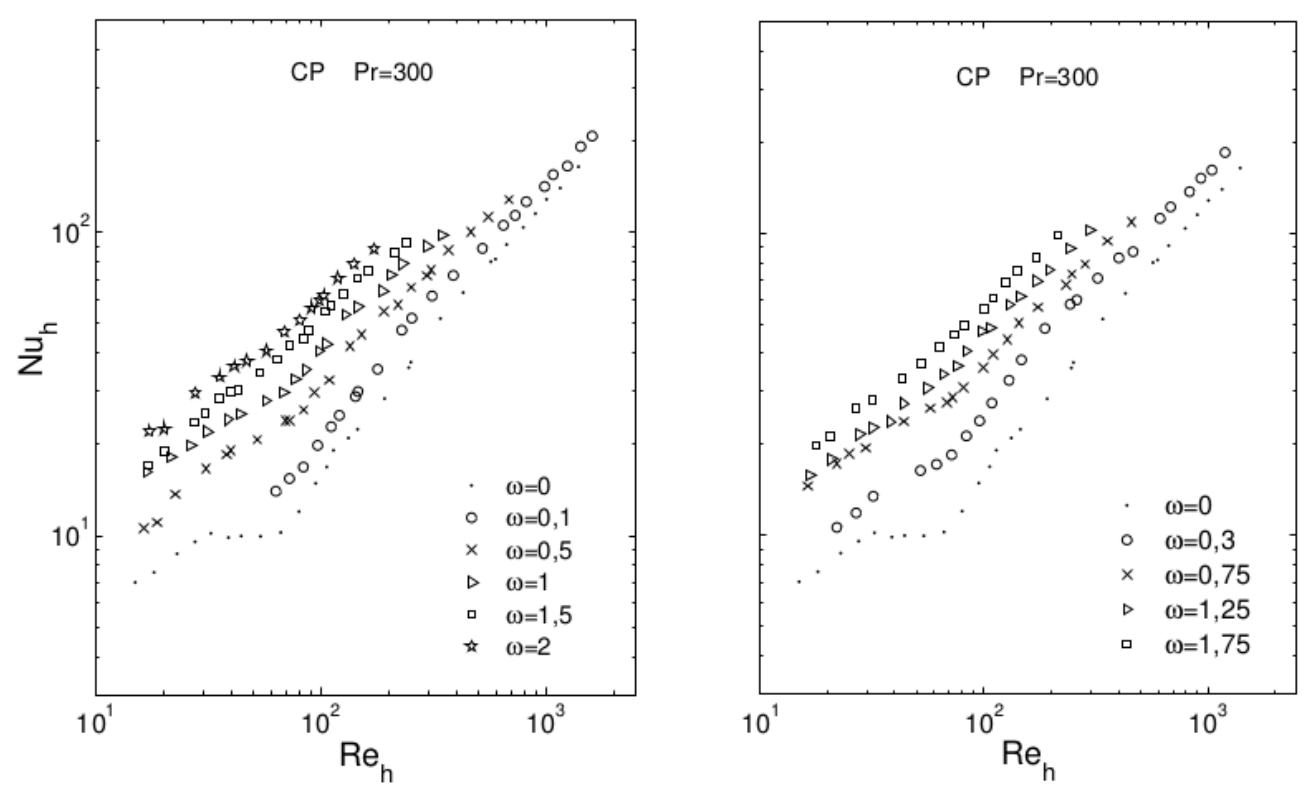

Figura 9.9: Número de Nusselt en régimen dinámico con fluidos newtonianos. (Solano, 2009a).

Siguiendo un procedimiento similar al del Apartado 9.2, para poder observar el efecto de la velocidad de rascado en la transmisión de calor, es necesario eliminar la variación del número de Prandtl generalizado. Para conseguirlo, primero es necesario obtener las correlaciones experimentales a las que se puede ajustar el número de Nusselt.

En primer lugar es necesario conocer las distintas regiones de comportamiento del flujo en función del número de Reynolds. Como base se puede partir de los resultados del Número de Nusselt existentes en la bibliografía para la geometría bajo estudio (véase Fig. 9.9). En dichos resultados se observan tres regiones en función del número de Reynolds ${ }^{1}$.

1. Para $R e<30$ el flujo es laminar.

2. Para $R e>60$ el flujo es turbulento.

3. Entre ambas regiones se produce una transición, donde el comportamiento varía con la velocidad de rascado.

La región de transición, en ensayos en régimen estático forma un escalón plano, mientras que con el rascador en movimiento la transición entre el flujo laminar y el turbulento parece ser más suave. Se observa además, que a mayores velocidades de rascado, la región turbulenta

\footnotetext{
${ }^{1} \mathrm{El}$ valor del número de Reynolds para un fluido newtoniano $n=1$, es independiente de $n$, para cualquiera de las definiciones de la viscosidad utilizadas en la presente investigación.
} 


\begin{tabular}{ccccccccc}
\hline Región & $R e_{g}$ & $a$ & $b$ & $c$ & $d$ & $e$ & $N$ & Error \\
\hline I & $1-4$ & 0,0212 & 0,6677 & 0,6102 & 1,2401 & 1,5544 & 26 & $15,1 \%$ \\
II & $4-30$ & 0,2584 & 0,5989 & 0,3702 & 0,6511 & 0,9300 & 147 & $6,6 \%$ \\
IV & $>50$ & 0,0566 & 0,8977 & 0,3820 & $2,2 \times 10^{-10}$ & 0,0179 & 24 & $21,7 \%$ \\
\hline
\end{tabular}

Tabla 9.5: Correlaciones experimentales de la transmisión de calor. Ec. 9.7. $N$ son las medidas experimentales en cada rango.

aparece a números de Reynolds menores, aunque esta variación no es muy significativa. Por ejemplo se puede observar que si $|\omega|=1,5$ el flujo turbulento se adelanta a $R e>50$.

Utilizando los margenes observados en los resultados obtenidos con fluidos newtonianos en régimen dinámico y dividiendo la región de flujo laminar en dos subregiones, tal y como indican los resultados obtenidos en régimen estático, se obtienen un total de cuatro regiones para el comportamiento del flujo al igual que ocurre en régimen estático. De este modo se obtienen correlaciones para tres de dichas regiones, omitiendo la zona de transición donde el comportamiento del flujo es cambiante. Los datos experimentales se ajustan adecuadamente a la Ec. 9.7. Los valores de las constantes aparecen en la Tabla 9.5 en función de la región del flujo.

$$
N u=a \operatorname{Re}_{g}^{b} \operatorname{Pr}_{g}^{c}(d+|\omega|)^{e} \Delta^{1 / 9}
$$

donde de nuevo se ha utilizado el factor $\Delta^{1 / 9}$. Como se vio anteriormente, para la geometría de tubo con eje, el valor promedio de dicho factor en régimen dinámico es prácticamente el mismo que en régimen estático. Cualitativamente se observa en las correlaciones un comportamiento similar al caso estático en cuanto a los números de Reynolds y Prandtl generalizados. Por otro lado el movimiento alternativo de rascado produce mejoras de transmisión de calor en todas las regiones del flujo.

Una vez obtenidas las correlaciones experimentales, se pueden trasladar los resultados del número de Nusselt a un Prandtl de referencia. Así pues, en la Fig. 9.10 se muestran los resultados trasladados a $\operatorname{Pr}_{g}=1000$. En ella además se diferencian los resultados obtenidos con los dos tipos de bomba utilizada. En los resultados no se aprecian diferencias debidas al uso de sendos sistemas de bombeo, con lo que se puede concluir que el sistema de bombeo no tiene influencia sobre la transmisión de calor. Además, en las situaciones de $|\omega|=1,5$, la desviación de las correlaciones obtenidas es mayor. Tal y como se vio en el Capítulo 7, para valores de $\omega>1$ en sentido equicorriente el flujo cambia significativamente y por lo tanto se debería obtener una correlación específica para $|\omega|>1$. No obstante, no es ese el objetivo del 

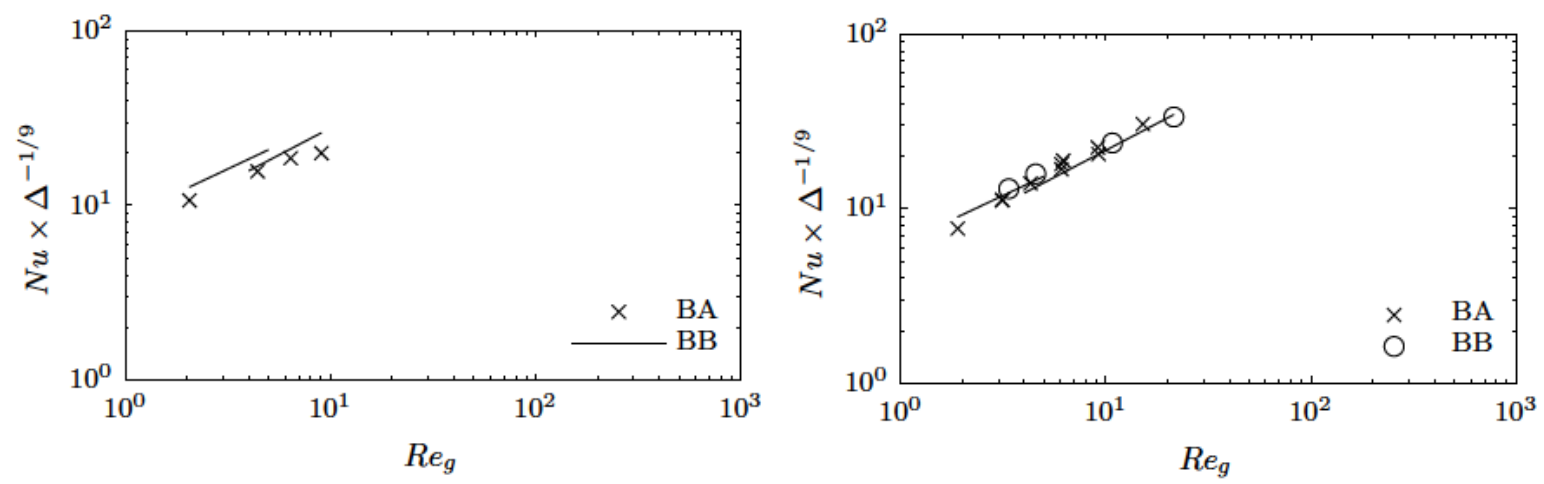

(a) $|\omega|=1,5$

(b) $|\omega|=1$
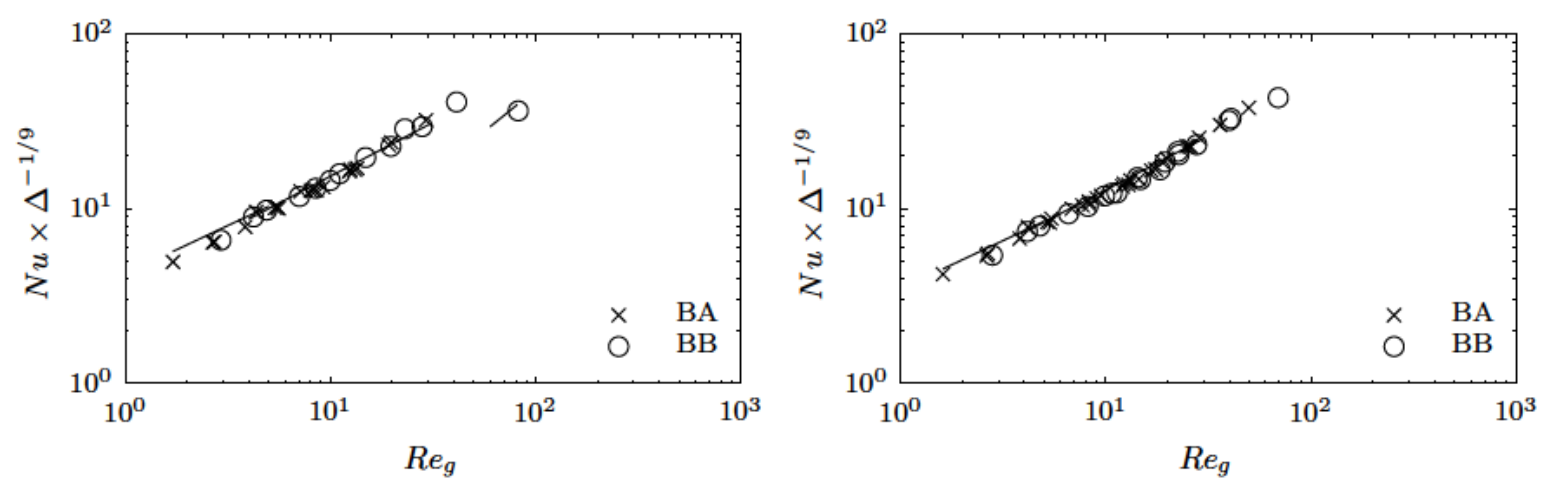

(c) $|\omega|=0,5$

(d) $|\omega|=0,3$
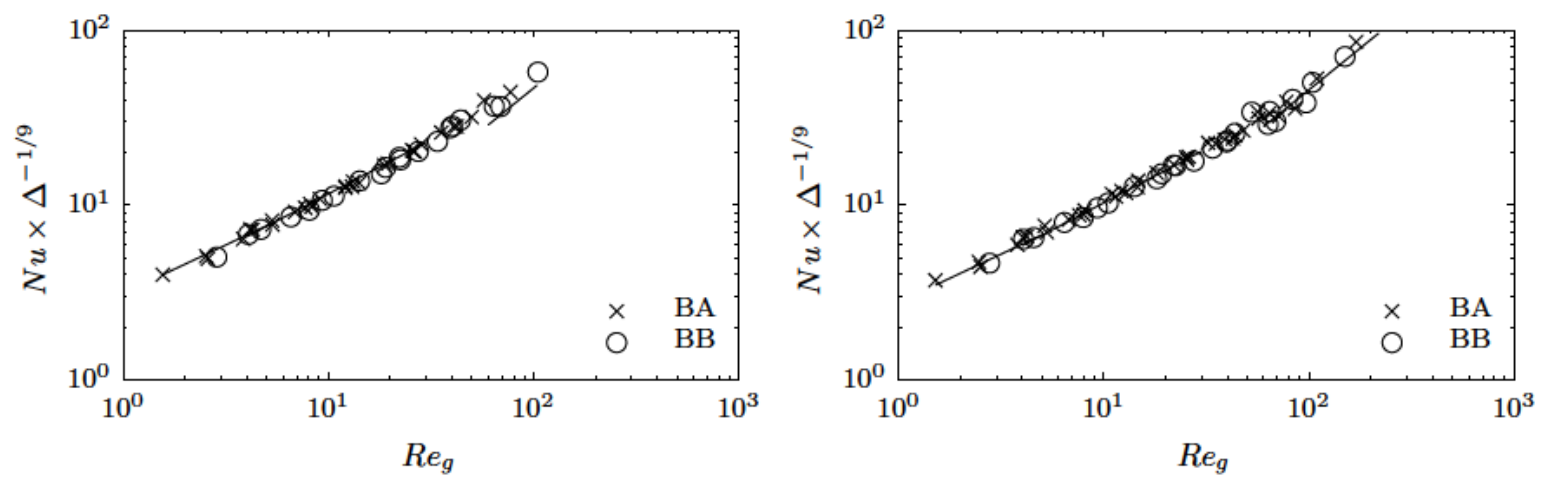

(e) $|\omega|=0,2$

(f) $|\omega|=0,1$

Figura 9.10: Medidas del número de Nusselt trasladadas a $\operatorname{Pr}_{g}=1000$. Ensayos con la bomba de engranajes grandes (BA) y de engranajes pequeños (BB). 

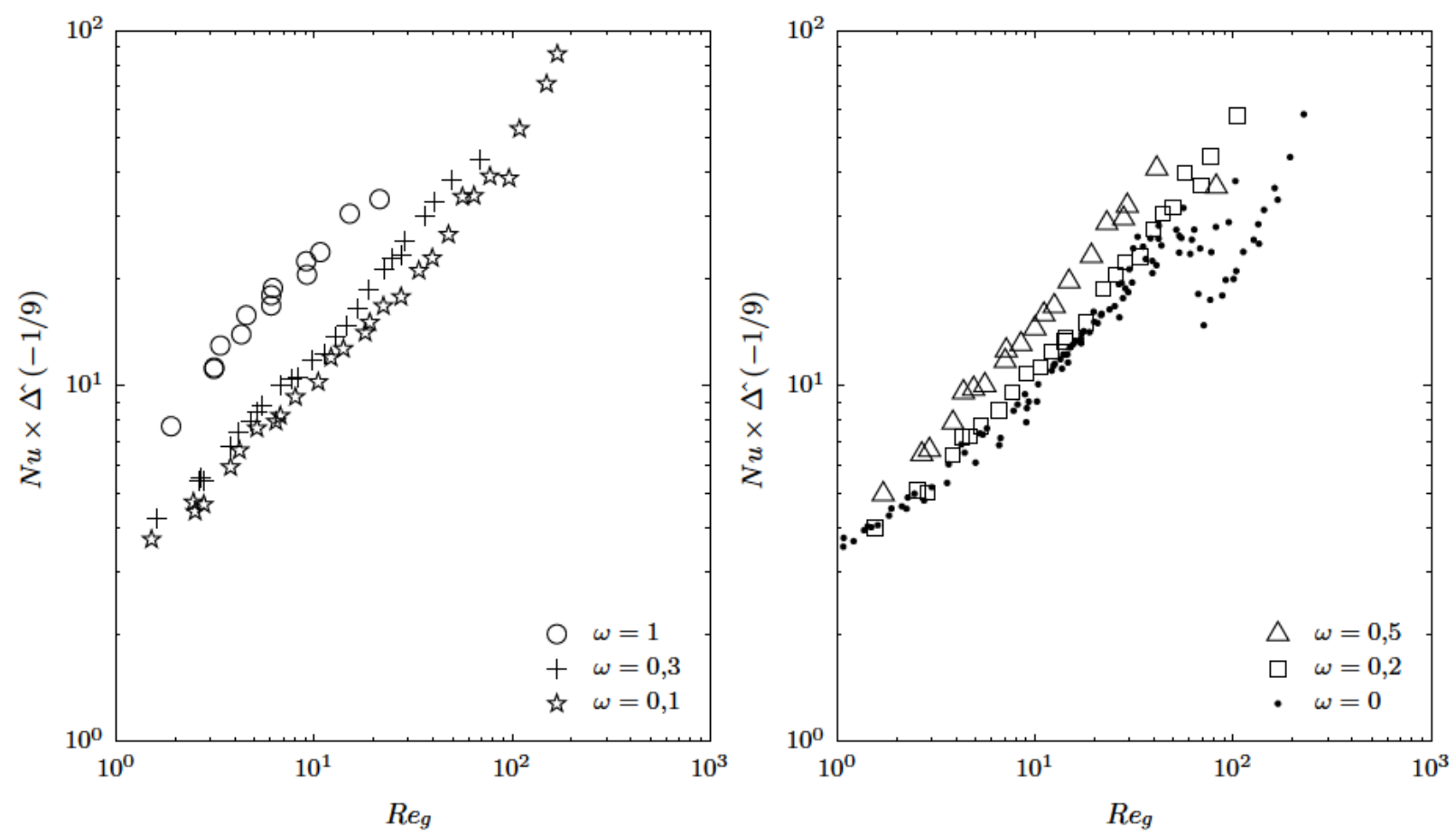

Figura 9.11: Efecto de la velocidad de rascado en el número de Nusselt. Datos trasladados a $\operatorname{Pr}_{g}=1000$.

presente estudio.

En la Fig. 9.11 se muestran resultados a diferentes velocidades de rascado a modo de comparativa, también trasladados a $P r_{g}=1000$. Tal y como se adelantaba, el movimiento del rascador produce mejoras en el número de Nusselt, siendo éstas mayores a medida que aumenta la velocidad de rascado $|\omega|$.

Finalmente se procede a la validación de los resultados comparándolos con los existentes en la bibliografía para fluidos newtonianos. Dicha comparación queda representada en la Fig. 9.12. La comparación resulta en algunos casos complicada por los diferentes rangos de números de Reynolds en los que se encuentran los datos. En régimen turbulento se aprecian ligeras diferencias entre los resultados obtenidos con fluidos no newtonianos y los existentes en la bibliografía. Cabe recordar que el método de generalización de la viscosidad seguido es estrictamente válido en la región laminar y pueden aparecer desviaciones en la región turbulenta. Además la definición de $\Delta$ está basada en el flujo laminar y su aplicación en la 

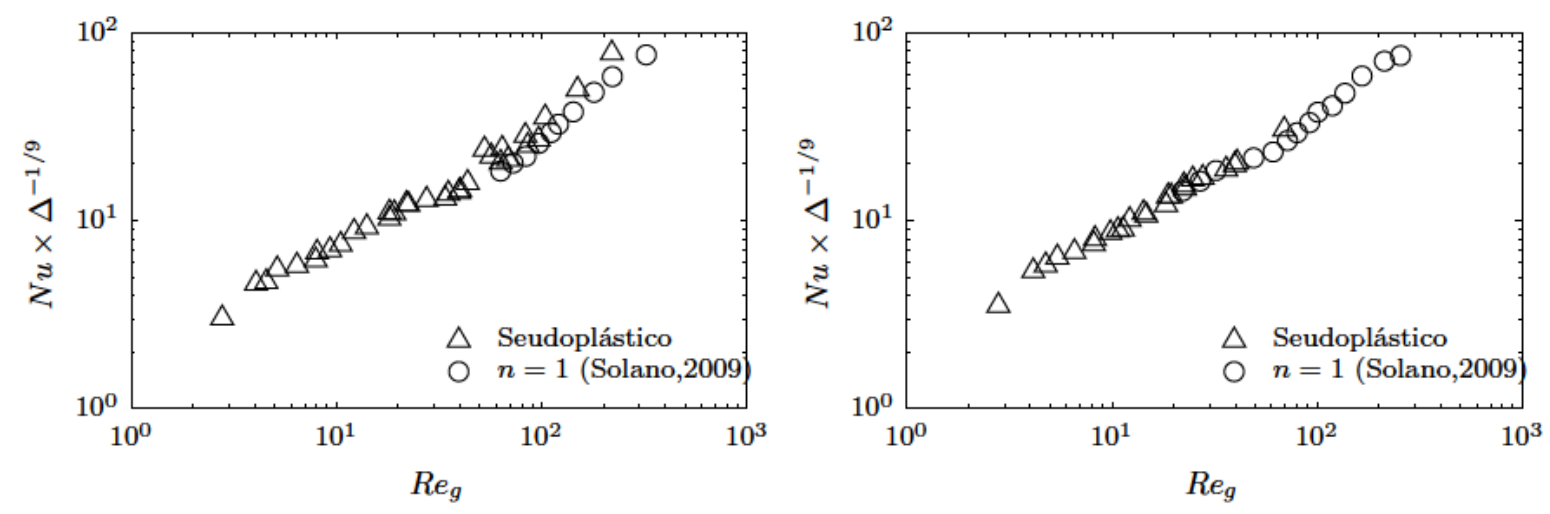

(a) $|\omega|=0,1$

(b) $|\omega|=0,3$
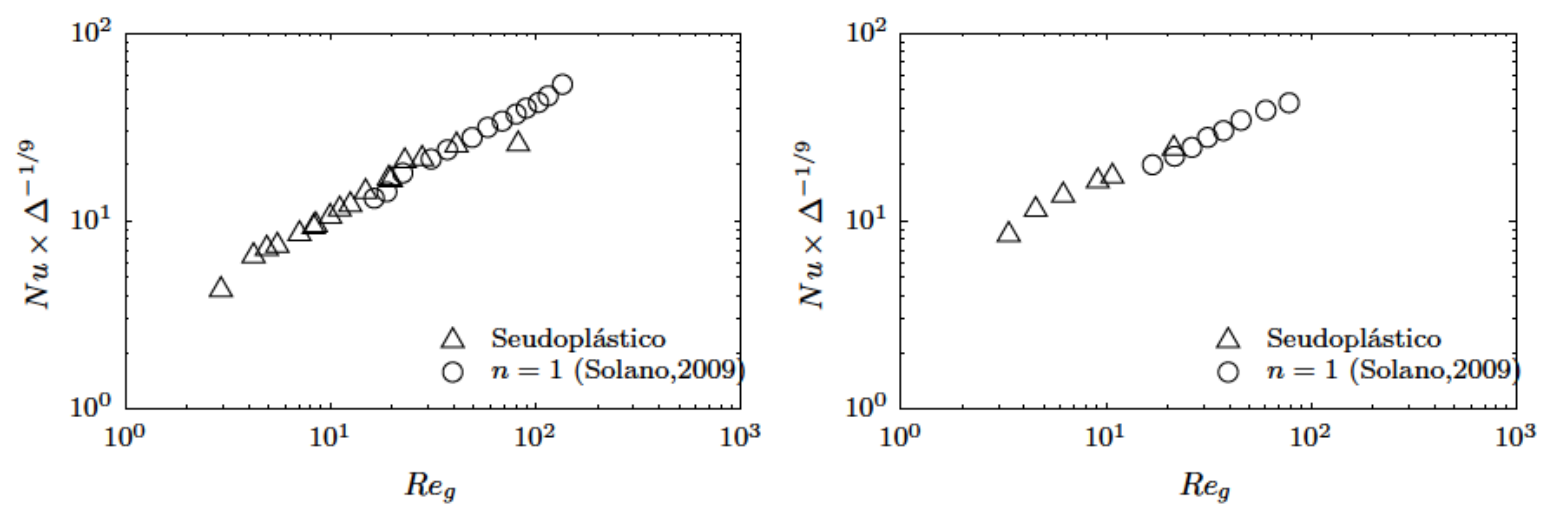

(c) $|\omega|=0,5$

(d) $|\omega|=1$

Figura 9.12: Comparación de resultados del número de Nusselt en fluidos seudoplásticos (trasladados a $\left.\operatorname{Pr}_{g}=450\right)$ con resultados para fluidos newtonianos existentes en la bibliografía $(\operatorname{Pr}=450)$. 
región turbulenta no es más que una aproximación. 


\subsection{Evaluación de las prestaciones}

Para evaluar las prestaciones del rascador en su funcionamiento con fluidos no newtonianos se debe realizar una comparativa respecto a la solución comercial más habitual. El problema reside en que en muchas de las aplicaciones en las que interesa el uso del intercambiador UNICUS, no existe una solución estándar, ya que el ensuciamiento puede ser tan importante que la alternativa de utilizar un intercambiador de carcasa y tubos es inviable. No obstante es necesaria una base comparativa, además de que el dispositivo se podría utilizar también con fluidos menos viscosos.

La solución adoptada es la de comparar las prestaciones del rascador en régimen estático frente a las dos geometrías simples que se vienen estudiando a lo largo de la tesis. Los resultados del rascador en régimen estático se comparan con las geometrías de:

- Tubo liso: se selecciona uno de diámetro igual al diámetro hidráulico del tubo con rascador: $D_{\text {Tubo }}=D_{h, \text { rascador }}$, que se denotará simplemente con $D_{h}$.

- Tubo con eje (sin rascadores), de diámetro hidráulico $D_{h}=D-d$. Siendo $D$ y $d$ las dimensiones del tubo y del eje del rascador. Esta comparación resulta ilustrativa para observar el efecto de los tacos rascadores.

En el estudio del régimen dinámico se realiza la comparativa entre dicho caso y la situación con el rascador estático. El objetivo es el de observar el efecto de accionar el dispositivo.

Los criterios de comparación utilizados son los criterios R1, R3 y R5 definidos por Bergles (1974).

- Criterio R1: Determina el aumento de la transferencia de calor para el mismo caudal circulante y superficie de intercambio $\left(Q_{s} / Q_{x}=1, \dot{A}_{s} / \dot{A}_{x}=1\right)$.

- Criterio R3: Determina el aumento de la transferencia de calor para la misma potencia consumida y superficie de intercambio $\left(\dot{W}_{s} / \dot{W}_{x}=1, \dot{A}_{s} / \dot{A}_{x}=1\right)$.

- Criterio R5: Determina la reducción de área de intercambio para la misma potencia consumida y calor total intercambiado $\left(\dot{W}_{s} / \dot{W}_{x}=1, q_{s} / q_{x}=1\right)$.

Los subíndices utilizados son: $s$ para el dispositivo con rascador en la situación a evaluar y $x$ para la geometría de referencia, ya sea ésta el tubo liso, el tubo con eje o el rascador en régimen estático. 
Los criterios de mejora propuestos por Bergles proporcionan una aproximación cuantitativa de los beneficios de las técnicas de mejora. Las simplificaciones consideradas en la evaluación de dichos criterios son las siguientes:

1. Se supone que el coeficiente de transmisión de calor interior es de orden inferior al exterior y a la resistividad térmica del tubo. Por lo tanto $U \approx h_{i}$.

2. No se consideran factores de ensuciamiento.

3. No se tiene en cuenta la variación de salto térmico que se produce al mejorar la transferencia de calor. Por tanto $\Delta T_{s} \approx \Delta T_{x}$, que se denotará simplemente como $\Delta T$.

De la definición de los criterios de mejora se deduce que el rascador insertado produce mejoras de la transferencia de calor si $R 1>1, R 3>1$ o $1 / R 5>1$ según sendos criterios. La evaluación de los mismos implica el cálculo del factor de fricción de Fanning y el número de Nusselt en la geometría de referencia, evaluados a un número de Reynolds que se deduce de la formulación de cada criterio.

Factor de fricción en las geometrías de referencia. En todos los casos estudiados, el flujo en la geometría de referencia es laminar y se puede obtener el factor de fricción $f_{x}$ como:

$$
f_{x}=\frac{2 \xi}{R e_{g, x}}
$$

utilizando la definición del Reynolds definida por Delplace y Leuliet (1995); Kozicki et al. (1966), según la Ec. 5.15. El valor de $\xi$ depende de la geometría utilizada: $\xi=8$ en tubo liso y $\xi=11,69$ para la geometría de tubo con eje utilizada $(\alpha=5 / 18)$. De forma análoga, para el tubo con rascador insertado se utiliza el método de generalización desarrollado en la presente investigación (Ec. 5.22).

Número de Nusselt en las geometrías de referencia. Al ser el flujo laminar, el número de Nusselt se obtiene haciendo uso del simulador numérico desarrollado en el Capítulo 3.

En este punto se plantea la importancia de la longitud del tubo de referencia considerado, ya que esto determina si el flujo está térmicamente desarrollado o no. En todos los casos se considera que la convección es puramente forzada. Se van a resolver dos casos:

- Tubo de longitud infinita donde la región de entrada pierde importancia. 
- Tubo de longitud finita. Al considerar el tubo finito, se plantea el uso de un intercambiador de calor con tubos de longitud idéntica (a escala) al intercambiador de calor UNICUS, de modo que la longitud de los tubos en la solución de referencia siempre será la misma, aunque para el criterio R5 puede variar el número de tubos $N$.

\subsubsection{Formulación de los criterios de comparación}

Los criterios de comparación establecidos por Bergles, establecen dos condiciones que definen la situación en la que se debe realizar la comparación. Así mismo establecen los parámetros a comparar.

De forma general para las comparativas a realizar, el criterio R1 establece como primera condición la igualdad de caudales circulantes, $Q_{s} / Q_{x}=1$, de donde se deduce que

$$
\frac{u_{b, x}}{u_{b, s}}=\frac{S_{x}}{S_{s}}
$$

siendo

$$
\frac{R e_{x}}{R e_{s}}=\left(\frac{u_{b, x}}{u_{b, s}}\right)^{2-n} \frac{\phi_{s}(n)}{\phi_{x}(n)}
$$

Las ecuaciones 9.8 y 9.9 se simplifican en función de la geometría de referencia utilizada en cada comparativa (véase Apartado A.6). Finalmente R1 se obtiene del cociente

$$
R 1=\frac{N u_{s}}{N u_{x}}
$$

Por otro lado, los criterios R3 y R5 comparten como primera condición la igualdad de potencias consumidas, $\dot{W}_{s} / \dot{W}_{x}=1$. De la formulación de dicha condición se deduce en el Apartado A.6 la siguiente relación entre los números de Reynolds de los casos a comparar

$$
\frac{R e_{g, x}}{R e_{g, s}}=\left[X_{x} \times \frac{f_{s}}{f_{x}} \times F_{D}\right]^{\frac{2-n}{3}}
$$

que también se puede expresar como

$$
\frac{u_{b, x}}{u_{b, s}}=\frac{P e_{x}}{P e_{s}}=\left(\frac{\phi_{x}(n)}{\phi_{s}(n)}\right)^{1 /(2-n)}\left[X_{x} \times \frac{f_{s}}{f_{x}} \times F_{D}\right]^{1 / 3}
$$

donde 
(a) Expresiones de $F_{D}$ en función del criterio de comparación y la geometría.

\begin{tabular}{lcc}
\hline Geom. referencia & Criterio & $F_{D}$ \\
\hline \multirow{2}{*}{ AN o rascador } & R3 & 1 \\
& R5 & $N u_{x} / N u_{s}$ \\
\multirow{2}{*}{ TL } & R3 & $1+\alpha=1+(d / D)$ \\
& R5 & $(1+\alpha) N u_{x} / N u_{s}$ \\
\hline
\end{tabular}

(b) Expresiones para la evaluación de los criterios de comparación.

\begin{tabular}{ccc}
\hline Geom. referencia & Criterio & Evaluación del criterio \\
\hline \multirow{2}{*}{ AN o rascador } & R3 & $N u_{s} / N u_{x}$ \\
& R5 & $N u_{x} / N u_{s}$ \\
TL & R3 & $N u_{s} / N u_{x}$ \\
& R5 & $D h N u_{x} /\left(N u_{s} D\right)$ \\
\hline
\end{tabular}

Tabla 9.6: Las expresiones de $F_{D}$ y de evaluación de los criterios de comparación se deducen a partir del planteamiento de dichos criterios en cada geometría. AN: Comparativa del rascador frente a tubo con eje o de sección anular; TL: comparativa del rascador frente a tubo liso; rascador: comparativa frente al tubo con rascador insertado en condiciones distintas a las del ensayo evaluado. Deducción y detalles de nomenclatura en Apartado A.6.

$$
X_{x}=\left(\left(\frac{\phi_{s}(n)}{\phi_{x}(n)}\right)^{3 /(2-n)}+P_{A} \times \frac{D_{h}}{2 \rho f_{s} S_{s} L_{r e f}}\left(\frac{\rho D_{h}^{n}}{m \phi_{x}(n) R e_{g, s}}\right)^{3 /(2-n)}\right)
$$

y

$$
F_{D}=\frac{S_{s} N_{s} L_{s}}{S_{x} N_{x} L_{x}}
$$

El factor $F_{D}$ es una relación entre las dimensiones de los tubos a comparar, que se obtiene de la formulación de la segunda condición de los criterios R3 y R5 aplicada a cada caso. El resultado se muestra en la Tabla 9.5(a).

Por último el propio criterio (R3 o R5) establece qué parámetro se debe comparar en cada caso. El planteamiento de dicha comparación lleva a los resultados mostrados en la Tabla 9.5(b).

Más detalles sobre la deducción de las expresiones de los criterios de comparación en el Apartado A.6. 


\subsubsection{Resultados en régimen estático}

En la evaluación de los criterios de mejora influyen muchos factores. Por ejemplo al aumentar $n$, el parámetro $\Delta$ disminuye en el tubo con rascador y en la geometría de referencia, mientras que también varía la relación $R e_{g, x} / R e_{g, s}$. Si aumenta el $\operatorname{Pr}$, aumentan $N u_{x}$ y $N u_{s}$. Por otro lado si la longitud de entrada térmica es importante, tendrá una gran influencia en el resultado final. De este modo, la evaluación de los criterios de mejora supone tener todos esos efectos en cuenta.

En aras de la claridad, en el presente apartado se muestran los resultados de una selección de los ensayos estáticos, que cubre fluidos de diferentes propiedades. Los ensayos seleccionados, que se representan en dos grupos son:

- TC5 y T11

- TC7, T10 y TC4

Los resultados de los ensayos se presentan en dicho orden en la leyenda y se referencian mediante el valor medio de la propiedad $n$ del fluido. El resto de propiedades se detallan en la Tabla 9.2 .

Tal y como se ha introducido anteriormente, a continuación se realizan comparativas frente a dos geometrías de referencia:

- Tubo con eje.

- Tubo liso.

Además se considerará los tubos de cada geometría de referencia tienen una longitud:

- infinita,

- o igual a la de los tubos de un intercambiador UNICUS comercial.

\subsubsection{Rascador frente a tubo con eje}

La justificación de realizar la presente comparativa es la de observar el efecto de los tacos rascadores en el flujo, ya que se comparan geometrías idénticas con o sin tacos.

Si la longitud del tubo de referencia se considera infinita y bajo la premisa de la existencia de convección forzada pura, la región de entrada térmica no tiene importancia. Según los 3 criterios de evaluación, el flujo comparable en la geometría de referencia se encuentra en 

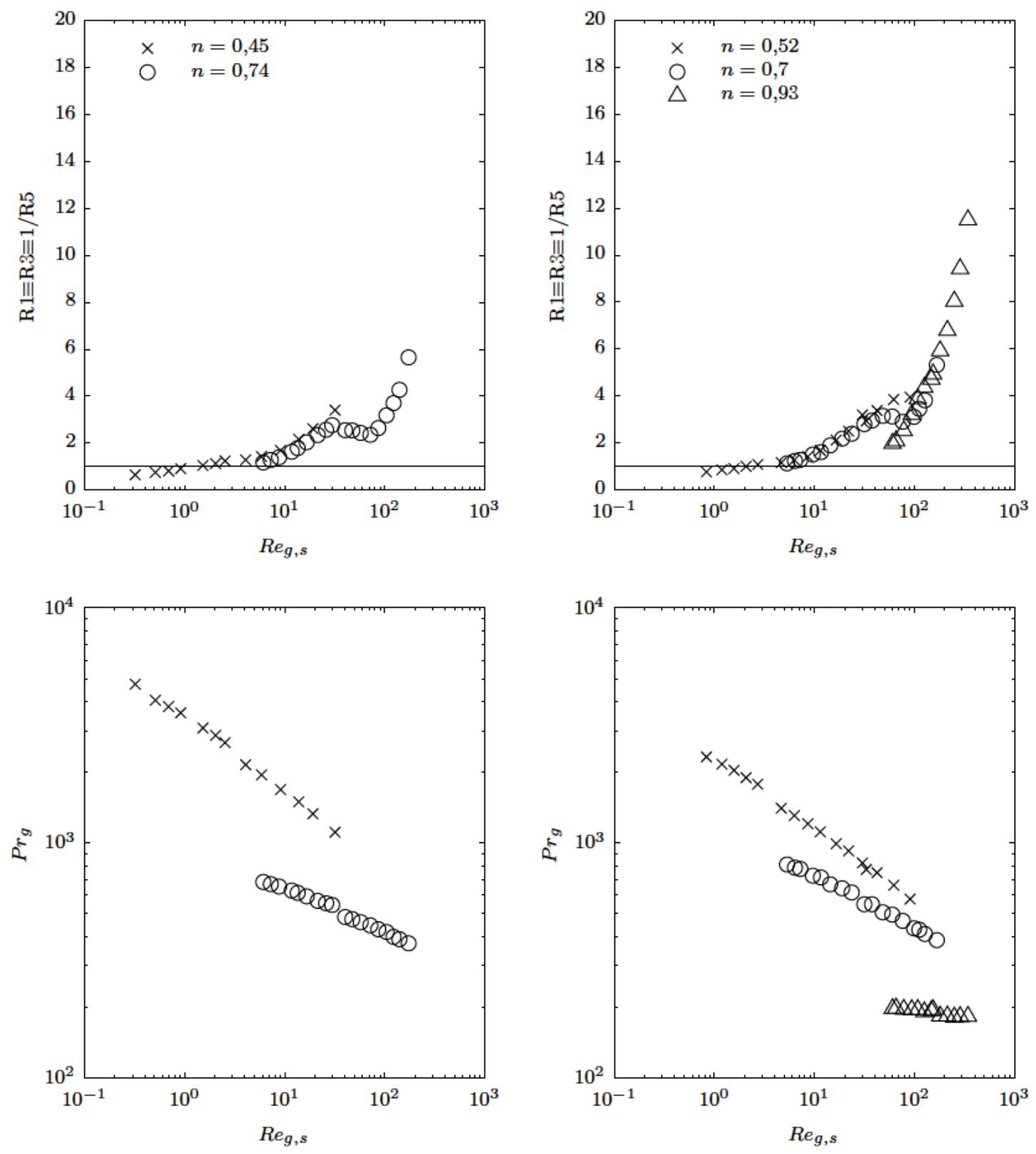

Figura 9.13: Criterios R1, R3 y R5 comparando los resultados en régimen estático $\left(v_{s}=0\right)$ frente a un tubo con eje de longitud infinita, donde la convección es puramente forzada. Para todos los ensayos realizados y los tres criterios utilizados, el flujo en la geometría de referencia se mantiene en la región laminar, lo que provoca que $R 1 \equiv R 3 \equiv 1 / R 5$. 

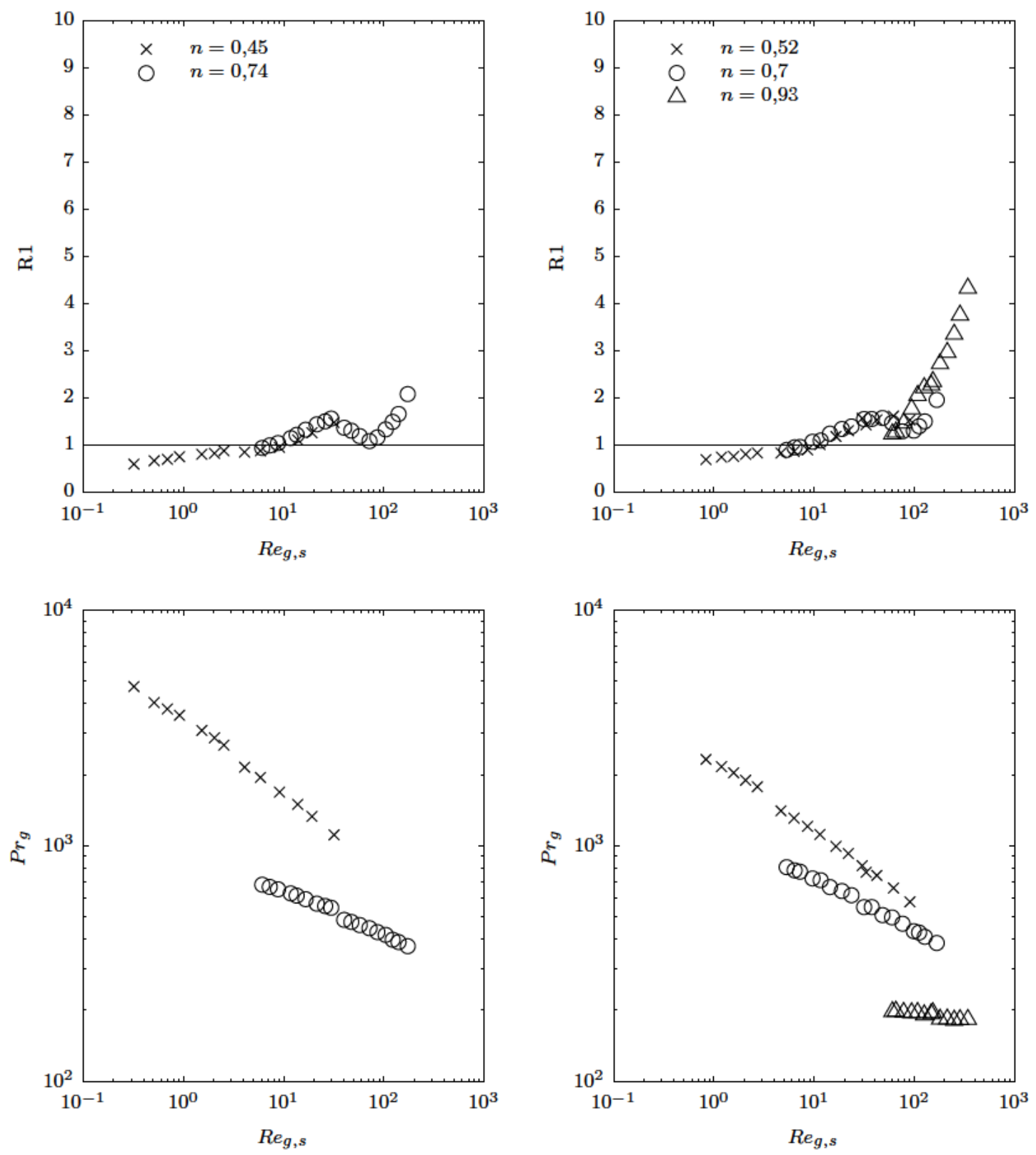

Figura 9.14: Criterio $\mathrm{R} 1$ comparando los resultados en régimen estático $\left(v_{s}=0\right)$ frente a un tubo con eje de longitud finita, donde la convección es puramente forzada. 

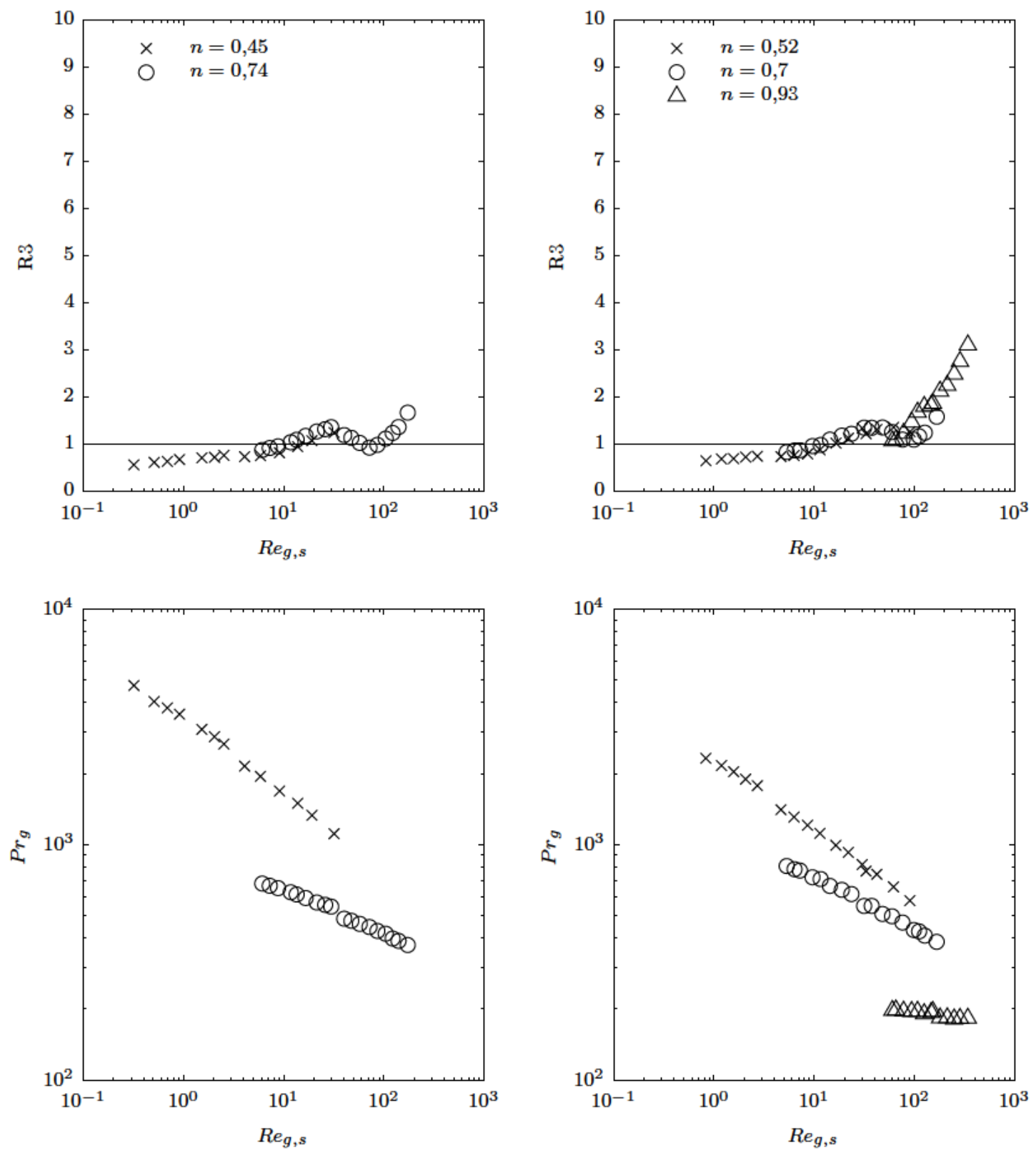

Figura 9.15: Criterio R3 comparando los resultados en régimen estático $\left(v_{s}=0\right)$ frente a un tubo con eje de longitud finita, donde la convección es puramente forzada. 

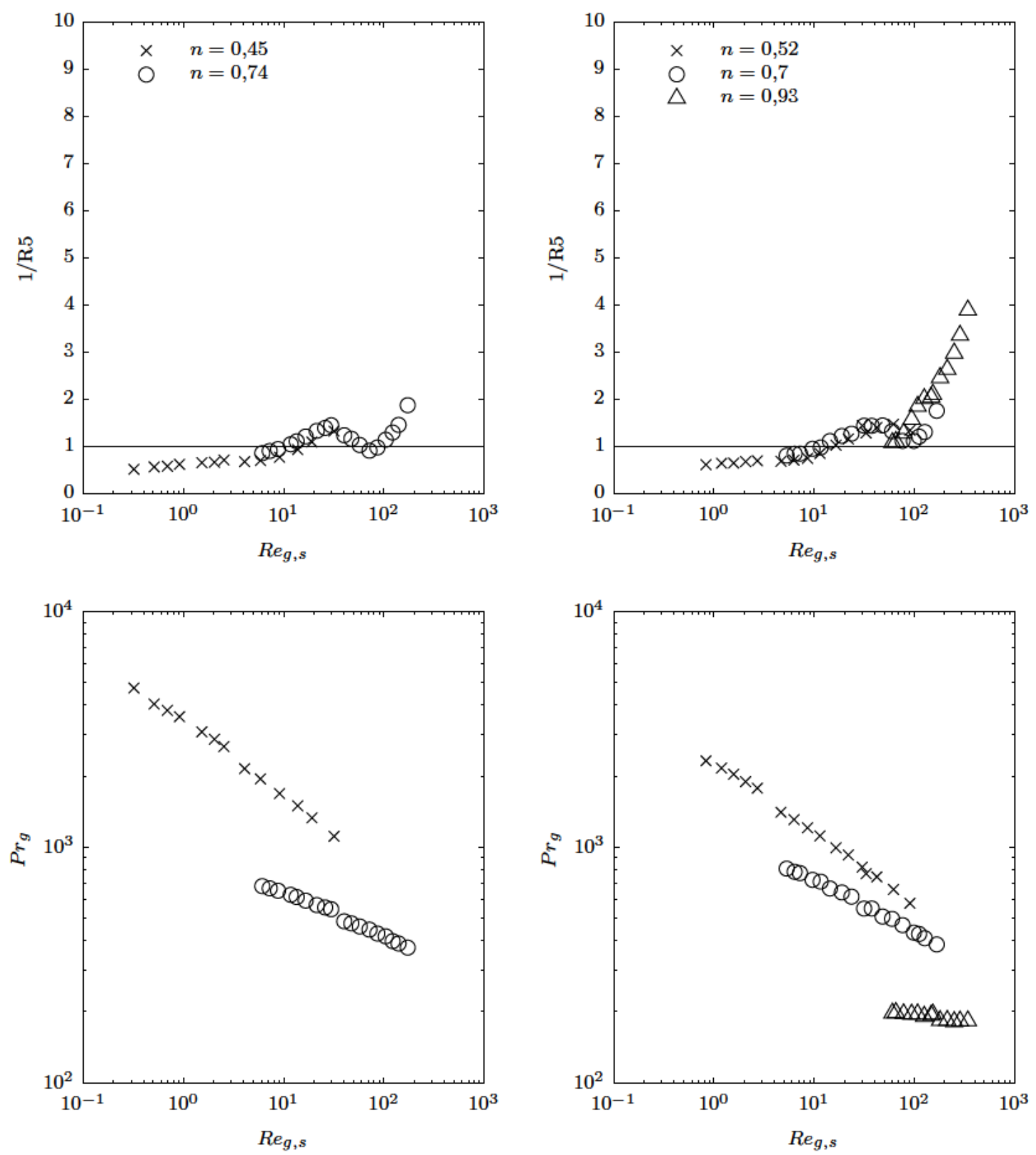

Figura 9.16: Criterio R5 comparando los resultados en régimen estático $\left(v_{s}=0\right)$ frente a un tubo con eje de longitud finita, donde la convección es puramente forzada. 
todos los casos en la región laminar. Esto provoca que los resultados de $R 1, R 3$ y $1 / R 5$ sean idénticos.

Los resultados se muestran en la Fig. 9.13 para cinco fluidos diferentes. Las diferencias entre los resultados de los diferentes fluidos son mínimas presentando todos un comportamiento similar. Para $R e_{g, s}>2$ los coeficientes de transferencia obtenidos mediante el método de mejora son mayores que en una geometría de tubo con eje, alcanzando valores más de 11 veces superiores en el tubo con rascador al utilizar los fluidos menos viscosos. Los ensayos se realizan a distintos números de $\operatorname{Pr}_{g}$ y tienen distintas propiedades reológicas, pero la suma de dichos factores no parece tener una gran influencia en los criterios evaluados. A la vista de los resultados y de forma general se puede afirmar que los tacos del rascador resultan beneficiosos para el proceso de intercambio para $R e_{g, s}>2$, si se comparan con una situación de convección forzada y flujo totalmente desarrollado.

Se considera ahora la situación en la que los tubos tienen longitud finita y son de proporciones iguales a las del UNICUS comercial. En todos los ensayos se comprueba que la longitud de entrada es importante. Para una tanda de ensayos, en los experimentos a menor caudal, la longitud de entrada cubre todo el tubo; en los ensayos a mayor caudal, la longitud de entrada es del orden de la longitud del tubo. En la región de entrada térmica el número de Nusselt es mayor al del flujo desarrollado y esto tiene una gran influencia en el resultado final.

Los resultados para el criterio R1 se muestran en la Fig. 9.14. Tal y como se puede observar, para todos los fluidos $R 1>1$ para $R e_{g, s}>10$, pudiendo llegar a valores de $R 1>4$ para los fluidos menos viscosos.

En las Fig. 9.15 se muestran los resultados del criterio R3. Éstos muestran que el rascador produce mejoras de la transferencia de calor para $R e_{g, s}>10$, dándose algunos casos en torno a $R e_{g, s} \approx 80$ donde la transferencia de calor es peor. Para obtener mejoras significativas el flujo en el tubo con rascador debe ser turbulento, consiguiéndose valores de R3 de más de 3 veces con el fluido menos viscoso. Con el fluido más viscoso únicamente se consiguen valores de $\mathrm{R} 3>1$ en el rango alto de caudal.

Los resultados del criterio R5 se muestran en la Fig. 9.16. El comportamiento general es similar al del criterio R3, aunque aparecen ciertas diferencias entre los fluidos y se pueden obtener reducciones de área de intercambio de casi 4 veces con el fluido menos viscoso. 


\subsubsection{Rascador frente a tubo liso}

En el presente apartado se realiza la comparación según los criterios R1, R3 y R5 del tubo con rascador frente al tubo liso, que será la geometría de referencia. Esta comparación resulta común en los estudios de intercambiadores de tubos mejorados, ya que representa la alternativa más sencilla y estándar.

En primer lugar se estudia la situación en la que el tubo tuviese longitud infinita, despreciando el efecto de la longitud de entrada. De nuevo se considera la convección forzada pura y de nuevo los resultados para R1, R3 y R5 están relacionados y se representan en la Fig. 9.17. Los resultados son muy similares a los del Apartado 9.4.2.1. En el presente caso, se producen mejoras de la Para $R e_{g, s}>2$ alcanzando mejoras en la transferencia de calor de hasta 13 veces para los criterios R1 y R3, así como reducciones del área de intercambio de hasta 18 veces según R5. Además no se observan diferencias significativas en los resultados para los diferentes fluidos.

Se considera ahora la situación en la que los tubos tienen longitud finita y son de proporciones iguales a las del UNICUS comercial. Al igual que en el caso anterior, en todos los ensayos se comprueba que la longitud de entrada es importante. En la Fig. 9.18 se presentan los resultados para el criterio R1. En ellos se observan valores de $R 1$ en torno a la unidad para para $20<R e_{g, s}<100$ y aumentos significativos a partir de $R e_{g, s}>100$, llegando a valores de $\mathrm{R} 1=3,5$. El criterio R3, representado en la Fig. 9.19, presenta un comportamiento muy similar a R1 aunque las mejoras de transferencia de calor son menores, alcanzando un máximo de $\mathrm{R} 3=2,8$ para el fluido menos viscoso (TC4, con $n=0,93$ ). La comparativa según el criterio R5, representada en la Fig. 9.20, resulta más favorable. Se alcanzan valores de $1 / R 5>1$ para valores de $R e_{g, s}>10$. La mayor reducción del área de intercambio para los fluidos menos viscosos y puede ser de hasta 4,5 veces.

La principal conclusión que se obtiene en el presente apartado es que el rascador puede producir significativas mejoras, bien en cuanto a la transferencia de calor, o bien reduciendo el área de intercambio necesaria. No obstante dichas mejoras dependen de las características reológicas del fluido de trabajo y del punto de funcionamiento. En general se consiguen mejoras de la transferencia de calor para $R e_{g, s}>10$.

\subsubsection{Resultados en régimen dinámico}

En el presente apartado se desea evaluar la conveniencia energética de accionar el rascador. Para ello se realiza la siguiente comparativa: 

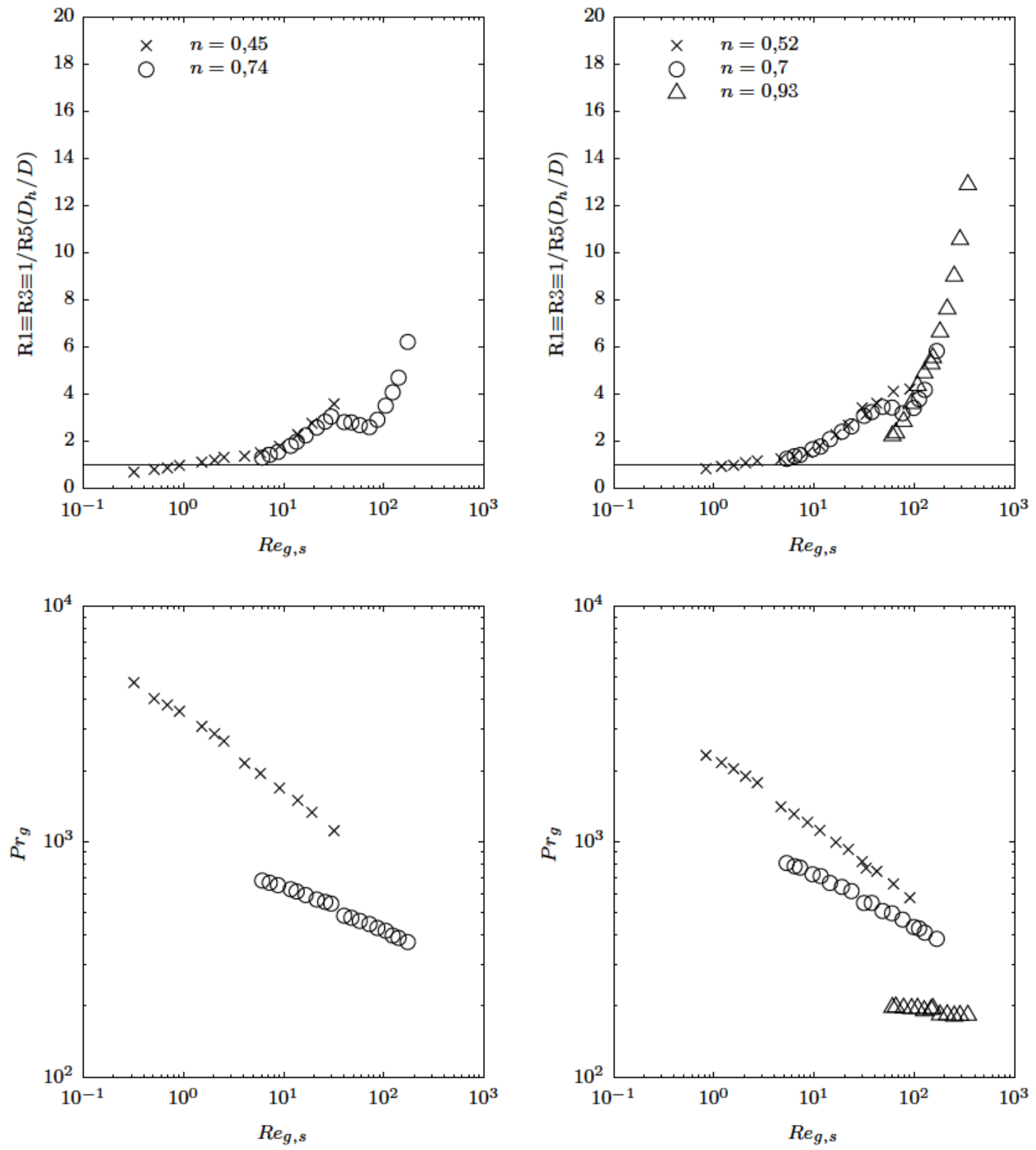

Figura 9.17: Criterio R1 comparando los resultados en régimen estático $\left(v_{s}=0\right)$ frente a un tubo liso de longitud infinita, donde la convección es puramente forzada. 

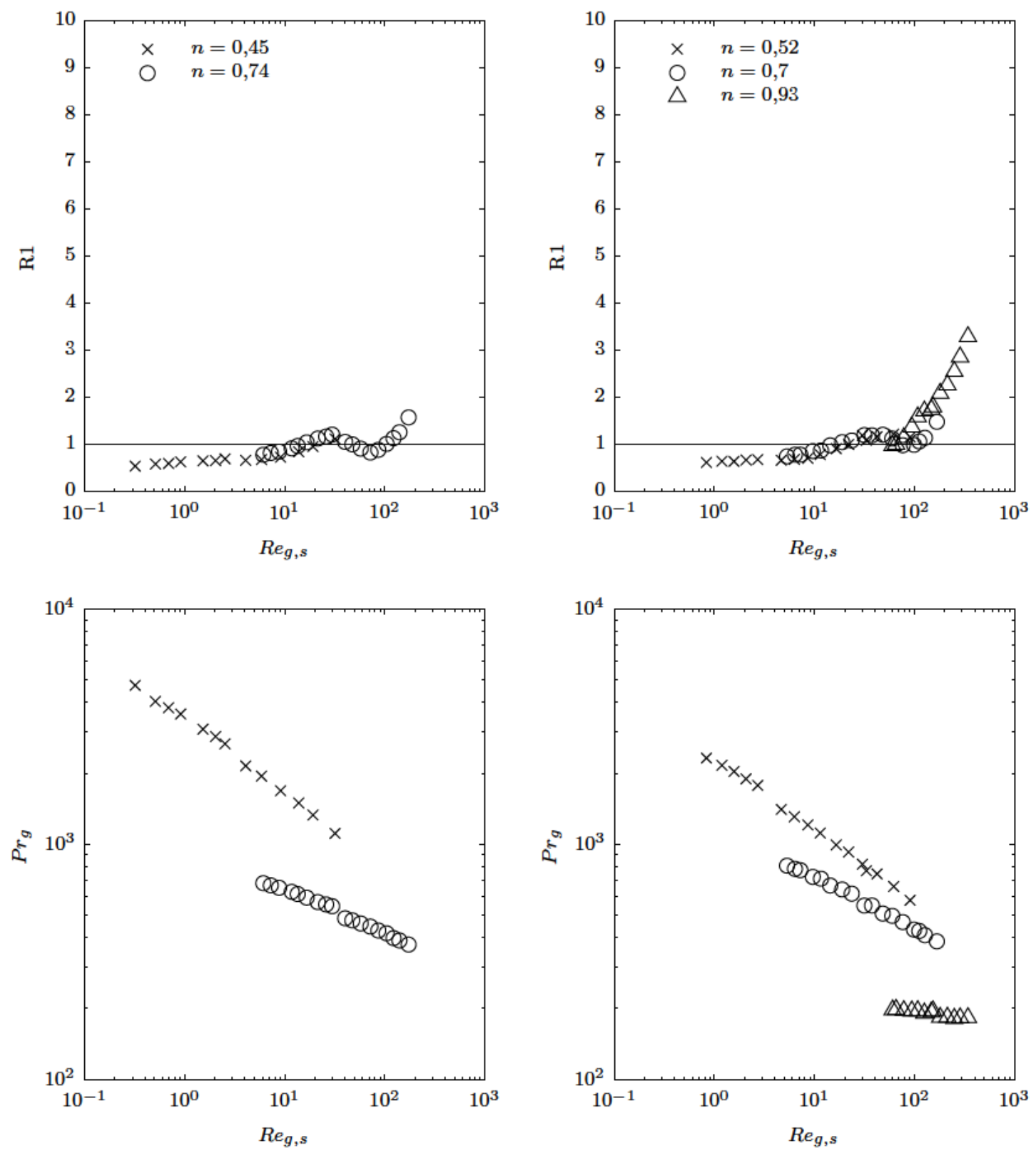

Figura 9.18: Criterio $\mathrm{R} 1$ comparando los resultados en régimen estático $\left(v_{s}=0\right)$ frente a un tubo liso de longitud finita, donde la convección es puramente forzada. 

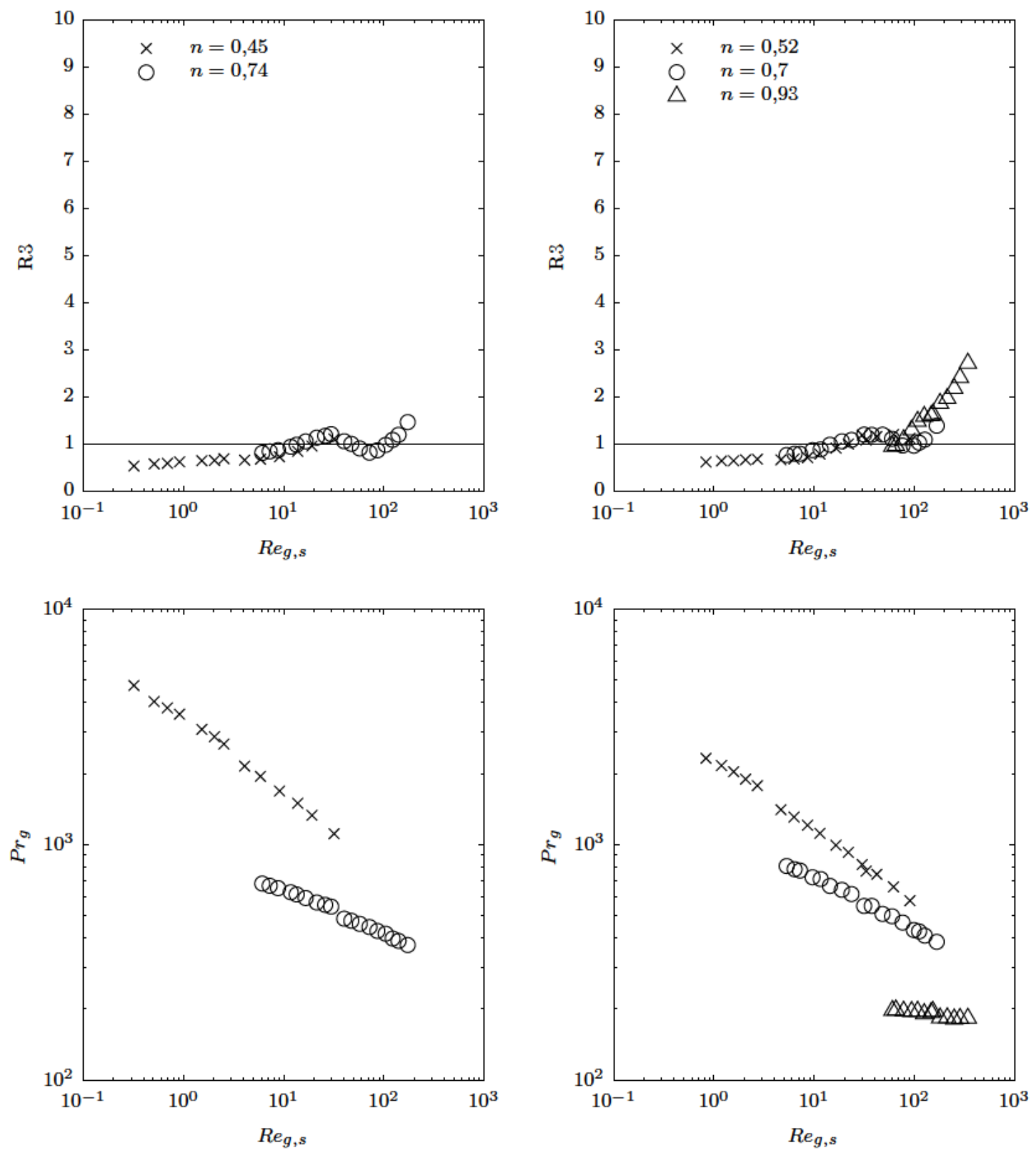

Figura 9.19: Criterio R3 comparando los resultados en régimen estático $\left(v_{s}=0\right)$ frente a un tubo liso de longitud finita, donde la convección es puramente forzada. 

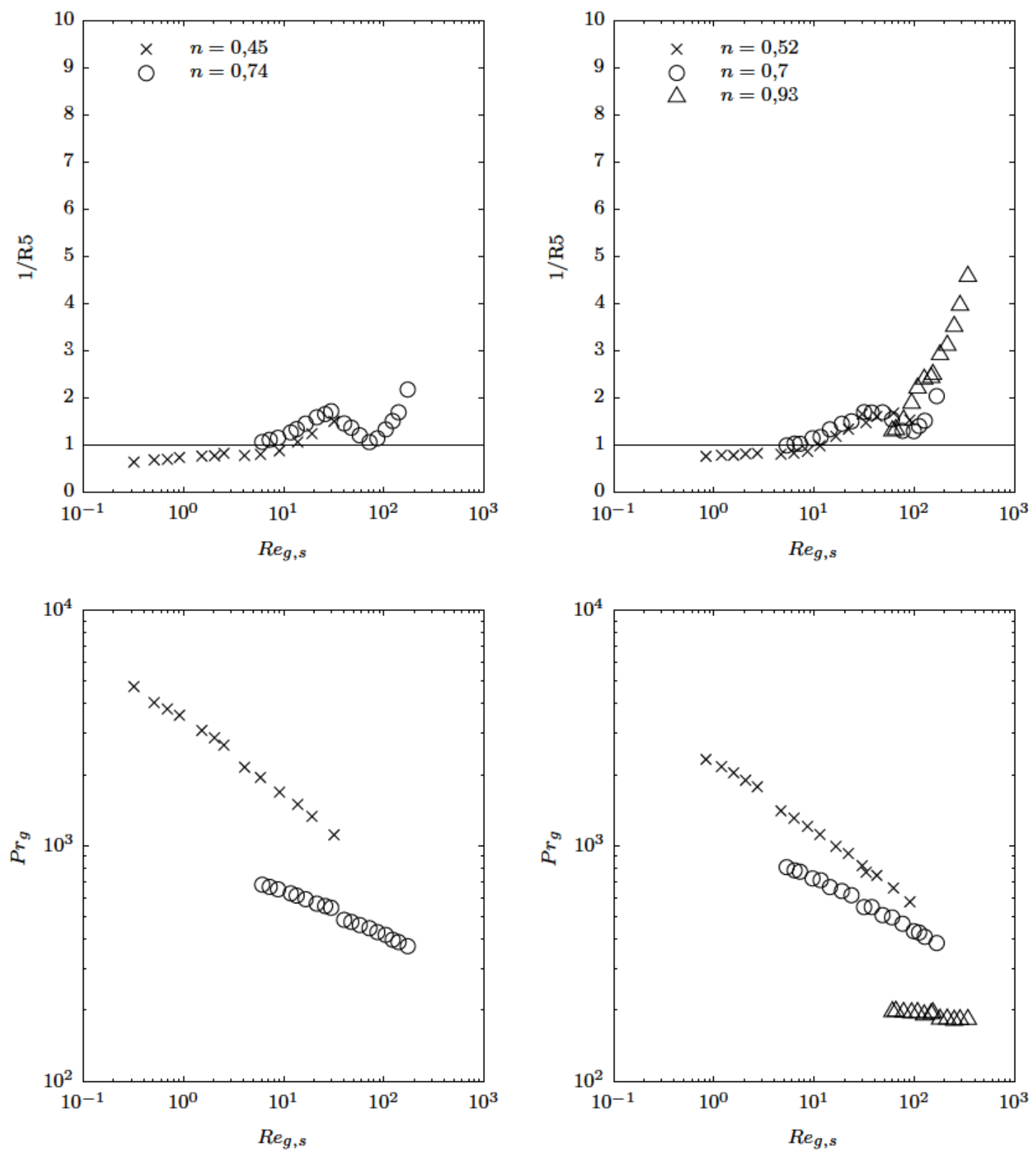

Figura 9.20: Criterio R5 comparando los resultados en régimen estático $\left(v_{s}=0\right)$ frente a un tubo liso de longitud finita, donde la convección es puramente forzada. 


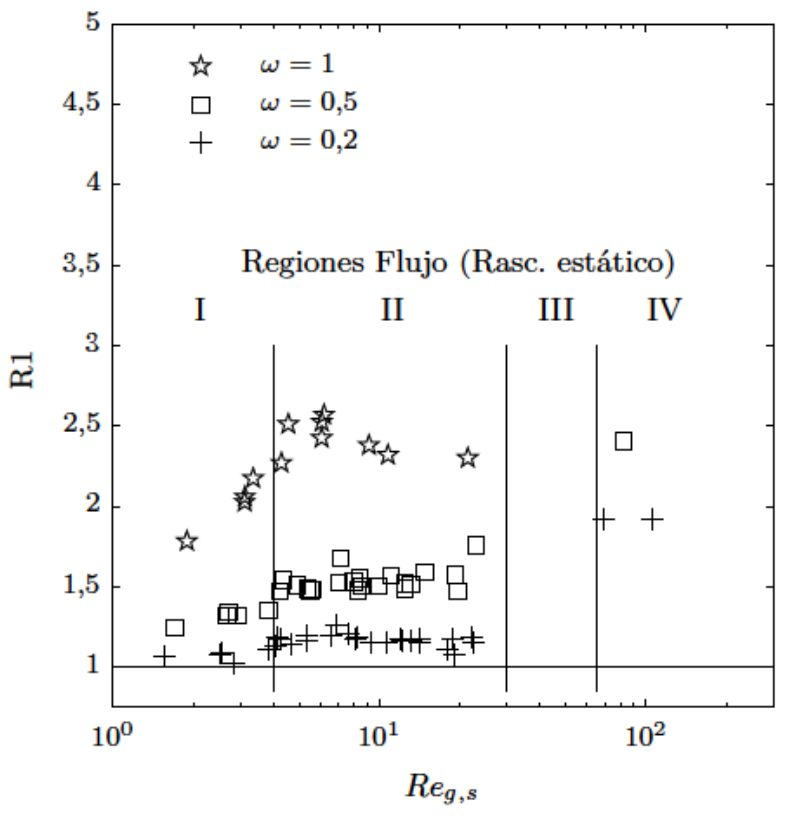

Figura 9.21: Criterio R1 comparando los resultados en régimen dinámico $\left(v_{s} \neq 0\right)$ a los resultados en régimen estático. Los datos mostrados corresponden a las medidas del número de Nusselt a los números de Prandtl de cada ensayo.

- Situación a evaluar: tubo con rascador en movimiento (subíndice $s$ ).

- Situación de referencia: tubo con rascador estático (subíndice $x$ )

En el presente apartado se evalúan los criterios de mejora R1 y R3, descartándose R5 que no aporta excesiva información adicional respecto a R3.

En la Fig. 9.21 se muestran los resultados del criterio R1 para diferentes velocidades de rascado $|\omega|$. Se encuentran representados ensayos de todas las tandas, con números de Prandtl diferentes. Tal y como muestra el criterio $\mathrm{R} 1$, el accionamiento del rascador produce mejoras de la transferencia de calor en todo el rango ensayado. Además dicha mejora aumenta al hacerlo el número de Reynolds generalizado $R e_{g, s}$. Además dichas mejoras son mayores para velocidades de rascado mayores. Se debe tener en cuenta que el criterio R1 no tiene en cuenta la potencia consumida. En procesos industriales reales, el accionamiento del rascador puede proporcionar un método de regulación del proceso sin necesidad de modificar el caudal de producto. 

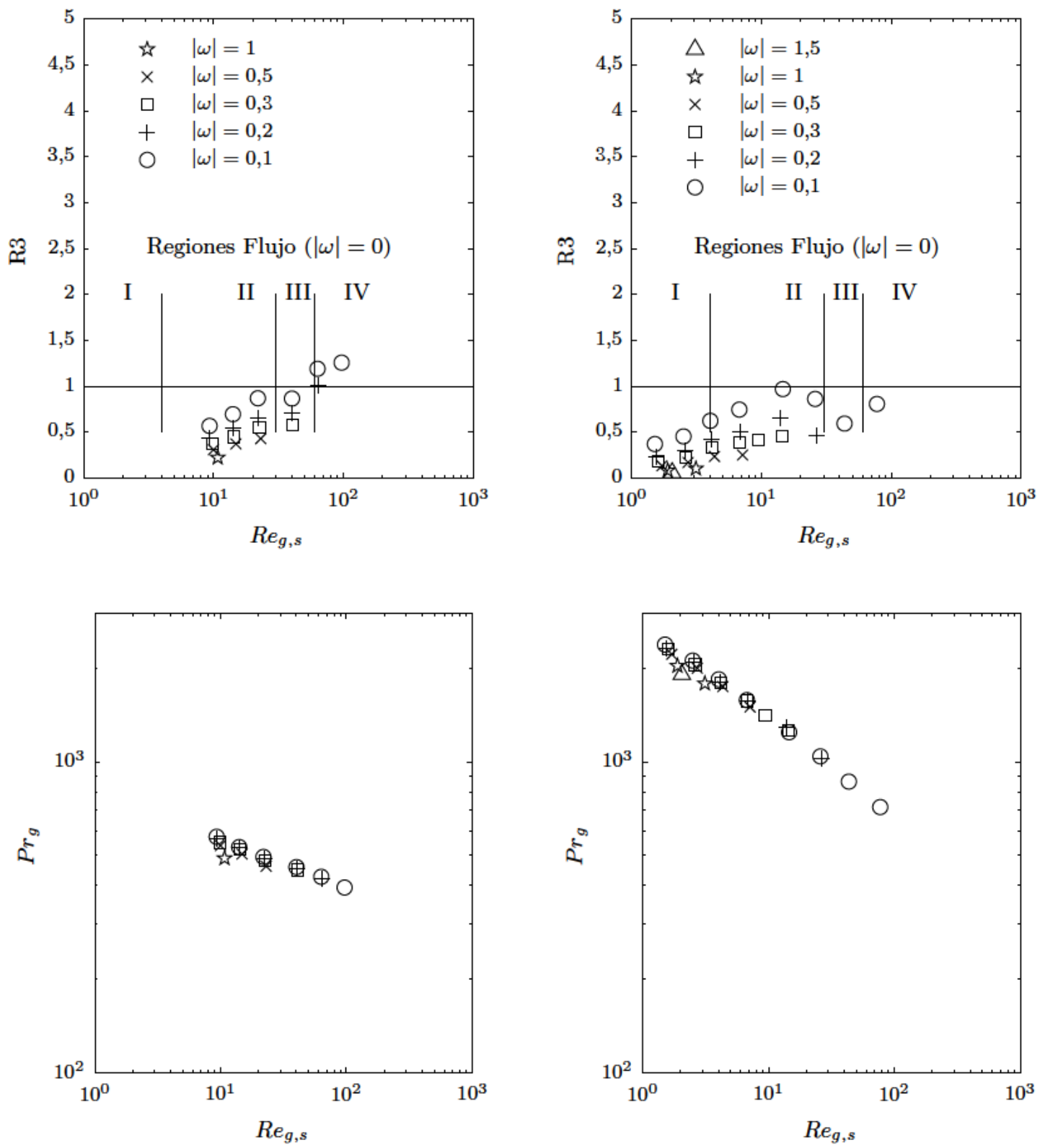

(a) TCD-3, $n \approx 0,81$ y $m \approx 0,2$

(b) TCD-7, $n \approx 0,52$ y $m \approx 2,6$

Figura 9.22: Criterio $\mathrm{R} 3$ comparando los resultados en régimen dinámico $\left(v_{s} \neq 0\right)$ frente a a los resultados en régimen estático. En el eje $x$ se representa $R e_{g, s}$ de los ensayos de régimen dinámico, que no se debe confundir con el número de Reynolds de la situación de referencia (rascador estático). 

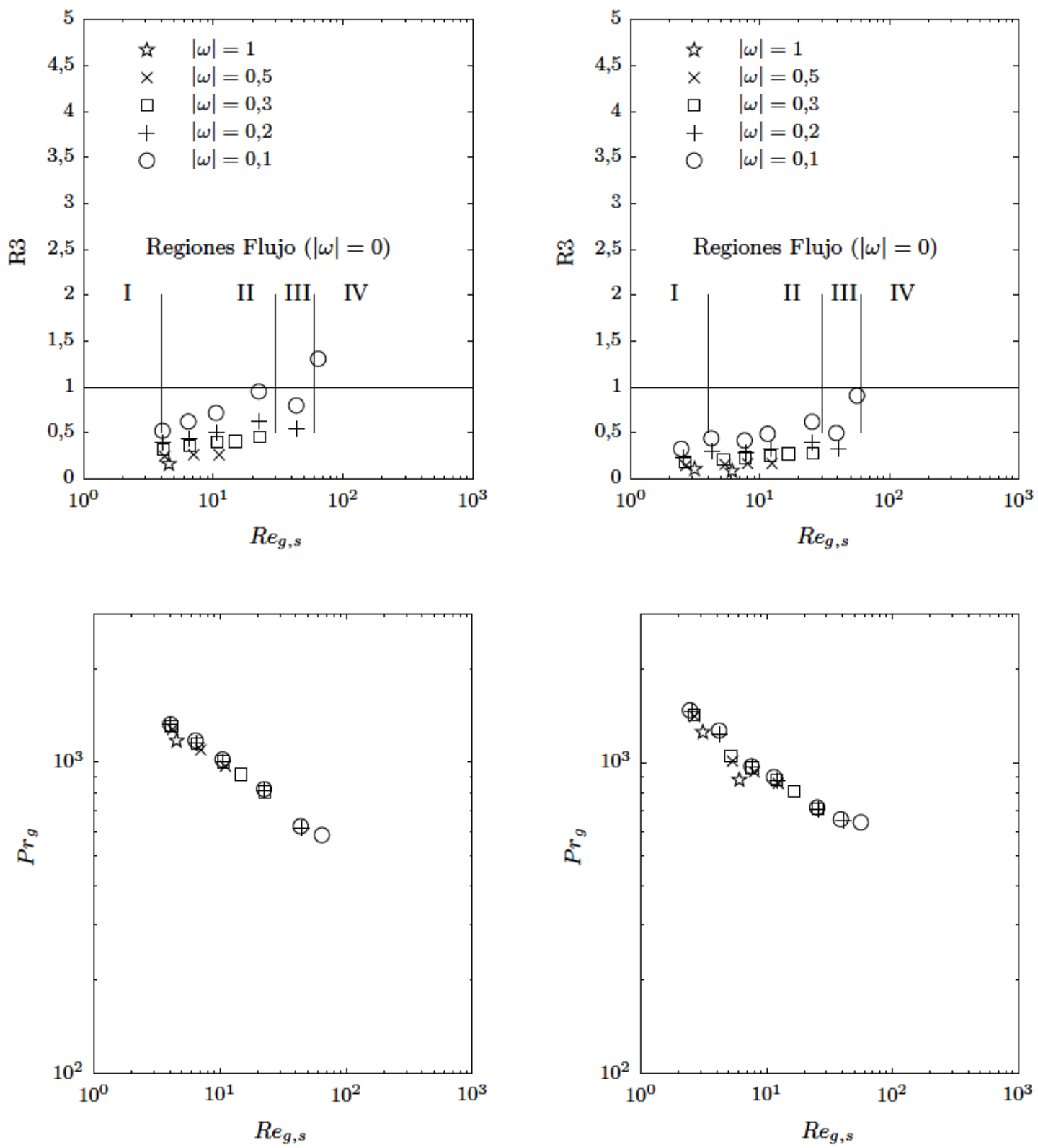

(a) TCD- $2, n \approx 0,61$ y $m \approx 1,1$

(b) TCD- $8, n \approx 0,93$ y $m \approx 0,06$

Figura 9.23: Criterio R3 comparando los resultados en régimen dinámico $\left(v_{s} \neq 0\right)$ frente a a los resultados en régimen estático. En el eje $x$ se representa $R e_{g, s}$ de los ensayos de régimen dinámico, que no se debe confundir con el número de Reynolds de la situación de referencia (rascador estático). 
En las figuras 9.22 y 9.23 se muestran los resultados para cuatro tandas de ensayos del criterio R3 (en el Apartado D.2 se adjuntan resultados adicionales). Dicho criterio establece que en las situaciones comparadas las potencias consumidas deben ser idénticas. De este modo la potencia necesaria de accionamiento del rascador implicará mayores números de Reynolds en la situación de referencia. En los resultados se observa, que las situaciones en las que interesa energéticamente el accionamiento del rascador se producen en la región IV donde el flujo en régimen de rascador dinámico es turbulento. Además únicamente se han obtenido valores de $\mathrm{R} 3>1$ para velocidades de rascado de $|\omega|=0,1 ; 0,2$. No obstante, es importante matizar que, debido a las limitaciones mecánicas del sistema de rascado, no ha sido posible ensayar a números de Reynolds grandes en régimen dinámico. Los resultados parecen indicar, que de haber sido posible ensayar a números de Reynolds mayores, se podrían haber obtenido más resultados favorables del criterio $\mathrm{R} 3$ para velocidades de rascado bajas y siempre en la región IV.

\subsection{Conclusiones}

Del análisis del proceso de transferencia de calor realizado en el capítulo, se extraen las siguientes conclusiones:

1. Se ha medido el coeficiente de convección medio, $h_{i}$ en procesos de intercambio de calor en el tubo, considerando distintos regímenes de funcionamiento. Se han estudiado por separado los casos en régimen estático y dinámico del rascador, abarcando números de Reynolds en el rango $R e_{g} \in[0,2 ; 400]$.

2. En régimen estático se observan las regiones laminar, de transición y turbulenta en función del número de Reynolds. La zona laminar a su vez está dividida en dos subregiones: a bajos números de Reynolds el Nusselt aumenta con $R e_{g}^{0,37}$, mientras que a partir de $R e_{g}>4$ la pendiente de la recta en ejes logarítmicos aumenta, de modo que $N u \sim R e_{g}^{0,64}$. En la región de transición, los resultados varían al hacerlo $n$ y $P r_{g}$, aunque para un mismo fluido el Nusselt se muestra prácticamente independiente del número de Reynolds. Por último, en la región turbulenta, el Nusselt es más sensible a la variación del número de Reynolds $N u \sim R e_{g}^{1,02}$.

3. Además se ha cuantificado el efecto de $n$ en el número de Nusselt, concluyendo que dicho efecto está prácticamente recogido por la definición de la viscosidad efectiva. 
Existe un pequeño efecto adicional de mejora de la transferencia de calor al disminuir $n$, de entorno al $2 \%$ para $n=0,5$ con respecto al fluido newtoniano.

4. En régimen dinámico se han detectado las regiones laminar y turbulenta, siendo la transición abrupta. Al igual que en el apartado estático la región laminar se divide en dos subregiones. En la primera de ellas, la influencia del $P r_{g}$ es algo superior y la del $R e_{g}$ algo inferior. En la región turbulenta $N u \sim \operatorname{Pr}_{g}^{0,44}$.

5. Se han evaluado las prestaciones del rascador de acuerdo a los criterios de mejora clásicos, obviando el significativo efecto del ensuciamiento. Los resultados muestran la importancia de la región de entrada en la geometría de referencia:

a) Si el tubo es largo y la región de entrada no es importante, el uso del rascador ofrece mejoras significativas respecto a las alternativas estudiadas en gran parte de los puntos de funcionamiento posibles.

b) En el caso contrario, en el que el tubo sea del orden de la longitud de entrada o de un orden inferior, el rascador también supone una mejora de acuerdo a los criterios clásicos, aunque de menor entidad.

6. En cuanto al accionamiento del rascador, se concluye que:

a) Produce mejoras en la transferencia de calor, lo cual supone un grado de libertad adicional en la regulación de procesos industriales. Se han detectado incrementos del número de Nusselt crecientes con la velocidad de rascado.

b) En comparación con la situación de rascador estático, y teniendo en cuenta el consumo de potencia de accionamiento, únicamente supone una mejora energética para números de Reynolds altos $R e_{g, s}>60$ y velocidades de rascado muy bajas $|\omega|=0,1$. 


\section{Capítulo 10}

\section{Aplicación industrial y conclusiones finales}

En el presente capítulo se presenta en primer lugar un resumen del cumplimiento de los objetivos de la tesis doctoral, que se desarrolla en mayor detalle en el apartado de conclusiones, donde además se evalúan los logros de la investigación y se describen las posibles nuevas vías de investigación a las que ésta puede dar lugar.

Así mismo, se presenta un esquema de la aplicación industrial de los resultados de la investigación. En él se detallan los pasos a seguir a la hora de valorar la conveniencia del uso del intercambiador de calor estudiado en función de las condiciones de trabajo.

\subsection{Resumen del cumplimiento de los objetivos}

En la presente investigación se ha estudiado el flujo en intercambiadores de calor mejorados con rascador de movimiento lineal alternativo, funcionando con fluidos no newtonianos. En concreto se ha estudiado el uso de un fluido seudplástico, el comportamiento no newtoniano con mayor presencia en la industria. Además se ha caracterizado el comportamiento termohidráulico de dichos fluidos en el intercambiador.

Para lograrlo, se ha desarrollado una metodología experimental específica para el ensayo de fluidos no newtonianos en intercambiadores de este tipo, descrita en los capítulos 5 y 6. Adicionalmente se han mejorado herramientas de análisis existentes, como el método de generalización de la viscosidad, extendiendo su aplicación a geometrías complejas. El código de simulación numérica desarrollado en el Capítulo 3 ha permitido un análisis preliminar 
del comportamiento del flujo en la geometría de tubo con eje. Los ensayos de visualización (Capítulo 7) han permitido la obtención del patrón del flujo en diferentes condiciones de funcionamiento, que se han comparado con las soluciones obtenidas por el modelo numérico en la geometría de tubo con eje ( $\sin$ tacos).

Finalmente los ensayos en la instalación de ensayos termohidráulicos han permitido la obtención de correlaciones experimentales, válidas para fluidos seudoplásticos que siguen el modelo Power law (Capítulos 8 y 9). Las correlaciones propuestas permiten la obtención de la potencia de bombeo necesaria, así como la potencia de accionamiento y el coeficiente de transmisión de calor en el intercambiador de calor mejorado estudiado. Así mismo, se ha analizado la conveniencia del uso del intercambiador estudiado en función de los parámetros del problema (Capítulo 9).

\subsection{Aplicación industrial de los resultados obtenidos}

En el presente apartado se detallan las ventajas del intercambiador de calor estudiado en aplicaciones industriales. El intercambiador UNICUS mostrado en la Fig. 10.1 se utiliza en las industrias petroquímica, cosmética y alimenticia principalmente. Éste puede trabajar con diferentes rascadores, donde el modelo de rascador estudiado está especialmente diseñado para trabajar con fluidos de alta viscosidad, con tolerancia a partículas grandes. En dichas aplicaciones el ensuciamiento del intercambiador puede llegar a ser muy costoso por diversas razones:

1. Pérdida de eficiencia del intercambiador. Se produce una disminución progresiva de la eficiencia en la transmisión de calor y un aumento de la pérdida de carga. En el Apartado 1.2.2 se muestra un ejemplo de cuantificación de dicho efecto para un intercambiador industrial.

2. Disminución de la calidad del producto. Especialmente en la industria alimenticia la higiene es primordial y la acumulación de ensuciamiento va radicalmente en contra de este principio.

3. Pérdida de productividad por las paradas de mantenimiento. Las paradas se producen de forma periódica para eliminar el ensuciamiento mediante productos de limpieza.

Así, en el procesado de productos alimenticios el intercambiador analizado supone una gran ventaja, ya que permite el procesado en continuo del producto, sin continuas interrupcio- 


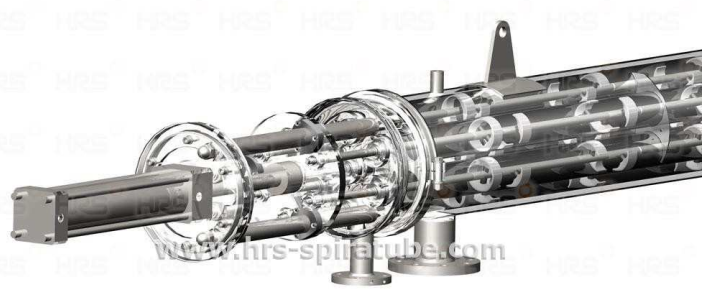

Figura 10.1: Intercambiador UNICUS de 7 tubos.

nes. Además, dependiendo del producto, es probable que los intercambiadores tradicionales no sean una alternativa y sea necesario un sistema autolimpiante.

Por otro lado, en las condiciones de trabajo en las que el UNICUS presenta un coeficiente de transmisión de calor más alto que un intercambiador de carcasa y tubo estándar, éste tiene las siguientes ventajas con respecto a su homólogo tradicional, aparte de la obvia:

- Una mejor transferencia de calor puede implicar menores necesidades de superficie de intercambio.

- Menor deterioro del producto para una misma $\Delta T$. En muchos productos utilizados en la industria alimenticia existe un límite de temperatura que el producto no debe superar por motivos de deterioro. Es el caso por ejemplo de las disoluciones de CMC estudiadas. Un mejor coeficiente de transmisión de calor, permite trabajar con un $\Delta T$ superior para una temperatura menor del producto en la pared de contacto. Esto permite transferir más calor al producto.

\subsubsection{Consulta de los resultados de la investigación.}

De lo expuesto anteriormente se deduce la necesidad de utilizar intercambiadores de calor mejorados con sistema automático de limpieza para determinadas aplicaciones. Ante esta situación, el ingeniero que debe diseñar el proceso se ve en la tesitura de seleccionar un 
modelo de intercambiador de calor y para ello necesita poder compararlos. Es en este punto donde el ingeniero necesita resultados como los que se presentan en esta investigación, que le permitirán decantarse por el sistema más adecuado a su aplicación.

En el presente apartado se detalla de forma concisa el procedimiento que se debe seguir para evaluar la conveniencia de utilizar el intercambiador de calor UNICUS, aplicado a fluidos newtonianos o seudoplásticos.

Dimensiones del intercambiador. El UNICUS tiene las siguientes dimensiones:

\begin{tabular}{cc}
\hline$L$ & $6 \mathrm{~m}$ \\
$D$ & $74 \mathrm{~mm}$ \\
$D_{h}$ & $56 \mathrm{~mm}$ \\
$N$ & $1 / 4 / 7$ tubos \\
\hline
\end{tabular}

Paso 1- Determinación de las necesidades. En primer lugar se deben evaluar las necesidades que existen en el proceso en cuanto a:

- Gasto másico del fluido. Es decir, velocidad a la que necesitamos procesar el producto.

- Necesidades de calor intercambiado por kilogramo de producto.

Ambas necesidades van a determinar el flujo de calor necesario.

Paso 2- Determinación de las propiedades del producto no newtoniano. Es necesario conocer las propiedades termofísicas del producto, tales como: $\rho, c_{p}, k, \ldots$.

Además debemos conocer la temperatura máxima a la que se puede calentar el producto sin degradarse y la temperatura media del fluido en el tubo. Tomando un margen de seguridad establecemos el $\Delta T$ al que trabaja el intercambiador.

Por último, si se trata de un fluido seudoplástico, es necesario conocer las propiedades reológicas del mismo, $m$ y $n$ a la temperatura de trabajo. Para ello se pueden seguir dos vías:

- Revisión bibliográfica. En la bibliografía existen numerosos estudios sobre las propiedades reológicas de todo tipo de productos: zumos, siropes, mayonesas, etc. Estos estudios no nos proporcionan el valor exacto de las propiedades reológicas, ya que varían según el origen de la materia prima, pero pueden ser una buena estimación. 
- Medición de las propiedades reológicas. La otra alternativa, es la de utilizar un reómetro. Se puede optar por seleccionar uno en el mercado o por construir uno similar al utilizado en la presente investigación (véase capítulo de metodología experimental).

Paso 3- Determinación del número de Reynolds y de Prandtl. Con los datos de velocidad media del flujo de la sección del tubo comercial, el gasto másico que es necesario trasegar y las propiedades termofísicas y reológicas del fluido se puede calcular el número de Reynolds:

$$
\begin{gathered}
u_{b}=G / \rho A \\
R e_{g}=\frac{\rho u_{b} D_{h}}{\mu_{g}} \\
P r_{g}=\frac{c_{p} \mu_{g}}{k}
\end{gathered}
$$

con

$$
\mu_{g}=m n^{d} c^{n-1}\left(\frac{u_{b}}{D_{h}}\right)^{n-1}
$$

donde $c$ y $d$ son constantes cuyo valor se indica en la Tabla 5.8, correspondientes a la Ec. 5.19.

Paso 4- Obtención de los números adimensionales de Fanning y Nusselt en régimen estático. En primer lugar se obtienen los parámetros de funcionamiento en régimen estático.

Obtención del factor de fricción. En función del número de Reynolds, debemos establecer en qué región del flujo nos encontramos. En consecuencia se puede utilizar una o las dos opciones descritas a continuación:

1. Utilizar la relación para flujo laminar:

$$
f=a / R e_{g}
$$

donde el valor de $a$ se encuentra en la Tabla 5.8. 
2. Utilizar los resultados experimentales representados en la Fig. 8.3, representada a continuación, válidos tanto para flujo laminar como turbulento.

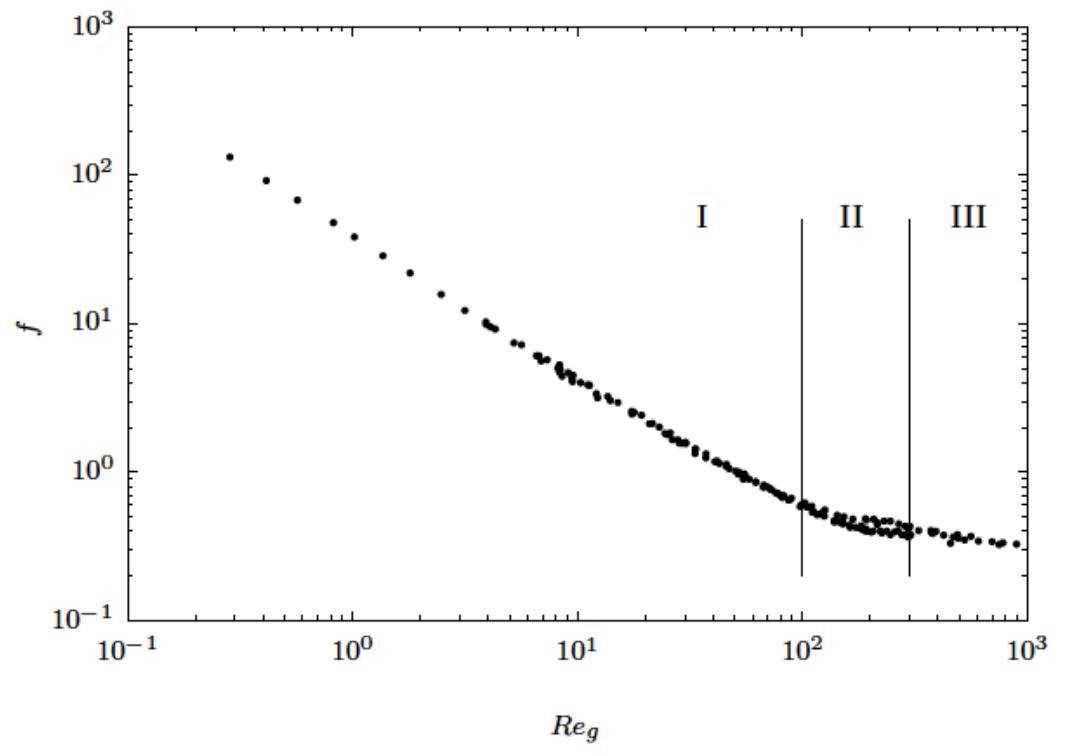

Obtención del Número de Nusselt. Al igual que en el paso anterior, lo primero es determinar la región del flujo en función del número de Reynolds. Para ello se puede consultar la Fig. 9.4.

Una vez seleccionada la región, la mejor opción es utilizar las correlaciones experimentales (Ec. 9.5), cuyos coeficientes, en función de la región del flujo, se detallan en la Tabla 9.3.

$$
N u=a \times R e_{g}^{b} \times P r_{g}^{c}
$$

Paso 5- Determinación de la potencia de bombeo necesaria. La potencia requerida por el sistema de bombeo se puede obtener a partir del número de Fanning:

$$
\dot{W}=Q \times \Delta p=u_{b} S \times \Delta p=f S N L 2 \rho u_{b}^{3} / D_{h}
$$

Paso 6- Flujo de calor. A partir del Número de Nusselt se puede calcular el flujo de calor transmitido al flujo.

$$
q=U A \Delta T
$$


obviamente el coeficiente global de intercambio de calor no sólo depende del coeficiente interior determinado en esta investigación. Así, el resto de parámetros de los que depende $U$ se obtienen de la forma habitual, mientras que $h_{i}$ se determina a partir del número de Nusselt:

$$
h_{i}=\frac{k N u}{D_{h}}
$$

Paso 7- Velocidad de rascado / frecuencia de rascado. A lo largo de la investigación se ha determinado que $h_{i}$ aumenta con la velocidad de rascado, sin embargo el accionamiento del rascador supone un consumo de energía adicional nada despreciable.

En principio, las necesidades de frecuencia con la que se acciona el rascador, o la velocidad de rascado las determina la velocidad de acumulación de ensuciamiento. Es imposible por lo tanto establecer una recomendación general para todos los procesos.

En general, a partir de las necesidades de limpieza, se obtiene una velocidad de rascado necesaria $v_{s}$. A partir de ella se pueden obtener los números adimensionales del paso 4 , teniendo en cuenta el efecto del rascador:

$$
\begin{gathered}
|\omega|=\frac{\left|v_{s}\right|}{u_{b}} \\
\beta=1-\frac{v_{s}}{u_{b}} \\
f=a \times R e_{g}^{b} \times \beta^{c}
\end{gathered}
$$

donde las constantes se obtienen de la Tabla 8.1.

$$
N u=a \operatorname{Re}_{g}^{b} \operatorname{Pr}_{g}^{c}(d+|\omega|)^{e}
$$

estando las constantes definidas en la Tabla 9.5.

Además, a la potencia de bombeo necesaria hay que sumar la potencia de accionamiento del sistema en función de $v_{s}$. Ésta depende en cierta medida del sistema hidráulico de accionamiento y otros parámetros variables. De modo que los resultados obtenidos en la presente investigación sirven de guía para los cálculos de dimensionado, pero no pueden ser tomados como universales. Para la estimación se puede utilizar la siguiente ecuación: 


$$
P_{A}=a_{p} \times v_{s}^{b_{p}}+\left(a_{s}(T)+a_{f} \beta^{b_{f}}+c_{f} m^{d_{f}}\right) v_{s}
$$

donde el valor de las constantes se puede obtener de la Tabla 8.4 y la Tabla 8.3.

\subsection{Conclusiones finales}

El presente trabajo supone el colofón a una línea de investigación que comenzó hace más de una década, llevada a cabo por investigadores de la Universidad Politécnica de Cartagena y la Universidad Miguel Hernández de Elche. En dicha línea, se han estudiado diferentes técnicas de mejora de la transferencia de calor en intercambiadores de calor de tubos trabajando principalmente con fluidos newtonianos. Actualmente se encuentra en marcha una investigación sobre el uso de muelles insertados en paneles solares térmicos en parrilla, cuyo objetivo es evaluar la posible mejora de la eficiencia de dichos sistemas.

En los subapartados sucesivos, se detallan las aportaciones genuinas de la presente investigación al conocimiento científico. Así mismo, se enumera la producción científica derivada de la investigación.

\subsubsection{Aportaciones en la descripción experimental del campo de velocidades}

1. Se ha obtenido el campo de velocidades mediante $\mathrm{PIV}^{1}$ en el tubo con rascador, en regímenes de rascado estático y dinámico, en direcciones equicorriente y contracorriente. Se han analizado diferentes velocidades de rascado, de modo que el factor de bloqueo ha variado en el rango de $\beta \in[-2,4]$. Para cada caso de estudio se han realizado ensayos en un rango de números de Reynolds en el rango de $R e_{g} \in[5,48]$.

2. Se ha comprobado que la estructura del flujo depende fuertemente del factor de bloqueo, apareciendo tres estructuras distintas para los siguientes rangos de valores del parámetro: $\beta<0, \beta=0 \mathrm{y} \beta>0$.

3. Los regímenes de funcionamiento con valores altos de $|\beta|$ provocan mayores gradientes de velocidades, recirculaciones y en general un mayor mezclado del flujo.

\footnotetext{
${ }^{1}$ Velocimetría por imágenes de partículas
} 
4. Mediante el ensayo a diferentes números de Reynolds y diferentes valores del Índice de comportamiento del flujo ( $n$ ), se ha comprobado la influencia que tiene sobre las estructuras de flujo el hecho de variar conjuntamente ambos parámetros. Al aumentar $R e_{g}$ y $n$ en general crecen las zonas de alta velocidad y aumentan las recirculaciones, en general mejoran las condiciones para un transferencia de calor más eficiente.

5. El efecto de variar $n$ y $R e_{g}$ por separado no se ha podido comprobar debido a las limitaciones experimentales existentes. La simulación del flujo en la geometría de tubos concéntricos ha servido para comprobar el efecto de variar $n$ en una geometría similar, sin embargo el efecto exacto en la geometría bajo estudio sigue siendo desconocido. Esta dificultad encontrada en los ensayos de visualización pone de manifiesto la utilidad del método de generalización de la viscosidad, el cuál permite obtener la caída de presión a partir de una curva independientemente del valor de $n$ del fluido.

\subsubsection{Aportaciones en la descripción del comportamiento termo- hidráulico}

1. Se ha estudiado la caída de presión del flujo isotermo en el tubo con rascador en régimen estático y dinámico. En dicho estudio se observan las regiones laminar, de transición y turbulenta del flujo. El estudio de la caída de presión promedio en el tubo con el rascador en movimiento, indica que dicha caída de presión promedio en un ciclo de rascado es aproximadamente igual a la caída de presión en régimen estático. La caída de presión medida está claramente determinada por el índice de comportamiento de flujo $n$, aunque dicho comportamiento está incluido en la definición de la viscosidad generalizada.

2. Se ha estudiado la potencia de accionamiento del rascador, desglosando los diferentes aportes a dicha potencia y cuantificándola completamente. En general la potencia del rascador aumenta con la velocidad de rascado. También influyen la temperatura del sistema (fluido, rascador, pared) y otros factores como el grado de presión del cojinete de sellado.

3. Se ha caracterizado la transferencia de calor en el tubo con rascador.

a) Se han estudiado los régimenes de rascado estático y dinámico, observándose una mejora de la transferencia de calor al aumentar la velocidad de rascado. 
b) En régimen estático, se han observado 2 regiones distintas en el flujo laminar, además de las regiones de transición y turbulenta.

c) Para obtener la influencia del índice de comportamiento de flujo $n$ se ha hecho uso del simulador numérico desarrollado, equiparando la situación en el tubo con rascador a la que se da en una geometría de tubo con eje.

d) Por último se han obtenido correlaciones del número de Nusselt en función de los números de Reynolds y de Prandtl generalizados.

4. Utilizando los criterios de mejora clásicos propuestos por Bergles, se ha realizado una comparativa del uso del rascador en régimen estático frente a dos geometrías de referencia: un tubo liso y un tubo con eje. En general, los diferentes criterios de comparación muestran las mejores prestaciones del tubo con rascador estático para números de Reynolds en el rascador mayores de 10. No obstante, la magnitud de las mejoras depende significativamente de la longitud del tubo de referencia en comparación con la longitud de entrada térmica.

5. El accionamiento del rascador supone un aumento del número de Nusselt con respecto al obtenido si el rascador se encuentra estático (criterio R1), siendo el aumento mayor a medida que aumenta la velocidad de rascado o el número de Reynolds. No obstante esta mejora tiene un precio alto en forma de consumo de potencia por parte del pistón. La formulación del criterio R3 únicamente ofrece resultados favorables para velocidades de rascado muy bajas y números de Reynolds por encima de 60 .

\subsubsection{Producción científica}

En una fase previa al estudio del tubo con rascador sobre el cual versa el presente documento, se estudió el flujo en el mismo tubo con un rascador similar, diseñado para trabajar con hielo líquido. Consiste en un eje sobre el que se encuentran montados discos rascadores con seis perforaciones que dejan pasar el fluido. Dicha investigación ha llevado a la publicación de los siguientes trabajos:

- D. Crespí, Experimental investigation of turbulence level in enhanced exchangers 2009. Heat Exchanger Fouling and Cleaning VIII. Schladming (Austria)

- Medida experimental del flujo turbulento en Intercambiadores de calor de superficie rascada 2010. Conferencia Internacional de Ingeniería Mecánica. Santiago de Cuba 
(Cuba)

- D. Crespí-Llorens, P. Martínez, P. Vicente, A. Viedma. Effect of the axial scraping velocity on enhanced heat exchangers. International journal heat and fluid flow. 2013. IF: 1,7 .

La investigación expuesta en el presente documento ha servido para producir los siguientes artículos de investigación:

- Medida experimental del flujo con rascadores alternativos y fluidos no newtonianos. 2012. IV Congreso iberoamericano ciencias y técnicas del frío.

- Medida del flujo con fluidos no newtonianos y rascadores alternativos. 2012. II Congreso encuentro del Área de Máquinas y Motores Térmicos del sureste de España. Murcia.

- D. Crespí-Llorens, P. Vicente, A. Viedma. Generalized Reynolds number and viscosity definitions for non-Newtonian fluid flow in ducts of non-uniform cross-section. Experimental Thermal and Fluid Science. 2015².

Además se espera la publicación de al menos dos artículos más, centrados en el comportamiento termohidráulico en regímenes estático y dinámico del rascador, así como en posteriores trabajos que sirvan para extender la aplicación del método de generalización a geometrías más dispares.

\footnotetext{
${ }^{2}$ Bajo proceso de revisión a fecha de presentación del presente documento
} 


\section{Bibliografía y Nomenclatura}





\section{Bibliografía}

(2001). Heat Exchanger Fouling in the Pre-heat Train of a Crude Oil Distillation Unit. 00016. ESDU International PLC.

Abdelrahim, K. A. y Ramaswamy, H. S. (1995). High temperature/pressure rheology of carboxymethyl cellulose (cmc). Food Research International, 28(3):285 290.

Abu-Jdayil, B. (2003). Modelling the time-dependent rheological behavior of semisolid foodstuffs. Journal of Food Engineering, 57(1):97 102.

Addio, L. D., Carotenuto, C., Natale, F. D., y Nigro, R. (2012). A new arrangement of blades in scraped surface heat exchangers for food pastes. Journal of Food Engineering, 108(1):143 149 .

Afonso, I. M., Cruz, P., Maia, J. M., y Melo, L. F. (2008). Simplified numerical simulation to obtain heat transfer correlations for stirred yoghurt in a plate heat exchanger. Food and Bioproducts Processing, 86(4):296 303.

Awuah, G., Ramaswamy, H. S., y Simpson, B. K. (1993). Surface heat transfer coefficients associated with heating of particulates in cmc solutions. Journal of Food Proc. Engineering, 16:39 57.

Balmforth, N. J., Craster, R. V., Rust, A. C., y Sassi, R. (2007). Viscoplastic flow over an inclined surface. Journal of Non-Newtonian Fluid Mechanics, 142(1-3):219 243. Viscoplastic fluids: From theory to application.

Barletta, A. y Magyari, E. (2007). Forced convection with viscous dissipation in the thermal entrance region of a circular duct with prescribed wall heat flux. International Journal of Heat and Mass Transfer, 50(1-2):26 35.

Barnes, H. (1989). J. Rheol., 33(329). 
Barnes, H. A. (1999). The yield stress a review or ' $\pi \alpha \nu \tau \alpha \rho \varepsilon \iota$ ' everything flows? Journal of Non-Newtonian Fluid Mechanics, 81(1-2):133 178.

Benchabane, A. y Bekkour, K. (2008). Rheological properties of carboxymethyl cellulose (cmc) solutions. Colloid and Polymer Science, 286(10):1173 1180.

Bergles, A. (1997). Heat transfer enhancement. the encouragement and acommodation of high heat fluxes. Journal of Heat Transfer.

Bergles, A. (2002). Exhft for fourth generation heat transfer technology. Experimental Thermal and Fluid Science.

Bergles., A. y Joshi, S. (1983). Low Reynolds Number Flow Heat Exchangers, chapter Experimental Verification of Analyses and Correlation of the Effects of Temperature Dependent Fluid Properties on Laminar Heat Transfer. Hemisphere.

Beuf, M., Rizzo, G., Leuliet, J., H.MÃCEller-Steinhagen, Karabelas, A., Yiantsios, S., y Benezech, T. (2003). Fouling and cleaning of modified stainless steel plate heat exchangers processing milk products. In Heat Exchanger Fouling and Cleaning: Fundamentals and Applications.

Bird, R. (1976). Annu. rev. fluid mech 8 (1976) 13.

Bird, R., Armstrong, R., y Hassager, O. (1987). Dynamics of polymeric liquids. Fluid Dynamics, volume 1. Hanser Publishers, New York, 2nd edition.

Bird, R. B. (1959). Zur theorie des wÃ€rme ÃCEbergangs an nicht-newtonsche flÃ(Essigkeiten bei laminarer rohrstrÃ $₫$ mung. Chemie Ingenieur Technik, 31(9):569 572.

Blel, W., Legentilhomme, P., Bénézech, T., y Fayolle, F. (2013). Cleanabilty study of a scraped surface heat exchanger. Food and Bioproducts Processing, 91(2):95 102.

Buckingham, E. (1914). On physically similar systems: illustrations of the use of dimensional equations. Physical Review, 4:345 376.

Cancela, M., Alvarez, E., y Maceiras, R. (2005). Effects of temperature and concentration on carboxymethylcellulose with sucrose rheology. Journal of Food Engineering, 71:419 424. 
Capobianchi, M. (2008). Pressure drop predictions for laminar flows of extended modified power law fluids in rectangular ducts. International Journal of Heat and Mass Transfer, 51(5-6):1393 1401.

Capobianchi, M. y Wagner, D. (2010). Heat transfer in laminar flows of extended modified power law fluids in rectangular ducts. International Journal of Heat and Mass Transfer, $53(1-3): 558 \quad 563$.

Carreu, P., Dekee, D., y Chhabra, R. (1997). Rheology of Polymeric Systems. Hanser Publishers.

Chandra, A. y Chhabra, R. (2011). Flow over and forced convection heat transfer in newtonian fluids from a semi-circular cylinder. International Journal of Heat and Mass Transfer, $54(1-3): 225 \quad 241$.

Chandra, A. y Chhabra, R. (2012). Laminar free convection from a horizontal semi-circular cylinder to power-law fluids. International Journal of Heat and Mass Transfer, 55(1112):2934 2944 .

Chebbi, R. (2002). Laminar ow of power-law uids in the entrance region of a pipe. Chemical Engineering Science, 57:4435 4443.

Chhabra, R. y Richardson, J. (1999). Non-newtonian flow in the process industries. Fundamentals and engineering applications. Butterworth-Heinemann.

Chhabra, R. y Richardson, J. (2008). Non Newtonian flow and applied rheology. Engineering aplications. Butterworth-Heinemann, 225 Wildwood Av., Woburn.

Cho, Y. y Harnett, J. P. (1982). Non-newtonian fluids in circular pipe flow. volume 15 of Advances in Heat Transfer, pages 59 141. Elsevier.

Cho, Y. y Hartnett, J. (1982). Non newtonian fluids in circular pipe flow. Advances in Heat Transfer, (15):59 141.

Choi, S. M., Kim, W. H., Coté, D., Park, C.-W., y Lee, H. (2011). Blood cell assisted in vivo particle image velocimetry using the confocal laser scanning microscope. The International Online Journal of Optics, 19:4357. 
Choudary, N. V. y Jasra, R. V. (1994). Densities of aqueous solutions of sodium bisulfite and sodium 2-methylalylsulfate. Journal of Chemical and Engineering Data, 39:181 183.

Churchill, S. W. y Chu, H. H. (1975). Correlating equations for laminar and turbulent free convection from a horizontal cylinder. International Journal of Heat and Mass Transfer, 18(9):1049 1053 .

Crespí-Llorens, D., Martínez, P., Vicente, P., y Viedma, A. (2013). Effect of the axial scraping velocity on enhanced heat exchangers. Int. J. Heat Fluid Flow.

Cui, H., Cai, M., yun Zhang, S., y Xiu, D. (2009). Numerical computation of pressure gradient for the flow of power-law fluid in annulus with inner cylinder executing a planetary motion. Journal of Hydrodynamics, Ser. B, 21(5):699 704.

Dehkordi, A. M. y Mohammadi, A. A. (2009). Transient forced convection with viscous dissipation to power-law fluids in thermal entrance region of circular ducts with constant wall heat flux. Energy Conversion and Management, 50(4):1062 1068.

Deissler, R. G. (1951). Analytical investigation of fully developed laminar flow in tubes with heat transfer with fluid properties variable along the radius. NACA, -:Technical Note 2410.

Delplace, F. y Leuliet, J. (1995). Generalized reynolds number for the flow of power law fluids in cylindrical ducts of arbitrary cross-section. The Chemical Engineering Journal and the Biochemical Engineering Journal, 56(2):33 37.

Dhiman, A. K. (2009). Heat transfer to power-law dilatant fluids in a channel with a built-in square cylinder. International Journal of Thermal Sciences, 48(8):1552 1563.

Ditchfield, C., Tadini, C., Singh, R., y Toledo, R. (2007). Heat transfer during thermal processing of a temperature dependent non-newtonian fluid in a tubular heat exchanger. Chemical Engineering and Processing, 46(5):472 476. Advances in the Application of Chemical Engineering Principles in Food Industry.

Doorne, C. y Westerweel, J. (2007). Measurement of laminar, transitional and turbulent pipe flow using stereoscopic-piv. Experiments in Fluids, 42(2):259 279.

Galindo-Rosales, F., Rubio-Hernández, F., y Sevilla, A. (2011). An apparent viscosity function for shear thickening fluids. Journal of Non-Newtonian Fluid Mechanics, 166(5-6):321 325. 
García, A. (2006). Mejora de la Transmisión de Calor en Tubos Circulares Lisos Mediante Elementos Insertados Estáticos. PhD thesis, UPCT.

García, A., Solano, J., Vicente, P., y Viedma, A. (2012). The influence of artificial roughness shape on heat transfer enhancement: Corrugated tubes, dimpled tubes and wire coils. Applied Thermal Engineering, 35(0):196 201.

Ghannam, M. T. y Esmail, M. N. (1996). Rheological properties of carboxymethyl cellulose. Journal of Applied Polymer Science, 64:289 301.

Gluck, D. F. (1959). The effect of turbulence promotion on newtonian and non-newtonian heat transfer rates. Master's thesis, University of Delaware.

Goddard, J. y Bashir, Y. (1990). Recent developments in structured continua II., chapter 2. Longman, London.

Grigull, U. (1956). WÃ €rmẽ̃(Ebergang an nicht-newtonsche flã(Essigkeiten bei laminarer rohrstr $\widetilde{A}$ mung. Chemie Ingenieur Technik, 28(8-9):553 556.

Guo, B. y Guo, C. (2009). The convergence of non-newtonian fluids to navier-stokes equations. Journal of Mathematical Analysis and Applications, 357(2):468 478.

Gupta y Rao, M. (1979). Heat transfer and friction characterisation of newtonian and powerlaw type of non-newtonian fluids in smooth and spirally corrugated tubes. Advance in enhanced heat transfer, ASME.

Han, H.-Z., Li, B.-X., Yu, B.-Y., He, Y.-R., y Li, F.-C. (2012). Numerical study of flow and heat transfer characteristics in outward convex corrugated tubes. International Journal of Heat and Mass Transfer, 55(25-26):7782 7802.

Hanks, R. y Larsen, K. (1979). The flow of power-law non-newtonian fluids in concentric annuli. Ind. Eng. Chem. Fundam., 18:33 35.

Hewitt, G. F., Shires, G. L., y Bott, T. R. (1994). Process Heat Transfer. CRC Press.

Holdsworth, S. (1993). Rheological models used for the prediction of the flow properties of food products: a literature review. Transactions of institute of chemical engineering, 71(3):139 179 . 
Igumentsev, T. I. y Nazmeev, Y. G. (1978). Intensification of convective heat exchange by spiral swirlers in the flow of anomalously viscous liquids in pipes. J. Eng. Phys., (35):890 894.

Illán, F. (2008). Caracterización experimental del comportamiento termohidráulico del hielo lãquido generado a partir de disoluciones de NaCl. Aplicación a cambiadores de calor. PhD thesis, Universidad Politécnica de Cartagena.

Irvine, T. J. y Karni, J. (1987). Non newtonian flow and heat transfer, chapter 5. John Wiley and sons, New York.

Joshi, S. y Bergles, A. (1980a). Experimental study of laminar heat transfer to in-tube flow of non-newtonian fluids. Journal of Heat Transfer, 102:397 401.

Joshi, S. y Bergles, A. (1980b). Heat transfer to laminar flow of non-newtonian pseudplastic fluids in tubes. Journal of Heat Transfer.

Joshi, S. D. (1978). Heat transfer in in-tube flow of non-Newtonian fluids. PhD thesis, Iowa State University, Ames, Iowa.

Joshi, S. D. y Bergles, A. E. (1979). Heat transfer in laminar in-tube flow of non-newtonian fluids, analitical and experimental studies. Technical Report HTL-18, Iowa State University, Ames, Iowa.

Kakaç, S., Shah, R., y Aung, W. (1987). Handbook of single-phase convective heat transfer. A Wiley Interscience publication. Wiley.

Kays, W. M., Crawford, M. E., y Weigand, B. (2005). Convective heat and mass transfer. McGraw-Hill, Boston, 4th. ed. edition.

Kozicki, W., Chou, C. H., y Tiu, C. (1966). Non-newtonian flow in ducts of arbitrary crosssectional shape. Chemical Engineering Science, 21(8):665 679.

Lagarrigue, S. y Alvarez, G. (2001). The rheology of starch dispersions at high temperatures and high shear rates: a review. Journal of Food Engineering, 50(4):189 202.

Liu, J. H., Yan, J. P., Gu, P. Y., Ye, L., y Pan, Z. R. (1992). Laminar flow and heat transfer to pseudoplastic fluids in pipes. Chemical Engineering and Processing, 31(4):247 253. 
Loulou, T., Peerhossaini, H., y Bardon, J. (1992). Étude experimentale de la conductivité thermique de fluides non- newtoniens sous cisaillement application aux solutions de carbopol 940. Int. J. Heat Mass Transfer, 35:2557.

Mabit, J., Belhamri, R., Fayolle, F., y Legrand, J. (2008). Development of a time temperature integrator for quantification of thermal treatment in scraped surface heat exchangers. Innovative Food Science and Emerging Technologies, 9(4):516 526.

Manglik, R., Bergles, A., y Joshi, S. (1988). Augmentation of heat transfer to laminar flow of non-newtonian fluids in uniformly heated tubes with twisted-tape inserts. In Elsevier, editor, 1st World Conf. Experimental Heat Transfer, Fluid Mechanics and Thermodynamics, pages 676 684, New York.

Marn, J. y Ternik, P. (2006). Laminar flow of a shear-thickening fluid in a pipe bend. Fluid Dynamics Research, 38(5):295 312.

Martínez, D., García, A., Solano, J., y Viedma, A. (2014). Heat transfer enhancement of laminar and transitional newtonian and non-newtonian flows in tubes with wire coil inserts. International Journal of Heat and Mass Transfer, 76:540 548.

Metzner, A. (1965). Heat transfer in non-newtonian fluids. In Hartnett, J. y T. F. Irvine, J., editors, Advances in Heat Transfer, volume 2, pages 357 397. Academic Press, New York.

Metzner, A. B. y Reed, J. C. (1955). Flow of non-newtonian fluids - correlation of the laminar, transition, and turbulent-flow regions. Aiche Journal, 1(4):434 440.

Muller-Steinhagen, H. (2000). Heat Exchanger Fouling: Mitigation and Cleaning Techniques - IChemE. Inst of Chemical Engineers UK.

Muñoz-Esparza, D. y Sanmiguel Rojas, E. (2011). Numerical simulations of the laminar flow in pipes with wire coil inserts. Computers \&f Fluids, 44(1):169 177.

Nazmeev, Y. G. (1979). Intensification of convective heat exchange in anomalously viscous media by the aplication of artificial periodic roughness. J. Eng. Phys., (36):16 20.

Nazmeev, Y. G. (1981). Intensification of convective heat exchange in the local swirling of an anomalously viscous fluid by screw swirlers. J. Eng. Phys., (41):1117 1120. 
Nirmalkar, N. y Chhabra, R. (2012). Forced convection in power-law fluids from an asymmetrically confined heated circular cylinder. International Journal of Heat and Mass Transfer, $55(1-3): 235 \quad 250$.

Nouar, C., Ouldrouis, M., y Salem, A. (1995). Developing laminar flow in the entrance region of annuli-reviewed and extension of standard resolution methods for the hydrodynamic problem. International Journal of Engineering Science, 33:1517 1534.

Papanastasiou, T. C. y Boudouvis, A. G. (1997). Flows of viscoplastic materials: Models and computations. Computers and Structures, 64(1-4):677 694. Computational Structures Technology.

Patil, A. G. (2000). Laminar flow heat transfer and pressure drop characteristics of powerlaw fluids inside tubes with varying width twisted tape inserts. Journal of Heat Transfer, 122(1):143 149 .

Patnana, V. K., Bharti, R. P., y Chhabra, R. P. (2010). Two-dimensional unsteady forced convection heat transfer in power-law fluids from a cylinder. International Journal of Heat and Mass Transfer, 53(19-20):4152 4167.

Pethkool, S., Eiamsa-ard, S., Kwankaomeng, S., y Promvonge, P. (2011). Turbulent heat transfer enhancement in a heat exchanger using helically corrugated tube. International Communications in Heat and Mass Transfer, 38(3):340 347.

Pilizota, V., Subaric, D., y Lovric, T. (1996). Rheological properties of cmc dispersions at low temperatures. Food technology and biotechnology, 34(2-3):87 90.

Poole, R. J. (2010). Development-length requirements for fully developed laminar flow in concentric annuli. Journal of Fluids Engineering, 132:064501.

Poole, R. J. y Ridley, B. S. (2007). Developement-length requirements for fully developed laminar pipe flow of inelastic non-newtonian liquids. Journal of Fluids Engineering, 129:1281 1287.

Pritchard, A. (1987). Fouling Science and Technology. L.F. Melo and T.R. Bott, and C.A. Bernardo, volume 145, chapter The economics of Fouling. Kluwer Academic Publishers. NATO ASI series E. 
Raffel, M., Willer, C., y Kompenhans, J. (2000). Particle Image Velocimetry: A practical guide. Springer.

Rainieri, S., Bozzoli, F., Cattani, L., y Pagliarini, G. (2013). Compound convective heat transfer enhancement in helically coiled wall corrugated tubes. International Journal of Heat and Mass Transfer, 59(0):353 362.

Rainieri, S. y Pagliarini, G. (1997). Convective heat transfer to orange juice in smooth and corrugated tubes. Heat Technology, (15):69 75.

Rainieri, S. y Pagliarini, G. (2002). Convective heat transfer to temperature dependent property fluids in the entry region of corrugated tubes. International Journal of Heat and Mass Transfer, 45(22):4525 4536.

Rajasekaran, S., Kubair, V., y Kuloor, N. (1966). Secondary flow of non-newtonian fluids in helical coils. Indian Journal of Technology, (4):33 35.

Rajasekaran, S., Kubair, V., y Kuloor, N. (1970). Heat transfer to non-newtonian fluids in coiled pipes in laminar flow. International Journal of Heat and Mass Transfer, (13):1583 1594.

Rao, M. A., Walter, R. H., y Cooley, H. J. (1981). Effect of heat treatment on the flow properties of aqueous guar gum and carboxymethyl cellulose ( $\mathrm{cmc}$ ) solutions. Journal of Food Science, 46:896 9.

Rao, P. K., Sasmal, C., Sahu, A., Chhabra, R., y Eswaran, V. (2011). Effect of power-law fluid behavior on momentum and heat transfer characteristics of an inclined square cylinder in steady flow regime. International Journal of Heat and Mass Transfer, 54(13-14):2854 2867.

Rennie, T. J. y Raghavan, G. V. (2007). Thermally dependent viscosity and non-newtonian flow in a double-pipe helical heat exchanger. Applied Thermal Engineering, 27(5-6):862 868.

Reynolds, W. C., Lunderg, R. E., y McCuen, P. A. (1963). Heat transfer in annular passages. hydrodynamically developed laminar flow with arbitrary prescribed wall temperature of heat flux. International Journal of Heat and Mass Transfer, 6:495 529. 
Rozzi, S., Massini, R., Paciello, G., Pagliarini, G., Rainieri, S., y Trifiro, A. (2007). Heat treatment of fluid foods in a shell and tube heat exchanger: Comparison between smooth and helically corrugated wall tubes. Journal of Food Engineering, 79(1):249 254 .

Sahu, A. K., Chhabra, R., y Eswaran, V. (2009). Effects of reynolds and prandtl numbers on heat transfer from a square cylinder in the unsteady flow regime. International Journal of Heat and Mass Transfer, 52(3-4):839 850.

Saraceno, L., Boccardi, G., Celata, G., Lazzarini, R., y Trinchieri, R. (2011). Development of two heat transfer correlations for a scraped surface heat exchanger in an ice-cream machine. Applied Thermal Engineering, 31(17-18):4106 4112. <ce:title>SET 2010 Special Issue $</$ ce:title $>$.

Sasmal, C. y Chhabra, R. (2012). Effect of orientation on laminar natural convection from a heated square cylinder in power-law liquids. International Journal of Thermal Sciences, 57(0):112 125 .

Scarano, F. y Reithmuller, M. (2000). Advances in iterative multigrid piv image processing. Experiments in Fluids, 29:51 60.

Skelland, A. H. P. (1967). Non-Newtonian Flow and Heat Transfer. Wiley, New York.

Solano, J. (2009a). Análisis del flujo y de la transmisión de calor en intercambiadores de superficie rascada por movimiento lineal alternativo. PhD thesis, UPCT.

Solano, J., GarcÃa, A., Vicente, P., y Viedma, A. (2011a). Flow field and heat transfer investigation in tubes of heat exchangers with motionless scrapers. Applied Thermal Engineering, 31(11-12):2013 2024.

Solano, J., GarcÃa, A., Vicente, P., y Viedma, A. (2011b). Flow pattern assessment in tubes of reciprocating scraped surface heat exchangers. International Journal of Thermal Sciences, 50(5):803 815 .

Solano, J. P. (2009b). Análisis del flujo y de la transmisión de calor en intercambiadores de superficie rascada por movimiento lineal alternativo. PhD thesis, Universidad Politécnica de Cartagena.

Steinhagen, R., MÃEEller-Steinhagen, H., y Maani, K. (1992). Problems and costs due to heat exchanger fouling in new zealand industries. Heat transfer engineering, 14(1):19 30 . 
Sun, K.-H., Pyle, D., Fitt, A., Please, C., Baines, M., y Hall-Taylor, N. (2003). Numerical study of $2 \mathrm{~d}$ heat transfer in a scraped surface heat exchanger. Computer and fluids, 33:869 880.

Ternik, P. (2010). New contributions on laminar flow of inelastic non-newtonian fluid in the two-dimensional symmetric expansion: Creeping and slowly moving flow conditions. Journal of Non-Newtonian Fluid Mechanics, 165(19-20):1400 1411.

Ternik, P., Marn, J., y Ẑuniĉ, Z. (2006). Non-newtonian fluid flow through a planar symmetric expansion: Shear-thickening fluids. Journal of Non-Newtonian Fluid Mechanics, 135(23):136 148 .

Thome, J. R. (1990). Enhanced Boiling Heat Transfer. Hemisphere Publ. Corp.

Tokpavi, D. L., Magnin, A., y Jay, P. (2008). Very slow flow of bingham viscoplastic fluid around a circular cylinder. Journal of Non-Newtonian Fluid Mechanics, 154(1):65 76.

Toledo, M. (1998). Measuring specific heat capacity. User com, 7:1 5.

Turian, R. M. (1964). Thermal phenomena and non-Newtonian viscometry. Ph. D. Thesis. University of Wisconsin, Madison, USA.

Vaschy, A. (1892). Sur les lois de similitude en physique. Annales Télégraphiques, 19:25 28.

Vicente, P. (2002). Técnicas de Mejora de la Transferencia Térmica en Tubos de Intercambiadores de Calor para Flujo MonofÃ jsico Laminar y Turbulento. PhD thesis, UPCT.

Vincent-Vela, C., Cuartas-Uribe, B., Álvarez-Blanco, S., Lora-García, J., y nos Rodríguez, E. B. (2010). Analysis of ultrafiltration processes with dilatant macromolecular solutions by means of dimensionless numbers and hydrodynamic parameters. Separation and Purification Technology, 75(3):332 339.

Webb, R. L. (2005). Principles of Enhanced Heat Transfer. Wiley Interscience.

Weltmann, R. N. (1943). Breakdown of tixotropic structure as function of time. Journal of Applied Physics, 14:343 50.

Westerweel, J. (2000). Theoretical analysis of the measurement precision in particle image velocimetry. Experiments in Fluids, 29(1):S003 S012. 
Wichterle, K. (2004). Heat transfer in temperature-dependent non newtonian flow. Chem. Eng. Process., (43):1223 1230.

Withers, J. G. (1980). Tube-side heat transfer and pressure drop for tubes having helical internal ridging with turbulent/transitional flow of single-phase fluid. part 1. single-helix ridging. Heat Transfer Engineering, 2:48 58.

Yang, X. H. y Zhu, W. L. (2007). Viscosity properties of sodium carboxymethylcellulose solutions. Cellulose.

Yataghene, M., Fayolle, F., y Legrand, J. (2009). Experimental and numerical analysis of heat transfer including viscous dissipation in a scraped surface heat exchanger. Chemical Engineering and Processing: Process Intensification, 48(10):1447 1458.

Yataghene, M., Francine, F., y Jack, L. (2011). Flow patterns analysis using experimental $\{\mathrm{PIV}\}$ technique inside scraped surface heat exchanger in continuous flow condition. Applied Thermal Engineering, 31(14-15):2855 2868.

Yataghene, M. y Legrand, J. (2013). A 3d-cfd model thermal analysis within a scraped surface heat exchanger. Computers \&6 Fluids, 71(0):380 399. 


\title{
Nomenclatura
}

\author{
$a, b, c, d, e \quad$ coeficientes de los distintos ajustes de la función $\phi(n)$. \\ $B_{m, e} \quad$ constante de proporcionalidad entre la temperatura y el logaritmo na- \\ tural de $m$ en la relación exponencial. \\ $B_{m, l} \quad$ constante de proporcionalidad entre la temperatura y $m$ en la relación \\ lineal. \\ $B_{n, l} \quad$ constante de proporcionalidad entre la temperatura y $n$. \\ $c_{p} \quad$ calor específico del fluido de trabajo. \\ D diámetro interior del tubo de ensayo. \\ $D_{h} \quad=D-d$ diámetro hidráulico del tubo de ensayo. \\ $D_{v} \quad$ diámetro interior del tubo liso (viscosímetro). \\ $D_{t e} \quad$ diámetro exterior del tubo de ensayo. \\ d diámetro del eje del rascador. \\ $d_{p} \quad$ diámetro de las perforaciones realizadas en el viscosímetro. \\ $e_{c} \quad$ número de píxels en la distancia $D_{h}$ en una imagen de PIV. \\ $e_{v} \quad$ espesor del tubo liso (viscosímetro). \\ $\overrightarrow{f_{m}} \quad$ fuerzas másicas.
}




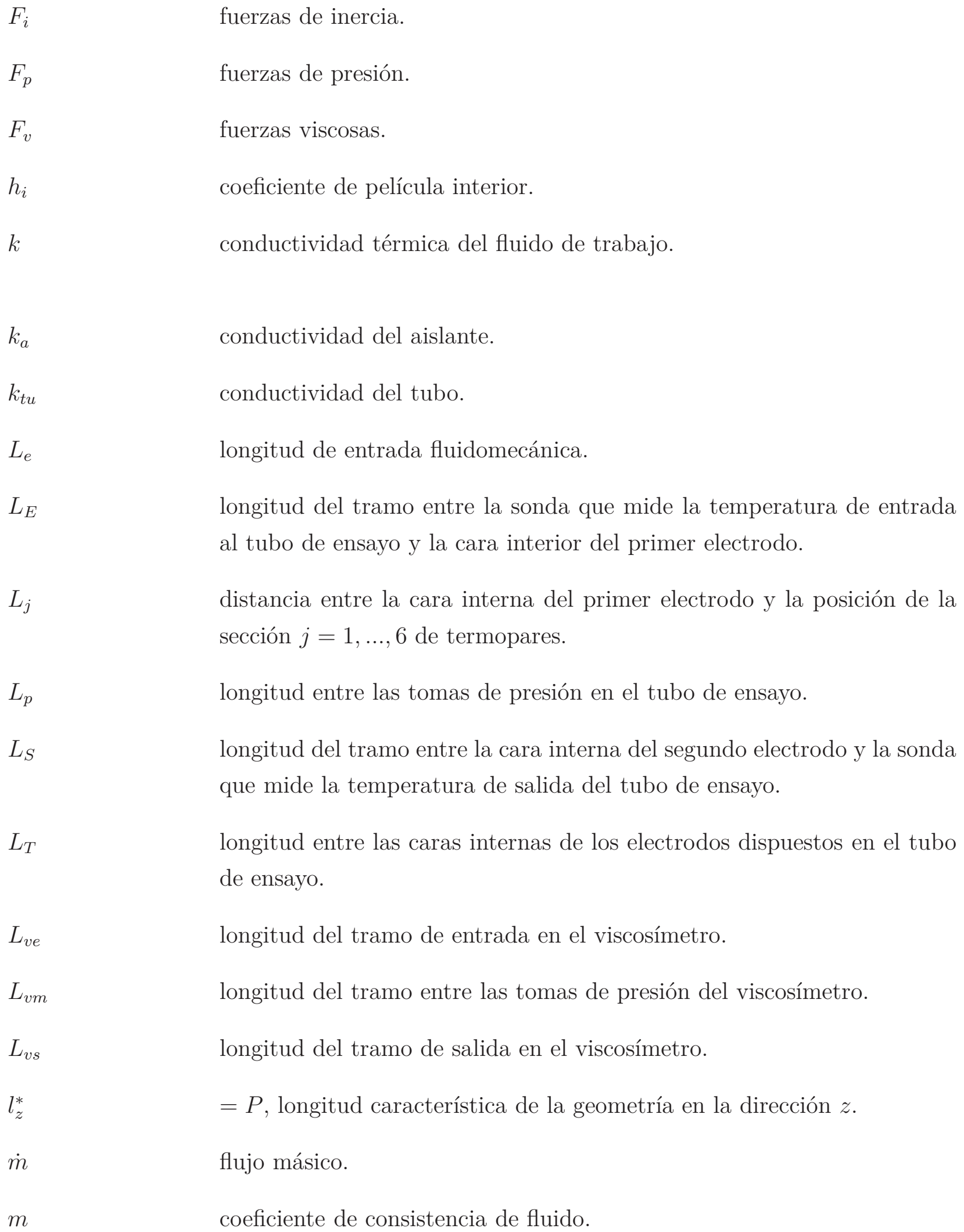


$M M$

N

$N_{S}$

$N_{p i x}$

$N_{T P}$

$N N$

n

P

$p, \Delta p, p_{L}$

$p_{1}$

$p_{2}$

$\dot{q}$

$\dot{q}_{f}, \dot{q}_{f}^{\prime \prime}$

$\dot{q}_{r}$

$\dot{q}_{r}$

$\dot{q}_{T}$

$\dot{q}_{p, e}, \dot{q}_{p}, \dot{q}_{p, s}$

$\dot{q}_{v o l}$ divisiones del mallado en dirección longitudinal (secciones).

número de veces que se toma una medida. En ensayos de PIV: número de pares de imágenes.

$=6$, número de secciones de termopares.

número de píxels contenidos en la distancia de la imagen correspondiente al diámetro hidráulico.

$=8$, número de termopares por secciones.

divisiones del mallado en dirección radial.

índice de comportamiento de flujo (adimensional).

longitud de paso en el rascador.

respectivamente: presión, caída de presión entre las tomas del tubo de ensayo y caída de presión por metro lineal en dicho tubo.

$=-0,14$, factor de corrección del coeficiente de transferencia de calor.

factor de corrección del factor de fricción.

flujo de calor.

flujo de calor aportado al fluido y flujo de calor aportado al fluido por metro cuadrado de superficie.

flujo de calor por radiación.

flujo de calor por reacciones químicas.

flujo de calor producido por efecto Joule.

flujo de calor perdido al ambiente a través de la superficie de los tramos de entrada, ensayo y salida del tubo de ensayo respectivamente.

calor por unidad de volumen generado en la pared del tubo por efecto Joule. 


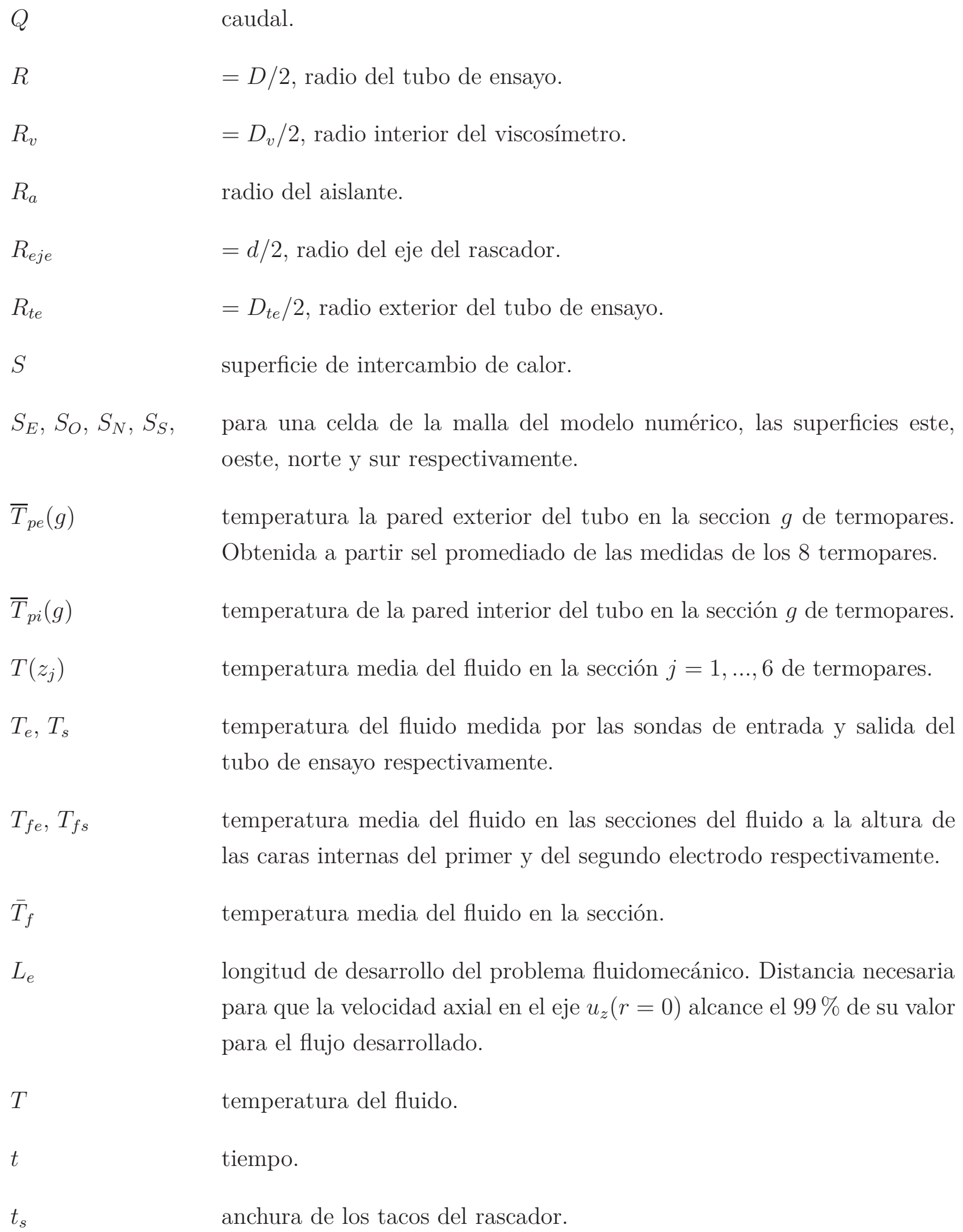


$U$

$u$

$u_{b}$

$u_{i}$

$\vec{v}$

$T_{r}$

$v_{s}$

$\Delta x_{i}$ $=\sum u_{i} / N$, velocidad en un punto del plano visualizado obtenida como resultado de promediar las velocidades obtenidas en ese punto para cada par de imágenes.

velocidad del fluido en una dirección del espacio indicada mediante el subíndice.

velocidad promedio del fluido a través de la sección de paso. Cociente entre el caudal y la sección de paso.

velocidad en un punto, en las direcciones z ó $r$, obtenida a partir de la aplicación del algoritmo de PIV al par de imágenes $i$.

vector de velocidad del fluido.

periodo de rascado.

velocidad de rascado.

desplazamiento medio en las direcciones z ó $r$ de las partículas trazadoras contenidas en un área de interrogación entre dos imágenes sucesivas.

\section{Números Adimensionales}

$\omega$

$N u$

$P e$

$\operatorname{Pr}$

$R e$ $=1-v_{s} / v_{b}$, factor de bloqueo.

$=v_{s} / v_{b}$, velocidad de rascado adimensional.

$=h_{i} D_{h} / k$ número adimensional de Nusselt.

$=\operatorname{Re} \operatorname{Pr}$ número adimensional de Peclet. Es el mismo para fluidos newtonianos o no newtonianos.

$=c_{p} \mu / k$ número adimensional de Prandtl.

$=\rho u_{b} D_{h} /(\mu)$, número adimensional de Reynolds.

\section{Símbolos griegos}


$\Gamma, \Psi$

$\mu$

$\mu_{0}, \mu_{\infty}$

$\mu_{g}$

$\phi^{\prime}(n)$

$\phi(n)$

$\phi_{v}$

$\rho$

$\tau$

$\tau_{w}$

$\xi$

$\xi_{a n}, \xi_{\text {exp }}$

$\overline{\bar{\tau}}^{\prime}$

\section{Subíndices}

$\infty$

$b$ funciones desconocidas.

viscosidad en un fluido newtoniano.

viscosidades extremas del fluido Power Law que aparecen ante esfuerzos muy pequeños o muy grandes.

viscosidad generalizada del flujo.

$=f \times R e_{b} /$ cte, función que relaciona factor de fricción de Fanning y el número de Reynolds básico,.

$=\frac{\phi^{\prime}(n)}{\phi^{\prime}(1)}$

disipación viscosa.

densidad del fluido de trabajo.

esfuerzo cortante.

esfuerzo cortante en la pared.

la mitad del producto de $f \times R e$ que tendría un fluido newtoniano en una geometría dada.

valores de $\xi$ para: la geometría de sección transversal anular (valor teórico) y la geometría de tubo con rascador (valor experimental), respectivamente.

matriz de esfuerzos tangenciales.

referentes al flujo desarrollado.

referente a la definición de la viscosidad obtenida a partir del análisis dimensional y a los números adimensionales que utilizan dicha definición. 
DL en geometrías símples de sección de paso constante utiliza la expresión de Delplace-Leuliet para definir el número de Reynolds y la viscosidad efectiva correspondiente.

$e c, c c$ referente a las direcciones de rascado equicorriente y contracorriente.

$g$ referente a la definición de la viscosidad generalizada que incluye la función $\phi(n)$ obtenida mediante correlaciones experimentales, así como a los números adimensionales que utilizan dicha definición.

$i \quad$ referente a un par de imágenes.

ko en tubo liso utiliza la expresión de Kozicky para definir la viscosidad efectiva y el número de Reynolds.

MR definiciones de la viscosidad y el número de Reynolds obtenidos por Metzner y Reed para geometrías de tubo liso.

$N \quad$ referente a un fluido newtoniano.

NN $\quad$ referente a un fluido no newtoniano.

$r, \theta, z \quad$ direcciones del espacio en coordenadas polares.

$S, N, E, O \quad$ referentes a las posiciones al sur, norte, este y oeste de una celda del mallado.

$\theta \quad=\left(T-T_{e j e}\right) k / q^{\prime \prime} D$, temperatura adimensional.

\section{Componentes de la base dimensional}

$\Theta \quad$ temperatura.

L longitud.

M masa.

Q energía transportada en forma de calor.

T tiempo. 
Apéndice 



\section{Apéndice A}

\section{Ensayos de transmisión de calor. Cálculos detallados y resultados adicionales}

En el presente capítulo se incluyen diversos algoritmos o procedimientos de cálculo integrados en el código de procesado de los ensayos de transmisión de calor realizados. Además se presenta el procedimiento de obtención del parámetro $\Delta$ aplicable al tubo con rascador. Por último se deduce la formulación de los criterios R3 y R5 aplicados a las comparaciones planteadas en el Capítulo 9.

\section{A.1. Procedimiento de cálculo de $\bar{T}_{f}\left(z_{i}\right)$ en las secciones de medida.}

En el presente apartado se detalla el cálculo de la temperatura media del fluido en una sección del tubo con rascador, intermedia entre las sondas de temperatura sitadas a la entrada y a la salida del mismo. Dicho procedimiento se utiliza para estimar la temperatura media del fluido en las secciones de termopares. Dichos termopares miden la temperatura de la pared exterior del tubo.

Balance tramo de entrada para obtener $T_{f e}$. El primer paso es obtener las propiedades del fluido, que son función de la temperatura del fluido: $c_{p}$ y $k$ a $T_{E}$. La densidad del fluido se miede mediante el caudalímetro de efecto Coriolis. El hecho de considerar la temperatura 
del fluido igual a $T_{E}$ tendrá una influencia inapreciable en el resultado. A continuación se obtiene $h_{i}$ aplicando correlaciones para flujo en tubos. El valor de $h_{i}$ será alto y no influirá significativamente en el resultado.

$$
\begin{gathered}
h_{i}=\frac{k}{D} \times 1,86 \times\left(\frac{D}{L_{E}} P e\right)^{1 / 3} \\
P e=\frac{\rho D_{h} u_{b} c_{p}}{k}
\end{gathered}
$$

El flujo de calor pasa del fluido al tubo por convección y atraviesa el tubo y después el aislante por conducción.

$$
R_{1}=\frac{1}{R h_{i}} ; \quad R_{2}=\frac{\log \left(R_{t e} / R\right)}{k_{t u}} ; \quad R_{3}=\frac{\log \left(R_{a} / R_{t e}\right)}{k_{a}}
$$

mientras que del aislante al ambiente se transmite por efecto de la convección. Para obtener el coeficiente convectivo $h_{e}$ y por lo tanto $R_{4}$ se utiliza el método iterativo descrito en el Apartado A.3.

$$
\left[h_{e}, R_{4}\right]=\text { ChurchillChu }\left(L_{E}, T_{e}, R_{1}, R_{2}, R_{3}\right)
$$

Una vez conocidos $R_{1}, R_{2}, R_{3}$ y $R_{4}$ se calcula el calor perdido en el primer tramo.

$$
\begin{gathered}
U=\frac{2 \pi}{R_{1}+R_{2}+R_{3}+R_{4}} \\
Q_{P, S E}=U L_{E}\left(T_{e}-T_{a m b}\right)
\end{gathered}
$$

y es inmediato obtener la temperatura media del fluido en la sección de transición entre el primer tramo y el segundo.

$$
\bar{T}_{f e}=T_{e}-\Delta T ; \Delta T=\frac{Q_{p, e}}{c_{p} \dot{m}}
$$


Calor perdido en la sección de ensayo. En la sección calentada existirá un flujo de calor de pérdidas al ambiente. Éste se puede obtener a partir de la temperatura media de la pared exterior del tubo $T_{p e}$, que se obtiene de promediar las medidas de los termpopares $T_{p e, i, j}$.

$$
T_{p e}=\frac{\sum_{j}^{N_{S}} \sum_{i}^{N_{T P}} T_{p e, i, j}}{N_{T P} \times N_{S}}
$$

Las resistencias que se encuentra el flujo de calor de pérdidas a su paso serán el aislante y el paso por convección al aire, que se vuelve a estimar utilizando el método del Apartado A.3. Una vez obtenidas éstas, el cálculo del flujo de calor de pérdidas en la sección de ensayo es inmediato.

$$
\begin{aligned}
& R_{3}=\frac{\log \left(R_{a} / R_{t e}\right)}{k_{a}} \\
& {\left[h_{e}, R_{4}\right]=\text { ChurchillChu }\left(L_{T}, T_{p e}, R_{1}=R_{2}=0, R_{3}\right)} \\
& \dot{q}_{p}=\frac{2 \pi}{R_{3}+R_{4}} \times L_{T}\left(T_{p e}-T_{a m b}\right)
\end{aligned}
$$

Balances de flujos de calor en la sección de ensayo. El calor total generado por efecto Joule en la sección de ensayo $\dot{q}_{T}$ viene dado por la tensión entre bornes y la intensidad que circula por el tubo. Una vez se ha obtenido el flujo de calor de pérdidas,

$$
\begin{gathered}
\dot{q}_{T}=V \times I \\
\dot{q}_{f}=\dot{q}_{T}-\dot{q}_{p} \\
\dot{q}_{f}^{\prime \prime}=\frac{\dot{q}_{f}}{S}
\end{gathered}
$$

Temperatura en la sección de ensayo Conocido el flujo de calor que recibe el fluido $\dot{q}_{f}$, se pueden obtener las temperaturas medias del fluido a la salida del tramo de ensayo $T_{f, s}$ 
y en cada una de las secciones de termopares $\bar{T}_{f}\left(z_{j}\right)$. Para ello es necesario conocer $c_{p}$ del fluido, que, sin cometer mucho error, se calcula para una temperatura $\left(T_{e}+T_{s}\right) / 2$.

$$
\begin{gathered}
T_{f s}=T_{f e}+\frac{\dot{q}_{f}}{S \dot{m} c_{p}} \\
S=\pi D L_{T}
\end{gathered}
$$

Para cada sección de termopares, $j$ :

$$
T_{f}\left(z_{j}\right)=T_{f e}+\left(T_{f s}-T_{f e}\right) \frac{L_{j}}{L_{T}}
$$

Tramo de salida. Comprobación de los cálculos. En el párrafo anterior se ha estimado el valor de $T_{f}\left(z_{j}\right)$ en cada sección. Para corroborar que la estimación es buena se hace uso de la lectura de la temperatura de salida $T_{s}$. A partir de ella, y estimando el flujo de calor perdido en el tramo de salida, se estima el valor de $T_{f s}$ (Ec. A.3) y se compara con el obtenido a partir de $T_{e}$ con la Ec. A.1. El valor de $R_{T}$ en el tramo de salida será el mismo que para el tramo de entrada.

$$
\begin{gathered}
\dot{q}_{p, s}=\frac{2 \pi}{R_{T}} L_{S}\left(T_{s}-T_{a m b}\right) \\
T_{f s}=T_{s}+\frac{\dot{q}_{p, s}}{\dot{m} c_{p}}
\end{gathered}
$$

Las desviaciones obtenidas en los ensayos son pequeñas, con lo que se confirma que el cálculo es bueno. Además, para obtener una estimación más precisa de los valores de $T_{f}\left(z_{j}\right)$ se obtienen también a partir de $T_{s}$ (Ec. A.4) y se promedian con los obtenidos a partir de $T_{e}$ (Ec. A.2).

$$
T_{f}\left(z_{j}\right)=T_{f s}-\left(T_{f s}-T_{f e}\right) \frac{L_{T}-L_{j}}{L_{T}}
$$


(5)
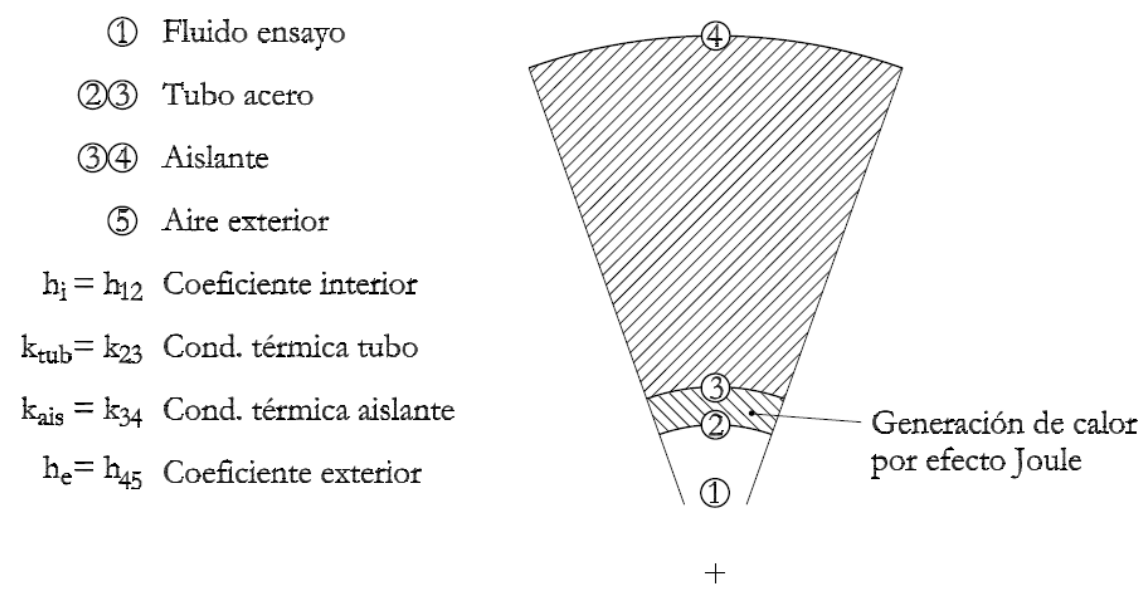

Figura A.1: Esquema del problema de conducción.

\section{A.2. Procedimiento de obtención de la temperatura de pared interior en presencia de un flujo de calor}

En el presente apartado se describe el método para estimar la temperatura de la pared interior del tubo, conocida la temperatura de la pared exterior (medida por los termopares), el flujo de calor y la temperatura del fluido. Este método ha sido descrito por Afshin J. Ghajar y Jae-yong Kim. El problema consiste en resolver el campo de temperaturas en las superficies sólidas de la pared del tubo y del aislante. Las condiciones de contorno del problema son de convección, donde se suponen conocidos los coeficientes de transmisión de calor interno y externo, $h_{i}, h_{e}$ así como la temperatura media del fluido en la sección transversal de medida $T_{f}\left(z_{j}\right)$ y la temperatura exterior $T_{a m b}$. En la pared del tubo se está generando cierto calor por unidad de volumen $q_{v o l}$ por efecto joule.

Se trata de un problema unidireccional estacionario con propiedades físicas constantes. La ecuación a resolver es la de conducción en dirección radial y en régimen estacionario:

$$
k \frac{1}{r} \frac{\partial}{\partial r}\left(r \frac{\partial T}{\partial r}\right)+q_{v o l}=0
$$

La hipótesis de régimen estacionario no merece discusión alguna. En cuanto a la hipótesis de flujo unidireccional se justifica por los siguientes motivos. En primer lugar, aunque existe un gradiente de temperaturas axial debido únicamente al calentamiento del fluido, el flujo de calor axial es completamente despreciable frente al flujo de calor en dirección radial. En 
cuanto al flujo de calor en dirección circunferencial, en flujo laminar existen gradientes de temperatura en esta direccion a causa de la convección natural existente, debida a los efectos de flotación. Sin embargo, el caudal circulante de fluido circulante por el tubo es suficientemente alto como para que la convección natural se pueda despreciar. Se ha comprobado que los resultados obtenidos mediante el modelo unidireccional son muy similares a los obtenidos mediante el modelo bidimensional (diferencias $<2 \%$ ).

Justificado el modelo unidireccional, las condiciones de contorno a aplicar son las siguientes:

$$
\begin{aligned}
r=R & \rightarrow k_{t u} \frac{\partial T}{\partial r}=-h_{i}\left(T_{f}\left(z_{j}\right)-T_{p i}\right) \\
r=R_{t e} & \rightarrow k_{t u} \frac{\partial T}{\partial r}=k_{a} \frac{\partial T}{\partial r} \\
r=R_{a} & \rightarrow k_{a} \frac{\partial T}{\partial r}=-h_{e}\left(T_{p e}-T_{a m b}\right)
\end{aligned}
$$

El calor generado por unidad de volumen se puede calcular a partir del calor total $\dot{q}_{T}$ considerando que el calor se genera uniformemente en el volumenn de la pared metálica del tubo.

$$
q_{v o l}=\frac{\dot{q}_{T}}{L_{T} \pi\left[R_{t e}^{2}-R^{2}\right]}
$$

Discretización de la ecuación diferencial. En la Fig. A.2 se muestra la discretización realizada. El dominio se divide en pequeños volúmenes diferenciales, situando un nodo central en cada volumen. Para cada uno de estos nodos se discretiza la ecuación diferencial planteándose de esta forma una ecuación algebraica para cada uno de los volúmentes en que se divide el dominio.

Adicionalmente se sitúan tres nodos adicionales para plantear las condiciones de contorno en $r=R$, en $r=R_{t u}$ y en $r=R_{a}$.

La ecuación a resolver en cada volumen finito será la siguiente:

$$
k \frac{T_{i+1}-T_{i}}{d_{n}} a_{n}-k \frac{T_{i}-T_{i-1}}{d_{s}} a_{s}+q_{v o l}=0
$$

Donde para el tubo, la conductividad térmica es $k=k_{t u b}$ y el calor generado es $q_{v o l}$ (Ec. A.5). En la región del aislante, la conductividad térmica es $k=k_{a}$ y el calor generado 


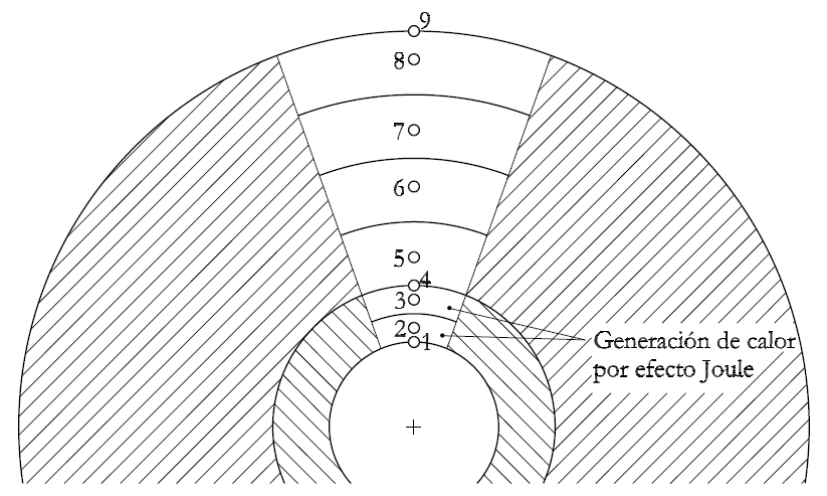

Figura A.2: Volumen finito en el cual se resuelve la ecuación diferencial.

es nulo.

Las condiciones de contorno discretizadas resultan:

$$
\begin{gathered}
r=R \rightarrow k_{t u} \frac{T_{i+1}-T_{i}}{d_{n}}=-h_{i}\left(T_{f}\left(z_{j}\right)-T_{i}\right) \\
r=R_{t e} \rightarrow k_{t u} \frac{T_{i}-T_{i-1}}{d_{s}}=k_{a} \frac{T_{i+1}-T_{i}}{d_{n}} \\
r=R_{a} \rightarrow k_{a} \frac{T_{i}-T_{i-1}}{d_{s}}=-h_{e}\left(T_{i}-T_{a m b}\right)
\end{gathered}
$$

\section{Ejemplo resuelto.}

Datos de entrada del problema:

- Diámetro del tubo, $D=0,018 \mathrm{~m}$

- Espesor del tubo, $R_{t u}=0,019 \mathrm{~m}$ y conductividad térmica del tubo, $k_{t u}=15 \mathrm{~W} / \mathrm{mK}$.

- Espesor del aislante, $R_{a}=0,037 \mathrm{~m}$ y conductividad térmica del aislante, $k_{a}=0,1 \mathrm{~W} / \mathrm{mK}$.

- Distancia entre electrodos $L_{T}=1 \mathrm{~m}$.

- Temperatura del fluido en el punto de medida, $T_{f}\left(z_{j}\right)=50^{\circ} \mathrm{C}$.

- Temperatura ambiente, $T_{a m b}=20^{\circ} \mathrm{C}$.

- Calor total suministrado, $\dot{q}_{T}=3000 \mathrm{~W}$.

- Coeficiente de película interior, $h_{i}=3000 \mathrm{~W} / \mathrm{m}^{2} \mathrm{~K}$ y exterior $h_{i}=10 \mathrm{~W} / \mathrm{m}^{2} \mathrm{~K}$. 


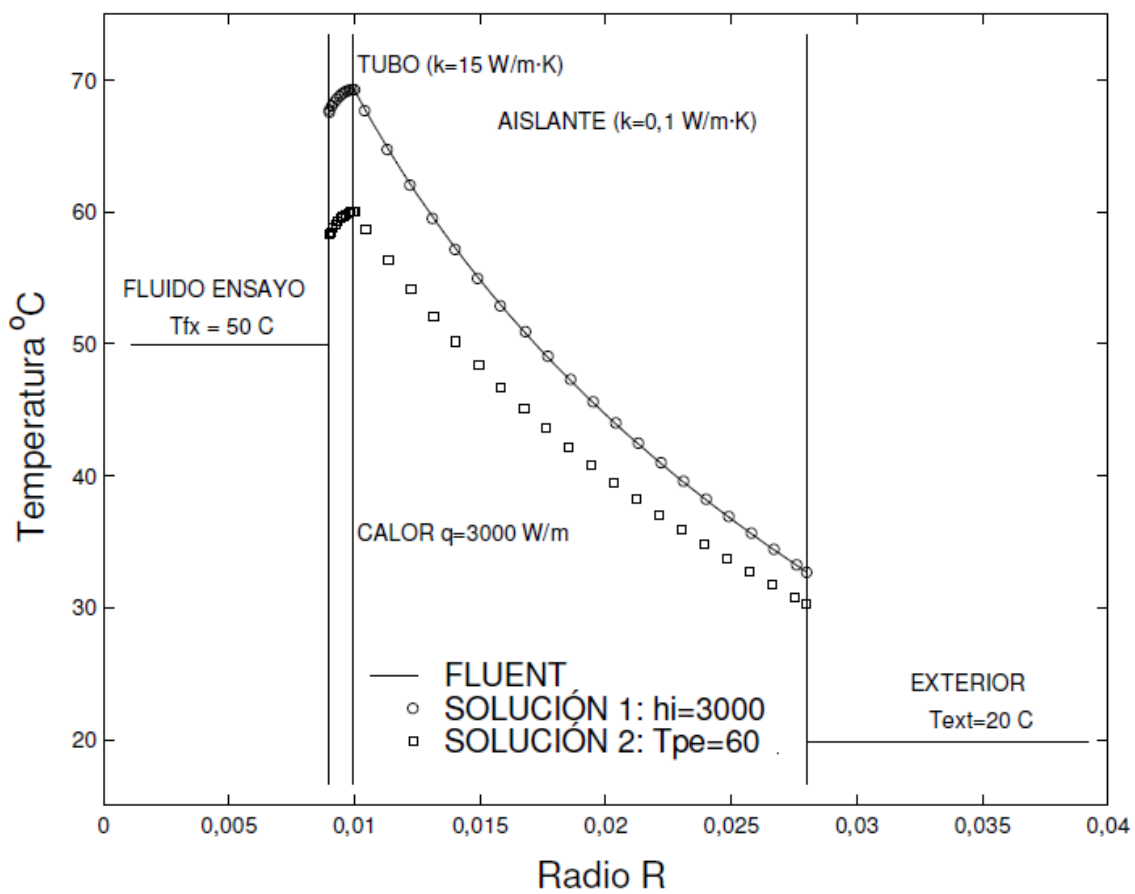

Figura A.3: Campo de temperatura de la región tubo-aislante. Círculos: solución mediante Matlab del problema propuesto con $h_{i}=3000 \mathrm{~W} / \mathrm{m}^{2} \mathrm{~K}$. Línea contínua: solución mediante Fluent. Cuadrados: solución mediante Matlab del problema modificado haciendo $T_{P I}=60^{\circ} \mathrm{C}$.

Este modelo se ha integrado como una subrutina en el programa de postprocesado de resultados experimentales de transmisión de calor. Además se realiza una modificación, ya que en el postproceso de los datos experimentales se conoce la temperatura de la pared exterior $T_{p e}$, siendo el coeficiente de transmisión de calor interior $h_{i}$ una incógnita.

Si en el ejemplo propuesto se impone que la temperatura de la pared exterior sea $T_{p e}=$ $60^{\circ} \mathrm{C}$, se obtiene el campo de temperaturas mostrado mediante cuadros en la Fig. A.3, donde el coeficiente de película interior calculado es $h_{i}=6363 \mathrm{~W} / \mathrm{m}^{2} \mathrm{~K}$. La figura muestra el campo de temperaturas obtenido en un ejemplo resuelto y la verificación del programa mediante Fluent.

\section{A.3. Algoritmo de Churchill y Chu}

El presente algoritmo fue publicado por Churchill y Chu (1975). Se trata de un proceso iterativo que permite obtener el valor del parámetro convectivo $h_{e}$ en el exterior de un tubo aislado. Como parámetros de entrada se necesitan, la temperatura del fluido, la longitud del 
tramo de tubería y los coeficientes resistentes que debe atravesar el flujo de calor.

$$
\left[h_{e}, R_{4}\right]=\text { ChurchillChu }\left(L, T, R_{1}, R_{2}, R_{3}\right)
$$

Además, son necesarias las propiedades del fluido ambiente (aire) a la temperatura medida $T_{a m b}: \alpha_{a}, \nu_{a}, \beta_{a}, P r_{a}=\nu_{a} / \alpha_{a}$.

El algoritmo comienza suponiendo un valor de $h_{e}=10$. Y crea un bucle hasta que la variación de $h_{e}$ sea $<0,001$.

A partir de $h_{e}$ calcula $R_{4}$ y en consecuencia el coeficiente $U$ y el flujo de calor existente.

$$
\begin{gathered}
R_{4}=\frac{1}{R_{A} h_{e}} ; R_{T}=R_{1}+R_{2}+R_{3}+R_{4} \\
U=\frac{2 \pi}{R_{T}} \\
\dot{q}=U * L\left(T-T_{a m b}\right)
\end{gathered}
$$

Definiendo $U_{4}$ se puede calcular la temperatura de la pared exterior sistema (en este caso la del aislante) a partir de la ambiente.

$$
\begin{gathered}
U_{4}=\frac{2 \pi}{R_{4}} \\
T_{p e, a}=T_{a m b}+\frac{\dot{q}}{U_{4} L}
\end{gathered}
$$

Si se define el número de Rayleigh para la convección libre exterior del siguiente modo:

$$
\text { Ray }=\frac{9,81 \beta_{a} \times\left|T_{p e, a}-T_{a m b}\right| \times\left(2 R_{a}\right)^{3}}{\nu_{a}, \alpha_{a}}
$$

Se obtiene finalmente el valor de $h_{e}^{\prime}$ :

$$
h_{e}^{\prime}=\frac{k_{a}}{2 R_{a}} \times \frac{0,6+\left(0,386 R a y^{1 / 6}\right)}{\left[1+\left(0,559 / P r_{a}\right)^{9 / 16}\right]^{8 / 27}}
$$


El valor obtenido para $h_{e}^{\prime}$ se compara con el supuesto al inicio del bucle $h_{e}$ y cuando la diferencia sea mínima termina el algoritmo:

$$
\left|h_{e}-h_{e}^{\prime}\right|<0,001
$$

\section{A.4. Procedimiento de cálculo para la calibración de los termopares}

En el presente apartado se detalla el algoritmo de cálculo utilizado en el procedimiento de calibración de los 48 termopares de contacto distribuidos en 6 secciones en la pared exterior del tubo.

Procedimiento de cálculo de $T_{p e}$ a partir de las medidas de las sondas RTD $T_{e} \mathbf{y}$ $T_{s}$

1. Cálculos intermedios

a) Temperatura media del fluido en la sección: $\bar{T}_{f}=T_{e}+\left(T_{s}-T_{e}\right)\left(L_{E} / L_{T}\right)$

b) Se obtienen las propiedades del aire y el agua a $\bar{T}_{f}: \rho, \mu, c_{p}, k_{a i r}, k_{w}, \operatorname{Pr}_{a}$.

c) Cálculo $\operatorname{Re}$ y $\operatorname{Pr}$ del agua en estas condiciones. $R e_{h}=\rho u_{b} D_{h} / \mu$.

2. Cálculo del número de Nusselt y $h_{i}$ a partir de las correlaciones ya conocidas. $N u_{h}=$ $\operatorname{Pr}^{m 1} *\left(C_{1} \operatorname{Re}_{h}^{m 2}+\frac{C_{2} \omega^{m 3}}{R e_{h}^{m 4}+C_{3}}\right) ; h_{i n t}=N u_{h} \frac{k}{D_{h}}$

3. Cálculo $h_{e}$ mediante el método de Churchill y Chu (1975) detallado en el Apartado A.3.

4. Calor perdido en la prueba

$$
\begin{gathered}
R_{1}=\frac{1}{R h_{i}} ; \quad R_{2}=\frac{\log \left(R_{t u} / R\right)}{k_{t u}} ; \quad R_{3}=\frac{\log \left(R_{a} / R_{t u}\right)}{k_{a}} ; R_{4}=\frac{1}{R_{a} h_{e}} \\
R_{T}=\sum_{i=1}^{4} R_{i}
\end{gathered}
$$




$$
\dot{q}_{p, c}=\frac{2 \pi L_{T}\left(\bar{T}_{f}-T_{a m b}\right)}{R_{T}}
$$

5. Salto de temperaturas fluido-superficie

$$
\begin{aligned}
\Delta T_{f-S} & =\frac{\dot{q}_{p, c}}{2 \pi L_{T}\left(R_{1}+R_{2}\right)} \\
T_{p e} & =\bar{T}_{f}-\Delta T_{f-S}
\end{aligned}
$$

6. La medida de cada termopar $T_{p e, i}$ se debe comparar con el valor de $T_{p e}$ calculado (Ec. A.6).

De este modo, cada termopar tendrá una curva de error asociada que se ajusta por mínimos cuadrados a un polinomio de grado 4. Los coeficientes del ajuste se utilizarán posteriormente para corregir las medidas de los termopares.

\section{A.5. Procedimiento de obtención de $\Delta$ en tubo con eje}

En el presente apartado se busca definir una expresión aproximada para tubo de sección anular o tubo con eje, similar a la definida en la Ec. 9.3 para tubo liso. El objetivo final sería aplicarla al tubo con rascador para cuantificar el efecto de la seudoplasticidad en la transmisión de calor.

\section{A.5.1. Obtención de $\Delta$}

En primer lugar, se desea obtener el valor de $\Delta$ (Ec. 9.1) para la geometría de tubo de sección anular. Tal y como se detalla en el Apartado 3.1, el perfil de velocidades de un fluido no newtoniano en dicha geometría no se puede obtener de forma analítica y se debe recurrir a métodos numéricos de integración o a la simulación numérica del flujo. En este caso se ha utilizado el modelo numérico desarrollado en el Capítulo 3 para obtener la derivada de la velocidad en dirección radial en la pared, $\left(\left.\frac{\partial u}{\partial r}\right|_{r=R}\right)_{n \neq 1}$. Para ello, se ha realizado en primer lugar una etapa de validación del método: se ha obtenido la derivada en dirección radial de la velocidad axial en la pared para un tubo liso, comparándolo posteriormente con la expresión teórica . La simulación se realiza con $N N=100$ celdas en dirección transversal al flujo. 


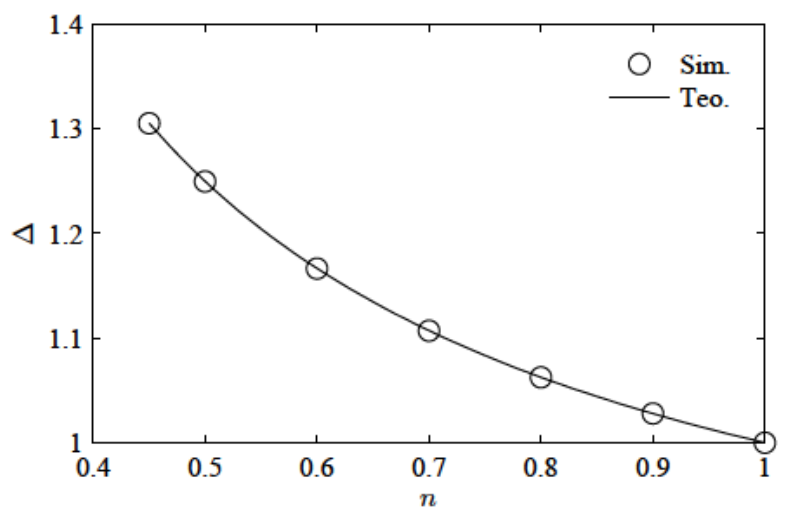

Figura A.4: Expresión de $\Delta$ en tubo liso. Comparación entre el valor teórico y el valor obtenido mediante simulación.

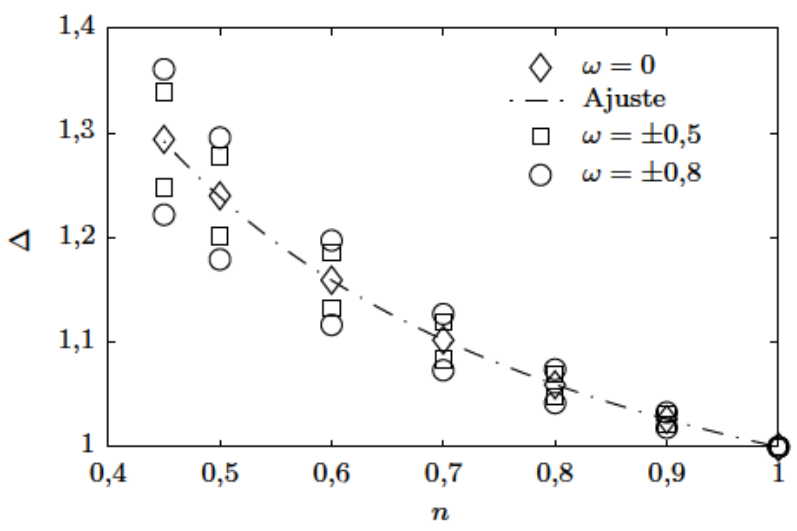

Figura A.5: Expresión de $\Delta$ en tubo con sección transversal anular. Se muestran los valores obtenidos mediante simulación numérica para varias velocidades de rascado, así como la representación de la Ec. A.7.

La comparación representada en la Fig. A.4 arroja desviaciones menores del 0,01\% en la estimación de $\Delta$.

A continuación se realiza la simulación en tubo de sección anular con $\alpha=5 / 18$ (relación de diámetros en la instalación experimental), estando el eje estático. Los resultados se ajustan correctamente a la siguiente ecuación con $a=7,532 \pm 0,001$ (intervalo de confianza del $95 \%$ )

$$
\Delta=\frac{24 n+a}{(24+a) n}
$$

Además se ha medio el efecto que provoca el movimiento del eje en $\Delta$. Se han realizado 


\begin{tabular}{cccccc}
\hline$n$ & $\Delta$ & $N u_{\infty}$ & $N u_{\infty} / N u_{\infty, n=1}$ & $\Delta^{1 / 9}$ & Error \\
\hline 0,45 & 1,294 & 5,068 & 1,031 & 1,029 & $-0,15 \%$ \\
0,50 & 1,240 & 5,039 & 1,025 & 1,024 & $-0,04 \%$ \\
0,60 & 1,159 & 4,995 & 1,016 & 1,017 & $0,09 \%$ \\
0,70 & 1,102 & 4,965 & 1,010 & 1,011 & $0,12 \%$ \\
0,80 & 1,059 & 4,944 & 1,005 & 1,006 & $0,11 \%$ \\
0,90 & 1,026 & 4,929 & 1,002 & 1,003 & $0,06 \%$ \\
1,00 & 1,000 & 4,918 & 1,000 & 1,000 & $0,00 \%$ \\
\hline
\end{tabular}

Cuadro A.1: Obtención del exponente de $\Delta$ que replica correctamente la gráfica de $N u_{\infty} / N u_{\infty, n=1}$

simulaciones similares para valores de $|\omega|=\left|v_{s}\right| / u_{b}<1$. Los resultados muestran diferencias entre los ciclos de rascado, aunque en promedio, el valor de $\Delta$ es prácticamente el mismo que con el eje estático. Se debe notar que al superar $|\omega|=1$, el valor promedio de $\Delta$ varía.

\section{A.5.2. Exponente de $\Delta$}

De forma análoga a 9.1.1, se desea conocer para tubo anular, la relación entre el número de Nusselt trabajando con un fluido seudoplástico y el número de Nusselt si se trabaja con uno newtoniano, $N u_{\infty} / N u_{\infty, n=1}$. En la Tabla A.1 se representa el valor de $N u_{\infty}$ obtenido para fluidos seudoplásticos en dicha geometría, así como la relación $N u_{\infty} / N u_{\infty, n=1}$. Los resultados de esta última relación se ajustan a un valor de $\Delta^{1 / 9}$ con un error del $0,15 \%$, estando $\Delta$ definido por la Ec. A.7 definida con anterioridad.

\section{A.6. Definición de criterios R1, R3 y R5 para las geo- metrías estudiadas}

En el presente apartado se detalla la formulación de los criterios R1, R3 y R5. Estos criterios se utilizan para comparar las prestaciones del del tubo con rascador frente a las prestaciones de tubos más simples:

- Tubo liso: de diámetro igual al diámetro hidráulico del tubo con rascador: $D_{\text {Tubo }}=$ $D_{h, \text { rascador }}$ que se denotará simplemente con $D_{h}$.

- Tubo con eje (sin rascadores), de diámetro hidráulico $D_{h}=D-d$. Siendo $D$ y $d$ las dimensiones del tubo y del eje del rascador. 
Los criterios de comparación utilizados son los criterios R1, R3 y R5 definidos por Bergles (1974).

- Criterio R1: Determina el aumento de la transferencia de calor para el mismo caudal circulante y superficie de intercambio. Formulando dichas condiciones de comparación:

- Condición 1: $Q_{s} / Q_{x}=1$

- Condición 2: $\dot{A}_{s} / \dot{A}_{x}=1$

- Criterio R3: Determina el aumento de la transferencia de calor para la misma potencia consumida y superficie de intercambio. Formulando dichas condiciones de comparación:

- Condición 1: $\dot{W}_{s} / \dot{W}_{x}=1$

- Condición 2: $\dot{A}_{s} / \dot{A}_{x}=1$

- Criterio R5: Determina la reducción del área de intercambio para la misma potencia consumida y calor total intercambiado:

- Condición 1: $\dot{W}_{s} / \dot{W}_{x}=1$

- Condición 2: $q_{s} / q_{x}=1$.

donde los subíndices indican: $s$ para el dispositivo con rascador que se desea evaluar y $x$ para la geometría de referencia considerada, ya sea ésta un tubo liso, un tubo con eje, o el propio rascador en condiciones diferentes.

En todos los casos estudiados, el flujo equivalente en la geometría de referencia es laminar y por lo tanto la caída de presión se puede obtener de forma analítica para el tubo liso y para el tubo con eje (Delplace y Leuliet, 1995; Kozicki et al., 1966), que expresadas de forma general queda:

$$
f \times R e_{D L}=2 \xi
$$

donde el valor de $\xi$ depende de la geometría utilizada: $\xi=8$ en tubo liso y $\xi=11,69$ para la geometría de tubo con eje utilizada $(\alpha=5 / 18)$. Para el resto del apartado se utilizará la generalización de Delplace y Leuliet (1995) (Ec. 5.15) para el tubo con eje y el tubo liso con los valores indicados de $\xi$ en cada caso. De forma análoga, para el tubo con rascador insertado se utiliza el método de generalización desarrollado en la presente investigación (Ec. 5.22). 
En los párrafos a continuación se plantea la primera condición correspondiente a cada criterio de mejora de forma general, de modo que en sucesivos apartados se particulariza para cada una de las comparativas realizadas.

Planteamiento de la "Condición 1" de R1 Dicha condición establece la igualdad de caudal circulante en las situaciones comparadas, que implica

$$
\frac{u_{b, x}}{u_{b, s}}=\frac{S_{x}}{S_{s}}
$$

siendo

$$
\frac{R e_{x}}{R e_{s}}=\left(\frac{u_{b, x}}{u_{b, s}}\right)^{2-n} \frac{\phi_{s}(n)}{\phi_{x}(n)}
$$

Planteamiento de la "Condición 1" común a R3 y R5 La "condición 1" establece igualdad de consumo de potencia entre las situaciones comparadas. Es decir, que se deben comparar situaciones en las que en el tubo con rascador se produzca el mismo consumo energético que en la geometría de referencia:

$$
\dot{W}_{x}=\dot{W}_{s}
$$

En ambos casos se produce un consumo de potencia asociado al bombeo del fluido,

$$
\dot{W}_{b}=Q \Delta p=u_{b} S \times \Delta p=f S N L 2 \rho u_{b}^{3} / D_{h}
$$

donde $\Delta p$ se ha despejado de la definición del número de Fanning y se ha sustituido. $S$ es la sección transversal al flujo, $N$ y $L$ son el número y longitud de los tubos respectivamente y $D_{h}$ es el diámetro hidráulico de la geometría.

Además de este consumo de potencia, habría que considerar la potencia de accionamiento del rascador, siempre que no se encuentre estático. La potencia de accionamiento se ha medido en cada uno de los experimentos realizados (véase Capítulo 8). Dichas medidas se han realizado en las condiciones de ensayo, pero obviamente la potencia de accionamiento puede variar si lo hace la longitud del tubo o el número de tubos. Para tener este factor en cuenta se ha considerado que la potencia de accionamiento es proporcional al número de tubos y a la longitud de estos, tomando como longitud de referencia la que tiene actualmente el tramo de tubo rascado $L_{r e f}=2,5 \mathrm{~m}$. Se denotará con $P_{A}$ a la medida experimental de potencia de accionamiento, que deberá ser corregida en función de $L_{s}$ y $N_{s}$. Así, la "condición 
2" queda expresada del siguiente modo

$$
f_{x} S_{x} N_{x} L_{x} 2 \rho u_{b, x}^{3} / D_{h}=f_{s} S_{s} N_{s} L_{s} 2 \rho u_{b, s}^{3} / D_{h}+P_{A} N_{s} \frac{L_{s}}{L_{r e f}}
$$

donde el término de la izquierda es $\dot{W}_{x}$ y a la derecha de la ecuación quedan las contribuciones de la potencia de bombeo y la potencia de accionamiento al consumo de potencia en el tubo con rascador $\dot{W}_{s}$.

dividiendo por $2 \rho$ :

$$
f_{x} S_{x} N_{x} L_{x} u_{b, x}^{3}=f_{s} S_{s} N_{s} L_{s} u_{b, s}^{3}+P_{A} N_{s} \frac{L_{s}}{L_{r e f}} \frac{D_{h}}{2 \rho}
$$

despejando $u_{b}$ de las definiciones del Reynolds generalizadas para cada geometría $u_{b}=$ $\left(\frac{R e_{g} m \phi(n)}{\rho D_{h}^{n}}\right)^{1 /(2-n)}$, se obtiene

$$
\begin{gathered}
f_{x} S_{x} N_{x} L_{x}\left(\frac{R e_{g, x} m \phi_{x}(n)}{\rho D_{h}^{n}}\right)^{3 /(2-n)}=f_{s} S_{s} N_{s} L_{s}\left(\frac{R e_{g, s} m \phi_{s}(n)}{\rho D_{h}^{n}}\right)^{3 /(2-n)}+P_{A} N_{s} \frac{L_{s}}{L_{r e f}} \times \frac{D_{h}}{2 \rho} \\
R e_{g, x}^{\frac{3}{2-n}}=\left(\operatorname{Re}_{g, s}^{\frac{3}{2-n}}\left(\frac{\phi_{s}(n)}{\phi_{x}(n)}\right)^{3 /(2-n)}+P_{A} \times \frac{D_{h}}{2 \rho f_{s} S_{s} L_{r e f}}\left(\frac{\rho D_{h}^{n}}{m \phi_{x}(n)}\right)^{3 /(2-n)}\right) \frac{f_{s}}{f_{x}} \times \frac{S_{s} N_{s} L_{s}}{S_{x} N_{x} L_{x}} \\
\frac{\operatorname{Re}_{g, x}}{R e_{g, s}}=\left[\left(\left(\frac{\phi_{s}(n)}{\phi_{x}(n)}\right)^{3 /(2-n)}+P_{A} \times \frac{D_{h}}{2 \rho f_{s} S_{s} L_{r e f} R e_{g, s}^{\frac{3}{2-n}}}\left(\frac{\rho D_{h}^{n}}{m \phi_{x}(n)}\right)^{3 /(2-n)}\right) \frac{f_{s}}{f_{x}} \times \frac{S_{s} N_{s} L_{s}}{S_{x} N_{x} L_{x}}\right]^{\frac{2-n}{3}}
\end{gathered}
$$

donde se denotará

$$
X_{x}=\left(\left(\frac{\phi_{s}(n)}{\phi_{x}(n)}\right)^{3 /(2-n)}+P_{A} \times \frac{D_{h}}{2 \rho f_{s} S_{s} L_{r e f}}\left(\frac{\rho D_{h}^{n}}{m \phi_{x}(n) R e_{g, s}}\right)^{3 /(2-n)}\right)
$$

y a la relación de dimensiones entre el rascador y el tubo de referencia

$$
F_{D}=\frac{S_{s} N_{s} L_{s}}{S_{x} N_{x} L_{x}}
$$

De este modo, la relación entre $R e_{g, x}$ y $R e_{g, s}$ queda 


$$
\frac{R e_{g, x}}{R e_{g, s}}=\left[X_{x} \times \frac{f_{s}}{f_{x}} \times F_{D}\right]^{\frac{2-n}{3}}
$$

que también se puede expresar como

$$
\frac{u_{b, x}}{u_{b, s}}=\frac{P e_{x}}{P e_{s}}=\left(\frac{\phi_{x}(n)}{\phi_{s}(n)}\right)^{1 /(2-n)}\left[X_{x} \times \frac{f_{s}}{f_{x}} \times F_{D}\right]^{1 / 3}
$$

$F_{D}$ depende del criterio de comparación formulado y de la geometría de referencia, así debe ser obtenido para cada criterio (R3 y R5) y cada geometría de referencia, lo cual se lleva a cabo en apartados sucesivos.

Por otro lado, resulta obvio que si el rascador no se acciona (régimen estático) la potencia de accionamiento es nula y la Ec. A.10 se simplifica.

Además, en la relación $f_{s} / f_{x}, f_{s}$ se ha medido en los ensayos y $f_{x}$ dependerá de si el flujo es laminar o turbulento. Como en todos los casos estudiados, resulta ser laminar:

- Flujo laminar en tubo liso: $f_{x}=16 / R e_{g, x}$.

- Flujo laminar en tubo con eje: $f_{x}=2 \xi_{a n} / R e_{g, x}$.

\section{A.6.1. Rascador frente a tubo con eje}

En este caso $S_{x}=S_{s}=S$ y por lo tanto para los criterios R3 y R 5

$$
F_{D}=\frac{N_{s} L_{s}}{N_{x} L_{x}}
$$

\section{A.6.1.1. Criterio R1}

El criterio R1, se calcula como

$$
R_{1}=\left(\frac{N u_{s}}{N u_{a n}}\right)
$$

Al tener la misma superficie de paso, de A.8 y A.9 se deduce que:

$$
\frac{u_{b, x}}{u_{b, s}}=1
$$




$$
\frac{R e_{g, x}}{R e_{g, s}}=\frac{\phi_{s}(n)}{\phi_{x}(n)}
$$

\section{A.6.1.2. Criterio R3}

El criterio R3, se calcula como

$$
R_{3}=\left(\frac{N u_{s}}{N u_{a n}}\right)
$$

implica la igualdad de superficies de intercambio como "condición 2", $A_{x}=A_{s}$ lo que lleva a $N_{x} L_{x}=N_{s} L_{s}$. Aplicando esta condición en la condición 1, previamente desarrollada (Ec. A.12):

$$
F_{D}=1
$$

\section{A.6.1.3. Criterio R5}

El criterio R5 se calcula como

$$
R_{5}=\left(\frac{A_{s}}{A_{x}}\right)=\left(\frac{N_{s} L_{s}}{N_{x} L_{x}}\right)=\left(\frac{N u_{x}}{N u_{s}}\right)
$$

donde la expresión más a la derecha se deduce a continuación de la "condición 2" del propio criterio (Ec. A.15). Dicha condición establece que $\dot{q}_{x}=\dot{q}_{s}$, donde si despreciamos la disipación provocada por el rascador,

$$
h_{x} N_{x} L_{x} \pi D \Delta T=h_{s} N_{s} L_{s} \pi D \Delta T
$$

Además, si sustituimos

$$
N u=\frac{h_{i} D_{h}}{k}
$$

en

$$
\frac{N u_{x} k}{D_{h}} N_{x} L_{x} \pi D \Delta T=\frac{N u_{s} k}{D_{h}} N_{s} L_{s} \pi D \Delta T
$$

y cancelando lo que es igual en ambos casos, se obtiene: 


$$
\begin{gathered}
N u_{x} N_{x} L_{x}=N u_{s} N_{s} L_{s} \\
\frac{N_{s} L_{s}}{N_{x} L_{x}}=\frac{N u_{x}}{N u_{s}}
\end{gathered}
$$

De la condición 1 (Ec. A.12)

$$
F_{D}=\frac{N u_{x}}{N u_{s}}
$$

\section{A.6.2. Rascador frente a tubo liso de mismo diámetro hidráulico}

En este caso $S_{x} \neq S_{s}$ y por lo tanto, la "condición 1", que establece la igualdad de consumo de potencia en ambas geometrías en los criterios R3 y R5, viene dada por la Ec. A.11 con

$$
F_{D}=\frac{S_{s} N_{s} L_{s}}{S_{x} N_{x} L_{x}}
$$

además entre ambas geometrías se ha establecido la condición de que tengan el mismo diámetro hidráulico $D_{h, s}=D_{h, x}$, con lo que el área transversal diferente $S_{x} \neq S_{s}$, existiendo la siguiente relación entre ambas:

$$
\begin{aligned}
S_{x} & =\frac{\pi D_{h}^{2}}{4}=\frac{\pi(D-d)^{2}}{4} \\
S_{s} & =\frac{\pi\left(D^{2}-d^{2}\right)}{4}=\frac{\pi(D-d)(D+d)}{4} \\
\frac{S_{x}}{S_{s}} & =\frac{(D-d)}{(D+d)}=\frac{D_{h}}{(D+d)}
\end{aligned}
$$

de modo que la "condición 1" (común a R3 y R5) en la Ec. A.11, $F_{D}$ se puede escribir del siguiente modo

$$
F_{D}=\frac{N_{s} L_{s}(D+d)}{N_{x} L_{x} D_{h}}
$$

En los siguientes apartados se calcula $F_{D}$ aplicando la "condición 2" para los criterios R3 y R5. 


\section{A.6.2.1. Criterio R1}

En este caso, para el criterio R1

$$
R_{1}=\left(\frac{N u_{s}}{N u_{x}}\right)
$$

la relación entre las superficies de paso dada por la Ec. A.17 lleva a las siguientes relaciones:

$$
\begin{gathered}
\frac{u_{b, x}}{u_{b, s}}=\frac{D+d}{D_{h}} \\
\frac{R e_{g, x}}{R e_{g, s}}=\left(\frac{D+d}{D_{h}}\right)^{2-n} \frac{\phi_{s}(n)}{\phi_{x}(n)}
\end{gathered}
$$

\section{A.6.2.2. Criterio R3}

El criterio R3, calculado como

$$
R_{3}=\left(\frac{N u_{s}}{N u_{x}}\right)
$$

establece como "condición 2" la igualdad de superficie de intercambio: $A_{x}=A_{s}$

$$
\begin{aligned}
\left(\pi D_{h} L N\right)_{x} & =(\pi D L N)_{s} \\
\frac{L_{x} N_{x}}{L_{s} N_{s}} & =\frac{D}{D_{h}}
\end{aligned}
$$

Aplicando la "condición 2" en la "condición 1" (Ec. A.11):

$$
F_{D}=\frac{D_{h}(D+d)}{D D_{h}}=(1+\alpha)
$$

siendo $\alpha=d / D$. 


\section{A.6.2.3. Criterio R5}

El criterio R5 se calcula como

$$
R_{5}=\left(\frac{A_{s}}{A_{x}}\right)=\left(\frac{N_{s} L_{s}}{N_{x} L_{x}}\right)=\left(\frac{D_{h} N u_{x}}{D N u_{s}}\right)
$$

donde la expresión más a la derecha se deduce a continuación de la "condición 2" planteada para el presente criterio (Ec. A.20). Dicha condición establece que $\dot{q}_{x}=\dot{q}_{s}$, donde se desprecia la disipación provocada por el rascador y siguiendo un procedimiento equivalente al del Apartado A.6.1.3,

$$
\frac{N u_{x} k}{D} N_{x} L_{x} \pi D \Delta T=\frac{N u_{s} k}{D_{h}} N_{s} L_{s} \pi D \Delta T
$$

simplificando a ambos lados,

$$
\begin{aligned}
N u_{x} N_{x} L_{x} & =\frac{D}{D_{h}} N u_{s} N_{s} L_{s} \\
\frac{N_{s} L_{s}}{N_{x} L_{x}} & =\frac{D_{h} N u_{x}}{D N u_{s}}
\end{aligned}
$$

Aplicando esta relación a la condición 1 (Ec. A.18):

$$
F_{D}=(1+\alpha) \frac{N u_{x}}{N u_{s}}
$$

\section{A.6.3. Rascador en régimen dinámico frente a estático}

En el presente apartado se deduce la formulación de los criterios R1, R3 y R5 al comparar las siguientes situaciones:

- Situación evaluada: tubo con el rascador en régimen dinámico (subíndice $s$ ).

- Situación de referencia: tubo con el rascador estático (subíndice $x$ ).

En este caso la superficie transversal en ambas situaciones es la misma,

$$
S_{s}=S_{x}
$$

Además la definición del número de Reynolds generalizada es idéntica y por tanto

$$
\phi_{s}(n)=\phi_{x}(n)
$$




\section{A.6.3.1. Criterio R1}

En este caso, para el criterio R1

$$
R_{1}=\left(\frac{N u_{s}}{N u_{x}}\right)
$$

haciendo uso de la Ec. A.22 y la Ec. A.23, se obtiene:

$$
\frac{u_{b, x}}{u_{b, s}}=\frac{R e_{g, x}}{R e_{g, s}}=1
$$

\section{A.6.3.2. Criterio R3}

El criterio R3, calculado como

$$
R_{3}=\left(\frac{N u_{s}}{N u_{x}}\right)
$$

establece como "condición 2" la igualdad de superficie de intercambio: $A_{x}=A_{s}$. Aplicala en la "condición 1" (Ec. A.11):

$$
F_{D}=1
$$

con $\phi_{s}(n) / \phi_{x}(n)=1$ en la definición de $X_{x}$

\section{A.6.3.3. Criterio R5}

El criterio R5 se calcula como

$$
R_{5}=\left(\frac{A_{s}}{A_{x}}\right)=\left(\frac{N_{s} L_{s}}{N_{x} L_{x}}\right)=\left(\frac{N u_{x}}{N u_{s}}\right)
$$

donde la expresión más a la derecha se deduce a continuación de la "condición 2" planteada para el presente criterio (Ec. A.25). Dicha condición establece que $\dot{q}_{x}=\dot{q}_{s}$, donde se desprecia la disipación provocada por el rascador y siguiendo un procedimiento equivalente al del Apartado A.6.1.3,

$$
\frac{N u_{x} k}{D} N_{x} L_{x} \pi D \Delta T=\frac{N u_{s} k}{D} N_{s} L_{s} \pi D \Delta T
$$

simplificando a ambos lados,

$$
N u_{x} N_{x} L_{x}=N u_{s} N_{s} L_{s}
$$




$$
\frac{N_{s} L_{s}}{N_{x} L_{x}}=\frac{N u_{x}}{N u_{s}}
$$

Aplicando esta relación a la condición 1 (Ec. A.18):

$$
F_{D}=\frac{N u_{x}}{N u_{s}}
$$




\section{Apéndice B}

\section{Modelo numérico. Discretización.}

En el presente capítulo se incluye el proceso de discretización de las ecuaciones de los problemas fluidomecánico y térmico, así como la solución analítica del problema térmico del flujo desarrollado en un tubo liso.

\section{B.1. Discretización de las ecuaciones}

Discretización de la ecuación de cantidad de movimiento. A continuación se detalla el método de discretización de la ecuación de cantidad de movimiento

$$
\frac{\partial p}{\partial z}=\frac{1}{r} \frac{\partial}{\partial r}\left(r m\left(\frac{\partial u_{z}}{\partial r}\right)^{n}\right)
$$

El método de volúmenes finitos implica escribir dicha ecuación en forma integral para un volumen de control:

$$
0=\int_{V_{c}}\left(-\frac{\partial p}{\partial z}\right) d V+\int_{V_{c}} \frac{1}{r} \frac{\partial}{\partial r}\left(r m\left(\frac{\partial u_{z}}{\partial r}\right)^{n}\right) d V
$$

donde mediante el teorema de la divergencia, la segunda integral se transforma en una de superficie, que para los volúmenes finitos considerados, atraviesa cuatro superficies. Además al ser el flujo completamente desarrollado, no hay flujos netos a través de las superficies oeste (1) y este (3):

$$
\int_{V_{c}} \frac{1}{r} \frac{\partial}{\partial r}\left(r m\left(\frac{\partial u_{z}}{\partial r}\right)^{n}\right) d V=\int_{V_{c}} \nabla\left(m\left(\frac{\partial u_{z}}{\partial r}\right)^{n}\right) d V=\int_{S_{c}} m\left(\frac{\partial u_{z}}{\partial r}\right)^{n} \vec{n} d S=
$$




$$
\begin{aligned}
=\int_{S_{O}} m\left(\frac{\partial u_{z}}{\partial r}\right)^{n} \vec{n} d S_{O}+\int_{S_{N}} m\left(\frac{\partial u_{z}}{\partial r}\right)^{n} \vec{n} d S_{N} & +\int_{S_{E}} m\left(\frac{\partial u_{z}}{\partial r}\right)^{n} \vec{n} d S_{E}+\int_{S_{S}} m\left(\frac{\partial u_{z}}{\partial r}\right)^{n} \vec{n} d S_{S} \\
& =\int_{S_{N}} m\left(\frac{\partial u_{z}}{\partial r}\right)^{n} \vec{n} d S_{N}+\int_{S_{S}} m\left(\frac{\partial u_{z}}{\partial r}\right)^{n} \vec{n} d S_{S}
\end{aligned}
$$

donde los diferenciales de las superficies inferior y superior son $d S_{N}=d S_{S}=d z . r . d \theta$, y por tanto:

$$
\begin{array}{r}
\int_{V_{c}} \frac{1}{r} \frac{\partial}{\partial r}\left(r m\left(\frac{\partial u_{z}}{\partial r}\right)^{n}\right) d V=-m\left(\frac{d u_{z}}{d r}\right)_{j-1 / 2}^{n} S_{S}+m\left(\frac{d u_{z}}{d r}\right)_{j+1 / 2}^{n} S_{N} \\
\text { discretizada: }:-m\left(\frac{u_{j}-u_{j-1}}{\Delta r}\right)^{n} S_{S}+m\left(\frac{u_{j+1}-u_{j}}{\Delta r}\right)^{n} S_{N}
\end{array}
$$

Por otro lado, la primera integral sería simplemente:

$$
\int_{V_{c}}\left(-\frac{\partial p}{\partial z}\right) d V=\left(-\frac{\partial p}{\partial z}\right) V=p_{L} V
$$

de modo que la ecuación de cantidad de movimiento discretizada queda

$$
\begin{gathered}
0=p_{L} V-m\left(\frac{u_{j}-u_{j-1}}{\Delta r_{S}}\right)^{n} S_{S}+m\left(\frac{u_{j+1}-u_{j}}{\Delta r_{N}}\right)^{n} S_{N} \\
0=p_{L} V-m\left(\frac{u_{j}-u_{j-1}}{\Delta r_{S}}\right)^{n-1}\left(\frac{u_{j}-u_{j-1}}{\Delta r_{S}}\right) S_{S}+m\left(\frac{u_{j+1}-u_{j}}{\Delta r_{N}}\right)^{n-1}\left(\frac{u_{j+1}-u_{j}}{\Delta r_{N}}\right) S_{N}
\end{gathered}
$$

donde si se define la viscosidad en las caras norte y sur de cada volumen finito del siguiente modo: $\mu_{S}=m\left(\frac{u_{j}-u_{j-1}}{\Delta r_{S}}\right)^{n-1}$ y $\mu_{N}=m\left(\frac{u_{j+1}-u_{j}}{\Delta r_{N}}\right)^{n-1}$, queda:

$$
0=p_{L} V-\mu_{S}\left(\frac{u_{j}-u_{j-1}}{\Delta r_{S}}\right) S_{S}+\mu_{N}\left(\frac{u_{j+1}-u_{j}}{\Delta r_{N}}\right) S_{N}
$$




\section{B.2. Importancia relativa de los términos de la ecua- ción de la energía}

Se desea evaluar la importancia del término de disipación viscosa en la ecuación de la

energía: $m\left(\partial u_{z} / \partial r\right)^{n+1}$. Para ello se realiza un estudio del orden de magnitud de cada uno de los términos para los valores más significativos de las distintas variables en el rango de ensayos realizados. Los distintos términos de la ecuación se cuantificarán utilizando las variables significativas del problema.

1. Término convectivo (T1):

$$
\rho c_{p} u_{z} \frac{\partial T}{\partial z} \sim \rho c_{p} u_{b} \frac{\Delta T}{L}
$$

2. Término conducción en dirección $r$ (T2):

$$
\frac{1}{r} \frac{\partial}{\partial r}\left(r k \frac{\partial T}{\partial r}\right) \sim k \times \frac{\Delta T}{\left(R-R_{e j e}\right)^{2}}
$$

3. Término conducción en dirección $z$ (T3):

$$
\frac{\partial}{\partial z}\left(k \frac{\partial T}{\partial z}\right) \sim k \times \frac{\Delta T}{L} \frac{1}{P}
$$

4. Término disipación viscosa (T4):

$$
m\left(\frac{\partial u_{z}}{\partial r}\right)^{n+1} \sim m\left(\frac{u_{b}}{R-R_{e j e}}\right)^{n+1}
$$

El caso en el cual la disipación viscosa será más importante vendrá dado para el fluido más viscoso. Por lo tanto se toman las propiedades de dicho fluido $m=4 \mathrm{~Pa} . \mathrm{s}^{n}, n=0,5$, $\rho=1000 \mathrm{~kg} / \mathrm{m}^{3}, c_{p}=4180 \mathrm{~J} / \mathrm{kgK}$ y $k=0,58 \mathrm{~W} / \mathrm{mK}$. En cuanto a los saltos térmicos existentes en los ensayos, estos se diseñan para que la diferencia térmica entre la pared y la temperatura media del fluido se encuentre en el rango $15-25^{\circ} \mathrm{C}$. Además se utiliza un valor de incremento térmico por metro de unos $8^{\circ} \mathrm{C} / \mathrm{m}$, aunque esto dependerá de muchos factores y simplemente es un valor orientativo.

En la Fig. B.1 se muestra la variación de los diferentes términos de la ecuación de la energía al variar el caudal circulante en los rangos ensayados. Tal y como se puede observar: el término 


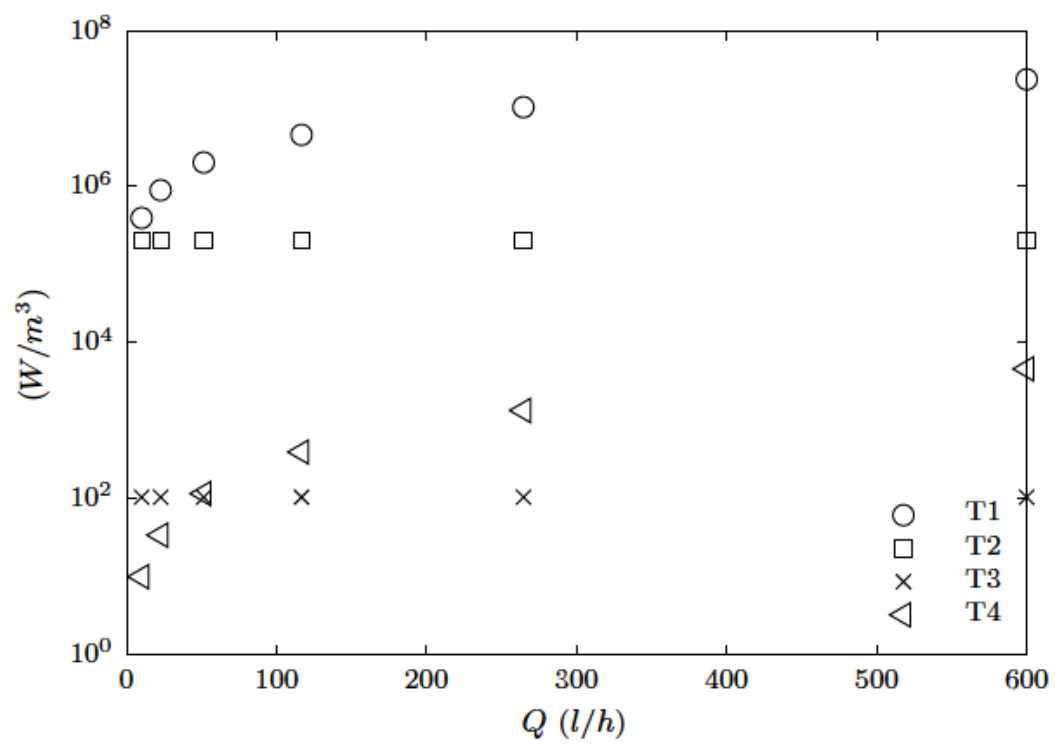

Figura B.1: Importancia de los diferentes términos en la ecuación de la energía.

convectivo es más importante al aumentar el caudal y el término de conducción en dirección radial se mantiene constante (por considerar constante la diferencia de temperaturas entre la pared y el fluido). Estos dos términos son los más importantes, mientras que el término de conducción en dirección axial dependerá del flujo de calor aportado, pero será de varios órdenes de magnitud inferior y por lo tanto puede despreciarse. Por último el término de disipación viscosa aumenta su importancia con el caudal y puede ser más significativo que el término de conducción axial en este tipo de fluidos. No obstante sigue teniendo una influencia baja.

De hecho , la solución para tubo liso de los efectos de la disipación viscosa se puede obtener analíticamente, según muestran Dehkordi y Mohammadi 2009, y viene dada por la siguiente expresión:

$$
\theta=\left(\frac{1+3 n}{n}\right)^{n}\left[8 z_{a}-0,5+0,5 *\left(\frac{1+3 n}{1+n}\right)\left(r^{*}\right)^{2}-\left(\frac{n}{1+n}\right)\left(r^{*}\right)^{1 /(n+3)}\right]
$$

donde $\theta$ es la temperatura adimensional definida como

$$
\theta=\frac{\left(T-T_{0}\right) k R^{n-1}}{m u_{b}^{n+1}}
$$




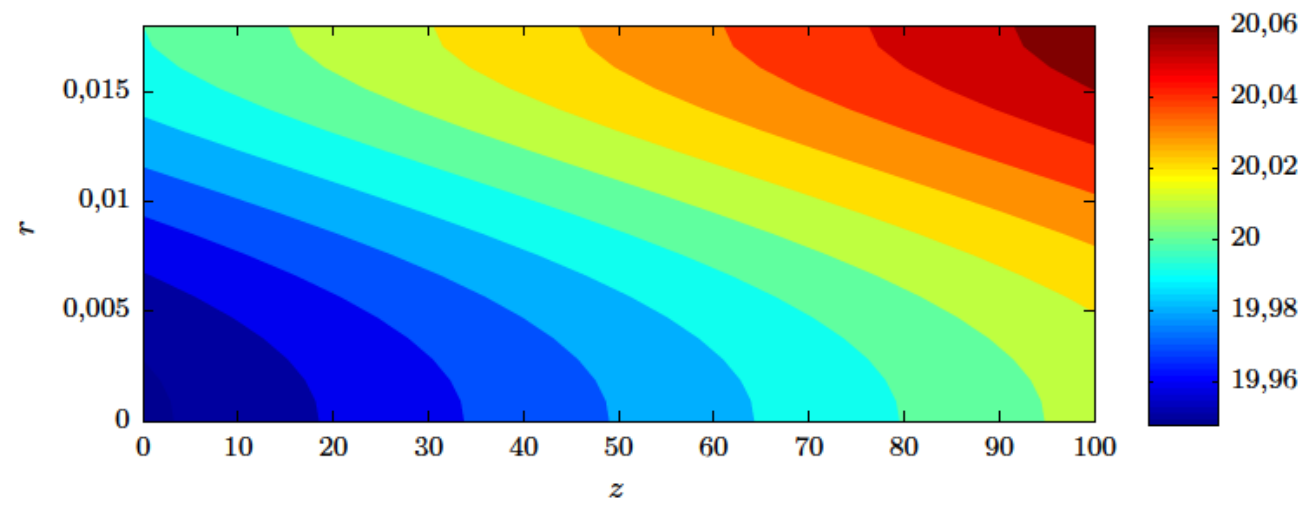

Figura B.2: Influencia de la disipación viscosa en el campo térmico en un tubo liso de 100m de longitud según la expresión de Molaei Dehkordi y Asghar Mohammadi (2008). Las variables consideradas son: $n=0,5 ; m=4$ Pa.s ${ }^{\mathrm{n}} ; Q=500 \mathrm{l} / \mathrm{h} ; T_{0}=20^{\circ} \mathrm{C} ; R=0,018 \mathrm{~m} ; L=100 \mathrm{~m}$.

$$
r^{*}=r / R ; \quad z^{*}=\frac{z \alpha}{4 u_{b} R^{2}}
$$

En la Fig. B.2 se muestra la influencia de la disipación viscosa en el campo térmico en un tubo liso de $100 \mathrm{~m}$. En ella se observan diferencias de $0,1^{\circ} \mathrm{C}$ debidas a la disipación viscosa en un tubo de $18 \mathrm{~mm}$ y $100 \mathrm{~m}$ de longitud. Por lo tanto se decide despreciar dicho efecto.

\section{B.3. Solución analítica del problema térmico en tubo liso}

La ecuación de la energía tiene la siguiente forma:

$$
\frac{\rho c_{p}}{k} u_{z} \frac{\partial T}{\partial z}=\frac{1}{r} \frac{\partial}{\partial r}\left(r \frac{\partial T}{\partial r}\right)
$$

donde si el flujo está totalmente desarrollado:

$$
\frac{\partial T}{\partial z}=\frac{\partial T_{s}}{\partial z}=\frac{\partial \bar{T}}{\partial z}=\frac{2 q^{\prime \prime}}{\rho u_{b} c_{p} R}
$$

sustituyendo en la ecuación anterior y conociendo la expresión analítica del perfil de veloci- 
dades:

$$
\begin{gathered}
\frac{\rho c_{p}}{k} u_{z} \frac{2 q^{\prime \prime}}{\rho u_{b} c_{p} R}=\frac{2 q^{\prime \prime}}{k R} \frac{u_{z}}{u_{b}}=\frac{1}{r} \frac{\partial}{\partial r}\left(r \frac{\partial T}{\partial r}\right) \\
\frac{2 q^{\prime \prime}}{k R}\left(\frac{3 n+1}{n+1}\right)\left(1-\left(\frac{r}{R}\right)^{(n+1) / n}\right)=\frac{1}{r} \frac{\partial}{\partial r}\left(r \frac{\partial T}{\partial r}\right) \\
\frac{2 q^{\prime \prime}}{k R}\left(\frac{3 n+1}{n+1}\right) r\left(1-\left(\frac{r}{R}\right)^{(n+1) / n}\right) d r=d\left(r \frac{\partial T}{\partial r}\right)
\end{gathered}
$$

La expresión anterior únicamente se puede integrar para valores de $1 / n$ que pertenezcan a los números naturales, de modo que existe solución analítica para el perfil de temperaturas para $n=1 ; 0,5 ; 1 / 3 ; 0,25 ; 1 / 5 ; \ldots$.

Se resuelve dicha integral para los 2 primeros valores de $n$, que son los que se encuentran en el rango de propiedades del fluido de trabajo.

\section{Caso $n=1$}

$$
\begin{gathered}
T=\frac{4 q^{\prime \prime}}{k R}\left(\frac{r^{2}}{2}-\frac{r^{4}}{4 R^{2}}+C\right)=r \frac{\partial T}{\partial r} \\
T=\frac{4 q^{\prime \prime}}{k R} \int\left(\frac{r}{2}-\frac{r^{3}}{4 R^{2}}+C / r\right) d r=d T \\
T=\frac{4 q^{\prime \prime}}{k R}\left(\frac{r^{2}}{4}-\frac{r^{4}}{16 R^{2}}+C \ln r\right)+C_{0}=T
\end{gathered}
$$

Aplicando las condiciones de contorno:

- $r=0, \partial T / \partial r=0 \rightarrow C=0$

- $r=0, T=T_{c} \rightarrow C_{0}=T_{c}$

$$
T=\frac{q^{\prime \prime} R}{k}\left(\frac{r}{R}\right)^{2}\left(1-\frac{1}{4}\left(\frac{r}{R}\right)^{2}\right)+T_{c}
$$




$$
T=\frac{q^{\prime \prime} D}{k 2}\left(\frac{r}{R}\right)^{2}\left(1-\frac{1}{4}\left(\frac{r}{R}\right)^{2}\right)+T_{c}
$$

En forma adimensional:

$$
\theta=\frac{T-T_{c}}{\frac{q^{\prime \prime} D}{k}}=\frac{\left(r^{*}\right)^{2}}{2}\left(1-\frac{\left(r^{*}\right)^{2}}{4}\right)
$$

Si como segunda condición para obtener $C_{0}$ se utiliza la temperatura de la pared interior $T_{p i}$ en $r=R$, se puede deducir el número de Nusselt del flujo desarrollado.

$$
T=-\frac{q^{\prime \prime} R}{k}\left[\frac{3}{4}-\left(\frac{r}{R}\right)^{2}\left(1-\frac{1}{4}\left(\frac{r}{R}\right)^{2}\right)\right]+T_{p i}
$$

Si se realiza la integral entre $0<r<R$ para obtener la temperatura media:

$$
\begin{gathered}
\bar{T}=\frac{1}{u_{b} A} \int_{0}^{R} u T d A=\frac{1}{\pi R^{2}} \int_{0}^{R} \frac{u}{u_{b}} T 2 \pi r d r=\frac{2}{R^{2}} \int_{0}^{R} \frac{u}{u_{b}} T r d r= \\
=\frac{2}{R^{2}} \int_{0}^{R} 2\left(1-\left(\frac{r}{R}\right)^{(n+1) / n}\right)\left[-\frac{q^{\prime \prime} R}{k}\left[\frac{3}{4}-\left(\frac{r}{R}\right)^{2}\left(1-\frac{1}{4}\left(\frac{r}{R}\right)^{2}\right)\right]+T_{p i}\right] r d r \\
\frac{4}{R^{2}} \int_{0}^{R}\left(1-\left(\frac{r}{R}\right)^{(n+1) / n}\right)\left[-\frac{q^{\prime \prime} R}{k}\left[\frac{3}{4}-\left(\frac{r}{R}\right)^{2}\left(1-\frac{1}{4}\left(\frac{r}{R}\right)^{2}\right)\right]+T_{p i}\right] r d r
\end{gathered}
$$

se puede separar en 2 integrales:

$$
\begin{gathered}
I_{1}=\frac{4}{R^{2}} \int_{0}^{R}\left(1-\left(\frac{r}{R}\right)^{2}\right) T_{p i} r d r=\frac{4 T_{p i}}{R^{2}} \int_{0}^{R}\left(r-\frac{r^{3}}{R^{2}}\right) d r=\frac{4 T_{p i}}{R^{2}}\left[\frac{r^{2}}{2}-\frac{r^{4}}{4 R^{2}}\right]_{0}^{R}= \\
I_{1}=4 T_{p i}\left[\frac{1}{2}-\frac{1}{4}\right]=T_{p i} \\
I_{2}=-\frac{q^{\prime \prime} R}{k} \frac{4}{R^{2}} \int_{0}^{R}\left(1-\left(\frac{r}{R}\right)^{2}\right)\left[\frac{3}{4}-\left(\frac{r}{R}\right)^{2}\left(1-\frac{1}{4}\left(\frac{r}{R}\right)^{2}\right)\right] r d r
\end{gathered}
$$




$$
\begin{gathered}
I_{2}=-\frac{4 q^{\prime \prime}}{R k} \int_{0}^{R}\left[\frac{3}{4}-\left(\frac{r}{R}\right)^{2}\left(1-\frac{1}{4}\left(\frac{r}{R}\right)^{2}\right)\right]-\left(\frac{r}{R}\right)^{2}\left[\frac{3}{4}-\left(\frac{r}{R}\right)^{2}\left(1-\frac{1}{4}\left(\frac{r}{R}\right)^{2}\right)\right] r d r \\
I_{2}=-\frac{4 q^{\prime \prime}}{R k} \int_{0}^{R}\left[\frac{3}{4} r-\frac{r^{3}}{R^{2}}+\frac{1}{4} \frac{r^{5}}{R^{4}}-\frac{3}{4} \frac{r^{3}}{R^{2}}+\left(\frac{r^{5}}{R^{4}}\right)-\frac{1}{4} \frac{r^{7}}{R^{6}}\right] d r \\
I_{2}=-\frac{4 q^{\prime \prime}}{R k}\left[\frac{3}{2 * 4} r^{2}-\frac{r^{4}}{4 R^{2}}+\frac{1}{4 * 6} \frac{r^{6}}{R^{4}}-\frac{3}{4 * 4} \frac{r^{4}}{R^{2}}+\frac{r^{6}}{6 R^{4}}-\frac{1}{4 * 8} \frac{r^{8}}{R^{6}}\right]_{0}^{R} \\
I_{2}=-\frac{4 q^{\prime \prime} R}{k}\left[\frac{3}{2 * 4}-\frac{1}{4}+\frac{1}{4 * 6}-\frac{3}{4 * 4}+\frac{1}{6}-\frac{1}{4 * 8}\right]=-\frac{4 q^{\prime \prime} R}{k}\left(\frac{11}{96}\right)=-\frac{11 q^{\prime \prime} R}{24 k} \\
\bar{T}=T_{p i}-\frac{11}{24} \frac{q^{\prime \prime} R}{k}
\end{gathered}
$$

sabiendo que el coeficiente de transmisión de calor se define como $q^{\prime \prime}=h\left(T_{p i}-\bar{T}\right)$, entonces

$$
h_{i}=\frac{24 k}{11 R}=\frac{48 k}{11 D}=4,36 \frac{k}{D}
$$

\section{Caso $n=0.5$}

$$
\begin{gathered}
\frac{2 q^{\prime \prime}}{k R}\left(\frac{1,5+1}{0,5+1}\right) r\left(1-\left(\frac{r}{R}\right)^{(0,5+1) / 0,5}\right) d r=d\left(r \frac{\partial T}{\partial r}\right) \\
\frac{5 q^{\prime \prime}}{1,5 k R} r\left(1-\left(\frac{r}{R}\right)^{3}\right) d r=d\left(r \frac{\partial T}{\partial r}\right) \\
\frac{5 q^{\prime \prime}}{1,5 k R}\left(\frac{r^{2}}{2}-\frac{r^{5}}{5 R^{3}}+C\right)=r \frac{\partial T}{\partial r}
\end{gathered}
$$




$$
\begin{aligned}
& \frac{5 q^{\prime \prime}}{1,5 k R} \int\left(\frac{r}{2}-\frac{r^{4}}{5 R^{3}}+C / r\right) d r=d T \\
& T=\frac{10 q^{\prime \prime}}{3 k R}\left(\frac{r^{2}}{4}-\frac{r^{5}}{25 R^{3}}+C \ln r\right)+C_{0}
\end{aligned}
$$

Aplicando las condiciones de contorno:

- $r=0, \partial T / \partial r=0 \rightarrow C=0$

- $r=0, T=T_{c} \rightarrow C_{0}=T_{c}$

$$
T=\frac{10 q^{\prime \prime} R}{3 k}\left(\frac{r}{R}\right)^{2}\left(\frac{1}{4}-\frac{1}{25}\left(\frac{r}{R}\right)^{3}\right)+T_{c}
$$

En forma adimensional:

$$
\theta=\frac{T-T_{c}}{\frac{q^{\prime \prime} R}{k}}=\frac{10}{3}\left(r^{*}\right)^{2}\left(\frac{1}{4}-\frac{\left(r^{*}\right)^{3}}{25}\right)
$$

Si como segunda condición para obtener $C_{0}$ se utiliza la temperatura de la pared interior $T_{p i}$ en $r=R$, se puede deducir el número de Nusselt del flujo desarrollado.

$$
\begin{gathered}
T_{p i}=\frac{10 q^{\prime \prime} R}{3 k}\left(\frac{1}{4}-\frac{1}{25}\right)+C_{0} \\
C_{0}=T_{p i}-\frac{10 q^{\prime \prime} R}{3 k}\left(\frac{1}{4}-\frac{1}{25}\right)=T_{p i}-\frac{10 q^{\prime \prime} R}{3 k}\left(\frac{25-4}{100}\right)=T_{p i}-\frac{10 q^{\prime \prime} R}{3 k}\left(\frac{21}{100}\right)
\end{gathered}
$$

además

$$
\begin{gathered}
T_{c}=T_{p i}-\frac{10 q^{\prime \prime} R}{3 k}\left(\frac{21}{100}\right) \\
T=-\frac{10 q^{\prime \prime} R}{3 k}\left[\frac{21}{100}-\left(\frac{r}{R}\right)^{2}\left(\frac{1}{4}-\frac{1}{25}\left(\frac{r}{R}\right)^{3}\right)\right]+T_{p i}
\end{gathered}
$$


Integrando T.u a lo largo del tubo:

$$
\begin{gathered}
\bar{T}=\frac{1}{u_{b} A} \int_{0}^{R} u T d A=\frac{1}{\pi R^{2}} \int_{0}^{R} \frac{u}{u_{b}} T 2 \pi r d r=\frac{2}{R^{2}} \int_{0}^{R} \frac{u}{u_{b}} T r d r= \\
=\frac{2}{R^{2}} \int_{0}^{R}\left(\frac{2,5}{1,5}\right)\left(1-\left(\frac{r}{R}\right)^{3}\right)\left[-\frac{10 q^{\prime \prime} R}{3 k}\left[\frac{21}{100}-\left(\frac{r}{R}\right)^{2}\left(\frac{1}{4}-\frac{1}{25}\left(\frac{r}{R}\right)^{3}\right)\right]+T_{p i}\right] r d r \\
=\frac{10}{3 R^{2}} \int_{0}^{R}\left(1-\left(\frac{r}{R}\right)^{3}\right)\left[-\frac{10 q^{\prime \prime} R}{3 k}\left[\frac{21}{100}-\left(\frac{r}{R}\right)^{2}\left(\frac{1}{4}-\frac{1}{25}\left(\frac{r}{R}\right)^{3}\right)\right]+T_{p i}\right] r d r
\end{gathered}
$$

se puede separar en 2 integrales:

$$
\begin{gathered}
I_{1}=\frac{10}{3 R^{2}} \int_{0}^{R}\left(1-\left(\frac{r}{R}\right)^{3}\right) T_{p i} r d r=\frac{10 T_{p i}}{3 R^{2}} \int_{0}^{R}\left(r-\frac{r^{4}}{R^{3}}\right) d r=\frac{10 T_{p i}}{3 R^{2}}\left[\frac{r^{2}}{2}-\frac{r^{5}}{5 R^{3}}\right]_{0}^{R}=T_{p i} \\
I_{2}=-\frac{10 q^{\prime \prime} R}{3 k} \frac{10}{3 R^{2}} \int_{0}^{R}\left(1-\left(\frac{r}{R}\right)^{3}\right)\left[\frac{21}{100}-\left(\frac{r}{R}\right)^{2}\left(\frac{1}{4}-\frac{1}{25}\left(\frac{r}{R}\right)^{3}\right)\right] r d r \\
I_{2}=-\frac{10^{2} q^{\prime \prime}}{3^{2} k R} \int_{0}^{R}\left[\frac{21}{100}-\left(\frac{r}{R}\right)^{2}\left(\frac{1}{4}-\frac{1}{25}\left(\frac{r}{R}\right)^{3}\right)-\left(\frac{r}{R}\right)^{3}\left(\frac{21}{100}-\left(\frac{r}{R}\right)^{2}\left(\frac{1}{4}-\frac{1}{25}\left(\frac{r}{R}\right)^{3}\right)\right)\right] r d r \\
I_{2}=-\frac{10^{2} q^{\prime \prime}}{3^{2} k R} \int_{0}^{R}\left[\frac{21}{100} r-\frac{r^{3}}{4 R^{2}}+\frac{1}{25} \frac{r^{6}}{R^{5}}-\frac{21}{100} \frac{r^{4}}{R^{3}}+\frac{r^{6}}{4 R^{5}}-\frac{1}{25} \frac{r^{9}}{R^{8}}\right] d r \\
I_{2}=-\frac{10^{2} q^{\prime \prime}}{3^{2} k R}\left[\frac{21}{2 * 100} r^{2}-\frac{r^{4}}{4 * 4 R^{2}}+\frac{1}{25 * 7} \frac{r^{7}}{R^{5}}-\frac{21}{5 * 100} \frac{r^{5}}{R^{3}}+\frac{r^{7}}{4 * 7 R^{5}}-\frac{1}{25 * 10} \frac{r^{10}}{R^{8}}\right]_{0}^{R} \\
\left.\frac{10^{2} q^{\prime \prime} R}{200}-\frac{1}{16}+\frac{1}{175}-\frac{21}{500}+\frac{1}{28}-\frac{1}{250}\right]=-\frac{10^{2} q^{\prime \prime} R}{3^{2} k} \frac{531}{14000}=-\frac{q^{\prime \prime} R}{140} \frac{q^{\prime \prime} R}{k}= \\
k
\end{gathered}
$$


sabiendo que el coeficiente de transmisión de calor se define como $q^{\prime \prime}=h\left(T_{p i}-\bar{T}\right)$, entonces

$$
h_{i}=\frac{140 k}{59 R}=\frac{280 k}{59 D}=4,746 \frac{k}{D}
$$

$$
N u=4,746
$$




\section{Apéndice $\mathrm{C}$}

\section{Visualización. Resultados adicionales.}

En el presente capítulo se presentan los resultados adicionales del patrón de flujo en régimen dinámico, no incluidos en el Capítulo 7. En las figuras C.1, C.2 y C.1 se muestra el campo fluido en régimen dinámico, en situaciones en las que el rascador se desplaza en sentido contracorriente, siendo $\beta=3 ; 2 ; 1,5$ respectivamente. Por otro lado, en la Fig. C.4 se muestra el campo de velocidades en dirección del rascador equicorriente con $\beta=1$.

Por último en la Tabla C.1 se muestran las propiedades reológicas del fluido utilizado en cada uno del total de ensayos de visualización dinámicos realizados en la investigación. 


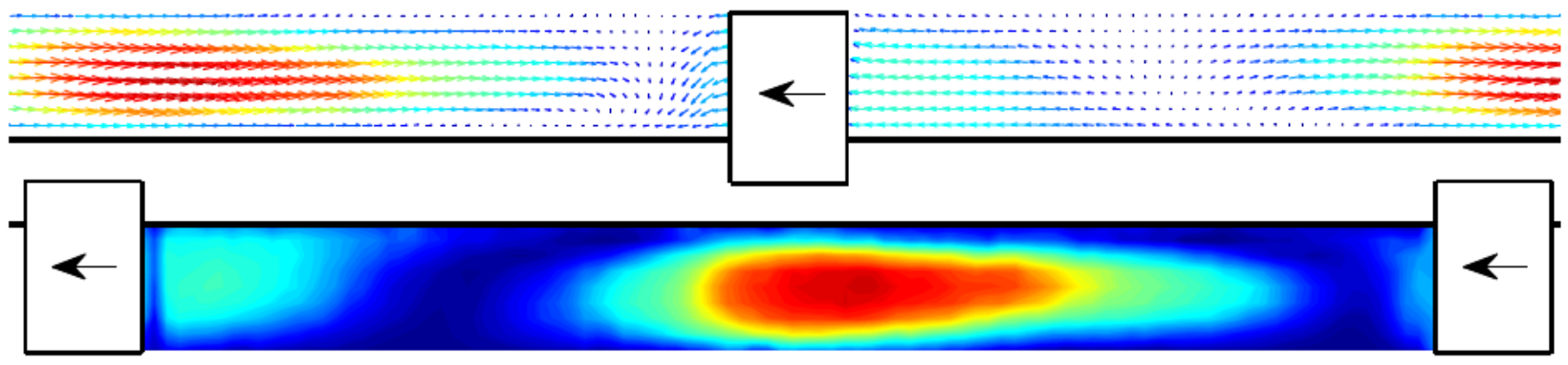

(a) $R e_{g}=13, n=0,65$.

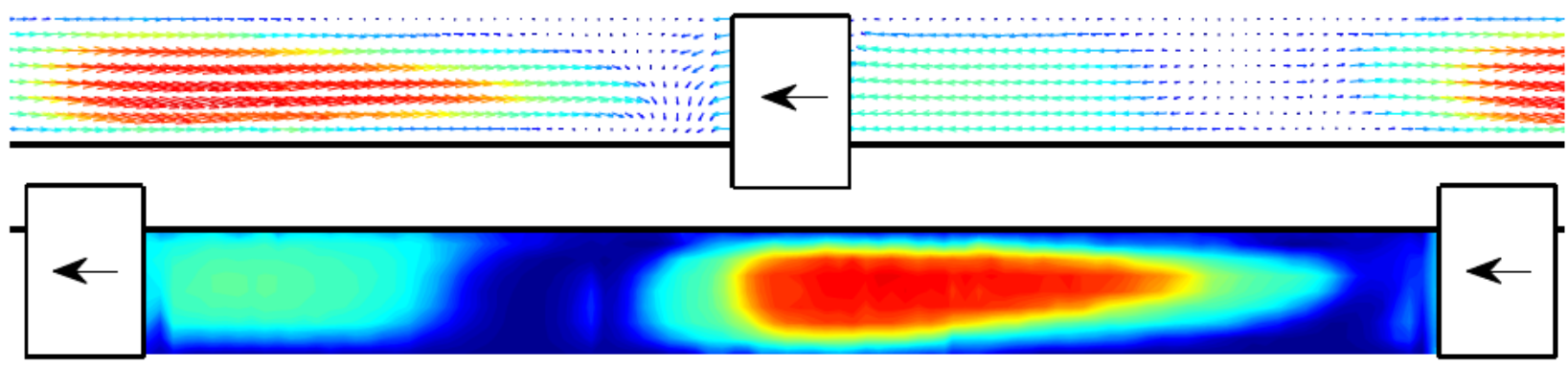

(b) $R e_{g}=26,4, n=0,78$.

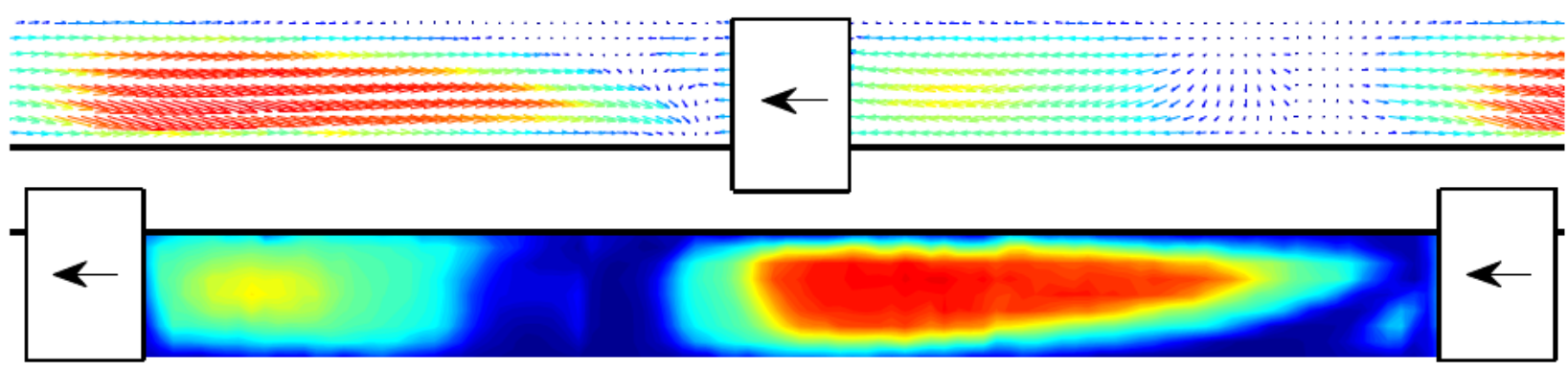

(c) $R e_{g}=44, n=0,88$.

\begin{tabular}{rrrrrrrrrrr}
0 & 0,3 & 0,6 & 0,9 & 1,2 & 1,5 & 1,8 & 2,1 & 2,4 & 2,7 & 3 \\
\hline & 1 & 1 & 1 & 1 & 1 & & & \\
\hline
\end{tabular}

Figura C.1: Caso dinámico contracorriente con $\beta=3$. 


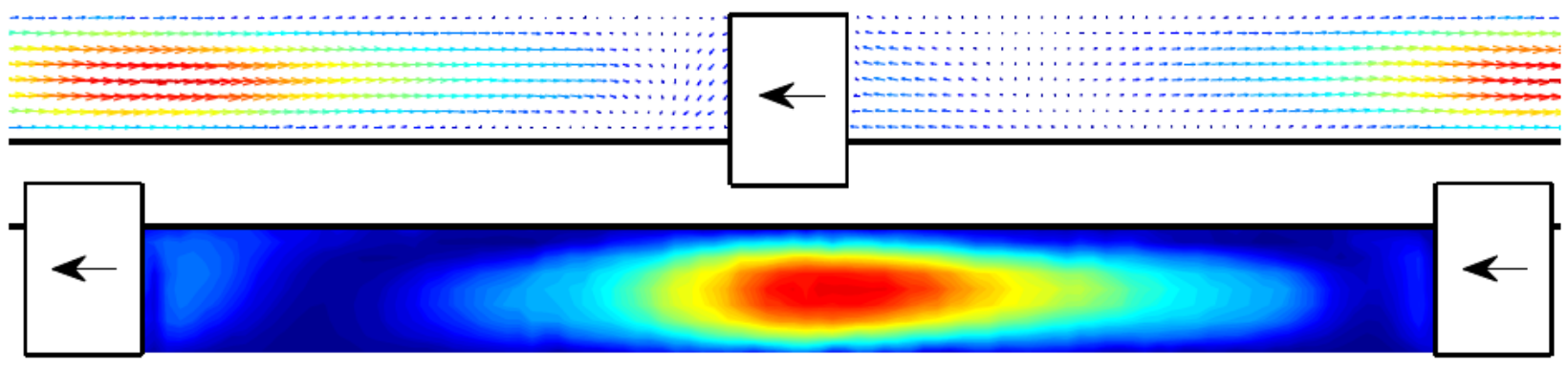

(a) $R e_{g}=13, n=0,64$.

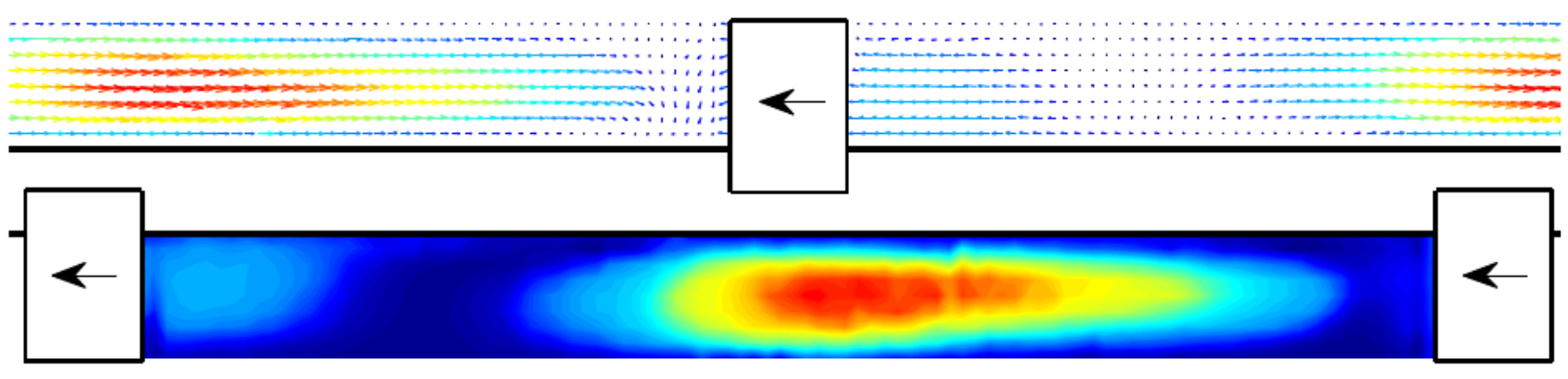

(b) $R e_{g}=25,4, n=0,77$.

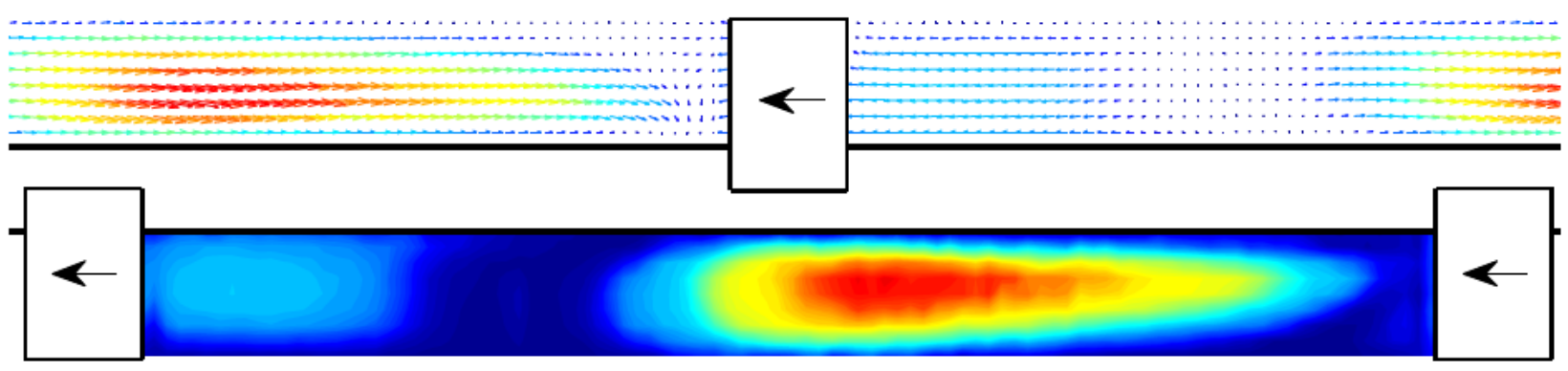

(c) $R e_{g}=44, n=0,88$.

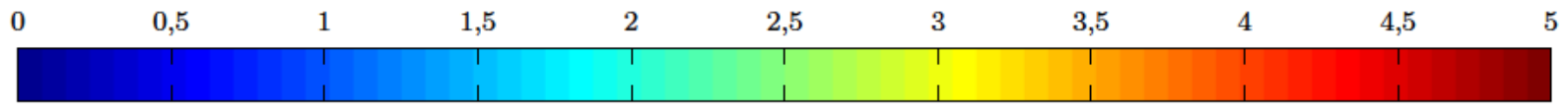

Figura C.2: Caso dinámico contracorriente con $\beta=2$. 


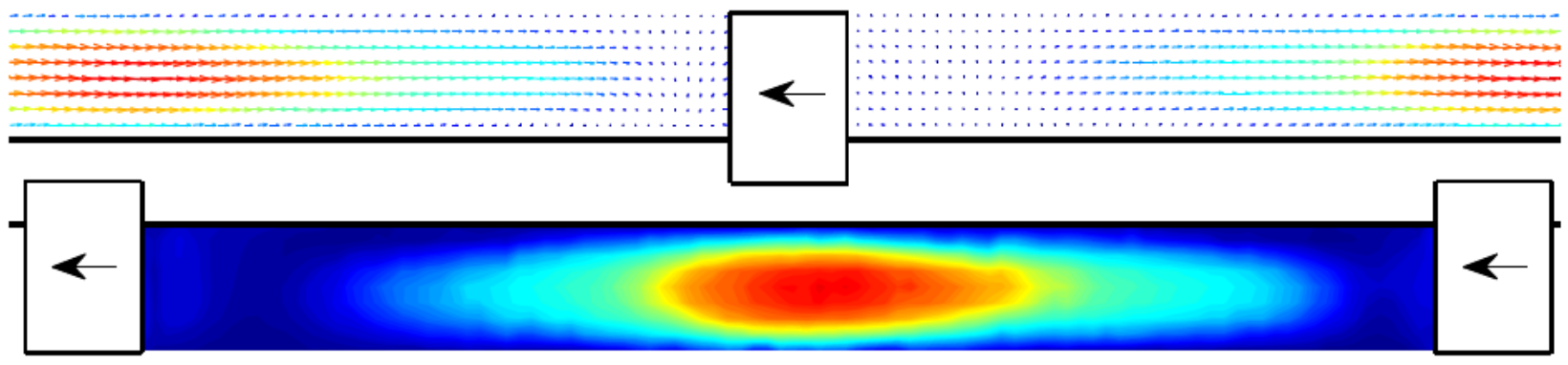

(a) $R e_{g}=12, n=0,62$.

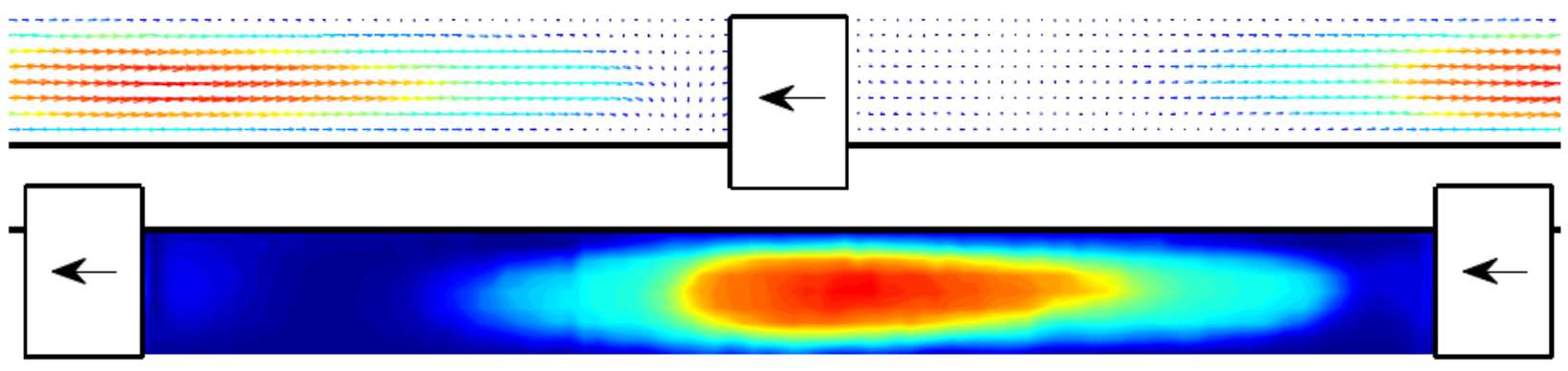

(b) $R e_{g}=24,6, n=0,76$.

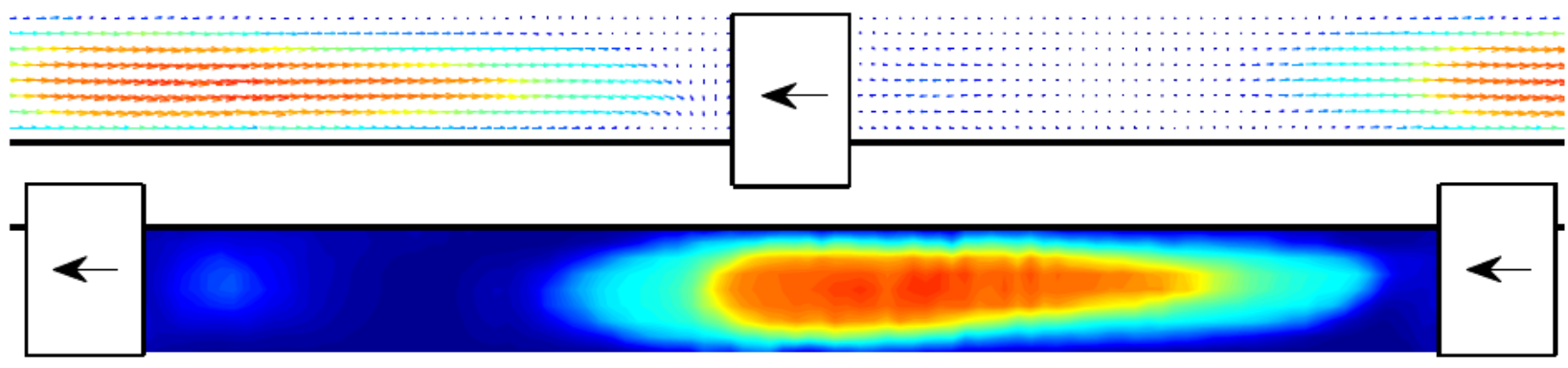

(c) $R e_{g}=43, n=0,87$.

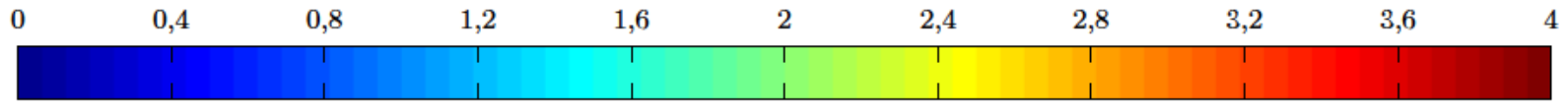

Figura C.3: Caso dinámico contracorriente con $\beta=1,5$. 


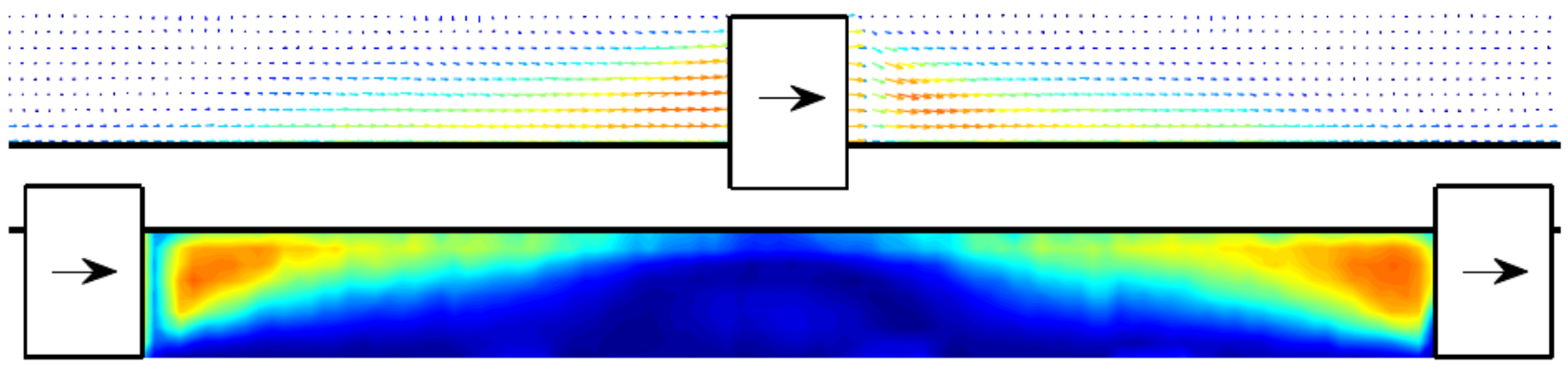

(a) $R e_{g}=12, n=0,62$.

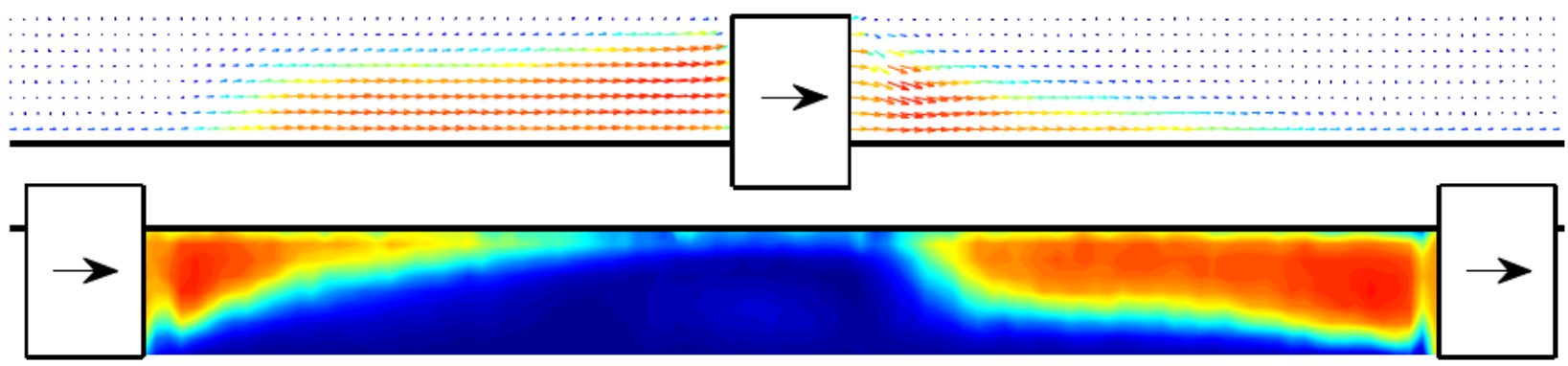

(b) $R e_{g}=28, n=0,79$.

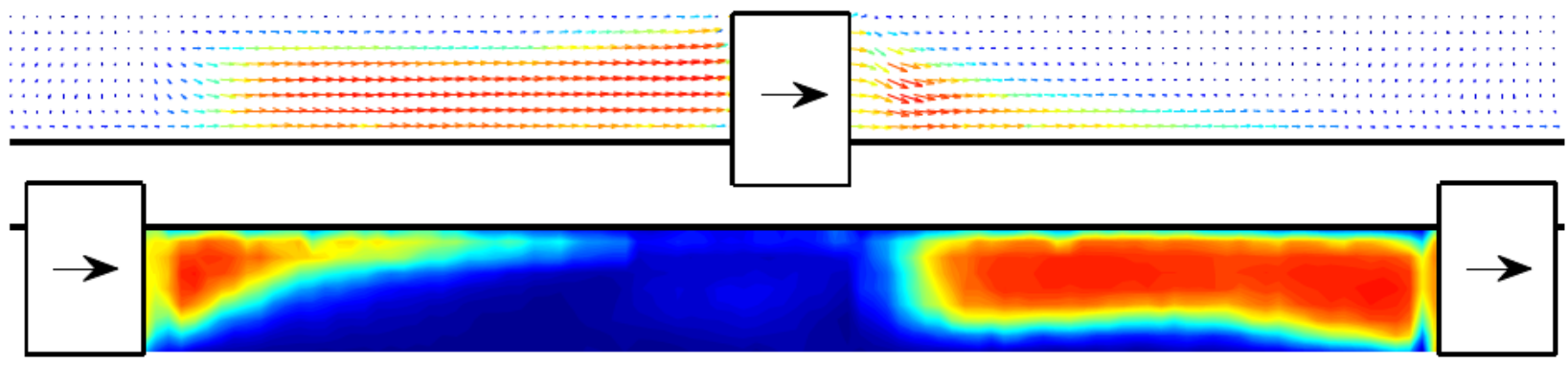

(c) $R e_{g}=48, n=0,87$.

\begin{tabular}{rrrrrrrrrrr}
0 & 0,3 & 0,6 & 0,9 & 1,2 & 1,5 & 1,8 & 2,1 & 2,4 & 2,7 & 3 \\
\hline & 1 & 1 & 1 & 1 & $\mid$ & 1 & 1 & 1
\end{tabular}

Figura C.4: Caso dinámico equicorriente con bloqueo negativo $\beta=-1$ variando el número de Reynolds. 


\begin{tabular}{ccccccc}
\hline$\omega=v_{s} / u_{b}$ & $\beta=1-\omega$ & $Q(\mathrm{l} / \mathrm{h})$ & $n$ & $m\left(\mathrm{~Pa} . \mathrm{s}^{\mathrm{n}}\right)$ & $R e_{b}$ & $R e_{g}$ \\
\hline 0,5 & 0,5 & 210 & 0,61 & 0,767 & 3.8482 & 11,5 \\
1 & 0 & 210 & 0,61 & 0,736 & 4.0103 & 12,0 \\
2 & -1 & 210 & 0,62 & 0,706 & 4.1321 & 12,1 \\
3 & -2 & 210 & 0,63 & 0,675 & 4.2716 & 12,3 \\
$-0,5$ & 1,5 & 210 & 0,62 & 0,689 & 4.2340 & 12,4 \\
-1 & 2 & 210 & 0,64 & 0,643 & 4.4320 & 12,5 \\
-2 & 3 & 210 & 0,65 & 0,597 & 4.7179 & 13,0 \\
-3 & 4 & 210 & 0,66 & 0,551 & 5.0523 & 13,6 \\
$-0,5$ & 1,5 & 226 & 0,76 & 0,231 & 11.7418 & 24,6 \\
-1 & 2 & 226 & 0,77 & 0,215 & 12.4597 & 25,4 \\
-2 & 3 & 226 & 0,78 & 0,199 & 13.2951 & 26,4 \\
-3 & 4 & 226 & 0,79 & 0,183 & 14.2787 & 27,5 \\
0,5 & 0,5 & 226 & 0,79 & 0,183 & 14.2787 & 27,5 \\
1 & 0 & 226 & 0,79 & 0,181 & 14.4365 & 27,8 \\
2 & -1 & 226 & 0,79 & 0,178 & 14.6798 & 28,3 \\
3 & -2 & 226 & 0,795 & 0,176 & 14.7546 & 28,0 \\
0,5 & 0,5 & 236 & 0,85 & 0,0805 & 31.6632 & 51,2 \\
1 & 0 & 236 & 0,86 & 0,0800 & 31.4536 & 49,4 \\
2 & -1 & 236 & 0,87 & 0,0794 & 31.2860 & 47,6 \\
3 & -2 & 236 & 0,87 & 0,0789 & 31.4842 & 47,9 \\
$-0,5$ & 1,5 & 236 & 0,87 & 0,089 & 27.9113 & 42,5 \\
-1 & 2 & 236 & 0,88 & 0,0821 & 29.8701 & 44,1 \\
-2 & 3 & 236 & 0,88 & 0,0815 & 30.0900 & 44,4 \\
-3 & 4 & 236 & 0,89 & 0,0810 & 29.8885 & 42,8 \\
\hline
\end{tabular}

Cuadro C.1: Propiedades reológicas del fluido correspondientes a los ensayos dinámicos. 


\section{Apéndice D}

\section{Resultados adicionales de la transmisión de calor y evaluación de prestaciones.}

En el presente Capítulo se incluyen los resultados complementarios a los presentados en el Capítulo 9 en cuanto a la transmisión de calor en el tubo con eje y la evaluación de prestaciones realizada.

\section{D.1. Resultados adicionales de transmisión de calor}

En el presente apartado se incluyen resultados adicionales de transmisión de calor en régimen de rascador estático y dinámico.

En la Fig. D.1 se muestran los resultados obtenidos en régimen estático, complementarios a la selección mostrada en la Fig. 9.3 del Capítulo 9.

En el presente apartado se muestran los resultados de los ensayos de transmisión de calor que no se han incluido en el Capítulo 9.

Las figuras D.2, D.3, D.4 y D.5 muestran los resultados individuales de las tandas de ensayos de transmisión de calor en régimen estático.

En las figuras D.6 y D.7 se encuentran los resultados de las mediciones del número de Nusselt en las condiciones de $\operatorname{Re}_{g}$ y $\operatorname{Pr}_{g}$ en que fueron medidas, para las tandas de ensayos en régimen dinámico TCD-2, TCD-3, TCD-5, TCD-6, TCD-8 y TCD-9. 


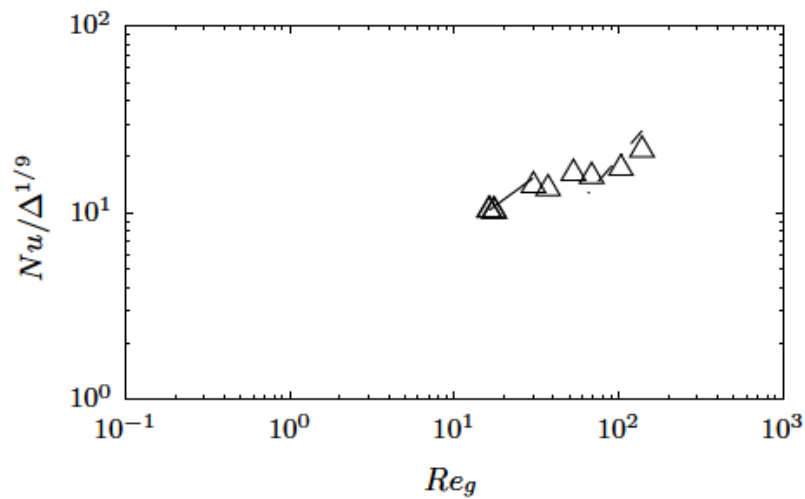

(a) TC1, $P r_{g, r e f}=500$

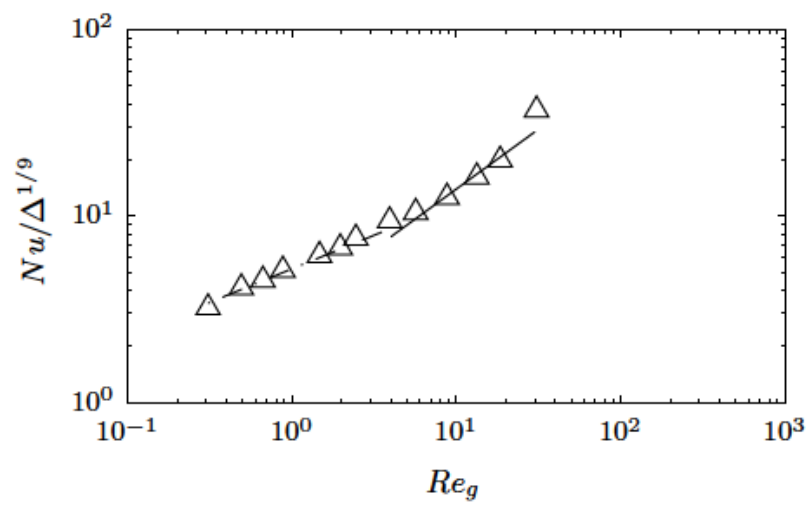

(c) TC5, $P r_{g, r e f}=2960$

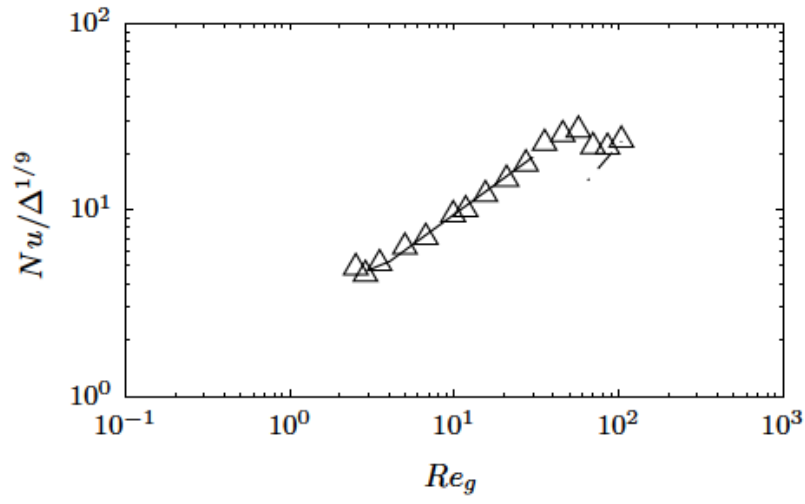

(e) $\mathrm{TC} 9, P r_{g, r e f}=940$

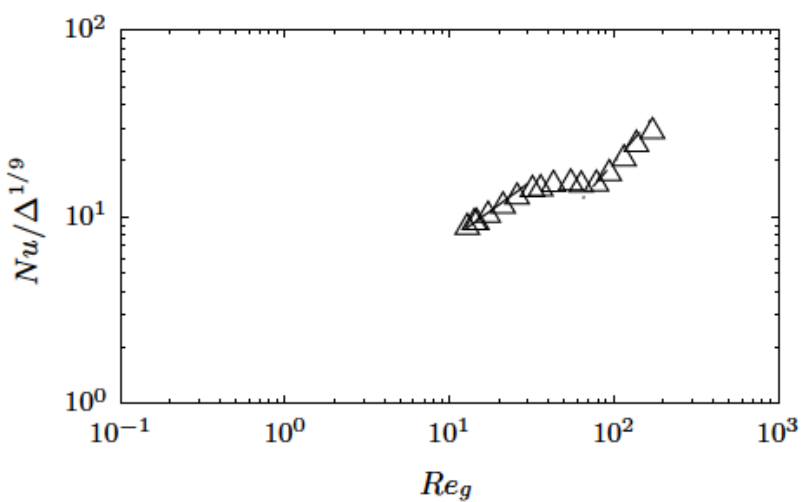

(b) $\mathrm{TC} 2, \operatorname{Pr}_{g, r e f}=470$

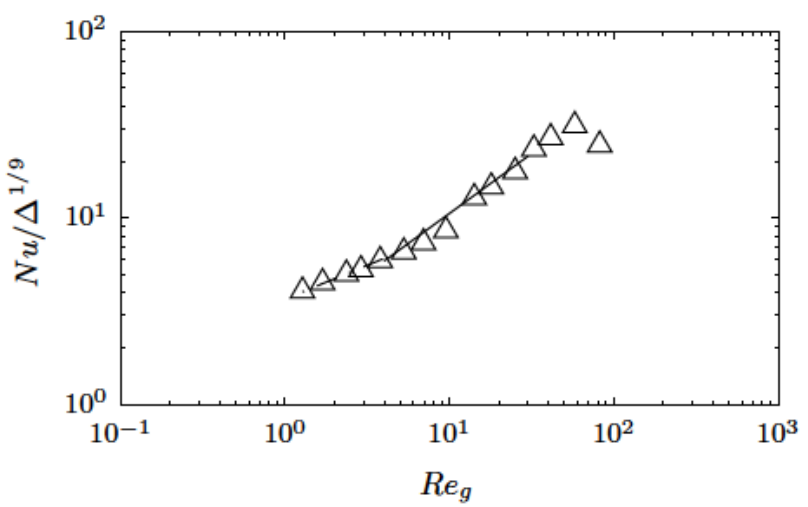

(d) $\mathrm{TC} 8, P r_{g, r e f}=1320$

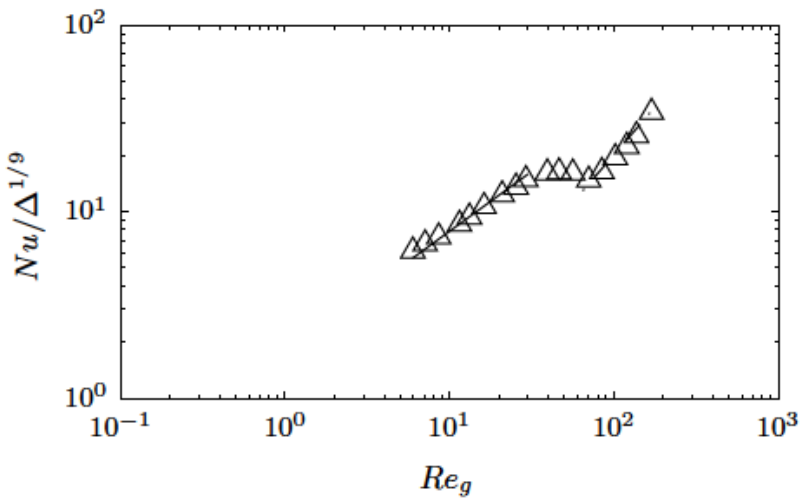

(f) $\mathrm{T} 11, P r_{g, \text { ref }}=550$

Figura D.1: Selección de tandas de ensayos trasladados al Prandtl medio de la tanda. 


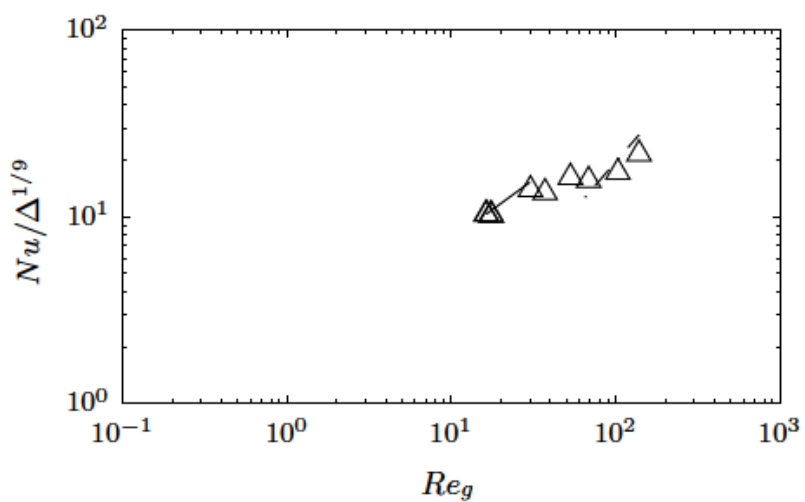

(a) TC1, $P r_{c, r e f}=500$

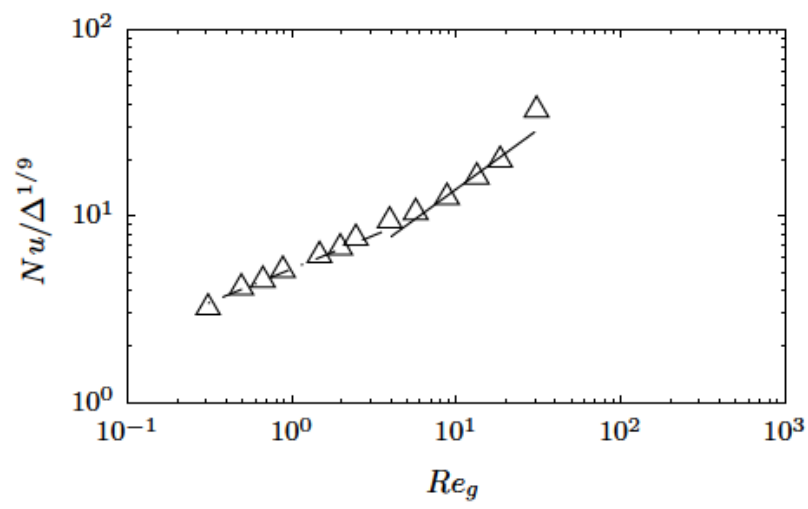

(c) TC5, $P r_{c, r e f}=2960$

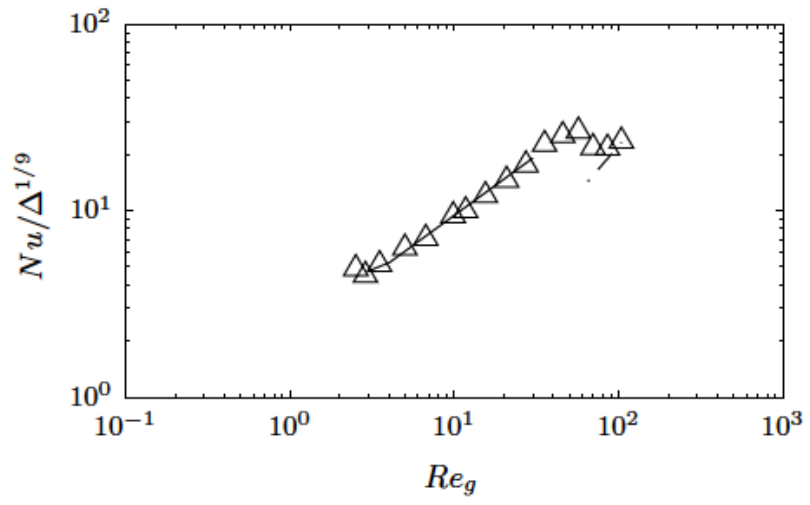

(e) $\mathrm{TC} 9, P r_{c, r e f}=940$

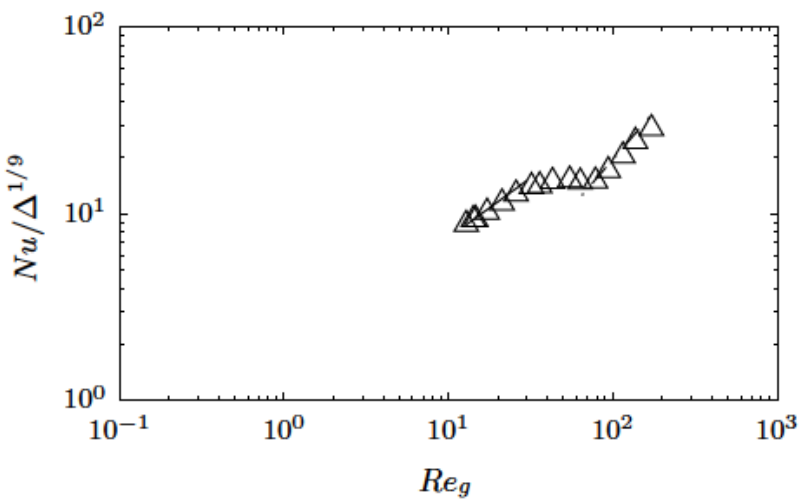

(b) TC2, $P r_{c, r e f}=470$

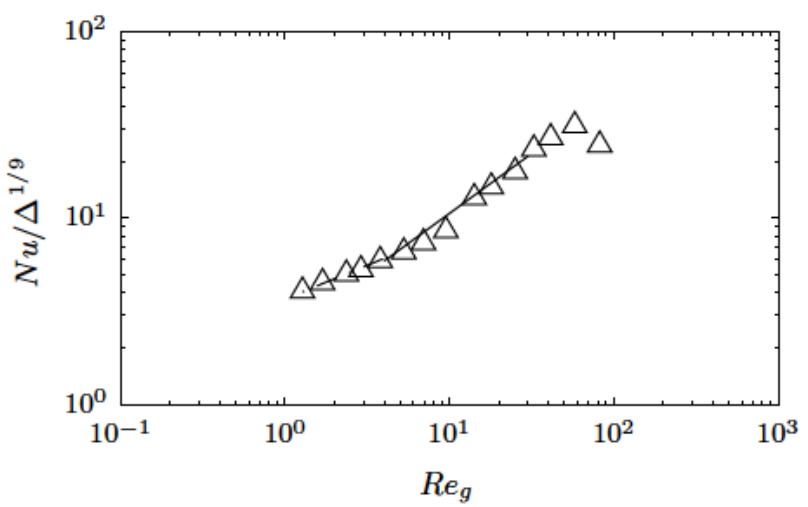

(d) $\mathrm{TC} 8, P r_{c, r e f}=1320$

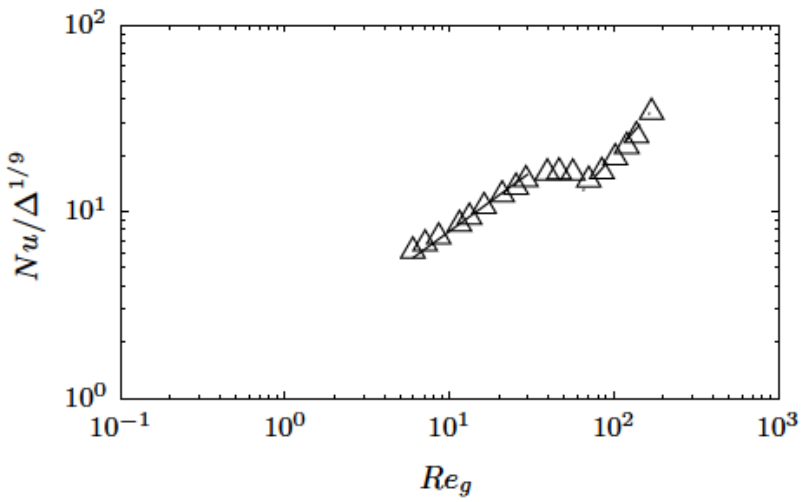

(f) $\mathrm{T} 11, P r_{c, r e f}=550$

Figura D.2: Selección de tandas de ensayos trasladados al Prandtl medio de la tanda. 

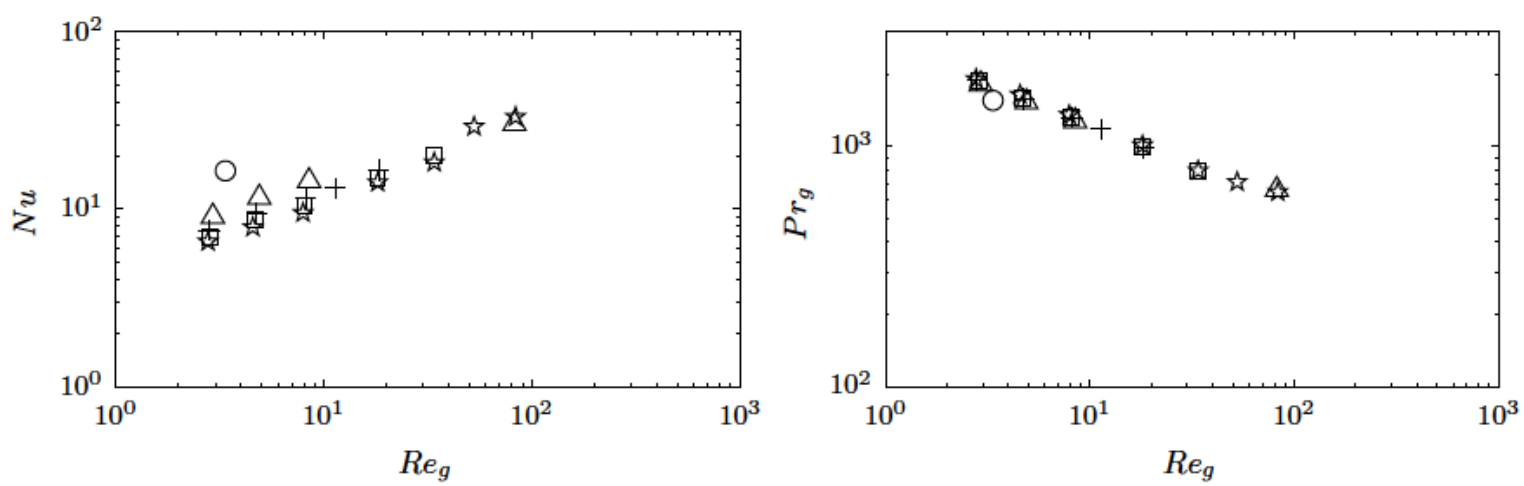

(a) $T C D-1$
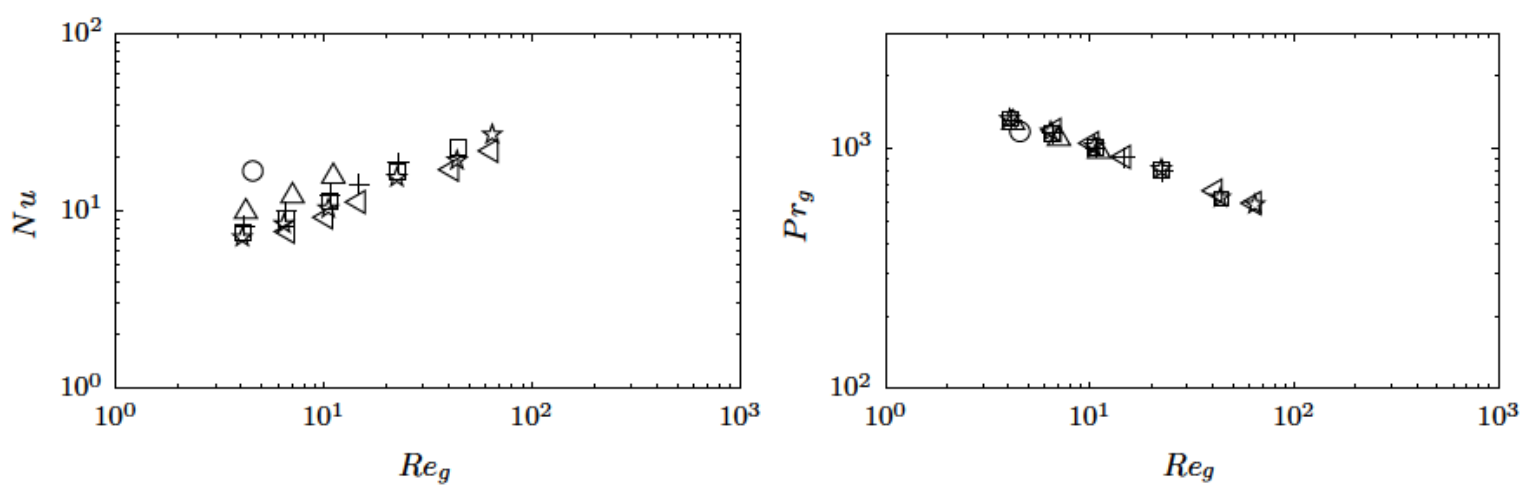

(b) $T C D-2$
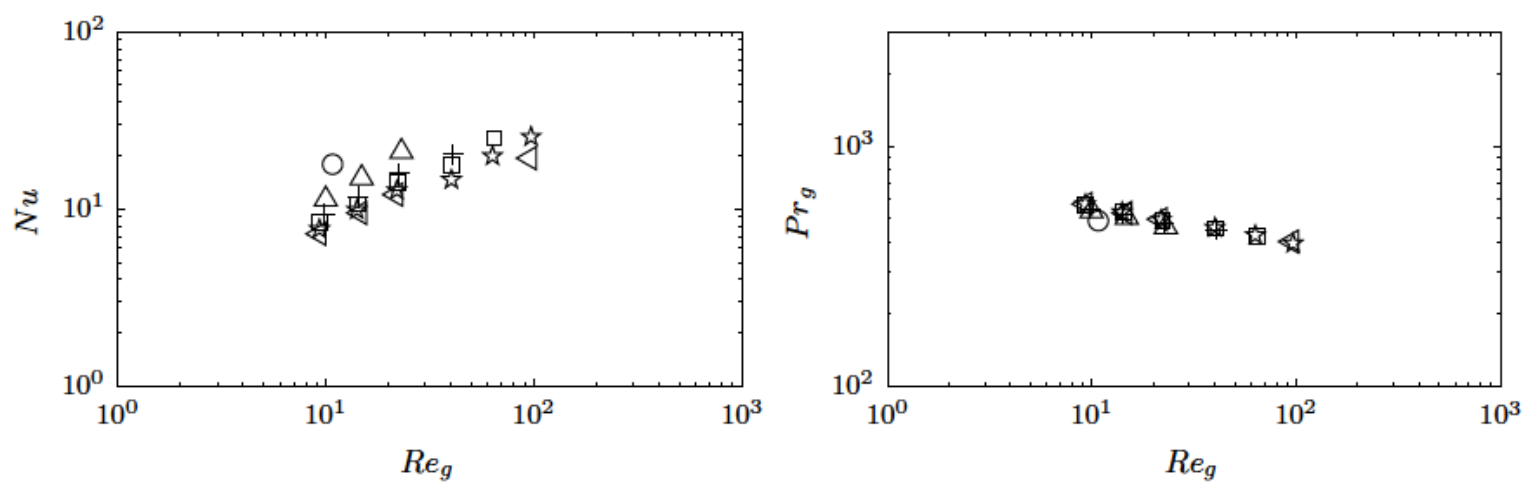

(c) $T C D-3$

\begin{tabular}{|c|c|c|c|c|c|c|c|}
\hline$x$ & $\omega=1,5$ & $\triangle$ & $\omega=0,5$ & $\square$ & $\omega=0,2$ & $\triangleleft$ & $\omega=0$ \\
\hline O & $\omega=1$ & + & $\omega=0,3$ & 七 & $\omega=0,1$ & & \\
\hline
\end{tabular}

Figura D.3: Medidas del número de Nusselt en las diferentes tandas de experimentos (I). 

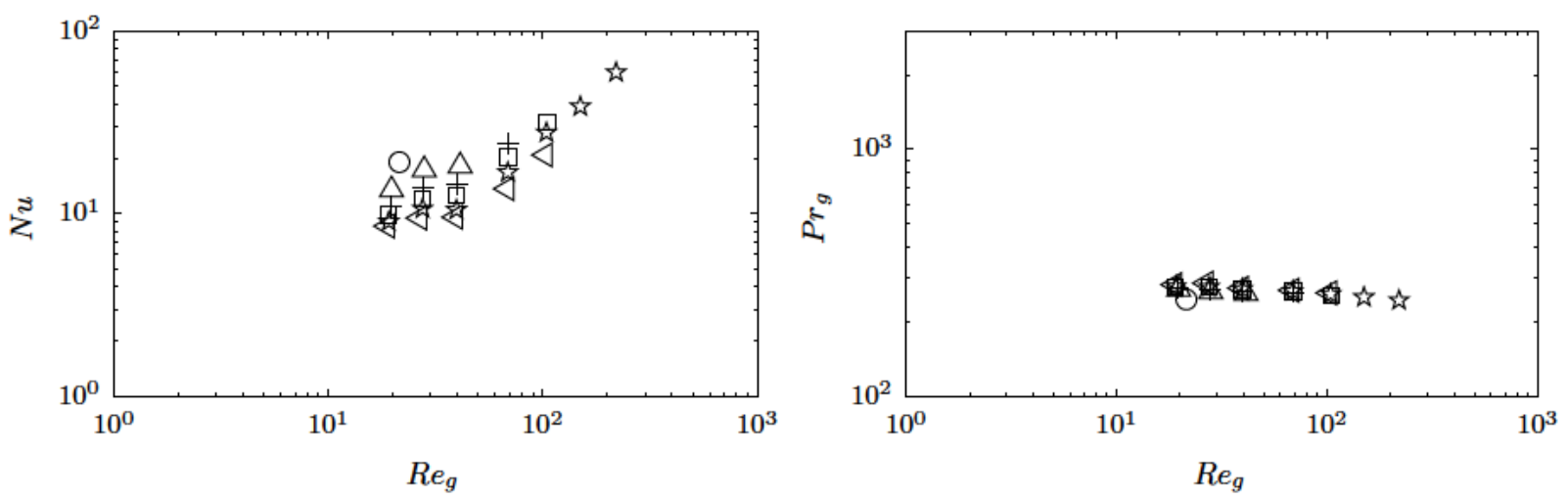

(a) $T C D-4$
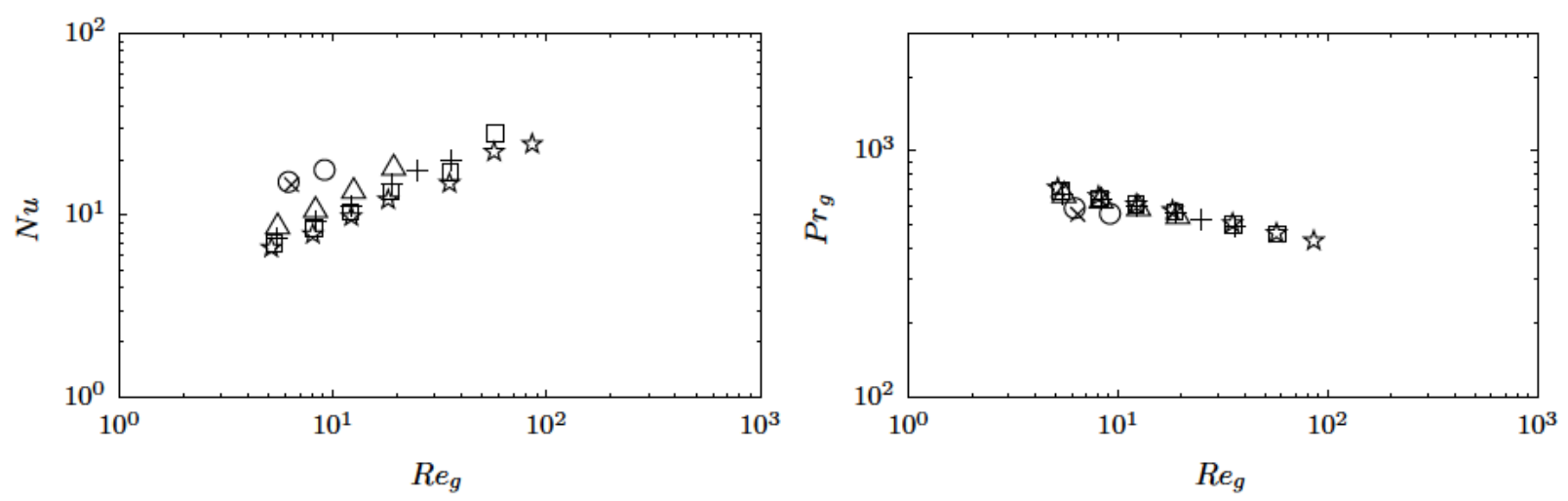

(b) $T C D-5$
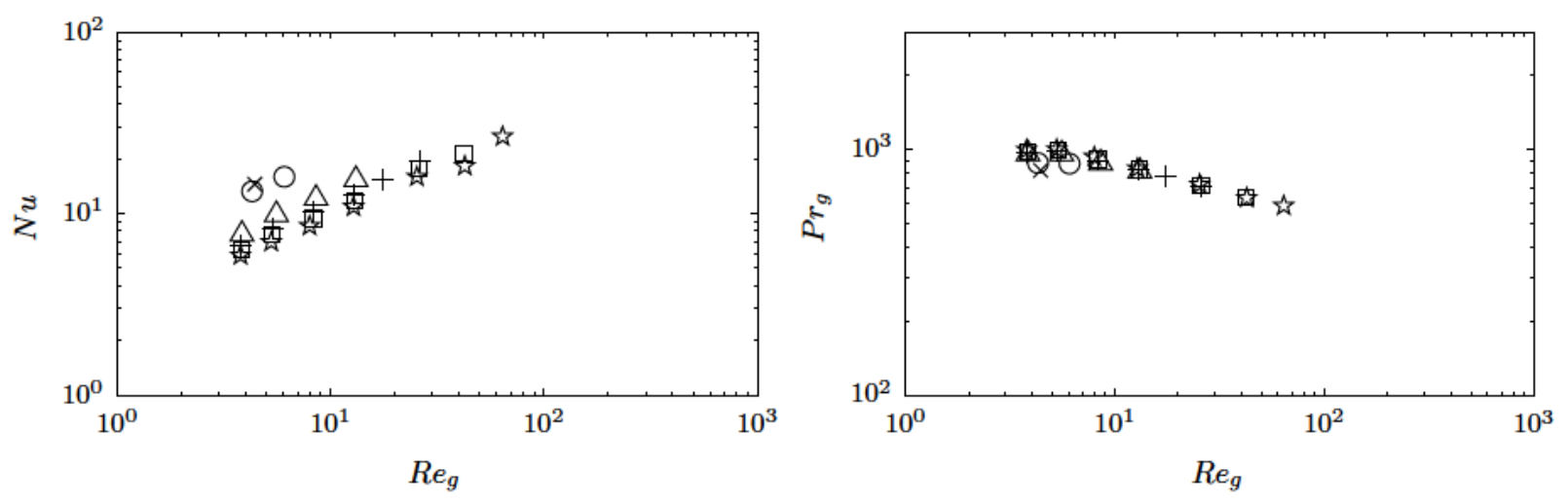

(c) $T C D-6$

\begin{tabular}{|c|c|c|c|c|c|c|c|}
\hline$x$ & $\omega=1,5$ & $\triangle$ & $\omega=0,5$ & 口 & $\omega=0,2$ & $\triangleleft$ & $\omega=0$ \\
\hline 0 & $\omega=1$ & + & $\omega=0,3$ & $\dot{4}$ & $\omega=0,1$ & & \\
\hline
\end{tabular}

Figura D.4: Medidas del número de Nusselt en las diferentes tandas de experimentos (II). 

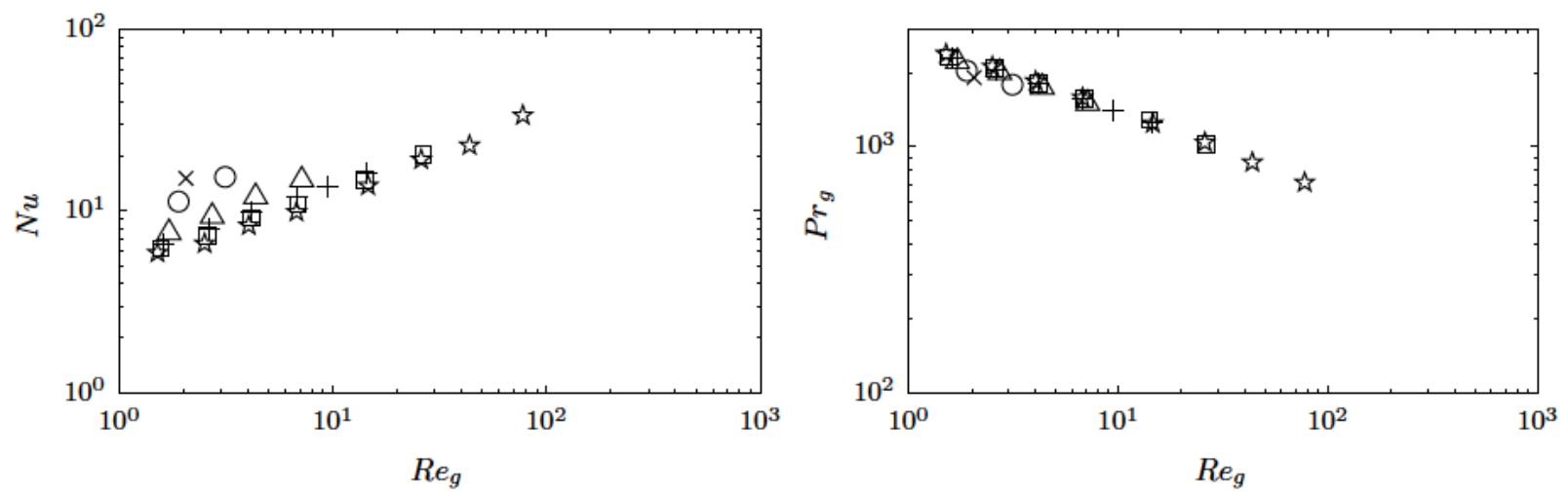

(a) $T C D-7$
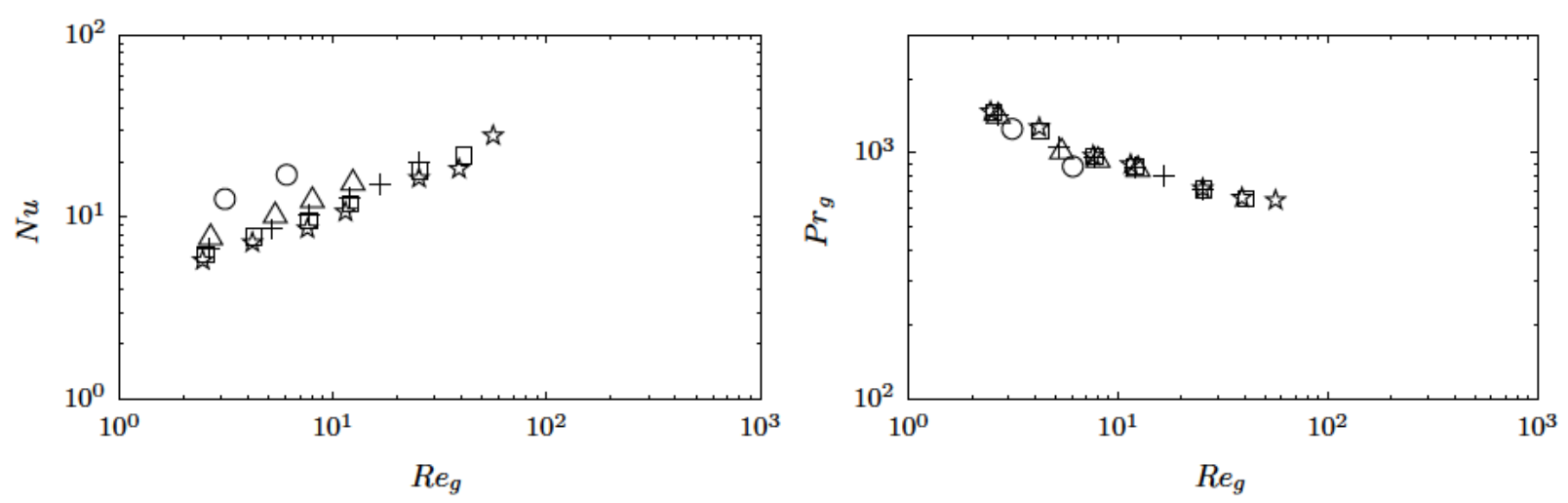

(b) $T C D-8$
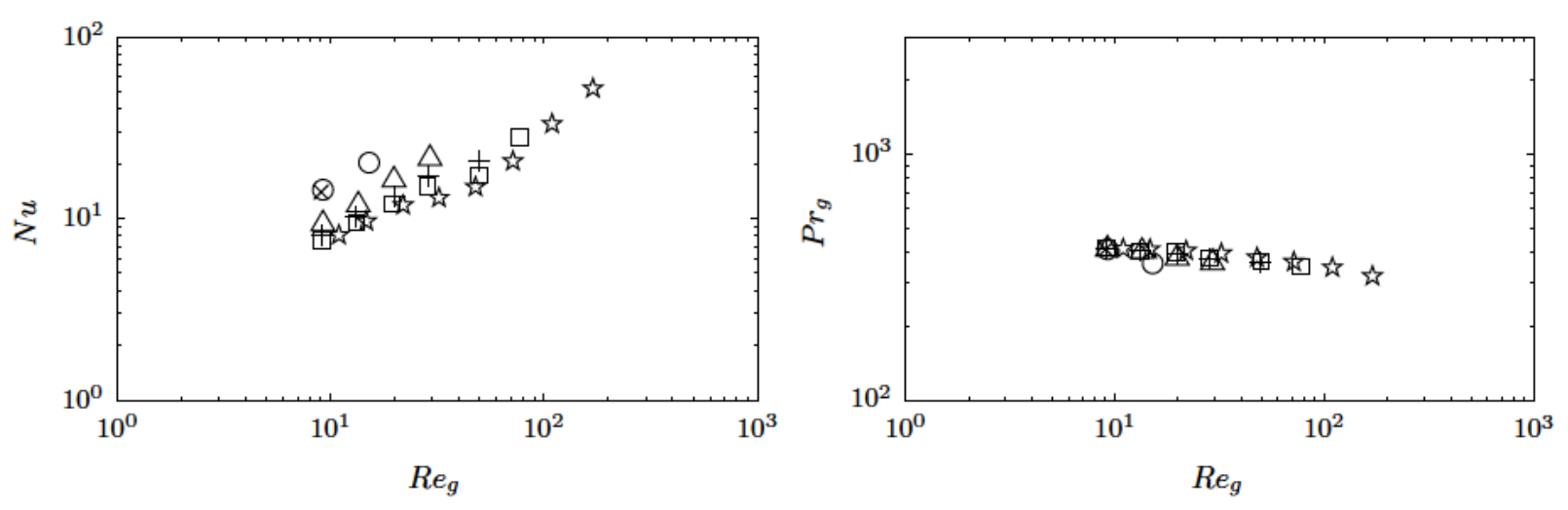

(c) $T C D-9$

$\left[\begin{array}{lllllll}\times & \omega=1,5 & \triangle & \omega=0,5 & & \square & \omega=0,2 \\ \circ & \omega=1 & + & \omega=0,3 & & \text { ४ } & \omega=0,1\end{array}\right]$

Figura D.5: Medidas del número de Nusselt en las diferentes tandas de experimentos (III). 

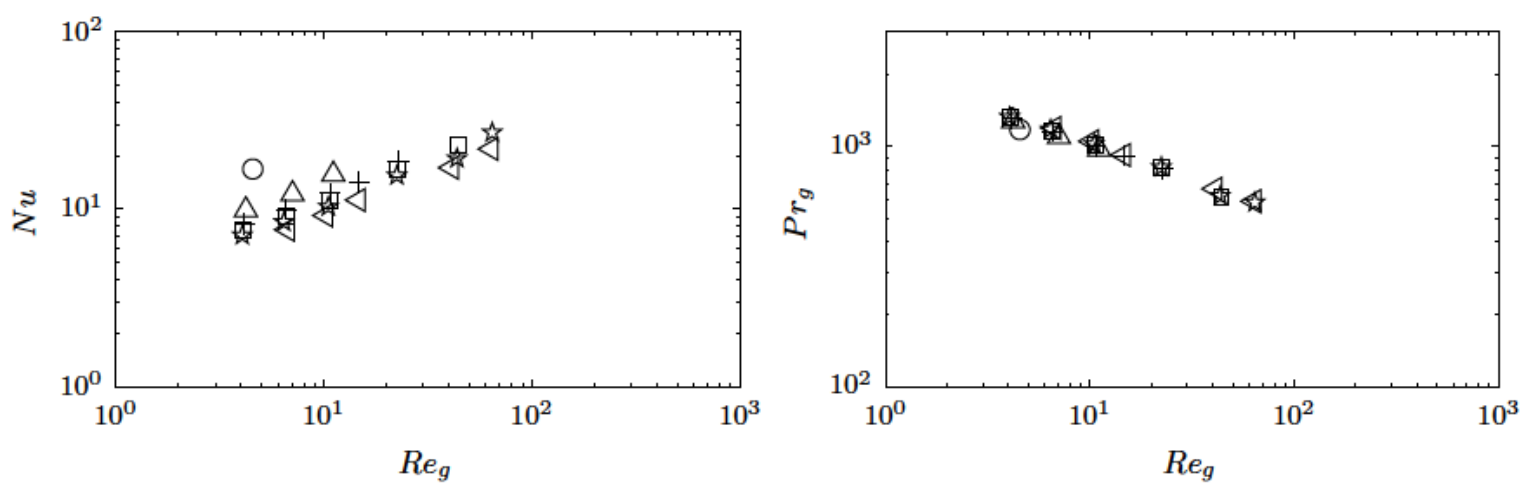

(a) $T C D-2(\mathrm{BB})$
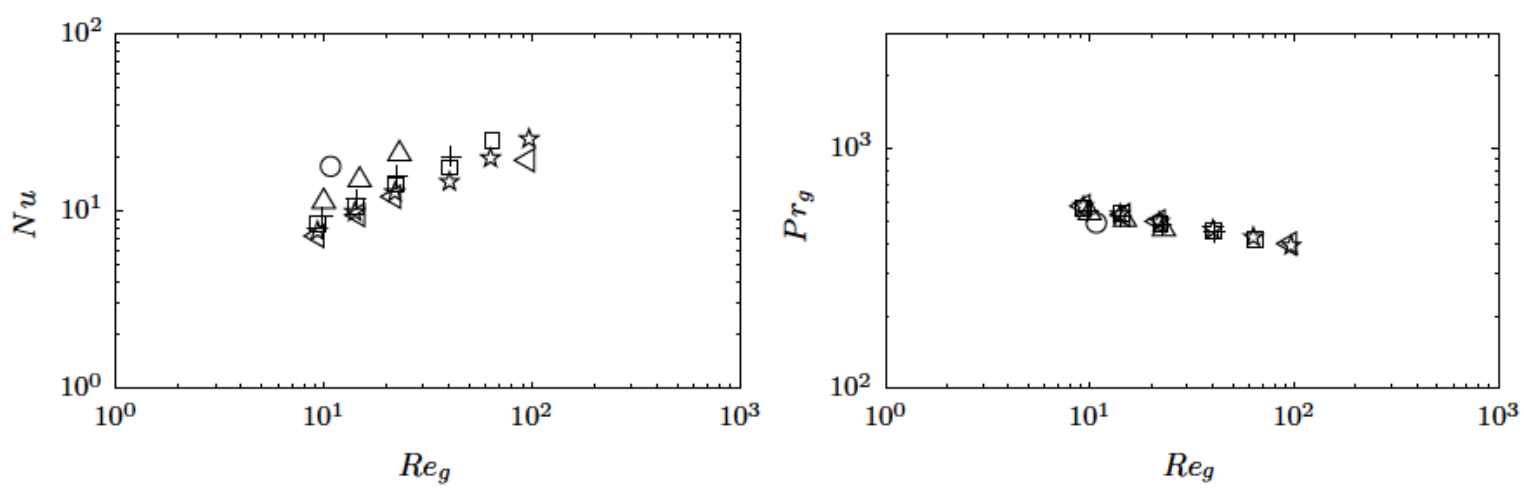

(b) $T C D-3(\mathrm{BB})$
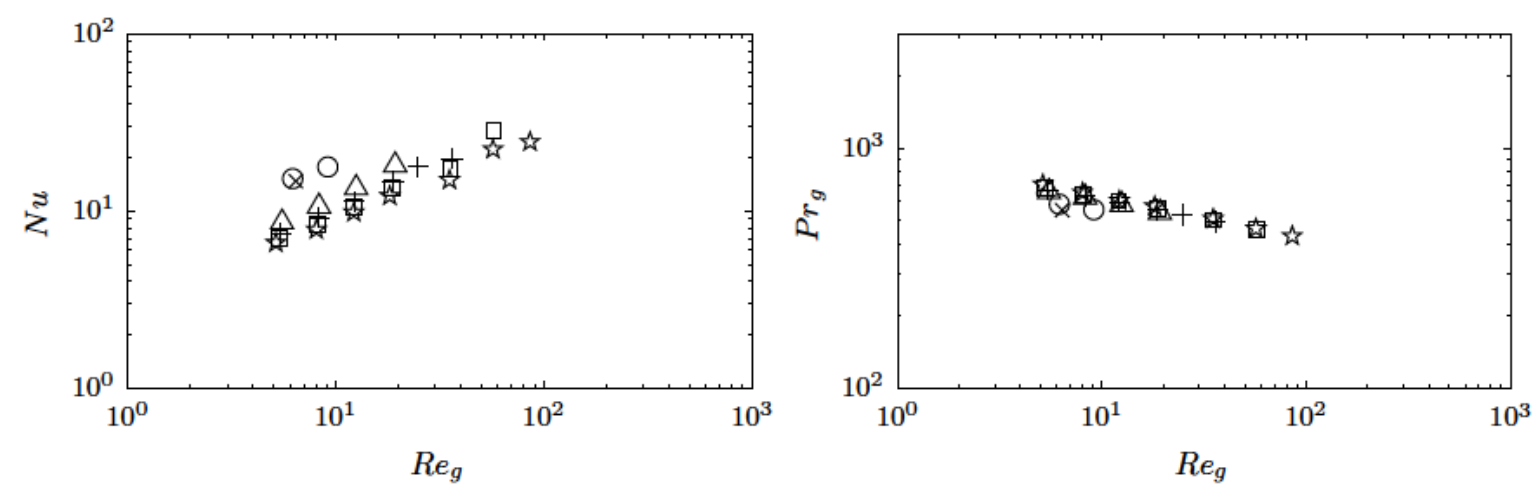

(c) $T C D-5$ (BA)

\begin{tabular}{|c|c|c|c|c|c|c|c|}
\hline$x$ & $\omega=1,5$ & $\triangle$ & $\omega=0,5$ & $\square$ & $\omega=0,2$ & $\triangleleft$ & $\omega=0$ \\
\hline 0 & $\omega=1$ & + & $\omega=0,3$ & th & $\omega=0,1$ & & \\
\hline
\end{tabular}

Figura D.6: Medidas del número de Nusselt en las diferentes tandas de experimentos (I). 

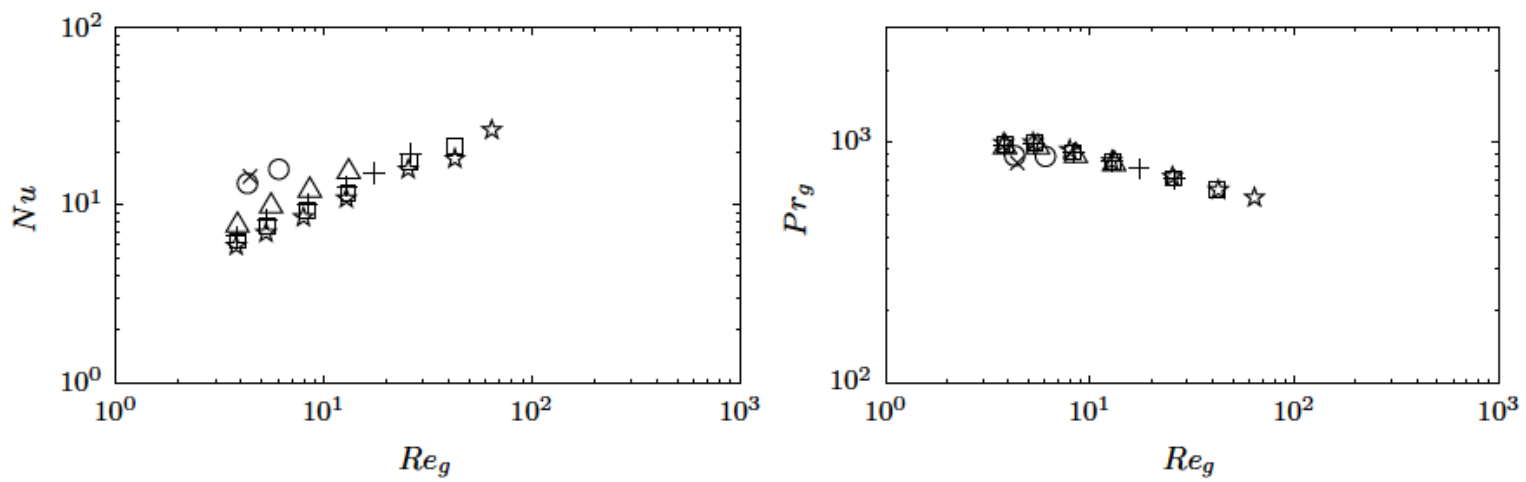

(a) $T C D-6(\mathrm{BB})$
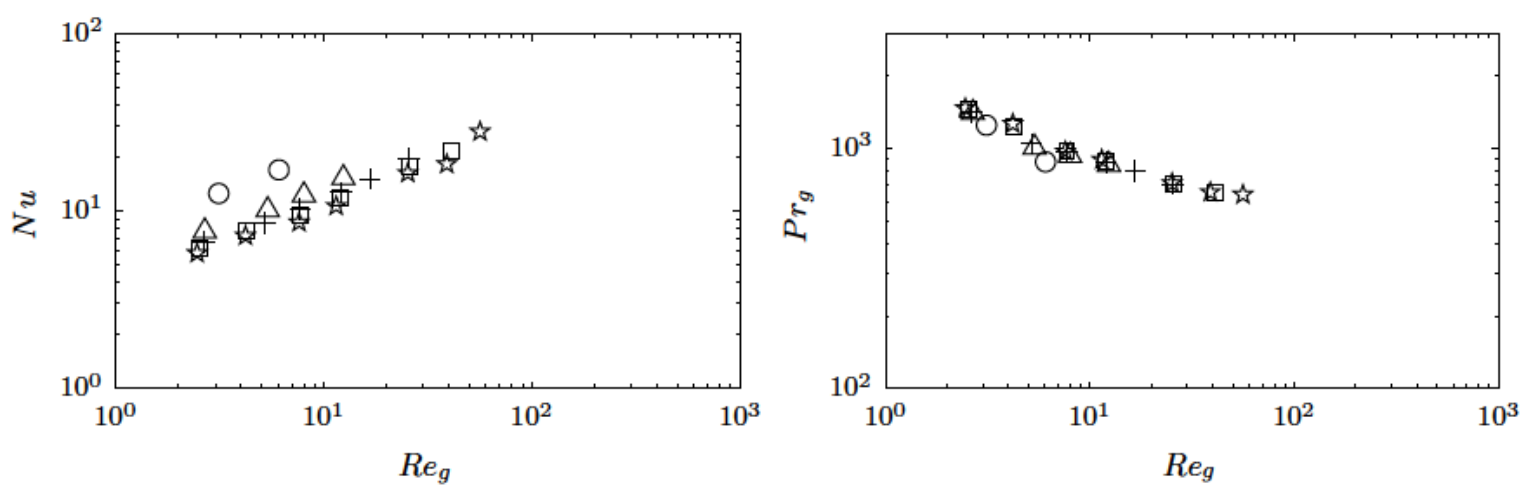

(b) $T C D-8(\mathrm{BB})$
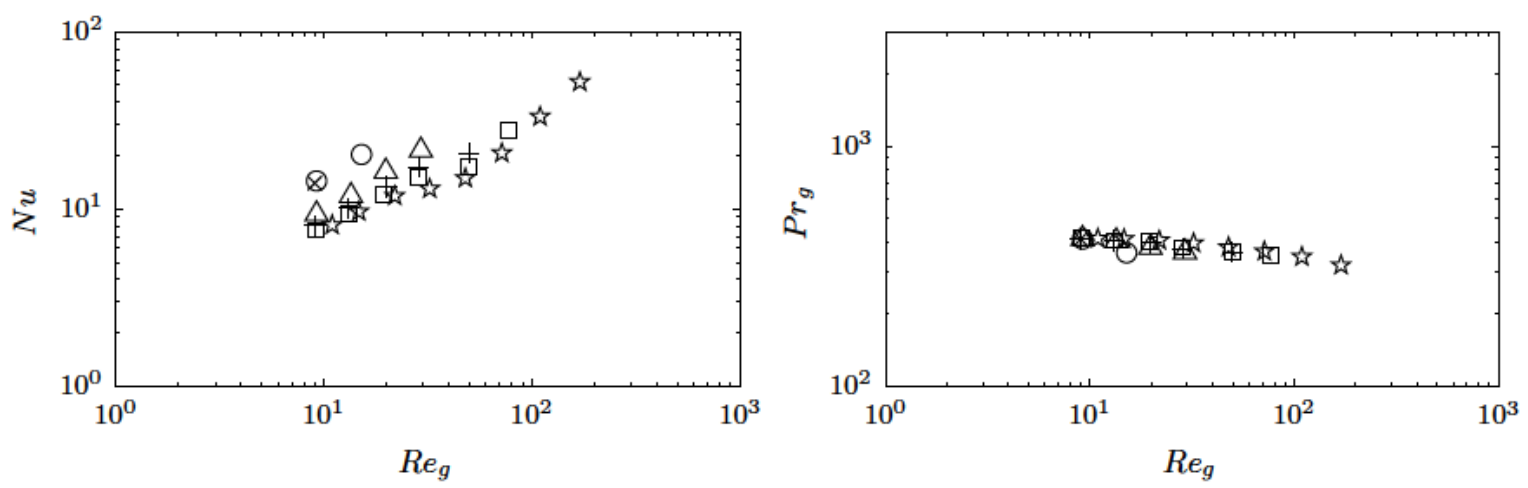

(c) $T C D-9(\mathrm{BA})$

\begin{tabular}{|c|c|c|c|c|c|c|c|}
\hline$x$ & $\omega=1,5$ & $\triangle$ & $\omega=0,5$ & $\square$ & $\omega=0,2$ & $\triangleleft$ & $\omega=0$ \\
\hline O & $\omega=1$ & + & $\omega=0,3$ & 七 & $\omega=0,1$ & & \\
\hline
\end{tabular}

Figura D.7: Medidas del número de Nusselt en las diferentes tandas de experimentos (I). 


\section{D.2. Evaluación de prestaciones. Resultados adiciona- les.}

En el presente apartado se incluyen resultados complementarios a los del Apartado 9.4. En las figuras D.8 y D.9 se muestra la evaluación de las prestaciones en régimen dinámico según el criterios R3 del rascador en régimen dinámico. Los resultados experimentales se comparan con la situación en la que el rascador se encuentra estático. 

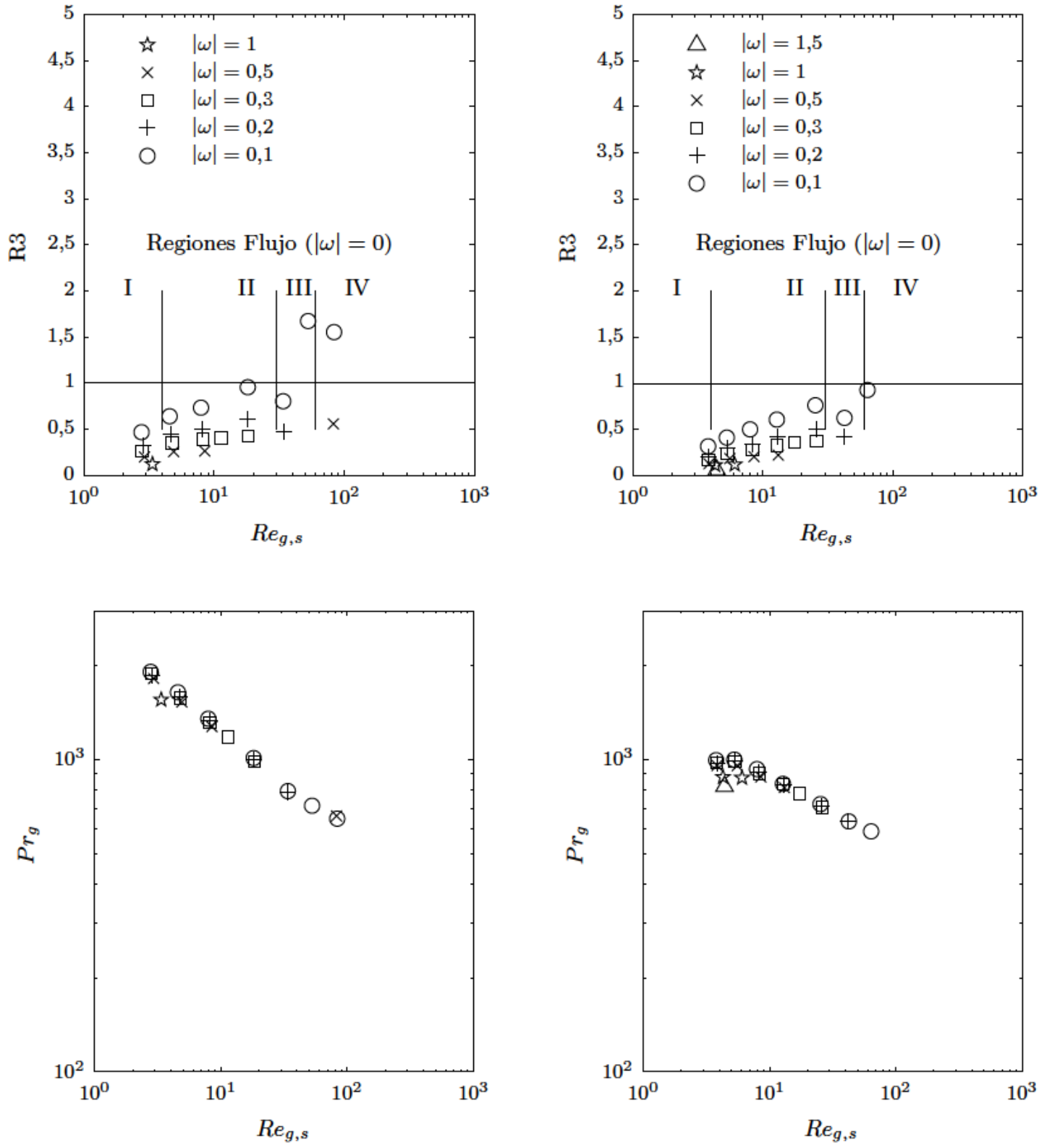
(a) TCD- $1, n \approx 0,5$ y $m \approx 2,4$
(b) TCD-6, $n \approx 0,7$ y $m \approx 0,61$

Figura D.8: Criterio R3 comparando los resultados en régimen dinámico $\left(v_{s} \neq 0\right)$ frente a a los resultados en régimen estático. 

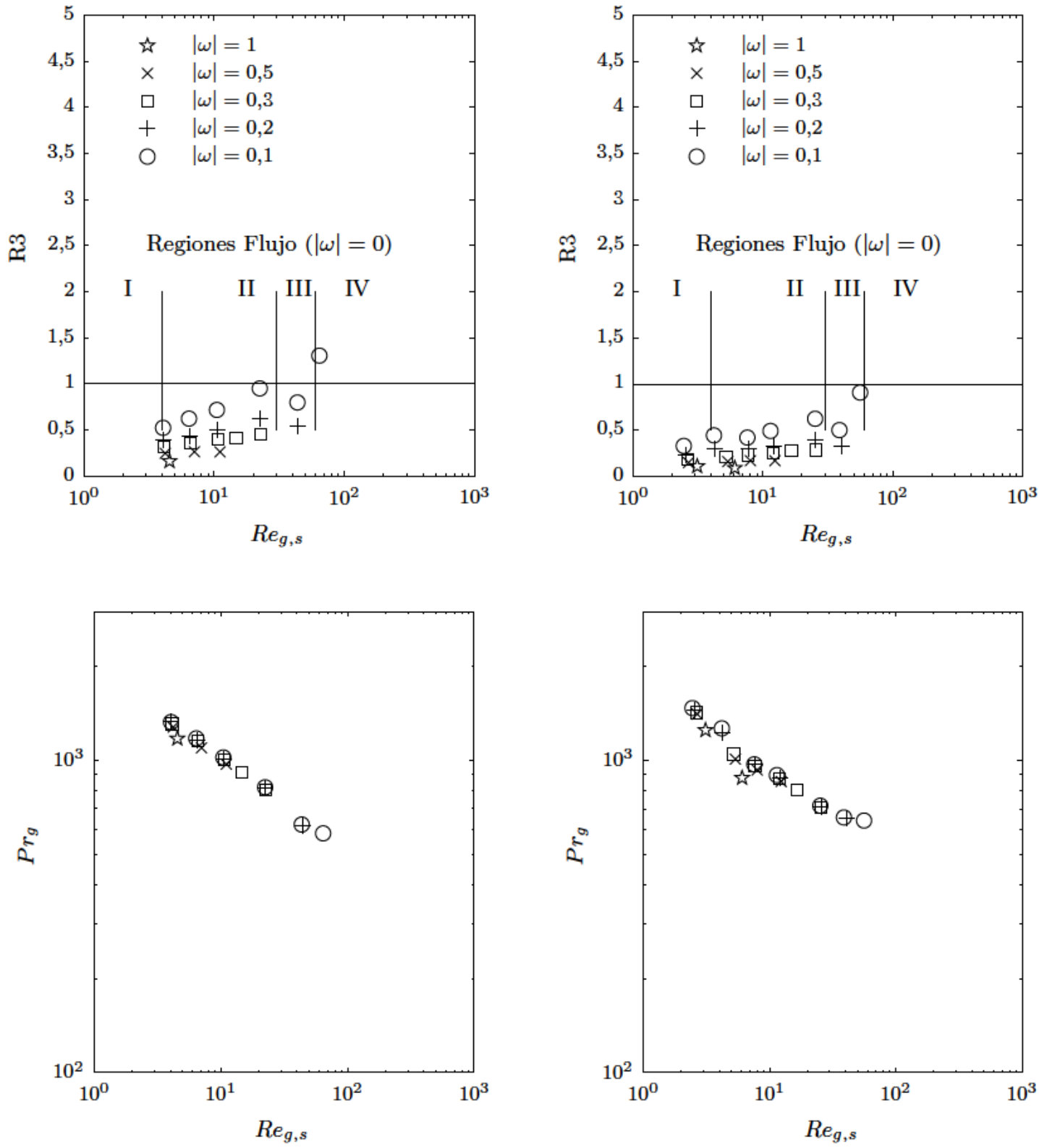
(a) TCD- $5, n \approx 0,75$ y $m \approx 0,35$
(b) TCD- $8, n \approx 0,63$ y $m \approx 1,1$

Figura D.9: Criterio R3 comparando los resultados en régimen dinámico $\left(v_{s} \neq 0\right)$ frente a a los resultados en régimen estático. 